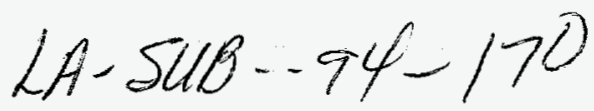

\title{
Evaluation and Compilation of Fission Product Yields 1993
}

T. R. England and B. F. Rider

Los Alamos National Laboratory

October, 1994

\begin{abstract}
This document is the latest in a series of compilations of fission yield data. Fission yield measurements reported in the open literature and calculated charge distributions have been used to produce a recommended set of yields for the fission products. The original data with reference sources, and the recommended yields are presented in tabular form. These include many nuclides which fission by neutrons at several energies. These energies include thermal energies ( $T$ ), fission spectrum energies (F), $14 \mathrm{meV}$ High Energy ( $\mathrm{H}$ or $\mathrm{HE}$ ), and spontaneous fission (S), in six sets of ten each. Set $A$ includes U235T, U235F, U235HE, U238F, U238HE, Pu239T, Pu239F, Pu241T, U233T, Th232F. Set B includes U233F, U233HE, U236F, Pu239H, Pu240F, Pu241F, Pu242F, Th232H, Np237F, Cf252S. Set $\mathrm{C}$ includes U234F, U237F, Pu240H, U234HE, U236HE, Pu238F, Am241F, Am243F, Np238F, Cm242F. Set D includes Th227T, Th229T, Pa231F, Am241T, Am241H, Am242MT, Cm245T, Cf249T, Cf251T, Es254T. Set E includes Cf250S, Cm244S, Cm248S, Es253S, Fm254S, Fm255T, Fm256S, Np237H, U232T, U238S. Set F includes Cm243T, Cm246S, Cm243F, Cm244F, Cm246F, Cm248F, Pu242H, Np237T, Pu240T, and $\mathrm{Pu} 242 \mathrm{~T}$ to complete fission product yield evaluations for 60 fissioning systems in all. This report also serves as the primary documentation for the second evaluation of yields in ENDF/B-VI released in 1993.

This report was originally intended to be printed full sized including all evaluated and compiled data. However, it contains more than 80,000 single spaced lines, most extending over 132 columns (about 1300 pages). The main text, tabular data in the text, evaluated chain yields, and an extensive, annotated bibliography are retained in this text. All evaluated and compiled data have been relegated to six appendices for the six groups of fissioning nuclides noted above. These are available to anyone having access to the internet. The anonymous node is t2.lanl.gov under the subnode yields. The files will also be available from BNL, IAEA and other sites. Please consult the "readme.yld" file in t2.lanl.gov yields node for additional information.
\end{abstract}

\section{General}

In recent years, there has been great interest in fission yields. They are used in the calculation of odd-even pairing effects, the number of prompt neutrons per fission (nubar), of delayed neutron 
precursor yields, and of delayed neutron spectra. Yields are used in the calculation of waste disposal inventories, and in the calculation of beta and gamma ray spectra of fission-product inventories. Fission yields are also used in the calculation of decay heat, especially in the time from 1 to 1000 seconds after a loss-of-coolant accident in a nuclear power plant. Such a calculation requires a value for the independent yield of every fission product nuclide with a half-life longer than a few tenths of a second. This work includes a recommended independent (RI) yield for every fission product nuclide to satisfy the requirements of decay heat calculations. The recommended independent yield values have been transmitted to the National Nuclear Data Center's Evaluated Nuclear Data File (ENDF/B-VI) at Brookhaven National Laboratory. The Cross Section Evaluation Working Group (CSEWG) Fission Product Yield and Decay Data Subcommittee has reviewed this work.

Several tests have been applied to the data including checks of atom, neutron, and proton balances. First, except for the very small ternary fission contribution the total of chain yields in the light and heavy mass peaks should each add to $100 \%$ or $200 \%$ when combined. Second, the average mass of the heavy fission product, plus the average of the light fission product, plus the average number of neutrons emitted in fission (nubar) should equal the mass of the fissioning nuclide plus the mass of the neutron inducing the fission. Third, the $\mathrm{Z}$ of each fission product, times the independent yield of that fission product, summed over all fission products including ternary fission products with $Z=1$ through 4 , and divided by 100 (to remove percent) should equal $\mathrm{Z}$ of the fissioning nuclide. These tests were satisfied for thermal fission, and within experimental error for other fission energies. Test results appear in the following table in order of the amount of data available (the order of appearance in this document):

Table 1.

Iton, Jentron and Proton Balances

\begin{tabular}{|c|c|c|c|c|c|c|c|c|c|}
\hline Inelide & $\begin{array}{l}\text { Sw of } \\
\text { Z*Yielda }\end{array}$ & $\begin{array}{l}\text { Itonic } \\
\text { Iunber }\end{array}$ & $\begin{array}{l}\text { Colpound } \\
\text { Juclens }\end{array}$ & $\begin{array}{l}\text { Minus } \mathbf{~ A V . ~} \\
\text { Light Hass }\end{array}$ & $\begin{array}{l}\text { Hinus IV. } \\
\text { Heav Iass }\end{array}$ & $\begin{array}{l}\text { Equals } \Delta p- \\
\text { parent Inbar }\end{array}$ & $\begin{array}{l}\text { Eralua } \\
\text { Iabar }\end{array}$ & ted & $\begin{array}{l}\text { Error on } \\
\text { Eval. Iuba }\end{array}$ \\
\hline U235t & 92.05 & 92.00 & 236 & -94.9072 & -138.6923 & 2.40 & 2.42 & - & 0.12 \\
\hline U235f & 92.00 & 92.00 & 236 & -95.0549 & -138.4740 & 2.47 & 2.47 & - & 0.12 \\
\hline U235h & 92.00 & 92.00 & 236 & -96.5733 & -135.3098 & 4.12 & 4.38 & $*$ & 0.22 \\
\hline U238x & 92.00 & 92.00 & 239 & -97.3361 & -138.7286 & 2.93 & 2.79 & - & 0.14 \\
\hline U238h & 92.00 & 92.00 & 239 & -97.8840 & -136.6967 & 4.42 & 4.42 & - & 0.22 \\
\hline Pu239t & 94.00 & 94.00 & 240 & -98.9910 & -138.1044 & 2.90 & 2.88 & $\bullet$ & 0.14 \\
\hline P.2391 & 94.00 & 94.00 & 240 & -98.9995 & -138.0987 & 2.90 & 2.94 & - & 0.15 \\
\hline Pu241t & 94.00 & 94.00 & 242 & -100.2773 & -138.7588 & 2.96 & 2.87 & $*$ & 0.14 \\
\hline U233t & 92.01 & 92.00 & 234 & -93.3624 & -138.1835 & 2.45 & 2.50 & - & 0.12 \\
\hline Th2321 & 90.00 & 90.00 & 233 & -91.0555 & -139.7144 & 2.23 & 2.42 & - & 0.12 \\
\hline U233f & 92.00 & 92.00 & 234 & -93.6925 & -137.7976 & 2.51 & 2.52 & - & 0.12 \\
\hline U233h & 91.99 & 92.00 & 234 & -95.9442 & -134.5816 & 3.47 & 4.27 & - & 0.21 \\
\hline U236f & 91.99 & 92.00 & 237 & -95.7290 & -138.5854 & 2.69 & 2.78 & - & 0.14 \\
\hline Pu239h & 93.99 & 94.00 & 240 & -100.1713 & -135.2287 & 4.60 & 4.90 & * & 0.25 \\
\hline Pu240f & 93.99 & 94.00 & 241 & -99.6395 & -138.3988 & 2.96 & 2.94 & $*$ & 0.15 \\
\hline Pu241f & 94.00 & 94.00 & 242 & -100.3500 & -138.6715 & 2.98 & 2.95 & - & 0.15 \\
\hline Po242f & 93.99 & 94.00 & 243 & -100.8390 & -138.8155 & 3.35 & 3.31 & - & 0.17 \\
\hline Th232h & 90.00 & 90.00 & 233 & -93.8282 & -136.2661 & 2.91 & 3.92 & - & 0.20 \\
\hline Ip237f & 93.00 & 93.00 & 238 & -97.1469 & -138.1594 & 2.69 & 3.05 & - & 0.15 \\
\hline Cf252: & 97.97 & 98.00 & 252 & -106.1231 & -141.9496 & 3.93 & 3.82 & ** & 0.12 \\
\hline U234I & 92.00 & 92.00 & 235 & -93.9101 & -138.3704 & 2.72 & 2.67 & $*$ & 0.13 \\
\hline U237f & 91.99 & 92.00 & 238 & $-96,5407$ & -138.7426 & 2.72 & $(2.74)$ & & --- \\
\hline Pz240h & 93.98 & 94.00 & 241 & -100.3305 & -136.0700 & 4.60 & $(4.62)$ & & \\
\hline
\end{tabular}




\section{DISCLAIMER}

This report was prepared as an account of work sponsored by an agency of the United States Government. Neither the United States Government nor any agency thereof, nor any of their employees, make any warranty, express or implied, or assumes any legal liability or responsibility for the accuracy, completeness, or usefulness of any information, apparatus, product, or process disclosed, or represents that its use would not infringe privately owned rights. Reference herein to any specific commercial product, process, or service by trade name, trademark, manufacturer, or otherwise does not necessarily constitute or imply its endorsement, recommendation, or favoring by the United States Government or any agency thereof. The views and opinions of authors expressed herein do not necessarily state or reflect those of the United States Government or any agency thereof. 


\section{DISCLAIMER}

Portions of this document may be illegible in electronic image products. Images are produced from the best available original document. 


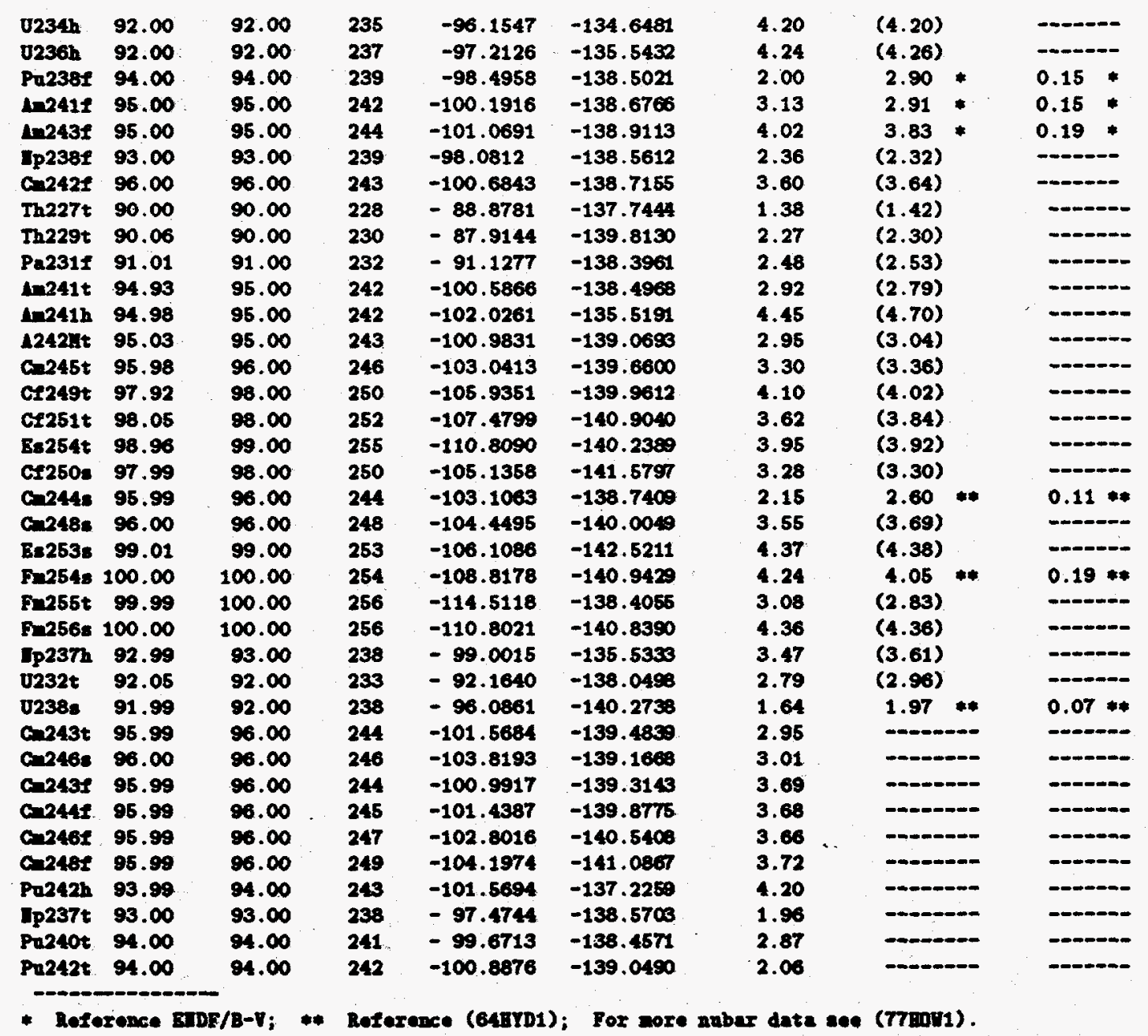

Yield are alse cheched to see if they wh to $100 \%$ per fisetion noder each peat. FiftJ-cae do sw to $100.00 \%$. U235t, u235t E 253s, Fups6s are off by $0.01 \%$, Pu240h, C1249t, and Ip237h by $0.04 \%$, U232t bJ $0.02 \%$ and ct251t bJ $0.05 \%$. For BIDF/B-YI, eron these andl deriaticin do not applJ because of the final noralization.

Independent yields are taken from a calculated charge distribution model. The model independent yields are normalized so their sum equals the chain yield. Large errors are given to the model yields. These model yields are merged statistically with weighted averages of measured yields. One set of cumulative yields is calculated by adding independent yields starting with the initial nuclide and ending with the chain yield. A second set of cumulative yields is calculated by starting with the chain yields and subtracting independent yields ending with the initial nuclide. These two sets are averaged using reciprocal variance weighting. The first set dominates the initial nuclide yield averages. The second set dominates the final chain member yield averages because of the small errors caused by the constraint imposed at $0 \%$ and $100 \%$ of the chain yield, respectively. The powerful and constrained merging technique and resulting error analysis used is that recommended by Professor B. I. Spinrad of Oregon State University. He was chairman of the Subcommittee for ENDF/B-V 
Fission Yield Errors (77SPI1). Other refinements treat delayed neutron emission, partition direct yields between metastable and ground state isomers, handle internal transition, and accomodate beta decay branching ratios.

\section{Light Ternary Fission Products}

A survey was made by Madland (77MAD1) of experimental information on the probability of ternary fission and the charge distribution of the light ternary fission products. A new prescription was presented for the ternary fission probability as a function of incident particle energy and certain compound nucleous properties. Based upon systematics, a method for obtaining charge distributions of the light ternary products was presented. Although Madland's study is yet incomplete, his current yield recommendations are listed together with the charge balances for all sixty fissionable nuclides. We have adjusted Wahl-recommended $\mathrm{Zp}$ values (88WAH1, 89WAH1) by a small adder to get a satisfactory charge balance. We have included the charge carried with the light ternary fission products because accurate calculation of decay heat requires a good charge balance.

Table 2.

Light Ternary Fiseicen Producte

\begin{tabular}{|c|c|c|c|c|c|c|c|c|c|c|}
\hline c1i & 2 & $\begin{array}{l}\text { Ternary } \\
\text { Yield, }\end{array}$ & $\begin{array}{l}\text { tandard } \\
\text { Der." }\end{array}$ & $\begin{array}{l}\text { B }(z=1) \\
\text { Tield, } y_{*}\end{array}$ & $\begin{array}{l}\text { He }(2=2) \\
\text { Yield, } X_{*}\end{array}$ & $\begin{array}{l}\text { Li }(2=3) \\
\text { Iield, } \% *\end{array}$ & $\begin{array}{l}\text { Be }(2=4) \\
\text { Yield, }, *\end{array}$ & $\begin{array}{l}\text { Ternery } \\
\text { Charge }\end{array}$ & $\begin{array}{l}\text { Binery } \\
\text { Cherge }\end{array}$ & $\begin{array}{l}\text { Totel } \\
\text { Charge }\end{array}$ \\
\hline U235t & $\infty$ & 2137 & 0196 & 1641 & 19755 & 0.00012 & 0.00002 & 0.0021 & 810 & 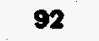 \\
\hline U235f & & 73 & 123 & 253 & 0.17395 & 0.00018 & 205 & 0.0036 & 91.9004 & 82 \\
\hline U235h & 6979 & 1456 & 0.0070 & 0.00974 & 0.13522 & 0.00014 & 0.00050 & .0028 & 1.9972 & 92 \\
\hline U238f & -0.01255 & 1667 & 0.0233 & 0.01115 & 0.15481 & 0.00016 & 0.00058 & 0.0032 & 91.9908 & 92 \\
\hline U238h & 1379 & 1258 & 0.0055 & 0.00841 & 0.11683 & 0.00012 & 0.00044 & 0.0024 & 91.0976 & 92 \\
\hline Pe239t & +0.01551 & 0.2326 & 0.0108 & 0.01556 & 0.21602 & 0.00022 & 0.00081 & 0.0045 & 93.9955 & 94 \\
\hline & & 92 & 0249 & 01399 & 0.18428 & 0.00020 & 0.00073 & 0.0041 & 93.9959 & 94 \\
\hline$P$ a & 860 & 2273 & 0145 & 0.01520 & 0.21100 & 0.00022 & 0.00078 & 0.0044 & 3.8956 & 94 \\
\hline D233t & -0.15331 & 4427 & 0.0094 & 0.01134 & 0.23010 & 0.00021 & 0.00104 & 0048 & .9952 & 92 \\
\hline Th232f & -0.09410 & 0.0840 & 0.0031 & 0.00562 & 0.07801 & 0.00008 & 0.00029 & .0016 & 384 & 90 \\
\hline 02331 & -0.00839 & 0.2257 & 0.0260 & 01509 & 0.20961 & 0.00022 & 0.00078 & 0.0044 & 91.9956 & 92 \\
\hline O233h & 6821 & 80 & 0.0 & 158 & 246 & 221 & 0.00076 & 042 & 91.9958 & 92 \\
\hline U2361 & 1958 & 00 & 0 & 1070 & 859 & 015 & 0.00055 & 0.0031 & .9969 & 92 \\
\hline Pn239h & -0.06819 & 0.2560 & 0.0640 & 712 & 3775 & 0.00025 & 0.00089 & 0.0050 & 3.9950 & 94 \\
\hline Pu240f & -0.00630 & 0.2400 & 0.0600 & 605 & 2289 & 0023 & 0.00083 & 0.0047 & .9953 & 4 \\
\hline Pe241: & 0078 & 0.2200 & 0.0 & 471 & 431 & 0021 & 076 & 0.0043 & 9957 & 4 \\
\hline & & 0 & 0. & 38 & 74 & 19 & 069 & 39 & 961 & 94 \\
\hline Th232h & -0 & 958 & 0.0 & 641 & 897 & 000 & 033 & 0.0019 & 9981 & 90 \\
\hline & & 200 & 0.0 & 471 & 431 & 021 & 076 & 0.0012 & 92.9958 & 93 \\
\hline CE252: & +0.05678 & 0.3469 & 0.0857 & 0.02830 & 0.31601 & 0.00051 & 0.00108 & 0.0067 & 97.9933 & 98 \\
\hline 02341 & +0.00820 & 0.2009 & 0.0502 & 0.01308 & 0.20479 & 0.00018 & 0.00070 & 0.0041 & 91.9959 & 92 \\
\hline U2371 & -0.01426 & 0.1405 & 0.0351 & 0.00915 & 0.13074 & 0.00013 & 0.00050 & 0.0027 & 91.9973 & 92 \\
\hline Pu240h & -0.01140 & 0.2369 & 0.0592 & 0.01542 & 0.22043 & 0.00021 & 0.00083 & 0.0046 & 93.9954 & 94 \\
\hline U234h & -0.00096 & 0.1997 & 0.0499 & 0.01300 & 0.18582 & 0.00018 & 0.00070 & 0.0039 & 91.9961 & 92 \\
\hline v236h & -0.00662 & 0.1625 & 0.0408 & 0.01058 & 0.15121 & 0.00015 & 0.00056 & 0.0032 & 91.9968 & 92 \\
\hline Pu2381 & -0.17529 & 0.2815 & 0.0704 & 0.01833 & 0.26194 & 0.00025 & 0.00098 & 0.0055 & 93.9945 & 94 \\
\hline $1-24$ & +0.04430 & 0.3016 & 0.0754 & 0.01963 & 0.28064 & 0.00027 & 0.00106 & 0.0059 & 94.9941 & 95 \\
\hline $\ln 24$ & +0.03270 & 0.2613 & 0.0653 & 0.01701 & 0.24314 & 0.00024 & 0.00091 & 0.0051 & 94.9949 & 95 \\
\hline D238 & +0.00516 & 0.2211 & 0.0553 & 0.01439 & 0.20573 & 0.00020 & $0.000 \mathrm{rt}$ & 0.0043 & 92.9957 & 93 \\
\hline $\cos 2421$ & -0.00877 & 0.3620 & 0.0905 & 0.02357 & 0.33684 & 0.00033 & 0.00127 & 0.0070 & 95.9930 & 96 \\
\hline Th22 & -0.00159 & 0.1808 & 0.0452 & 0.01209 & 0.16791 & 0.00017 & 0.00061 & 0.0035 & 89. & 90 \\
\hline Th229 & -0.01446 & 0.1405 & 0.0351 & 0.00940 & 0.13050 & 0.00013 & 0.00048 & 0.0027 & 89.9973 & 90 \\
\hline Pa2311 & -0.00939 & .1808 & 0.0452 & 0.01209 & 0.16791 & 0.00017 & 0.00061 & 0.0035 & 90.9965 & 91 \\
\hline
\end{tabular}




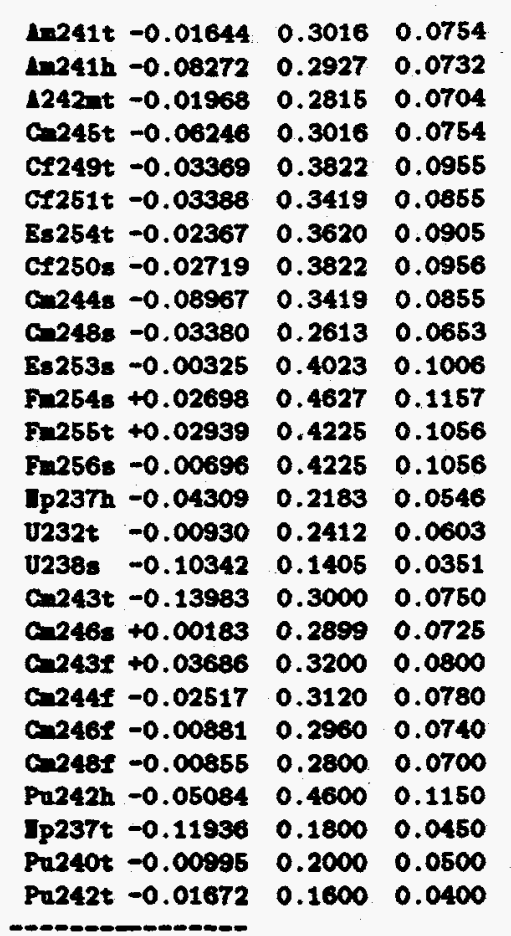

- Fron Reference (77.MD) $\begin{array}{llllll}0.02018 & 0.28014 & 0.00029 & 0.00103 & 0.0059\end{array}$

$\begin{array}{llllll}0.01958 & 0.27186 & 0.00029 & 0.00100 & 0.0057\end{array}$

$\begin{array}{lllllll}0.01883 & 0.26144 & 0.00027 & 0.00096 & 0.0054\end{array}$

$\begin{array}{llllll}0.02018 & 0.28014 & 0.00029 & 0.00103 & 0.0059\end{array}$

$\begin{array}{llllll}0.02557 & 0.35497 & 0.00037 & 0.00130 & 0.0074\end{array}$

$\begin{array}{llllll}0.02287 & 0.31756 & 0.00033 & 0.00116 & 0.0066\end{array}$

$\begin{array}{llllll}0.02422 & 0.33626 & 0.00036 & 0.00123 & 0.0070\end{array}$

$\begin{array}{lllllll}0.02166 & 0.35330 & 0.00021 & 0.00111 & 0.0073\end{array}$

$\begin{array}{lllllll}0.02465 & 0.31606 & 0.00019 & 0.00099 & 0.0066\end{array}$

$\begin{array}{llllll}0.01885 & 0.24159 & 0.00015 & 0.00076 & 0.0051\end{array}$

$\begin{array}{lllllll}0.02901 & 0.37191 & 0.00023 & 0.00117 & 0.0078\end{array}$

$\begin{array}{lllllll}0.03337 & 0.42777 & 0.00026 & 0.00134 & 0.0090\end{array}$

$\begin{array}{lllllll}0.03046 & 0.39053 & 0.00024 & 0.00123 & 0.0082\end{array}$

$\begin{array}{llllll}0.03046 & 0.39053 & 0.00024 & 0.00123 & 0.0082\end{array}$

$\begin{array}{llllll}0.01574 & 0.20180 & 0.00012 & 0.00063 & 0.0042\end{array}$

$\begin{array}{lllllll}0.01739 & 0.22297 & 0.00014 & 0.00070 & 0.0047\end{array}$

$\begin{array}{llllll}0.01013 & 0.12988 & 0.00008 & 0.00041 & 0.0027\end{array}$

$\begin{array}{llllll}0.02163 & 0.27733 & 0.00017 & 0.00087 & 0.0058\end{array}$

$\begin{array}{llllll}0.02090 & 0.26799 & 0.00016 & 0.00084 & 0.0058\end{array}$

$\begin{array}{llllll}0.02307 & 0.29582 & 0.00093 & 0.00093 & 0.0062\end{array}$

$\begin{array}{llllll}0.02249 & 0.28842 & 0.00017 & 0.00090 & 0.0062\end{array}$

$\begin{array}{lllllll}0.02134 & 0.27363 & 0.00017 & 0.00086 & 0.0057\end{array}$

$\begin{array}{llllll}0.02019 & 0.25884 & 0.00016 & 0.00081 & 0.0054\end{array}$

$\begin{array}{llllll}0.03317 & 0.42524 & 0.00026 & 0.00133 & 0.0089\end{array}$

$\begin{array}{llllll}0.01298 & 0.16639 & 0.00010 & 0.00052 & 0.0035\end{array}$

$\begin{array}{llllll}0.01442 & 0.18489 & 0.00012 & 0.00058 & 0.0039\end{array}$

$\begin{array}{llllll}0.01153 & 0.14790 & 0.00009 & 0.00046 & 0.0031\end{array}$
94.9941

94.9943

94.9946

95.9941

97.9926

97.9934

98.9930

97.9927

95.9934

95.9949

98.9922

99.9910

99.9918

99.9918

92.9968

91.9953

91.9973

95.9942

95.9944

95.9938

95.9938

95.9943

95.9946

93.9911

92.9965

93.9961

93.9969
95

95

95

98

98

98

99

98

96

96

99

100

100

100

93

92

92

96

88

96

98

96

86

94

93

94

94

\section{Treatment of Data}

The original values reported in the literature have been tabulated with the reference value (if any) against which they were determined. An updated value was then calculated by using the current recommended value for the fission yield of the reference nuclide from the file. All the updated values were adjusted by a small adder to ensure that the chain yields would total $100 \%$ except for the small difference between iterations. To find this adder, the variance of the sum is obtained by summing the variances of each chain yield. Any difference from $100 \%$ is apportioned to each chain yield in the proportion its variance bears to the total variance. That is, to a chain yield whose variance contributes $10 \%$ to the total variance, is added $10 \%$ of the total difference from $100 \%$. This method results in a negligible adjustment to any accurately determined absolute yield. It ensures that the adjustment is made mainly in the lesser known yields where most of the error lies. Yet these lesser known values are adjusted by only a fraction of their standard deviation or well within their experimental error.

The relative standard deviation reported in the literature is not allowed to be smaller than $0.5 \%$ for mass spectrometric measurements or $5 \%$ for $\mathrm{Ge}(\mathrm{Li})$-era radiochemical measurements made since 1965. A lower limit of $10 \%$ is set for sodium-iodide-era measurements between 1955 and 1965 , and $20 \%$ for Geiger-counter-era measurements before 1955 . Estimates are generally no better than $\pm 10 \%$ and are defaulted at $\pm 30 \%$. If the relative standard deviation is not reported in the literature, and is not supplied by the evaluator, it defaults to three times these lower limits. If separate plus and minus errors are reported, the smaller value plus two-thirds the difference is used as the relative standard deviation. For relative values, the resultant error is combined statistically with the error in the recommended yield of the reference nuclide from the previous iteration to give an error of the 
updated value. For absolute values, a $2 \%$ upper limit of conceivable systematic error is combined with the reported random error. Average experimental independent yields and experimental cumulative yields are determined for each nuclide. The individual values in the average are weighted by the inverse square of the relative standard deviation. If more than the above standardized treatment is required, a special treatment number is assigned so that these various cases can be individually treated. Detailed meanings of the special treatment numbers are given in a later section.

Various compilers/evaluators (74MEE1, 73WAL1, 73CRO1, 73NET2) have agreed within experimental error on all but a few yields, principally: Pu239T, Ru-103; Th232F, Cs-137; U235T, I-129, and Te-130. These discrepancies have been resolved by later measurements. Spinrad and Wu (78SPI1) have developed a general correlation for independent fission product yield uncertainties. Measurements of independent fission product yields from thermal-neutron fission of U235 (79 values), Pu239 (48 values), U233 (41 values), Pu241 (21 values) were compared to expected yields from the semiempirical model used in our evaluation. A general correlation between experimental to theoretical ratio and the distance of the nuclide from $\mathrm{Z}_{\mathrm{p}}$ (most probable charge in a fission product mass chain), was prepared. This correlation serves as a basis for assigning uncertainties to theoretical yield estimates. The FORTRAN code correlation is $\operatorname{ABS}(\mathrm{LN}(\mathrm{YT}(\mathrm{I}) / \mathrm{YE}(\mathrm{I})))=0.143+0.108 *(\mathrm{Z}(\mathrm{I})-\mathrm{Zp})$ **2, where YT and YE are theoretical and experimental fractional independent yields of nuclide(I). The $Z(I)$ and $\mathrm{Zp}$ are charge of nuclide(I) and the most probable charge for that mass chain, respectively. Further details on the independent yield model have been published (74WOL1, 76MAD1, 76MAD2).

For this edition, all published values previously not used (NU) have been reevaluated. Several previously unused values were found consistent with new measurements and are now used. All values that remain unused are given an explanation of why in the note column (by symbols described under note definitions). Many are merely superseded by later work of the same author or are upper limits. Others are outliers that are inconsistent with the model or other measurements.

When our independent yields were used, calculations of decay heat for U-235 thermal neutron fission have shown excellent agreement with experimental measurement from 20 to 100,000 seconds after reactor shutdown (77BJE1, 92ENG3).

\section{Half-Lives}

The half-lives were taken from the 14th edition of the General Electric Chart of the Nuclides (89WAL1) unless otherwise noted. Half-lives in ENDF/B-VI decay files may differ in some cases. Half-lives are not important to the yield evaluation but we have tried to provide accurate values.

\section{Decay Chains and Branching Fractions}

The three rows of numbers tabulated immediately below the half-lives are the percent of the precursor nuclides that decay to the labeled nuclide. The precursors covered by these three rows are, respectively, the ground state nuclide with an atomic number one unit smaller than the listed nuclide (Z-1G); the first isomeric nuclide with an atomic number one unit smaller than the listed nuclide (Z-1M); and the first isomer of the nuclide listed, i.e., same atomic number (Z-0M). The branching fractions permit beta-decay and isomeric transitions to be considered. If the value listed is negative, the nuclide decays by positron emission or electron capture to the Z-1 nuclide. The value listed is then the percentage of the atoms of the listed nuclide that decay to the respective $\mathrm{Z}-1$ isomers. Charged particle emission was not considered. 


\section{Calculated Yields}

A Gaussian charge distribution was calculated by using the most probable charge and Gaussian width parameter listed at the extreme left-hand side of the tabular section. These $\mathrm{Zp}$ values were obtained using preliminary ENDF/B-VI data as described in $89 \mathrm{WAH} 1$ and by subsequent private communications from A. C. Wahl to the authors. A small adder was applied to every $\mathrm{Zp}$ to adjust the proton balance to equal the atomic number. Sigma values vary by mass as listed by A. C. Wahl (89WAH1). Where isomerism occurs, the independent yield of each isomer depends on a non-linear function of the angular momentum of each isomer. Madland (76MAD2) finds such a model to predict the isomer split (metastable/ground state) within plus or minus 50\%. The Madland Model is used to divide isomeric yields between metastable and ground states except where the isomeric yields have been measured. Where angular momentum for each isomer is not known, it is divided equally between the ground state and a single metastable state. The decay chains listed at the top of the tables were then used to obtain fractional cumulative yields. These yields were modified by the neutron and proton odd-even effect (76MAD1) and renormalized to unity. Pairing effects applied to the Gaussian model are given in Table 3.

Table 3.

Ievtren and Proton Odd-Bren Effect

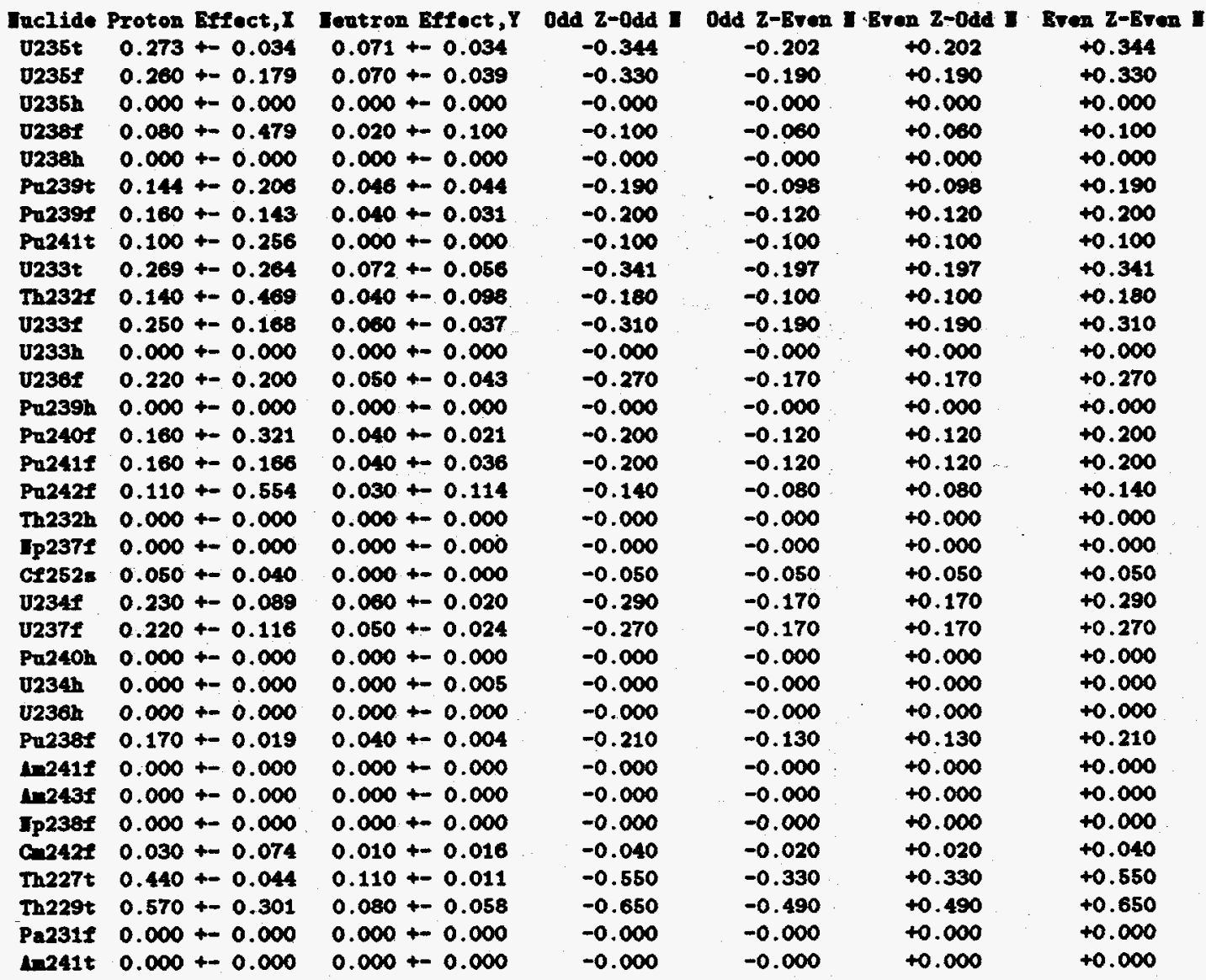




\begin{tabular}{|c|c|c|}
\hline & & \\
\hline 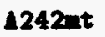 & 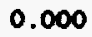 & $+\infty 0$ \\
\hline & 0 & 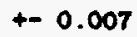 \\
\hline - & & $+\infty$ \\
\hline $251 t$ & & +0 \\
\hline $254 t$ & & +-0 \\
\hline $250=$ & & +-0 \\
\hline 2448 & & +0 \\
\hline 2488 & & $+\infty$ \\
\hline $253=$ & & +-0 \\
\hline 2548 & 0. & $+\infty$ \\
\hline $1255 t$ & & $+\infty$ \\
\hline 3 & & \\
\hline $237 \mathrm{~h}$ & & +-0 \\
\hline $232 t$ & & $+\infty$ \\
\hline 每 & & +- \\
\hline $243 t$ & & +0 \\
\hline 2485 & 0. & +0 . \\
\hline 2243 & & + \\
\hline 2448 & & $+\infty 0$ \\
\hline 2464 & 0. & +0.002 \\
\hline 2485 & 0. & + \\
\hline n.242h & 0. & +0.000 \\
\hline p37 & & $t-0.000$ \\
\hline 240 & 0.1 & +0.017 \\
\hline 242 & 0.14 & .01 \\
\hline
\end{tabular}

$0.000+-0.000$

$0.000+-0.000$

$0.020+-0.002$

$0.000+0.000$

$0.000+-0.000$

$0.000+0.000$

$0.000+-0.000$

$0.050+-0.005$

$0.000+-0.000$

$0.000+0.000$

$0.000+0.000$

$0.000+0.000$

$0.000+-0.000$

$0.000+0.000$

$0.050+-0.005$

$0.000+0.000$

$0.015+0.002$

$0.030+0.003$

$0.010+0.001$

$0.010+0.001$

$0.000+0.000$

$0.000+0.000$

$0.000+0.000$

$0.000+0.000$

$0.040+-0.004$

$0.035+0.004$

$\begin{array}{ll}-0.000 & -0.000 \\ -0.000 & -0.000 \\ -0.090 & -0.050 \\ -0.000 & -0.000 \\ -0.000 & -0.000 \\ -0.000 & -0.000 \\ -0.050 & -0.050 \\ -0.230 & -0.130 \\ -0.000 & -0.000 \\ -0.000 & -0.000 \\ -0.000 & -0.000 \\ -0.000 & -0.000 \\ -0.000 & -0.000 \\ -0.000 & -0.000 \\ -0.240 & -0.140 \\ -0.080 & -0.080 \\ -0.075 & -0.045 \\ -0.150 & -0.090 \\ -0.060 & -0.040 \\ -0.060 & -0.040 \\ -0.020 & -0.020 \\ -0.020 & -0.020 \\ -0.000 & -0.000 \\ -0.000 & -0.000 \\ -0.210 & -0.130 \\ -0.175 & -0.105\end{array}$

$+0.000$

$+0.000$

$+0.000$

$+0.050$

$+0.000$

$+0.000$

$+0.000$

$+0.050$

$+0.130$

$+0.000$

$+0.000$

$+0.000$

$+0.000$

$+0.000$

$+0.000$

$+0.140$

$+0.080$

$+0.045$

$+0.090$

$+0.040$

$+0.040$

$+0.020$

$+0.020$

$+0.000$

$+0.000$

$+0.130$

$+0.105$
$+0.000$

$+0.090$

$+0.000$

$+0.000$

$+0.000$

$+0.050$

$+0.230$

$+0.000$

$+0.000$

$+0.000$

$\$ 0.000$

$+0.000$

$+0.000$

$+0.240$

$+0.080$

$+0.075$

$+0.150$

$+0.060$

$+0.060$

$+0.020$

$+0.020$

$+0.000$

$+0.000$

$+0.210$

$+0.175$

The calculated independent yield division between metastable and ground states of isomers for known spin isomers were as follows:

Table 4.

Indopendont Y1eld Division botween retesteble and Oround states of Isomers Having Inom Spin Stater.

\begin{tabular}{|c|c|c|c|c|c|c|c|c|c|c|}
\hline Isotope & $\begin{array}{l}\text { Heta- } \\
\text { State } \\
\text { Spin }\end{array}$ & $\begin{array}{l}\text { Ground } \\
\text { state } \\
\text { Spin }\end{array}$ & $\mathrm{J} \mathrm{x}-\mathrm{J0}$ & $\begin{array}{l}\text { Integer } \\
\text { Odd/Bren }\end{array}$ & $\begin{array}{l}\text { JDJG J }=+ \\
\text { JKKJG=- }\end{array}$ & $\begin{array}{l}\text { Odd or } \\
\text { Eren } 1\end{array}$ & $\begin{array}{l}\text { Formala } \\
\text { Vumber* }\end{array}$ & $\begin{array}{l}\text { Frac. of Yield } \\
\text { Thermal }\end{array}$ & $\begin{array}{l}\text { Motastablo } \\
\text { Fast }\end{array}$ & $\begin{array}{l}(\mathrm{H} / \mathrm{H}+\mathrm{G}) \\
\text { High E. }\end{array}$ \\
\hline $68 \mathrm{Cu}$ & 6 & 1 & 5 & 0 & + & E & 7 & 0.70 & 0.73 & 0.82 \\
\hline 69zn & $9 / 2$ & $1 / 2$ & 4 & E & + & o & 1 & 0.81 & 0.83 & 0.89 \\
\hline 7000 & 5 & 1 & 4 & $\mathbf{E}$ & + & $\mathbf{E}$ & 5 & 0.75 & 0.78 & 0.85 \\
\hline $712 \mathrm{n}$ & $9 / 2$ & $1 / 2$ & 4 & E & + & 0 & 1 & 0.81 & 0.83 & 0.89 \\
\hline 735 & $1 / 2$ & $9 / 2$ & 4 & $\mathbf{E}$ & - & 0 & 2 & 0.19 & 0.17 & 0.11 \\
\hline 7360 & $1 / 2$ & $9 / 2$ & 4 & B & - & 0 & 2 & 0.19 & 0.17 & 0.11 \\
\hline 741s & 5 & 2 & 3 & 0 & + & E & 7 & 0.70 & 0.73 & 0.82 \\
\hline 756 & $7 / 2$ & $1 / 2$ & 3 & 0 & + & 0 & 3 & 0.87 & 0.88 & 0.92 \\
\hline $77 B x$ & $9 / 2$ & $3 / 2$ & 3 & 0 & + & 0 & 3 & 0.77 & 0.79 & 0.86 \\
\hline T7Ge & $1 / 2$ & $7 / 2$ & 3 & 0 & - & 0 & 4 & 0.13 & 0.12 & 0.07 \\
\hline 775 & $7 / 2$ & $1 / 2$ & 3 & 0 & + & 0 & 3 & 0.87 & 0.88 & 0.92 \\
\hline 79Se & $1 / 2$ & $7 / 2$ & 3 & 0 & - & o & 4 & 0.13 & 0.12 & 0.07 \\
\hline $791 \mathrm{I}$ & $7 / 2$ & $1 / 2$ & 3 & 0 & + & 0 & 3 & 0.87 & 0.88 & 0.92 \\
\hline $79 B x$ & $9 / 2$ & $3 / 2$ & 3 & 0 & + & 0 & 3 & 0.77 & 0.79 & 0.86 \\
\hline $80 \mathrm{Br}$ & 5 & 1 & 4 & E & + & E & 5 & 0.75 & 0.78 & 0.85 \\
\hline $81 \mathrm{Rb}$ & $9 / 2$ & $3 / 2$ & 3 & 0 & + & 0 & 3 & 0.77 & 0.79 & 0.86 \\
\hline 81se & $7 / 2$ & $1 / 2$ & 3 & 0 & + & 0 & 3 & 0.87 & 0.88 & 0.92 \\
\hline 81Kr & $1 / 2$ & $7 / 2$ & 3 & 0 & - & 0 & 4 & 0.13 & 0.12 & 0.07 \\
\hline $82 \mathrm{Ab}$ & 5 & 1 & 4 & E & + & $\mathbf{E}$ & 5 & 0.30 & 0.27 & 0.18 \\
\hline 82Br & 2 & 5 & 3 & 0 & - & E & 8 & 0.30 & 0.27 & 0.18 \\
\hline 835 & $1 / 2$ & $9 / 2$ & 4 & $\mathbf{E}$ & - & o & 2 & 0.19 & 0.17 & 0.11 \\
\hline 83II & $1 / 2$ & $9 / 2$ & 4 & E & - & 0 & 2 & 0.19 & 0.17 & 0.11 \\
\hline
\end{tabular}




\begin{tabular}{|c|c|c|c|c|c|c|c|c|c|c|}
\hline 84BI & 6 & 2 & 4 & $\mathbf{E}$ & + & E & 5 & 0.64 & 0.68 & 0.78 \\
\hline $84 Y$ & 1 & 4 & 3 & 0 & - & E & 8 & 0.19 & 0.17 & 0.11 \\
\hline 84Rb & 6 & 2 & 4 & E & + & $\boldsymbol{E}$ & 5 & 0.64 & 0.68 & 0.78 \\
\hline 85R r & $1 / 2$ & $9 / 2$ & 4 & $\mathbf{E}$ & - & 0 & 2 & 0.19 & 0.17 & 0.11 \\
\hline $855 x$ & $1 / 2$ & $9 / 2$ & 4 & $\mathbf{E}$ & - & 0 & 2 & 0.19 & 0.17 & 0.11 \\
\hline $85 Y$ & $1 / 2$ & $9 / 2$ & 4 & $\mathbf{E}$ & - & 0 & 2 & 0.19 & 0.17 & 0.11 \\
\hline $852 \mathrm{x}$ & $1 / 2$ & $7 / 2$ & 3 & 0 & - & 0 & 4 & 0.13 & 0.12 & 0.07 \\
\hline $86 \mathrm{Ab}$ & 6 & 2 & 4 & E & + & $\mathbf{E}$ & 5 & 0.64 & 0.68 & 0.78 \\
\hline $86 Y$ & 8 & 4 & 4 & $\mathbf{E}$ & + & $\mathbf{E}$ & 5 & 0.42 & 0.47 & 0.61 \\
\hline $879 x$ & $1 / 2$ & $9 / 2$ & 4 & $\mathbf{E}$ & - & 0 & 2 & 0.19 & 0.17 & 0.11 \\
\hline $87 Y$ & $9 / 2$ & $1 / 2$ & 4 & $\mathbf{E}$ & + & 0 & 1 & 0.81 & 0.83 & 0.89 \\
\hline $89 Y$ & $9 / 2$ & $1 / 2$ & 4 & E & + & 0 & 1 & 0.81 & 0.83 & 0.89 \\
\hline $89 Z I$ & $1 / 2$ & $9 / 2$ & 4 & $\mathbf{E}$ & - & 0 & 2 & 0.19 & 0.17 & 0.11 \\
\hline 8917 & $1 / 2$ & $9 / 2$ & 4 & E & - & 0 & 2 & 0.19 & $0: 17$ & 0.11 \\
\hline 9ORb & 4 & 1 & 3 & 0 & + & $\mathbf{B}$ & 7 & 0.81 & 0.83 & 0.89 \\
\hline $90 y$ & 7 & 2 & 5 & 0 & + & $\mathbf{E}$ & 7 & 0.59 & 0.63 & 0.74 \\
\hline $902 x$ & 5 & 0 & 5 & 0 & + & B & 7 & 0.81 & 0.83 & 0.89 \\
\hline $901 \mathrm{~b}$ & 4 & 8 & 4 & E & - & $\mathbf{E}$ & 6 & 0.58 & 0.53 & 0.39 \\
\hline $91 Y$. & $9 / 2$ & $1 / 2$ & 4 & $\mathbf{E}$ & + & 0 & 1 & 0.81 & 0.83 & 0.89 \\
\hline 91Ib & $1 / 2$ & $9 / 2$ & 4 & $\mathbf{E}$ & - & 0 & 2 & 0.19 & 0.17 & 0.11 \\
\hline 9110 & $1 / 2$ & $9 / 2$ & 4 & $\mathbf{E}$ & - & 0 & 2 & 0.19 & 0.17 & 0.11 \\
\hline 921B & 2 & $T$ & 5 & 0 & - & E & 8 & 0.41 & 0.37 & 0.26 \\
\hline 931b & $1 / 2$ & $9 / 2$ & 4 & $\mathbf{E}$ & - & 0 & 2 & 0.19 & 0.17 & 0.11 \\
\hline 93:0 & $21 / 2$ & $5 / 2$ & 8 & E & + & 0 & 1 & 0.38 & 0.43 & 0.58 \\
\hline $93 T c$ & $1 / 2$ & $9 / 2$ & 4 & $\mathbf{E}$ & - & 0 & 2 & 0.19 & 0.17 & 0.11 \\
\hline 947 & 3 & 6 & 3 & 0 & - & $\mathbf{E}$ & 8 & 0.41 & 0.37 & 0.26 \\
\hline 9ATC & 2 & 7 & 5 & 0 & - & E & 8 & 0.41 & 0.37 & 0.26 \\
\hline 951b. & $1 / 2$ & $9 / 2$ & 4 & $\mathbf{E}$ & - & 0 & 2 & 0,19 & 0.17 & 0.11 \\
\hline 95TC & $1 / 2$ & $9 / 2$ & 4 & $\mathbf{E}$ & - & 0 & 2 & 0.19 & 0.17 & 0.11 \\
\hline $96 Y$ & 3 & 0 & 3 & $\mathbf{0}$ & + & $\mathbf{E}$ & $T$ & 0.80 & 0.91 & 0.94 \\
\hline 967c. & 4 & 7 & 3 & 0 & - & E & 8. & 0.53 & 0.48 & 0.34 \\
\hline 9716 & $1 / 2$ & $9 / 2$ & 4 & E & - & 0 & 2 & 0.19 & 0.17 & 0.11 \\
\hline 97Te & $1 / 2$ & $9 / 2$ & 4 & $\mathbf{E}$ & - & 0 & 2 & 0.19 & 0.17 & 0.11 \\
\hline 9816 & 1 & 5 & 4 & $\mathbf{B}$ & - & $\mathbf{E}$ & 6 & 0.25 & 0.22 & 0.15 \\
\hline 99Tc & $1 / 2$ & $9 / 2$ & 4 & $\mathbf{E}$ & - & 0 & 2 & 0.19 & 0.17 & 0.11 \\
\hline $99 \mathrm{hh}$ & $9 / 2$ & $1 / 2$ & 4 & E & + & 0 & 1 & 0.81 & 0.83 & 0.89 \\
\hline $991 \mathrm{~b}$ & $1 / 2$ & $9 / 2$ & 4 & $\mathbf{B}$ & - & $\mathbf{0}$ & 2 & 0.19 & 0.17 & 0.11 \\
\hline 101Rh & $9 / 2$ & $1 / 2$ & 4 & $\mathbf{E}$ & + & 0 & 1 & 0.81 & 0.83 & 0.89 \\
\hline 1032h & $7 / 2$ & $1 / 2$ & 3 & 0 & + & 0 & 3 & 0.87 & 0.88 & 0.92 \\
\hline $1034 \mathrm{~g}$ & $1 / 2$ & $T / 2$ & 3 & 0 & - & 0 & 4 & 0.13 & 0.12 & 0.07 \\
\hline $104 \mathrm{~h}$ & 5 & 1 & 4 & E & + & E & 5 & 0.75 & 0.78 & 0.85 \\
\hline $1044_{8}$ & 2 & 5 & 3 & 0 & - & B & 8 & 0.30 & 0.27 & 0.18 \\
\hline $105 \mathrm{hh}$ & $1 / 2$ & $T / 2$ & 3 & 0 & - & 0 & 4 & 0.13 & 0.12 & 0.07 \\
\hline $105 A_{6}$ & $7 / 2$ & $1 / 2$ & 3 & 0 & + & 0 & 3 & 0.87 & 0.88 & 0.92 \\
\hline $1084 \mathrm{~g}$ & 6 & 1 & 5 & 0 & + & $\mathbf{B}$ & 7 & 0.70 & 0.73 & 0.82 \\
\hline $107 / \mathrm{g}$ & $7 / 2$ & $1 / 2$ & 3 & 0 & + & 0 & 3 & 0.87 & 0.88 & 0.92 \\
\hline $107 \mathrm{Pd}$ & $11 / 2$ & $5 / 2$ & 3 & 0 & + & $\mathbf{0}$ & 3 & 0.65 & 0.69 & 0.79 \\
\hline $108 A_{8}$ & 6 & 1 & 5 & 0 & + & $\mathbf{E}$ & 7 & 0.70 & 0.73 & 0.82 \\
\hline $100 \mathrm{Pd}$ & $11 / 2$ & $5 / 2$ & 3 & 0 & + & 0 & 3. & 0.65 & 0.69 & 0.79 \\
\hline $1094 \mathrm{~g}$ & $7 / 2$ & $1 / 2$ & 3 & $\mathbf{0}$ & + & 0 & 3 & 0.87 & 0.88 & 0.92 \\
\hline $109 \mathrm{In}$ & $1 / 2$ & $9 / 2$ & 4 & $\mathbf{E}$ & - & 0 & 2 & 0.19 & 0.17 & 0.11 \\
\hline $110{ }_{18}$ & 6 & 1 & 5 & 0 & + & E & 7 & 0.70 & 0.73 & 0.82 \\
\hline $111 P d$ & $11 / 2$ & $5 / 2$ & 3 & 0 & + & 0 & 3 & 0.65 & 0.69 & 0.79 \\
\hline $1114 \mathrm{~g}$ & $7 / 2$ & $1 / 2$ & 3 & 0 & + & 0 & 3 & 0.87 & 0.88 & 0.92 \\
\hline $111 \mathrm{~cd}$ & $11 / 2$ & $1 / 2$ & 5 & 0 & + & 0 & 3 & 0.77 & 0.79 & 0.86 \\
\hline 111In & $1 / 2$ & $9 / 2$ & 4 & E & - & 0 & 2 & 0.19 & 0.17 & 0.11 \\
\hline $112 I n$ & 4 & 1 & 3 & 0 & + & E & 7 & 0.81 & 0.83 & 0.89 \\
\hline $1134_{\mathrm{g}}$ & $7 / 2$ & $1 / 2$ & 3 & 0 & + & 0 & 3 & 0.87 & 0.88 & 0.92 \\
\hline $1131 n$ & $1 / 2$ & $9 / 2$ & 4 & E & - & 0 & 2 & 0.19 & 0.17 & 0.11 \\
\hline $113 \mathrm{Cd}$ & $11 / 2$ & $1 / 2$ & 5 & 0 & + & o & 3 & 0.77 & 0.79 & 0.86 \\
\hline $1135 n$ & $7 / 2$ & $1 / 2$ & 3 & 0 & + & 0 & 3 & 0.87 & 0.88 & 0.92 \\
\hline $114 I n$ & 5 & 1 & 4 & $\mathbf{E}$ & + & E & 5 & 0.75 & 0.78 & 0.85 \\
\hline $115 \mathrm{Cd}$ & $11 / 2$ & $1 / 2$ & 5 & 0 & + & 0 & 3 & 0.77 & 0.79 & 0.86 \\
\hline $115 I n$ & $1 / 2$ & $9 / 2$ & 4 & E & - & 0 & 2 & 0.19 & 0.17 & 0.11 \\
\hline $117 \mathrm{Cd}$ & $11 / 2$ & $1 / 2$ & 5 & 0 & + & 0 & 3 & 0.77 & 0.79 & 0.86 \\
\hline 117 In & $1 / 2$ & $9 / 2$ & 4 & $\mathbf{E}$ & - & 0 & 2 & 0.19 & 0.17 & 0.11 \\
\hline
\end{tabular}




\begin{tabular}{|c|c|c|c|c|c|c|c|c|c|c|}
\hline $117 \mathrm{Sn}$ & $11 / 2$ & $1 / 2$ & 5 & 0 & + & 0 & 3 & $0 . \pi 7$ & 0.79 & 0.86 \\
\hline $118 I n$ & 1 & 5 & 4 & B & - & $\boldsymbol{E}$ & 6 & 0.25 & 0.22 & 0.15 \\
\hline $118 s b$ & 8 & 1 & 7 & 0 & + & $\mathbf{E}$ & 7 & 0.59 & 0.63 & 0.74 \\
\hline $119 \mathrm{In}$ & $1 / 2$ & $9 / 2$ & 4 & E & - & 0 & 2 & 0.19 & 0.17 & 0.11 \\
\hline $1198 n$ & $11 / 2$ & $1 / 2$ & 5 & 0 & + & 0 & 3 & 0.77 & 0.79 & 0.86 \\
\hline 119Te & $11 / 2$ & $1 / 2$ & 5 & 0 & + & 0 & 3 & 0.77 & 0.79 & 0.86 \\
\hline $120 \mathrm{sb}$ & 8 & 1 & 7 & 0 & + & B & 7 & 0.59 & 0.63 & 0.74 \\
\hline $121 \mathrm{In}$ & $1 / 2$ & $9 / 2$ & 4 & B & - & 0 & 2 & 0.19 & 0.17 & 0.11 \\
\hline $121 \mathrm{sn}$ & $11 / 2$ & $3 / 2$ & 4 & $\mathbf{E}$ & - & 0 & 1 & 0.71 & 0.74 & 0.82 \\
\hline 121Te & $11 / 2$ & $1 / 2$ & 5 & o & + & 0 & 3 & 0.77 & 0.79 & 0.86 \\
\hline $122 \mathrm{sb}$ & 8 & 2. & 6 & E & + & $\mathbf{E}$ & 5 & 0.53 & 0.57 & 0.70 \\
\hline 123In & $1 / 2$ & $9 / 2$ & 4 & E & - & D & 2 & 0.19 & 0.17 & 0.11 \\
\hline 123Te & $11 / 2$ & $1 / 2$ & 5 & 0 & + & D & 3 & 0.77 & 0.79 & 0.86 \\
\hline $1239 n$ & $3 / 2$ & $11 / 2$ & 4 & $\mathbf{E}$ & - & 0 & 2 & 0.29 & 0.26 & 0.18 \\
\hline $1255 n$ & $3 / 2$ & $11 / 2$ & 4 & E & - & D & 2 & 0.29 & 0.26 & 0.18 \\
\hline 125T: & $11 / 2$ & $1 / 2$ & 5 & 0 & + & 0 & 3 & 0.77 & 0.79 & 0.86 \\
\hline $125 x_{0}$ & $9 / 2$ & $1 / 2$ & 4 & $\mathbf{B}$ & + & 0 & 1 & 0.81 & 0.83 & 0.89 \\
\hline $1268 b$ & 5 & 8 & 3 & 0 & - & E & 8 & 0.63 & 0.58 & 0.43 \\
\hline $127 \mathrm{sn}$ & $3 / 2$ & $11 / 2$ & 4 & E & - & 0 & 2 & 0.29 & 0.26 & 0.18 \\
\hline 127Te & $11 / 2$ & $3 / 2$ & 4 & E & + & 0 & 1 & 0.71 & 0.74 & 0.82 \\
\hline $127 \times$ & $9 / 2$ & $1 / 2$ & 4 & E & + & 0 & 1 & 0.81 & 0.83 & 0.89 \\
\hline $1285 b$ & 5 & 8 & 3 & 0 & - & E & 8 & 0.63 & 0.58 & 0.43 \\
\hline $1298 n$ & $11 / 2$ & $3 / 2$ & 4 & B & + & 0 & 1 & 0.71 & 0.74 & 0.82 \\
\hline 129T. & $11 / 2$ & $3 / 2$ & 4 & $\mathbf{E}$ & + & 0 & 1 & 0.71 & 0.74 & 0.82 \\
\hline $129 x_{0}$ & $11 / 2$ & $1 / 2$ & 5 & 0 & + & 0 & 3 & 0.77 & 0.79 & 0.86 \\
\hline $1298 \mathrm{~B}$ & $11 / 2$ & $1 / 2$ & 6 & 0 & + & 0 & 3 & 0.77 & 0.79 & 0.86 \\
\hline $1308 n$ & 7 & 0 & 7 & 0 & + & B & 7 & 0.70 & 0.73 & 0.82 \\
\hline $130 s b$ & 5 & 8 & 3 & 0 & - & $\mathbf{E}$ & 8 & 0.63 & 0.58 & 0.43 \\
\hline $130 \mathrm{I}$ & 2 & 5 & 3 & 0 & - & E & 8 & 0.30 & 0.27 & 0.18 \\
\hline 131T. & $11 / 2$ & $3 / 2$ & 4 & E & + & 0 & 1 & 0.71 & 0.74 & 0.82 \\
\hline 13120 & $11 / 2$ & $3 / 2$ & 4 & B & + & 0 & 1 & 0.71 & 0.74 & 0.82 \\
\hline $1328 b$ & 8 & 4 & 4 & $E$ & + & $\mathbf{E}$ & 5 & 0.42 & 0.47 & 0.61 \\
\hline 133Te & $11 / 2$ & $3 / 2$ & 4 & $\mathbf{E}$ & + & 0 & 1 & 0.71 & 0.74 & 0.82 \\
\hline $133 I$ & $19 / 2$ & $7 / 2$ & 6 & $\mathbf{E}$ & + & 0 & 1 & 0.38 & 0.43 & 0.58 \\
\hline $133 x_{0}$ & $11 / 2$ & $3 / 2$ & 4 & E & + & 0 & 1 & 0.71 & 0.74 & 0.82 \\
\hline $133 \mathrm{Ba}$ & $11 / 2$ & $1 / 2$ & 5 & 0 & + & 0 & 3 & 0.77 & 0.79 & 0.86 \\
\hline $134 I$ & 8 & 4 & 4 & E & + & $\mathbf{E}$ & 5 & 0.42 & 0.47 & 0.61 \\
\hline $134 x=$ & 7 & 0 & 7 & 0 & + & $\mathbf{E}$ & 7 & 0.70 & 0.73 & 0.82 \\
\hline 1340 & 8 & 4 & 4 & E & + & $\mathbf{B}$ & 5 & 0.42 & 0.47 & 0.61 \\
\hline 1358a & $11 / 2$ & $3 / 2$ & 4 & $\mathbf{E}$ & + & 0 & 1 & 0.71 & 0.74 & 0.82 \\
\hline $135 x$ & $11 / 2$ & $3 / 2$ & 4 & $\mathbf{E}$ & + & 0 & 1 & 0.71 & 0.74 & 0.82 \\
\hline $135 \mathrm{C}$ & $19 / 2$ & $7 / 2$ & 6 & 0 & + & 0 & 1 & 0.38 & 0.43 & 0.58 \\
\hline $1368 \mathrm{a}$ & 7 & 0 & $T$ & 0 & + & $\mathbf{E}$ & 7 & 0.70 & 0.73 & 0.82 \\
\hline $137 \mathrm{Ba}$ & $11 / 2$ & $3 / 2$ & 4 & E & + & 0 & 1 & 0.71 & 0.74 & 0.82 \\
\hline $137 \mathrm{C}$ & $11 / 2$ & $3 / 2$ & 4 & B & + & 0 & 1 & 0.71 & 0.74 & 0.82 \\
\hline 1390 & $11 / 2$ & $3 / 2$ & 4 & $\mathbf{E}$ & + & 0 & 1 & 0.71 & 0.74 & 0.82 \\
\hline 141IId & $11 / 2$ & $3 / 2$ & 4 & $\mathbf{E}$ & + & 0 & 1 & 0.71 & 0.74 & 0.82 \\
\hline 141s & $11 / 2$ & $1 / 2$ & 5 & 0 & + & 0 & 3 & 0.77 & 0.79 & 0.86 \\
\hline 142Pr & 5 & 2 & 3 & 0 & + & $\mathbf{E}$ & 7 & 0.70 & 0.73 & 0.82 \\
\hline $143 s \mathrm{~m}$ & $11 / 2$ & $3 / 2$ & 4 & E & + & 0 & 1 & 0.71 & 0.74 & 0.82 \\
\hline 144PI & 3 & 0 & 3 & 0 & + & E & 7 & 0.90 & 0.91 & 0.94 \\
\hline $148 \mathrm{Pm}$ & 6 & 1 & 5 & D & + & B & 7 & 0.70 & 0.73 & 0.82 \\
\hline $1545 \mathrm{n}$ & 8 & 3 & 5 & 0 & + & E & 7 & 0.47 & 0.52 & 0.66 \\
\hline $156 \mathrm{~Tb}$ & 0 & 3 & 3 & 0 & - & $\overline{\mathbf{E}}$ & 8 & 0.10 & 0.09 & 0.06 \\
\hline 15880 & 2 & 5 & 3 & 0 & - & $\mathbf{B}$ & 8 & 0.30 & 0.27 & 0.18 \\
\hline $158 \mathrm{~Tb}$ & 0 & 3 & 3 & 0 & - & E & 8 & 0.10 & 0.09 & 0.06 \\
\hline 159Ho & $1 / 2$ & $7 / 2$ & 3 & 0 & - & 0 & 4 & 0.13 & 0.12 & 0.07 \\
\hline $160 \mathrm{Ho}$ & 2 & 5 & 3 & 0 & - & B & 8 & 0.30 & 0.27 & 0.18 \\
\hline 161Ho & $1 / 2$ & $7 / 2$ & 3 & 0 & - & 0 & 4 & 0.13 & 0.12 & 0.07 \\
\hline 162Io & 6 & 1 & 5 & 0 & + & $\mathbf{B}$ & 7 & 0.70 & 0.73 & 0.82 \\
\hline $163 \mathrm{H}^{\circ}$ & $1 / 2$ & $7 / 2$ & 3 & 0 & - & 0 & 4 & 0.13 & 0.12 & 0.07 \\
\hline $164 \mathrm{H}_{0}$ & 6 & 1 & 5 & 0 & + & $\mathbf{E}$ & 7 & 0.70 & 0.73 & 0.82 \\
\hline 165Dy & $1 / 2$ & $7 / 2$ & 3 & 0 & - & 0 & 4 & 0.13 & 0.12 & 0.07 \\
\hline 16680 & 7 & 0 & 7 & 0 & + & $\mathbf{E}$ & 7 & 0.70 & 0.73 & 0.82 \\
\hline $167 \mathrm{Ex}$ & $1 / 2$ & $T / 2$ & 3 & 0 & - & $\mathbf{0}$ & 4 & 0.13 & 0.12 & 0.07 \\
\hline $169 \mathrm{Yb}$ & $1 / 2$ & $7 / 2$ & 3 & 0 & - & 0 & 4 & 0.13 & 0.12 & 0.07 \\
\hline
\end{tabular}




$\begin{array}{ccccccccccc}\text { 169Ln } & 1 / 2 & 7 / 2 & 3 & 0 & - & 0 & 4 & 0.13 & 0.12 & 0.07 \\ \text { 17OLE } & 4 & 0 & 4 & \mathrm{~B} & + & \mathrm{B} & 5 & 0.85 & 0.86 & 0.91\end{array}$

* Reference (76H12, 77IUD2).

\section{Delayed Neutron Emission}

Delayed neutron emission is included for the first time in this compilation. Delayed neutron precursors, which from the early days have been divided into six half-life groups, are by now well-defined. A total of 271 delayed neutron precursors identified to date are listed together with the approximate half-life group, percent probability of neutron emission of daughter nuclides, and precursor half-lives ranging from 0.074 to 58.2 seconds as proposed for ENDF/B-VI as follows:

Table 5

271 Delayed Ientron Precursors, Their Ealf-lires and Delayed Ientron Eniszion Probabilities (Pn), and Uncertainties (dPu) Is Found in Table I of Roference 86EJG1 (updated on Jenenry 7, 1987).

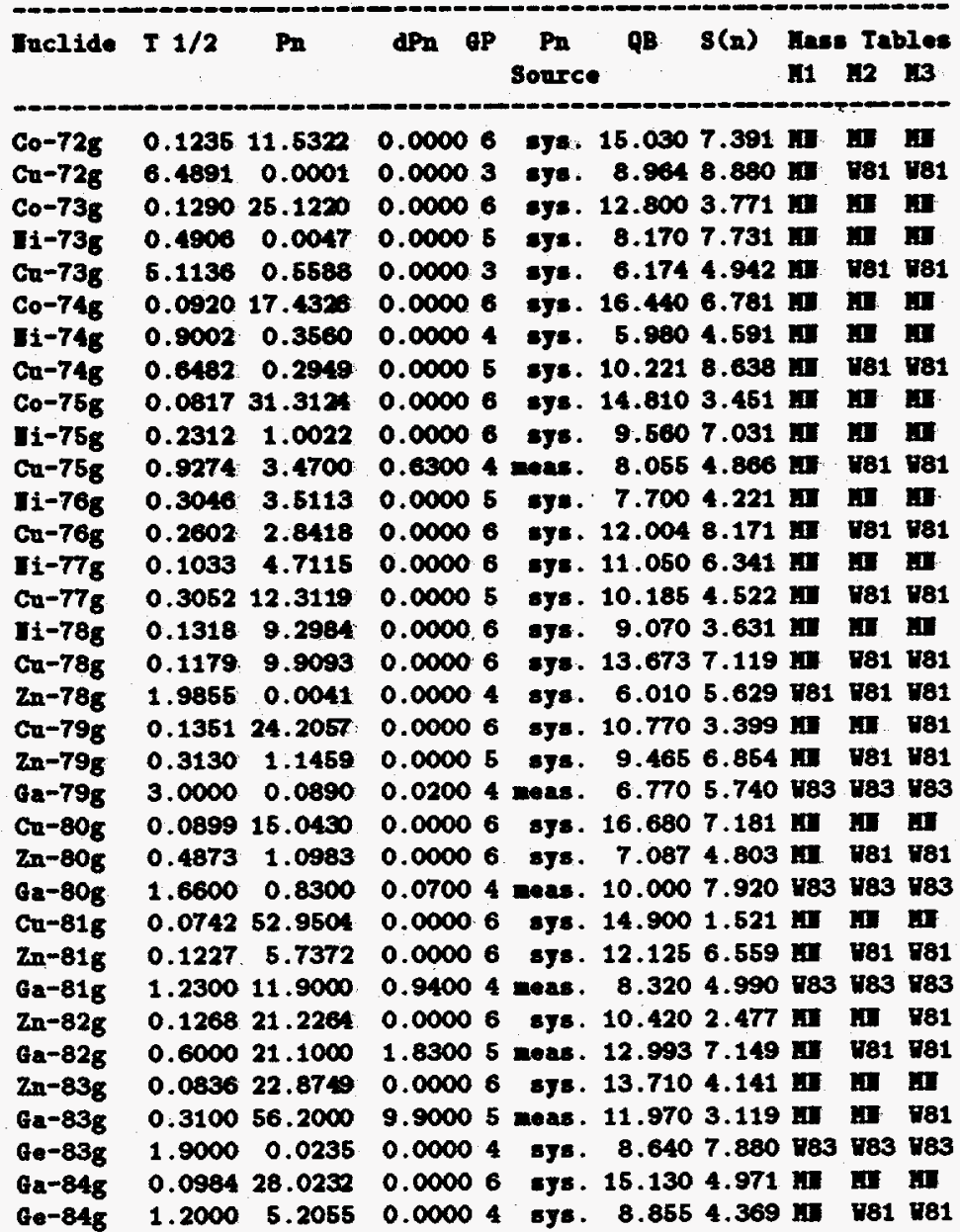

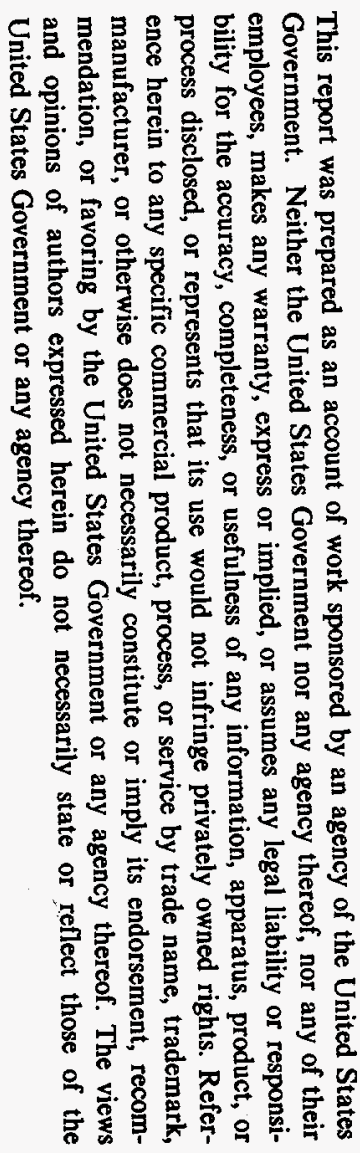




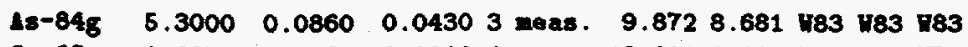

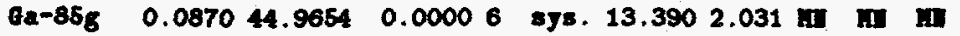
Ge-85g $0.250016 .4540 \quad 0.00006$ BET 11.0504 .226 मा II v81

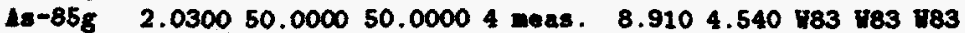
Ge-86g $0.247015 .2148 \quad 0.00006$ sje. 9.4502 .911 fil an HI

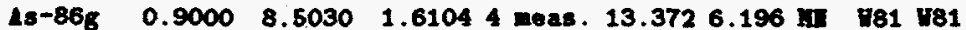

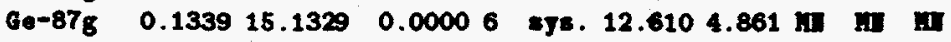

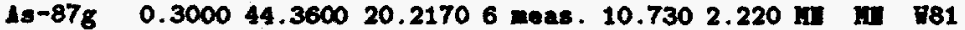
$\begin{array}{lllllll}\text { Se-87g } & 5.6000 & 0.1880 & 0.0210 & 3 \text { meas. } & 7.170 & 6.310 \text { y83 v83 } 183\end{array}$

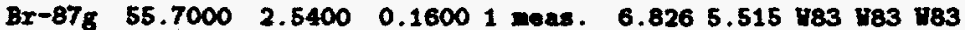
6e-88g 0.129021 .65510 .00006 sje. 10.8502 .531 in al $48-88 \mathrm{~g} \quad 0.134819 .9068 \quad 0.00006$ s5s. 13.7305 .531 al at

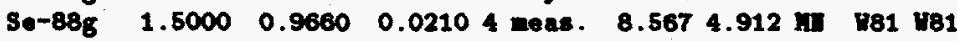

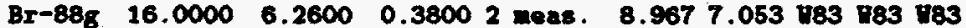

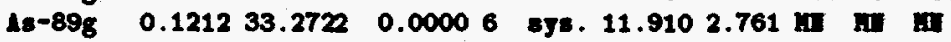

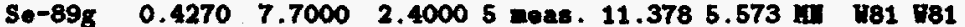

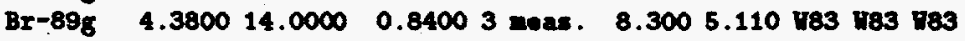
$18-90 \mathrm{~g} \quad 0.091124 .3493 \quad 0.00006$ a7s. 15.0805 .291 हा हा ता

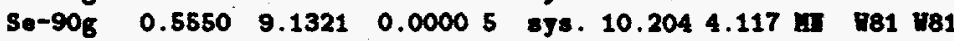

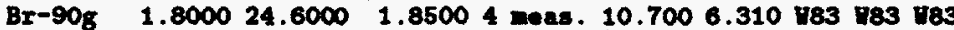

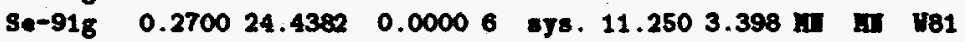
Br-91g 0.600018 .10001 .48005 neas. 11.7954 .493 II 181 v81

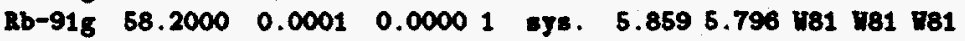
Se-92g 0.168213 .23330 .00006 g5s. 9.4803 .181 h 4 iा $B x-92 \mathrm{~g} \quad 0.360042 .73449 .74645$ meas. $13.8635 .350 \mathrm{II}$ v81 181

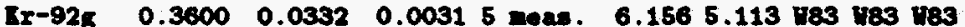

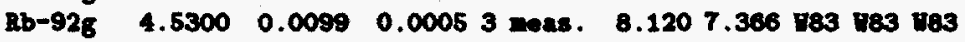
Se-938 0.096812 .03210 .00006 s78. 12.4405 .271 II aा ता

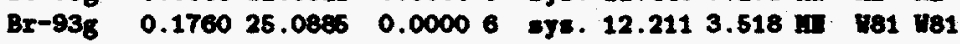

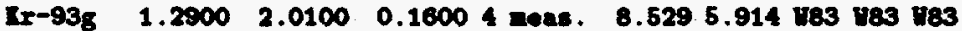

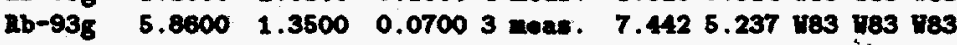
BI-948 0.110829 .80350 .0000 o are. 13.5804 .411 it in 181

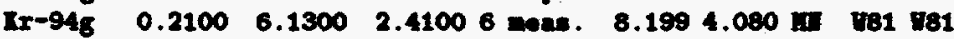
lb-948 2.760010 .00000 .5000 anas. 10.3076 .786 ves ves ves Br-95g $0.108927 .0797 \quad 0.00006$ s5. 11.9903 .271 E. II II Ir-958 0.7800 .7 .60510 .00005 BETL 10.0785 .151 HI 181 t81

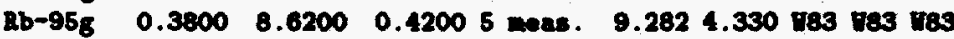
Br-96g 0.088821 .91950 .00006 s7e. 14.8005 .491 an at in

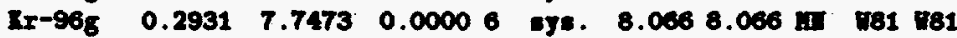
Ib-96g $0.204014 .0000 \quad 0.71006$ neas. 11.7506 .860 v83 u83 183

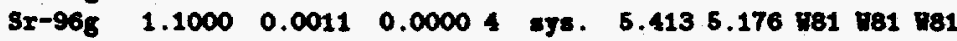

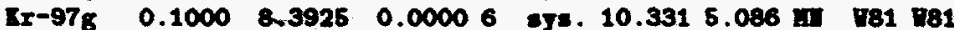
$\mathrm{Bb}-97_{\mathrm{g}} 0.170028 .60001 .48006$ meas. 10.6203 .980 घ83 $\mathrm{n83}$ tes

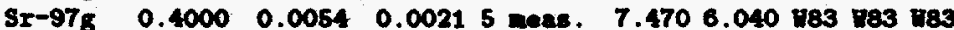

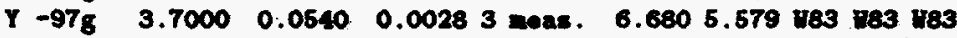
Y $-97 \mathrm{E} \quad 1.1100 \quad 0.1090 .0 .03004$ neas. 0.0000 .000 Y97

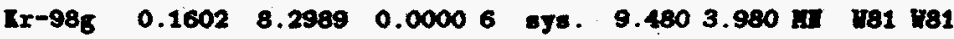
$\mathrm{Ab}-98 \mathrm{~g} 0.110013 .30001 .20006$ mas. 12.4305 .760 v83 ve3 v83

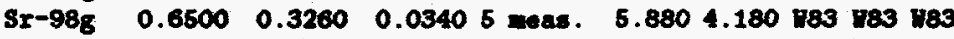

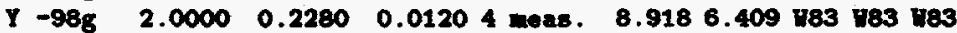
$\begin{array}{llllll}Y & -98 & 0.6500 & 0.2280 & 0.96005 \text { meas. } 0.000 & 0.000 \gamma 98\end{array}$ $\mathrm{Rb}-99 \mathrm{~g} 0.145017 .1000,4.20006$ maas, 11.3203 .760 v83 v83 v83

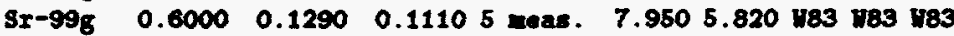

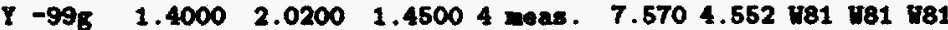

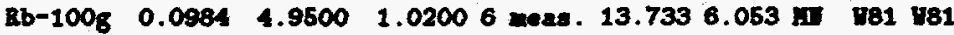

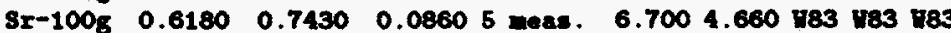

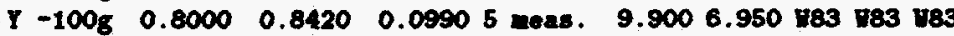

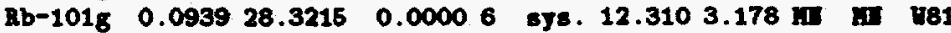

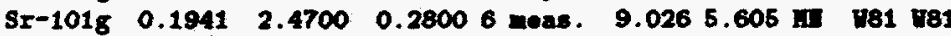

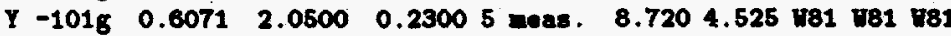

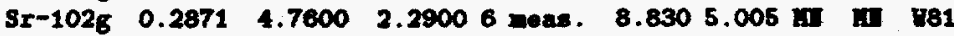
Y $-102 \mathrm{~g} \quad 0.9000 \quad 5.9400 \quad 1.71004$ meas. $10.4426 .727 \mathrm{fl}$ 181 181

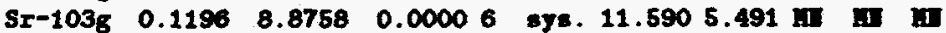

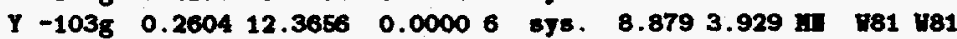

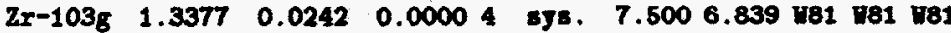

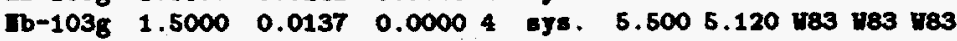




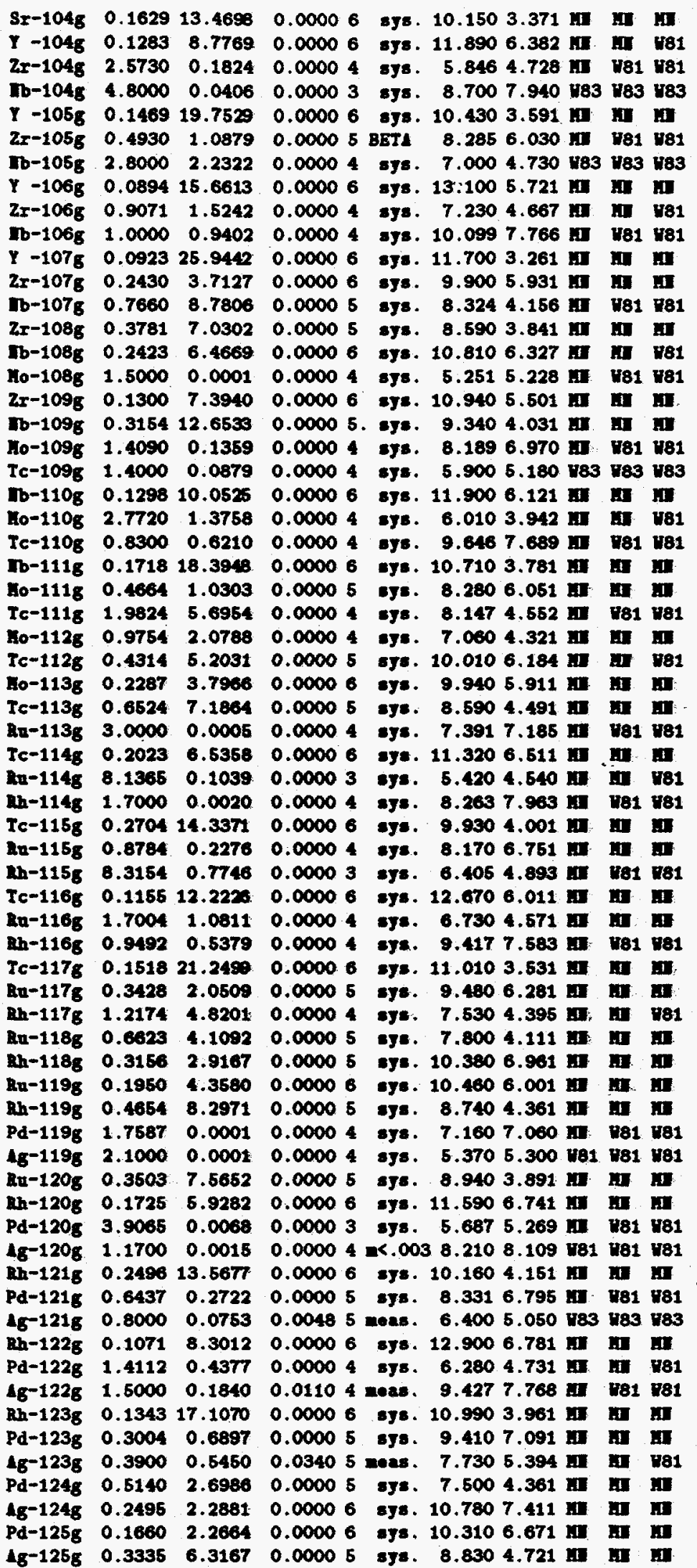




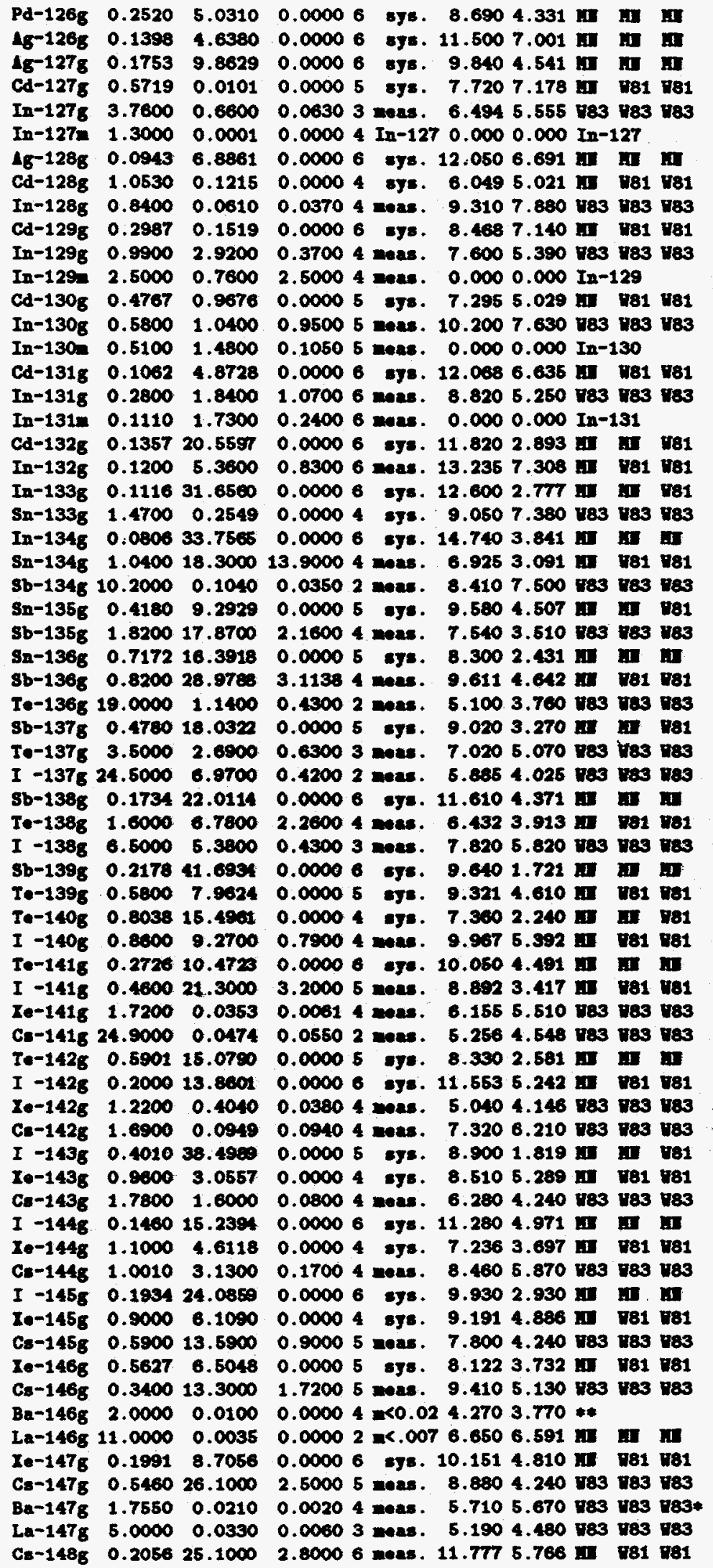




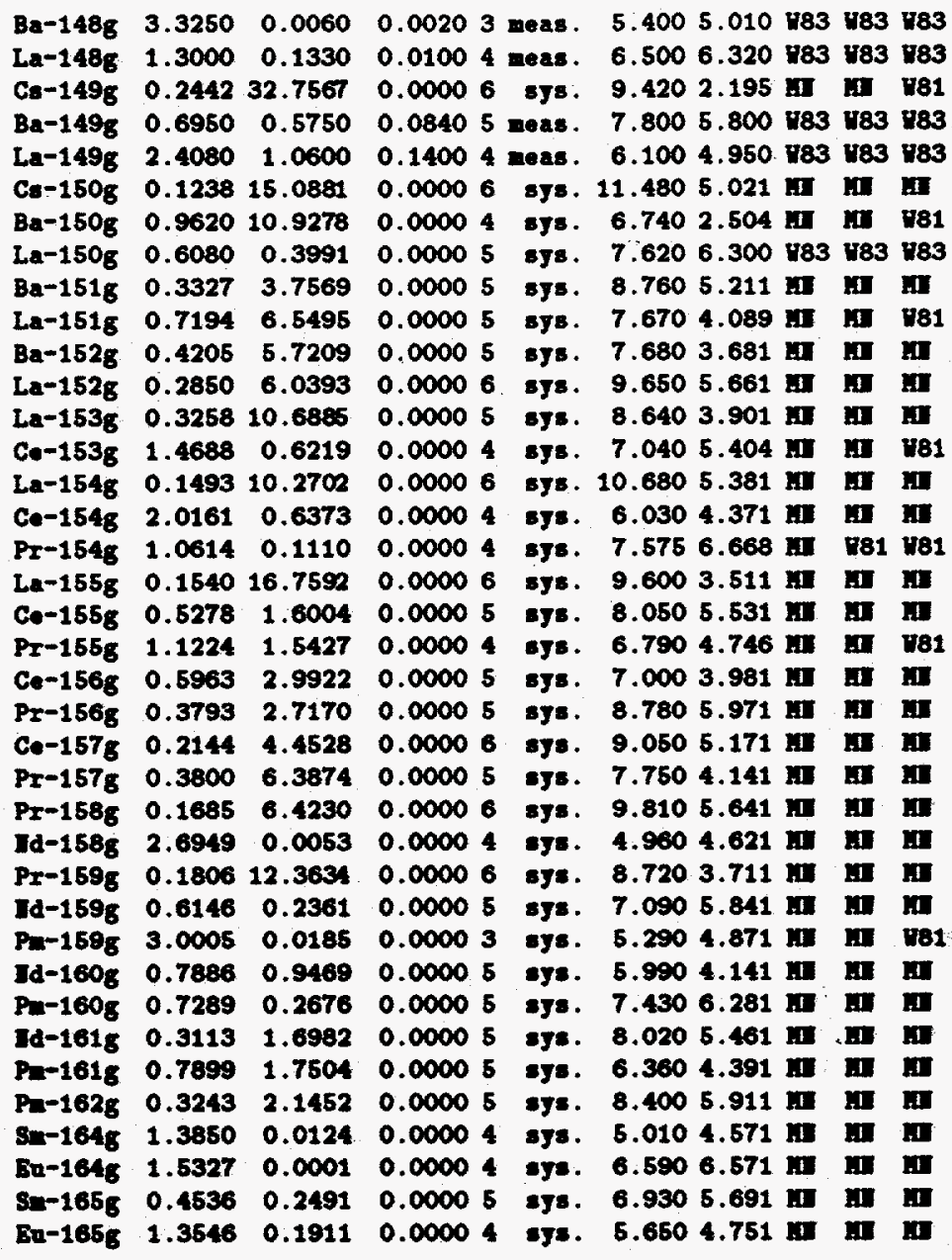

This table contains the latest eraluated Pa valnes (1/7/87). values indicated as derived fros spotenatics are based on a lenst equares fit of the eralnated $P_{2}$ velnes to the paranoters in the Herrmann-lisetz equation as given in Iratz, K.-L., and Bermann, O., "Systemetics of Ieutron Bnission Probsbilities fron Delayed Ieutron Precursors," Z. Physik 263, 435 (1973). This equatican can be expressed: $\quad P_{n}=a[(q B-s(n)) /(G B-z)] * * b$, where $(b=3.44)$ and $(a=54.0)$ from a nez fit to the evaluated Pn's resulting fron the Birminghen meeting Sept 1986 and $I=0$ for oreneven precursor, 13/1**1/2 for eren-odd precursor, and $26 / 1 * * 1 / 2$ for odd-odd precureor.

T 1/2 - half life in seconds, for nuclides dith erap spec taken Iron EDE/B-V

Pn - probability of delajed neatron omission in percent

dPa - uncertainty in Pn value (0.0000 for calculated ralues)

GP - lists which of six tenporal groups the nuclide probably belongs in.

QB - Maximi beta decay energy of precursor (neV)

$S(n)$ - noutron binding onorgy in daughter (neV)

II source of mass of 2 ,

12 source of mass of $z+1,4$

III - Hoeller-Tix

v81 - Vapstra81 


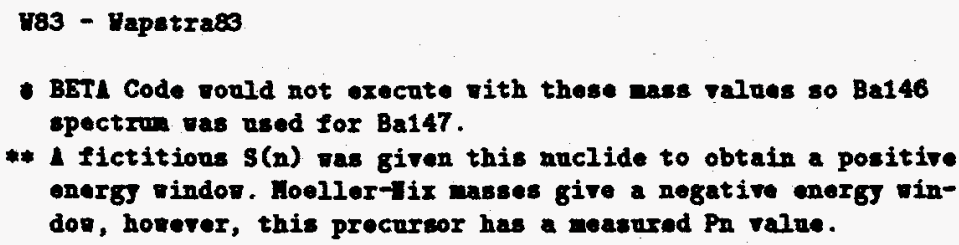

Some thought was given to the method of including delayed neutron emission for more than seventy of the most important nuclides in the existing format. One problem faced is that Pn data is given for the precursors but we need to know it when processing the daughter. For instance, As- 85 decays to Se- 85 by beta decay and Se- 84 by neutron emission. Se- 84 is processed first since masses are processed one at a time in increasing order. A special box was created similar to a metastable state but labeled SE84D (for delayed neutron emission product). Pn for its formation from As-85 is listed in the third row of its decay scheme (Z-0 is the only empty space), and the RC row contains the absolute yield calculated from Pn multiplied by the As- 85 cumulative yield from the previous iteration. Se- $84 \mathrm{~g}$ contains the information in the third row of its decay scheme that $100.0 \%$ of $\mathrm{Dn}$ emission (Z-0 box) adds to Se-84 $\mathrm{g}$ cumulative yield. When processing mass $85, \mathrm{Se}-85$ has listed in the first row of its decay scheme that $68.8 \%(1-\mathrm{Pn})$ of $\mathrm{Z}-1 \mathrm{~g}$ (As-85) is to be added to its cumulative yield, instead of $100 \%$ (the other $31.2 \%$ having left this mass chain for mass chain 84 ). The facts are now correctly represented. The independent yields are all before delayed neutron emission. The cumulative yields are after delayed neutron emission. Also, the chain yield is redefined as the sum of the independent yields plus delayed neutron decay gains from the $A+1$ chain, minus the delayed neutron decay losses to the A-1 chain. Now the chain yields retain their traditional after-delayed-neutron-emission value as measured by traditional radiochemistry and mass spectrometry. A special problem arises for online mass spectrometers that measure instantaneous mass yields such as Hiawatha (76DII1). These pre-delayed-neutron chain yields must be corrected to post-delayed-neutron chain yields by hand.

One can predict the delayed neutron yield from delayed neutron precursor yields multiplied by probability of neutron emission, $(P n)$ summed over all 271 precursors. If the yields are correct, good agreement should be found between predicted and measured delayed neutron emission. Agreements for most types of fission evaluated are as follows:

Tablo 6 .

Delajed Ientron Yields per 100 Fissions

Calculated Valnes Veing Yields and Pn Valnes Listed in this Report

\begin{tabular}{|c|c|c|c|c|c|c|c|c|c|c|}
\hline $\begin{array}{l}\text { Fissionable } \\
\text { Inclide }\end{array}$ & $\begin{array}{c}\text { Using Pn } \\
\text { and ye }\end{array}$ & $\begin{array}{l}\text { From } \\
\text { Bral }\end{array}$ & $\begin{array}{l}\text { Yield } \\
\text { Ination }\end{array}$ & $\begin{array}{l}\text { valdo } \\
\text { Fit }\end{array}$ & $\begin{array}{c}\text { Fissionable } \\
\text { Inclide }\end{array}$ & $\begin{array}{r}\text { Uei } \\
\text { an }\end{array}$ & $\begin{array}{l}{ }_{8} P_{2} \\
J_{c}\end{array}$ & $\begin{array}{l}\text { Frol } \\
\text { Eral }\end{array}$ & $\begin{array}{l}\text { Yield } \\
\text { Ination }\end{array}$ & $\begin{array}{l}\text { tialdo } \\
\text { Fit }\end{array}$ \\
\hline v235t & $1.80+-0.17$ & .67 & +-0.08 & 1.60 & Th227t & 0.59 & +0.10 & 0.59 & +-0.07 & 0.72 \\
\hline U2351 & $1.70+0.16$ & 1.66 & +-0.09 & 1.50 & Th229t & 1.09 & $t-0.19$ & 1.06 & +-0.10 & 1.53 \\
\hline U235h & $1.24+0.19$ & 1.18 & +0.11 & --- & Pa231I & 1.34 & +-0.20 & 1.32 & +-0.12 & 1.04 \\
\hline U238I & $4.06+-0.34$ & 3.85 & +-0.23 & 4.60 & $1+241 t$ & 0.55 & +-0.07 & 0.53 & +0.06 & 0.46 \\
\hline U236h & $2.72+-0.27$ & 2.53 & +0.16 & -- & in241h & 0.37 & +-0.07 & 0.34 & +0.04 & $--\infty$ \\
\hline Pu239t & $0.70+-0.04$ & 0.68 & +0.03 & 0.68 & $\ln 242 t$ & 0.74 & +-0.09 & 0.72 & +-0.07 & 0.67 \\
\hline Pu2391 & $0.61+0.07$ & 0.60 & +0.05 & 0.68 & a.245t & 0.70 & +0.08 & 0.67 & +-0.06 & 0.66 \\
\hline Pn.241t & $1.29+-0.08$ & 1.24 & +-0.06 & 1.45 & Cr249t & 0.28 & +-0.04 & 0.25 & +-0.03 & 0.30 \\
\hline v233t & $0.71+-0.08$ & 0.70 & +0.06 & 0.70 & Ce251t & 0.82 & +-0.09 & 0.76 & +0.08 & 0.65 \\
\hline Ih232f & $5.92+-0.97$ & 5.78 & +0.43 & 4.70 & Es254t & 0.77 & +-0.09 & 0.71 & +0.07 & 0.64 \\
\hline U233f & $0.67+-0.09$ & 0.68 & +0.07 & 0.70 & C12508 & 0.42 & +-0.05 & 0.41 & +0.05 & 0.30 \\
\hline 0233h & $0.70+-0.12$ & 0.67 & +0.08 & $-\infty$ & Con 2448 & 0.43 & +-0.06 & 0.42 & +0.05 & 0.31 \\
\hline
\end{tabular}




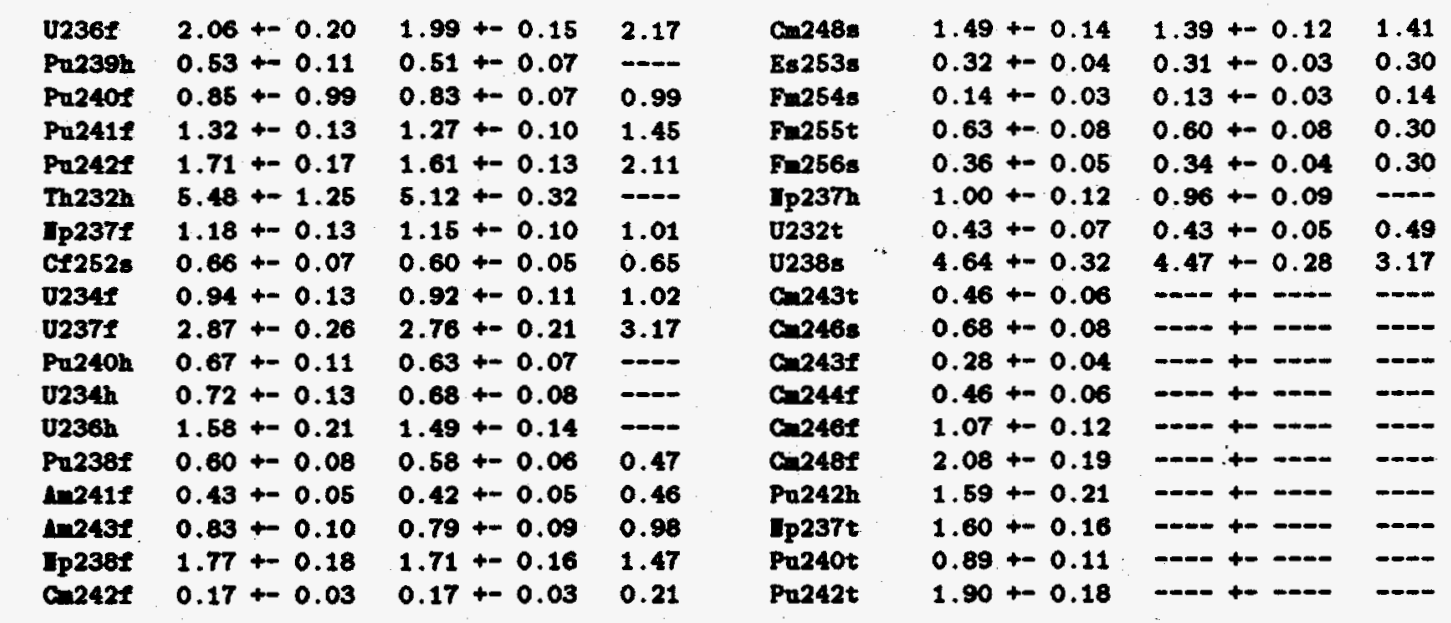

The calculation of delayed neutrons is very sensitive to the amount of odd-even effect (proton and neutron pairing effect) applied. U235 and Pu239 delayed neutron calculations are in good agreement. U238F and TH232F are low. These have large pairing effects that result from the use of fission neutron energies that are very close to large fission threshold energies. The model for energy dependence of pairing tends to blow-up near the fission threshold and is very sensitive to assumed fission neutron energy. Inadequate pairing treatment may therefore account for these poorer agreements.

The moving force behind the 1993 revision was decay heat calculation by summation codes such as CINDER-10 that use fission yields, decay constants, and heat of disintegration ( $Q$ values). A test of goodness for independent yields is whether such summation agrees with experiment. The original 1974 fit was good except at 10 and 15 seconds where calculations were low. An important thrust of the 1978 revision was to improve the fit below 15 seconds. Four sources of error in ENDF/B-IV yields were suspected to be responsible for the $4 \%$ underestimate at 10 seconds:

1. Ternary Fission was not treated. Ternary fission carries a small amount of charge away. This means Zp's must be lowered a bit.

2. Charge balance tests showed some $\mathrm{Zp}$ values were slightly high. If $\mathrm{Zp}$ is reduced to a proper value, the yields on the left shoulder of the Gaussian charge distribution rise abruptly where very shortlived energetic nuclides are found far from stability. The right shoulder yields fall where long-lived low energy nuclides are found near stability.

3. Independent yields were arbitrarily split 50-50 between short lived metastable states and long-lived ground states. In several measured cases like $\mathrm{Xe}-133, \mathrm{Xe}-135$ and $\mathrm{Te}-133$, the short half-life (high energy) metastable state is favored.

4. Delayed neutrons were not treated, and 264 out of 271 of the delayed neutron precursors have half-lives under 10 seconds.

By correcting these shortcomings, ENDF/B-VI (ENDF-349) yields have greatly improved agreement down to 6 seconds. The refinement effort appears to have been worth while. It has contributed toward development of a new ANS Decay Heat Standard with errors reduced from $20 \%$ in the 1973 Standard to under $2 \%$ in the 1993 Standard. 


\section{Recommended Yields}

The weighted average experimental independent yields, the weighted average experimental cumulative yields, and the calculated cumulative yields (where no data were available) were combined statistically to form a single, self-consistent recommended value. The following is a summary of the procedure used to obtain the recommended values. The calculated charge distribution was used only when no data were available. Even then it was normalized to the nearest experimentally determined yields to ensure the experimental and recommended values will closely agree. A large error was assumed for the calculated yields to ensure any respectable data would dominate. The contributions of all precursors were added. The total precursor contribution was then subtracted from the experimental cumulative yield when available or the normalized calculated yield to obtain an independent yield. (Note: independent yields so obtained which are less than $0.1 \%$ are given no weight and negative values are discarded). This independent yield was then averaged with the experimental independent yield (if available) or the calculated independent yield and stored for later use. A cumulative yield was then obtained by adding the precursor contribution to the independent yield just obtained. This cumulative yield was averaged with the experimental cumulative yields (if available) or the calculated cumulative yield to obtain a cumulative yield. This cumulative yield was stored as input for the next member of the chain. After this procedure had been done for all members of the chain, the chain yield was obtained by adding the stored cumulative yields of all stable nuclides. The stored independent yields were then normalized so that their total equals the chain yield (after adjustment for delayed neutron emission). The recommended cumulative yields were obtained by adding the independent yield of all precursors to the independent yield of the nuclide. The total of the chain yields of each peak was then obtained. The difference between $100 \%$ and this total was distributed among the chain yields in proportion to their variances. This method ensures the reported chain yields will total $100 \%$. This procedure preserves the independent yield significance of the differences between the recommended yields. It also allows unstable nuclides to affect the chain yields if independent yields have been determined, or if the calculated charge distribution shows the yield of the nuclide to be very near the chain yield.

\section{ENDF/B-VI and Mass Chain Yields}

As noted, the evaluated and compiled yields and distribution parameters (sigma, Zp, branchings, etc) are available for 60 yield sets in groups of 10 fissioning systems in the six appendices. All measured data are also listed in the appendices. Any user consulting these files should also check the reference indicated there in the annotated bibliography to possibly explain the reason for any change (update) to measured data.

We are making the compiled data freely available to users. These data have been collected over several decades and updated or corrected as noted in the bibliography and appendices. We request that users of these computer files acknowledge this printed document and also inform the authors of any errors or new data.

The recommended and cumulative yields ( $\mathrm{RI}$ and $\mathrm{RC}$ lines in the appendices) are the basis for the second evaluation and release of ENDF/B-VI (1993). Values in ENDF/B-VI have been extended to cover all nuclides in the ENDF/B-VI decay files and also four charge units from Zp, but the extension does not alter the values in this document for the specific nuclides listed in the appendices. 
ENDF/B-VI does not contain mass chain yields and these are difficult to determine from the RI and $\mathrm{RC}$ values using decay branchings from decay files. Tables 7 through 12 below list the chain yields. The letters $a-p$ following the yield values indicate the accuracy of weighted yields; these are defined in a later section of this document.

Table 7

Sot $\triangle$ Hass Chain Yields

nass n235t u235t u235he u238t u238he pu239t pu239f pu241t u233t th2321

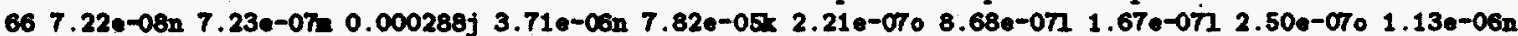
67 3.61e-07a 2.32e--0en 0.000604 j 2.15e-05n 0.0001391 4.48e-07n $2.87 e-061 \quad 5.05 e-071$ 1.12e-06e 3.90e-06n $687.16 e-07 n$ 4.03e-06n $0.000887 \mathrm{k}$ 2.80e-06n 0.0002991 1.62e-06n 8.42e-061 1.41e-061 3.43e-06e 1.43e-05n

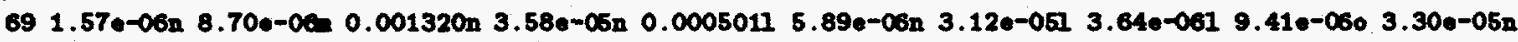
70 3.62e-0e 1.89e-05 $0.002361 k$ 4.41e-05=0.0009011 1.99e-05= 8.69e-051 1.40e-051 3.67e-05e 6.54e-05= 71 8.38n-06n 4.65e-05n 0.0039461 4.95e-05n 0.001592 3.67e-05n $0.000203 n$ 5.24e-051 0.00016200 .000166

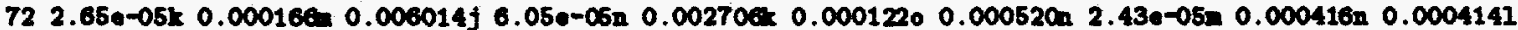

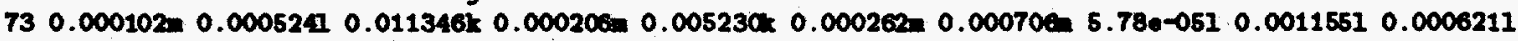
$740.000339=0.001190=0.017011 k 0.000277 \mathrm{n} 0.00800610 .000638 \mathrm{n} \quad 0.00173619 .480-05=0.002708 \mathrm{n}=0.0011541$

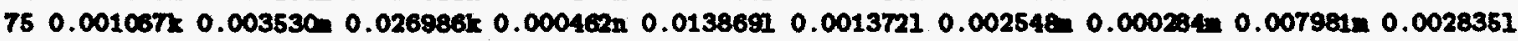

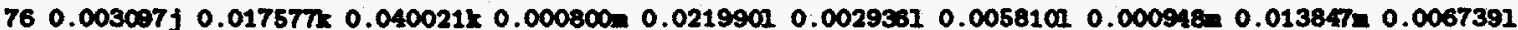
$770.007961 j \quad 0.031808 \quad 0.068594 k 0.003301 k 0.031293 j \quad 0.007235 k 0.012692 j \quad 0.001806 \quad 0.0259691 \quad 0.011765 j$

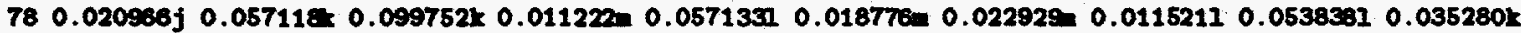

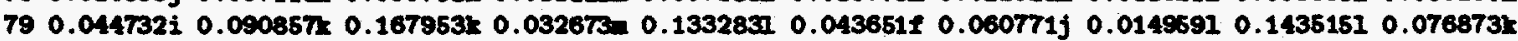

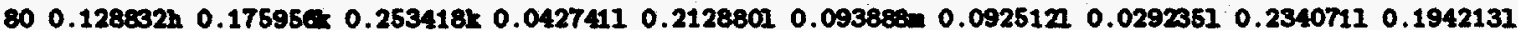

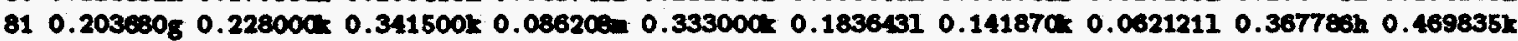

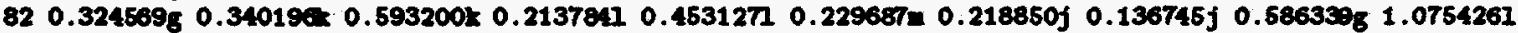

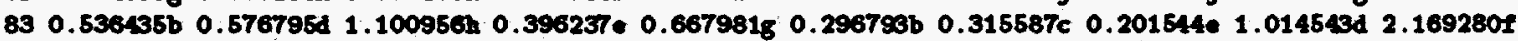

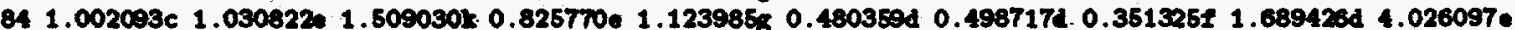

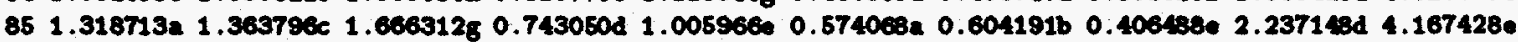

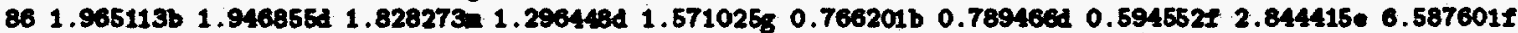

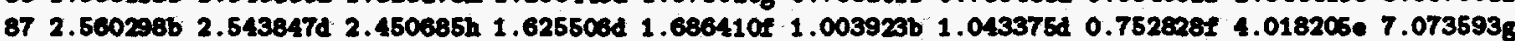

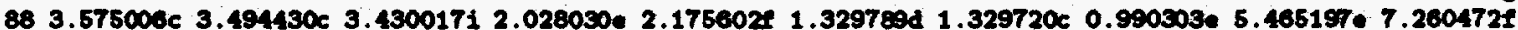

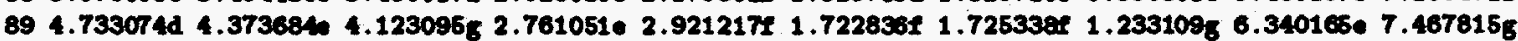

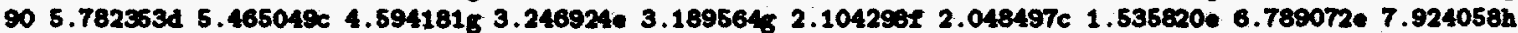

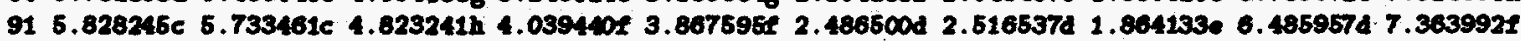

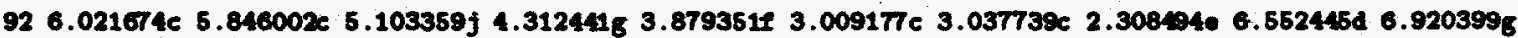

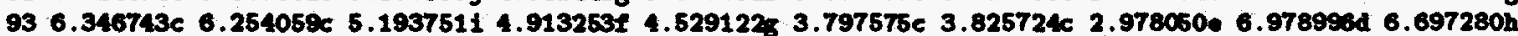

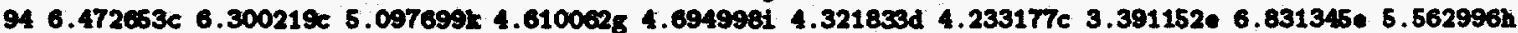

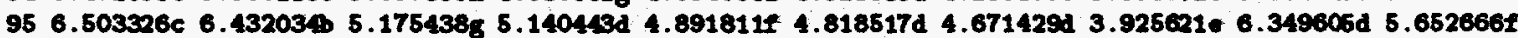
96 6.340230d 6.202490c 5.207079j 6.017320h 5.575470 4.893751d 4.856321c 4.401147* 5.678962d 4.7767641

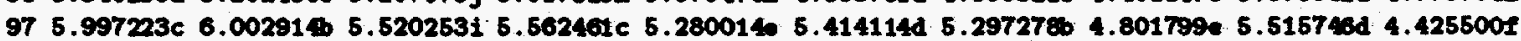
98 5.790300c 5.923745b 5.4363961 5.944993d 5.46424k 5.830892d 5.633049c 5.035685t 5.189312d 3.680042i 99 6.109164d 5.943191d 5.136274g 6.1681920 5.705377d 6.211806e 5.982639P 5.9618351 4.910973e 2.9440601

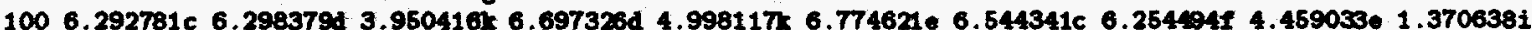

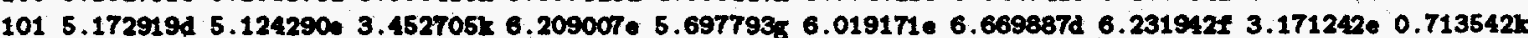
102 4.298778d 4.359081d 3.2540261 6.447346d 4.621668j 6.126339e 6.731884d 6.655272t 2.402804d 0.367805k $1033.031149 \mathrm{~d} 3.243933$ b $3.205137 \mathrm{~g} 6.275279$ e $4.615807 \mathrm{f}$ 6.994895 $6.829040 \mathrm{e}$ 6.776879g $1.573207 \mathrm{f} 0.155461 \mathrm{~h}$

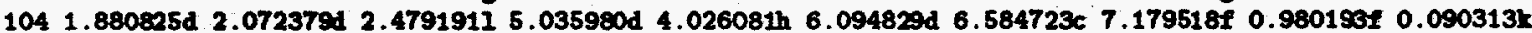
1050.964228 . $1.196323 \mathrm{~g} 1.872265 \mathrm{~h} 4.051243$ 3.2158810 $5.643815 \mathrm{f}$ 5.361104g $6.074350 \mathrm{~g} 0.495809 \mathrm{~g} 0.051481 \mathrm{~g}$

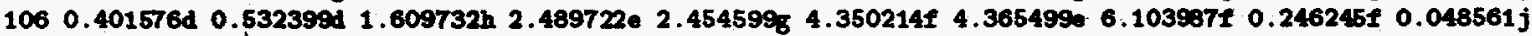
$1070.146202 \mathrm{~g} \quad 0.302329 \mathrm{k} 1.285484 \mathrm{k} 1.445866 \mathrm{j} 1.694368 \mathrm{i} 3.330057 \mathrm{~h} 3.221088 \mathrm{i} 4.886322 \mathrm{j} \quad 0.114532 \mathrm{~h} 0.051075 \mathrm{k}$ $1080.054129 \mathrm{~h} 0.1308161$ 1.178097 0.6014201 1.2224611 $2.163956 \mathrm{~h} 2.002769 \mathrm{j} 3.769599 \mathrm{j} 0.07576 \mathrm{hh} 0.0613471$ $1090.031223 i$ i $0.081546 \mathrm{~h} 1.173138 \mathrm{i} 0.252140 \mathrm{i} 1.122826 \mathrm{i} 1.477898 \mathrm{~h} 1.036285 \mathrm{~h} 2.587597 \mathrm{~h} 0.0394061 \quad 0.066215 \mathrm{~h}$ $110 \quad 0.025445 \mathrm{~h} \quad 0.064915 \mathrm{I} \quad 1.083704 \mathrm{~K} \quad 0.1407601$ 1.027439k $0.645132 \mathrm{i} \quad 0.647332 \mathrm{j} 1.325114 \mathrm{j} \quad 0.038664 \mathrm{~h} \quad 0.0709971$

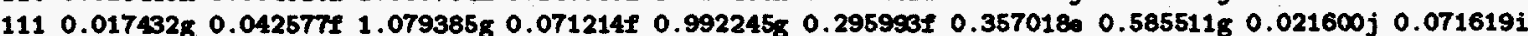
$1120.013043 \mathrm{~h} 0.037712 f 1.075936 \mathrm{j} \quad 0.05590 \mathrm{~h} 1.032693 \mathrm{~g} \quad 0.128508 f \quad 0.188927 f \quad 0.218486 \mathrm{~g} \quad 0.013304 \mathrm{j} \quad 0.078263 i$ $1130.014205 \mathrm{~h} 0.032614 \mathrm{~g} 1.091408 \mathrm{j} 0.046111 \mathrm{k} 0.914336 \mathrm{i} \quad 0.081664 \mathrm{~g} \quad 0.127662 \mathrm{f} 0.151924 \mathrm{~h} \quad 0.013562 \mathrm{k} 0.078066 \mathrm{~h}$ $1140.011833 \mathrm{~h} \quad 0.032294 \mathrm{~g} 1.057132 \mathrm{k} \quad 0.03925110 .718885 \mathrm{k} \quad 0.060254 \mathrm{~g} \quad 0.094265 \mathrm{~F} \quad 0.071086 \mathrm{~m} \quad 0.012860 \mathrm{k} \quad 0.0734231$ $\begin{array}{lllllllllll}115 & 0.012584 \mathrm{~g} & 0.033878 \mathrm{~g} & 1.091665 \mathrm{~g} & 0.037548 \mathrm{f} & 0.857044 \mathrm{~g} & 0.042600 \mathrm{i} & 0.081408 \mathrm{~h} & 0.038087 \mathrm{j} & 0.0149821 & 0.077003 \mathrm{~g}\end{array}$ $1160.013249 \mathrm{~h} 0.03400 \mathrm{k}$ l. $1.056328 \mathrm{k} \quad 0.039314 \mathrm{k} 0.67563 \mathrm{k} \quad 0.050678 \mathrm{j} \quad 0.060358 \mathrm{~g} \quad 0.027787 \mathrm{n} 0.01320210 .0738011$ $1170.012762 \mathrm{~h} 0.036819 \mathrm{j} 1.065332 \mathrm{j} \quad 0.037732 \mathrm{k} 0.604700 \mathrm{~h} \quad 0.044459 \mathrm{j} \quad 0.069871 \mathrm{j} \quad 0.023626 \times 3 \times .014124 \mathrm{k} \quad 0.073822 \mathrm{~g}$ $1180.011346 \mathrm{j} 0.033632 \mathrm{j} 1.081950 \mathrm{k} \quad 0.043176 \mathrm{k} \quad 0.831012 \mathrm{k} \quad 0.032488 \mathrm{k} \quad 0.059861 \mathrm{k} \quad 0.023157 \mathrm{n} \quad 0.015417 \mathrm{k} \quad 0.063281 \mathrm{k}$ 
$1190.012874 \mathrm{j} \quad 0.035020 \mathrm{j} 1.084139 \mathrm{k} \quad 0.039720 \mathrm{k} \quad 0.725951 \mathrm{k} \quad 0.032249 \mathrm{j} \quad 0.055017 \mathrm{k} \quad 0.023156 \mathrm{n} \quad 0.01855810 .0572101$

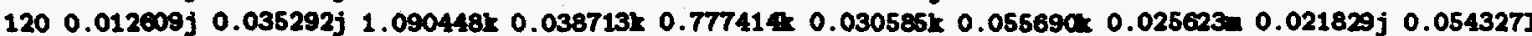
$1210.013047 \mathrm{i} \quad 0.038811 \mathrm{j} 1.054143 \mathrm{~h} 0.036927 \mathrm{j} \quad 0.833461 \mathrm{~h} 0.037808 \mathrm{j} \quad 0.063088 \mathrm{k} \quad 0.025882 \mathrm{n} 0.023082 \mathrm{j} \quad 0.048821 \mathrm{i}$

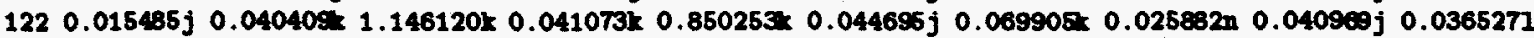
$123 \quad 0.015658 \mathrm{~g} \quad 0.044063 \mathrm{k} 1.192724 \mathrm{k} \quad 0.04453410 .921818 \mathrm{k} \quad 0.0441201 \quad 0.076809 \mathrm{k} \quad 0.026704 \mathrm{n} \quad 0.060415 \mathrm{~m} \quad 0.0293541$

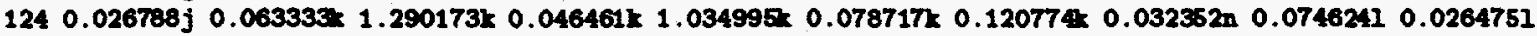
$1250.034022 \mathrm{f} 0.068030 \mathrm{i} 1.460497 \mathrm{j} 0.048524 \mathrm{i} 1.196325 \mathrm{~h} 0.111659 \mathrm{j} 0.177846 \mathrm{j} 0.046901 \mathrm{j} 0.116962 \mathrm{j} 0.032954 \mathrm{k}$

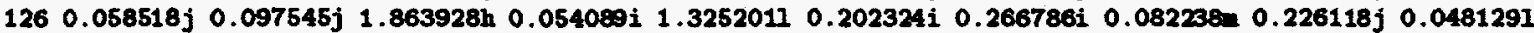
$1270.156991 \mathrm{~g} \quad 0.306279 \mathrm{f} 2.179644 \mathrm{~g} \quad 0.136294 \mathrm{~h} 1.493819 \mathrm{i} 0.506298 \mathrm{i} 0.500864 \mathrm{j} \quad 0.23238 \mathrm{~h} \quad 0.55545 \mathrm{~h} \quad 0.101355 \mathrm{~h}$

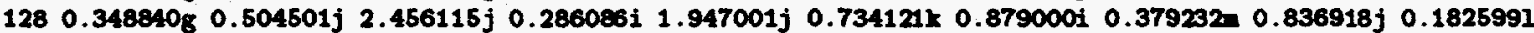
$1290.543386 \mathrm{~d} 0.83517 \mathrm{Ch} 3.375012 \mathrm{j} 1.011337 \mathrm{i} 2.077459 \mathrm{i} 1.371454 \mathrm{~h} 1.461065 \mathrm{i} 0.819952 \mathrm{~m} 1.591337 \mathrm{l} 0.252076 \mathrm{~h}$ 1301.810633 f 1.79463 th $3.599778 j$ 1.913653i 3.1492531 2.361688j 2.4540951 1.818825k 2.091632k $0.945582 k$ $1312.890898 \mathrm{~b} 3.219551 \mathrm{c} 4.103087 \mathrm{~g} 3.290794 \mathrm{~d} 3.992440$ 3.866439b $3.878424 \mathrm{~b} .100837$ 3.604300 $1.620949 \mathrm{f}$

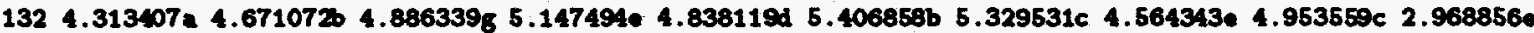

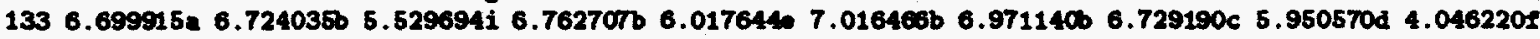

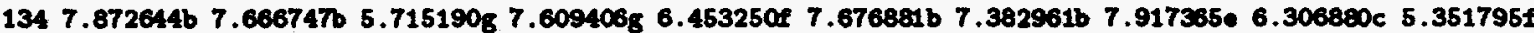
1356.539455 a 6.601362x 5.729764i 6.967573e 5.841928e 7.621001c 7.554585b 7.170T71d 6.2652230 5.528784f

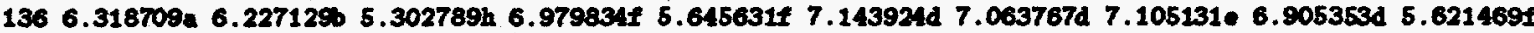
1376.188944 b $6.221562 \mathrm{a} 4.948814 \mathrm{~g}$ 6.052506c $5.146585 \mathrm{f}$ 6.613842b $6.590226 \mathrm{~b}$ 6.650916 6.757385c 5.839457h $1386.788035 b$ 6.680526c 4.593901i 5.762461 e 4.883586g 6.124533d 6.126624c 6.605149d 5.907377 6.449821i

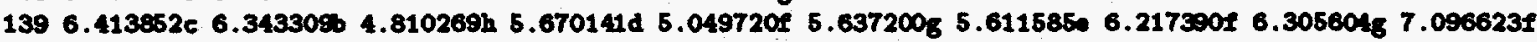

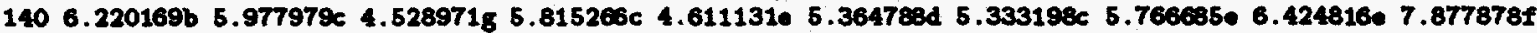

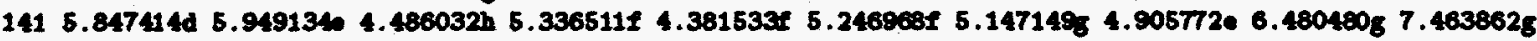
$1425.849283 b 5.542190 d 4.205015 i$ 4.585776d 4.061520g 4.928967b 4.754411c 4.746982d 6.670707• 6.5403818

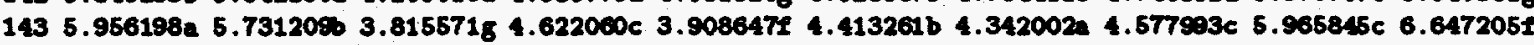
$1445.499964 \mathrm{a} 5.268694 \mathrm{c} 3.172470 \mathrm{~g} 4.547939 \mathrm{c} 3.72281 \mathrm{~g}$ 3.739785a $3.672940 \mathrm{c} 4.227394 \mathrm{c} 4.726134 \mathrm{c} 7.916097 \mathrm{~g}$

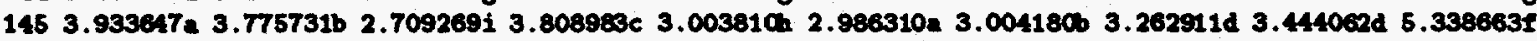
$1462.997113 \mathrm{a} 2.921372 \mathrm{~b} 2.198985 \mathrm{k} 3.445628$ c $2.436894 \mathrm{i}$ 2.458148a $2.460051 \mathrm{~b} 2.769456 \mathrm{c} .585897 \mathrm{c} 4.554332 \mathrm{~g}$ $1472.246886 \mathrm{c} 2.138887 \mathrm{c} 1.623358 \mathrm{~g} 2.692719 \mathrm{c} 2.091141 \mathrm{f} 2.002981 \mathrm{~d} 1.990639 \mathrm{c} 2.28494961 .738404 \mathrm{~g} 2.978662 \mathrm{~g}$ $1481.673680 \mathrm{e} 1.683446 \mathrm{~b} 1.197989 \mathrm{k} 2.112486 \mathrm{c} 1.730509 \mathrm{l} 1.642120 \mathrm{~b} 1.681454 \mathrm{~m} 1.832103 \mathrm{c} 1.301585 \mathrm{~d} 2.005246 \mathrm{~g}$ $1491.081705 d 1.036973 \mathrm{c} 0.8132821$ 1.625277d $1.458148 \mathrm{i} 1.216561 \mathrm{c} 1.241118 \mathrm{~b} 1.474076 \mathrm{0.7781346} 1.083641 \mathrm{~h}$

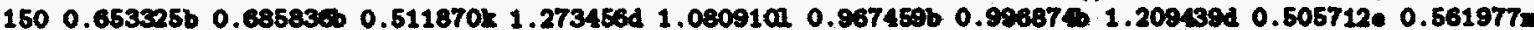

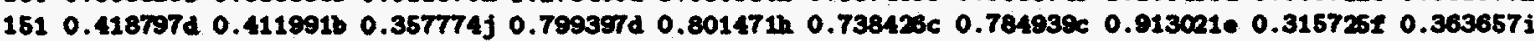

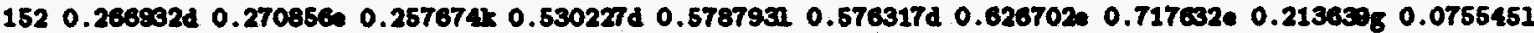

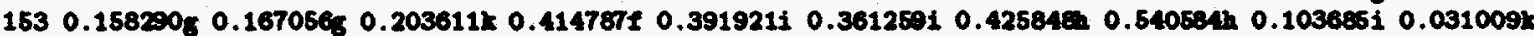

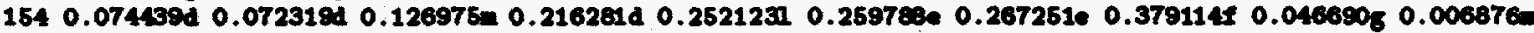

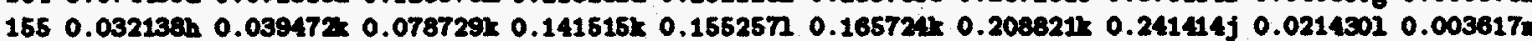

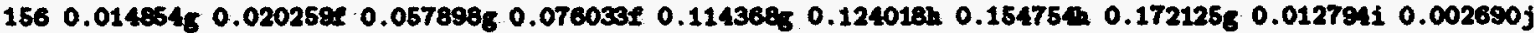

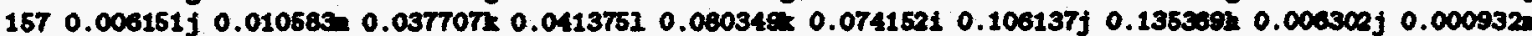

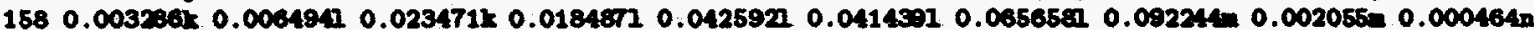

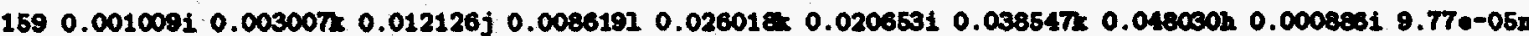

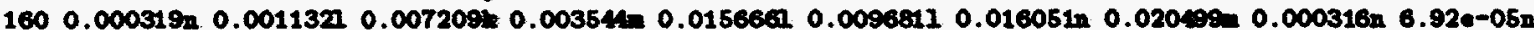

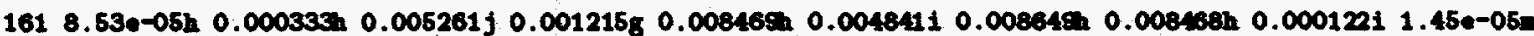

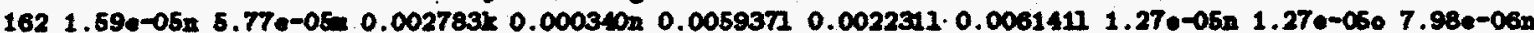

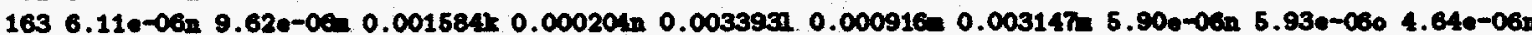

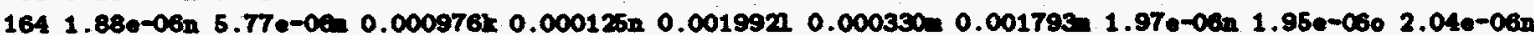
$1659.52 e-07 n$ 2.31e-0en 0.000538k 7.66e-05n 0.00109n 0.00013An 0.000897n 6.15e-07m 6.32e-07n 3.53e-07m

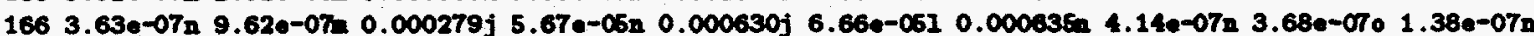
$1672.47 e-07 n$ 3.85e-07n 0.000185k 4.35e-061 0.000369i 1.52e-05n 0.000267n 1.04e-07n 5.09e-080 9.77e-08n

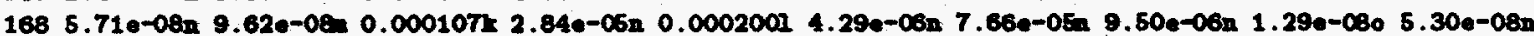

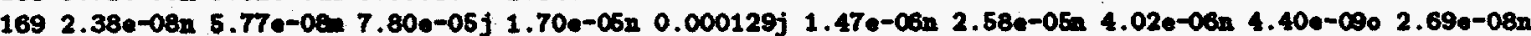
$1705.01 e-09 n$ 1.92e-0en 3.25e-05k 1.02e-05n 5.95e-051 3.18e-07n 7.66e-0en 1.64e-0en 1.62e-00n 1.07e-08n $1712.35 e-09 \mathrm{n}$ 6.73e-09 1.76e-05k 5.72e-06n 3.30e-001 $1.57 e-07 \mathrm{n} 2.58 e-0 e n$ 2.19e-07n 4.47e-10e 4.64e-09n

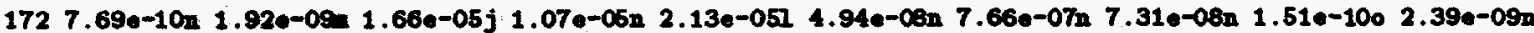

Table 8

Set B Hass Chein Yields

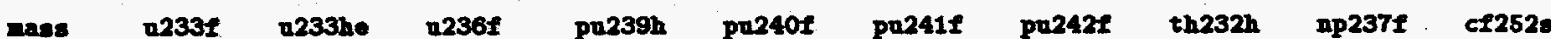
66 3.99e-07n 0.000670 8.30e-07n 6.23e-051 8.88e-07n 1.88e-07n 2.90e-0en 0.000129i $1.87 e-071$ 5.26e-08n $671.60 e-06 n$ 0.0015151 2.08e-06n 9.82e-051 1.33e-0en 1.31e-06n 5.79e-06n 0.000224j 3.73e-0rn $1.44 e-07 \mathrm{n}$ $684.88 e-06 \mathrm{n} 0.001808 \mathrm{n} 4.15 \mathrm{e}-06 \mathrm{n} 0.0002171$ 3.11e-06n $2.82 \mathrm{e}-06 \mathrm{n}$ 8.69e-06n $0.000760 \mathrm{j} 1.88 \mathrm{e}-061$ 3.84e-07n $691.33 e-05 n \quad 0.002900 n$ 9.34e-06n 0.0003711 4.88e-0en $1.03 e-05 n$ 1.16e-05n $0.001515 j$ 6.89e-081 9.91e-07n $705.32 e-05 n \quad 0.0047042 .08 e-05 n$ 0.0006701 1.19e-0En 3.28e-05n 2.90e-08n 0.002783j 2.44e-051 2.39e-06m 
$710.000231 n \quad 0.007445 n$ 5.19e-05n $0.00119613 .41 e-05 n \quad 8.45 e-05 n$ 5.79e-05n $0.005304 j 6.19 e-0516.02 e-06 n$

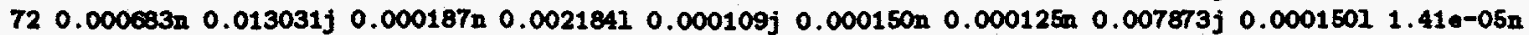

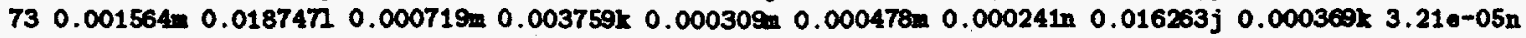

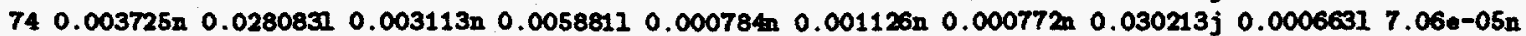
$\begin{array}{lllllllllll}75 & 0.011085 n & 0.043497 & 0.008302 n & 0.0102301 & 0.001706 n & 0.002816 n & 0.001158 n & 0.053042 j & 0.0013181 & 0.000152 n\end{array}$ $\begin{array}{lllllllllllllllll}76 & 0.019703 n & 0.066968 n & 0.022832 n & 0.0164181 & 0.004092 n & 0.005632 n & 0.002413 n & 0.090643 j & 0.0060121 & 0.000317 n\end{array}$ $770.037259=0.101957 \mathrm{~m} \quad 0.045174 \mathrm{~m} 0.023704 \mathrm{k} \quad 0.01078 \mathrm{~m} \quad 0.00969310 .006828 \mathrm{~m} \quad 0.130970 \mathrm{j} \quad 0.010440 \mathrm{k} 0.000625 \mathrm{~m}$ $\begin{array}{lllllllllllll}78 & 0.062079 \mathrm{n} & 0.154923 \mathrm{n} & 0.058119 \mathrm{n} & 0.0415121 & 0.02221 \mathrm{~m} & 0.0184161 & 0.013517 \mathrm{n} & 0.249312 \mathrm{j} & 0.024370 \mathrm{k} & 0.002061 \mathrm{k}\end{array}$ $\begin{array}{llllllllllll}79 & 0.097555 n & 0.2308431 & 0.114166 n & 0.086286 k & 0.041693 & 0.0358641 & 0.033796 n & 0.413505 j & 0.056444 k & 0.003436 n\end{array}$ $\begin{array}{lllllllllllllllll}80 & 0.2118191 & 0.3376401 & 0.186862 n & 0.1574121 & 0.071108 & 0.0649441 & 0.055138 m & 0.691637 & 0.111391 k & 0.004784 n\end{array}$

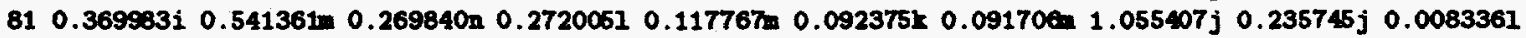
$820.592212 \mathrm{j} \quad 0.837895 \mathrm{~m} \quad 0.384297 \mathrm{n} \quad 0.34982810 .173119 \mathrm{a} \quad 0.142856 \mathrm{j} \quad 0.125578 \mathrm{n} 1.371988 \mathrm{j} \quad 0.357483 \mathrm{k} \quad 0.0152771$

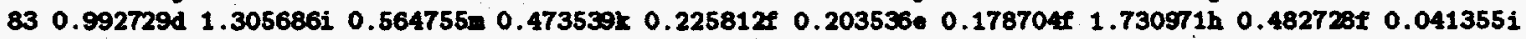
84 1.646913d 2.055871i $1.041023=0.7975971 \quad 0.387259$ I 0.356864 e 0.344098 2.405313h $0.764156 f \quad 0.0658241$ $852.120474 \mathrm{~d} 2.128544 \mathrm{j} 1.404166 \mathrm{~h} 0.965547 \mathrm{~h} \quad 0.433496 \mathrm{~g} \quad 0.446553 \mathrm{f} \quad 0.323157 \mathrm{f}$ 4.504963h $0.94352910 .109031 \mathrm{i}$ $862.791283 \mathrm{~d} 2.6824771 .769318 \mathrm{~m} 1.135089 \mathrm{k} \quad 0.626292 f \quad 0.599326 \mathrm{e} \quad 0.541052 f \quad 5.387893 \mathrm{j} 1.312144 \mathrm{~g} \quad 0.1273911$ 87 3.83997od 3.384983i $2.300375 \mathrm{~h} 1.311613 \mathrm{~h} 0.855547 \mathrm{~g} \quad 0.801998 \mathrm{f} 0.63334 \mathrm{Ch} 4.972307 \mathrm{~g} 1.748054 \mathrm{~g} 0.2149491$ $885.074510 \mathrm{~d} 3.972427 \mathrm{j} 2.903340 \mathrm{~h} 1.905361 \mathrm{j} 0.974526 \mathrm{~h} 0.988938$ e $0.834913 \mathrm{~F} \quad 5.344973 \mathrm{~h} 2.160300_{\mathrm{g}} 0.316107 \mathrm{j}$ $895.752346 \mathrm{~g} 4.624521 \mathrm{i} 3.866 \mathrm{T71i} 2.068536 \mathrm{~h} 1.409931 \mathrm{i} 1.282172 \mathrm{i} 1.098664 \mathrm{j} \quad 5.935102 \mathrm{~g} 2.540039 \mathrm{~h} 0.363233_{\mathrm{g}}$ $906.386977 \mathrm{~d} 4.758131 \mathrm{j} 4.72682812 .1027541 \mathrm{~K} 1.798823 \mathrm{f} 1.550924 \mathrm{f} 1.360012 \mathrm{f}$ 6.084759g $3.341144 \mathrm{f} 0.5224651$ $916.449272 \mathrm{~d} 5.17840215 .500850 \mathrm{~g} 2.237309 \mathrm{j} 2.190363 \mathrm{~F} 1.928825 \mathrm{f} 1.801399 \mathrm{~g}$ 5.991665g $3.926816 \mathrm{f} \quad 0.597083 \mathrm{~g}$ 92 6.541788d $5.475885 i$ 5.611012h $2.914485 \mathrm{k} 2.680322 \mathrm{f} 2.394422 \mathrm{f} 2.151179 \mathrm{~g} 5.515768 \mathrm{~g} 4.385161 \mathrm{f} 0.683939 \mathrm{~g}$

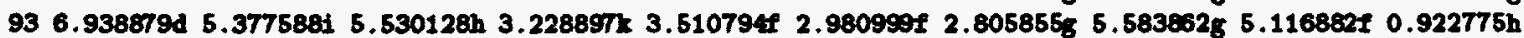
$946.754053 \mathrm{~d} 5.02821 \mathrm{n} 5.022972 \mathrm{i} 3.488761 \mathrm{k}$ 3.931243f $3.305810 \mathrm{I}$ 3.126856 $5.355038 \mathrm{j} 5.135931 \pm 1.082168 \mathrm{i}$ 95 6.282346d 5.053958h 6.357054i 3.928726i 4.438210f 3.791982f $3.624745 f$ 4.938007i 5.669297 . $1.242835 f$

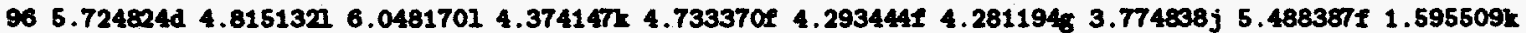

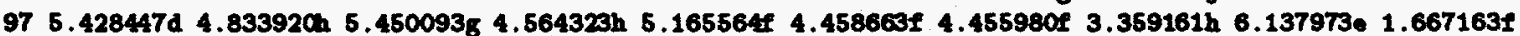

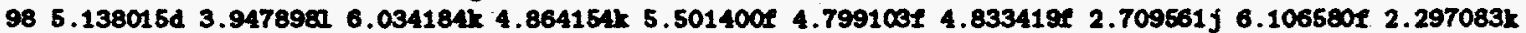
$994.698149 \mathrm{~g} 3.781740 \mathrm{~h} 5.815444 \mathrm{~g} 4.749738 \mathrm{~h} 5.930922 \mathrm{~g}$ 5.528992h 5.4378071 2.0484521 6.118047g 2.6320671

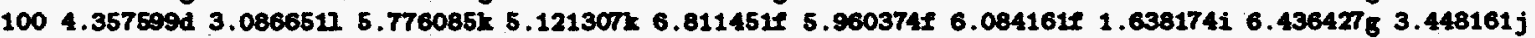

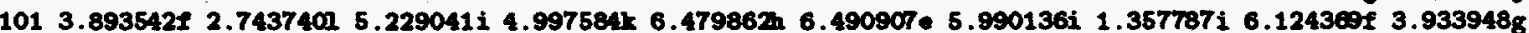

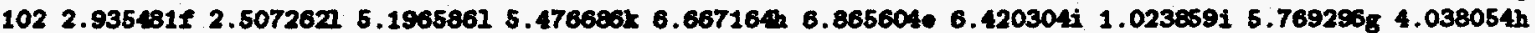

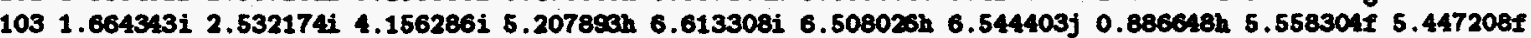

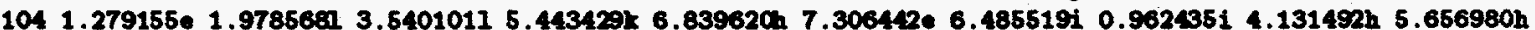
$1050.855466 i$ 1.804765i $2.465547 \mathrm{~h} 4.274868 \mathrm{~h}$ 5.584536i 6.6834181 6.966055h $1.081533 \mathrm{~h} 3.103525 \mathrm{~g} 6.2116261$

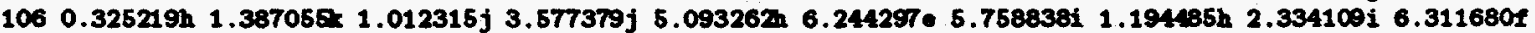

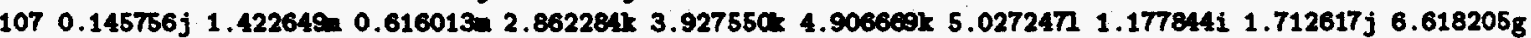
$1080.097559 \mathrm{n} 1.431957 \mathrm{n} 0.384001 \mathrm{n} 2.398210 \mathrm{k}$ 2.795168k $3.541954 \mathrm{k} 4.3973091$ 1.279823i $1.29686616 .109400 \mathrm{~g}$ $1090.085292 \mathrm{~m}$ 1.503548k $0.154096 \mathrm{~m} 2.476479 \mathrm{i} 1.746191 \mathrm{i}$ 2.35297m $3.194055 \mathrm{k} 1.413551 \mathrm{~h} 0.637762 \mathrm{j} 5.940418 \mathrm{~g}$ $1100.079815 \mathrm{n} 1.321089 \mathrm{n} 0.114152 \mathrm{n} 1.744318 \mathrm{k} 0.9410131$ 1.399562 j $2.07760811 .38225410 .300653 \mathrm{k}$ 5.903153k

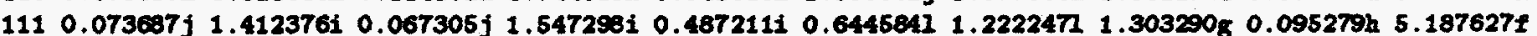
1120.0648571 1.690540i $0.052925 \mathrm{n} 1.392305 \mathrm{i} \quad 0.227972 \mathrm{i} \quad 0.31778610 .65357 \mathrm{en} 1.471980 \mathrm{~h} \quad 0.073460 \mathrm{j} 4.135488 \mathrm{~g}$ 1130.0626311 1.702444i $0.041067 \mathrm{~m} 1.295266 \mathrm{k} \quad 0.14958 \mathrm{~K} \quad 0.2132411 \quad 0.318057 \mathrm{~m}$ 1.418936h $0.051558 \mathrm{j} 4.782829 \mathrm{~h}$ 1140.05587 on 1.228653 n 0.036321 n $1.256600 \mathrm{k} 0.091121=0.117393 \mathrm{k}$ o.133050 1.33101610 .0628341 3.328426k

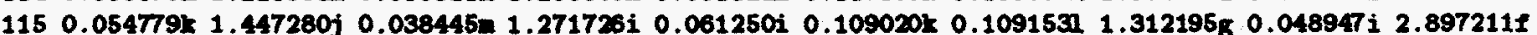
$1160.065484 \mathrm{n} 1.3817611 \quad 0.037359 \mathrm{n} 1.157196 \mathrm{k} \quad 0.064827 \mathrm{~m} \quad 0.0969281 \quad 0.097549 \mathrm{n} \quad 1.272370 \mathrm{i} \quad 0.047787 \mathrm{k}$ 2.127287 k 1170.065723 j 1.32099110 .0376981 1.151458k $0.06375270 .088045 k 0.0982671$ 1.429681h $0.043023 k$ k $1.500654 i$

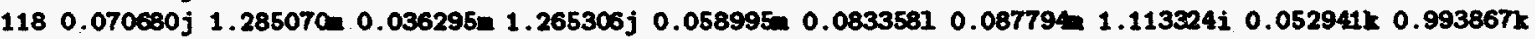

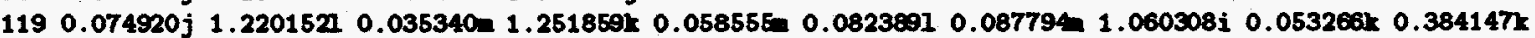

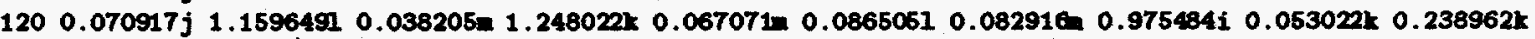

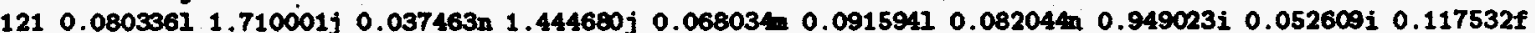
$1220.072647 \mathrm{j} 1.235136 \quad 0.050575 \mathrm{n} 1.460716 \mathrm{k} \quad 0.0759960 .0946511 \quad 0.083866 \mathrm{n} \quad 0.848247 \mathrm{i} \quad 0.060728 \mathrm{k} \quad 0.0884641$ $1230.075808 \mathrm{n} 1.35124510 .071178 \mathrm{n} 1.729587 \mathrm{~K} \quad 0.08694 \mathrm{Cm} \quad 0.10177110 .088422 \mathrm{n} \quad 0.805834 \mathrm{i} \quad 0.075697 \mathrm{k} \quad 0.040869 \mathrm{j}$

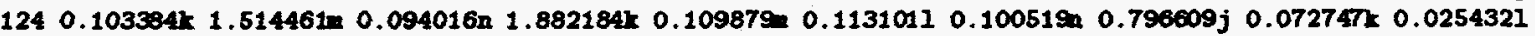
$1250.146139 \mathrm{~g} 1.516257 \mathrm{i} \quad 0.164285 \mathrm{~m} 1.954902 \mathrm{j} \quad 0.089289 \mathrm{i} \quad 0.151116 \mathrm{k} \quad 0.128898 \mathrm{k} \quad 0.720982 \mathrm{i} \quad 0.131045 \mathrm{~h} \quad 0.017685 \mathrm{j}$ $1260.284589 \mathrm{i} 2.312017 \quad 0.243428=2.5479331 \quad 0.271673 k \quad 0.223951 k 0.1683511 \quad 0.878031 i \quad 0.165173 k \quad 0.027712 k$

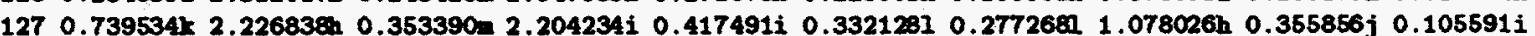

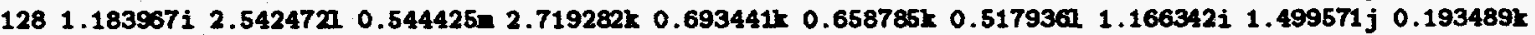
129 1.737216i $2.83362510 .930972 \mathrm{j} 3.387362 k$ 1.1494831 $1.167150 \mathrm{j} 1.230159 \mathrm{j}$ 1.252821h $1.759487 \mathrm{i} 0.588805 \mathrm{~h}$ $1302.79297613 .20009111 .74298614 .472196 k 2.03106 \% 1.863027$ j $2.005994 k 2.445798 j 2.726681$ j 0.8471241 $1313.742352 \mathrm{~d} 4.066951 \mathrm{~h} 2.949756 \mathrm{f} 4.365749 \mathrm{~g} 3.520040 \mathrm{~g} 3.362428 \mathrm{f} 3.106460 \mathrm{f} 2.815254 \mathrm{~h} 3.600055 \mathrm{~d} 1.599812 \mathrm{~h}$ 1325.037372 e $3.98349 \mathrm{hh} 4.376397 \mathrm{f} 5.231196 \mathrm{~g} 5.041959 \mathrm{j} 4.709868 \mathrm{f} 4.483763 \mathrm{~h}$ 3.428572g $4.853792 \mathrm{~d} 2.152595 \mathrm{~g}$ $1336.038073 d 4.560393 i$ 6.893372h $4.865743 j 6.919886$ e 6.459724 e $6.649572 d$ 4.663337i 6.4688641 3.152104g $1346.314475 \mathrm{~d} 4.985265 \mathrm{i} 7.740875 \mathrm{f}$ 6.009275h $7.486179 \mathrm{~g}$ 7.544606 $7.430162 \mathrm{~d}$ 6.642892h $7.072826 \mathrm{e}$ 3.899700h 135 6.423164d 5.133530i 6.079545f 6.376440k 7.442543e 7.069647 6.99719ef $5.246944 \mathrm{~g} 7.259396 f 4.190145 \mathrm{~g}$ 1367.039554 e $5.70862916 .592126 \mathrm{~g} 6.243613 \mathrm{~g} 6.681315 f \quad 6.687501 f 6.877002 \mathrm{f} \quad 6.579760 \mathrm{k} 6.712400$ e $4.191617 \mathrm{j}$ 


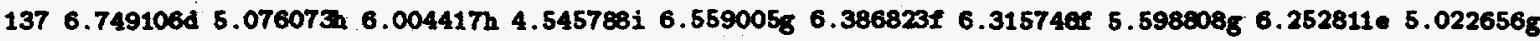
1386.575755 6.396232 6.638414k 4.131454k 6.449165f 6.147261i 6.195603F 5.7607648 6.135233f 5.5615458

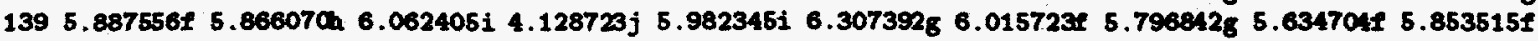

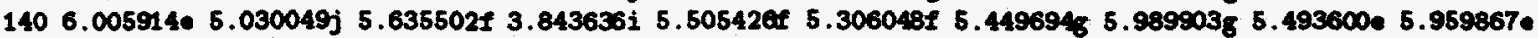

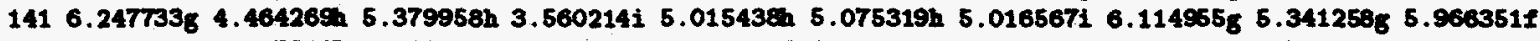

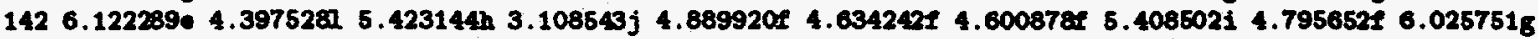

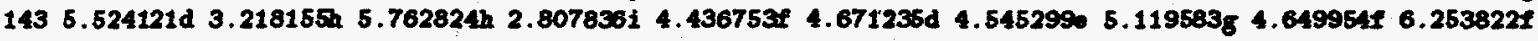

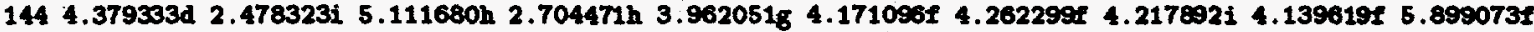
$1453.199751 \mathrm{~d} 2.025202 \mathrm{j} 4.152905 \mathrm{i} 2.158065 \mathrm{j} 3.075099 \mathrm{f} 3.326898 \mathrm{~d} 3.369844 \mathrm{2} .630949 \mathrm{~h}$ 3.450362f $5.05151 \mathrm{hh}$ $1462.381593 d 1.62910 \mathrm{~m} 3.462719 \mathrm{j} 1.743971 \mathrm{z} 2.55308202 .805788 d 2.908089$ 2.453127j $2.799534 \mathrm{f} 4.433891 \mathrm{~h}$

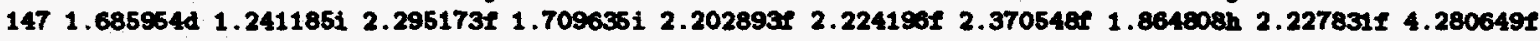

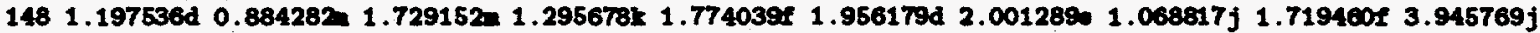

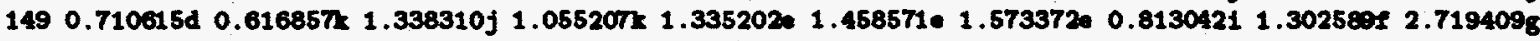
$1500.46985900 .4460700 .739914 \mathrm{n} 0.9937591$ 1.085382f $1.230379 d 1.31990500 .410517 \mathrm{j} 0.993970 \mathrm{f} 2.444907 \mathrm{k}$

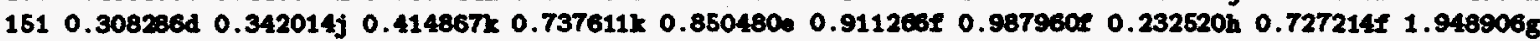

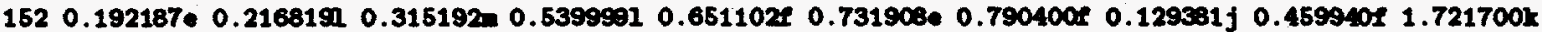

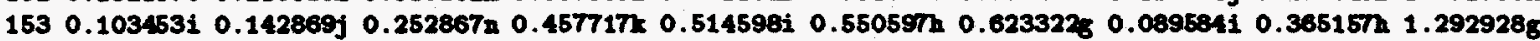

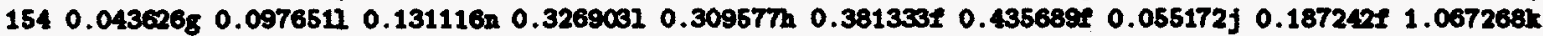
$1550.0227420 \quad 0.06537 q$ a $0.093655 \mathrm{n} \quad 0.233150 \mathrm{k} \quad 0.235513 \mathrm{i} \quad 0.3358451 \quad 0.328165 \mathrm{a} \quad 0.031658 \mathrm{j} \quad 0.141933 \mathrm{k} \quad 0.792890 \mathrm{~h}$

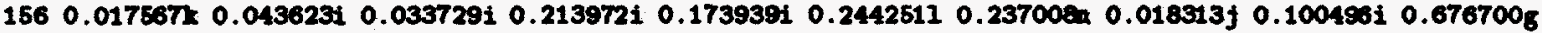
$1570.0101221 \quad 0.027761 \mathrm{k} 0.02341 \mathrm{An} 0.113299 \mathrm{k} 0.130276 \mathrm{j} \quad 0.1628341 \quad 0.164082 \mathrm{~m} \quad 0.010612 \mathrm{j} \quad 0.03221510 .537819 \mathrm{~h}$

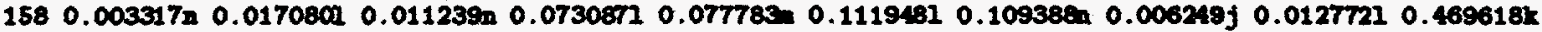

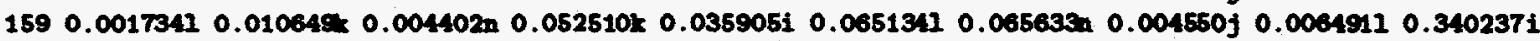
$160 \quad 0.000419 n \quad 0.0071621 \quad 0.001967 n \quad 0.0353861 \quad 0.01940120 .0396921 \quad 0.041021 n \quad 0.001724 \mathrm{j} \quad 0.00250810 .285805 \mathrm{k}$

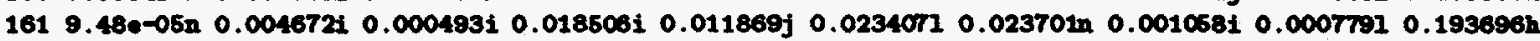

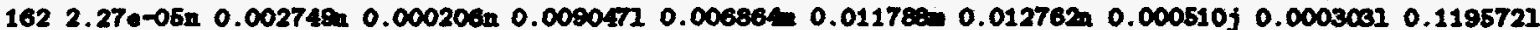

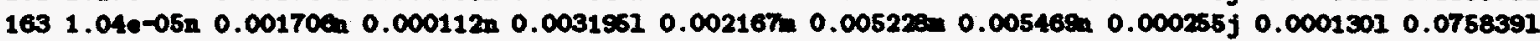

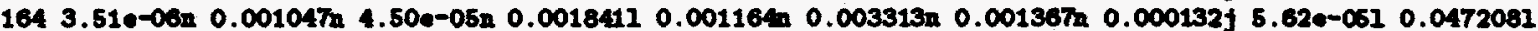

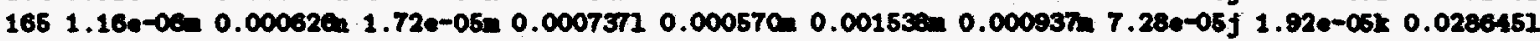

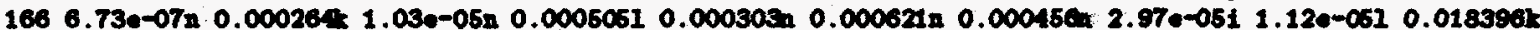

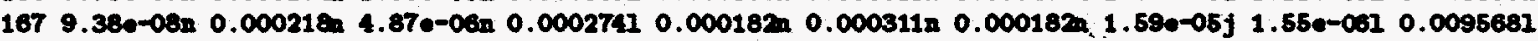

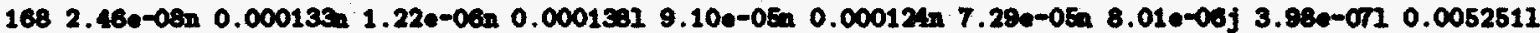
$1698.24 \bullet-09 n$ 8.29e-05 3.00e-07n 6.42e-051 9.27e-051 5.90e-05n 4.38e-05n 3.88e-08j $1.30 e-071$ 0.001669n

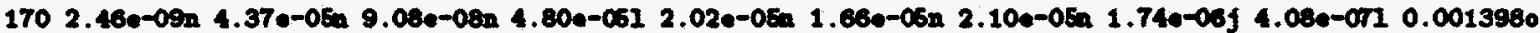

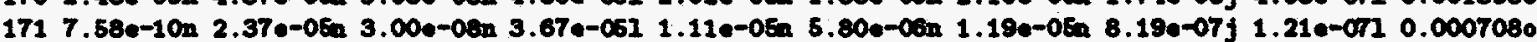

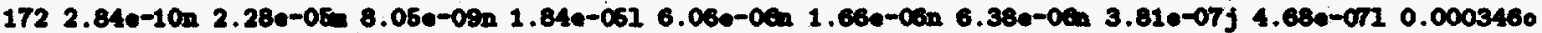

Table 9

Set C Hass Gain Yielde

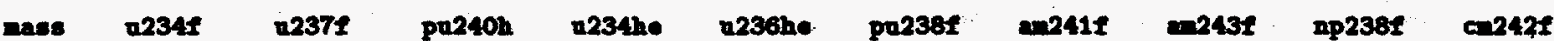

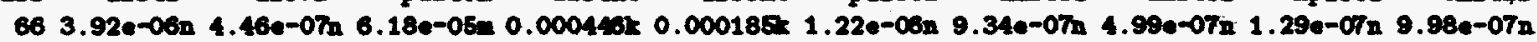

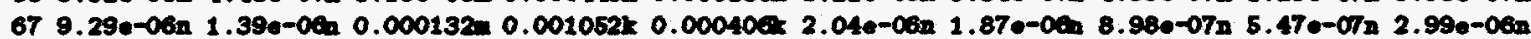
$682.68 e-05 n$ 1.98e-06n $0.000245 n 0.001472 n \quad 0.000787 n$ 6.12e-06n $5.71 e-06 n$ 2.20e-06n 2.29e-06n 3.99e-06n 69 6.19e-05n 5.96e-0en $0.000501 \mathrm{n} 0.002060 \mathrm{n} 0.00109 \mathrm{n}$ 2.04e-05n 9.34e-0en 4.99e-06n 6.97e-06n 5.99e-06n $700.000207 n$ 1.19e-0En $0.001002 n \quad 0.003435 n$ 0.001994n 7.14e-05n 2.08e-05n 1.60e-05n 1.59e-05n 1.50e-05n

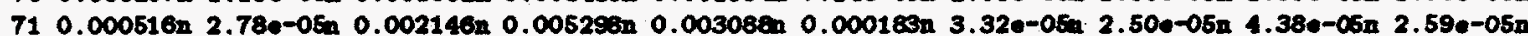

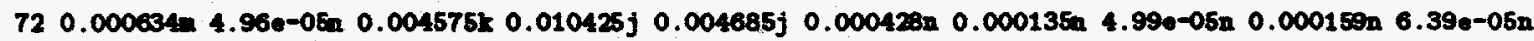

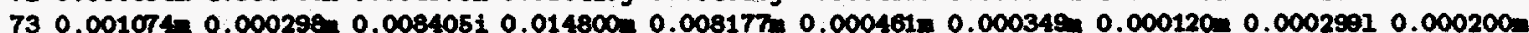

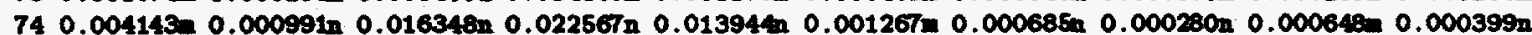
$\begin{array}{lllllllllll}75 & 0.011764 n & 0.003172 n & 0.032695 n & 0.034342 n & 0.019920 n & 0.002436 n & 0.001350 n & 0.000699 n & 0.002890 & 0.000898 n\end{array}$

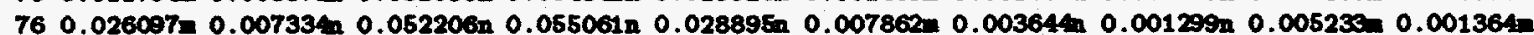

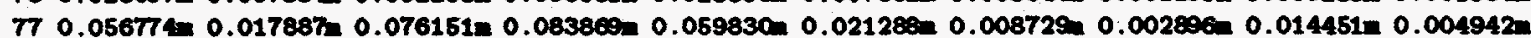
$780.088793 n \quad 0.046588 n \quad 0.110352 n \quad 0.135411 n \quad 0.109573 n \quad 0.036494 n \quad 0.018485 n \quad 0.004992 n \quad 0.030398 n 0.012980 n$

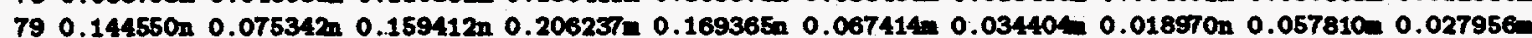

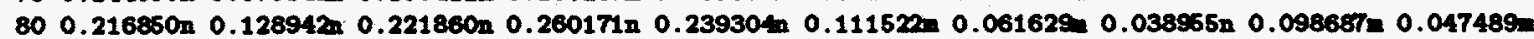

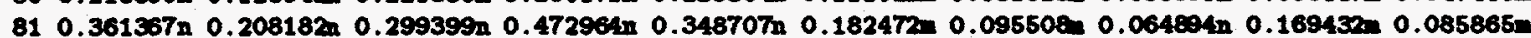

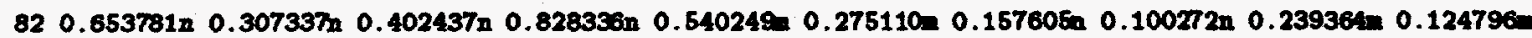

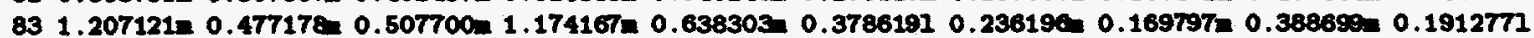

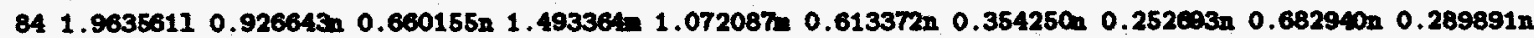

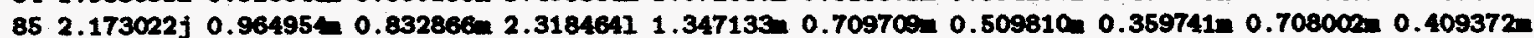

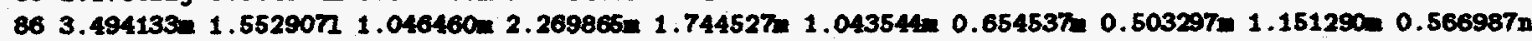

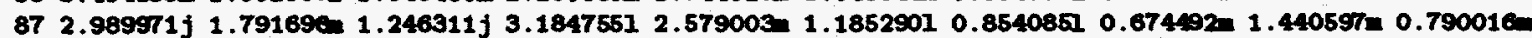

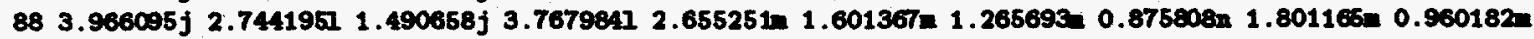


895.38994613 .3892451 1.747368i 4.3000281 3.659138j 2.074393m $1.357625 \mathrm{~m} 1.124441 \mathrm{~m} 2.3006141 \quad 1.219797 \mathrm{~m}$ 906.0388631 3.9154341 $1.952523 i$ 4.7070811 3.820010i 2.4346801 1.715407 $1.369531 \mathrm{~m} 2.6674051 \quad 1.517704 \mathrm{~m}$ $916.425058 \mathrm{j} 4.386102 \mathrm{Z}$ 2.450160h 5.0029941 4.254236t 2.9488121 2.126664i $1.748210 \mathrm{n}$ 3.2237501 $1.887072 \mathrm{~m}$ $926.110431 \mathrm{j} 4.81068412 .872168 \mathrm{~h} \quad 5.1835371 \quad 4.8180801 \quad 3.47765512 .939793 \mathrm{j} 2.106981 \mathrm{~m} 3.67786812 .2696591$ 93 6.155856i 5.2995362 .8969501 5.042664k 5.069331 j 4.11273612 .8187772 .57896214 .38003312 .6611081 $946.41868215 .4640651 \quad 3.4938181 \quad 5.1658011 \quad 5.3616081 \quad 4.7408671$ 3.688954 $3.143605=4.84638113 .3580671$ $956.334472 j \quad 5.6106861$ 3.697669i 5.032528 j $5.261609 j 4.42413113 .751411 i 3.73942615 .04469914 .0156321$ 96 6.2246291 5.8041281 4.1352221 5.2921761 5.3075191 5.3370941 4.7406491 4.4531581 5.4219061 4.6337651 $976.03874815 .87993814 .276969 i 5.042643 j 5.445944 j 5.65611014 .636896 i$ 4.6779291 5.65034514 .8449371 98 5.7666171 5.938378x 4.7593991 4.8576421 5.2252981 5.6221031 5.4455341 5.0872621 5.6973421 5.2054081 99 5.075879i 6.197933k 4.868966i 4.254718j 5.126146j 6.112515k 5.398710i 5.398364k 6.164795k 5.427610k 1004.1361381 5.941237k 5.2077081 3.5088731 4.7906201 6.1088821 5.909581k 5.4456011 6.0954361 5.6492501 $\begin{array}{lllllllllll}101 & 3.3942071 & 5.8040441 & 5.3979721 & 3.167290 & 4.2015781 & 6.0481021 & 6.1041941 & 5.9247191 & 6.0555291 & 5.9533341\end{array}$ $1022.669934 \mathrm{~m} .68501 \mathrm{~g}$ 5.4386041 2.970021 3.55305616 .13920216 .06363215 .99493816 .14574315 .9733851

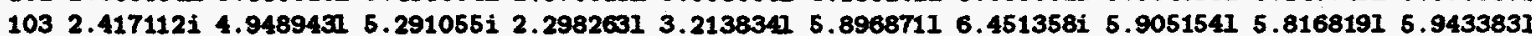
$1041.3216091 \quad 4.6392301 \quad 5.21849112 .084273 \quad 2.8201321 \quad 5.7067251 \quad 6.02405315 .99053315 .70729415 .9451201$ $1051.237440 \mathrm{i} 2.6885361$ 4.754474g 2.132680k 2.504589j 4.762554k $5.3358241 \quad 5.866728 \mathrm{k} 4.2405141$ 5.785440k $1060.429079 \mathrm{~L} 2.0607841$ 4.021683i $1.529381 \mathrm{n} 1.814851 \mathrm{~m} 4.24073914 .81345915 .62498613 .84082115 .4838601$ $1070.268439 \mathrm{n} 1.122885 \mathrm{~m}$ 3.147594n $1.233372 \mathrm{n} 1.585501 \mathrm{~m}$ 3.010741= 5.12589315 .24532412 .44415915 .1342541 $1080.185862 n$ n 0.495590 n 2.6386141 1.420996n 1.376317 2.1406231 4.11715414 .9361901 1.6564061 4.4850871

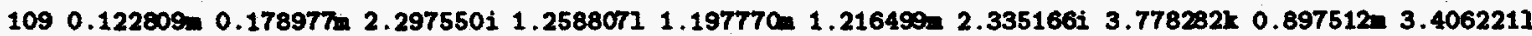

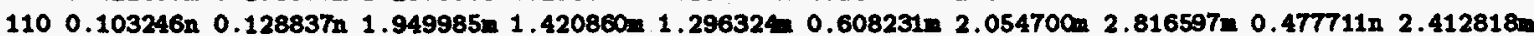
$1110.058195=0.069377 \mathrm{n} 1.671646 \mathrm{i} 1.318350 \mathrm{j} 1.106070 \mathrm{j} 0.305820 \mathrm{n} 1.001643 \mathrm{i} 1.747796 \mathrm{n} 0.229225 \mathrm{n} 1.427738 \mathrm{~m}$ $1120.072272 n \quad 0.059466 \mathrm{n} 1.568710 \mathrm{i} 1.471315 \mathrm{j} 1.160652 \mathrm{j} \quad 0.163104 \mathrm{n} 0.743581 \mathrm{i} \quad 0.928829 \mathrm{~m} 0.129380 \mathrm{n} 0.738830 \mathrm{~m}$

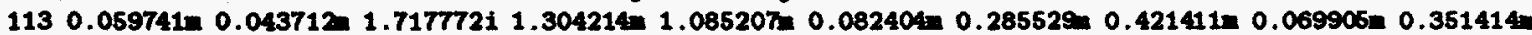
$1140.058850 \mathrm{n} 0.03667 \mathrm{n} 1.248885 \mathrm{~m} 1.324603 \mathrm{n} 1.047029 \mathrm{n} \quad 0.081552 \mathrm{n} \quad 0.134957 \mathrm{n} \quad 0.219608 \mathrm{n} 0.073647 \mathrm{n} 0.179597 \mathrm{n}$ $1160.068516 \mathrm{k} 0.041579 \mathrm{n} 1.19307 \mathrm{~g}_{\mathrm{g}} 1.328335 \mathrm{k} 1.044624 \mathrm{k} 0.083435 \mathrm{~m} 0.169600 \mathrm{i} \quad 0.101991 \mathrm{~m} 0.071175 \mathrm{~m} 0.110575 \mathrm{~m}$

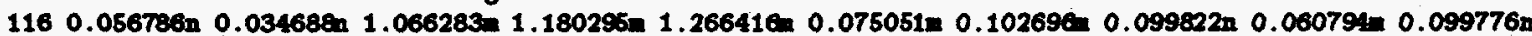

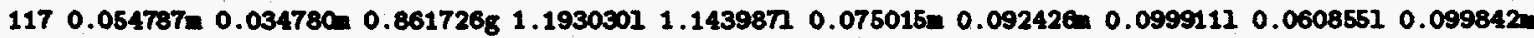

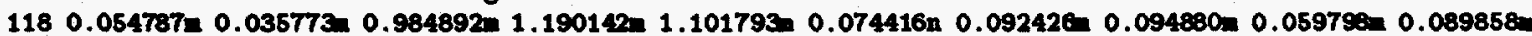

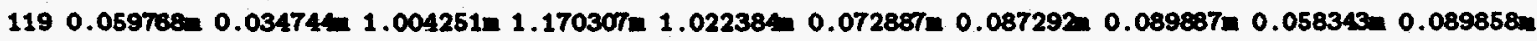

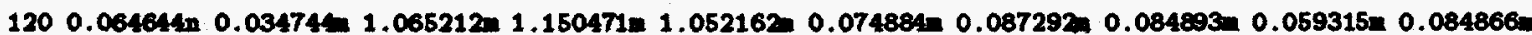
$1210.069617 \mathrm{n} \quad 0.036618 \mathrm{n} 1.166651 \mathrm{n} 1.33894 \mathrm{n}$ 1.16142E $0.077829 \mathrm{n} 0.093612 \mathrm{n} \quad 0.084850 \mathrm{n} \quad 0.061501 \mathrm{n} 0.082838 \mathrm{n}$

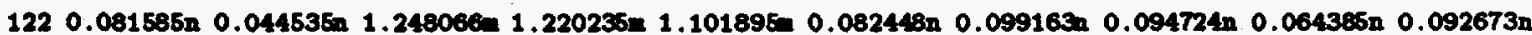
$1230.099453 \mathrm{n} \quad 0.056410 \mathrm{n} 1.369443 \mathrm{~m} 1.299239 \mathrm{~m} 1.17127 \mathrm{n}$ a $0.092797 \mathrm{n} \quad 0.114414 \mathrm{n} \quad 0.099669 \mathrm{n} \quad 0.072071 \mathrm{n} \quad 0.107203 \mathrm{n}$ $1240.119440 \mathrm{n} 0.071312 \mathrm{~m} 1.536602 \mathrm{n} 1.430212 \mathrm{n} 1.29325 \mathrm{n} \quad 0.109895 \mathrm{n} \quad 0.12552 \mathrm{n} \quad 0.119679 \mathrm{n} \quad 0.083231 \mathrm{n} \quad 0.118397 \mathrm{n}$

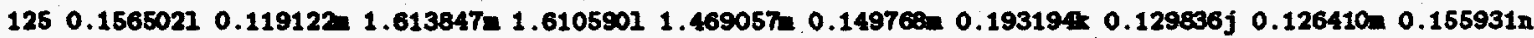
$\begin{array}{llllllllllllll}126 & 0.2592561 & 0.177882 & 2.0663751 & 2.1360811 & 1.5964790 .2303621 & 0.2616741 & 0.2309881 & 0.184736 & 0.2689081\end{array}$ $1270.388771 \mathrm{j} \quad 0.248168 \mathrm{~m}$ 2.0021281 2.1971691 $1.955418 \mathrm{~m} \quad 0.369425 \mathrm{~m} \quad 0.380497 \mathrm{~m} \quad 0.349178 \mathrm{~m} \quad 0.272261 \mathrm{~m} \quad 0.373166 \mathrm{~m}$ $1280.647501 \mathrm{n} 0.574035 \mathrm{n} 2.91904112 .510235 \mathrm{~m} 2.234180 \mathrm{n} \quad 0.659086 \mathrm{n} \quad 0.613680 \mathrm{n} \quad 0.568125 \mathrm{n} \quad 0.586200 \mathrm{n} 0.604254 \mathrm{n}$

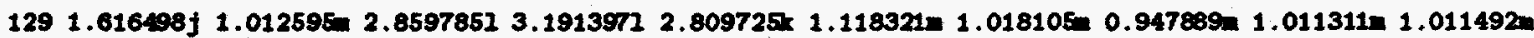
$1302.540781 \mathrm{n} 2.029719 \mathrm{n} 4.3094791$ 3.478196 $3.35067512 .227635 \mathrm{~m}$ 1.867115m $1.736782 \mathrm{~m}$ 2.090704 $1.906686 \mathrm{~m}$ 1313.735887 j 3.2134561 4.460339i 3.7083361 3.8099841 3.91571914 .1683281 3.1947241 $3.476196 j 3.4554961$ $1324.364303 \mathrm{j} 4.7552711$ 4.590675k $5.261580 \mathrm{k} 4.873230 \mathrm{~K}$.3041961 $4.321860 \mathrm{j} 4.7221761$ 5.147919k 4.8870631

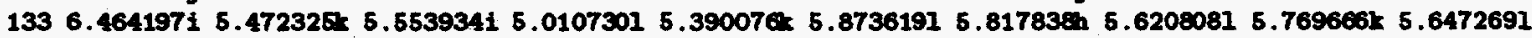
$1345.749837 \mathrm{j} 6.81900415 .473597 \mathrm{k}$ 5.542541k 5.8448951 6.6049411 7.828394 6.237599k 8.045231 j 6.2964521 $1355.581853 k 6.624940 \times 5.341134 h \quad 5.4597931 \quad 6.57362516 .74525216 .506050 h \quad 6.77218216 .429053 j 6.7648611$ $1366.49210816 .718522 x \quad 5.77637316 .50016016 .1097201 \quad 7.4207001$ 7.7963771 7.4572411 7.278368k 8.3029741 $1375.86258816 .46697 \mathrm{si}$ 4.541609i 5.0319601 5.139651i $6.5103391 \quad 5.838064 i \quad 7.15265616 .55569616 .9813471$ $1386.58717716 .28765615 .445086 k 5.2618421 \quad 5.07149516 .29619116 .6554786 .60598816 .19292916 .4410331$ $1396.00410616 .0684291 \quad 4.5837501 \quad 5.2792361 \quad 5.2035291 \quad 5.72401716 .751272 i \quad 5.95088815 .73003615 .7756291$ $1405.787173 h$ 6.640428 $3.853162 i$ 3.930930j $4.118092 j$ 5.6143201 4.9414428 5.4816151 5.7866801 5.3709601 $1416.505636 \mathrm{j} 5.57509213 .587640 \mathrm{i} 3.680841 \mathrm{j} 4.054839 \mathrm{j} 4.78516214 .719010 \mathrm{i} 4.95243014 .93355014 .7686461$ $1425.990981 j \quad 5.4568451 \quad 3.899261 j$ 4.3256881 4.2624991 5.8752721 5.4485421 5.2463261 5.7717461 5.3819581 $1436.142069 \mathrm{j} 4.8168761$ 3.102701i 3.285722j $3.939119 \mathrm{j} 4.53545113 .888470 \mathrm{i}$ 5.2025901 4.37998614 .9660821 $1445.443792 j 4.85257912 .671651 i 2.695224 j 3.486658 j 3.92587613 .414493 i \quad 4.15368114 .14053913 .9491471$ 1453.9890721 3.6320611 2.687350k 2.340618 2.551063m 3.2364641 3.4478231 3.41437413 .26522513 .2975301

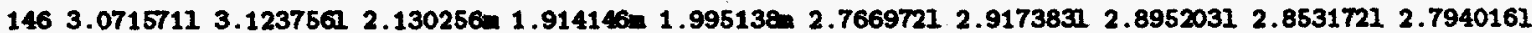
$1472.017734 \mathrm{j} 2.6163921 .7859361$ 1.282374h $1.732623 \mathrm{~h} 2.236537 \mathrm{~m} 2.149320 \mathrm{i} 2.33612912 .392061 \mathrm{~h} 2.180079 \mathrm{~m}$ $1481.434487 \mathrm{~m} 1.856962 \mathrm{~m} 1.511467 \mathrm{~m} 1.047696 \mathrm{n} 1.27061 \mathrm{~m} 1.757320 \mathrm{~m} 1.902551 \mathrm{~m} 1.865712 \mathrm{~m} 1.770249 \mathrm{~m} 1.806850 \mathrm{~m}$ $1491.035806 \mathrm{k} 1.4425501$ 1.367703h $0.622681 \mathrm{n} 0.880697 \mathrm{n} 1.597527 \mathrm{~m} 1.570523 \mathrm{i} 1.556353 \mathrm{~m} 1.750311 \mathrm{~m} 1.492667 \mathrm{~m}$ $1500.637331 \mathrm{n} 1.042320 \mathrm{n} 1.069353 \mathrm{n} 0.483257 \mathrm{n} 0.684734 \mathrm{n} 0.982394 \mathrm{n} 1.239334 \mathrm{n} 1.233071 \mathrm{n} 0.903318 \mathrm{n} 1.241071 \mathrm{n}$

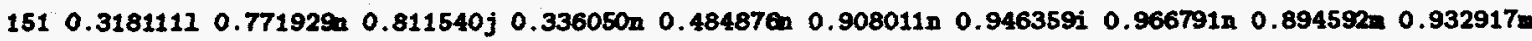
$1520.248632 n \quad 0.524515 n \quad 0.653045 n \quad 0.237211 n \quad 0.35623$ n $0.678513 n \quad 0.811300 n \quad 0.797353 n \quad 0.643832 n \quad 0.750385 n$ $1530.1487611 \quad 0.346378 \mathrm{n} \quad 0.580414 i \quad 0.181944 \mathrm{k} \quad 0.279672 \mathrm{k} \quad 0.468972 \mathrm{n} \quad 0.629203 \mathrm{i} \quad 0.588048 \mathrm{n} \quad 0.432424 \mathrm{n} \quad 0.545759 \mathrm{n}$ $1540.080563 n \quad 0.188033 n \quad 0.400489 n \quad 0.089192 n \quad 0.141543 n \quad 0.319398 n \quad 0.426601 n \quad 0.418833 n \quad 0.288283 n \quad 0.407957 n$ 
$1550.050721 n \quad 0.118758$ n $0.279493 i \quad 0.064245 n \quad 0.092028 n \quad 0.239475 n \quad 0.3984771 \quad 0.308974 n \quad 0.201793 n \quad 0.292371 n$ $1560.020835 \mathrm{n} \quad 0.057400 \mathrm{n} \quad 0.22609210 .047118 \mathrm{j} 0.070480 \mathrm{j} 0.159650 \mathrm{n} 0.2624641 \quad 0.219272 \mathrm{n} 0.12492 \mathrm{n} \quad 0.204680 \mathrm{n}$

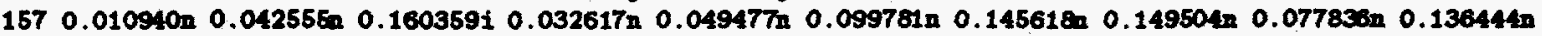

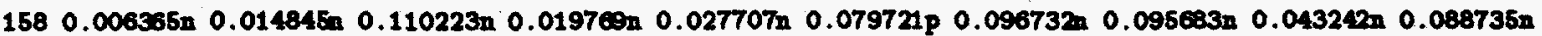

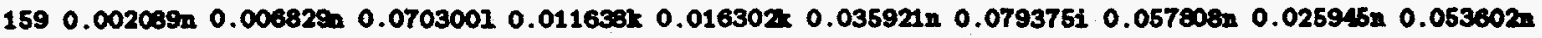
$160 \quad 0.001095 \mathrm{n} 0.002730=0.052202 \mathrm{n} 0.007020 \mathrm{n} 0.009921 \mathrm{n} 0.019994 \mathrm{n} 0.063022 \mathrm{n} 0.034972 \mathrm{n} 0.013453 \mathrm{n} 0.034201 \mathrm{n}$

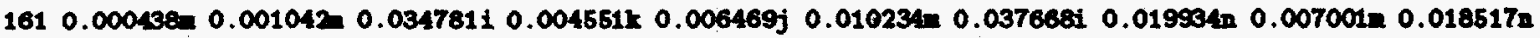
$1620.000159 \mathrm{n} 0.000297 \mathrm{n} 0.023470 \mathrm{n} 0.002767 \mathrm{n} 0.00385 \mathrm{~h}$ 0.003744m $0.008741 \mathrm{~m} 0.008480 \mathrm{~m} 0.002917 \mathrm{n} 0.007365$

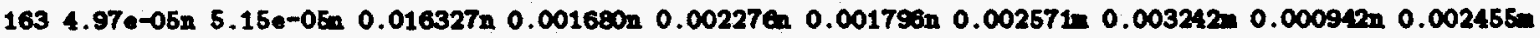

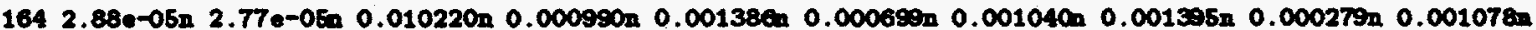

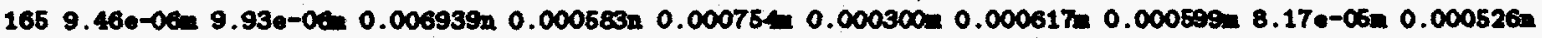

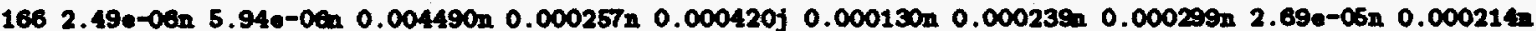

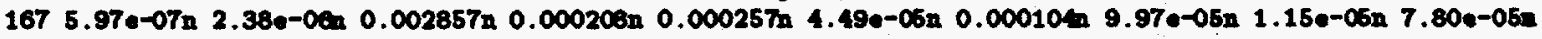
$1682.69 e-07 n$ 1.09e-06n $0.001837 n$ 0.000119n $0.000148 n 6.98 e-06 n \quad 4.16 e-05 n$ 5.58e-05n 4.13e-06n 2.44e-05n $1699.45 e-08 n$ 4.16e-07n $0.00115417 .91 e-05 n \quad 0.000119$ 3.99e-06n $1.46 e-05 n$ 2.39e-05n 1.25e-06n 1.46e-05n $1702.49 e-08 n$ 8.41e-08n $0.000741 n$ 3.91e-05n 4.46e-05n 1.70e-03n 8.33e-0en 1.30e-05n 3.08e-07n 1.01e-05n $1717.96 e-09 n 2.28 e-08 n \quad 0.000531 n$ 2.08e-05n 2.47e-05n 6.98e-07n 4.89e-0en 3.49e-06n 6.73e-08n 4.48e-06n

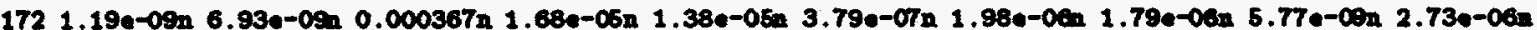

Tab10 10

Set D rase Ghain Yields

nas th227t th229t pa2311 w241t andih a242t crest c1249t cf251t es254t $668.53 e-08 n$ 3.02e-0en 9.85e-05n 1.36e-07n 0.000338n 9.57e-06n 1.04e-06n 3.00e-07n 9.36e-05n 9.39e-09n 67 2.16e-0Tn 7.18e-0an 0.000177n 2.62e-07n 0.000726n 1.91e-05n 2.000-0en 5.01e-07n 0.000140n 1.88e-08n 68 8.23e-07n 2.71e-0n $0.000345 n$ 5.81e-07n 0.001162 2.87e-06n 4.180-06n 3.00e-06n 0.000187n 3.75e-08n

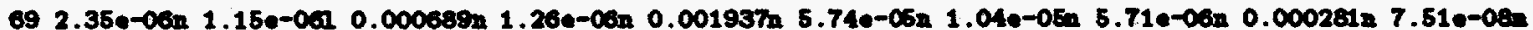
70 5.19e-06n 3.64e-0al 0.001280n 4.55e-00a 0.003200n 9.57e-06a 2.61e-05a 3.06e-05n 0.000374m 1.41e-07m

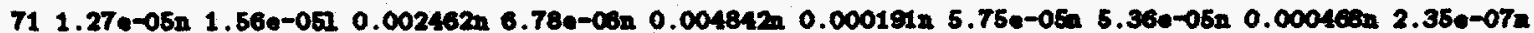

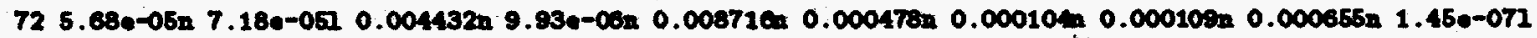

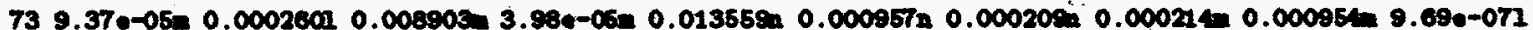

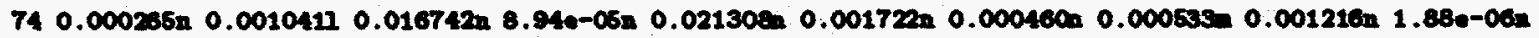

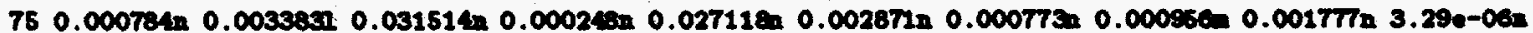

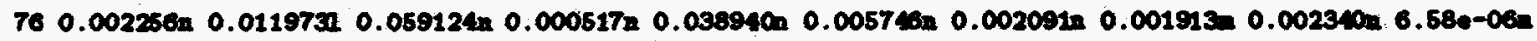

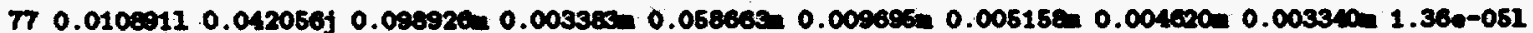

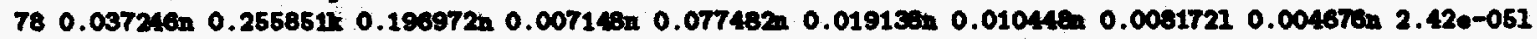

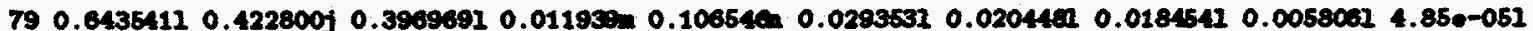

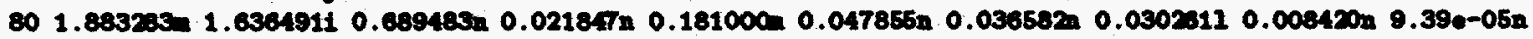

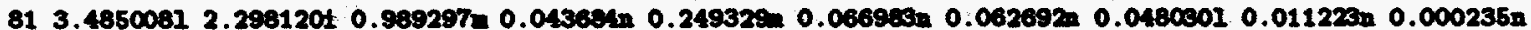

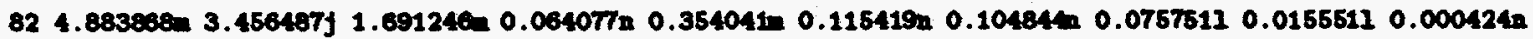

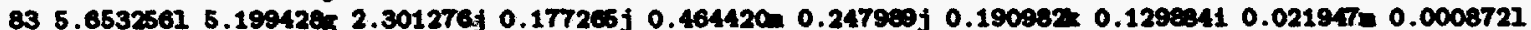

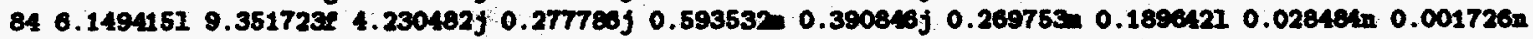

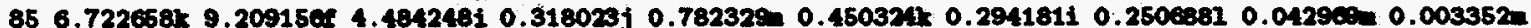

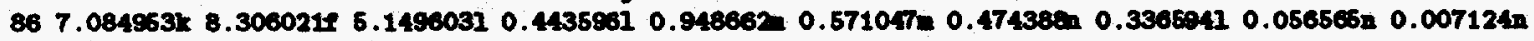

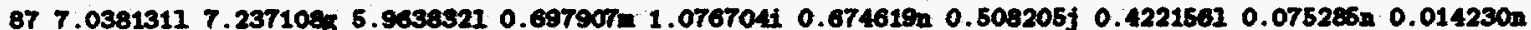

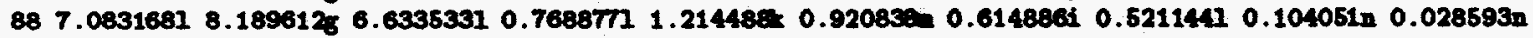

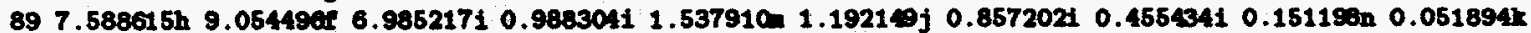

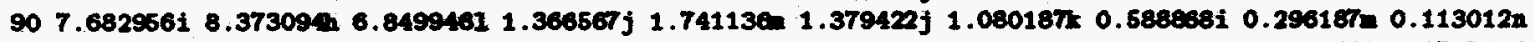

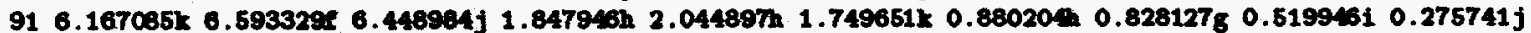
924.74028815 .985047 g 6.7738571 2.010441h $2.217020 \mathrm{j} 2.101637 \mathrm{i} 1.290145 \mathrm{j} 0.891135 \mathrm{~h} 0.581024 \mathrm{n} 0.357619 \mathrm{n}$ 934.3737771 1.844053 $6.71518012 .6871431 \quad 2.4243421$ 2.579150j 1.8166351 1.078319h 0.70168910 .470869 943.6644741 4.111386j 6.3595881 3.1926901 2.598156i 2.721451= 1.639377i 1.197019h 0.749811n 0.565227n $953.382430 \mathrm{j} 2.676360 \mathrm{f}$ 6.356236t $3.977545 \mathrm{~g}$ 3.124817i $3.294279 \mathrm{j} 2.351206 \mathrm{~h} 1.506089 \mathrm{f} 0.978900 \mathrm{j} 0.708960 \mathrm{j}$

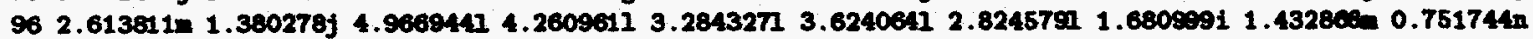
$972.3824401 \quad 0.731037 \mathrm{~g}$ 4.353557h $5.267398 \mathrm{~g} 3.545127 \mathrm{j} 4.280808 \mathrm{j} 2.896429 \mathrm{~h} 2.0856081$ 1.6497461 $0.975564 \mathrm{j}$

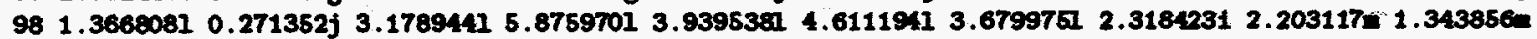
$991.432854 \mathrm{f} 0.152071 \mathrm{~h} 2.534050 \mathrm{~h} 6.624390 \mathrm{~g} 4.336901 \mathrm{t}$.3494241 4.169585e $2.800711 \mathrm{~g} .288263 \mathrm{~h} 2.2977051$

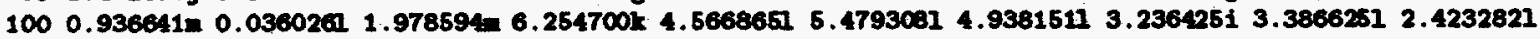

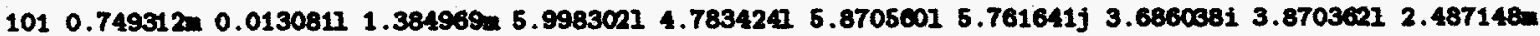
1020.5817250 .00655210 .6430326 .1778471 4.891798 6.6536341 5.956845 4.21424814 .25763212 .7142421

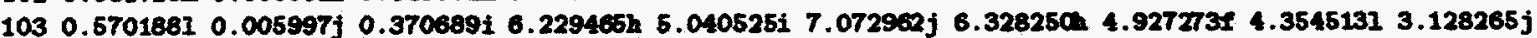

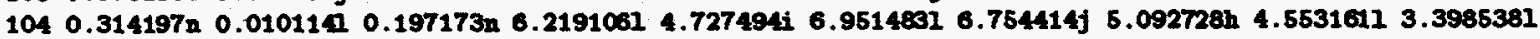

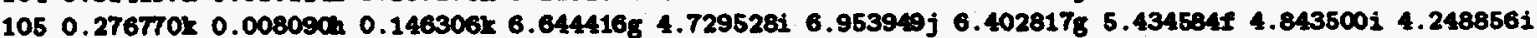

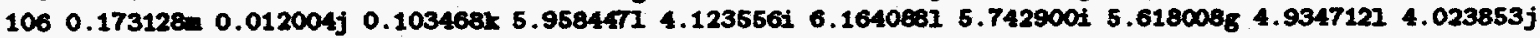

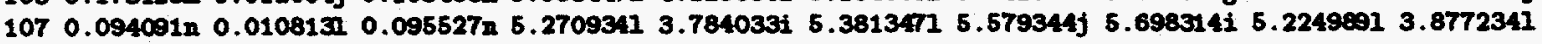


$108 \quad 0.053909 n \quad 0.01174210 .078791 n 4.2547391 \quad 3.513967$ A.1097451 $5.74371515 .725655 i \quad 5.32262113 .6840611$ $1090.033753 \mathrm{~m} 0.012898 \mathrm{k} 0.079651 \mathrm{j} 2.494585 \mathrm{i} 3.222369 \mathrm{i} 3.749192 \mathrm{j} 4.658722 \mathrm{j} 5.579277 \mathrm{~h} 5.225069 \mathrm{i} .721595 \mathrm{j}$

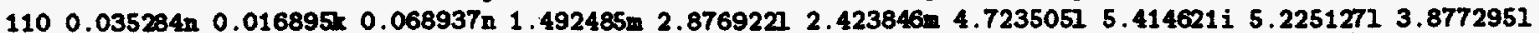
$1110.04098910 .021650 \mathrm{i} 0.095282 \mathrm{j} 0.951136 \mathrm{~h} 2.332104 \mathrm{i} 1.534712 \mathrm{j} 4.010336 \mathrm{i} 5.500346 \mathrm{~h} 5.017987 \mathrm{~h} 4.192541 \mathrm{~h}$ $1120.02871310 .020836 i \quad 0.058512 j \quad 0.559857 i \quad 2.423159 i \quad 0.642989 j 3.006297$ F $4.925475 \mathrm{~h} 5.334010 i$ 4.342348j

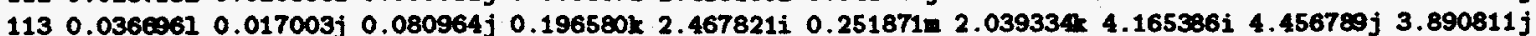
$1140.030304 \mathrm{n} 0.021486 \mathrm{n} \quad 0.061059 \mathrm{n} 0.148919 \mathrm{n} 1.838109 \mathrm{n} 0.105254 \mathrm{n} 1.093787 \mathrm{n}$ 3.624160i 4.60897814 .9434761 $1150.030215 \mathrm{~m} \quad 0.019833 \mathrm{i} \quad 0.083305 \mathrm{j} \quad 0.051124 \mathrm{~h} 1.720210 \mathrm{i} \quad 0.073742 \mathrm{j} \quad 0.559542 \mathrm{~h} 2.986375 \mathrm{~g} 4.559190 \mathrm{~g} 5.572666 \mathrm{~h}$

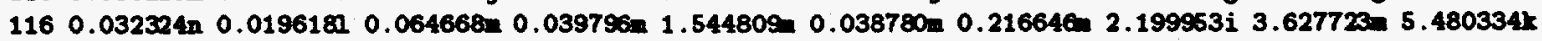

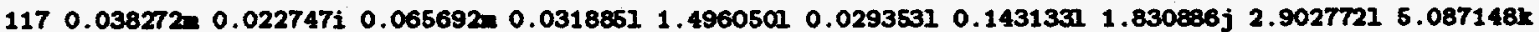

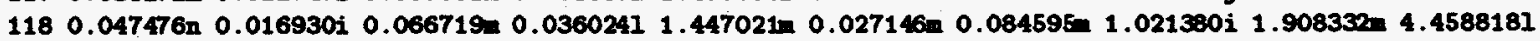

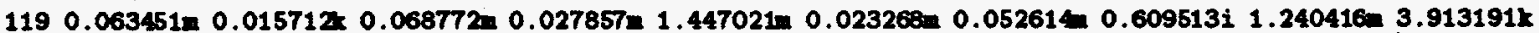

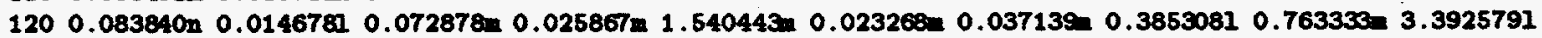

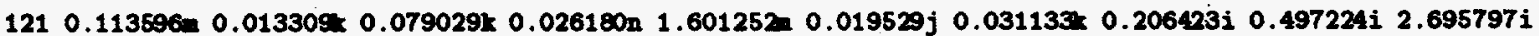
$1220.109142 \mathrm{n} \quad 0.013673 \mathrm{x} \quad 0.087210 \mathrm{n} \quad 0.029351 \mathrm{n} 1.78851 \mathrm{~m} \quad 0.032850 \mathrm{n} \quad 0.053311 \mathrm{n} \quad 0.18026610 .280555 \mathrm{n} 2.295830 \mathrm{~m}$

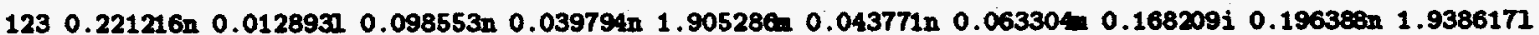
$1240.313720 \mathrm{n} 0.01110510 .116361 \mathrm{n} 0.050377 \mathrm{n}$ 2.296014 $0.054787 \mathrm{n} 0.071014 \mathrm{n} 0.13742810 .187420 \mathrm{n} 1.339261 \mathrm{n}$

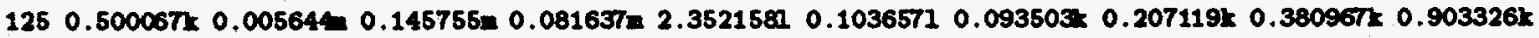
$\begin{array}{llllllllllllll}126 & 0.5691491 & 0.019045 j & 0.1932711 & 0.1701001 & 3.578009 & 0.1691261 & 0.1619911 & 0.4461291 & 0.5067191 & 0.8451231\end{array}$ 1270.67310810 .038914 h 0.291509 m $0.585649 \mathrm{~h} 2.321208 \mathrm{i} \quad 0.26666 \mathrm{~m}$ o.486344j $0.588926 \mathrm{j} 0.608631 \mathrm{j} 0.944831 \mathrm{j}$

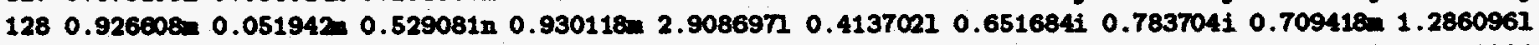
$1291.428327 \mathrm{~K} 0.118843 \mathrm{i} 1.034453 \mathrm{~h} 1.361734 \mathrm{~h} 3.60407 \mathrm{~h} 1.471990 \mathrm{~m} 1.270584 \mathrm{j} 1.630072 \mathrm{j} 0.929560 \mathrm{i} 1.960259 \mathrm{j}$ $1301.864048=0.417244 \times 2.084777=2.233984=4.59520912 .004841=1.850324 \mathrm{~m} 1.9741701$ 1.216432n $2.461702 \mathrm{~m}$

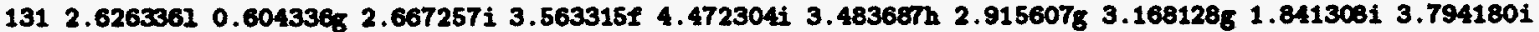

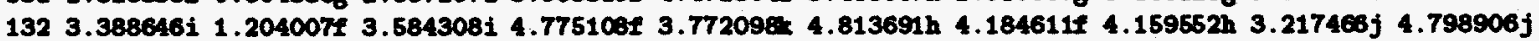
$1334.83907 \mathrm{~h}$ 2.966538F $5.092815 \mathrm{i}$ 5.507650 4.630996h $5.982260 \mathrm{i}$ 5.467867g $5.249503 \mathrm{~g} 3.911973 \mathrm{j} 5.058292 \mathrm{t}$ 1345.7298901 5.968143F 6.375640k 7.239502g 5.281205k 7.0033361 7.100712h 5.3905745 4.8542121 5.3546481 135 6.7766841 5.806931f 6.672920i 7.012588g 4.741971j 6.654152h 6.800380g 5.420276h 4.432428j $5.476295 j$ 1367.6661711 5.9745471 7.4761111 7.930157 G 6.2168851 6.553652k 5.041344j 6.662877k 5.6658151 5.1351961

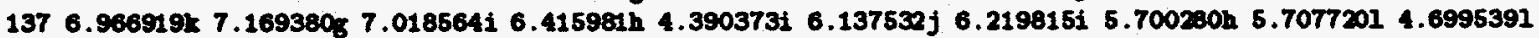
1387.2485731 7.888810 7.45263716 .9344481 4.404919 $5.97597516 .173800 \mathrm{~h}$.399914h 5.77718314 .4740471

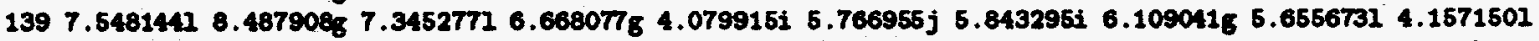

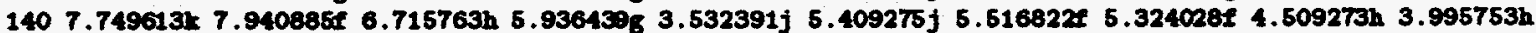
$1417.640609 \mathrm{j} 7.461594 \mathrm{f}$ 6.838927i 4.791970 $3.243333 \mathrm{i} 5.244436 \mathrm{j} 5.159751 \mathrm{~h}$ 5.431640h $4.918617 \mathrm{j} 4.583781 \mathrm{i}$ 1427.4489231 T.300350 6.2081051 4.720582t 3.0385311 4.490711j $4.731230 \mathrm{~h} 4.39359965 .4646041$ 4.5925521

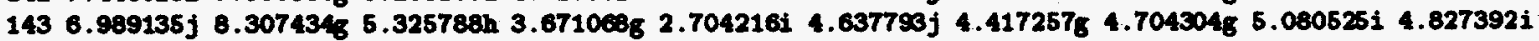
$1445.966224 j 9.758022 \mathrm{~h} 4.6944761$ 3.488406h $2.525339 \mathrm{i} 4.425765 \mathrm{j} 4.298894 \mathrm{j} 4.317734 \mathrm{~h} 5.05088313 .889585 \mathrm{j}$ $1453.10563215 .016667 j 3.208685 j 3.31313312 .2498593 .3508681$ 3.036074j $3.537418 \mathrm{k} 4.94703413 .6610601$

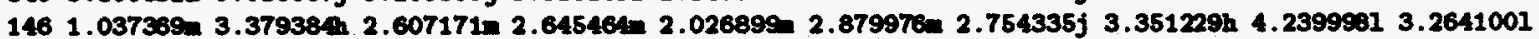

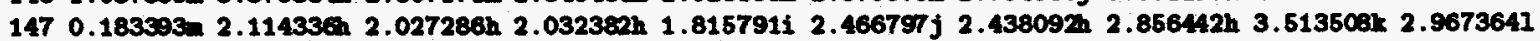

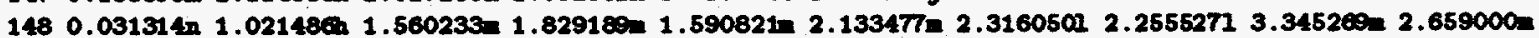
$1490.010101 \mathrm{n} 0.58823 \mathrm{Ch} 0.921756 \mathrm{i} 1.432518 \mathrm{i} 1.305875 \mathrm{i} 1.719593 \mathrm{j} 1.950343 \mathrm{j} 2.053920 \mathrm{~h}$ 3.020187 $2.264642 \mathrm{~m}$

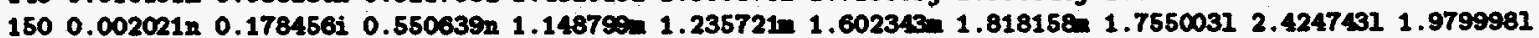
$1510.000505 \mathrm{n} 0.03799 \mathrm{~h} 0.316407 \mathrm{n} 0.83947 \mathrm{j}$ 0.897233i $1.218078 \mathrm{j} 1.14281 \mathrm{~h} 1.604719 \mathrm{~h} 2.0190511$ 1.700240k $1520.000202 \mathrm{n} 0.00975610 .179470 \mathrm{n} 0.72257 \mathrm{nn} 0.815177 \mathrm{n} 1.023991 \mathrm{n} 1.222758 \mathrm{n} 1.25587511 .520199 \mathrm{n} 1.624615 \mathrm{~m}$

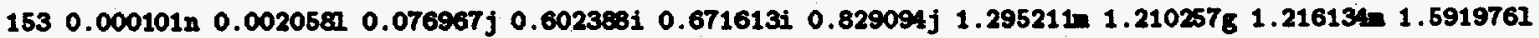

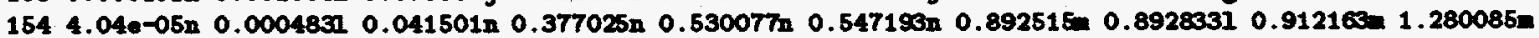
$1551.01 e-05 n$ 9.84e-051 0.020748n $0.307721 k \quad 0.341757 i \quad 0.361109 n \quad 0.696315 \mathrm{n} 0.616094 i \quad 0.713341 \mathrm{n} 0.984615 \mathrm{~m}$

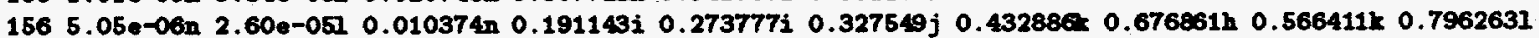
$1571.52 e-06 \mathrm{n} 7.16 e-061 \quad 0.005187 \mathrm{n} \quad 0.164262 \mathrm{j} \quad 0.179285 \mathrm{j} \quad 0.155052 \mathrm{k} 0.447993 \mathrm{n} 0.421298 \mathrm{i} 0.407619 \mathrm{n} 0.754309 \mathrm{k}$ 158 5.76e-07n 1.79 -061 $0.003216 \mathrm{n} 0.085871 \mathrm{n} \quad 0.153996 \mathrm{n} \quad 0.08754 \mathrm{n} \quad 0.328393 \mathrm{n} \quad 0.38103210 .305713 \mathrm{n} 0.684770 \mathrm{n}$

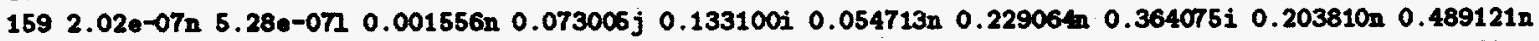
$1601.01 e-08 n$ 1.34e-0n $0.000737 n \quad 0.034595 n \quad 0.087645 n \quad 0.038331 n \quad 0.179528 n \quad 0.2329671 \quad 0.152907 n \quad 0.391501 n$ $1616.06 e-09 n \quad 3.58 e-081 \quad 0.000373 n \quad 0.022102 j \quad 0.060727 i \quad 0.0201501 \quad 0.119600 n \quad 0.193116 j \quad 0.101904 n \quad 0.293473 n$ $1623.03 e-09 n$ 8.86e-09n $0.000197 n \quad 0.010472 n \quad 0.034647 n \quad 0.013131 n 0.079666 n \quad 0.110678 n \quad 0.081523 n \quad 0.195649 n$ $1631.01 \mathrm{e}-09 \mathrm{n} 2.68 \mathrm{e}-09 \mathrm{n} \quad 0.000104 \mathrm{n} \quad 0.002827 \mathrm{n} \quad 0.024462 \mathrm{n} \quad 0.006566 \mathrm{n} \quad 0.054732 \mathrm{n} \quad 0.066861 \mathrm{n} \quad 0.061142 \mathrm{n} \quad 0.097824 \mathrm{n}$ 164 6.06e-10n 8.95e-101 4.15e-05n $0.000325 n \quad 0.01745$ n $0.002189 n \quad 0.024934 n \quad 0.043472 n \quad 0.040762 n \quad 0.047934 n$ $1653.02 \mathrm{e}-10 \mathrm{~m}$ 2.68e-101 2.05e-05n $0.000100 \mathrm{~m} \quad 0.010190 \mathrm{n} \quad 0.001067 \mathrm{~m} \quad 0.010904 \mathrm{~m} 0.028031 \mathrm{~m} 0.030403 \mathrm{~m} 0.019692 \mathrm{~m}$ $1661.01 e-10 n$ 7.16e-11I 9.34 e-06n 6.81e-05n $0.007133 n \quad 0.000547 n \quad 0.004460 n \quad 0.016692 n \quad 0.020381 n \quad 0.008804 n$ $167 \quad 6.06 e-11 n$ 1.79e-111 $3.63 e-06 n$ 3.04e-06n 0.00407 n $0.000219 n \quad 0.002230 n \quad 0.007394 n \quad 0.015285 n \quad 0.003913 n$

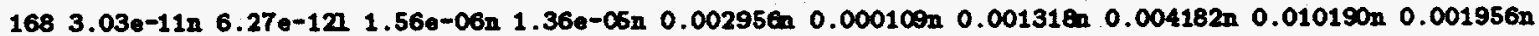
$1691.01 e-11 n$ i.79e-1n 6.22e-07n 5.86e-06n 0.001936 n $8.75 e-05 n \quad 0.000598$ n $0.001687 n \quad 0.008152 n$ n $0.000978 n$ $170 \quad 6.06 e-12 n$ 1.61e-1n $3.11 e-07 n$ 1.68e-06n $0.001165 n \quad 4.38 e-05 n \quad 0.000294 n \quad 0.000863 n \quad 0.006115 n \quad 0.000489 n$ $171 \quad 3.02 e-121$ 1.34e-12n $1.56 e-07 n$ 3.25e-07n $0.000774 n$ 2.19e-05n $0.000172 n \quad 0.000481 n \quad 0.004076 n$ n.000196n $1721.11 e-121 \quad 1.16 e-121.04 e-07 n$ 1.05e-07n $0.000510 n \quad 1.09 e-05 n \quad 8.01 e-05 n \quad 0.000363 n \quad 0.003057 n$ 9.78e-05n 
Table 11

Sot $E$ Hass Chain Yielda

nase c1250: ca244s ca248s es253s fm2548 fa255t fa256s np237h v232t v238s 660.001985 n 3.96e-05a $0.000439 n$ 0.000267n 2.83e-05n 0.000158 n $0.000101 n 0.005000$ n $0.006996 n$ 8.13e-11n $670.002457 n$ 6.57e-05n $0.000549 n \quad 0.000359 n$ 3.84e-08n $0.000205 n \quad 0.000162 n \quad 0.006999 n 0.008991 n 2.45 e-10 n$ $680.002948 n$ 9.96e-05n $0.000769 n 0.000513 n$ n $5.26 e-0.5 n 0.000252 n 0.000222 n 0.008999 n 0.012991 n$ 8.13e-10n

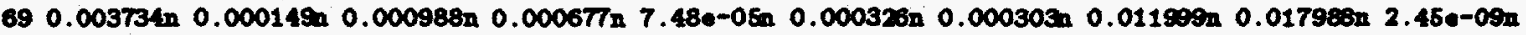

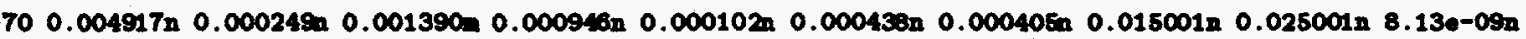

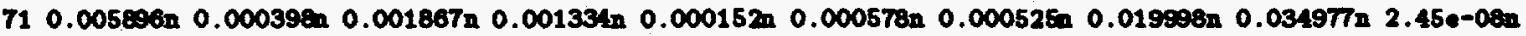
$720.007861 n \quad 0.000657 n \quad 0.002416 n \quad 0.001847 n \quad 0.000202 n \quad 0.000746 n \quad 0.000707 n \quad 0.024998 n \quad 0.046967 n$ 8.13n-08n

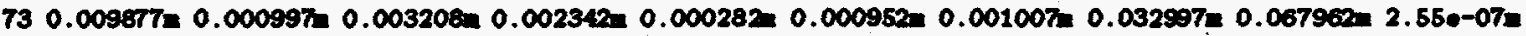

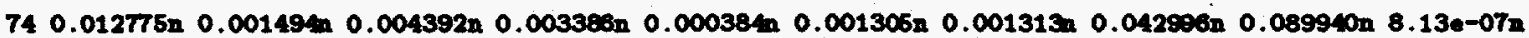

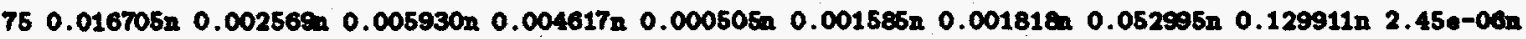
$760.019719 n \quad 0.003989 n \quad 0.007689 n \quad 0.006199 n \quad 0.000722 n \quad 0.002149 n \quad 0.002540 n \quad 0.070053 n \quad 0.170962 n$ 8.13n-06n

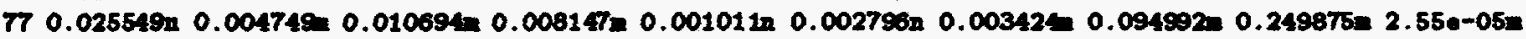

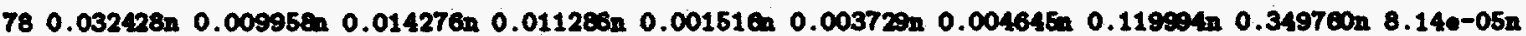

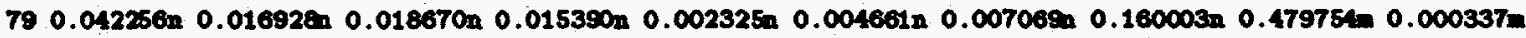

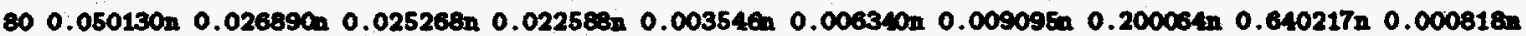
$810.068788 \mathrm{n} \quad 0.044810 \mathrm{n} \quad 0.035143 \mathrm{n} \quad 0.030780 \mathrm{n} \quad 0.005054 \mathrm{n} \quad 0.008017 \mathrm{n} \quad 0.012118 \mathrm{n} \quad 0.259968 \mathrm{n} \quad 0.799466 \mathrm{n} \quad 0.004026 \mathrm{n}$

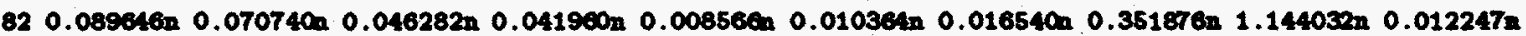

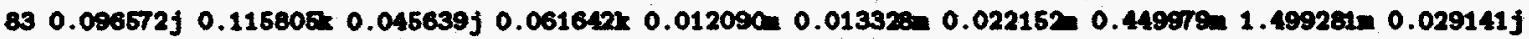

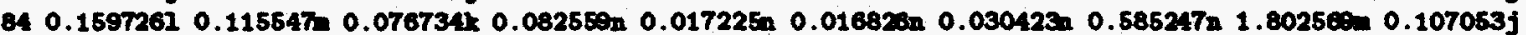

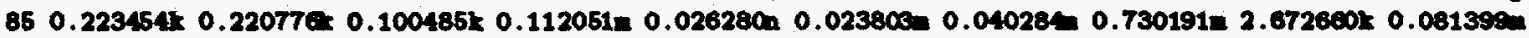

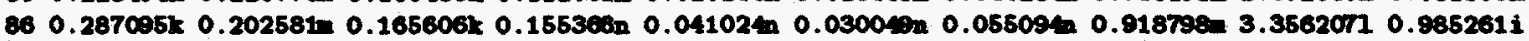

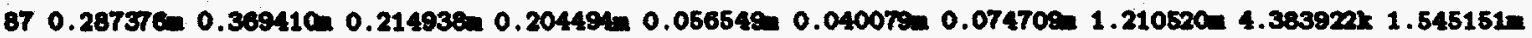

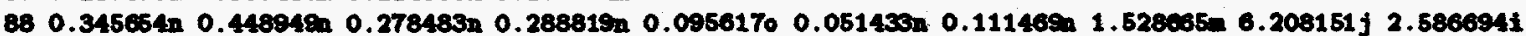

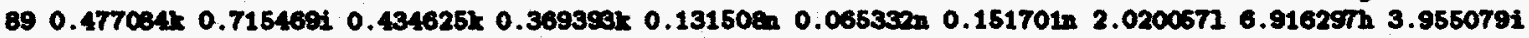

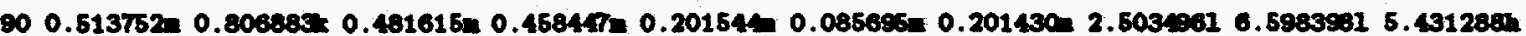
$910.5665101 \quad 0.824968 \times 0.58627310 .532615 \mathrm{j} \quad 0.394977 \mathrm{j} 0.11186 \mathrm{~m} \quad 0.2421461$ 2.669318k $7.157881 \mathrm{~h}$ 5.820960h

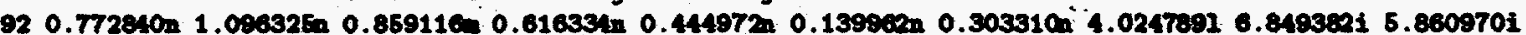

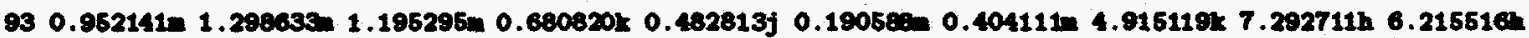

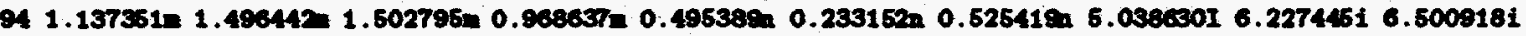
951.4815121 1.62770n 1.6278021 1.1460341 $0.52189210 .307643 n$ o.666565 5.541081 6.19964h 6.6065631

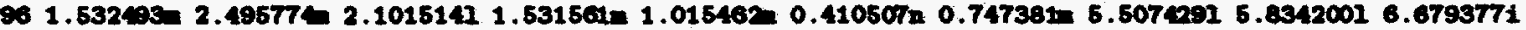

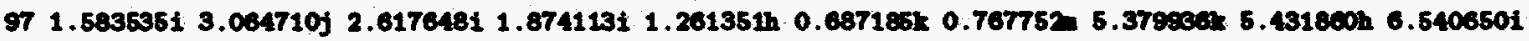

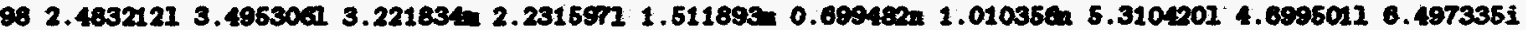

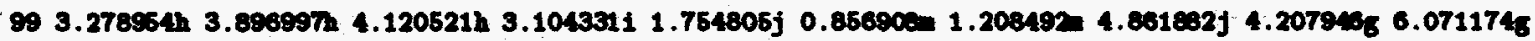
1003.5689511 4.9896601 4.5109501 3.4452681 2.218987 0.952051 n 1.510748 n 5.2001821 3.000601n 6.130801i

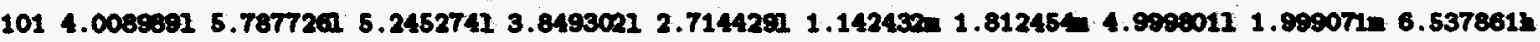

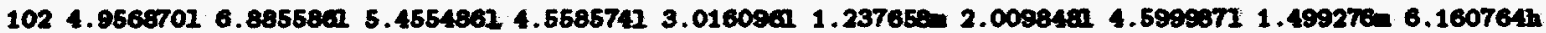

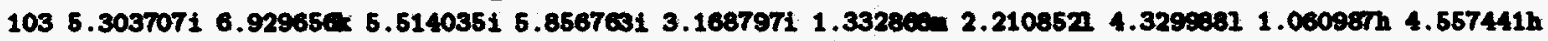

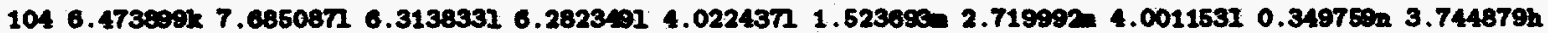

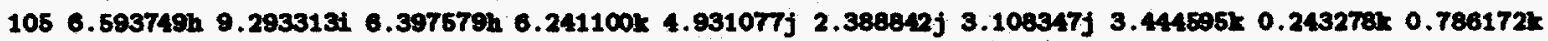
$1066.82554817 .397746 .16943516 .531293 j 5.564657 j \quad 2.4151921$ 3.617618 $2.9998311 \quad 0.099931 n 0.269772 \times$ 107 T.270417k 6.9851861 6.2943291 6.2804601 5.42886n 2.6084081 4.52202n 2.499859 $0.025982 \mathrm{n} 0.139032 \mathrm{n}$

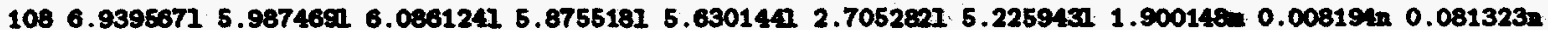

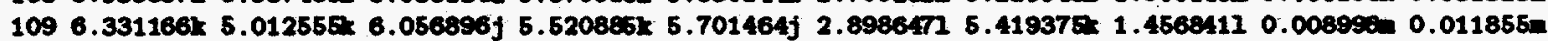

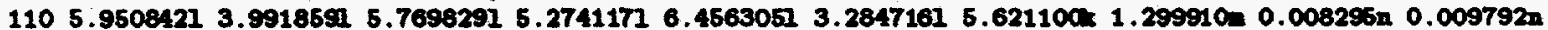

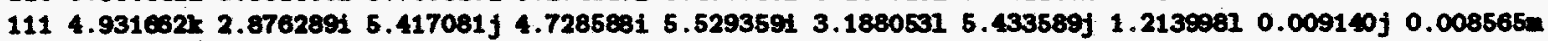
1124.0952001 2.04534CK 4.6833081 3.977335k 6.228977i 3.62535015 .6274111 1.2116921 $0.010992 \mathrm{n} 0.005859 \mathrm{n}$

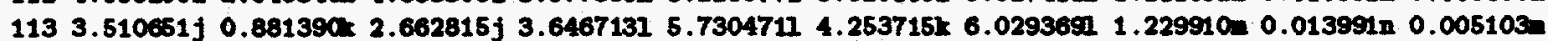
$1142.46958 \mathrm{~m} \quad 0.39830 \mathrm{~m} 2.09811013 .24200615 .03247414 .83038516 .02960 \mathrm{n} 1.229892 \mathrm{n} 0.015960 \mathrm{n} 0.004110 \mathrm{n}$ $1152.049625 \mathrm{~h} 0.25777 \mathrm{c}$ 1.387753h $2.828023 \mathrm{~h} 5.078004 \mathrm{i}$ 5.591322 j 6.131334j $1.229247 \mathrm{~h} 0.018780 \mathrm{j} 0.003827 \mathrm{~m}$ $1161.191441 \mathrm{n} 0.069704 \mathrm{n} 0.076888 \mathrm{n}$ 2.571044 $4.244323 \mathrm{n}$ 5.662013k $5.65700 \mathrm{k}$ 1.22996en $0.015477=0.003554 \mathrm{n}$

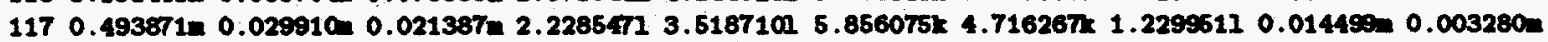

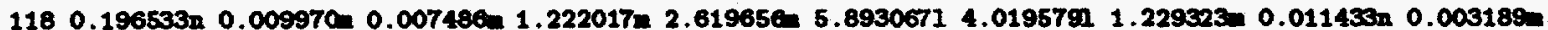

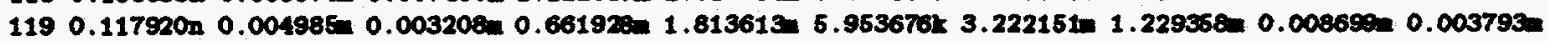

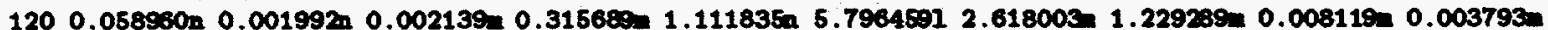

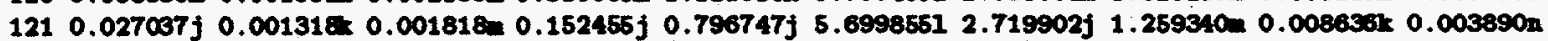

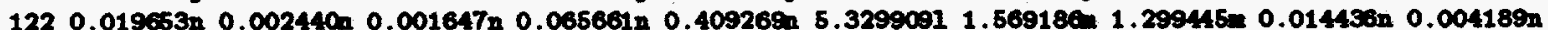

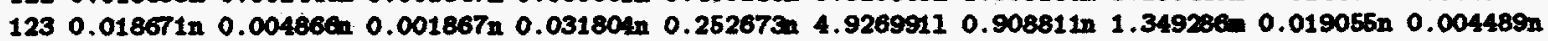

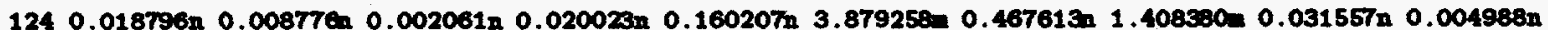

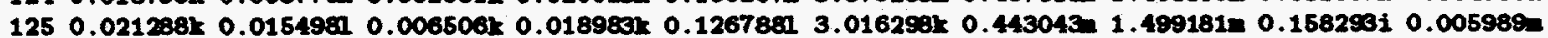

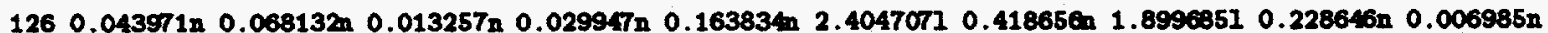


$1270.113078 \mathrm{k} \quad 0.198664 j \quad 0.031151 \mathrm{j} \quad 0.063365 \mathrm{j} \quad 0.238890 \mathrm{k} 2.321930 \mathrm{j} \quad 0.42933512 .501147 \mathrm{k} 0.382894 \mathrm{j} \quad 0.013975 \mathrm{~m}$ $1280.214964 n \quad 0.486668 n \quad 0.101962 n \quad 0.154564 n \quad 0.455129 n$ 2.1689701 $0.570901 n$ 2.899035n $1.063250 n 0.019952 n$ $1290.396905 \mathrm{k} 0.660480 \mathrm{j} 0.322215 \mathrm{k} 0.310467 \mathrm{j} 0.871424 \mathrm{j} 2.264065 \mathrm{j} 0.858857 \mathrm{l}$ 3.2988291 $1.765107 \mathrm{j} 0.026590 \mathrm{k}$ $1300.880002 \mathrm{n} 1.991361 \mathrm{i} 2.027769 \mathrm{n} 0.580676 \mathrm{n} 1.5075042 .482584 \mathrm{~m} 1.546477 \mathrm{n}$ 3.615708n 3.012895 $0.139653 \mathrm{n}$ 131 1.704686h $3.00401282 .620447 \mathrm{~h} 1.136256 \mathrm{k} 2.440037 \mathrm{i} 3.214969 \mathrm{k} 2.537032 \mathrm{~h} 4.090617 \mathrm{~h} 3.748439 \mathrm{~h} 0.448890 \mathrm{~h}$ $1322.459597 \mathrm{~h} 4.19422 \mathrm{sh} 4.335317 \mathrm{~h} 2.528404 \mathrm{k} 3.520872 \mathrm{~h} 4.808938 \mathrm{j} 4.024000 \mathrm{k}$ 4.397509h $5.170557 \mathrm{i} .326248 \mathrm{f}$ 133 3.904174h S.660986 5.264997i 2.669811k 4.341222i 5.422682j 3.8276731 4.4983601 5.803819h 5.601202f $1344.159653 \mathrm{~h} 6.81296 \mathrm{~h} 4.993017 \mathrm{k} 2.95238215 .18887015 .85515114 .1025461$ 5.447073h $5.235405 \mathrm{k} 4.837292 \mathrm{e}$ $1354.642911 \mathrm{k} 7.483203 \mathrm{~g} 5.741358 \mathrm{i} 4.702590 \mathrm{k} 4.478733 \mathrm{i} .163349 \mathrm{j} 6.211316 \mathrm{x}$.9994211 5.319465h 5.213117f

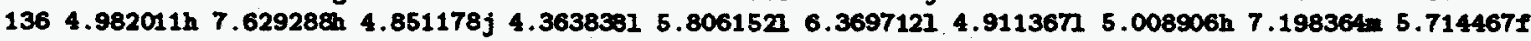
$1375.45360917 .92323816 .076900 \mathrm{i} 4.5305371$ 5.5427041 6.2851121 5.0899511 5.1131611 7.804503i 5.809433h 1385.7366401 7.663838i 6.6341071 4.8203721 5.7325761 6.0575701 5.2759001 5.0035791 5.639774j 6.440974i 1396.191587 i $6.997414 i$ 6.221661i 5.870703k 4.987087k 5.6598101 6.098468k 4.764126k 7.120304h 6.840348i $1405.249179 i$ 6.101880h 5.820549i 5.660593i 5.543736h 4.828491k 5.960553j 4.826781h 7.304308h 6.798937f $1415.849432 i 5.619863 \mathrm{~h} 5.607301 \mathrm{i} 5.740320 \mathrm{k} 5.478458 \mathrm{i} 4.62149215 .75749914 .79881616 .913198 \mathrm{~h} 6.890223 i$

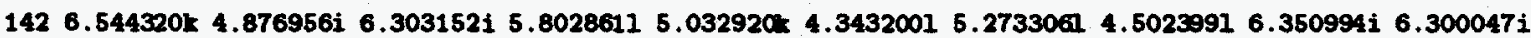
$1435.565486 \mathrm{i} 3.99577 \mathrm{Th} 5.484139 \mathrm{k} 6.442620 \mathrm{i} 5.454272 \mathrm{i}$ 3.138478k 4.880064 $3.54271715 .085506 \mathrm{~g} 7.160881 \mathrm{~h}$ $1444.743435 i$ 3.675941i $5.828263 i$ 5.107928k $4.846000 j$ 3.2069511 4.4892111 2.9995621 $4.329340 i 6.212791 i$ 1454.94283313 .0853551 4.4438571 5.79981814 .106912 2.8290231 3.9030431 2.498738 $3.11545615 .997633 i$

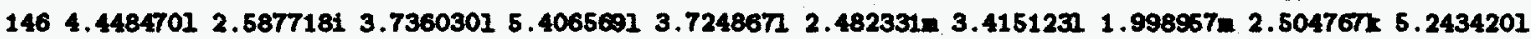
1473.8144931 1.981320i $3.130742 \mathrm{i} 4.816761 \mathrm{I}$ 3.447090 $2.022638 \mathrm{~m}$ 3.1223931 1.7019241 1.205412h $4.399052 \mathrm{j}$

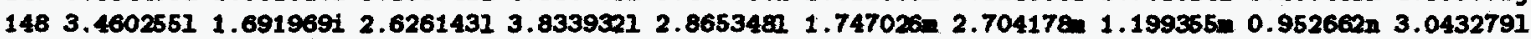
1493.2573191 1.451882i $2.027575 n$ 3.131921k 2.8569801 1.468327k 2.653692 $0.899318 \mathrm{n} 0.666917 \mathrm{n} 2.201026 \mathrm{i}$ $1502.367200 \mathrm{~m} 0.9974061$ 1.521120 $2.465120 \mathrm{~m} 2.201135 \mathrm{~m} 1.287714 \mathrm{~m} 2.238920 \mathrm{~m} 0.702004 \mathrm{n} 0.511157 \mathrm{n} 0.998215 \mathrm{n}$ 1512.0717941 0.696698i $1.184067 \mathrm{i} 1.907814 \mathrm{k} 1.685685 \mathrm{n} 1.285991 \mathrm{k} 2.0500031 \quad 0.529598 \mathrm{n} 0.342982 \mathrm{n} 0.199498 \mathrm{n}$ $1521.574084 \mathrm{~m} 0.5971651 \quad 0.968502 \mathrm{n} 1.561535 \mathrm{~m} 1.49837 \mathrm{~m} 1.103257 \mathrm{~m} 1.641670 \mathrm{n} \quad 0.399692 \mathrm{n} 0.266765 \mathrm{n} 0.049874 \mathrm{n}$ $1531.21510910 .5005611 \quad 0.792720 \mathrm{i} 1.482217 \mathrm{z} 1.3851761 \quad 0.9980791 \mathrm{~L} 1.352259 \mathrm{n} \quad 0.31482510 .190543 \mathrm{n} 0.019950 \mathrm{n}$

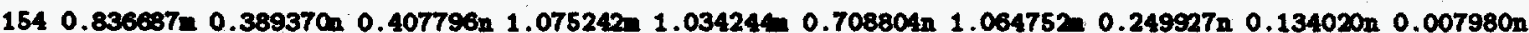

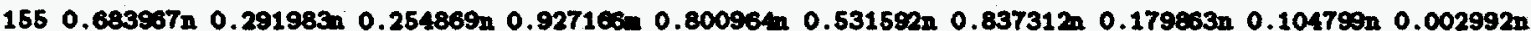

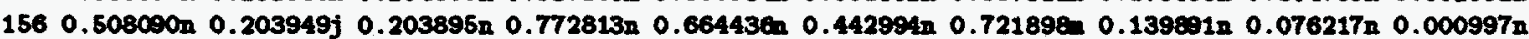

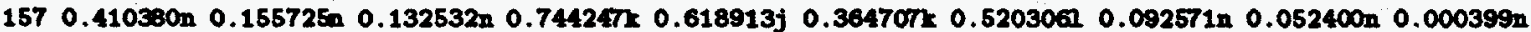

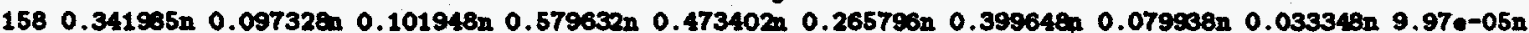

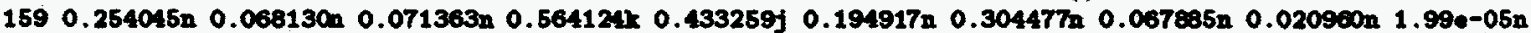
$160 \quad 0.215609 \mathrm{n} \quad 0.043847 \mathrm{n} \quad 0.050979 \mathrm{n} \quad 0.439806 \mathrm{n} \quad 0.218077 \mathrm{n} \quad 0.150646 \mathrm{n} \quad 0.231067 \mathrm{n} \quad 0.050143 \mathrm{n} \quad 0.01454 \mathrm{n} 4.99 \mathrm{e}-06 \mathrm{n}$

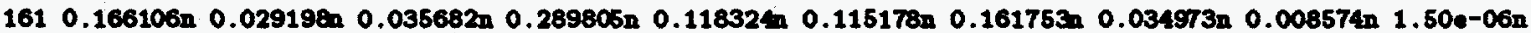

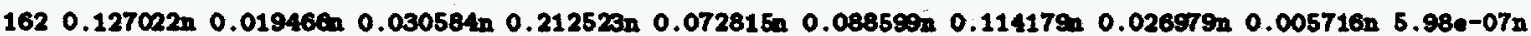
$1630.107481 \mathrm{n} \quad 0.011679 \mathrm{n} 0.023448 \mathrm{n} 0.144905 \mathrm{n} 0.054617 \mathrm{n} 0.070879 \mathrm{n} 0.079926 \mathrm{n} 0.019984 \mathrm{n} 0.003525 \mathrm{n} 9.97 \mathrm{~m}-08 \mathrm{n}$ $1640.078176 \mathrm{n} 0.007786 \mathrm{n} 0.018351 \mathrm{n} 0.106390 \mathrm{n} 0.03839 \mathrm{~m} \quad 0.053169 \mathrm{n} 0.057130 \mathrm{n} 0.015990 \mathrm{n} 0.002384 \mathrm{n} 2.99 \mathrm{a}-08 \mathrm{n}$

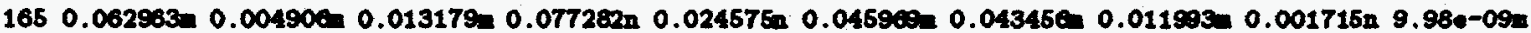

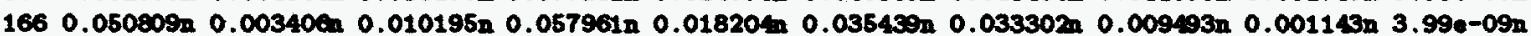

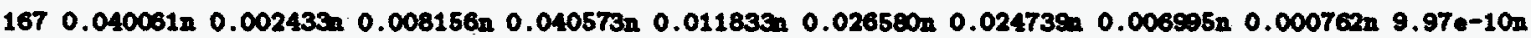
$1680.032244 \mathrm{n} 0.001460 \mathrm{n} 0.008117 \mathrm{n} 0.028981 \mathrm{n} \quad 0.005462 \mathrm{n} \quad 0.017720 \mathrm{n} 0.019030 \mathrm{n} \quad 0.005496 \mathrm{n} 0.000476 \mathrm{n}$ 3.99e-10n $1690.024427 \mathrm{n} \quad 0.000973 \mathrm{n} 0.004588 \mathrm{n} \quad 0.022218 \mathrm{~m} \quad 0.003641 \mathrm{n} \quad 0.015062 \mathrm{n} \quad 0.013321 \mathrm{n} \quad 0.004197 \mathrm{n} \quad 0.000305 \mathrm{n} 1.99 \mathrm{e}-10 \mathrm{n}$

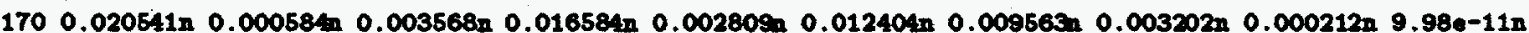

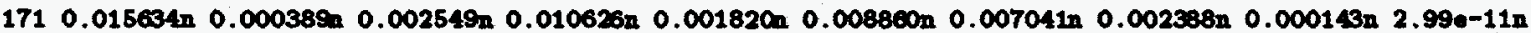
$1720.012702 n \quad 0.000292 n \quad 0.002039 n \quad 0.007728 n \quad 0.000910 n \quad 0.007088 n \quad 0.004757 n \quad 0.001899 n$ 9.53e-05n 9.98e-12n

Table 12

Sot F Hass Chain Yields

mass cm243t cn2468 cm243f cm244f cm246f cm248f pref2h np237t pu240t pu242t $663.42 e-06 n$ 2.16e-050 9.50e-06n 7.30e-06n 4.11e-06n 2.08e-06n 2.08e-07n 4.88e-08n 1.60e-07n 4.97e-08n $678.56 e-06 n \quad 0.00044101 .80 e-05 n$ 1.30e-05n 7.53e-06n 3.66e-06n 4.17e-07n 8.78e-08n 3.10e-07n 9.95e-08n $682.12 e-05 n \quad 0.0004210 \quad 3.40 e-05 n \quad 2.50 e-05 n \quad 1.40 e-05 n \quad 6.83 e-06 n$ 7.33e-07n $4.88 e-07 n$ 9.39e-07n 2.29e-07n $694.70 e-05 n \quad 0.00081506 .70 e-05 n \quad 4.80 e-06 n \quad 2.91 e-05 n \quad 1.29 e-06 n \quad 1.56 e-06 n \quad 1.95 e-06 n \quad 2.98 e-06 n \quad 4.97 e-07 n$ $708.56 e-05 n \quad 0.0008010 \quad 0.000140 n$ 9.61e-05n $5.72 e-05 n$ 2.58e-05n 3.12e-06n 5.85e-06n 9.99e-06n $1.79 e-06 n$ $710.000170 n$ o.0011070 $0.000270 n$ 0.000150n $0.000110 n$ 5.25e-05n 5.22e-06n 1.46e-05n $1.80 e-05 n$ 2.69e-06n $720.000376 n \quad 0.0014990 \quad 0.000530 n$ 0.000380n $0.000231 n \quad 0.000100 n$ 1.14e-05n 2.93e-05n $5.99 e-05 n$ 9.95e-06n

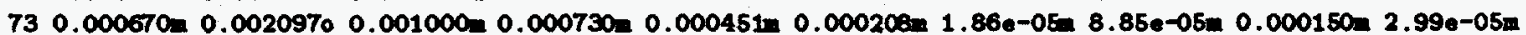
$74 \begin{array}{llllllllll}0.001712 n & 0.0028710 & 0.002000 n & 0.001401 n & 0.000863 n & 0.000396 n & 5.22 e-05 n & 0.000263 n & 0.000300 n & 7.96 e-05 n\end{array}$

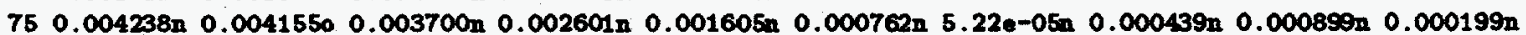
$\begin{array}{lllllllllllll}76 & 0.008582 n & 0.0057070 & 0.006647 n & 0.004818 n & 0.003012 n & 0.001386 n & 0.000209 n & 0.001854 n & 0.001793 n & 0.000418 n\end{array}$

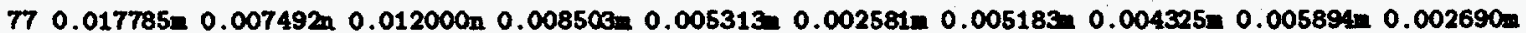

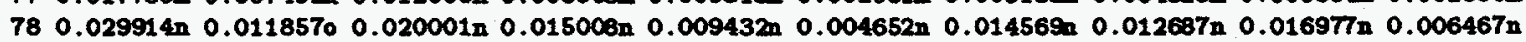


$\begin{array}{lllllllllll}79 & 0.051272 n & 0.0171500 & 0.034002 n & 0.025013 n & 0.016057 n & 0.008020 n & 0.029249 n & 0.026350 n & 0.015980 n & 0.007464 n\end{array}$

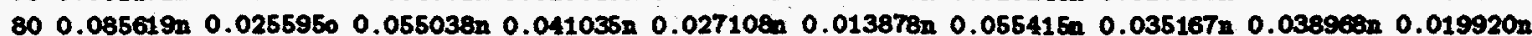
$810.158714 n \quad 0.0392000 \quad 0.087004 n \quad 0.066034 n \quad 0.043153 n \quad 0.022777 n \quad 0.091987 \mathrm{n} \quad 0.117108 \mathrm{n} \quad 0.109853 \mathrm{n} \quad 0.040801 \mathrm{n}$

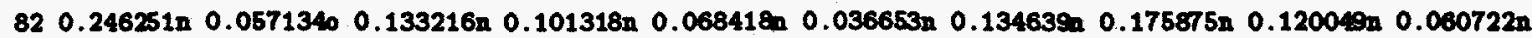

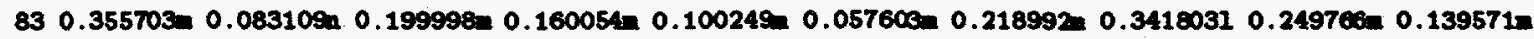

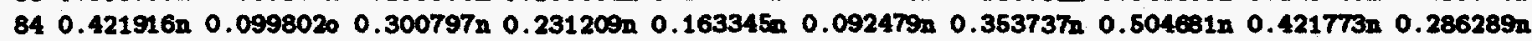

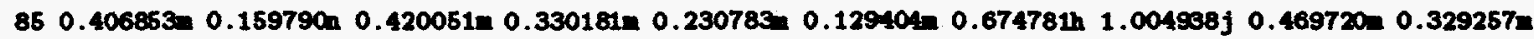
$860.550524 \mathrm{~m}=0.184031 \mathrm{n} 0.585856 \mathrm{~m} 0.464337 \mathrm{n} 0.324214 \mathrm{n} 0.190041 \mathrm{n} 0.830466 \mathrm{n} 1.141117 \mathrm{~m} 0.684139 \mathrm{n} 0.501334 \mathrm{n}$

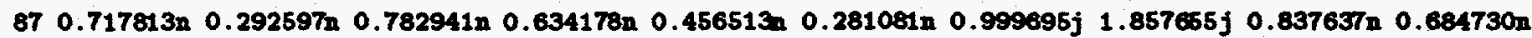
$880.776808 \mathrm{n} 0.364701 \mathrm{n} 1.003472$ n $0.844165 \mathrm{n} 0.620736 \mathrm{n} 0.385740 \mathrm{n} 1.180816 \mathrm{j} 2.253053 \mathrm{j} 1.201259 \mathrm{n} 0.868460 \mathrm{n}$ 89 1.058497n $0.569279 n$ 1.301320 $1.103167 \mathrm{~m} 0.808538 \mathrm{n} 0.526878 \mathrm{~m} 1.345949 \mathrm{k}$ 2.8196111 $1.324100 \mathrm{~m} 0.924308 \mathrm{n}$ $901.136520=0.635261 \mathrm{~m}$ 1.600331m $1.401007 \mathrm{n}$ 1.004433 $0.677513 \mathrm{~m}$ 1.533371m 3.4427021 1.8405621 $1.238810 \mathrm{~m}$

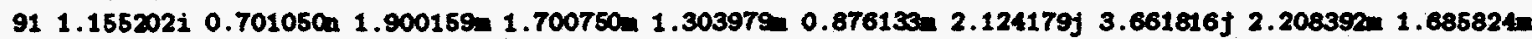
$921.37756310 .969182 \mathrm{~m} 2.303857 \mathrm{~m} 2.006985 \mathrm{~m}$ 1.610599n $1.096403 \mathrm{~m} 2.397422 \mathrm{j} 4.17569812 .68383412 .047368 \mathrm{~m}$ $931.794693 \mathrm{k} 1.243862 \mathrm{n} 2.60843012 .4179541$ 1.9471001 $1.441356 \mathrm{a} 2.647926 \mathrm{t}$.824462 3.46029112 .6834961

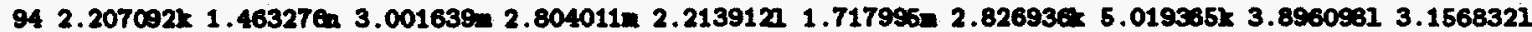

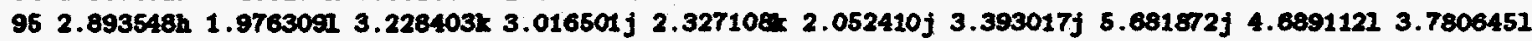
963.34658312 .278779 a.6220521 3.6094721 2.9091381 2.2961731 3.8575481 5.6751361 4.73049514 .2557941 97 3.960518h 2.363480 L.0007121 4.1020791 3.3077642 .68942614 .25029215 .85332715 .16895314 .5132591

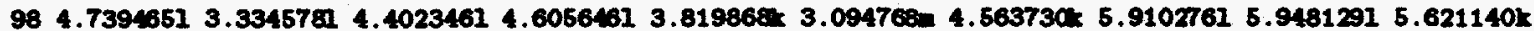
$995.021762 \mathrm{~h} 4.225438 \mathrm{j}$ 4.900341k 5.1018161 4.308126 3.594600k 5.1279361 6.6120581 5.968915k 5.995629k 100 5.4296811 4.716195 5.503917k 5.702185k 4.9083351 4.085070k 5.0645281 5.3445931 6.6953681 6.148659k

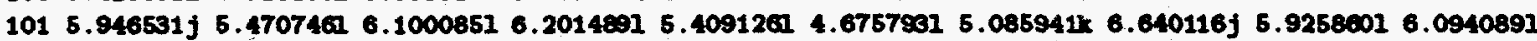

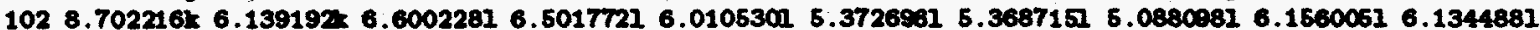

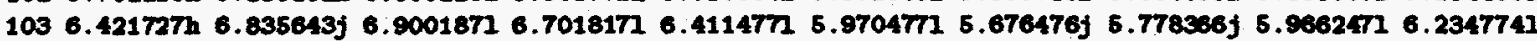

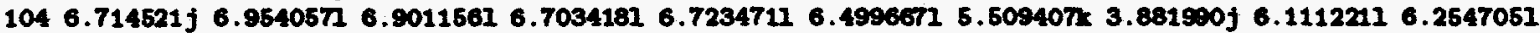
105 6.383824h 6.795922j 6.600092t 6.401184t 6.808940h 6.879703t 5.550813i 2.6473811 5.6564191 6.0063711 $1065.9100931 \times 6.73169916 .00008415 .90141716 .011154$ 6.9839931 5.0844543 .29891015 .2683215 .9345061

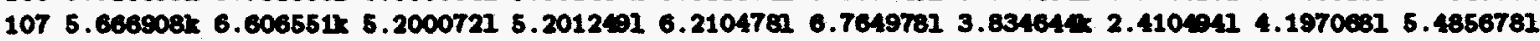
1084.30633716 .007434 4.2001351 4.301147 5.51019616 .38962013 .03944111 .77869013 .26795214 .4496841

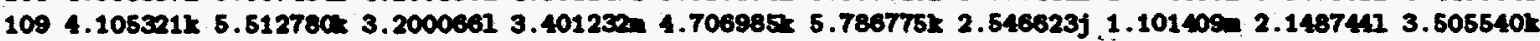

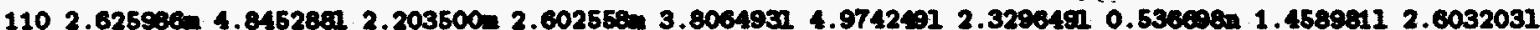
111 1.8492401 4.30942n 1.500030 1.8004582 2.9049011 4.0788841 2.234185j 0.136014n $0.39944 \mathrm{~m} 1.105912 \mathrm{~m}$

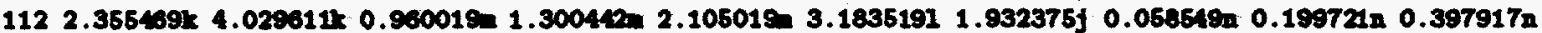

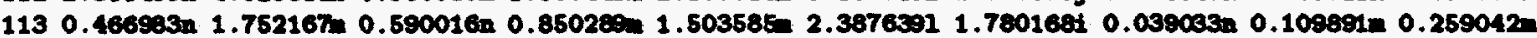

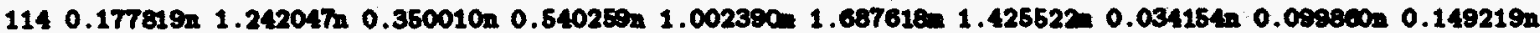

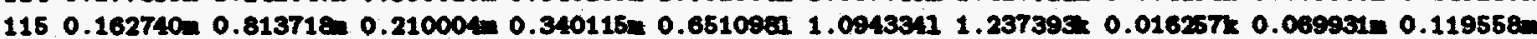

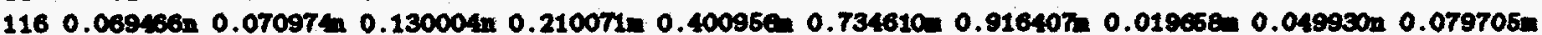

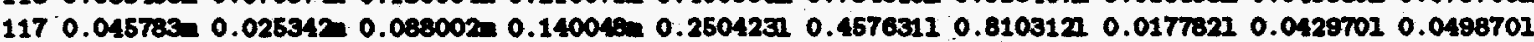

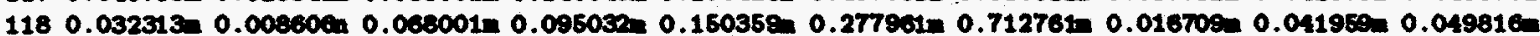

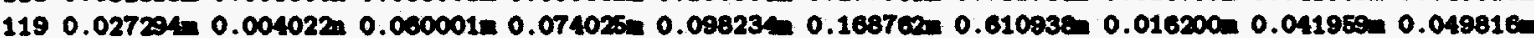
120 0.035216 0.00203 in $0.057001=0.065022$ a $0.070167=0.109190=0.610938$ a $0.014294=0.030865=0.035867=$

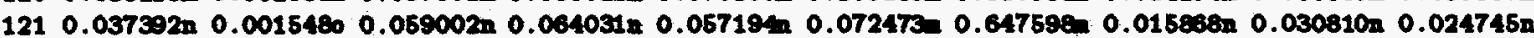

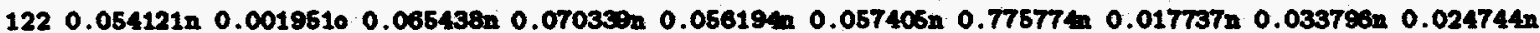

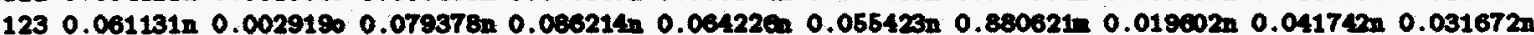

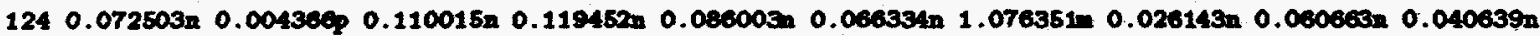

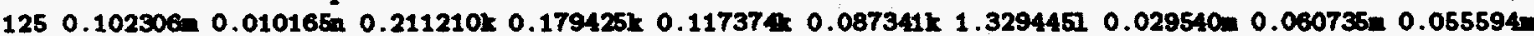
$1260.168261 \mathrm{n} \quad 0.0346410 \quad 0.297670 \mathrm{n} 0.287386 \mathrm{n} \quad 0.207515 \mathrm{n} 0.148175 \mathrm{n} 1.565842 \mathrm{n} \quad 0.070961 \mathrm{n} 0.139148 \mathrm{n} 0.118791 \mathrm{n}$

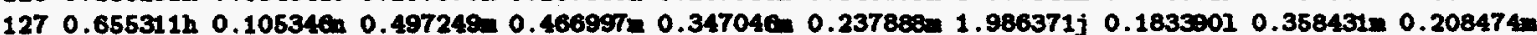
$1280.745795 \mathrm{k} \quad 0.2508490 \quad 0.825492 \mathrm{n} \quad 0.745302 \mathrm{n} \quad 0.553344 \mathrm{n} \quad 0.3950 \mathrm{mn} 2.4620891 \quad 0.522738 \mathrm{n} \quad 0.696985 \mathrm{n} \quad 0.415731 \mathrm{n}$

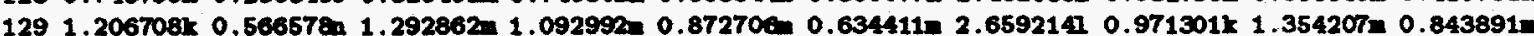

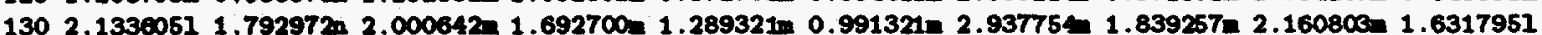
$1313.193492 \mathrm{~g} 3.325675 \mathrm{j} 2.8887221$ 2.3891621 1.8886961 $1.48690 \mathrm{~m}$ 3.781217i 3.156840h 3.33971112 .6166411 $1324.773685 h$ 4.654923j 3.8848351 3.1855481 2.67725e 2.081571n $5.269045 \mathrm{~g} 4.56773614 .81515113 .9695681$ $1335.742512 \mathrm{~h} 5.705270 \mathrm{f} 4.8809481$ 3.9819431 3.4792931 2.8828211 5.6596361 6.476310i 6.7155201 5.8601161

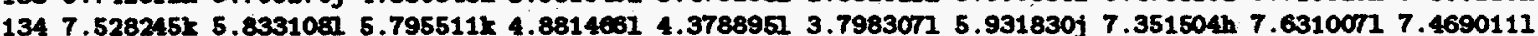
$1355.908389 k$ 6.626351k 6.4752041 5.6752321 6.2738261 4.6966onk 6.1775741 7.6760001 7.2342691 7.494485k 1364.8689461 7.000599 T.4690681 6.5984121 6.215476k 5.7561341 5.556258k 7.1781581 7.026604k 7.390275k 137 6.3172391 6.759442 6.813590j 6.687470j 6.515968j 6.363644j 5.1608031 6.1757141 6.5567741 6.7214151 1385.8133231 6.6554201 6.6833421 6.7724421 6.7790511 6.7461411 $5.230965 j 6.22477016 .4085601$ 6.6166751 $1396.800054 k$ 6.2928641 6.1769561 6.5704611 6.7619381 6.9731031 4.893587j 6.1313471 5.9626521 6.3540391 140 5.540412h 6.368098 $5.67809816 .27207516 .56224016 .86436214 .87938716 .016882 j 5.70347916 .0226041$ 141 5.149223k 5.32366t 4.850189k 5.925597k 5.929272k 6.617429j 4.823173j 6.018260j 5.4938171 5.2951561 1423.77388315 .5310541 4.5828621 5.2780361 5.6783931 6.0943681 4.310172j 4.972978j 4.7912171 4.6775121 $1434.285902 i \quad 5.3142011$ 4.0842311 4.7788421 4.9741971 5.5870201 3.632369j 5.036034j 4.4877401 4.5472771 144 3.721052I $5.051618 \times 3.699217 \mathrm{k} 4.023911 \mathrm{k} 4.673161 \mathrm{k}$ 5.054390j $3.166505 \mathrm{j} 4.84566413 .92886414 .1954331$ 
1453.2734511 3.5115381 3.3867801 3.7828491 3.9763881 4.2751631 2.5605661 3.6059661 $3.07061013 .435676 \mathrm{~m}$

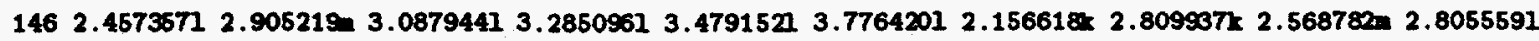

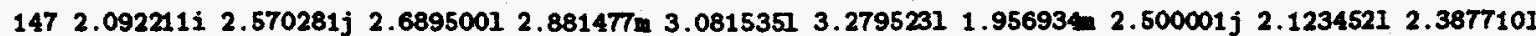

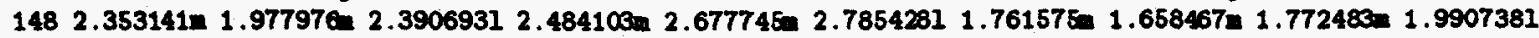
149 1.984747 m 1.543240n 1.989001m 2.0905181 2.280648 2.379099m 1.592938k 1.5471691 1.393918 $1.598404 \mathrm{~m}$

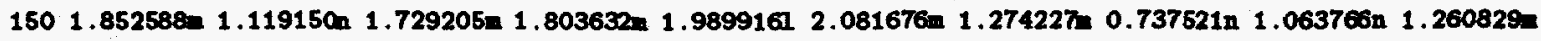
151 1.244853i $0.857558 \mathrm{n} 1.392300 \mathrm{~m} 1.490419 \mathrm{~m} \quad 1.685679 \mathrm{~m} 1.685082 \mathrm{~m} \quad 0.905197 \mathrm{k} \quad 0.7418381 \quad 0.854712 \mathrm{n} 1.022541 \mathrm{~m}$ $1521.237907 \mathrm{~m} \quad 0.57129701 .093945 \mathrm{~m} 1.192334 \mathrm{~m} \quad 1.388207 \mathrm{~m} \quad 1.387714 \mathrm{~m} \quad 0.872593 \mathrm{~m} \quad 0.364031 \mathrm{n} \quad 0.655942 \mathrm{n} \quad 0.801704 \mathrm{n}$ $1531.309522 \mathrm{n} \quad 0.58957$ in $0.843389 \mathrm{n} \quad 0.904188 \mathrm{~m} 1.090734 \mathrm{n} 1.189469 \mathrm{n} \quad 0.659293 \mathrm{n} \quad 0.196017 \mathrm{n} \quad 0.457172 \mathrm{n} \quad 0.623548 \mathrm{n}$ $1540.904141 \mathrm{~m} \quad 0.3356500 \quad 0.637940 \mathrm{n} \quad 0.686477 \mathrm{~m} \quad 0.849812 \mathrm{n} \quad 0.941665 \mathrm{~m} \quad 0.455775 \mathrm{n} \quad 0.104821 \mathrm{~m} \quad 0.327975 \mathrm{n} \quad 0.435494 \mathrm{n}$ $\begin{array}{llllllllllllll}155 & 0.708376 n & 0.2285190 & 0.456422 n & 0.5268421 & 0.693182 l & 0.7378961 & 0.368428 n & 0.078616 n & 0.258401 n & 0.316723 n\end{array}$ $\begin{array}{lllllllllll}156 & 0.445058 n & 0.1812080 & 0.327433 n & 0.366658 n & 0.484163 n & 0.562926 n & 0.271473 n & 0.061457 n & 0.139139 n & 0.237542 n\end{array}$ $\begin{array}{lllllllllll}157 & 0.455385 n & 0.1205080 & 0.228212 n & 0.257652 n & 0.355711 n & 0.414788 n & 0.178397 n & 0.024299 n & 0.099385 n & 0.168259 n\end{array}$ $\begin{array}{lllllllllll}158 & 0.344903 n & 0.0830170 & 0.148850 n & 0.178378 n & 0.247022 n & 0.306153 n & 0.119255 n & 0.012864 n & 0.072551 n & 0.118771 n\end{array}$ $\begin{array}{lllllllllll}159 & 0.233372 n & 0.0580220 & 0.099222 n & 0.118916 n & 0.177856 n & 0.217270 n & 0.073589 n & 0.001027 n & 0.044723 n & 0.079181 n\end{array}$

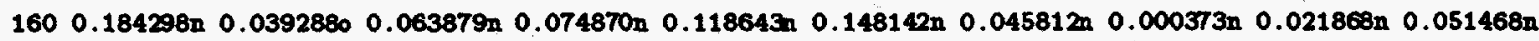

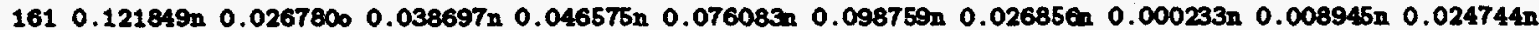

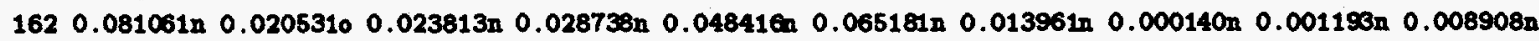

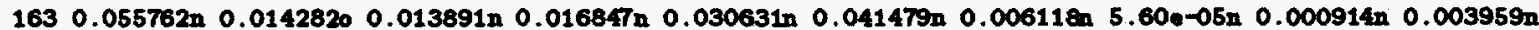
$1640.025414 n \quad 0.0107120 \quad 0.007849 n \quad 0.009913 n \quad 0.018774 n \quad 0.025677 n \quad 0.003054 n \quad 2.33 e-05 n \quad 0.000318 n \quad 0.001485 n$

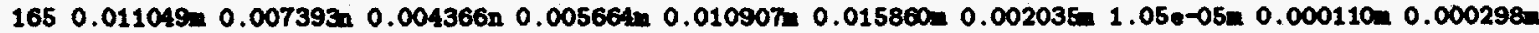

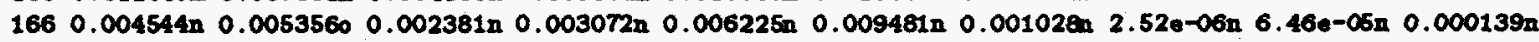
$1670.002272 n \quad 0.0044630 \quad 0.001290 n \quad 0.001685 n \quad 0.003557 n \quad 0.005432 n \quad 0.000392 n \quad 9.33 e-07 n$ 2.39e-05n 3.96e-05n

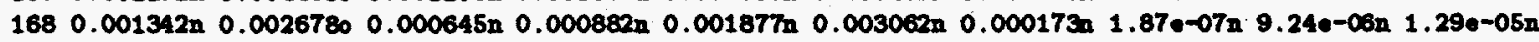
$1690.000609 n \quad 0.0017850 \quad 0.000327 n \quad 0.000456 n \quad 0.000988 n \quad 0.001679 n \quad 9.68 e-05 n \quad 7.00 e-08 n \quad 3.68 e-06 n$ 3.46e-06n

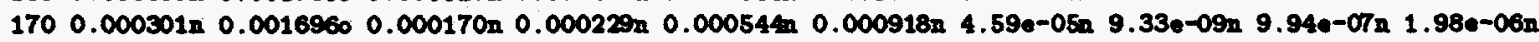
$1710.000176 n \quad 0.00116008 .24 e-05 n \quad 0.000119 n \quad 0.000277 n \quad 0.000484 n \quad 1.97 e-05 n \quad 4.67 e-09 n$ 2.39e-07n 9.90e-07n $1729.57 e-05 n \quad 0.00098204 .17 e-05 n \quad 5.85 e-05 n \quad 0.000138 n \quad 0.000247 n \quad 1.53 e-05 n \quad 9.33 e-10 n \quad 7.65 e-03 n \quad 4.95 e-07 n$

\section{Future Work}

Because of continuing work on fission yield data, no compilation can be regarded as a final work. By applying computer techniques, it is possible to produce future revisions faster. It is not possible to examine the data well enough or gain enough knowledge of the work by each contributor to eliminate all possible mistakes or omissions. However, it is now easier to correct errors and to ensure random new typographical errors will not occur. If all errors found are reported to the authors, it will be possible to minimize errors in later issues of this document.

\section{Energy Definitions}

A fission neutron spectrum is one giving a Cd-115 ground state $\mathrm{R}$-value of 2.8 for $235 \mathrm{U}$ fission using Mo-99 as reference nuclide. As an expedient, yields measured in the core of a fast reactor have been pooled with fission neutron spectrum yields. Selected yields measured in monoenergetic neutron energies between 0.5 and $2.0 \mathrm{meV}$ have also been pooled with fission neutron spectrum yields. The definitions of thermal and high energy neutrons are consistent with general usage. Thermal neutrons are obtained from a reactor and are assumed to have been moderated to thermal equilibrium. The high energy neutrons are obtained from the $\mathrm{H} 3(\mathrm{~d}, \mathrm{n}) \mathrm{He} 4$ reaction. The actual energies quoted vary from 14 to $15 \mathrm{meV}$ with $14.7 \mathrm{meV}$ being the most often listed mean value. Detailed descriptions of the neutron sources are given in the reference section. 


\section{Special Treatment Numbers}

- 1. The data were multiplied by the ratio of the Mo-99 yield in U-235 thermal fission to the Mo-99 yield in U-233 thermal fission (6.06:4.80) to remove the original normalization; then they were treated as $\mathrm{R}$-values.

- 2. Reported data (66ARA1) for Sb-126 are the sum of the independent yields of both isomers. The ratio 1.0 to 1.72 corrects for the estimated isomer yield ratio. No renormalization was made.

- 3. The preceding comment applies except the ratio equals 0.5555 .

- 4. The measurements were relative to Mo-99, $\mathrm{Ba}-139$, and $\mathrm{Ba}-140$ equal to $6.25,6.42$, and 6.25 , respectively. We assumed they were averaged and corrected for the ratio (Mo-99+ Ba-139+ $\mathrm{Ba}-140)$ to $(6.25+6.42+6.25)$. No other renormalization was made.

- 5. The value is measured relative to the total number of fissions determined from the B-10-toB-11 ratio, and is multiplied by 100 to obtain percent:

- 6. The data were renormalized by using the formula: Yield(mz,ma,mf $)=($ Yield(mz,ma,norf $)$ * 6.14) / (Yield(norz,nora,norf)) where $\mathrm{mz}$, ma, and $\mathrm{mf}$ are the atomic number $(\mathrm{Z})$, mass atomic weight (A), and fissionable material (F), respectively, of the measured nuclide and norz, nora, and norf are the atomic number $(\mathrm{Z})$, atomic weight $(\mathrm{A})$, and fissionable material $(\mathrm{F})$, listed for the normalizing nuclide.

- 7. Values listed are $\mathrm{R}$-values.

- 8. The data were multiplied by 6.14:6.32 to remove the original Mo-99 normalizing values; then they were treated as $\mathbf{R}$-values.

- 9. Values were relative to both $\mathrm{Mo-99}$ and $\mathrm{Ba}-140$ renormalized by the $(\mathrm{Mo}-99+\mathrm{Ba}-140)$ to $(6.1+5.4)$ ratio.

- 10. Average of 10 values relative to Mo-99 and 4 values relative to Ba-140. Renormalized by $\left(4^{*} \mathrm{Mo}-99+10 * \mathrm{Ba}-140\right) /\left(4^{*} 6.1+10 * 5.4\right)$.

- 11. The data were multiplied by the yield of Mo-99 in U-235 thermal fission to the Mo-99 yield in U-235 fission with fission spectrum neutrons $(6.14: 6.1)$ to remove the original normalizing values; then they were treated as $R$-values.

- 12. The data were multiplied by the yield ratio of Mo-99 in U-235 thermal fission to the Mo-99 yield in fission with $8 \mathrm{meV}$ neutrons $(6.14: 5.4)$ to remove the original normalizing values; then they were treated as R-values.

- 13. The data were multiplied by the ratio of the Mo-99 yield in U-235 thermal fission to the Mo99 yield in U-238 fission spectrum fission $(6.14: 6.2)$ to remove the original normalizing values; then they were treated as $\mathrm{R}$-values.

- 14. The data were measured relative to the independent yield and were multiplied by the independent yield in the file. 
- 15. The data were measured relative to the sum of the yields of both isomers and were renormalized to the sum of isomer yields in the file.

- 16. The data were corrected to a Sr-90 half-life of 28.9 years.

- 17. The data were measured relative to $\mathrm{Sn}-121$ and $\mathrm{Sn}-125$ yields and were renormalized through the ratio $(\mathrm{Sn}-121+\mathrm{Sn}-125):(0.0122+0.0116)$.

- 18. The listed absolute independent yield values (IN) were calculated from the author's total isotopic yield and the isomer yield ratio $(\mathrm{M} / \mathrm{M}+\mathrm{G})$ predicted by $76 \mathrm{MAD} 2$ (77DEN1 if measured). For relative or fractional independent yields, see special treatment 40 that normalizes to the reference nuclide or multiplies by chain yield, as appropriate.

- 19. The values were measured relative to $6 \mathrm{Sr}-89$ determinations and $2 \mathrm{Mo-99}$ values. The $\mathrm{Sr}-90$ half-life was corrected from 19.9 to 28.9 years. The correction ratio was: $\left(6{ }^{*} \mathrm{Sr}-89+2\right.$ * Mo-99) / $(6 * 4.08+2 * 5.98) *(28.9 / 19.9)$.

- 20. The value was multiplied by 100 to give percent.

- 21. The Sr-90 half-life was corrected from 19.9 to 28.9 years. The values were also renormalized to the latest value of the reference nuclide.

- 22. The data were multiplied by the ratio of the Sr-89 yield in U-235 thermal fission to the Sr-89 yield in Th-232 pile neutron fission (4.6:6.7) to remove the original normalization; then they were treated as R-values.

- 23. The Sr-90 half-life was corrected from 25 to 28.9 years. The value was also renormalized to the latest value of the reference nuclide in the file.

- 24. The data were multiplied by the ratio of the Mo-99 yield in U-235 thermal fission to the Mo-99 yield in U-233 thermal fission (6.06:4.8) to remove the original normalization. Then they were treated as $\mathrm{R}$-values.

- 25. The data were multiplied by the ratio of the Cs-137 yield in the $\mathrm{Pu}-239$ thermal fission to the Cs-137 yield in the $\mathrm{Pu}-241$ thermal fission (6.60:6.62) to remove the original normalization; then they were treated as R-values.

- 26. The data were multiplied by the ratio of the Mo-99 yield in U-235 thermal fission to the Mo-99 yield in U-233 thermal fission (6.06:4.75) to remove the original normalization; then they were treated as $\mathrm{R}$-values.

- 27. The reported value was measured relative to the sum of the Sm-151 and Sm-152 yields; then we renormalized it to the sum of the yields of Sm-151 and Sm-152 in the file.

- 28. The data were multiplied by the ratio of the Mo-99 yield in U-235 thermal fission to the Mo-99 yield in Th-229 thermal fission (6.06:0.16) to remove the original normalization; then they were treated as $\mathrm{R}$-values.

- 29. The listed normalizing values were obtained by adjusting the heavy mass peak yields to $100 \%$.

- 30 . The data were multiplied by 6.4 , the assumed mass 136 yield. 
- 31. The data were multiplied by the ratio of the Mo-99 yield in U-235 thermal fission to the Mo-99 yield in $\mathrm{Pu}-239$ thermal fission (6.16:6.79) to remove the original normalization; then they were treated as $R$-values.

- 32. The data were multiplied by the ratio of the Mo-99 yield in U-235 thermal fission to the Mo-99 yield in U-232 thermal fission (6.25:4.15) to remove the original normalization; then they were treated as $\mathrm{R}$-values.

- 33. The standard error is stated. The standard deviation is obtained by multiplying the standard error by 1.47 .

- 34. The $\mathrm{Kr}-85$ half-life was corrected from 9.2 to 10.76 years.

- 35. The Cs-137 half-life was corrected from 33 to 30 years.

- 36. The error was reassigned for this work.

- 37. The value reported is the sum of the metastable and ground states. One-half of the total yield is arbitrarily assigned to each state.

- 38. Measured relative to the sum of the independent yields of both isomers. This treatment is not yet functioning because the sum of independent yields is not stored or available to the computer.

- 39. The listed total relative or fractional isomer yield is the sum of the metastable and ground states. The fraction of this total assigned by computer to the metastable state is 0.71 for $\mathrm{Xe}-133, \mathrm{Xe}-135$, Te-133, Te-131 thermal fission as predicted by 76MAD2. For general cases (other than $M / M+G=0.71$ ) see special treatment number 40 . For total yields expressed as absolute independent yields (IN), rather than relative or fractional independent (FI) see special treatment number 18 that bypasses normalization to reference nuclide or multiplication by chain yield as appropriate.

- 40. The listed relative or fractional independent yields (IN or FI) were calculated from the author's total isotopic yield and the isomer yield ratio $(M / M+G)$ predicted by 76MAD2 (77DEN1 if measured). For absolute independent yields, see special treatment 18 that bypasses normalization to reference nuclide or multiplication by the chain yield.

\section{R-Values}

For yields that were originally measured by the $\mathrm{R}$-method, the original $\mathrm{R}$-values were mathematically reconstructed. An updated yield value was then obtained from the usual $R$-value method equation. A discussion of the $R$-method is found in (56FOR1). This is a ratio method used to cancel counter geometry. Radioactivities of specific fission products in, say $\mathrm{Pu} 239$, to the corresponding radioactivity produced in U235 in the same irradiation are ratioed. The relative fissions in each foil are normalized by the relative fissions as determined from a nuclide, like Mo-99. If the yields of U235 are assumed to be well-known, the corresponding yield in Pu239 can be calculated from the ratios (designated R-values). 
Symbols

Fissile material and inducing species in the order of appearance in this compilation

\begin{tabular}{|c|c|c|c|c|c|}
\hline 1 & $\mathrm{U} 235 \mathrm{~T}$ & Uranium & 235 & Thermal neutrons & \\
\hline 2 & $\mathrm{U} 235 \mathrm{~F}$ & Uranium & 235 & Fission spectrum neutrons & \\
\hline 3 & U235HE & Uranium & 235 & High energy neutrons ( 14.7 & $\mathrm{MeV})$ \\
\hline 4 & $\mathrm{U} 238 \mathrm{~F}$ & Uranium & 238 & Fission spectrum neutrons & \\
\hline 5 & U238HE & Uranium & 238 & High energy neutron (14.7 & $\mathrm{MeV})$ \\
\hline 6 & PU239T & Plutonium & 239 & Thermal neutrons & \\
\hline 7 & PU239F & Plutonium & 239 & Fission spectrum neutrons & \\
\hline 8 & PU241T & Plutonium & 241 & Thermal neutrons & \\
\hline 9 & $\mathrm{U} 233 \mathrm{~T}$ & Uranium & 233 & Thermal neutrons & \\
\hline 10 & TH232E & Thorium & 232 & Fission spectrum neutrons & \\
\hline 11 & $\mathrm{U} 233 \mathrm{~F}$ & Uranium & 233 & Fission spectrum neutrons & \\
\hline 12 & U233HE & Uranium & 233 & High energy neutrons (14.7 & $\mathrm{MeV})$ \\
\hline 13 & $\mathrm{U} 236 \mathrm{~F}$ & Uranium & 236 & Fission spectrum neutrons & \\
\hline 14 & PU239H & Plutonium & 239 & High energy neutrons (14.7 & $\mathrm{MeV})$ \\
\hline 15 & PU240F & Plutonium & 240 & Fission spectrum neutrons & \\
\hline 16 & PU241F & Plutonium & 241 & Fission spectrum neutrons & \\
\hline 17 & PU242F & Plutonium & 242 & Fission spectrum neutrons & \\
\hline 18 & TH232H & Thorium & 232 & High energy neutrons ( 14.7 & $\mathrm{MeV})$ \\
\hline 19 & NP237E & Neptunium & 237 & Fission spectrum neutrons & \\
\hline 20 & CF252S & Californium & 252 & Spontaneous fission & \\
\hline 21 & $\mathrm{U} 234 \mathrm{~F}$ & Uranium & 234 & Fission spectrum neutrons & \\
\hline 22 & U237F & Uranium & 237 & Fission spectrum neutrons & \\
\hline 23 & PU240H & Plutonium & 240 & High energy neutrons ( 14.7 & $\mathrm{MeV})$ \\
\hline 24 & U234HE & Uranium & 234 & High energy neutrons (14.7 & $\mathrm{MeV})$ \\
\hline 25 & U236 HE & Uranium & 236 & High energy neutrons ( 14.7 & $\mathrm{MeV})$ \\
\hline 26 & PU238F & Plutonium & 238 & Fission spectrum neutrons & \\
\hline 27 & AM241F & Americium & 241 & Fission spectrum neutrons & \\
\hline 28 & AM243F & Americium & 243 & Fission spectrum neutrons & \\
\hline 29 & NP238F & Neptunium & 238 & Fission spectrum neutrons & \\
\hline 30 & CM242F & Curium & 242 & Fission spectrum neutrons & \\
\hline 31 & TH227T & Thorium & 227 & Thermal neutrons & \\
\hline 32 & TH229T & Thorium & 229 & Thermal neutrons & \\
\hline 33 & PA231F & Protactinium & 231 & Fission spectrum neutrons & \\
\hline 34 & AM241F & Americium & 241 & Thermal neutrons & \\
\hline 35 & Am241H & Americium & 241 & High energy neutrons (14.7 & $\mathrm{MeV})$ \\
\hline 36 & A242MT & Americium & $242 \mathrm{M}$ & Thermal neutrons & \\
\hline 37 & CK245T & Curium & 245 & Thermal neutrons & \\
\hline 38 & CF249T & Californium & 249 & Thermal neutrons & \\
\hline 39 & CF251T & Californium & 251 & Thermal neutrons & \\
\hline 40 & ES254T & Einsteinium & 254 & Thermal neutrons & \\
\hline 41 & CF250S & Californium & 250 & Spontaneous fission & \\
\hline
\end{tabular}




$\begin{array}{lllll}42 & \text { CM244S } & \text { Curium } & 244 & \text { Spontaneous fission } \\ 43 & \text { CM248S } & \text { Curium } & 248 & \text { Spontaneous fission } \\ 44 & \text { ES253S } & \text { Einsteinium } & 253 & \text { Spontaneous fission } \\ 45 & \text { FM254S } & \text { Fermium } & 254 & \text { Spontaneous fission } \\ 46 & \text { FM255T } & \text { Fermium } & 255 & \text { Thermal neutron fission } \\ 47 & \text { FM256S } & \text { Fermium } & 256 & \text { Spontaneous fission } \\ 48 & \text { NP237H } & \text { Neptunium } & 237 & \text { High energy neutrons (14.7 MeV) } \\ 49 & \text { U232T } & \text { Uranium } & 232 & \text { Thermal neutron fission } \\ 50 & \text { U238S } & \text { Uranium } & 238 & \text { Spontaneous fission } \\ 51 & \text { CM243T } & \text { Curium } & 243 & \text { Thermal neutron fission } \\ 52 & \text { CK246S } & \text { Curium } & 246 & \text { Spontaneous fission } \\ 53 & \text { CM243F } & \text { Curium } & 243 & \text { Fission spectrum neutrons } \\ 54 & \text { CH244F } & \text { Curium } & 244 & \text { Fission spectrum neutrons } \\ 55 & \text { CK246F } & \text { Curium } & 246 & \text { Fission spectrum neutrons } \\ 56 & \text { CM248F } & \text { Curium } & 248 & \text { Fission spectrum neutrons } \\ 57 & \text { PU242H } & \text { Plutonium } & 242 & \text { High energy neutrons (14.7 MeV) } \\ 58 & \text { MP237T } & \text { Neptunium } & 237 & \text { Thermal neutrons } \\ 59 & \text { PU240T } & \text { Plutonium } & 240 & \text { Thermal neutrons } \\ 60 & \text { PU242T } & \text { Plutonium } & 242 & \text { Thermal neutrons }\end{array}$

Symbols Used in Tabular Section Pages

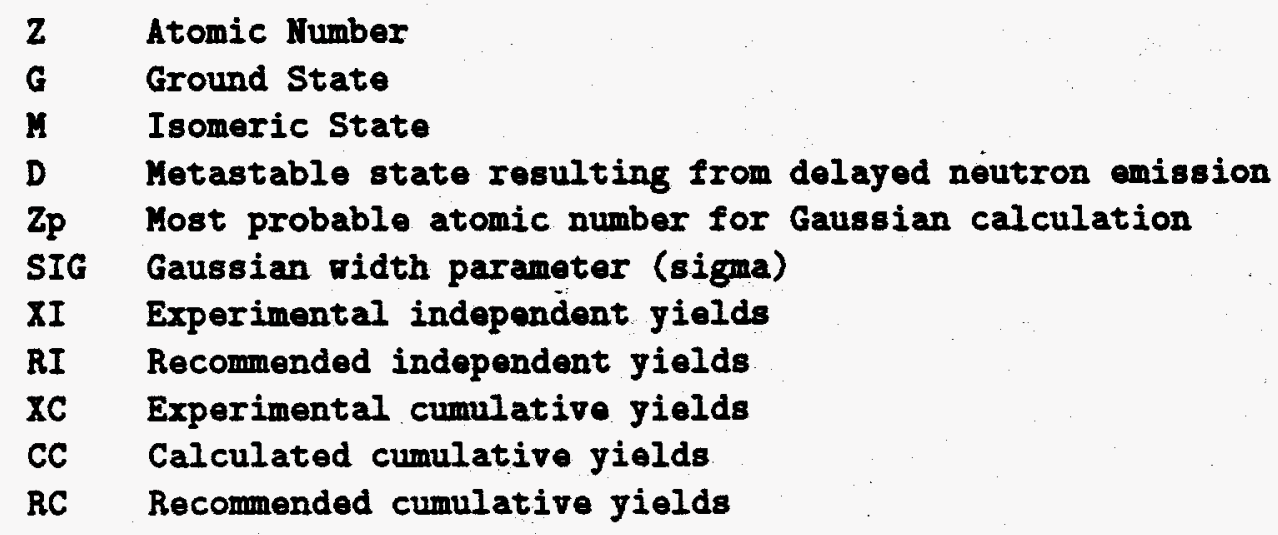

Symbols To Indicate Accuracy of Weighted Average Numbers

$\begin{array}{ccc}\text { Letter } & \text { Range, } \% & \text { Error Stored in File, } \% \\ \text { A } & <0.35 & 0.35 \\ \text { B } & 0.35-0.50 & 0.50 \\ \text { C } & 0.50-0.70 & 0.70 \\ \text { D } & 0.70-1.00 & 1.00 \\ \text { E } & 1.00-1.40 & 1.40\end{array}$




$\begin{array}{lcc}\text { F } & 1.40-2.00 & 2.00 \\ \text { G } & 2.00-2.80 & 2.80 \\ \text { H } & 2.80-4.00 & 4.00 \\ \text { I } & 4-6 & 6 \\ \text { J } & 6-8 & 8 \\ \text { K } & 8-11 & 11 \\ \text { L } & 11-16 & 16 \\ \text { M } & 16-23 & 23 \\ \text { N } & 23-32 & 32 \\ \text { O } & 32-45 & 45 \\ \text { P } & 45 \text { and up } & >64\end{array}$

The reader should note that the letter $\mathrm{P}$ stands for all errors greater than $45 \%$. The letter $\mathrm{P}$ should suggest "poorly" known. The modeled independent yields greater than $1 \%$, are assumed to have $32 \%$ error. Those between 0.5 and 1.0 are assumed to have $64 \%$ error and those $<0.5 \%$ are assumed to have $100 \%$ error. The latter two cases, (although weighted as indicated) both translate as $P$ in every output table. It is recognized that even smaller independent yields have even larger than $100 \%$ error. Indeed, independent yields in the range of 1.0e-9 to 1.0e-12 may be difficult to predict to better than a factor of 100. For this reason, all yields less than 1.0e-12 are blanked out.

\section{Half-Lives}

$\begin{array}{ll}\text { S } & \text { Seconds } \\ \text { M } & \text { Minutes } \\ \text { H } & \text { Hours } \\ \text { D } & \text { Days } \\ \text { Y } & \text { Years } \\ \text { L } & \text { Common } 10 \mathrm{~g} \text { of jears }\end{array}$

The half-life also may be listed as short, long, or stable where appropriate.

\section{Symbols To Indicate Measurement Techniques}

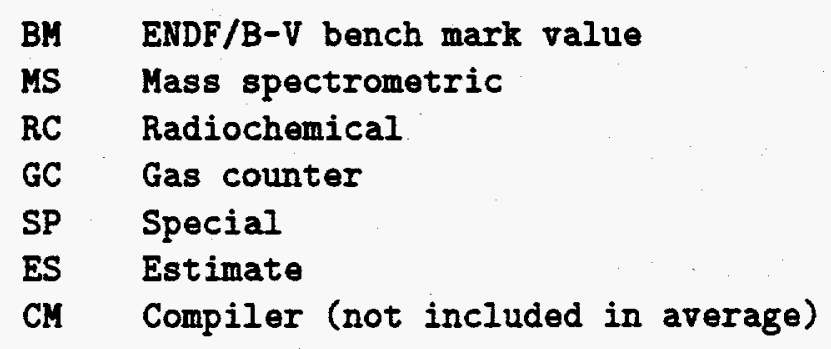

Measurement Type 
CU

IN

FC

FI

RE
Cumulative

Independent

Fractional Cumulative

Fractional Independent

Relative (arbitrary units)

Note Symbols

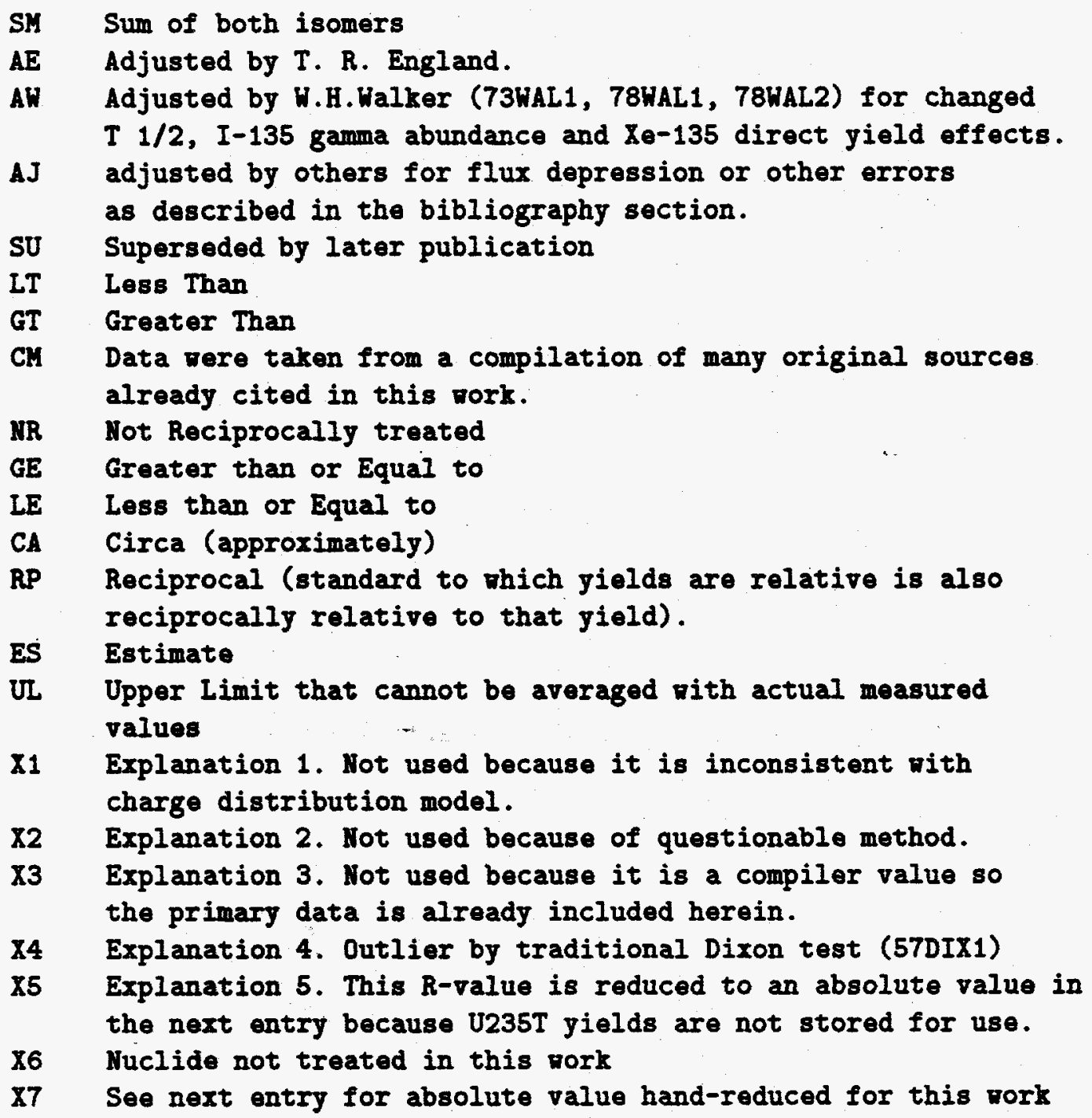

\section{Acknowledgements}

The authors are grateful to the scientists who made helpful suggestions and forwarded their latest published and unpublished work. We also appreciate the contributions and recommendations of members of the IAEA Coordinated Research Committe for yield evaluation methods and data (Meinhart 
Lammer, Secretary) formed in 1991. The fission yields recommended in this work appear in the Evaluated Nuclear Data File (ENDF/B-VI) at the Brookhaven National Laboratory. To improve the evaluation for use in calculation of decay heat, revisions were recommended by the ENDF/B-VI Fission Yield Subcommittee. These revisions included modeled direct yield metastable vs. ground state splits (76MAD2), odd-even proton and neutron pairing effects (76MAD1), and the delayed neutron yields treated so that all independent yields are before delayed neutron emission and all cumulative yields are after delayed neutron emission (77ENG3). The authors are grateful for the cooperation of Mike James, David Weaver and Robert Mills of the UK with whom they have exchanged computer data banks and for the encouragement received from the IAEA Fission Product Nuclear Data Panels at Bologna, Italy, 26-30 November 1973, and Petten, Netherlands, 5-9 September 1977.

We especially appreciate the generosity of the General Electric Company in making early versions of codes and data compiled through 1981 available to Los Alamos for continued use in evaluations. Since 1981, the effort has been supported at Los Alamos by the U. S. DoE. Some brief review articles on fission yields appear in the literature. However, the authors believe that the user should have access to both the evaluated yields and the data on which they are based. This belief arose because no abstracting journal has a category "fission yield" so data remain widely scattered in letters, papers, reports, articles, books, and proceedings. Thus, the data have been collected only with difficulty and are not readily available to the user. Large bulk and consequent publication cost rule out journal publication. However, since 1977, it has been available from the authors on microfiche of the highest quality that are easily read, inexpensive, and conveniently stored. As noted in the text the data are now being made available on the internet.

The authors are grateful for the guidance and recommendations of the Fission Yield Subcommittee of CSEWG that is the functional body responsible for the preparation of the Evaluated Nuclear Data File for the USA National Nuclear Data Center at Brookhaven National Laboratory. Members of that Yield Subcommittee have included: T. R. England, chairman; D. G. Madland, J. R. Liaw, B. I. Spinrad, R. E. Schenter, D. M. Gilliam, J. P. Unik, V. Mc Lane, Y. Harker, P. L. Reeder, B. W. Wehring, W. J. Maeck, B. F. Rider, W. B. Wilson, A. C. Wahl, W. H. Walker, and K. Wolfsberg. This Fission Yield and Decay Data Subcommittee is a Sub-group of the Cross Section Evaluation Group (CSEWG) of the Evaluated Nuclear Data File (ENDF) maintained at Brookhaven National Laboratory.

Much credit is owed M.E. Meek who originated the Vallecitos Compilation of Fission Yields, and who was senior author on the first six editions. His interest was in values for the unmeasured short lived $\mathrm{Kr}$ and $\mathrm{Xe}$ isotopes for use of fission off-gas analyses to estimate fuel integrity. It was his decision to computerize the evaluation and he wrote the computer program to do so. More than thirty additional experimentally determined half-lives became available in the under 10 second region from ENDF/B-V summarized by $80 \mathrm{ENG}$. These have been included here. Together with newly evaluated $Q$ values, they almost close any discrepancy in decay heating values and give excellent agreement between theoretical decay heating values by summation and those by experiment throughout the period of 1 10,000 seconds after shut down. This period spans, indeed far exceeds, the period of time important in LOCA (loss of coolant accident) analysis. Inquiries should be directed to the subcommittee chairman, T. R. England, Los Alamos National Laboratory, Post Office Box 1663, Los Alamos, New Mexico, 87545, U.S.A. 


\author{
Annotated Bibliography \\ for \\ ENDF-349, LA-UR-94-3106
}

39ABE1 Abelson, P., "Cleavage of the Uranium Nucleous," Phys. Rev. 55, 1 (1939). Phys. Rev., 56, 418 (1939). $10 \mathrm{hr}$. Te127, $70 \mathrm{~min}$. Te-129, 8 day I-131 are independent proof of fission as proposed by $39 \mathrm{HAH} 1$.

39AND1 Anderson, H. L., Booth, E. T., Dunning, J. R., Fermi, E., Glasoe, G. N., Slack, F. G., "The Fission of Uranium," Phys. Rev. 55, 511-512 (1939). Pupin Physics Laboratories, Columbia University. Thermal neutron fission is due to isotope $\mathrm{U}-235$ as suggested by $39 \mathrm{BOH} 1$ and 39BOH2. Fission efficiency follows a $1 / \mathrm{v}$ law.

39BOH1 Bohr, N., "Resonance in Uranium and Thorium Disintegrations and the Phenomenon of Nuclear Fission," Phys. Rev., 55, 418 (1939).

39BOH2 Bohr, N., Wheeler, J. A., “The Mechanism of Nuclear Fission,” Phys. Rev. 56, 1124 (1939).

39FRI1 Frisch, O., "Physical Evidence for the Division of Heavy Nuclei Under Neutron Bombardment," Nature, 143, 276 (1939).

39HAH1 Hahn, O., Strassmann, F., "Neutron-Induced Radioactivity of Uranium," Naturwiss., Vol. 27, Page 11, (1939). Discovery of fission by identification of fission products after neutron bombardment of uranium.

39HAH2 Hahn, O., Strassmann, F., "Fission Products of $U$ and Th," Ann. de Physik 36, 3-4, 368-372 (Oct 1939). Kr and Xe are products of fission.

39HAH3 Hahn, O., Strassmann, F., “Zur Frage der Existenz der 'Trans-Urane'," Naturwiss., 27, 451-453 (1939). Neutron bombardment of uranium made not just transuranic species but a 2.5 $\mathrm{hr}$ activity which was iodine and $66 \mathrm{hr}$ activity which was molybdenum.

39HAH4 Hahn, O., Strassmann, F., "Weitere Spaltprodukte aus der Bestrahlung des Urans mit Neutronen," Naturwiss., 27, 529-534 (1939). Rb, Cs, I, Br are also products of fission.

39HAH5 Hahn, O., Strassmann, F., Fluegge, S., "Uber einige Bruchstucke beim Zerplaten des Thoriums," Naturwiss., 27, 544-547 (1939).

39HAH6 Hahn, O., Strassmann, F., "Collapse of $U$ and Th Nuclei into Lighter Atoms," Phys. Zeits. 40, 673-680 (Nov 15, 1939). $3 \mathrm{hr} \mathrm{Kr}$ and $14 \mathrm{~min} \mathrm{Ba}$ is proven by chemical reaction. Chains of Xe-Cs-Ba; Sr-Kr; Sb-Te-I identified. The word collapse used instead of the word 'fission' later used by 39MEI1.

39HAL1 Halban, H. von Junior, Joliot, F., Kowarski, L., "Number of Neutrons Liberated in the Nuclear Explosion of Uranium," Nature, 143, 680, (1939). 
39HAL2 Halban, H. von Junior, Joliot, F., Kowarski, L., "Liberation of Neutrons in the Nuclear Explosion of Uranium," Nature 143, 470 (1939).

39LAN1 Langsdorf, A. Jr., "Fission Products of Thorium," Phys. Rev. 56, 205 (15 July 1939). 1 page. $18 \mathrm{~min} \mathrm{Kr}-88$ identified.

39LAR1 Lark-Horovitz, K., Risser, J. R., Smith, R. N., "Nuclear Excitation of Indium with Alpha Particles," Phys. Rev. 55, 878 (1 May 1939). In-115m decays with half life of 4.1 hours.

39LIB1 Libby, W. F., Lee, D. D., “Energies of Soft Beta-Rays of Rb and Other Bodies. Method for Their Determination," Phys. Rev. 55, 245-251 (1 Feb 1939).

39MCM1 Mc Millan, E., Anderson, H. L., Booth, E. T., Dunning, J. R., Fermi, E., Glasoe, G. N., Slack, F. G., "Radioactive Recoils from Uranium Activated by Neutrons," Phys. Rev. 55, $510-511$ (1939). Fission products range is $1.7 \mathrm{~cm}$. in air.

39MEI1 Meitner, L., Frisch, O. R., "Disintegration of Uranium by Neutrons a New Type of Nuclear Reaction," Nature 143: 239-240 (11 Feb 1939). To explain Hahn and Strassmann's proven barium formation upon bombardment of uranium with neutrons, a liquid-drop model for fission is proposed in which ca. $200 \mathrm{meV}$ is released from the difference in packing fraction between uranium and the elements in the middle of the periodic system. First used the word 'fission' for this event.

39NIE1 Nier, A. O., "The Isotopic Constitution of Uranium and Half Lives of the Uranium Isotopes," Phys. Rev. 55, 150-153 (1939).

39PEI1 Peierls, R., "Critical Conditions in Neutron Multiplication," Proc. Camb. Phil. Soc. 35,610 (1939).

39ROB1 Roberts, R. B., and Kuper, J. B. H., "Uranium and Atomic Power," J. Appl. Phys. 10, 612 (1939).

39SEA1 Seaborg, G. T., Segre, E., "Nuclear Isomerism in Element 43," Phys. Rev. 55, 808-814 (1 May 1939).

39SZI1 Szilard, L., and Zinn, W., "Instantaneous Emission of Fast Neutrons in the Interaction of Slow Neutrons with Uranium," Phys. Rev., 55, 799-800 (1939).

40AND1 Anderson, H., Fermi, E., Grosse, A. von, "Branching Ratios in the Fission of U(235)," Phys. Rev. 59, 52-66 (Jan 1 1941). First quantitative fission product yield measurements in thermal neutron bombardment of U235.

40HAH1 Hahn, O., Strassmann, F., "New Method for Separation of Active Fission-Products from U and Th," Naturwiss. Vol. 28, pages 54, 61, 455, 543 (1940).

40LAN1 Langsdorf, A. Jr., Segre, E., “Nuclear Isomerism in Se and Kr," Phys. Rev. 57, 105-110 (Jan 15, 1940). A 2.3 hour Br product occurs in fission. Probably a daughter of Se-83. Br-83 and $\mathrm{Kr}-83$ also produced in fission of $\mathrm{U}$ and $\mathrm{Th}$.

40MEI1 Meitner, L., "Uber das Verhalten Einiger Seltenen Erden bei Neutronenbestrahlung," Arkiv. Mat. Astron. Fysik, 27A(17), pages 1-18 (28 Juni 1940). Rare Earths Dy-165, Ho-166, Sm-153 found in fission. 
40NIE1 Nier, A. O., Booth, E. T., Dunning, J. R., Grosse, A. V., "Nuclear Fission of Separated Uranium Isotopes," Phys. Rev. 57, 748 (1940).

40NIE2 Nier, A. O., Booth, E. T., Dunning, J. R., Grosse, A. V., "Further Experiments on Fission of Separated Uranium Isotopes," Phys. Rev. 57, 748 (1940).

40STR1 Strassmann, R., Hahn, O., "Kurzlebige Brom- und Jod-Isotope bei der Uranspaltung," Naturwiss. 28, 817-820 (27 Dezember 1940).

40TUR1 Turner, L. A., "Nuclear Fission," Rev. Mod. Phys. 12, 1-29 (1940). First review article of about 50 radioactive fission products identified to date. 133 refs.

41HAH1 Hahn, O., Strassmann, F., "Formation of Zirconium and Protactinium by Bombardment of Thorium and Uranium with Neutrons," Naturwiss. Vol. 29, Pages 285, 369 (1941) and Z. Physik, 117, 789 (1941).

41HAH2 Hahn, O., Strassmann, F., “On the Molybdenum Isotopes from the Fission of Uranium," Naturwiss., 29, 369-370 (June 13, 1941).

41MOU1 Moussa, A., and Goldstein, L., "Radioactive Bromine Isotopes from Uranium Fission," Phys. Rev. 60, 534-535 (1941).

42HAH1 Hahn, O., Strassmann, F., "Uber Die bei der Uranspaltung Aufretenden Kurzleigen Barium- und Lanthan-Isotope," Naturwiss. Vol.30, Page 324-328 (29 May 1942).

43HAH1 Hahn, O., Strassmann, F., "Uber Die bei der Uranspaltung Auftretenden Activen Strontium- und Yttrium-Isotope," Naturwiss. Vol. 31, Pages 249-250, also page 499 (21 May 1943); And Z. Physik 121, 729 (1943). About Kr-Rb-Sr-Y-Zr chains of mass 89, 90 and above.

46GRU1 Grummitt, W. E., and Wilkinson, G., "Fission Products of U235," Nature, 158, 163 (Aug. $3,1946)$.

46SIE1 Siegel, J. M., "Nuclei Formed in Fission, Decay Characteristics, Fission Yields, and Chain Relationships," J. Am. Chem. Soc., 68, 2411-2442 (1946). Revs. Modern Phys. 18, 513-544 (1946). Second review article on 160 radiaactive fission products recognized at that time. Complete bibliography of over 300 sources. Valuable historical record. With complete two humped fission yield curve for U235T before fine structure was suspected.

47ARN1 Arnold, J. R., and Sugarman, N., "Short-Lived Isomeric States of Se83 and Ge77," J. Chem. Phys., 15, 703-712 (1947).

47GRU1 Grummitt, W. E., Gueron, J., Wilkinson, G., and Yaffe, L., "Fission Yields of Ba139 and Ba140 in Neutron Fission of U235 and U238," Can. J. Research, 25B, 364 (1947). Chem. Abstracts, 42: 0452c (1948). Superseded by 51GRU1.

47STE1 Steinberg, E. P., Seiler, J. A., Goldstein, A., and Dudley, A., "Fission Yields in Uranium233," MDDC-1632, (Jan. 6, 1948). Revised in 1954 by Steinberg in ref. 55STE1. CSISRS90004. 
47THO1 Thode, H. G., and Graham, R. L., "A Mass Spectrometer Investigation of the Isotopes of Xenon and Krypton Resulting from the Fission of U-235 by Thermal Neutrons," Can. J. Res., 25A, No. 1, 1-14 (1947). See 48TH01.

47YAF1 Yaffe, L., and Mackintosh, C. E., "Fission Yields of Masses 131, 132, 134, and 136 Formed in Neutron Fission of U," Can. J. Res., 25B, 371 (1947). Chem. Abstracts, 42: 0452e (1948).

48GRU1 Grummitt, W. E., and Wilkinson, G., "Fission Products of U233," Nature, 161, 520 (1948). Replaced by ref. 51GRU1.

48THO1 Thode, H. G., "Mass Spectrometry and Nuclear Chemistry," Nucleonics, 3, No. 3, 14-24 (March 1948). NSA 01: 01283 (1948).

49ARR1 Arrol, W. J., Chackett, K. F., and Epstein, S., "The Extraction and Purification of Xenon and Krypton Isotopes from Neutron Irradiated Uranium," Can. J. Res., 27B, 757-763 (1949). See 48TH01. 53KAT2 on pages 69-70 (footnote 30) questions complete recovery of Xe in this work. A low $\mathrm{Xe} / \mathrm{Kr}$ ratio would result.

49C001 Cook, G. B., AERE C/R 424 (1949).

49GLE1 Glendenin, L. E., Ph. D. Thesis, Massachusetts Institute of Technology, (Aug. 1, 1949), op. cit. ref. 51COR1, pg. 2008.

49GLE2 Glendenin, L. E., "The Distribution of Nuclear Charge in Fission," Laboratory for Nuclear Science, Massachusetts Institute of Technology, Technical Report No. 35 (December 1949 ) and unpublished work cited in ref. 55STE1. NSA 04: 01546 (1950). Isomeric independent yields are here split per 76MAD2. CSISRS-90008.

49ING1 Inghram, M. G., Hess, D. C., Reynolds, J., "Relative Yields of Fission Cesium Isotopes," Phys. Rev., 76, 1717 (1949).

49KOC1 Koch, J., Kofoed-Hansen, O., Kristensen, P., and Drost-Hansen, W., "Measurements on Radioactive Krypton Isotopes from Fission After Mass-Spectrometric Separation," Phys. Rev., 76, 279 (1949).

49SAK1 Sakakura, A. Y., B. S. Thesis, Massachusetts Institute of Technology, (June 1, 1949), op. cit., ref. 51COR1, pg. 2008. CSISRS-90010.

49SEG1 Segre, E., Helmholtz, A. C., “Nuclear Isomerism,” Revs. Mod. Phys. 21, 271-304 (1949).

49STA1 Stanley, C. W., and Katcoff, S., "The Properties of 86-Second I136," J. Chem. Phys., 17, 653 (1949). Chem. Abstracts, 43: 8872f (1949).

49THO1 Thode, H. G., and Shields, R. B., "Mass Spectrometry," Reports on Progress in Physics, 12, 1-21 (1949). Supersedes 47THO1 and 48THO1. Is superseded by 55WAN1.

49THO2 Thode, H. G., Private communication to C. D. Coryell (1949). op. cit. ref. 51COR1, page 2008.

49WAY1 Way, K., and Dismuke, N., "Fission Product Yields," AECD-2817 (Aug 29, 1949). Compilation. 
50COR1 Coryell, C. D., Sakakura, A. Y., and Ross, A. M., "Fission Yields of 65-Day Zr95 and 17-Hour Zr87: Search for Other Zr and Nb Fission Chains," Phys. Rev., 77, 755 (Mar. 1, 1950).

50ING1 Inghram, M. G., Hayden, R. J., and Hess, D. C., "U235 Fission Yields in the Rare Earth Region," Phys. Rev. 79, 271-274 (July 15, 1950). CSISRS-90013.

50MAC1 Macnamara, J., Collins, C. B., and Thode, H. G., "Fission Yield of Xe133 and Fine Structure in the Mass Yield Curve," Phys. Rev., 78, 129 (1950). CSISRS-90014.

50MAC2 Macnamara, J., and Thode, H. G., "The Isotopes of Xenon and Krypton in Pitchblende and the Spontaneous Fission of U-238," Phys. Rev., 80, 471-472 (1950).

50MIT1 MIT Staff "MIT Quarterly Report," NP-1857 (1950), pg. 25, Sb yields in U235T fission replaced by later values in 53PAP1.

50NAG1 Nagle, D., and Zacharias, J. R., Private communication 51COR2.

50NID1 Niday, J. B., and Turkevich, A., "Fission Yield Curve of Th-232 with Pile Neutrons," AECD-2862, June 16, 1950. (Relative to Sr89 assumed to be 6.00\%). NSA 04: 05026 (1950). CSISRS-90015.

50PAP1 Pappas, A. C., and Coryell, C. D., Annual Report, Laboratory for Nuclear Science, Mas: sachusetts Institute of Technology, July 1, 1950. CSISRS-90016.

50STE1 Steinberg, E. P., Private communication to C. D. Coryell (1950) op. cit. ref. 51COR1 pg. 2008. CSISRS-90017.

51ALL1 Allen, K. W., and Dewan, J. T., "The Emission of Short-Ranged Charged Particles in the Slow Neutron Fission of Uranium," Phys. Rev., 82, No. 4, 527-530 (May 15, 1951). Not coded.

51BAL1 Ballou, N. E., Novey, T. B., Engelkemeir, D. W., Brady, E. L., and Seiler, J. A., "Characteristics of 57d Y91," Paper 75, pp. 675-677 loc. cit. ref. 51COR1. From progress report CC-920, 4, (Sept 1943). CSISRS-90018.

51BUR1 Burgus, W. H., and Ballou, N. E., "Characteristics of 28d Ce141," Paper 180, pp. 11841187, loc. cit. ref. 51COR1. From R. P. Schuman, Progress Report CN-2929, 5, April (1945). CSISRS-90019.

51BUR2 Burgus, W. H., Winsberg, L., Seiler, J. A., and Rubinson, W., “Characteristics of 275d Ce144," Paper 184, pp. 1195-1199, loc. cit. ref. 51COR1. CSISRS-90020.

51 COO1 Cook, G. B., Ph. D. Thesis, Cambridge, 1951.

51COR1 Coryell, C. D., and Sugarman, N., editors, Radiochemical Studies: The Fission Products, National Nuclear Energy Series, Manhattan Project Technical Section, Division IV - Plutonium Project Record, Mc Graw-Hill Book Co., Inc., New York, New York, 1951, Vol 9, Part V, Book 2 and 3. 
51COR2 Coryell, C. D., and Sugarman, N., Appendix B, pp. 2003-2011, loc. cit. ref. 51COR1. Compiler. All values referenced directly. Supersedes J. Am. Chem. Soc. 68, 2411 (1946) and MDDC-1632 (Nov 1, 1947). From Progress Report CN-1958 (August 1944). CSISRS-90021.

51DIL1 Dillard, C. R., Adams, R. M., Finston, H., and Turkevich, A., "Fission Yield of 20m Y," Paper 79, pp. 692-694. loc. cit. ref. 51COR1.

51ENG1 Engelkemeir, D. W., Novey, T. B., and Schover, D. S., "Determination of Absolute Slow Neutron Fission Yields in U235 (I)," Paper 205, pp. 1334-1343, loc. cit. ref. 51COR1 Book 3.

51ENG2 Engelkemeir, D. W., Seiler, J. A., Steinberg, E. P., and Winsberg, L., "Thermal-Neutron Fission Yields in U235," Paper 217, pp. 1372-1374, loc. cit. ref. 51COR1, Book 3. Replaced by 52ENG1. From Progress Report CC-3420 (1946). CSISRS-90022.

51ENG3 Engelkemeir, D. W., Seiler, J. A., Steinberg, E. P., and Winsberg, L., "Fission Yields in U238," Paper 318, pp. 1375-1377, loc. cit. ref. 51COR1, Book 3. Report on earlier experiment 1944-1946. CSISRS-90023.

51FEL1 Feldman, M. H., Glendenin, L. E., and Edwards, R. R., "Identification and Yield of 36 Hr. Br82 in Fission," Paper 62 pp. 598-602 loc. cit. ref. 51COR1. Value re-normalized to Br-83. CSISRS-90024.

51FEL2 Feldman, M. H., and Glendenin, L. E., "Identification and Yield of 19.5 Day Rb86 in Fission," Paper 71, pp. 654-659 loc. cit. ref. 51COR1. CSISRS-90025.

51FIN1 Finkle, B., Hoagland, E. J., Katcoff, S., and Sugarman, N., "Note on the Fission Yield of 67h Mo-99," Paper 96, pg. 770 loc. cit. ref. 51COR1. This is a revision of earlier work 1944-1946. CSISRS-90026.

51FRE1 Freedman, M. S., and Engelkemeir, D. W., "Determination of Absolute Slow-Neutron Fission Yields in U235 (II)," Paper 206, pp. 1344-1348, loc. cit. ref. 51COR1, Book 3. Ba-140 U235T error assigned and value corrected for flux depression in Table C1 73WAL3. From Progress Report CC-1331, 4 (Feb 1944). CSISRS-90027.

51GLE1 Glendenin, L. E., "Absence of Long-Lived Selenium in Fission II. Search for Se79," Paper 61, pp. 596-597 loc. cit. ref. 51COR1. From Progress Report MON-N-6, 9 (Aug 1945). CSISRS-90028.

51GLE2 Glendenin, L. E., and Steinberg, E. P., "Long-Lived Ruthenium-Rhodium Chains in Fission," Paper 103, pp. 793-804 loc. cit. ref. 51COR1. CSISRS-90029.

51GLE3 Glendenin, L. E., Metcalf, R. P., “Characteristics of 6.7h I135 (I)," Paper 140, pp. 9921004 loc. cit. ref. 51COR1.

51GLE4 Glendenin, L. E., "Further Study of the 13d Cs Activity," Paper 159, pp. 1092-1095, loc. cit. ref. 51COR1. Based on earlier unpublished work. CSISRS-90030.

51GLE5 Glendenin, L. E., Steinberg, E. P., Inghram, M. G., and Hess, D. C., "Nuclear Structure in Fission," Phys. Rev., 84, 860-861 (1951). 
51GLE6 Glendenin, L. E., Footnote to ref. 51KAT2 page 591 loc. cit. ref. 51COR1. From Progress Report MON-N-15, 12 (Sept 1945). CSISRS-90031.

51GLE7 Glendenin, L. E., Footnote to ref. 51NOV2 page 979 loc. cit. ref. 51COR1. CSISRS-90032.

51GOL1 Goldstein, A., Schuman, R. P., and Rubinson, W., "Fission Yields of Members of Chain 141," Paper 181, pp. 1188-1190, loc. cit. ref. 51COR1.

51GRU1 Grummitt, W. E., and Wilkinson, G., "Thermal Neutron Fission Yields of U233 and U235," CRC-470 (1951). Many of these early measurements are not included where they are inconsistent with all subsequent data. CSISRS-90033. EXFOR-13054.

51HOA1 Hoagland, E. J., and Sugarman, N., "Discovery of 10-Yr Kr85 in Fission," Paper 69, pp. 635-641 loc. cit. ref. 51COR1. Corrected $\mathrm{Kr}-85$ from 9.2 year to 10.76 year half life. CSISRS-90034.

51HOA2 Hoagland, E. J., and Katcoff, S., "Fission Yield of 2.7-Hr Sr92 and Radiations of 3.5-Hr Y92," Paper 73, pp. 660-662, loc. cit. ref. 51COR1.

51HOA3 Hoagland, E. J., and Sugarman, N., "Independent Fission Yield of 9.2h Xe135," Paper 147, pp. 1035-1042, loc. cit. ref. 51COR1. Independent yields of Xe-135 divided between isomers per 76MAD2. CSISRS-90035.

51KAT1 Katcoff, S., Finkle, B., and Sugarman, N., "Characteristics of 33m Br84," Paper 58, pp. 587-590 loc. cit. ref. 51COR1.

51KAT2 Katcoff, S., Finkle, B., and Sugarman, N., "Fission Yield of 2.4h Br83 and Absence of Long-Lived Bromine in Fission," Paper 59, pp. 591-592, loc. cit. ref. 51COR1.

51KAT3 Katcoff, S., “Fission Yield of 30h Te131m," Paper 137, pp. 980-981 loc. cit. ref. 51COR1. CSISRS-90036.

51KAT4 Katcoff, S., Finkle, B., Hoagland, E. J., Sugarman, N., Glendenin, L. E., and Metcalf, R. P., "Characteristics of 54m I," Paper 138, pp. 982-983 loc. cit. ref. 51COR1.

51KAT5 Katcoff, S., Dillard, C. R., Finston, H., Finkle, B., Seiler, J. A., and Sugarman, N., "Characteristics of 6.7h I135," Paper 141, pp. 1005-1016, loc. cit. ref. 51COR1.

51KAT6 Katcoff, S., Finkle, B., Sugarman, N., “Fission Yield of 85m Ba139," Paper 161, pp. 10971100 , loc. cit. ref. 51COR1. CSISRS-90037.

51KAT7 Katcoff, S., Finkle, B., and Sugarman, N., "Fission Yield of 33h Ce143," Paper 176, p. 1167 , loc. cit. ref. 51COR1.

51KAT8 Katcoff, S., Finkle, B., Sugarman, N., Glendenin, L. E., and Winsberg, L., “Characteristics of I-131," Paper 143, pp. 1017-1022, loc. cit. ref. 51COR1 data reported in 51ENG3. CSISRS-90038.

51LEA1 Leader, G. R., "Study of Long-Lived Tin in Fission," Paper 130, loc. cit. ref. 51COR1.

51LEA2 Leader, G. R., and Sullivan, W. H., "Study of Long-Lived Antimony in Fission (II)," Paper 133, pp. 934-946 loc. cit. ref. 51COR1. CSISRS-90039. 
51MAR1 Marinsky, J. A., and Glendenin, L. E., "Identification and Characteristics of 11d Nd147," Paper 191, pp. 1229-1242, loc. cit. ref. 51COR1.

51MAR2 Marinsky, J. A., and Glendenin, L. E., "Discovery, Identification, and Characteristics of 47h Pm149," Paper 193, pp. 1254-1263, loc. cit. ref. 51COR1.

51MET1 Metcalf, R. P., "Study of 43d Cd115 in Fission," Paper 125, pp. 891-894 loc. cit. ref. 51COR1. CSISRS-90040.

51MET2 Metcalf, R. P., "Study of the Chain 2.33d Cd115 - 4.5h In115m," Paper 127, pp. 898-904 loc. cit. ref. 51COR1. CSISRS-90041.

51MET3 Metcalf, R. P., "Study of the Chain 2.83h Cd117 - 1.95h In117," Paper 128, 905-909 loc. cit. ref. 51COR1. CSISRS-90042.

51NOV1 Novey, T. B., Engelkemeir, D. W., Brady, E. L., and Glendenin, L. E., "Characteristics of 53-Day Sr89," Paper 76, pp. 678-681, loc. cit. ref. 51COR1. CSISRS-90018.

51NOV2 Novey, T. B., "Radiations from the Isomeric Pair, 32d Te129m - 72m Te129," Paper 136, pp. 976-979 loc. cit. ref. 51COR1. (Corrected in ref. 55STE1 to be Te-127m $=0.056 \%$ ).

51SCH1 Schuman, R. P., “Independent Fission Yield of 33h Ce143," Paper 182, pp. 1191-1192, loc. cit. ref. 51COR1.

51SEI1 Seiler, J. A., “Palladium - Silver Chains in Fission," Paper 119, pp. 860-870, loc. cit. ref. 51COR1. CSISRS-90044.

51SE12 Seiler, J. A., “Isotopes of Tin in Fission," Paper 129, pp. 910-918 loc. ref. 51COR1. $121 \mathrm{~g}-\mathrm{Sn}$ yield for U235T RCCU is considered as an estimate with little weight since it is stated impurities were present. CSISRS-90045.

51SIE1 Siegel, J. M., and Glendenin, L. E., "Zinc and Gallium Activities in Uranium Fission," Paper 53 pp. 549-565 in National Nuclear Energy Series, ref. 51COR1. CSISRS-90046.

51STA1 Stanley, C. W., and Glendenin, L. E., "Study of Long-Lived Antimony in Fission (III)," Paper 134, pp. 947-957 loc. cit. ref. 51COR1. CSISRS-90047.

51STE1 Steinberg, E. P., and Engelkemeir, D. W., "Short-Lived Germanium and Arsenic Fission Activities," Paper 54, pp. 566-579 loc. cit. ref. 51COR1. CSISRS-90048.

51STE2 Steinberg, E. P., and Glendenin, L. E., "Characteristics of 7.6 d Ag111," Paper 123, pp. 877-880 loc. cit. 51COR1. CSISRS-90049.

51STE3 Steinberg, E. P., and Freedman, M. S., "Summary of Results of Fission-Yield Experiments," Paper 219, pp. 1378-1390, loc. cit. ref. 51COR1, Book 3. CSISRS-90050.

51STE4 Steinberg, E. P., Footnote to ref. 51SEI2 page 913 loc. cit. ref. 51COR1. CSISRS-90051.

51SUG1 Sugarman, N., "Independent Fission Yield of La140," Paper 170, pp. 1139-1143, loc. cit. ref. 51COR1. CSISRS-90052.

51SUL1 Sullivan, W. H., Sleight, N. R., and Gladrow, E. M., "Characterization of 4.5h Ru Produced in Fission," Paper 105, pp. 808-812 loc. cit. ref. 51COR1. CSISRS-90053. 
51SUL2 Sullivan, W. H., and Katcoff, S., “Characteristics of 22h I-133," Paper 142, pp. 1015-1016, loc. cit. ref. 51COR1.

51SWA1 Swartout, J. A., and Sullivan, W. H., "Absence of Long Lived Rhodium in Fission II. Upper Limit to the Fission Yield of 210d Rh102," Paper 118, pp. 856-859, loc. cit. ref. 51COR1. CSISRS-90054.

51TUR1 Turkevich, A., and Niday, J. B., “Radiochemical Studies on the Fission of Th232 with Pile Neutrons," Phys. Rev., 84, No. 1 52-60 (1951). CSISRS-90055.

51WAT1 Waters, D. E., and Hume, D. N., "Comparison of Oxalate and Fluoride Methods for Determination of Zirconium Activity in Fission," Paper 247, pp. 1507-1509, loc. cit. ref. 51COR1, Book 3. CSISRS-90056.

51WIN1 Winsberg, L., "Study of $25 \mathrm{~m}$ Sm155 in Fission," Paper 196, pp. 1284-1291, loc. cit. ref. 51COR1. CSISRS-90057.

51WIN2 Winsberg, L., "Study of 60m Eu158 and 15.4h Eu157 Activities in Fission," Paper 197 pp. 1292-1301, loc. cit. ref. 51COR1. CSISRS-90058.

51WIN3 Winsberg, L., "Study of the Fission Chain 10hr Sm156 - 15.4d Eu156," Paper 198, pp. 1302-1310, loc. cit. ref. 51COR1. CSISRS-90059.

51WIN4 Winsberg, L., "Study of $2 y$ Eu155 in Fission," Paper 199, pp. 1311-1315, loc. cit. ref. 51COR1.

52ENG1 Engelkemeir, D. W., Freedman, M. S., Steinberg, E. P., Seiler, J. A., and Winsberg, L., "Fission Yields in Uranium-235 and Uranium-238," ANL-4927 (1952). Cs-137 corrected from 33year to 30 year half life. Ba-140 U235T error assigned and value corrected for flux depression in Table C1 73WAL3. CSISRS-90060.

52MEI1 Meitner, L., "Spaltung und Schalenmodell der Atomkerne," Arkiv. Fysik. 4: 383-386 (1952). Shell model. 11 refs.

52WAH1 Wahl, A. C., and Bonner, N. A., "Genetic Relationships and Fission Yields of Members of the Mass-115 Decay Chain," Phys. Rev., 85, 570-577 (Feb. 15, 1952). CSISRS-90061.

53BAR1 Bartholomew, R. M., Brown, F., Hawkings, R. C., Merritt, W. F., and Yaffe, L., “The Fission Yield of I131 in the Thermal Neutron Fission of U235," Can. J. Chem., 31, 120-125 (1953). CSISRS-90062.

53BRO1 Brown, F., Yaffe, L., "The Independent Yield of Xe135 Produced in the Fission of Natural Uranium by Pile Neutrons," Can. J. Chem., 31, 242-249 (1953). Independent yields of Xe-135 divided between isomers per 76MAD2. CSISRS-90063. EXFOR-13464.

53CUN1 Cuninghame, J. G., "The Chain 79 As - 79m Se in Slow Neutron Fission of 235U," Philosophical Magazine, 44, 900-906 (1953).

53FLE1 Fleming, W. H., Thode, H. G., "Neutron and Spontaneous Fission in Uranium Ores," Phys. Rev., 92(2), 378-382 (1953). 
53FOR1 Ford, G. P., and Stanley, C. W., "The Fraction of the Mass 141 Chain Formed Independently as La141 in the Thermal Neutron Fission of U235," AECD-3551 (1953). NSA 07: 06619 (1953). CSISRS-90065.

53FOR2 Ford, G. P., “Nuclear Charge Distribution -I: 235 U 14 meV Neutron Fission,” AECD3597, also called LADC-1200 3 pages (February 11, 1953). CSISRS-90066.

53HAR1 Hardwick, W. H., “The Fission Yields of Ru103 and Ru106," Phys. Rev., 92, 1072-1073 (November 15, 1953).

53KAT1 Katcoff, S., and Rubinson, W., "Yield of Xe133 in the Thermal Neutron Fission of U235," Phys. Rev., 91, 1458-1469 (Sept 15, 1953). Supersedes BNL-1485 (1953). Independent yields of Xe-135 divided between isomers per 76MAD2. CSISRS-90068.

53KAT2 Katcoff, S., and Rubinson, W., "Yield of Kr-85 and Xe-133 in the Thermal Neutron Fission of U-235 and Yield of $\mathrm{Kr}-85$ in the Thermal Neutron Fission of Pu-239," BNL-1652, Pages 53-54, (1953). NSA 16: 32388 (1962). CSISRS-90069.

53PAP1 Pappas, A. C., "A Radiochemical Study of Fission Yields in the Region of Shell Perturbations and the Effect of Closed Shells in Fission," MIT-LNS-63 or AECU 2806 (1953). Nucl. Sci. Abs. 8: 2273 (1954). CSISRS-90070. EXFOR-13461.

53REE1 Reed, G. W., and Turkevich, A., "Uranium-235 Thermal Neutron Fission Yields," Phys. Rev., 92, 1473-1481 (Dec. 15, 1953). Ba-140 U235T error assigned from Table C1 in 73WAL3. CSISRS-90071.

53STE1 Stehney, A. F., Sugarman, N., "Characteristics of Br87, a Delayed Neutron Activity," Phys. Rev., 89(1), 194-203 (1953). EXFOR-13225.

53SUG1 Sugarman, N., "Genetics of the Ge78-As78 fission chain," Phys. Rev., 89, No. 3, 570-572 (1953). CSISRS-90072.

53TER1 Terrell, J., Scott, W. E., Gilmore, J. S., and Minkkinen, C. O., "Yields of Mo99 from Fission of U-235 and U-238," Phys. Rev., 92, 1091 (1953). (Abstract). CSISRS-90073.

53TUR1 Turkevich, A., Niday, J. B., and Tompkins, A., "Radiochemical Studies on the Fission of Th-232 with Li+D Neutrons," Phys. Rev., 89, No. 3, 552-555 (1953).

53WET1 Wetherill, G. W., "Spontaneous Fission Yields from Uranium and Thorium," Phys. Rev., 92, No. 4, 907-912 (1953).

53WIL1 Wiles, D. R., Smith, B. W., Horsley, R., and Thode, H. G., "Fission Yields of the Stable and Long-Lived Isotopes of Cesium, Rubidium and Strontium and Nuclear Shell Structure," Can. J. Phys., 31, 419-431 (1953), See ref. 55PET1. CSISRS-90074.

53YAF1 Yaffe, L., Day, A. E., and Greer, B. A., "The Fission Yield of Te134," Can. J. Chem., 31, 48-54 (1953). CSISRS-90075.

54FLE1 Fleming, W. H., Tomlinson, R. H., and Thode, H. G., "The Fission Yield of the Stable and Long-Lived Isotopes of Xenon, Cesium and Krypton in Neutron Fission of U-233," Can. J. Phys., 32, 522-529 (1954). CSISRS-90076. 
54FRE1 Freiling, E. C., Bunney, L. R., and Ballou, N. E., "Identification of Gadolinium and Terbium Radioisotopes as Fission Products of U-235," Phys. Rev., 96, No. 1, 102 (Oct. 1, 1954). CSISRS-90077.

54KEL1 Keller, R. N., Steinberg, E. P., and Glendenin, L. E., "Yields of Fission Products from U-238 Irradiated with Fission Spectrum Neùtrons," Phys. Rev., 94, 969-974 (1954). Samples were sealed in cadmium-lined aluminum capsules and irradiated in a hollow uranium rod in the Oak Ridge pile. Cs-137 corrected from 33 year to 30 year half life. CSISRS-90078.

54KUR1 Kuroda, P. K., and Edwards, R. R., "Radiochemical Measurements of the Natural Fission Rate of Uranium," J. Chem. Phys., 22, 1940 (1954) as reported by 60KEN1.

54PET1 Petrow, H. G., and Rocco, G., "Fission Yield of Gd159 and Tb161," Phys. Rev., 96, No. 6, 1614 (Dec. 15, 1954). CSISRS-90079.

54STE1 Steinberg, E. P., Glendenin, L. E., Inghram, M. G., and Hayden, R. J., "Fine Structure in U233 Fission," Phys. Rev., 95, 867-68 (1954). As removed from graph by ref. 62FER1. CSISRS-90080.

54STE2 Steinberg, E. P., and Glendenin, L. E., "Radiochemical Investigation of the Spontaneous Fission of Cm242,” Phys. Rev., 95, No. 2, 431-39 (1954). Replaces AECD-3520 (1953).

54WIL1 Wiles, D. R., Coryell, C. D., "Fission Yield Fine Structure in the Mass Region 99-106," Phys. Rev., 96, No. 3, 696-702 (1954). CSISRS-90081.

54YAF1 Yaffe, L., Thode, H. G., Merritt, W. F., Hawkings, R. C., Brown, F., and Bartholomew, R. M., "Determination of the Absolute Fission Yield of Ba140 in Thermal Neutron Fission of U235," Can. J. Chem., 32, 1017-1024 (1954). CSISRS-90082.

55BLA1 Blades, A. T., and Thode, H. G., "The Branching Ratio of Kr86m from Fission Yield Studies," Z. Naturforsch., 10A, 838-840 (1955). CSISRS-90083. EXFOR-13462.

55BL01 Blomeke, J. O., "Nuclear Properties of U-235 Fission Products," ORNL- 1783 (1955). Superseded by 57BLO1.

55BRO1 Brown, F., “The Yield of Cs-137 in Pile Neutron Fission of Natural Uranium," J. Inorg. Nucl. Chem., 1, 248-252 (1955). relative to Ba-140 6.32\%.

55CO01 Cook, G. B., Personal communication to E. P. Steinberg and L. E. Glendenin, ref. 55STE1. Values are from Cook, G. B., Ph. D. Thesis, Cambridge, 1951. Isomeric independent yields are here split per 77MAD2.

55COR1 Coryell, C. D., and Winchester, J. W., Progress Report, Laboratory for Nuclear Science, Massachusetts Institute of Technology, Cambridge, Mass. (Aug. 31, 1955) as cited in ref. 60KAT1. CSISRS-90086. EXFOR-13465.

55GLE1 Glendenin, L. E., Steinberg, E. P., Flynn, K. F., Hayden, R. J., and Inghram, M. G., unpublished work cited in ref. 55STE1. CSISRS-90087.

55GLE2 Glendenin, L. E., and Steinberg, E. P., "Fission Yields in Spontaneous Fission of Cf-252," J. Inorg. Nucl. Chem., 1, 45-48 (1955). 
55KUK1 Kukavadze, G. M., Anikina, M. P., Goldin, L. L., Ershler, B. V., "Yields of Various Nd Isotopes and Ce Isotopes in Fission of 233U," AEC-TR-2435 Part 2, Pages 125-127 (July 1, 1955). Superseded by 59ANI1.

55LIT1 Littler, D. J., "Long Term Reactivity Changes in Natural Uranium Reactors," Proceedings of the International Conference on the Peaceful Uses of Atomic Energy, Geneva, Switzerland 1955 Vol. 5, 141-144 (United Nations, New York 1956). Harwell, U. K. Measurement of Sm-149 by pile oscillator assuming a 76700 barn cross section.

55MEL1 Melaika, E. A., Parker, M. J., Petruska, J. A., and Tomlinson, R. H., "The Relative Abundances of Neodymium and Samarium Isotopes in the Thermal Neutron Fission of U235 and U233," Can. J. Chem, 33, 830-837 (1955). U233 Sm adjusted by 73WAL1. CSISRS-90089.

55PAP1 Pappas, A. C., “The Distribution of Nuclear Charge in Low Energy Fission," Proceedings of the International Conference of the Peaceful Uses of Atomic Energy, Geneva, Vol. 7, 19-26 (United Nations, New York, 1955).

55PET1 Petruska, J. A., Melaika, E. A., and Tomlinson, R. H., "The Fission Yields of the Cesium Isotopes Formed in the Thermal Neutron Fission of U235 and the Neutron Absorption Cross Section of Xe-135," Can. J. Phys., 33, 640-649 (1955). 137Cs decreased $0.8 \%$ for change in decay correction by change of $t 1 / 2$ of $137 \mathrm{Cs}$ from 26.6 to 30.2 years per 73WAL1. CSISRS- 90090.

55PET2 Petruska, J. A., Thode, H. G., and Tomlinson, R. H., "The Absolute Fission Yields of Twenty-Eight Mass Chains in the Thermal Neutron Fission of U-235," Can. J. Phys., 33, 693-706 (1955). CSISRS-90091.

55REE1 Reed, G. W., “U235 Thermal Neutron Fission Yields at Masses 90 and 91," Phys. Rev., 98, 1327-1329 (June 1, 1955) Corrected to Sr-90 half-life of 28.8 Y. CSISRS-90092.

55STE1 Steinberg, E. P., and Glendenin, L. E., "Survey of Radiochemical Studies of the Fission Products," Proceedings of the International Conference on the Peaceful Uses of Atomic Energy, Geneva, Switzerland, United Nations, New York, New York, 1955, Vol. VII, p 3. Supersedes ANL-4680, Page 50, (1950).

55WAH1 Wahl, A. C., "Fission of U-235 by 14-meV Neutrons: Nuclear Charge Distributions and Yield Fine Structure," Phys. Rev., 99, 730-739 (1955). Cumulative yields relative to $\mathrm{U}-235$ thermal yield of same nuclide. CSISRS-90093.

55WAN1 Wanless, R. K., and Thode, H. G., "The Fission Yields of Isotopes of Xenon and Krypton in the Neutron Fission of U235 and U238," Can. J. Phys., 33, 541-544 (1955). CSISRS-90094. EXFOR-13389.

56BAR1 Bartholomew, R. M., and Baerg, A. P., "The Yield of Cs138 in Thermal Neutron Fission of U235," Can. J. Chem, 34, 201-205 (1956) relative to Ba140 yield of 6.32\%. CSISRS-90095.

56BLA1 Blades, A. T., Fleming, W. H., and Thode, H. G., "The Ratio of Xenon to Krypton in U235 Fission," Can. J. Chem, 34, 233-237 (1956). CSISRS-90096. EXFOR-13391. 
56CHE1 Chenley, R. B., Osmond, R. G., and Perry, S. G., "The Separation of Rhodium-105 from Mixed Fission Products," AERE C/R 1870 (1956).

56FLE1 Fleming, W. H., and Thode, H. G., "The Relative Yields of the Isotopes of Xenon in Plutonium Fission," Can. J. Chem, 34, 193-200 (1956). 136Xe decreased 3.2\% to account for higher 135 to 136 yield ratio than assumed in correction for Xe-135 capture per 73WAL1. CSISRS-90097.

56FOR1 Ford, G. P., and Gilmore, J. S., "Mass Yields from Fission by Neutrons between Thermal and $14.7 \mathrm{meV}$," University of California Los Alamos, Albuquerque, New Mexico, LA$1997,(1956)$. Fission spectrum neutrons were obtained by irradiation inside an enriched U-235 capsule. CSISRS-90098.

56HER1 Herrmann, G., Strassmann, F., "Strontium and Yttrium Isotopes from Uranium Fission," (In German) Z. Naturforsch., 11A, 946-954 (1956).

56KEN1 Kennett, T. J., and Thode, H. G., "Mass Spectrometrically Determined Independent Yields of I-128, I-130, Br-80, and Br-82 for U-233, U-235, and Pu-239 Fission," Phys. Rev., 103, 323-327 (1956). CSISRS-90100. EXFOR-13463.

56KUR1 Kuroda, P. K., Edwards, R. R., and Ashizawa, F. T., "Radiochemical Measurements of the Natural Fission Rate of Uranium and the Natural Occurrence of the Short-Lived Iodine Isotopes," Jour. Chem. Phys., 25, 603 (1956).

56PAP1 Pappas, A. C., Wiles, D. R., "New Short-Lived Isotopes of Tin Found in Fission," J. Inorg. Nucl. Chem., 2, 69-78 (1956).

56PUR1 Purkayastha, B. C., and Martin, G. R., "The Yields of 1291 in Natural and in NeutronInduced Fission of Uranium," Can. J. Chem., 34, 293-300 (1956). EXFOR-21393.

56RAM1 Ramaniah, M. V., Doctoral thesis, Washington Univ., St. Louis, Mo. (1956).

56ROB1 Robinson, M. T., and Krause, J. F., "The Yields of the Chemical Elements in Thermal Neutron Fission of U-235," Nucl. Sci. and Engr. 1, 216-221 (1956). Calculated elemental yields from Blomeke, J. O., ORNL-1783 (1955). See 57BLO1 for later version.

56RUS1 Russell, I. J., Ph. D. Dissertation, University of Chicago, (1956). Cited in 61KUR1.

56WAL1 Walker, W. H., "Fission Product Accumulation," CRRP-634 (15 March 1956).

56WIL1 Wiles, D. M., Petruska, J. A., and Tomlinson, R. H., "Some Cumulative Yields of Isotopes Formed in the Thermal Neutron Fission of Pu239," Can. J. Chem, 34, 227-232 (1956). Ref. 59FIC1 says that these results are in error due to fractionation. $147 \mathrm{Sm}$ increased $7 \%$ by recorrection for a change in $\mathrm{t} 1 / 2$ of $147 \mathrm{Pm}$ from 2.52 to 2.62 years and taking account of holdup in 11.06 day $147 \mathrm{Nd}$ per 73WAL1. CSISRS-90103.

57ALE1 Alexander, J. M., and Coryell, C. D., "Nuclear Charge Distribution in the Fission of Uranium and Thorium with $13.6 \mathrm{meV}$ Deuterons," Phys. Rev., 108, No. 5, 1274-1280 (1957). Isomeric independent yields are here split per 76MAD2. Also contains fission of Th232 with fast neutrons as well as deuterons. CSISRS-90104. 
57ANI1 Anikina, M. P., and Ershler, B. V., "The 90Sr Yield from the Fission of 233U," Sov. J. Nucl. Energy, 6, 169 (1957). Translated by L. Bovey from Atomnaya Energiya, 3, 275 (1957). NSA 12: 5687 (1958).

57ASH1 Ashizawa, F. T., Kuroda, P. K., "The Occurrence of the Short-Lived Iodine Isotopes in Natural and in Depleted Uranium Salts,". J. Inorg. Nucl. Chem., 5, 12-22 (1957) as reported by $60 \mathrm{KEN} 1$.

57BAE1 Baerg, A. P., and Bartholomew, R. M., "Yields in U-235 Thermal Neutron Fission," Can. J. Chem, 35, 980-985 (1957). Relative to Ba-140 (6.32\%). CSISRS-90106.

57BAY1 Bayhurst, B. P., “Fission Yields of Sr90," TID 5787, (1957). Used half-life of 28 yrs. This was corrected to 28.8 yrs. before averaging. CSISRS- 90107.

57BAY2 Bayhurst, B. P., Browne, C. I., Ford, G. P., Gilmore, J. S., Knobeloch, G. W., Lang, E. J., Melnick, M. A., Orth, C. J., "Resonance Fission of U235," Phys. Rev., 107, 325 (1957). U235T values computed from ratio of fission product activity to Mo99 activity by $73 \mathrm{CRO}$.

57BLO1 Blomeke, J. O., and Todd, M. F., “Uranium-235 Fission Product Production as a Function of Thermal Neutron Flux, Irradiation Time, and Decay Time, I. Atomic Concentrations and Gross Totals," ORNL-2127 Part 1 Vol.1, Table 2 (1957). A compilation of U235T yields. Supersedes ORNL-1783 (1955).

57CRO1 Crouthamel, C. E., Kafalas, P., Stupegia, D., “Chemical Engineering Division Summary Report for July, August, and September 1957," ANL-5789, Pages 80-81 (December 18, 1957). NSA 14: 01429 (1960). CSISRS-90108.

57CUN1 Cuninghame, J. G., "The Mass Yield Curve for Fission of Natural Uranium by 14 meV Neutrons," J. Inorg. Nucl. Chem., 5, 1-5 (1957).

57CUN2 Cuninghame, J. G., "The Mass Yield Curve for Fission of Am241 by Pile Neutrons," J. Inorg. Nucl. Chem., 4, 1-7 (1957).

57DIL1 Dillon, I. G., "Estimation of Fission Product Spectra in Discharged Fuel from Fast Reactors," Nucl. Sci. and Engr. 2, 567-581 (1957). Calculated inventory from early data. Not coded.

57DIX1 Dixon, W. J., Massey, F. J., “An Introduction to Statistical Analysis,” (2nd Ed.) New York: Mc Graw-Hill Book Company, 1957.

57GOR1 Gorshkov, V. K., Ivanov, R. N., Kukavadze, G. M., and Reformatsky, I. A., "The Yield of U235 Fission Products in the Rare Earth Region," Sov. J. At. Energy, 3, 729-733 (1957). A translation of Atomnaya Energiya 3, 11 (1957). The Sov. J. At. Energ. (English translation) has a faulty table. NSA 12: 378 (1958). Superseded by 59ANI1. EXFOR-40363.

57GRU1 Grummitt, W. E., and Milton, G. M., "The Independent Yields of 140La, 90Y, and 91Y in the Thermal Neutron Fission of $235 \mathrm{U}$ and $233 \mathrm{U}$," J. Inorg. Nucl. Chem., 5, 93-104 (1957). Isomeric independent yields are here split per 76MAD2. CSISRS-90111.

57HAY1 Hay, I. W., and Newton, T. D., "Estimated Fission Fragment Masses," Can. J. Phys., 35, 195-207 (1957). Calculated theoretical fission product masses, no experimental yields given. 
57HOP1 Hopkins, G. R., and Jamieson, C. P., "Measurement of the Xe-135 Cross Section and U235 Fission Yield of I135," J. Appl. Phys., 28, 1362-1363 (1957).

57IVA1 Ivanov, R. N., Gorshkov, V. K., Anikina, M. P., Kukavadze, G. M., Ershler, B. V., "Yields of Some Heavy Fragments in the Fission of 233U," Translation of Atomnaya Energiya, 3, 546-547 (1957). See 58ANI1 Sov. J. At. Energy, 3,1436-1438 (1957). See also NSA 12: 11193 (1958). NSA 14: 11127 (1960). Summarized in 59ANI1.

57KAF1 Kafalas, P., and Crouthamel, C. E., "The Absolute Yield of Cs137 in Fast-Neutron Fission of U235 and Pu239," J. Inorg. Nucl. Chem., 4, 239-244 (1957) and its errata J. Inorg. Nucl. Chem., 5, 92 (1957). Cs-137 corrected from 33 year to 30 year half life. CSISRS90113.

57KEE1 Keepin, G. R., Wilmett, T. F., and Ziegler, R. K., "Delayed Neutrons from Fissionable Isotopes of Uranium, Plutonium, and Thorium,” Phys. Rev., 107, 1044 (1957).

57KEN1 Kennett, T. J., and Thode, H. G., "The Cumulative Yields of the Krypton and Xenon Isotopes Produced in the Fast Neutron Fission of Th232," Can. J. Phys., 35, 969-979 (1957). CSISRS-90114. EXFOR-13463.

57KRI1 Krizhansky, L. M., Maly, Ya., Murin, A. N., and Preobrazhensky, B. K., “Rare-Earth Isotope Yields in the Fission of Pu239 by Pile Neutrons," Sov. J. At. Energ., 2, 334-336 (1957). Uncorrected Sm values used as recommended by ref. 59FIC1. Translated from Atomnaya Energiya, 2, 276 (1957). Also appears in J. Nucl. Energy, 6, 260 (1957). 144Ce decreased 1\% for change in $t 1 / 2$ of $144 \mathrm{Ce}$ from 282 days to 284.4 days per 73WAL1.

57NAS1 Nasuhoglu, R., Raboy, S., Ringo, G. R., Glendenin, L. E., and Steinberg, E. P., "Mass Distribution of U235 by Resonance Neutrons," Phys. Rev., 108, 1522-1523 (Dec. 15, 1957). CSISRS-90116.

57SAT1 Sattizahn, J. E., Kahn, M., and Knight, J. D., "Short-Lived Bromine and Selenium Nuclides from Fission," American Physical Society, Bulletin 11, No. 2, Pg. 197 (1957). CSISRS-90117.

57SUG1 Sugihara, T. T., Drevinsky, P. J., Troianello, E. J., Alexander, J. M., "Fission Yields of Natural Uranium with Deuterons of 5, 10, and $13.6 \mathrm{meV}$, Deuteron Capture and Competition with Stripping," Phys. Rev., 108(5), 1264-1273 (1957).

58ANI1 Anikina, M. P., Ivanov, R. N., Kukavadze, G. M., and Ershler, B. V., "Half-Life of Sr90 and its Yield in U233 Fission," Sov. J. At. Energy, 4, 270-271 (1958). Translation of Atomnaya Energiya 4, 198 (1958). NSA 12: 8110 (1958).

58ASH1 Ashizawa, F. T., Kuroda, P. K., "The Occurrence of the Short-Lived Iodine Isotopes in Natural and Depleted Uranium Salts," J. Inorg. Nucl. Chem., 5, 12 (1958). EXFOR13472. Superseded by 61 KUR1.

58CUN1 Cuninghame, J. G., "Some Further Fission Yields in Spontaneous Fission of 252Cf, J. Inorg. Nucl. Chem., 6, 181-183 (1958).

58GUS1 Gusev, N., Mashkovich, V., and Obrintsev, G., "Gamma Emission of Radioactive Isotope and Fission Products," Moscow Phys-Math Press (1958) as quoted in ref. 63ZYS1. 
58KAT1 Katcoff, S., "Fission-Product Yields from U, Th, and Pu," Nucleonics 16, No. 4, 78-85, (1958). Precursor to 60KAT1.

58KRI1 Krizhansky, L. M., and Murin, A. N., "Sr-90 and Sr-88 Yields in Pu-239 Fission by Reactor Neutrons," Sov. J. At. Energ., 4, 95-96 (1958). Originally in Russian, Atomnaya Energiya, 4, 77 (1958).

58MAL1 Maly, J., Knobloch, V., Imrisova, D., Prasil, Z., and Urbanec, Z., "Stanoveni Stepneho Vytezku 141Ba a 142Ba," Chem. Listy, 52, 918-925 (1958), Coll. Czechosl. Chem. Commun., 23, 1886 (1958).

58PAR1 Parker, P. L., and Kuroda, P. K., "The Occurrence of Molybdenum-89 in Natural and in Depleted Uranium Salts and the Spontaneous Fission Half-Life of Uranium-238," J. Inorg. Nucl. Chem., 5, 153-158 (1958) as reported by 60KEN1. EXFOR-13471.

58PRO1 Protopopov, A. N., Tolmachev, G. M., Ushatskii, V. N., Venediktova, R. V., Krisiuk, I. S., Rodionova, L. P., and Iokovleva, G. V., "Distribution of Fragments by Mass in Fission of U-235, U-238, and Pu-239 by Neutrons of $14.6 \mathrm{meV}$ Energy," Sov. J. At. Energ. 5, 963-968 (1958). English translation. See also J. Nucl. Energy A, 10, 80-85 (1959). Originally appeared in Atomnaya Energiya 5, 130 (1958) in Russian.

58ROB1 Robinson, M. T., Brooksbank, W. A. Jr., Reynolds, S. A., Wright, H. W., and Handley, T. H., "The Behavior of Fission Products in Molten Fluoride Reactor Fuels," Nucl. Sci. and Engr., 4, 288-296 (1958).

58WAH1 Wahl, A. C., "Nuclear-Charge Distribution in Fission: Cumulative Yields of ShortLived Krypton and Xenon Isotopes from Thermal-Neutron Fission of U235," J. Inorg. Nucl. Chem., 6, 263-277 (1958). See also refs. 69WAH1 and 66RAO1 for corrections.

59ALB1 Albenesius, E. L., "Tritium as a Product of Fission," Phys. Rev. Ltrs. (3) 274-275 (Sept 15, 1959). NSA 13: 21495 (1959).

59ANI1 Anikina, M. P., Aron, P. M., Gorshkov, V. K., Ivanov, R. N., Krizhansky, L. M., Kukavadze, G. M., Murin, A. N., Reformatsky, I. A., and Ershler, B. V., "Mass-Spectroscopic Studies of Fission Products of Uranium-233, Uranium-235, and Plutonium-239," Proceedings of the Second International Conference on the Peaceful Uses of Atomic Energy, Vol. 15, pp. 446-448, United Nations, New York, New York, 1959.

59BAK1 Bak, M. A., Burorkov, S. S., I'inskaya, T. A., Petrov, Yu. G., Petrzhak, K. A., Solntsev, V. N., Sorokina, A. V., Yushatskii, V. N., "Yields of Ru-103 and Ru-106 in Fission of U-235 and Pu-239 by Fast Neutrons," Atomnaya Energiya 6, 577 (1959): Sov. J. At. Energ., 6, 429-430, (1959).

59BAR1 Bartholomew, R. M., Martin, J. S., and Baerg, A. P., "Some Yields in Thermal Fission of U-233 and Pu-239," Can. J. Chem., 37, 660-663 (1959). EXFOR-13474.

59BID1 Bidinosti, D. R., Fickel, H. R., and Tomlinson, R. H., "Mass-Spectrometric Studies of Pile Poisons and Their Yields in the Fission of Uranium-233, Uranium-235, and Plutonium-239," Proceedings of the Second International Conference on the Peaceful Uses of Atomic Energy, Vol. 15, pp. 459-463, United Nations, New York, New York, 1959. Preliminary 
state-of-the-art values at Mc Master Univ. All data later published elsewhere, for example 59FIC2. See 61BID1.

59BOL1 Bolles, R. C., and Ballou, N. E., "Calculated Activities and Abundances of U-235 Fission Products," Nucl. Sci. and Engr., 5, 156-185 (1959). Calculations only. No experimental yields given.

59BUN1 Bunney, L. R., Scadden, E. M., Abriam, J. O., Ballou, N. E., "Extension of the FissionProduct Region and Yields of Heavy Products in the Neutron Fission of Plutonium239," Proceedings of the Second International Conference on the Peaceful Uses of Atomic Energy, United Nations, New York, New York, (1959), Vol. 15, pp. 444-445. Replaces USNRDLTR- 268 (1958).

59BUN2 Bunney, L. R., Scadden, E. M., Abriam, J. O., and Ballou, N. E., "Radiochemical Studies of the Fast Neutron Fission of Uranium-235 and Uranium-238," Proceedings of the Second United Nations International Conference on the Peaceful Uses of Atomic Energy, Vol. 15, 449-451 (1959). Neutrons were obtained by bombardment of beryllium with $12 \mathrm{meV}$ protons and filtered through cadmium foil.

59CHU1 Chu, Y. Y., "Charged-Particle-Induced Fission: A Mass Spectrometric Study," University of California Lawrence Radiation Laboratory, Berkeley, California, UCRL-8926, 1959, page 33. Relative values normalized to $\mathrm{Nd}-145$.

59CR01 Crouch, E. A. C., and Swainbank, I. G., "Rare Earth Fission Product Yields from Highly Irradiated Plutonium-239," Proceedings of the Second International Conference on the Peaceful Uses of Atomic Energy, 15, 464-468 (United Nations, New York, 1959). Not Corrected for cross-sections. Not usable for fission yields without burnup correction.

59FAI1 Fairhall, A. W., Jensen, R. C., and Neuzil, E. F., "Fission Studies of Elements Lighter than Thorium," Proceedings of the Second International Conference on the Peaceful Uses of Atomic Energy, Vol. 15, pp. 452-458 United Nations, New York, (1959).

59FER1 Ferguson, R. L., Thesis, Dept. of Chemistry, Washington Univ. (Jan 1959). Cited in 64HYD1 pages 146-147.

59FIC1 Fickel, H. R., and Tomlinson, R. H., "The Cumulative Fission Yields of Light Maw Fragments in the Thermal Fission of Pu-239," Can. J. Phys., 37, 916-925 (1959). PU239T $\mathrm{Rb} 85$ reduced $11 \%$ to recorrect for $\mathrm{Kr} 85$ holdup and $\mathrm{Ru}-103$ raised $7.7 \%$ after discarding one sample for natural $R u$ contamination. These adjustments recommended by $73 W A L 1$.

59FIC2 Fickel, H. R., and Tomlinson, R. H., "The Cumulative Fission Yields of 21 Heavy Mass Nuclides Produced in the Thermal Fission of Pu-239," Can. J. Phys., 37, 926-930 (1959). 144Ce decreased $1.7 \%$ for change in $t 1 / 2$ of 144 Ce from 278 to 284.4 days per 73 WALl. Mass 135 yield is superseded by 61BAY1.

59FRI1 Fritze, K., Mc Mullen, C. C., and Thode, H. G., "Absolute Yields of the Isotopes of Xenon and Krypton in the Neutron Fission of Plutonium-239," Proceedings of the Second International Conference on the Peaceful Uses of Atomic Energy, United Nations New York, New York, 1959. Vol. 15, pp. 436-439. 
59GOR1 Gorshkov, V. K., and Anikina, M. P., "Fine Structure in the Yield Curve for Fission Fragments of U-233," Atomnaya Energiya, 7, No. 2, 144-147 (1959). NSA 13: 20529 (1959).

59HEM1 Hemmendinger, A., "Fission Cross-Sections at meV Excitations," Proceedings of the Second International Conference on the Peaceful Uses of Atomic Energy Vol, 15, pp. 344-352, United Nations, New York, (1959).

59HEY1 Heydegger, H. R., and Kuroda, P. K., "Natural Occurrence of the Short-Lived Barium and Strontium Isotopes," J. Inorg. Nucl. Chem., 12, 12-17 (1959) as reported by 60KEN1. EXFOR-13470.

59KJE1 Kjelberg, A., and Pappas, A. C., "Fast Radiochemical Isolation of Fission Product Arsenic and the Independent Yield of Arsenic-78 in Thermal Neutron Fission of Uranium-235," J. Inorg. Nucl. Chem., 11, 173-180 (1959).

59KNI1 Knight, J. D., Hoffman, D. C., Dropesky, B. J., and Frasco, D. L., "Radiations of $93 \mathrm{Y}$ and 94Y and Half-Lives of 93Sr and 94Sr," J. Inorg. Nucl. Chem., 10, 183-197 (1959).

59LEA1 Leachman, R. B., "The Fission Process-Mechanisms and Data," Proceedings of the Second International Conference on the Peaceful Uses of Atomic Energy, Vol. 15, pp. 229-243, United Nations, New York (1959).

59MAR1 Marmol, P. del, Ph. D. Thesis in chemistry, Massachusetts Institute of Technology (January 1959). In author index del Marmol, P., reads (Marmol, P. del ).

59MER1 Merckx, K. R., "Fission Product Yield of Inert Gases," HW-60431, 2 pages, (May 19, 1959). Early compilation not coded.

59NET1 Nethaway, D. R., Ph. D. Thesis, Washington Univ. (September 1959). Cited in 64HYD1 pages 146-147.

59REG1 Regier, R. B., Burgus, W. H., and Tromp, R. L., "Ratio of Asymmetric to Symmetric Fission of U233 as a Function of Neutron Energy," Phys. Rev., 113, 1589-1592 (Mar. $15,1959)$. These ratios and $R$ values have been inverted to be consistent with our normalization treatment.

59ROE1 Roeland, L. W., Bollinger, L. M., and Thomas, G. E., "Mass Distribution in Fission," Proceedings of the Second International Conference on the Peaceful Uses of Atomic Energy, Vol. 15, pp. 440-443, United Nations, New York, (1959).

59TR01 Troutner, D. E., Ph. D. Thesis, Washington Univ. (1959). Cited in 74NET1. Isomeric independent yields are here split per 76MAD2.

59WAH1 Wahl, A. C., "14-Year 113m-Cd," J. Inorg. Nucl. Chem., 10, 1-3 (1959).

59WOL1 Wolfsberg, K., Ph. D. Thesis, Washington Univ. (1959). Cited in 64HYD1 pages 146-147.

60ALB1 Albenesius, E. L., Ondrejcin, R. S., "Nuclear Fission Produces Tritium," Nucleonics, 18, No. 9, 100 (September 1960).

60BAE1 Baerg, A. P., Bartholomew, R. M., and Betts, R. H., "Search for Independent Formation of Cs134m in Fission," Can. J. Chem., 38, 2147-2151 (1960). EXFOR-13473. 
60BON1 Bonyushkin, E. K., Zamyatnin, Yu. S., Kirin, I. S., Martynov, N. P., Skvortsov, E. A., and Yushatskii, V. N., "Fission Product Yields from the Fission of U-235 and U-238 with Fast Neutrons," Translated by the U. S. Joint Publications Research Service, New York, New York, AEC-TR-4682, (1960). Available from the Office of Technical Services, Dept. of Commerce, Washington, D. C. Neutrons were obtained from enriched U-235 placed in a reactor thermal column.

60COL1 Coleman, R. F., Hawker, B. E., and Perkin, J. L., "The Mass-Yield Curve for Fission of Np-237 by 14.5 meV Neutrons," J. Inorg. Nucl. Chem., 14, 8-13 (1960).

60CRO1 Croall, I. F., "Collected Independent Fission Yields for Thermal Neutron Fission of U-233, Pu-239, and U-235, $14 \mathrm{meV}$ Neutron Fission of U-235, Pile Neutron Fission of U-235, Pile Neutron Fission of U-238, Th-232, and Am-241, and Spontaneous Fission of Cm-242," AERE-R 3209 (1960).

60FLY1 Flynn, K. F., Unpublished work at Argonne National Laboratory cited by 60CRO1.

60GLE1 Glendenin, L. E., Private communication to I. F. Croall cited in 60CRO1. Isomeric independent yields are here split per 76MAD2.

60GRU1 Grummitt, W. E., Nussis, M., Lahaie, G., Contribution No. 16 at Third Symposium on Nucleat and Radiochemistry, Chalk River, Ontario, (Sept. 1960). Isomeric independent yields are here split per 77MAD2. Cited in 71DEN1, 74NET1, 75FLY1, 78WAH2.

60HYD1 Hyde, E. K., “A Review of Nuclear Fission; Part Two -Fission Phenomena at Moderate and High Energy," UCRL-9065, 257 pages (February 1960). Compiler.

60KAT1 Katcoff, S., “Fission-Product Yields from Neutron-Induced Fission," Nucleonics, 18, No. 11, 201-208 (November 1960).

60KEN1 Kenna, B. T., and Kuroda, P. K., "The Ratio of Induced Fission vs. Spontaneous Fission in Pitchblende and Natural Occurrence of Radiochlorine," J. Inorg. Nucl. Chem., 16, 1-7 (1960).

60KRI1 Krisyuk, I. T., Lepnev, G. P., Platunova, N. B., "Yields of Cs139 and Ba139 in U238 Fission by 14 meV Neutrons," Radiokhimiya 2, 743-745 (1960).

60KRI2 Krisyuk, I. T., Platunova, N. B., Protopopov, A. N., "Determination of Certain Individual Fragment Yields in U235 Fission by $14 \mathrm{meV}$ Neutrons," Radiokhimiya 2, 746-748 (1960). (In Russian). Isomeric independent yields are here split per 76MAD2.

60MAR1 Marinsky, J. A., and Eichler, E., "A New Fission Product: 74Ga," J. Inorg. Nucl. Chem., $12,223-227$ (1960). Relative to $\mathrm{Ba}-140$ yield of $6.32 \%$.

60NEP1 Nephew, E. A., "Thermal and Resonance Absorption Cross Sections of the U233, U235, and Pu239 Fission Products," ORNL-2869 pp. 4-5 (1960). Compilation of yields.

60NER1 Nervik, W. E., “Spontaneous Fission Yields of Cf252," Phys. Rev., 119, No. 5, 1685-1690 (September 1, 1960). 
60PET1 Petrzhak, K. A., Tolmachev, G. M., Yushatskii, V. N., Bak, M. A., Blinova, N. I., Burgorkov, S. S., Moskal'kova, Z. A., Petrov, Yu. G., Sorokina, A. V., Osipova, V. V., Chernysheva, L. P., and Shiryaeva, L. V., "Yields of a Number of Fission Products in the Fission of U-235, U-238, and Pu-239 by Neutrons," Translated by the U. S. Joint Publications Research Service, New York, New York, AEC-TR-4696, 1960. Available from the Office of Technical Services, Dept. of Commerce, Washington D. C. Neutrons were obtained from enriched U-235 placed in a reactor thermal column.

60REG1 Regier, R. B., Burgus, W. H., Tromp, R. L., and Sorensen, B. H., "Ratio of Asymmetric to Symmetric Fission of Pu239 and Pu241 as a Function of Neutron Energy," Phys. Rev., 119, 2017-2020 (Sept. 15, 1960). These ratios have been inverted for consistency with our treatment.

60SAN1 Santry, D. C., and Yaffe, L., "Absolute Thermal Neutron Fission Yields of U233," Can. J. Chem, 38, 421-438 (1960). EXFOR-13476.

60SAN2 Santry, D. C., and Yaffe, L., "The Yield of Ba-140 in the Thermal Neutron Fission of U235," Can. J. Phys., 38, 464-466 (1960). Relative to Co59 (n, gamma) Co60, Sigma = 36.3 barns. Ba-140 U235T error assigned and value renormalized to a sigma fission of 557 barns for U235 and 37.2 barns sigma absorption for Co-59 as in Table C1 73WAL3.

60SAT1 Sattizahn, J. E., Knight, J. D., Kahn, M., "Short-Lived Bromine and Selenium Nuclides from Fission," J. Inorg. Nucl. Chem., 12, 206-222 (1960). Selenium-87 assumed to be selenium-86, according to L. Tomlinson and M. H. Hurdus, ref. 68TOM1. EXFOR-13475.

60STE1 Stevenson, P. C., Hicks, H. G., Armstrong, J. C., Jr., Gunn, S. R., “Calorimetric Determination of the Average Total Kinetic Energy of Fragments from Fission of U235 and U238 in 14meV Neutrons," Phys. Rev., 117, 186-191 (Jan. 1, 1960).

60TOM1 Tomlinson, R. H., Private communication to S. Katcoff (tentative values). See 60KAT1.

60VLA1 Vlasov, V. A., Zysin, Yu. A., Kirin, I. S., Lbov, A. A., Osyaeva, L. I., Sel'chenkov, L. I., "Yield of Some Fragments During the Fission of Th-232 by $14.3 \mathrm{meV}$ Neutrons," Report No. AEC-TR-4665 (1960). NSA 16: 2500 (1962).

60WAL1 Walker, W. H., "Yields and Effective Cross Sections of Fission Products and PseudoFission-Products," Atomic Energy of Canada Limited, Chalk River, Ontario, Canada, AECL No. 1054, (March 1960).

60WOL1 Wolfsberg, K., Nethaway, D. R., Malan, H. P., and Wahl, A. C., "Identification of 142Xe and Measurement of Its Cumulative Yield from Thermal Neutron Fission of $235 \mathrm{U}$," J. Inorg. Nucl. Chem., 12, 201-205 (1960). EXFOR-13477.

60YEL1 Yellin, E., Ph. D. Thesis in chemistry, Massachusetts Institute of Technology (June 1960).

60YOU1 Young, B. G., and Thode, H. G., "Absolute Yields of the Xenon and Krypton Isotopes in U238 Spontaneous Fission," Can. J. Phys., 38, No. 1, 1-9 (Jan. 1960),

61BAY1 Bayly, J. G., Duret, M. F., Poulsen, N. B., and Tomlinson, R. H., "The Xe-135 Yield in Thermal Fission of Pu-239," Can. J. Phys., 39, 1391-1393 (1961). EXFOR-13057. 
61BID1 Bidinosti, D. R., Irish, D. E., and Tomlinson, R. H., "The Thermal Neutron Fission Yields of U233," Can. J. Chem, 39, 628-634 (1961). Increased Sr-89 by $2.5 \%$ due to change in decay correction moving $\mathrm{t} 1 / 2$ of $\mathrm{Sr}-89$ from 52.0 to $50.8 \mathrm{~d}$ and Mo-95 increased $0.6 \%$ due to change in $\mathrm{t} 1 / 2$ of $\mathrm{Zr}-95$ from 65.0 to $65.5 \mathrm{~d}$ per $73 \mathrm{WAL} 1$. Other values adjusted by $62 \mathrm{FER} 1$. EXFOR13208.

61BON1 Bonyushkin, E. K., Zamyatnin, Yu. S., Spector, V. V., Rachev, V. V., Negina, V. R., and Zamyatnina, V. N., "Fragment Yield in the Fission of U233 and Pu239 by Fast Neutrons," Atomnaya Energiya, 10, No. 1, 13-18 (1961). For an English translation see Sov. J. Atom. Energy, 10, 10-15 (1961). AEC-TR-4682 Is another English translation.

61COR1 Coryell, C. D., Kaplan, M., and Fink, R. D., "Search for Correlations of Most Probable Charge, $\mathrm{Zp}$, in Primary Fission Fragments with Composition and Excitation Energy," Can. J. Chem, 39, 646-663 (1961).

61CR01 Croall, I. F., "The Independent Yield of $\mathrm{Nb}-86$ in the Thermal Neutron Fission of U-233, U-235, and Pu-239," J. Inorg. Nucl. Chem., 16, 358-361 (1961). CSISRS-90202.

61CRO2 Croall, I. F., “A Search for 106m-Rh in Low Energy Fission,” AERE-R3879 (November 1961). 6 pages. Independent Yields of $106 \mathrm{~m}-\mathrm{Rh}$ in $\mathrm{U}-233, \mathrm{Pu}-239$ thermal fission. CSISRS90203. Isomeric independent yields are here split per 76MAD2.

61CUN1 Cuninghame, J. G., Kitt, G. P., and Rae, E. R., "The Ratio of Asymmetric to Symmetric Fission P.Wave Neutron Fission of U-235," Nucl. Phys., 27, 154-165 (1961).

61GLE1 Glendenin, L. E., Private communication to H. R. von Gunten, 1961, See 61GUN1.

61GOR1 Gorshkov, V. K., and Anikina, M. P., "The Fine-Structure in the Fission Yield Curve for 233U," J. Nucl. Energy, Part A, 13, 198-200 (1961).

61GRU1 Grummitt, W. E., and Milton, G. M., "The Independent Yield of 136Cs in the Thermal Neutron Fission of 235U, 233U, and 239Pu," J. Inorg. Nucl. Chem., 20, 6-11 (1961). EXFOR-13209.

61HER1 Herrmann, G., "Results of Chemical Investigations in Nuclear Fission," (In German), Habilitationsschrift, Univ. Mainz, (1961).

61KUR1 Kuroda, P. K., and Menon, M. P., "Determination of Trace Quantities of Fission Products in Nonirradiated Natural and Depleted Uranium Salts," Nucl. Sci. and Engr., 10, 70-74 (1961). EXFOR-13058.

61LAI1 Laidler, J. B., Brown, F., "Mass Distribution in the Spontaneous Fission of 240Pu," AWRE-49/61 (December 1, 1961). Published in J. Inorg. Nucl. Chem. 24, 1485 (1962).

61LEV1 Levy, H. B., Hicks, H. G., Nervik, W. E., Stevenson, P. C., Niday, J. B., and Armstrong, J. C., Jr., "Radiochemical Studies of Neutron Fission of U235 and U238 and the Two-Mode Fission Hypothesis," Phys. Rev., 124, 544-551 (1961). Data as reported in ref. 62WAL1. Fission spectrum neutrons were obtained by bombardment of beryllium with $12 \mathrm{meV}$ protons. Independent yields of Xe-135 divided between isomers per 76MAD2. EXFOR-13059. 
61LEV2 Levine, M. M., "Equal Charge Displacement Rule in Fission Product Poisoning," Nucl. Sci. and Engr., 9, 495-499 (1961). U233T Xe-135 independent yield of 0.78 is divided between isomers in the ratio predicted by 76MAD2. PU239T Xe135 independent yield of 0.58 is divided between isomers in the ratio predicted by 76MAD2.

61MAE1 Maeck, W. J., Kussy, M. E., and Rein, J. E., "Solvent Extraction Method for the Radiochemical Determination of Zinc," Anal. Chem. 33, No. 2, 235-236 (Feb. 1961). EXFOR-13210.

61MAR1 Marsh, S. F., Maeck, W. J., Booman, G. L., and Rein, J. E., "Improved 2-Thenoyltrifluoroacetone Extraction Method for Radiozirconium," Anal. Chem., 33, No. 7, 870872 (June 1961). EXFOR-13211.

61MIL1 Milton, J. C. D., and Fraser, J. S., "Prompt Fission Yields and Total Kinetic Energy Behavior from Time-of-Flight Measurements," Phys. Rev. Letters, 7, No. 2, 67-69 (July $15,1961)$.

61NEW1 Newson, H. W., "Symmetric and Asymmetric Fission," Phys. Rev, 122, 1224-1226 (May $15,1961)$. Calculated symmetric fission at $2.8 \mathrm{meV}$ neutron energy for Th232.

61ROY1 Roy, J. C., "On the Production of Be7, Mg28, and Ni66 in the Slow Neutron Fission of U235," Can. J. Phys., 39, 315-325, (1961). EXFOR-13060.

61VAL1 Vallis, D. G., and Thomas, A. O., "Light Mass Yield in the $14 \mathrm{meV}$ Neutron Fission of U235," Atomic Weapons Research Establishment Report No. AWRE-0-58/61.

61WAT1 Watson, J. C., "High Energy Alpha Particles and Tritons from the Spontaneous Fission of Californium-252," Phys. Rev. 121, 230-231 (Jan 1961). NSA 15: 6856 (1961).

62APO1 Apollonova, A. N., Krisyuk, I. T., Ushatskii, V. N., "Determination of Individual Yields of $A=139$ Isobars in Uranium Fission by $14 \mathrm{meV}$ Neutrons," Radiokhimiya 4, 587-591 (1962). For English translation see Sov. Radiochem. 4, 515 (1962). NSA 17: 21076 (1963).

62APO2 Apollonova, A. N., Krisyuk, I. T., Ushatskii, V. N., "Partial Yields of Isobars with a Mass Number of 138," Radiokhimiya 4, 711-714 (1962). For English translation see Sov. Radiochem. 4, 631 (1962). NSA 17: 15306 (1963).

62BRO1 Broom, K. M., “14.7 meV Neutron-Induced Fission of U-238," Phys. Rev., 126, 627-631 (1962). EXFOR-13061.

62BUR1 Burgus, W. H., "Radiochemically Determined Ratios of Asymmetric to Symmetric Fission of U233, Pu239, and U235 as a Function of Neutron Energy," IDO-16797 23 pages (July 30, 1962). CSISRS-90217. EXFOR-13098.

62COR1 Coryell, C. D., Storms, H. A., Fink, R. D., "Charge Distribution in Nuclear Fission: Studies with Energy Deposit of about $22 \mathrm{meV}$," Trans. Am. Nuclear Soc. 5, No. 1, 2324 (June 1962). NSA 16: 24351 (1962). Independent yields of Xe-135 divided between isomers per 76MAD2. EXFOR-13062.

62CRO1 Croall, I. F., and Willis, H. H., “The Fission Products Se-81 and Se-83," J. Inorg. Nucl. Chem., 24, 221-227 (1962). Supersedes AERE-R4148 (1962). 
62FAL1 Faler, K. T., and Tromp, R. L., "Variation in U-235 Mass Yields at Neutron Energies Below 0.5 eV," Trans. Amer. Nucl. Soc., 5, 22 (1962). Peak-to-valley ratios inverted to Ag111/Mo99 for consistency with our normalization treatment. EXFOR-13063. Superseded by 63FAL1.

62FAR1 Farrar, H., and Tomlinson, R. H., "Cumulative Yields of the Heavy Fragments in U-235 Thermal Neutron Fission," Nucl. Phys., 34, 367-381 (1962). 139Ba decreased $1.0 \%$ due to change in $t-1 / 2$ of $139 \mathrm{Ba}$ from 82.9 to 83.2 minutes per 73 WAL1. EXFOR-13064.

62FAR2 Farrar, H., Fickel, H. R., and Tomlinson, R. H., "Cumulative Yields of Light Fragments in U-235 Thermal Neutron Fission," Can. J. Phys., 40, 1017-1026 (1962). EXFOR-13065.

62FAR3 Farrar, H., and Tomlinson, R. H., "Structure in the Mass-Yield Distribution for U235 Fission," Can. J. Phys., 40, 943-953 (1962). (Theoretical treatment only; No new data reported).

62FER1 Ferguson, R. L., and O'Kelley, G. D., "A Survey and Evaluation of U-233 Fission Yield Data," ORNL-3305 (based on internal memo CF-62-3-7) (Central Files, March 1962).

62FRO1 Froehner, F. H., "Production and Investigation of Negative Fission Product Ions," Z. Physik, 170, 62-75 (1962). (in German); Chem. Abst. 58:6400g (1963), NSA 17:11746 (1963). Independent yields of Te-135 and I-135 relative to Te-134 independent yield for U235 thermal neutron induced fission.

62GAR1 Garrison, J. D., and Roos, B. W., "Fission-Product Capture Cross Sections," Nucl. Sci. and Engr., 12, 115-134 (1962). A compilation of yields for U233T, U235T, and PU239T.

62GLE1 Glendenin, L. E., Flynn, K. F., and Bollinger, L. M., "Ratio of Symmetric to Asymmetric Fission for Resonance Neutron Fission of U-235," Trans. Amer. Nucl. Soc., 5, 20-21 (1962). EXFOR-13066.

62GLE2 Glendenin, L. E., Steinberg, E. P., Talat-Erben, M., Private communication to 62WAH1 Table $\mathrm{V}$ footnote superscript $\mathrm{g}$. for fractional independent yield of Te131m of 0.18 for U233T. CSISRS90225. EXFOR-13099.

62GRA1 Gray Jr., J., and Hagemann, F. T., Unpublished work. See "A Thermal Neutron Fission Counter for Nuclear and Analytical Measurements,” Rev. Sci. Instr., 33, No. 11, 1258-1264 (1962). Fission measurements were made with this counter and $\mathrm{Kr}-85$ measurements were made with the absolute gas counter described in $80 \mathrm{JAF} 1$. (which see).

62HAG1 Hagebo, E., Kjelberg, A., and Pappas, A. C., "Radiochemical Studies of Isotopes of Antimony and Tin in the Mass Region 127-130," J. Inorg. Nucl. Chem., 24, 117-131 (1962). $1 \mathrm{hr}$ Sn-129 doesn't exist; ref. 68PAP1.

62HIC1 Hicks, H. G., Levy, H. B., Nervik, W. E., Stevenson, P. C., Niday, J. B., and Armstrong, J. C., Jr., "Further Radiochemical Studies of the U236* Compound Nucleus," Phys. Rev., 128, No. 2, 700-707 (1962). EXFOR-13091.

62HYD1 Hyde, E. K., "A Review of Nuclear Fission; Part I - Fission Phenomena at Low Energy," UCRL-9036-Rev. (April 1962). 356 pages, Compiler. 
62LAI1 Laidler, J. B., Brown, F., "Mass Distribution in the Spontaneous Fission of Pu240," J. Inorg. Nucl. Chem., 24, pp. 1485-1494 (1962). EXFOR-21482.

62NOB1 Nobles, R. A., "Long Range Particles from Nuclear Fission," Phys. Rev. 126: 1508-1513 (May 15, 1962). NSA 62: 21420 (1962).

620RT1 Orth, C. J., (Private communication to A. C. Wahl and D. R. Nethaway) reported as ref. 6 in 63WAH1. Authors $0.0008 \%$ U235T RCCU $121 \mathrm{~m}-\mathrm{Sn}$ assumes a 25 year $\mathrm{t} 1 / 2$. This corrects to $0.0016 \%$ for 60 year $t 1 / 2$ reported in 71ERD1 as noted in 73WAH1. CSISRS-90229. EXFOR13222.

62SLO1 Sloth, E. N., Horrocks, D. L., Boyce, E. J., and Studier, M. H., "Tritium in the Thermal Neutron Fission of Uranium-235," J. Inorg. Nucl. Chem., 24, 337-341 (April 1962).

62STO1 Storms, H. A., “Independent Fission Yields of Some Xenon Isotopes," Ph. D. Thesis, Massachusetts Institute of Technology (September 1962), Charles D. Coryell, Thesis supervisor, published as 65STO1.

62TER1 Terrell, J., "Neutron Yields from Individual Fission Fragments," Phys. Rev. 127, 880-904 (1962).

62VAL1 Vallis, D. G., Thomas, A. O., "Light Mass Yield in the $14 \mathrm{meV}$ Neutron Fission of U235," AWRE-0-58/61. A 1961 report distributed January 1962. See 61VAL1 for values.

62VlA1 Vlasov, V. A., Zysin, Yu. A., Kirin, I. S., Lbov, A. A., Osyaeva, L. I., Sel'chenkov, L. I., "Yields of Some Fragments during the Fission of 232Th by $14.3 \mathrm{meV}$ Neutrons," AEC-TR-4665, 12 pages (July 1, 1960). NSA 16: 2500 (1962).

62WAH1 Wahl, A. C., Ferguson, R. L., Nethaway, D. R., Troutner, D. E., and Wolfsberg, K., "NuclearCharge Distribution in Low-Energy Fission," Phys. Rev., 126, 1112-1127 (1962). (As adjusted by 69WAH1). Isomeric independent yields are here split per 76MAD2. EXFOR-13097.

62WAL1 Walker, W. H., "Fission Product Poisoning from the Fast Fission of U-238," Atomic Energy of Canada Limited, Chalk River, Ontario, Canada, CRRP-1090, (1962). Also called AECL-1537.

63ANL1 ANL Staff, "Reactor Physics Constants," ANL-5800 Second Edition, Section 1, Pages 628 (July 1963). A compilation of fission yields for U235T, U233T, PU239T, PU239F, U233F, TH232F, U235HE, and TH232F. With 193 refs.

63BRA1 Brandt, R., Thompson, S. G., Gatti, R. C., and Phillips, L., “Mass and Energy Distributions in the Spontaneous Fission of Some Heavy Isotopes," Phys. Rev., 131, No. 6, 2617-2624 (15 September, 1963). Mass yield graphs for Cf-252, Fm-254, Cf-254, Es-253, Cf-250, Cm-248; No digital data; Not coded.

63BUN1 Bunney, L. R., and Scadden, E. M., "Heavy Mass Yields in the Slow Neutron Fission of U-233," USNRDL-TR-679 (12 Sept. 1963) precursor to 65BUN1.

63COW1 Cowan, G. A., Bayhurst, B. P., and Prestwood, R. J., "Symmetry of Neutron Induced U235 Fission at Individual Resonances," Peaceful Nuclear Explosions, Los Alamos, New Mexico, PNE-114F, 18 pages, (February 1963). Ag111/Mo99 ratios for 36 resonances from 8.8 $\mathrm{eV}$ to $40 \mathrm{eV}$. Not coded. 
63CRO1 Croall, I. F., and Willis, H. H., "The Yields of the Isomers of Se-81 and Se-83 in the Thermal Neutron Fission of Pu-239," J. Inorg. Nucl. Chem., 25, 1213-1218 (1963).

63CRO2 Crook, J. M., and Voigt, A. F., "Radiochemical Studies of Some Pile-Neutron Fission Yields of Thorium-232," IS-558 (Oct. 1, 1963). NSA 18: 09311 (1964). CSISRS-90232. EXFOR-13200.

63DAV1 Davies, J. H., and Yaffe, L., "Nuclear Charge Dispersion in the High-Energy Fission of Uranium," Can. J. Phys., 41, 762-783 (1963).

63FAL1 Faler, K. T., and Tromp, R. L., "Variation in U235 Mass Yields at Neutron Energies Below 0.5 meV," Phys. Rev., 131, No. 4, 1746-1749 (15 Aug 1963). EXFOR-13092. Journal publication of 62FAL1.

63FRA1 Fraser, J. S., Milton, J. C. D., Bowman, H. R., and Thompson, S. G., "Precise Kinetic Energy Measurements and Fine Structure in the Spontaneous Fission of Cf252," Can. J. Physics, 41, 2080-2097 (1963). Wings and valley values are not used because of incomplete mass resolution making these effectively upper limits due to uncorrected background scattering (see 87WAH1). EXFOR-13067.

63HAG1 Hagebo, E., “The Pile Neutron Fission Yields of Sb-127 and Sb-129," J. Inorg. Nucl: Chem., 25, 615-617 (1963).

63IYE1 Iyer, R. H., Mathews, C. K., Ravindran, N., Rengan, K., Singh, D. V., Ramaniah, M. V., and Sharma, H. D., “Fission of 232Th by Reactor Neutrons: Mass-Yield Curve," J. Inorg. Nucl. Chem., 25, 465-472 (1963).

63KIR1 Kirby, L. J., "Fission Yield Studies with Pu-239 and Pu-241," in "Hanford Radiological Sciences Research and Development Annual Report for 1962," HW-77609 pp. 3.1 - 3.2 (1963). CSISRS-90237. EXFOR-13201.

63LER1 Lerner, J. L., "Half-Life of 85Kr," J. Inorg. Nucl. Chem., 25, 749-757 (1963).

63MCH1 Mc Hugh, J. A., "Mass-Spectrometric Study of Fission from the U236 Compound Nucleus at Moderate Excitations," UCRL-10673, Pages 30-31 Feb. 1963, Th-232 partially coded. See also ref. 66MCH1. CSISRS-90238. EXFOR-13202.

63ROB1 Roberts, F. P., Wheelwright, E. J., van Tuyl, H. H., "Detection of 146Pm Among the Products of Uranium Fission," J. Inorg. Nucl. Chem., 25, 1298-1300 (1963). Ref. 63ZYS1 states that $\mathrm{Pm}-146$ value should be treated as less than value since no correction for $146 \mathrm{Pm}(\mathrm{n}$, gamma) was made. EXFOR-13212.

63TRO1 Troutner, D. E., Ferguson, R. L., and O'Kelley, G. D., "Yields and Half-Lives in the Mass99 Fission Product Chain," Phys. Rev., 130, 1466-1470 (May 15, 1963). EXFOR-13093.

63TRO2 Troutner, D. E., Wahl, A. C., and Ferguson, R. L., "Independent Fission Yield of Sb-127," ORNL-3488, pages 8-9, (June 20, 1963). EXFOR-13068.

63WAH1 Wahl, A. C., and Nethaway, D. R., "Nuclear Charge Distribution in Symmetric Fission Sn121-Independent Yield," Phys. Rev., 131, 830-831 (15 July 1963). Sn-121 data corrected in ref. 69WAH1. EXFOR-13094. 
63WEA1 Weaver, L. E., Strom, P. O., and Killeen, P. A., "Estimated Total Chain and Independent Fission Yields for Several Neutron Induced Fission Processes," U. S. Naval Radiological Defense Laboratory, San Francisco, California. USNRDL-TR-633, (March 5, 1963).

63ZYS1 Zysin, Yu. A., Lbov, A. A., and Sel'chenkov, L. I., "Fission Product Yields and Their Mass Distribution," Translation from 1963 Russian text, Consultants Bureau Enterprises, Inc., 227 W. 17th St., N. Y., N. Y. 10011 (1964).

64BEM1 Bemis, C. E., Gordon, G. E., and Coryell, C. D., "Half-Life of Sb-135 and Evidence on the Half-Life of Sb-134," J. Inorg. Nucl. Chem., 26, 213-218 (1964).

64BRO1 Broom, K. M., “2.95-meV and $14.8 \mathrm{meV}$ Neutron-Induced Fission of Th232," Phys. Rev., 133, No. 48, B874-B883 (1964). EXFOR-13095.

64COW1 Cowan, G. A., "R-Values for the Fission $235 \mathrm{U}, 238 \mathrm{U}$, and $239 \mathrm{Pu}$ by $14 \mathrm{meV}$ and Fast Neutrons," Private Communication to E. A. C. Crouch (March 1, 1964). Superseded by 76FOR1. CSISRS-90244.

64CRO1 Croall, I. F., and Willis, H. H., "Some Yields in the Thermal and Epicadmium-Neutron Fission of Pu-239," Physics and Chemistry of Fission, Vol. 1, International Atomic Energy Agency, Vienna 1965, pp. 355-365. Also Atomic Energy Research Establishment, Harwell, U. K. AERE-R-4723, (Sept. 1964). EXFOR-21464.

64CUN1 Cuninghame, J. G. Report AERE-R-4727 January 1964. Later Published as 74CUN1.

64DUR1 Duret, M. F., Durham, R. W., Lounsbury, M., Okazaki, A., Walker, W. H., Ward, A. G., Pease, L., "Fuel Burn-up and Reactivity Changes," 3rd International Conference on Peaceful Uses of Atomic Energy, A/Conf. 28/p/26 Geneva (1964). See Proceedings 3rd Geneva Conference, Vol. 3, Pages 347-356, United Nations, N. Y., N. Y. (1965). Preliminary results for 65OKA1. EXFOR-13069.

64FAR1 Farrar, H., Clarke, W. B., Thode, H. G., and Tomlinson, R. H., "Cumulative Yields of the Heavy Fragments in the Thermal-Neutron Fission of 241Pu," Can. J. Phys., 42, $2063-2079$ (1964). $147 \mathrm{Sm}$ increased $3 \%$ recorrection for change in the $1 / 2$ of $147 \mathrm{Pm}$ from 2.52 to 2.62 years. $138 \mathrm{Xe}$ decreased $0.8 \%$ due to change in $\mathrm{t} 1 / 2$ of $138 \mathrm{Xe}$ from 14.0 to 14.2 minutes and $150 \mathrm{Nd}$ results for two samples differed by $4.4 \%$. Only the value with the smaller standard deviation is used here per 73WAL1. EXFOR-13070.

64HER1 Herrmann, G., "25 Years of Nuclear Fission, Methods and Results of Chemical Studies: 1. History of Discovery, Fission Products," Radiochim. Acta, 3, No. 4, 169-185 (1964). (In German).

64HOR1 Horrocks, D. L., "Tritium Produced During Spontaneous Fission of Californium-252," Phys. Rev. 134, B1219 (1964). NSA 18: 26644 (1964).

64HYD1 Hyde, E. K., “The Nuclear Properties of the Heavy Elements III: Fission Phenomena," Prentice-Hall, Englewood Cliffs, New Jersey, (1964).

64JAM1 James, R. H., Martin, G. R., and Silvester, D. J., "Radiochemical Studies of Fission Induced by 14.7 meV Neutrons," Radiochim. Acta, 3, 76-80 (1964). 
64KIM1 Kimura, K., “The Independent Yield of Ag112 in U235 Fission," MIT-2098, No. 64, Pages 34-35 (May 1, 1964). CSISRS-90249. EXFOR-13203.

64LYL1 Lyle, S. J., Martin, G. R., and Whitley, J. E., "Radiochemical Studies of Fission Induced by 14.7 meV Neutrons," Radiochim. Acta, 3, 80-87 (1964).

64MAT1 Mathews, C. K., "Cumulative Yields in the Fast Neutron Fission of U238," Ph. D. Thesis, Mc Master University, Hamilton, Ontario, Canada (October 1964). Also reported in EANDC(CAN)23L. Published as 72MAT1.

64MEN1 Menon, M. P., and Kuroda, P. K., "Spontaneous and $14.7 \mathrm{meV}$ Neutron Induced Fission Yields of Uranium-238 in the Rare Earth Region," J. Inorg. Nucl. Chem., 26, 401-408 (1964). EXFOR-13223.

640TH1 Other, A. N., "R-Values for the Fission of $235 U, 238 U, 239 P u$ by 14 meV and Fast Neutrons," This is a private communication to E. A. C. Crouch (March 1, 1964). $R$ value here reduced to absolute yield assuming $\mathrm{PU} 239 \mathrm{H}$ Mo99 yield $=4.58$ and U235T Mo99 Sr89, Ag111, Cd115m, Cs136, Ba140, Ce144, Nd147, Eu156, Tb161 of 6.14, 4.48, 0.019, 0.00146, $0.00502,6.305,5.50,2.25,0.0134,0.000087$. These are Los Alamos Cockcroft-Walton Generator irradiations with $14 \mathrm{MeV}$ neutrons. These data later published with other work in 76FOR1 as Column 1, Table IV Pages 21-22. CSISRS-90252.

64RID1 Rider, B. F., Ruiz, C. P., Peterson, J. P., Jr., and Smith, F. R., “Accurate Nuclear Fuel Burnup Analysis, Eleventh Quarterly Progress Report, June 1964 - August 1964," General Electric Company, Pleasanton, California, GEAP-4716, 1964. See experimental capsule GEV-1, Page 26. Where $n v=2.0 \mathrm{e} 13$ and $n v t=1.0 \mathrm{e} 20$. Data relative to total $\mathrm{Nd}-148$ taken as 1.000. assuming 300 barns as a spectrum averaged neutron cross section for Nd-147, only 0.993 of Nd-148 is fission caused. CSISRS-90253. EXFOR-13204.

64TER1 Tercho, G. P., Marinsky, J. A., “The Fission Nuclide, 1.6 min. 104Mo," J. Inorg. Nucl. Chem., 26, 1129-1138, (1964). EXFOR-13071.

64TR01 Troutner, D. E., Wahl, A. C., and Ferguson, R. L., "Independent Fission Yield of Sb127," Phys. Rev., 134, B1027-B1029 (8 June 1964). EXFOR-13096.

64WAL1 Walker, W. H., "The Effect of New Data on Reactor Poisoning by Non-Saturating Fission Products," CRRP-1185 (AECL-2111) Nov. 1964.

64WAL2 Walter, F. J., Schmitt, H. W., and Neiler, J. H., "Fragment Mass Distributions for Thermal-Neutron-Induced Fission of Pu238 and Pu241," Phys. Rev., 133, No. 6B, B1500-B1502 (1964).

64WOL1 Wolfsberg, K., “A Method for Estimating Fractional Yields from Low- and Medium Energy Neutron Induced Fission," Los Alamos Scientific Laboratory of the University of California, Albuquerque, New Mexico, LA-3169, 1964.

64WYT1 Wyttenbach, A., Gunten, H. R. von, and Dulakas, H., "Fission of 232Th by Reactor Neutrons: Fission Yields of Some Mass-Chains," Radiochim. Acta, 3, No. 3, 118-121 (1964). Data superseded by 65WYT1. NSA 19: 10351 (1965). In author index, von Gunten, H. R., reads (Gunten, H. R. von ). 
65ACC1 Accinni, F., and Ciuffolotti, L., "The Ba140 Yields for Thermal and Fast Fission in U235 and U238," CISE-R-171 Centro Informazioni Studi Esperienze, Milano (October 1965). Topical Report No. 11, 15 pages + 5 figures NSA 24: 05415 (1970). CSISRS-90257.

65AMI1 Amiel, S., "Delayed Neutrons and Photoneutrons from Fission Products," Physics and Chemistry of Fission, Vol. II, International Atomic Energy Agency, Vienna 1965, pp. 171-196.

65AND1 Anderson, C. A., "Fission Product Yields from Fast (ca. $1 \mathrm{meV}$ ) Neutron Fission of Pu-239," Los Alamos Scientific Laboratory of the University of California, Albuquerque, New Mexico, LA-3383, (July 1965).

65BAL1 Balcarczyk, L., Keratschev, P., and Lanzel, E., "Determination of Thermal and Epithermal Fission of 233U," Nukleonik, 7, 169-171 (1965).

65BIG1 Bigham, C. B., Okazaki, A., Walker, W. H., "The Direct Yield of Xe-135 in the Fission of U-233, U-235, Pu239 and Pu-241," Trans. Amer. Nucl. Soc., 8, No. 1, 11 (1965). Supersedes EANDC(CAN)23L5 (1965). Independent yields of Xe-135 divided between isomers per 76MAD2. EXFOR-13072.

65BUN1 Bunney, L. R., and Scadden, E. M., "Heavy Mass Yield in the Slow Neutron Fission of U-233 and U-235," J. Inorg. Nucl. Chem., 27, 273-279 (1965). EXFOR-13213.

65BUN2 Bunney, L. R., and Scadden, E. M., "Mass Yields in the Fast Neutron Fission of 233U," J. Inorg. Nucl. Chem., 27, 1183-1189 (1965). EXFOR-13214.

65CRO1 Crowther, P., and Eldridge, J. S., "Decay of 99-Mo - 99m-Tc," Nucl. Phys., 66, 472-480 (1965).

65CR02 Croall, I. F., "Yields from Neutron Induced Fission," Atomic Energy Research Establishment, Harwell, U. K., AERE-R-5086, 1965. Reprinted Jan. 1968. NSA 22: 20559 (1968).

65DIG1 Diggle, W. R., and Blackadder, W. H., “Gamma Scanning for Burnup," Nucleonics 23, No. 3, 71 (March, 1965).

65ENG1 England, T. R., "Time-Dependent Fission-Product Thermal and Resonance Absorption Cross Sections," Bettis Atomic Power Laboratory, Pittsburgh, Pennsylvania, WAPDTM-333, Addendum No. 1, January 1965.

65FOR1 Ford, G. P., Leachman, R. B., "Fission Mass Yield Studies," Physics and Chemistry of Fission, Vol. 1, IAEA, 333-345 (1965). EXFOR-13073. Graphical presentation. See 65FOR2 for digital data.

65FOR2 Ford, G. P., and Leachman, R. B., "Fission Mass Yield Dependence on Angular Momentum," Phys. Rev., 137, No. 4B, B826-B836 (22 February 1965). Partially coded. EXFOR13077.

65FOR3 Ford, G. P., Leachman, R. B., Los Alamos Scientific Laboratory, University of California, Chain yields in U235T of 0.024 for Mass $109 ; 0.014$ for mass $111 ; 0.012$ for mass 112 Table II Page 28 of Private communication to A. C. Wahl. Cited as Reference 62 in 69WAH1. CSISRS-90264. EXFOR-13221. 
65HER1 Herrmann, G., “25 years of Nuclear Fission, Methods and Results of Chemical Studies; 2, Methods," Radiochim. Acta, 4, No. 4, 173-188 (1965). (In German).

65HOR1 Horrocks, D. L., "Use of Tritium to Measure Burnup of Fissionable Nuclides," Amer. Nucl. Soc., 8, No. 1, 12 (1965). Compiler. Not coded.

65IYE1 Iyer, R. S., Jain, H. C., Namboodiri, M. N., Rajkishore, M. R., Ramaniah, M. V., Rao, C. L., Ravindran, N., and Sharma, H. D., "Neutron Induced Fission of Actinium-227, Protactinium-231, and Neptunium-237: Mass Distribution," Physics and Chemistry of Fission, Vol. 1, International Atomic Energy Agency, Vienua, Pages 439-448 (1965).

65KAT1 Katcoff, S., and Rubinson, W., "Yields of 85Kr in Thermal Neutron Fission of $235 \mathrm{U}$ and 239Pu," J. Inorg. Nucl. Chem. 27, 1447-1450 (1965). EXFOR-13215.

65KON1 Konecny, E., Opower, H., Gunther, H., and Gobel, H., "Primary Distribution of Nuclear Charge for Fission-Fragment Masses 132, 134, 136, and 137 from Thermal Fission of U235," Physics and Chemistry of Fission, Vol. 1 International Atomic Energy Agency, Vienna, Pages 401-413, (1965). Early data, the majority of which are not consistent with later values so none of these early data are used here until reasons for inconsistency are found. Perhaps non-uniform detector thickness as described under 74SIE2 is the reason for inconsistency.

65MAE1 Maeck, W. J., and Rein, J. E., editors, "Burnup Determination of Nuclear Fuels - Project Report for the Quarter January 1 - March 31, 1965," Phillips Petroleum Company, Idaho Falls, Idaho, IDO-14660. (June 1965). See Capsule 6-4-1..

65MAE2 Maeck, W. J., Abernathey, R. M., and Rein, J. E., "Absolute U-235 Thermal-Fission Yields of Cs-137 and Stable $\mathrm{Kr}, \mathrm{Xe}, \mathrm{Nd}$, and $\mathrm{Sm}$ Isotopes," Transactions of the American Nuclear Society 1965 Annual Meeting, Gatlinburg, Tennessee, June 21-25 (1965). Superseded by ref. 70LIS1.

65MAE3 Maeck, W. J., and Rein, J. E., "Burnup Determinations of Nuclear Fuels: Project Report for the Quarter July 1 - September 30, 1965," USAEC Doc. ID0-14667 (1965), Summarized in ref. 70LIS1.

65MAR1 Marsden, D. A., Yaffe, L., “Mass Distribution in Thermal Neutron Fission of Pu-239," Can. J. Phys. 43, 249-267 (1965). isomeric independent yields are here split per 76MAD2. EXFOR-13074.

65MCH1 Mc Hugh, J. A., "Independent Yields of Rb86, Cs132, Cs134, and Cs136 in the Thermal Neutron Fission of U235," KAPL-3113, 5 pages (September 30, 1965). Superseded by $66 \mathrm{MCH} 1$.

65MO 1 Mo, T., and Kuroda, P. K., "Yields of Zirconium Isotopes from Spontaneous and 14.7 meV Neutron-Induced Fission of Uranium-238," J. Inorg. Nucl. Chem., 27, 503-508 (1965). EXFOR-13075.

65NET1 Nethaway, D. R., and Levy, H. B., "Effect of Increasing Excitation Energy on Nuclear Charge Distribution in Fission," Phys. Rev., 139, B1505 - B1513 (Sept. 20, 1965). Later summarized in $74 \mathrm{NET} 1$ with assigned errors. Isomeric independent yields are here split per 76MAD2. EXFOR-13078. 
65NIE1 Niece, L. H., "Independent Yields of $\mathrm{Zr-95}$ from Thermal Neutron Fission of U235 and U-233," ORNL-TM-1333 (1965). precursor to 70NIE1. Footnote to Chapter I introduction reads: "The FRACTION [emphasis here] of each isobar formed at fission (after neutron emission, but before beta decay) is called [here] the independent yield...." This is a redefinition for his work not usually used. Conventionally, this is called fractional independent yield.

650KA1 Okazaki, A., and Walker, W. H., "Measurement of the Yields of I-135 and Ba-140 in the Fission of U-233, U-235, Pu-239, and Pu-241," Can. J. Phys., 43, 1036-1049 (1965). EXFOR-13076.

65PRU1 Prussin, S. G., and Meinke, W. W., "Isolation and Identification of 12.4 Minute Te133g," Radiochim. Acta, 4, No. 2, 79-82 (June 1965).

65RAN1 Rangarajan, C., Mishra, U. C., Lalit, B. Y., Gopalakrishnan, S., Sadasivan, S., "Fission Products Data and Its Application in Studying Fallout from Nuclear Weapon Tests," AEET-209 (1965). Atomic Energy Establishment Bombay, India.

65RAO1 Rao, M. N., and Shahani, C. J., "Ruthenium and Rhodium Isotopes in Spontaneous Fission of Uranium-238," J. Inorg. Nucl. Chem., 27, 2679-2681 (1965).

65RIC1 Rickard, R. R., Goeking, C. F., and Wyatt, E. I., "Mass-Fission-Yield Curve for Americium241," Nucl. Sci. and Engr., 23, 115-118 (1965). EXFOR-13079.

65RID1 Rider, B. F., Ruiz, C. P., Peterson, J. P., Jr., and Smith, F. R., “Accurate Nuclear Fuel Burnup Analysis, Sixteenth Quarterly Progress Report, September 1965 - November 1965," General Electric Company, Pleasanton, California, GEAP-5060, December 1, 1965, Page 7. U235T data relative to total Nd-148 from 3 capsules, 6-6-1, GEV-1, GEV-2 at nv = $6 \mathrm{e} 13,2 \mathrm{e} 13,1 \mathrm{e} 13$ and $\mathrm{nvt}=2.4 \mathrm{e} 20,1.0 \mathrm{e} 20,4.0 \mathrm{e} 19$, respectively. Assuming 300 barn spectrum averaged neutron cross section for $\mathrm{Nd}-147,0.982,0.993,0.993$, or average of 0.989 of $\mathrm{Nd}-148$ is fission caused. CSISRS-90274. EXFOR-13205.

65RID2 Rider, B. F., Ruiz, C. P., Peterson, J. P., Jr., and Smith, F. R., "Accurate Nuclear Fuel Burnup Analysis, Fourteenth Quarterly Progress Report," March - May 1965, General Electric Company, Pleasanton, California, GEAP-4893 June 1, 1965, page 19. Experimental capsule GEV-2. For capsules of PU239T, U233T, U235T, nv=1.0e13, 1.75e13, 2.25e13 and nvt $=4.0 \mathrm{e} 19,7.0 \mathrm{e} 19,9.0 \mathrm{e} 19$, respectively. Data relative to total Nd-148 taken as 1.000 . Assuming a 300 barn spectrum averaged neutron cross section for $\mathrm{Nd}-147$, only $0.9964,0.9946,0.9928$ of Nd-148 is fission caused in the PU239T, U233T, U235T capsules, respectively. CSISRS- 90275. EXFOR-13206.

65SAR1 Sarantites, D. G., Gordon, G. E., and Coryell, C. D., "Ratios of Independent Yield Isomers Te-131 - Te-131m in Fission," Phys. Rev., 138, B353-B364 (1965). EXFOR-13080.

65SCH1 Schmitt, H. W., Kiker, W. E., and Williams, C. W., "Precision Measurements of Correlated Energies and Velocities of Cf-252 Fragments," Phys. Rev., 137, No. 4B, B837-B847 (22 Feb. 1965). Wings and valley values are not used because of incomplete mass resolution causing these to be effectively upper limits from uncorrected high background scattering. See 87WAH1 evaluation. EXFOR-13081. 
65SCH2 Schroeder, I. G., Deruytter, A. J., Moore, J. A., "Relative Probability of Ternary Fission with Emission of Long Range Alpha Particles in the Thermal-Neutron Fission of U235," Phys. Rev. 137(3), B519-B523 (Feb 8 1965). Ternary Fission.

65ST01 Storms, H. A., and Coryell, C. D., "Independent Fission Yields of Some Xenon Isotopes," Paper from 150th Meeting American Chemical Society, Atlantic City, New Jersey, September, 1965. NSA 20: 30533 (1966). CONF-650902-22. Gmelin, AED-CONF-65-235-16. Publication of Ph. D. Thesis, M. I. T. (62STO1). Supersedes NY0-2669, Page 19, (1962). U233T Xe-135 fractional independent yield of 0.099 is divided between isomers in the ratio predicted by 76MAD2. EXFOR-13224.

65STR1 Strom, P. O., Grant, G. R., and Pappas, A. C., "Independent Yields of 124Sb and 126Sb in Thermal Neutron Induced Fission and Their Implication on the Empirical $Z_{p}$ Function," Can. J. Chem, 43, 2493-2507 (1965).

65WAH1 Wahl, A. C., "Mass and Charge Distribution in Low Energy Fission," Physics and Chemistry of Fission, Vol. I, IAEA, Vienna, Austria (1965).

65WEI1 Weiss, H. V., and Ballou, N. E., "Mass and Cumulative Yields in Near Symmetric Fission of U235 with Thermal Neutrons," Part 1, The 121-Mass Chain, Physics and Chemistry of Fission Vol I, International Atomic Energy Agency, Vienna, Austria, (1965) pp. 423-437. See also ref. 65WEI2.

65WEI2 Weiss, H. V., "Near-Symmetric Fission-Identification and Yield of Cd121," Phys. Rev., 139, B304-B306 (26 July 1965) EXFOR-13082. Reports same data as ref. 65WEI1.

65WEI3 Weiss, H. V., and Ballou, N. E., “Rapid Separation of Indium from Tin by Sublimation, Formation of 121In in Fission of $235 \mathrm{U}, "$ J. Inorg. Nucl. Chem., 27, 1917-1923 (1965). EXFOR-13216.

65WOL1 Wolfsberg, K., "Nuclear Charge Distribution in Fission: Fractional Yields of Krypton and Xenon Isotopes from Thermal Neutron Fission of U-233 and Pu-239 and from 14-meV Neutron Fission of U-235 and U-238," Phys. Rev., 137, B929-B935 (1965). EXFOR-13084.

65WYT1 Wyttenbach, A., and Gunten, H. R. von, "Fission Yields of Some Isotopes in the Fission of Th232 by Reactor Neutrons," Physics and Chemistry of Fission, Vol. 1, International Atomic Energy Agency, Vienna, Pages 415-421 (1965). NSA 20: 17860 (1966). In author index, von Gunten, H. R., reads (Gunten, H. R. von ).

66AND1 Anderson, I. O., and Brune, D., "Independent Fission Yields of Caesium-134," Nature, 211, 618-619 (August 6, 1966).

66ARA1 Aras, N. K., and Gordon, G. E., "The Fractional Chain Yield of Sb-126 in Thermal Neutron Fission of U-235," J. Inorg. Nucl. Chem., 28, 763-770 (1966). Measured relative to $\mathrm{Sb}-127=0.13$ percent. Replaces MIT-905-52, Page 21 (1965). EXFOR-13085.

66BAE1 Baeckmann, A. von, Feuerstein, H., “Technetium from Fission: II” (In German), Rz diochim. Acta, 5, No. 4, 234-235 (1966). 
66BEY1 Beyer, W. H., "Handbook of Tables for Probability and Statistics," The Chemical Rubber Co., Cleveland, Ohio, Table VII.4, page 267 (1966). Library of Congress Card 6617301.

66BR01 Brown, M. G., Lyle, S. J., and Martin, G. R., "Radiochemical Studies of Fission Induced by $14.7 \mathrm{meV}$ Neutrons," Radiochim. Acta, 6, No. 8, 16-20 (Aug. 1966).

66COW1 Cowan, G. A., Bayhurst, B. P., Prestwood, R. J., Gilmore, J. S., and Knobeloch, G. W., "Symmetry of Neutron Induced Pu-239 Fission at Individual Resonances," Phys. Rev., 144, 979-983 (1966).

66CUN1 Cuninghame, J. G., Fritze, K., Lynn, J. E., and Webster, C. B., "The Ratio of Asymmetric to Symmetric Fission of Pu-239 by Neutrons of Energies from $30 \mathrm{keV}$ to $14.7 \mathrm{meV}$," Nucl. Phys., 84, 49-61 (1966). EXFOR-22053 (Dec 22, 1988). EXFOR-22053.

66DAN1 Daniels, W. R., and Hoffman, D. C., "Fission Yields of Eu-156, Eu-157, Eu-158, and Eu-159," Phys. Rev., 145, 911-914 (March 1966). EXFOR-13086.

66DYA1 D'yachenko, P. P., Kuz'minov, B. D., Smirnov, V. I., Chernukhin, V. L., and Chubarov, S. I., "Kinetic Energies of Fragments with Various Masses in the Fission of U235 by Thermal and Fast Neutrons," Sov. J. Nucl. Phys., 2, No. 1, 65-67 (January 1966). Translation of J. Nucl. Phys. (USSR) 2, 92-96 (July 1965). Graphic plot not coded.

66ERD1 Erdal, B. R., Ph. D. Thesis, Washington University, St. Louis, 1966, University Microfilm No. 67-7035. Fission yields including U235T. Publications extracted from this thesis include 69ERD1 and 71ERD1. CSISRS-90290. EXFOR-13207. EXFOR-13225.

66FLY1 Flynn, K. F., Glendenin, L. E., Fractional Cumulative yield of Rb-84 is $(2.4 \pm 0.02) x 10^{-9}$ for U235T Table II Page 26, Private communication to A. C. Wahl. Cited as Reference 36 in 69WAH1 (1966). Isomeric independent yields split per 76MAD2. CSISRS-90291. EXFOR13220.

66FRI1 Fritze, K., "The Yield of $72 \mathrm{Zn}$ in the Thermal and Epi-Cadmium Neutron Fission of 239Pu," Radiochim. Acta, 5, No. 1, 57-58 (1966). EXFOR-13219.

66GAN1 Ganapathy, R., and Kuroda, P. K., "Mass Distribution in the Fission of 232Th by 14.8 meV Neutrons," J. Inorg. Nucl. Chem., 28, 2071-2074 (1966). EXFOR-13087.

66GAN2 Ganapathy, R., Thochi, H., and Kuroda, P. K., “New Isomer 5.3h Mo-103," Phys. Rev., 151, 960-963 (1966). EXFOR-13088.

66GAN3 Ganapathy, R., and Thochi, H., "Yields of Molybdenum Isotopes in the Fission of Th232 and U-238 by $14.8 \mathrm{meV}$ Neutrons," J. Inorg. Nucl. Chem., 28, 3071-3073 (1966). EXFOR-13089.

66GOE1 Goeking, C. F., O’Kelley, G. D., Private communication to 69WAH1 (1966).

66GOR1 Gordon, G. E., Harvey, J. W., and Nakahara, H., "Measuring Fission Spectra with Semiconductor Detectors," Nucleonics 24, No. 12, 62-67 (1966). Replaces MIT-905-68 (1966). $\mathrm{Xe}-135$ value appears to neglect direct yield of Xe-135 and assumes all came from decay of $\mathrm{I}-135$. If cooling period was 15 hours, yield corrects to $5.85 \%$, but if cooling period were 30 hours, yield 
corrects to $6.24 \%$. More information is essential to obtain a usable Xe135 yield from equation 2 in 71OKA1 (78WAL1). EXFOR-13217.

66GRA1 Grau, A., "Study on the Distribution of Nuclear Charge in the Fission Chain of Uranium-238," Title translated from the original German, Doctoral thesis, a facsimile report reproduced by the U. S. Atomic Energy Commission, Division of Technical Information, Oak Ridge National Laboratory, Oak Ridge, Tennessee, NP-16155, July 13, 1966. Pages 75-76 of text and Page 84 for Summary. CSISRS-90297. EXFOR-13218.

66HAR1 Harvey, J. W., Clarke, W. B., Thode, H. G., and Tomlinson, R. H., “The Thermal-Neutron Fission Yields of 229Th," Can. J. Phys., 44, 1011-1019 (1966). EXFOR-13090.

66KON1 Konecny, E., Gunther, H., and Siegert, G., "Fine Structure in Fission Fragment Charge Distribution," Arkiv Fysik, 36, No. 40, 319-323 (1966). Also, Proceedings of the Lysekil Symposium, Session VI, Nuclides Far Off the Stability Line, Paper 7, CONF-668017, (1966).

66LAR1 Larsen, R. P., and Meyer, R. J., Chemical Engineering Division Semi-Annual Report, January - June 1966, Argonne National Laboratory, Argonne, llinois, ANL-7225, November 1966, page 232. CSISRS-90300. EXFOR-13226.

66LIS1 Lisman, F. L., Private communication to B. F. Rider, Letter FLL-8-66, (Oct. 19, 1966), superseded by ref. 70LIS1.

66MAE1 Maeck, W. J., Abernathey, R. M., and Rein, J. E., "Burnup Determination of Nuclear Fuels, Project Report for the Quarter October 1 - December 31, 1965," USAEC Doc. IDO-14676 (May 1966). Data summarized in 70LIS1.

66MAE2 Maeck, W. J., and Rein, J. E., “Burnup Determination of Nuclear Fuels,” IDO-14681 (Feb 1967). Superseded by 70LIS1.

66MAR1 Marti, K., Eberhardt, P., Geiss, J., Z. Naturforsch. A, 21, 398 (1966). Meteoritic fission yields from data of 66ROW1 attributed to Pu-244 by 71ALE1. CSISRS-90301.

66MAR2 Marshall, M., and Scobie, J., "The Emission of Alpha Particles and Tritons in the Thermal Neutron Fission of 235U," Physics Letters, 23, No. 10, 583-585 (5 December 1966).

66MCH1 Mc Hugh, J. A., "The Independent Yields of Rb-86, Cs-132, Cs-134, and Cs-136 in the Thermal Neutron Fission of U-233," J. Inorg. Nucl. Chem., 28, 1787-1790 (1966). Isomeric independent yields are here split per 76MAD2. CSISRS-90302.

66MEN1 Menke, H., and Herrmann, G., "Fission Yields of Iodine Isotopes 131 to 135 from Fission of Uranium 238," (In German), Radiochim. Acta, 6, No. 2, 76-80 (1966).

66NIS1 Nisle, R. G., and Stepan, I. E., "Yields of I135 in the fission of $233 \mathrm{U}$ and $239 \mathrm{Pu}$ Relative to that for 235U," Nucl. Sci. and Engr. 25, 93-96 (1966). CSISRS-90304.

66NOR1 Norenberg, W., “Theory of Mean Primary Charge Distribution in Low Energy Fission of Even-Even Nuclei," Z. Physik, 197, 246-261 (1966). 
66NOR2 Norris, A. E., and Wahl, A. C., "Nuclear Charge Distribution in Fission: Y92, Y93, Y94, and Y95 Independent Yields," Phys. Rev., 146 926-931 (June 17, 1966). (as adjusted by ref. 69WAH1). CSISRS-90305.

660KA1 Okazaki, A., Walker, W. H., and Bigham, C. B., "The Ratio of the Direct to the Cumulative Yield of $135 \mathrm{Xe}$ in the Thermal Neutron Fission of 233U, 235U, 239Pu and 241Pu," Can. J. Phys., 44, 237-246 (1966).

660ND1 Ondrejcin, R. S., “Thermal Fission Yield of 137Cs in 233U," J. Inorg. Nucl. Chem., 28, 1763-1767 (1966). CSISRS-90306.

66RAO1 Rao, M. N., and Kuroda, P. K., "Decay Constant and Mass-Yield Curve for the Spontaneous Fission of Uranium-238," Phys. Rev., 147, No. 3, 884-886 (1966). CSISRS-90307.

66RAV1 Ravindran, N., Flynn, K. F., and Glendenin, L. E., "Mass Distribution in the Fission of 229Th," J. Inorg. Nucl. Chem. 28, 921-924 (1966). CSISRS-90308.

66RID1 Rider, B. F., Ruiz, C. P., Peterson, J. P., Jr., and Smith, F. R., "Accurate Nuclear Fuel Burnup Analysis, Nineteenth Quarterly Progress Report, June - August 1866," General Electric Company, Pleasanton, California, GEAP-5270 (September 1966), Page 6, U233T yields relative to total Nd-148 in capsule 6-6-3, GEV-1, GEV-2 where nv $=6 \mathrm{e} 13,2 \mathrm{el3}, 1 \mathrm{e} 13$ and nvt $=2.4 \mathrm{e} 20,1.0 \mathrm{e} 20,4.0 \mathrm{e} 19$, respectively. Assuming a 300 barn spectrum averaged neutron cross section for Nd-147, only $0.982,0.993,0.995$ or an average of 0.9898 of $\mathrm{Nd}-148$ is fission caused. CSISRS-90309.

66RID2 Rider, B. F., Ruiz, C. P., Peterson, J. P., Jr., and Smith, F. R., “Accurate Nuclear Fuel Burnup Analysis, Twentieth Quarterly Progress Report," September - November 1966, General Electric Company, Pleasanton, California, GEAP-5403, (1966), Page 6. PU239T data relative to total Nd-148 from 3 capsules, 6-6-1, GEV-1, GEV-2 at nv $=6 \mathrm{e} 13,2 \mathrm{e} 13,1 \mathrm{e} 13$ and nvt $=2.4 \mathrm{e} 20,1.0 \mathrm{e} 20,4.0 \mathrm{e} 19$, respectively. Assuming a 300 barn spectrum averaged neutron cross section for Nd-147, only $0.982,0.993,0.996$ or an average of 0.990 of $\mathrm{Nd}-148$ is fission caused. CSISRS-90310.

66ROW1 Rowe, M. W., Bogard, D. D., J. Geophys. Res., 71, 4183 (1966). Meteoritic fission yields attributed to $\mathrm{Pu}-244$ by $71 \mathrm{ALE} 1$.

66RUN1 Runnalls, N. G., "Yields from Highly Asymmetric Fission: Fractional Cumulative Yields of Ba-143, Ba-144, and Ce-147 from Thermal-Neutron Induced Fission of U-235," Ph. D. Thesis, University of Missouri, Columbia (1966). See 69RUN1 and 69WAH1. CSISRS-90311.

66SIL1 Silbert, M. D., and Tomlinson, R. H., "Yields of Fission-Recoil Bromine by DelayedNeutron Studies," Radiochim. Acta, 5, No. 4, 223-227 (1966). CSISRS-90312.

66STR1 Strom, P. O., Love, D. L., Greendale, A. E., Delucchi, A. A., Sam, D., and Ballou, N. E., "Nuclear Charge Distribution of Fission Product Chains of Mass Numbers 131133," Phys. Rev., 144, 984-993 (1966). CSISRS-90313.

66UME1 Umezawa, H., "148Pm as a Primary Product of Thermal Neutron Fission of $233 \mathrm{U}$ and 235 U," JAERI-1103 (Feb. 1966). 
66WAH1 Wahl, A. C., Norris, A. E., and Ferguson, R. L., "Nuclear Charge Distribution in Fission: Rb92 and Rb93 Independent Yields," Phys. Rev., 146, 931-934 (June 27, 1966). CSISRS90315.

66WEI1 Weiss, H. V., and Reichert, W. L., "Pd115 Yield in Thermal Neutron Fission of U235," J. Inorg. Nucl. Chem., 28, 2067-2070 (1966). CSISRS-90316.

66WOG1 Wogman, N. A., Powers, J. A., and Cobble, J. W., "Charge Distribution in the Fission of Np237 and Pu239 with Intermediate-Energy Helium Ions," Phys. Rev., 152, No. 3, 1088-1095 (1966). Not coded.

66YOS1 Yoshida, H., Paiss, Y., and Amiel, S., “Determination of Independent Yields of $131 \mathrm{I}$ and $133 I$ in Thermal Neutron Fission of 235U," IA-1128, pp. 63-64 (January 1, 1966).

67BEN1 Bennett, M. J., and Stein, W. E., "Kinetic Energies of Fragments from Seven Fission Reactions at Low Excitation Energies," Phys. Rev., 156, No. 4, 1277-1283 (20 April 1967).

67BIR1 Birgul, O., and Lyle, S. J., "Tin and Antimony Isotopes of Mass 129 in Fission," Radiochim. Acta, 8, 9-12 (1967). Sb-129 values are low by loss of $\mathrm{Sb}$ on evaporation with $\mathrm{HCl}$, corrected in 71BIR1.

67BOR1 Borisova, N. I., Dubrovina, S. M., Novgorodtseva, V. I., Pchelin, V. A., Shigin, V. A., Shubko, V. M., "Variation of Mass Distributions of 238U Fission Fragments with Neutron Energy," Yadern. Fiz., 6, 454-461, (September 1967). In Russian. At 1.5, 5.0, and $18 \mathrm{meV}$ Ratios of $115 \mathrm{Cd}$ and $77 \mathrm{As}$ to $99 \mathrm{Mo}$ and $140 \mathrm{Ba}$. NSA 22: 01668 (1968).

67BOR2 Borden, K. D., Thochi, H., and Ward, T., "Gaseous Fission Products from the U238 Spontaneous Fission," ORO-3235-12, page 21 (January 31, 1967). Preliminary results. CSISRS-90320.

67BOR3 Borden, K. D., "Photofission of Th-232 Induced by $17.5 \mathrm{meV}$ Monoenergetic Gamma Rays," ORO-3235-12, Page 18 (January 31, 1967). Work to be done.

67BOR4 Borikwa, H. E., et. al., Fizika Vol 6, pg. 454 (1967). Gives peak to valley for U238F. Peak $\mathrm{Mo99}=(6.92+/-0.69)$ valley $111 \mathrm{Ag}=(0.0513+/-0.0103) ; 115 \mathrm{Cd}=(0.0256+/-0.0051)$. Cited by 85CHI1. CSISRS-90321.

67BRE1 Bresesti, M., Burei, G., Ferrari, P., and Moretto, L. G., "Radiochemical Determination of Fission Yields for the Fission of Th-232 by Pile Neutrons," J. Inorg. Nucl. Chem., 29, 1189-1196 (1967).

67BR01 Brown, L. C., Wahl, A. C., "Chemical Behavior of Tin Fission Products," J. Inorg. Nucl. Chem., 29, 2133-2145 (1967). A. C. Wahl (73WAH1) says hot atom method is not reliable ( $\mathrm{x} 2)$ and this reference data should not be used. CSISRS-90323.

67CHW1 Chwaszczewska, J., Dakowski, M., Krogulski, T., Piasecki, E., Przyborski, W., and Sowinski, M., "Emission of Long Range Charged Particles in the Fission of $235 \mathrm{U}$ by Thermal Neutrons," Physics Letters, 24B, No. 2, 87-89 (23 Jan. 1967). 
67COS1 Cosper, S. W., Cerny, J., Gatti, R. C., "Long-Range Particles of Z=1 to 4 Emitted During the Spontaneous Fission of Cf-252," Phys. Rev. 154, 1193-1206 (Feb 20, 1967). NSA 21: 19274 (1967). NSA 21: 19274 (1967).

67CR01 Crouch, E. A. C., "Calculated Independent Yields in Thermal Fission of U-235, Pu239, Pu-241, and U-233," Atomic Energy Research Establishment, Harwell, U. K., AERER-5488, (1967).

67DAK1 Dakowski, M., Chwaszczewska, J., Krogulski, T., Piasecki, E., and Sowinski, M., "Energy Spectra of Long Range Particles from the Thermal Neutron Fission of 235U," Physics Letters, 25B, No. 3, 213-214 (21 August, 1967).

67FAS1 Fasching, J. L., and Coryell, C. D., "Independent Fission Yields of La and Ce Isotopes," Massachusetts Institute of Technology, Laboratory for Nuclear Science, Report MIT-905-108, pp. 21-23, (Dec. 31, 1967). CSISRS-90324. Later published according to CSISRS-90324 as (J,BAP,16,516(BH2),7104); American Physical Society, Bulletin 16, p. 516 (Apr 1971).

67GAN1 Ganapathy, R., Mo, T., Meason, J. L., "Fission of $233 \mathrm{U}$ by Thermal Neutrons," J. Inorg. Nucl. Chem., 29, 257-261 (1967). CSISRS-90325.

67GAN2 Ganapathy, R., and Kuroda, P. K., "Mass Yield Distribution of the Iodine Isotopes from the Spontaneous Fission of Plutonium-242 and the Existence of Plutonium244 in the Early Solar System," Earth and Planetary Science Letters, Vol 3, 89-93 (1967). CSISRS-90326.

67GUN1 Gunten, H. R. von, Flynn, K. F., and Glendenin, L. E., "Distribution of Mass and Charge in Fission of 245 Cm," Phys. Rev. 161, No. 4, pp. 1192-1195 (1967). In author index, von Gunten, H. R., reads (Gunten, H. R. von ). CSISRS-90327.

67GUN2 Gunten, H. R. von, and Hermann, H., "Absolute Fission-Yields of 99Mo and 139Ba in the Thermal Neutron Fission of 235U," Radiochimica Acta, 8, 112-114 (Nov. 1967). In author index, von Gunten, H. R., reads (Gunten, H. R. von ).

67HOH1 Hohenberg, C. M., Munk, M. N., Reynolds, J., J. Geophys. Res., 72, 3139 (1967). Meteoritic fission yields attributed by 71ALE1 to $\mathrm{Pu}-244$.

67ISH1 Ishimori, T., Ueno, K., Kimura, K., Akatsu, E., Kobayashi, Y., Ono, R., and Hoshi, M., "The Spontaneous Fission of Uranium-238," Radiochim. Acta, 7, No. 2/3, 95-103 (1967).

67JAD1 Jadhav, A. V., Ramaniah, M. V., Rao, C. L., Shahani, C. J., "Cumulative Yields of 99Mo, 105Rh and 132Te in the Spontaneous Fission of 238U," Nukleonik, 9, 43-46 (1967). NSA 21: 25541 (1967).

67KON1 Konecny, E., Gunther, H., Siegert, G., Winter, L., "Pairing Effect in Fission Fragment Charge Distributions," Nucl. Phys. A100, 465-472 (1967).

67LED1 Lederer, C. M., Hollander, J. M., and Perlman, I., Table of Isotopes, Sixth edition, John M. Wiley and Sons, New York, New York, 1967.

67LIS1 Lisman, F. L., Maeck, W. J., and Rein, J. E., "Burnup Determination of Nuclear Fuels," Idaho Nuclear Corporation, Idaho Falls, Idaho, IN-1113, October 1967 and IN-1157 rev. (February 1968); Superseded by 70LIS1. 
67LIS2 Lisman, F. L., Maeck, W. J., Foster, R. E., Jr., and Rein, J. E., editors, "Burnup Determination of Nuclear Fuel," Report for the Quarter July 1 - September 30, 1966, Idaho Nuclear Corporation, Idaho Falls, Idaho IN-1064, (March 1967). See experimental capsule 6-4-1.

67MAR1 Marmol, P. del, and Mevergnies, M. N. de, "Investigation of Delayed Neutron Precursors of As, Sb, and Ge," J. Inorg. Nucl. Chem., 19, 273-279 (1967). In author index, de Mevergnies, M. N., reads (Mevergnies, M. N. de ). In author index del Marmol, P., reads (Marmol, P. del ).

67MEA1 Meason, J. L., and Ganapathy, R., "Fission Yields of Kr and Xe by Mass Spectroscopy," ORO-3235-12, Page 18 (January 31, 1967). Work to be done.

67MIT1 MIT staff, Massachusetts Institute of Technology, “Decay of 6.7-Hour 135I to the Levels of 135Xe," Laboratory for Nuclear Science, Chemistry Progress Report MIT-905-108 pp. 10-12 (Dec. 31, 1967).

67MO 1 Mo, T., and Rao, M. N., “Mass Distribution Near the Region of Symmetric Fission of Th232 by $14.8 \mathrm{meV}$ Neutrons," ORO-3235-12, page 19 (January 31, 1967). Replaced by $68 \mathrm{MO} 1$.

67MO 2 Mo, T., "Independent Yield of Sb124 in $14.8 \mathrm{meV}$ Neutron-Induced Fission of Th: 232," ORO-3235-12, Page 20 (January 31, 1967). Replaced by 69RA01.

67PAR1 Parsa, B., Wenzel, A., and Gordon, G. E., "Fission Yield and Branching Ratio of 133Sb in Fission," Massachusetts Institute of Technology Laboratory for Nuclear Science Report MIT-905-108, pg. 20 (Dec. 31, 1967).

67RAM1 Ramaniah, M. V., Namboodiri, M. N., and Ravindran, N., "Yields of Some Rare Earth Nuclides in the Neutron Fission of 231 Pa and. 237Np," pp. 197-204 of Proceedings of the Nuclear and Radiation Chemistry Symposium, Poona, March 6-9, 1967. CONF-670335, Bhabha Atomic Research Centre, Trombay, Bombay, India. 1967. NSA 23: 01893 (1969).

67RAO1 Rao, M. N., "Radiochemical Investigation of 238U Spontaneous Fission," Radiochim. Acta, 8, No. 1, 12-15 (1967).

67RAO2 Rao, M. N., "Zirconium-97 in the Spontaneous Fission of Uranium-238," J. Inorg. Nucl. Chem., 29, 863-864 (1967).

67RID1 Rider, B. F., Ruiz, C. P., Peterson, J. P., Jr., and Smith, F. R., "Accurate Nuclear Fuel Burnup Analyses, Twenty-Second Quarterly Progress and Final Report," General Electric Company, Pleasanton, California, GEAP-5505, June 30, 1967. On page 21 U238F Nd150 was miscalculated. Since recalculated by B. F. Rider. (PU239T Ce142 should read 4.93 because of typographical error). On page $8 \mathrm{PU} 241 \mathrm{~T}$ yields in capsule 6-6-5 where $\mathrm{nv}=6.0 \mathrm{e} 13$ and nvt $=2.4 \mathrm{e} 20$ assuming $\mathrm{Nd}-147$ spectrum-averaged neutron cross section to be 300 barns, only 0.982 of $\mathrm{Nd}-148$ is fission caused. CSISRS-90334.

67RID2 Rider, B. F., Ruiz, C. P., Peterson, J. P., Jr., Smith, F. R., "A Survey and Evaluation of Thermal Fission Yields for Pu-239, U-233 and Pu-241,’ GEAP-5356 (Mar. 1, 1967).

67ROC1 Roche, M. F., Ferguson, R. L., Troutner, D. E., ORNL-4164, Page 10, “Chemistry Div. Annual Report," Sept. 1967. NSA 21: 45085 (1967). CSISRS-90335. 
67SAB1 Sabu, D. D., and Rao, M. N., "U238 Spontaneous Fission Yields of Ru103 and Ba140," ORO-3235-12, Page 21 (January 31, 1967). Replaced by 71SAB1.

67SCH1 Schwartz, L. L., Data quoted in UCRL-50243 (Vol. I) "Atom Fraction of the Total Chain Yield Versus Time," pp. I-2 to I-7 (March 31, 1967). CSISRS-90336.

67SHA1 Shank, R. C., and Staff, Annual Report of the Analytical Chemistry Branch for 1966, Idaho Nuclear Corporation, Idaho Falls, Idaho, IN-1063, April 1967, p. 27. Superseded by 70LIS1.

67STE1 Stella, R., Crespi, V., Maxia, V., "Yields for the $A=141$ to $A=153$ Region in the Reactor Neutron Fission of 237Np," Ric. Sci., 37, 347-353 (1967).

67STE2 Stella, R., Dicasa, M., and Maxia, V., "Yields for the 131-135 Mass Region in the Reactor Neutron Fission of 237Np," Ric. Sci. 37, 354-356 (1967).

67STE3 Stella, R., Dicasa, M., and Maxia, V., “95Zr, $97 \mathrm{Zr}$ and $99 \mathrm{Mo}$ Yields in the Reactor Fission of 237Np," Ric. Sci., 37, 357-359 (1967). R value reduced here to absolute yield assuming NP237F Ba140 yield $=5.50$ and U235T yields of Ba140, Zr95, Zr97, Mo99, of 6.305, $6.455,5.961$, and 6.142 .

67TRA1 Trammell, M. R., Henninger, W. A., "Nuclear Data Library for the Fission Product Program," WANL-TME-574 (Rev. 1) (Nov. 17, 1966). 53 pp. U235T compilation. NSA 22: 27091 (1968).

67VIL1 Villarreal, R., “The Separation and Spectrophotometric Determination of Technetium for the Determination of Burnup in Fissium," Masters Thesis, Univ. of Idaho, May 1967, as cited in 70EBE1. CSISRS-90339.

67WAH1 Wahlgren, M. A., Stewart, D. C., Lawless, F. R., Hines, J. J., Flynn, K. F., and Lerner, J. L., "Decay of 10.4-Min Gd162," Phys. Rev. 153, 1310-1311 (1967).

67WAL1 Walker, W. H., Fission-Product Absorption in Thermal Reactors, in "Nuclear Data for Reactors," International Atomic Energy Agency, Vienna, Austria, (1967), Vol. 1, pp. 521-536. Available from the U. S. Atomic Energy Commission Office of Technical Services, Washington, D. C. CONF-661014.

67WHE1 Whetstone, S. L. Jr., and Thomas, T. D., "Light Charged Particles from Spontaneous Fission of Cf252," Phys. Rev. 154, 1174 (1967).

67WUN1 Wunderlich, F., "Measurement of Independent Iodine Fission Yields in Thermal Fission of 235U," Radiochim. Acta, 7, 105-114 (1967). (In German).

68ALE1 Alexander, P., Lau, J. P., “Nuclear Structure in 133, 135Xe and 133, 135Cs," J. Nucl. Phys. A121, 612-624 (1968). Xe135m/Xe135g $=0.095 \pm 0.005$. Branching ratio $=0.095 / 1.095$ $=0.08676$. This is consistent with ind. yield of Xe135m=1.7 times Xe135g (69YEL1).

68AMI1 Amiel, S., Yellin, E., Gilat, J., Katcoff, S., and Oron, M., "On-Line Studies of Short-Lived Noble Gases," IA-1190, 98-99 (July; 1968). Kr-94 and Xe-143 observed directly; No yield given. 
68AND1 Anderson, J. L., Freid, S. H., and Choppin, G. R., "Charge Distribution in the Fission of 238U," J. Inorg. Nucl. Chem., 30, 3167-3172 (1968). $9.5 \mathrm{meV}$ and $11.3 \mathrm{meV}$ proton and 11.5 meV deuteron fission. Not coded.

68AND2 Andreev, W. N., et. al., Yadern. Fiz., 8, 38 (1968). Ternary Fission (8Li, 8He, 10Be etc.).

68ARI1 Arino, H., and Kuroda, P. K., "Spontaneous Fission-Produced Strontium Isotopes in Uranium Minerals and Salts," J. Inorg. Nucl. Chem., 30, 677-683 (168). NSA 22: 39656 (1968). CSISRS-90341.

68BAC1 Bachmann, K., "Determination of the Independent Yield of Pm-148m and Pm-148 in the Thermal Fission of U235," (In German). Radiochim. Acta, 9, Heft 1, 27-31 (1968). NSA 22: 42065 (1969).

68BEL1 Beller, L. S., and Maddison, D. W., "Relative 238U Fission Yields by Gamma Ray Analysis," ANS Transactions, 11, No. 2, 607-8 (1968). With digital data privately communicated to B. F. Rider. NSA 23: 04014 (1969). CSISRS-90343.

68BOR1 Borisova, N. I., Zenkova, R. A., Kurchatov, B. V., Morozov, L. N., Novgorodtseva, V. I., Pchelin, V. A., Chistyakov, L. V., Shubko, V. M., "Radiochemical Investigation of the Th229 Fission by Slow Neutrons," Yadern. Fiz., 8, 695-703 (1968). NSA 23: 08980 (1968). See Sov. J. Nucl. Phys. 8, 404 (1968).

68BOR2 Borisova, N. I., Dubrovina, S. M., "Variation of Mass Distribution of 238U Fission Fragments with Neutron Energy," Sov. J. Nucl. Phys., 6, 331 (1968).

68BOR3 Boroughs, G. L., Craven, C. W. Jr., Drake, M. K., "A Set of Evaluated Nuclear Data for 233U," GA-8854 (Aug. 27, 1968). NSA 23: 10998 (1969). F. P. yields for ENDF/B-I.

68CEN1 Cenacchi, G., "A Compilation of Nuclear Data on the Thermal Fission Products of U-233, U-235 and Pu238," Comitato Nazionale Energia Nucleare, RT/FIMA(68)4, Roma 1968. Replaces Ceicia e Cuetura CEC(68)6, (1968).

68CIU1 Ciuffolotti, L., "The Ba140 Yields for Thermal and Fast Fission of U235 and U238," Energia Nucleare, 15, 272-276 (April 1968). EXFOR-21483.

68DEL1 Delucchi, A. A., Greendale, A. E., Strom, P. O., "Cumulative Fission Yield and HalfLife of Sb-134," Phys. Rev., 173, 1159-1165 (September 20, 1968). NSA 22: 50613 (1968). CSISRS-90347.

68DUD1 Dudey, N. D., “Low-Mass Fission Yield Studies,” pp. 106-107 in Chemical Engineering Division Research Highlights, May 1967-April 1968, ANL-7450 (1968).

68DJA1 D'Jachenko, P. P., Kuz'minov, B. D., “Neutron Fission of U-235 at the Excitation Energy of Compound Nucleous $22 \mathrm{MeV}$," Yadern. Fiz. 7(1), 36 (Jul 1968). EXFOR-40234.

68DJA2 D'Jachenko, P. P., Kuz'minov, B. D., Tarasko, M. Z., “Energy and Mass Distribution of Fragments at the Fission of U-235 by Monoenergetic Neutrons in the Energy Interval 5-7 MeV," Yadern. Fiz., 8(2), 286 (Aug 1968). EXFOR-40235.

68EDW1 Edwards, R. R., "A Review of Recent Studies of Nondestructive Assay Methods for Irradiated Nuclear Fuels," Nuclear Applications, 4, 245-259 (Apr. 1968). 
68EWB1 Ewbank, W. B., to M. E. Meek, Personal communication, "Nuclear Data Project," January $29,1968$.

68FRE1 Freid, S. H., Anderson, J. L., and Choppin, G. R., "Charge Distribution in the Fission of 232Th," J. Inorg. Nucl. Chem., 30, 3155-3165 (1968). CSISRS-90348.

68GAN1 Ganapathy, R., "Distribution of Mass in the Fission of Bismuth-209, Thorium-232, Uranium-233 and Plutonium-242," Ph. D. Dissertation, University of Arkansas (1968). NSA 22: 53216 (1968). DA/B 29, 319 (1968). See also refs. 66GAN1, 67GAN1. CSISRS-90349.

68GEV1 Gevaert, L. H., "14 meV Neutron-Induced Fission and $25 \mathrm{meV}$ Photo-Fission of 232Th and 238U," Thesis Univ. of Toronto (1968). NSA 26: 27056 (1972). Later published as 70GEV1.

68GOR1 Gorman, D. J., and Tomlinson, R. H., "Cumulative Yields in the 14-meV Neutron Fission of U-238," Can. J. Chem, 46, 1663-1672 (1968). NSA 22: 35325 (1968). CSISRS90350.

68GRE1 Greendale, A. E., Delucchi, A. A., "Fission Yields of Several Iodine Isotopes and HalfLife and Fission Yield of 135 Te," 29 pp. USNRDL-TR-69-11 (AD 686041) (December 31, 1968). NSA 23: 49379 (1969). CSISRS-90351.

68HAR1 Harvey, J. W., Clarke, W. B., Gorman, D. J., and Tomlinson, R. H., "Reactor-Neutron Fission Yields of 232Th," Can. J. Chem., 46, 2911-2915 (1968). CSISRS-90352.

68KOH1 Kohler, W., Private communication to W. N. Mc Elroy from International Atomic Energy Agency, Distribution Programme (1968). See 70MCE1. CSISRS-90353.

68KRI1 Krisyuk, I. T., Protopopov, A. N., and Usacheva, N. T., "Concerning the Fine Structure of the Fragment Mass Yield Curve in the Fission of U235 by 14-meV Neutrons," Soviet J. Nucl. Phys., 6, No. 5, 669-670 (May 1968). Submitted Nov. 12, 1966, Yad. Fiz. 5, 919-920 (1968).

68LEE1 Lee, C., Amiel, S., and Yellin, E., "Cumulative and Independent Yields in Thermal Neutron Fission of 233U," IA-1168, 67-68 (April 1968).

68LIS1 Lisman, F. L., Private communication to B. F. Rider. Letter FLL-1-68, Idaho Nuclear Corp., (March 6, 1968). See also IN-1189 page III (July 1968). NSA 22: 44680 (1968). Data later superseded by ref. 70LIS1.

68LIS2 Lisman, F. L., Maeck, W. J., and Rein, J. E., "Burnup Determination of Nuclear Fuels," USAEC Doc. IN-1178 (April 1968). Data superseded by ref 70LIS1. NSA 22: 25129 (1968).

68LIS3 Lisman, F. L., Maeck, W. J., and Rein, J. E., "Burnup Determination of Nuclear Fuels," USAEC Document IN-1215, (August, 1968), Summarized in ref. 70LIS1.

68LIS4 Lisman, F. L., Maeck, W. J., and Rein, J. E., "Burnup Determination of Nuclear Fuels," USAEC Document IN-1207 (July 1968). Data relative to Nd148 $=1.75$ summarized in ref. 70 LIS1. 
68LYL1 Lyle, S. J., Martin, G. R., and Rahman, Md. M., "Some Chain Yields in the Asymmetric Fission of Thorium-232 Induced by 3 and $14.7 \mathrm{meV}$ Neutrons," Radiochim. Acta, 9, 90-94 (1968). NSA 23: 04007 (1969).

68MAR1 Marmol, P. del, "Identification of New Arsenic Isotopes in Fission: 83As and 84As," J. Inorg. Nucl. Chem., 30, 2873-2880 (1968). NSA 23: 05861 (1969). In author index, del Marmol, P., reads (Marmol, P. del ).

68MCH1 Mc Hugh, J. A., and Michel, M. C., "Ratio of 85Kr Isomers Produced in Medium Energy Fission," J. Inorg. Nucl. Chem., 30, 673-676 (1968). NSA 23: 15486 (1969).

68MCH2 Mc Hugh, J. A., and Michel, M. C., "Fission Fragment Mass and Charge Distribution for the Moderately Excited U-236 Compound Nucleous," Phys. Rev., 172, No. 4, 1160-1175 (1968). See also 63MCH1. CSISRS-90358.

68MEE1 Meek, M. E., and Rider, B. F., "Summary of Fission Product Yields for U235, U238, Pu239, and Pu241 at Thermal, Fission Spectrum and 14meV Neutron Energies," General Electric Company Report APED-5398A (October 1, 1968). NSA 23: 34784 (1969).

68MO 1 Mo, T., and Rao, M. N., "Symmetric Fission of 232Th by 14 meV Neutrons," J. Inorg. Nucl. Chem., 30, 345-347 (1968). NSA 22: 20598 (1968). Replaces ORO-3235-12, page 19 (January 31,1967 ). For Te-129 the 70 minute isomer appears to have been measured (77CRO1). CSISRS-90359.

68NAM1 Namboodiri, M. N., Ravindran, N., Rajagopalan, M., and Ramaniah, M. V., “Mass Distribution in the Reactor Neutron Fission of $231 \mathrm{~Pa}$ and $237 \mathrm{~Np}$," J. Inorg. Nucl. Chem., 30, 2305-2310 (1968). NSA 23: 01886 (1969).

68NIS1 Nisle, R. G., and Stepan, I. E., "Measurement of I135 Yield Ratios and Fission Product Cross-Sections in the Advanced Reactivity Measurement Facilities," Nucl. Sci. and Engr., 31, 241-246 (1968). CSISRS-90361.

68NOT1 Notea, A., "Charge Distribution in Thermal Neutron Fission of 235U," IA-1168, 65-67 (1968). Precursor to 69NOT1.

68PAP1 Pappas, A. C., Strom, P. O., and Westgaard, L., "Existence of a 1 hr 129Sn Isomer Not Confirmed," J. Inorg. Nucl. Chem. 30 890-891 (1968).

68ROC1 Roche, M. F., Ferguson, R. L., and Troutner, D. E., "Nuclear Charge Distribution in Fission; Yield of Palladium-115 from Thermal-Neutron Fission of Uranium-235," ORNL-3994, pp. 7-8 (May 20, 1966). See also M. F. Roche, "The Independent Yield of $112 \mathrm{Ag}$ and the Cumulative Yield of $115 \mathrm{Pd}$ in Thermal-Neutron Induced $233 \mathrm{U}$ and 235U Fission," (TID-24500) Thesis St. Louis Univ., Mo. (Jan 1968) NSA 22: 32579 (1968). Dissertation abstracts B 29, 927 (Sept 1968). CSISRS-90363.

68RUD1 Rudy, C., Vandenbosch, R., and Ratcliffe, C. T., “Relative Independent Yields for $95 \mathrm{Nb}$ and $95 \mathrm{~m}$ Nb from Low Energy Fission," J. Inorg. Nucl. Chem., 30, 365-368 (1968). 10 meV proton induced 235U fission. NSA 22: 18218 (1968). CSISRS-90364. 
68SER1 Sergachev, A. I., Vorobjeva, V. G., Kuz'minov, B. D., Mikhaylov, V. B., Tarasko, M. Z., "Influence of Intermediate States of the Fissionable Nucleous Th-232 on Mass and Kinetic Energy Distributions of Fragments," Yadern. Fiz. 7(4), 778 (April 1968). EXFOR-40173.

68SHA1 Shank, R. C., and Staff, "Annual Report for the Analytical Chemistry Branch for Period Ending June 30, 1968," IN-1203 (Sept. 1968). Data superseded by ref. 70LIS1.

68SIN1 Sinclair, V. M., "Analytical Aspects of Chemical Studies on Irradiated Oxide Nuclear Fuels," UKAEA Report TRG-1735(d) (1968). Data later published as 69DAV1.

68THE1 Thein, M., Rao, M. N., and Kuroda, P. K., "Mass Distribution in 14.8 meV NeutronInduced Fission of 232Th," J. Inorg. Nucl. Chem., 30, 1145-1150 (1968). NSA 22: 44704 (1968). Originally appeared as ORO-3235-29 (1967). From thesis NSA 23: 13278 (1969). DA/B 29, 1809 (1968). CSISRS-90366.

68TOM1 Tomlinson, L., and Hurdus, M. H., "Delayed Neutron Precursors III, Selenium-87," J. Inorg. Nucl. Chem., 30, 1995-2002 (1968). NSA 22: 48543 (1968). Se87 yield superseded by author's recent work 71TOM1.

68WEC1 Wechsler, M. S., Private communication from Oak Ridge National Laboratory to W. N. Mc Elroy (1968). See 70MCE1. EXFOR-90368.

68WEI1 Weiss, H. V., Elzie, J. L., and Fresco, J. M., "Identification and Yield of 5.0-Sec 117Pd in the Thermal Neutron Fission of 235U," Phys. Rev., 172, 1269-1271 (August 20, 1968). NSA 22: 44736 (1968). CSISRS-90369.

68WEI2 Weiss, H. V., Fresco, J. M., and Reichert, W. L., "Identification of 5.3-Sec 118Ag as a Product of 235U Fission," Phys. Rev., 172, 1266-1269 (August 20, 1968). NSA 22: 44735 (1968).

68WIS1 Wish, L., "Thermal-Neutron Fission of 235U: Identification and Fractional Chain Yield of 17-Sec 77Ga," Phys. Rev., 172, 1262-1266 (August 20, 1968). NSA 22: 44733 (1968). CSISRS-90370.

69ADA1 Adamov, B. M., "Long Range Particles in Fission," Second IAEA Symposium on Physics and Chemistry of Fission, STI/PUB/234, pages 900-901 (1969). Abstract. Determined relative probabilities of $14 \mathrm{meV}$ neutron-induced fission of $232 \mathrm{Th}, 233 \mathrm{U}, 238 \mathrm{U}$, and $237 \mathrm{~Np}$ involving long range particles and in $235 \mathrm{U}$ by slow neutrons, and proton and triton yields per 100 alpha particles for these plus $233 \mathrm{U}$ and $235 \mathrm{U}$ by slow neutrons and spontaneous fission of $244 \mathrm{Cm}$. Not coded.

69AMI1 Amiel, S., Braun, C., Ehrenberg, B., Feldstein, H., Nir-El, Y., Oron, M., and Yellin, E., "Independent Yields and Distributions of Isotopes of $\mathrm{Br}, \mathrm{Kr}, \mathrm{I}$, and $\mathrm{Xe}$ in Thermal Neutron Fission of U-235 Studied with an On-Line Separator," Paper SM-122/38 Second IAEA Symposium on the Physics and Chemistry of Fission, Vienna 1969 STI/PUB-234 p. 948. Abstract without data.

69AUM1 Aumann, D. C., Flynn, K. F., Gindler, J. E., and Glendenin, L. E., "Formation of Pd-111 Isomers in Neutron Fission of Several Heavy Nuclides," J. Inorg. Nucl. Chem., 31, 1935-1945 (1969). NSA 23: 37227 (1969). CSISRS-90371. 
69BIR1 Birgul, O., Lyle, S. J., and Sellars, J., "Fine Structure in Nuclear Fission, Part II. Yields from $14.8 \mathrm{meV}$ Neutron Induced Fission of 231Pa in the Mass Region 131-135," Radiochim. Acta, 12, No. 2, 66-69 (1969). NSA 24: 01845 (1970).

69BIR2 Birgul, O., and Lyle, S. J., "Radiochemical Studies of 3 and $14.8 \mathrm{meV}$ Neutron Induced Fission of 231Pa," Radiochim. Acta, 11, No. 2, 108-112 (1969). NSA 23: 40052 (1969). EXFOR-21731.

69BLA1 Blachot, J., Carraz, L. C., Cavallini, P., Gadelle, A., and Moussa, A., "Application of New Fast Chemical Separations to the Determination of Charge Distribution in LowEnergy Fission," In French. IAEA 2nd Symposium on the Physics and Chemistry of Fission, Vienna (July 1969) STI/PUB/234 pp. 803-811. CONF-690708. Te-131m FI split between metastable and ground state in ratio of $(2 j+1)$ where $j$ is nuclear spin. Later split per 76MAD2.

69BLO1 Blocki, J., Chwaszczewska, J., Dakowski, M., Krogulski, T., Piasecki, E., Sowinski, M., Stegner, A., Tys, J., "Li and Be Nuclei Emitted During the Thermal Neutron Fission of 235U," Nucl. Phys. A127, 495-502 (1969). EXFOR-30317.

69BOH1 Bohn, E. M., Wehring, B. W., and Wyman, M. E., "Prompt K X-Rays as a Function of Fragment Mass and Total Kinetic Energy in the Thermal Fission of U235," Phys. Rev., 188, 1909-1915 (Dec. 20, 1969).

69BOR1 Borden, K. D., Kuroda, P. K., “Mass Distribution in the Fission of $233 \mathrm{U}$ by $14.8 \mathrm{meV}$ Neutrons," J. Inorg. Nucl. Chem., 31, 2623-2625 (1969). NSA 23: 40065 (1969). For thesis see NSA 23: 11007 (1969). Dissertation Abstracts B 29, 1621 (Nov 1968). CSISRS-90375.

69BRA1 Braun, C., and Amiel, S., "Separation of Short-Lived Bromine and Iodine Fission Products, and Measurement of Their Nuclear Properties," Research Laboratories Report January- December 1968, LA-1190, Israel Atomic Energy Commission, July 1969, pp. 104-106. Superseded by 69BRA2.

69BRA2 Braun, C., and Amiel, S., "Determination of Independent Fission Yields of Short-Lived Halogens in the Thermal Fission of 235U," IA-1218, 93-94 (1969).

69CAM1 Cambiaghi, M., Fossati, F., Pinelli, T., "Long-Range Alpha-Particles and Light Charged Nuclei in the Neutron-Induced Fission of 233U," Nuovo Cim. 59B 236-244 (Feb 11, 1969). NSA 23: 15484 (1969).

69CAV1 Cavallari, F., Cambiaghi, M., Fossati, F., Pinelli, T., "Emission of Light Charged Nuclei in the N-Thermal Fission of 238Pu," Second IAEA Symposium on Physics and Chemistry of Fission, STI/PUB/234, Pages 891-892 (1969).

$69 \mathrm{CEN1}$ Cenacchi, G., Compilation of nuclear data on the fission products of $233 \mathrm{U}, 235 \mathrm{U}$, and $239 \mathrm{Pu}$, (Comitato Nationale per l'Energia Nucleare, Rome, Italy) Report No. (RT/FIMA-(68)4), 1968. NSA 23: 37196 (1969).

69CRO1 Crouch, E. A. C., "Calculated Independent Yields in Thermal Neutron Fission of 233U, 235U, 239Pu, 241Pu, and in Fission of 232Th, 238U, and 240Pu," AERE-R6056 (1969). NSA 23: 32922 (1969). 
69CRO2 Croall, I. F., and Willis, H. H., "Yields from the Thermal Neutron Induced Fission of 241Pu," AERE-R-6154 (1969). NSA 24: 03914 (1970).

69DAN1 Dange, S. P., Jain, H. C., Manohar, S. B., Satyaprakash, K., Ramaniah, M. V., Ramaswami, A., Rengan, K., (Bhabha Atomic Research Centre, Trombay, India), "Fission Yields and Recoil Ranges Determined by a Ge(Li) Detector," Paper SM-122/97 pp. 741-757 of Physics and Chemistry of Fission, Vienna; International Atomic Energy Agency (1969). From 2nd Conference on Physics and Chemistry of Fission, Vienna, Austria. See STI/PUB-234; CONF-690708. NSA 24: 30803 (1970). Superseded by 71RAM1.

69DAV1 Davies, W., "Absolute Measurements of Fission Yields for 235 Uranium and 239 Plutonium in the Dounreay Fast Reactor," Radiochim. Acta 12, 173-178 (1969).

69DEN1 Denschlag, H. O., "Independent Yield of 1351 in the Thermal Neutron Fission of 235U, The Half-Life of 135Te," J. Inorg. Nucl. Chem., 31, 1873-1882 (1969). NSA 23: 37226 (1969).

69DEN2 Denschlag, H. O., Qaim, S. M., Eckhardt, W., and Herrmann, G., "Charge Distribution in Low-Energy Fission Reactions," Paper SM-122/26 Second IAEA Symposium on the Physics and Chemistry of Fission, Vienna, 1969, STI/PUB-234 pp. 945-946 (December 1969). Superseded by 70QAI1.

69DJA1 D'jachenko, P. P., Kuz'minov, B. D., Lajtai, A., "Kinetic Energy of Fragments at the Fission of U-235 by Neutrons in the Range of 0-0.6 MeV," Report YFI-8, 7 (Dec 1969). EXFOR-40017. Energy dependent yields.

69EHR1 Ehrenberg, B., Feldstein, H., Oron, M., and Amiel, S., "Primary Mass Distribution of Kr Isotopes Formed in the Thermal Fission of 235U," IA-1218, 96-97 (1969). NSA 25: 41833 (1971).

69ERD1 Erdal, B. R., Williams, J. C., and Wahl, A. C., "Cumulative Yields of Tin and Antimony Nuclides from Thermal- Neutron Fission of U235," J. Inorg. Nucl. Chem., 31, 2993-3003 (Oct. 1969). NSA 24: 01866 (1970). CSISRS-90383.

69ERD2 Erdal, B. R., Wahl, A. C., and Dropesky, B. J., "Short-Lived 123Sn and 125Sn from Thermal-Neutron Fission of 235U," J. Inorg. Nucl. Chem., 31, 3005-3009 (Oct. 1969). NSA 24: 06905 (1970). Replaces Chicago Operation Office Rept. COO-1162-32 (1967). CSISRS90384.

69FAS1 Fasching, J. L., and Coryell, C. D., "The Fractional Independent Chain Yield of 284Day 144Ce," in "Chemistry Progress Report December 31, 1969," Massachusetts Institute of Technology, Cambridge, Mass., MIT-905-154, pp. 15-17, (Dec 1969). CSISRS-90385.

69FEL1 Feldstein, H., and Amiel, S., "Rapid Separation of Fission Product Antimony," IA-1190, 100-101 (July 1969). For determination of fission yields of Sb isotopes with half-lives $2 \mathrm{sec}(\mathrm{e}$. g. $135 \mathrm{Sb})$ and longer. No yields given.

69FLY1 Flynn, K. F., and Gunten, H. R. von, "Distribution of Mass and Charge in the Fission of Cf-249 with Thermal Neutrons," Helvetica Chimica Acta, 52, Fasc. 8, 2216-2221 (1969). In German. In author index, von Gunten, H. R., reads (Gunten, H. R. von ). 
69FLY2 Flynn, K. F., and Gunten, H. R. von, "Distribution of Mass and Charge in the Fission of 227Th," Paper SM-122/1 Second IAEA Symposium on the Physics and Chemistry of Fission, Vienna, 1969, STI/PUB-234 pp. $731-739$ (December 1969). In author index, von Gunten, $H$. R., reads (Gunten, H. R. von ). EXFOR-21496.

69GOE1 Goennenwein, F., Holubarsch, W., Pfeiffer, E., "Mäss and Energy Distributions of Fission Fragments from 232Th $(n, f)$ by meV Neutrons," Second IAEA Symposium on Physics and Chemistry of Fission, STI/PUB/234, Page 944 (1969). Peak-to-valley is 560/1, 122/1, 45/1 at $1.92,2.97$ and $4.81 \mathrm{meV}$. Not coded.

69GUN1 Gunten, H. R. von, Flynn, K. F., and Glendenin, L. E., "Independent Yields of Br-82, Rb86, Cs-136, and Pm-150 in Spontaneous Fission of Cf-252," J. Inorg. Nucl. Chem., 31, 3357-3361 (1969). NSA 24: 06932 (1970). In author index, von Gunten, H. R., reads Gunten, H. R. von CSISRS-90388.

69GUN2 Gunten, H. R. von, "Distribution of Mass in Spontaneous and Neutron-Induced Fission," Actinides Rev., 275-298 (1969). NSA 24: 08896 (1970). In author index, von Gunten, H. R., reads (Gunten, H. R. von ).

69GUN3 Gunnink, R., Niday, J. B., Anderson, R. P., Meyer, R. A., USAEC Report UCID-15439 (1969).

69HAR1 Harbour, R. M., and Troutner, D. E., "Existence of 1-Min. Nb-101 Not Conflrmed," J. Inorg. Nucl. Chem., 31, 2273-2275 (1969). NSA 23: 40056 (1969).

69HAR2 Harbour, R. M., "Nuclear Charge Distribution: Fractional Cumulative Yields of $99 \mathrm{Nb}$ from the Low-Energy Fission of 233U, 235U, 238U, and 252Cf. Mass Distribution: Cumulative Yields of Masses 95, 99, 103, 105, 131, 132, 133, 140 141, 143, 144, and 147 from 252Cf Fission," Ph. D. Thesis, Univ. of Missouri, Columbia, Mo. (1969). University Microfilms Order No. 69-16076. NSA 24: 18416 (1970). For publications see 69HAR1, 71HAR1 and 71HAR2.

69HAS1 Hastings, J. D., Troutner, D. E., and Ferguson, R. L.; "Fractional Cumulative Yields of Mo-103, Mo-105, and Mo-106 from Thermal - Neutron Induced Fission of U-235," Radiochimica Acta, 11, No. 1, 51-56 (1969). See preliminary data in ORNL-3994 pg. 11 (May 20, 1966). NSA 23: 31006 (1969). CSISRS-90389.

69HAW1 Hawkings, R. C., Edwards, W. J., and Olmstead, W. J., "Independent Yield of Mass-135 Xenons in the Thermal Neutron Fission of $233 U, 235 U, 239 P u$ and $241 P u$," Atomic Energy of Canada Limited PR-CMA-10 Sec. 2.4.2 Relative to I-135 yields. Superseded by ref. 71HAW1.

69ISA1 Isak, T., and Yellin, E., "Separation and Determination of the Independent Yield of 90Y in Thermal Fission of $233 \mathrm{U}$ and $235 \mathrm{U}$," Research Laboratories Report January December 1968, IA-1190, Israel Atomic Energy Commission, (July 1969), pp. 101-102.

69KAN1 Kandil, A. T., “Charged Particle Fission of U-238," Ph. D. Dissertation Florida State University (1969). Not coded. NSA 25: 10052 (1971). University Microfilms Order No. 70 11131 
69KR01 Krogulski, T., Chwaszczewska, J., Dakowski, M., Piasecki, E., Sowinski, M., Tys, J., "Emission of Light Nuclei in Thermal Neutron Fission of Pu230," Second IAEA Symposium on Physics and Chemistry of Fission, STI/PUB/234, Page 893 (1969). Published in Nuclear Physics A128, 219 (April 1969). EXFOR-30320.

69LAEI Laeter, J. R. de, and Thode, H. G., "Relative Yields of Stable Tin Isotopes in NeutronInduced Fission," Can. J. Phys., 47, No. 13, 1409-1414 (1969). NSA 23: 40077 (1969). In author index, de Laeter, J. R., reads (Laeter, J. R. de ). Superseded by 83ROS1 in which the same authors redetermine tin isotopes. CSISRS-90391.

69LAR1 Larsen, R. P., Private communication (Dec. 18, 1969). Thermal neutron U-235 fission yield for Tc-99. Also, EBR-1 fast reactor U-235 fission yields normalized to $6.20 \%$ for Cs-137. CSISRS90392. CSISRS-90392.

69LIS1 Lisman, F. L., Maeck, W. J., Rein, J. E., Foster, R. E., Jr., Abernathey, R. M., Delmore, J. E., Emel, W. A., Kussy, M. E., Mc Atee, R. E., Workman, G. D., "Burnup Determination of Nuclear Fuels Project Report for the Quarter April 1-June 30, 1968 and Final Report," IN-1277 (Mar. 1969).

69LYL1 Lyle, S. J., and Sellars, J., "Fine Structure in Nuclear Fission, Part I. Yields from 3 and $14.8 \mathrm{meV}$ Neutron Induced Fission of 238U and 232Th in the Mass Region 131-135," Radiochim. Acta, 12, No. 1, 43-48 (1969). NSA 23: 44930 (1969). EXFOR-22062 (Feb 7, 1989).

69MEA1 Meadows, J. W., "Correlation of Mass, Energy, and Angle in MeV-Neutron-Induced Fission of U-235 and U-238," Phys. Rev. 177(4), 1817-1825 (Jan 20 1969).

69M001 Moore, B. M., "Short-Lived Delayed Gamma-Ray Emission from Fast Fission of Plutonium," LA-4257 (Oct. 1969). NSA 24: 06886 (1970). Dissertation Abstracts B 31, 3444 (Dec 1970). CSISRS-90394.

69MUK1 Mukherji, S., "Nuclear Charge Distribution in Fission," Nuclear Physics A129 (1969) 297304. A new prescription for calculating $\mathrm{Zp}$ with a given fragment from U235T. FI of fragment against $\mathrm{N} / \mathrm{Z}$ yields a single parabola with peak at $\mathrm{N} / \mathrm{Z}=1.587+/-0.005$.

69MUN1 Munze, R., Grosse-Ruyken, H., and Wagner, G., "Studies on Ternary Fission of Uranium, Part 6: Identification of Fission Chain 166Dy - 166Ho - 166 Er and Determination of the Fission Yield," Kernenergie, 12, No. 12, 380-382 (1969). (In German). NSA 24: 26669 (1970). Replaces Kernenergie, 5, 472 (1962); and Kernenergie, 5, 564 (1962).

69NET1 Nethaway, D. R., Mendoza, B., and Voss, T. E., "Low-Yield Products From Fission of Th232, U235, and U238 with 14.8 meV Neutrons," Phys. Rev., 182, No. 4, 1251-1259 (20 June 1969). NSA 24: 06936 (1970). 166Dy U235HE has typographical error on exponent in Table II. Correct exponent is given in Table IV, (that is, 2.8e-6). CSISRS-90396.

69NOT1 Notea, A., "Distribution of Nuclear Charge in Low Energy Fission of Transthorium Elements," Phys. Rev., 182, 1331-1340 (20 June 1969). See also IA-1190, 93-98 (July 1969).

69NOT2 Notea, A., and Nir, D., "Mass Distribution in Low-Energy Fission by Gamma Analysis," Paper 122/36 Second IAEA Symposium on the Physics and Chemistry of Fission, Vienna 1969 STI/PUB-234 pg. 947. Abstract without data. 
69PAR1 Parsa, B., Gordon, G. E., and Wenzel, A., "Branching Ratio of 133Sb Decay to 133Te Isomers,” J. Inorg. Nucl. Chem., 31, 585-590 (1969). CSISRS-90397.

69PET1 Petrzhak, K. A., Teplykh, V. F., and Pan'yan, M. G., “Mass Spectrometric Determination of Relative Yields of Xenon Isotopes During Fission of Natural Uranium by Neutrons with Energy $14.7 \mathrm{meV}, "$ JPRS-54305, pp: 92-97, NSA 26: 14086 (1972). Translated from Byull. Tsentra. Yadern. Dannym. No. 6, 266-271 (1969).

69PIL1 Pillay, K. K. S., Meyer, R. J., and Larsen, R. P., "Determination of the Fast Fission Yield of Pr-141 Using Neutron Activation Analysis," J. Radioanal. Chem., 3, 233-243 (1969). Supersedes ANL-7375 pp. 178-179. NSA 22: 27477 (1968). CSISRS-90398.

69PRI1 Prince, A., "Nuclear and Physical Properties of Californium-252," BNL-50168(T-530), 91 pages (April 1969). Compiler.

69RAO1 Rao, S. A., Rao, M. N., and Kuroda, P. K., "Independent Yields of 124Sb, 126Sb, and 136Cs in 14.8-meV Neutron- Induced Fission of Thorium and Uranium," J. Inorg. Nucl. Chem., 31, 591-597 (1969). NSA 23: 17116 (1969). Replaces ORO-3235-12, page 20 (January 31, 1967). Isomeric independent yields are here split per 77MAD2. Cs136 independent yield seriously in error (see 75LAM1). CSISRS-90399.

69RAS1 Rasmussen, J. O., Norenberg, W., Mang, H. C., "Model for Calculating the Angular Momentum Distribution of Fission Products," Nucl. Phys. A136, 465-480 (1969).

69ROD1 Rodgers, R. C., "Correlation and Analysis of the Mass, Eriergy and Radiation Emission Characteristics of Fission Fragments Resulting from Thermal Neutron Capture by U-233, U-235 and Pu-239," Ph. D. Thesis Catholic University of America (1969). Theoretical. Dissertation Abstracts B 30, 2222 (Nov 1969).

69RUN1 Runnalls, N. G., Troutner, D. E., and Ferguson, $\dot{R}$. L., "Charge Distribution in Fission: Fractional Cumulative Fission Yields of Barium-143 and -144 from ThermalNeutron Induced Fission of Uranium-235," Phys. Rev., 179, 1188-1193 (March 20, 1969). CSISRS-90400.

69SAR1 Sarkar, R., "Predicted Mass Yields from the Fission of 238U Induced by Neutrons of Various Energies," Proceedings of the Nuclear Physics and Solid State Physics Symposium, Univ. of Roorkee, Dec. 28-31 (1969). Vol II Bombay Dept of Atomic Energy (1969). CONF691208 (Vol 2) pp. 226-230. NSA 25: 50788 (1971).

69SRI1 Srinivasan, B., Alexander, E. C., Jr., Manuel, O. K., and Troutner, D. E., "Xenon and Krypton from the Spontaneous Fission of Cf-252," Phys. Rev., 179, No. 4, 1166-1169 (20 March 1969). NSA 23: 31037 (1969). CSISRS-90401.

69STE1 Stella, R., Moretto, L. G., Maxia, V., Dicasa, M., Crespi, V., and Rollier, M. A., “Mass Distribution in the Fission of $237 \mathrm{~Np}$ with Epicadmium Neutrons," J. Inorg. Nucl. Chem., 31, 3739-3745 (1969). NSA 24: 15915 (1970). EXFOR-21412.

69STR1 Strutinsky, V. M., and Pauli, H. C., "Shell-Structure Effects in the Fissioning Nucleus, A Review," IAEA-SM-122/203, pages 155-181 CONF 690708. 
69THI1 Thind, K. S., Tracy, B. L., Thode, H. G., Tomlinson, R. H., "The Effects of Nuclear Excitation of Prompt Fragments on the Independent Yields and Neutron Yields in Fission," Paper SM-122/50 Second IAEA Symposium on the Physics and Chemistry of Fission, Vienna, 1969, STI/PUB-234 pp. 845-860 (December 1969). Compilation. NSA 24: 30910 (1970).

69THI2 Thind, K. S., and Tomlinson, R. H., "Fine Structure in the Region of Symmetric Mass Distribution for Cumulative Fission Yields," Can. J. Phys., 47, 275-278 (1969). Theoretical predictions, no data given. NSA 23: 13303 (1969).

69TON1 Tong, S. L., and Fritze, K., "The Yield of 72Zn in the Epi-Sm Fission of 239Pu," Radiochim. Acta, 12, No. 4, 179-182 (1969). NSA 24: 20389 (1970). CSISRS-90403.

69VOR1 Vorobiev, A. A., Seleverstov, D. M., Grachov, V. T., Kondurov, I. A., Nikitin, A. M., Yegorov, A. I., and Zalite, Yu. K., "Light Nuclei from 233U Neutron Fission," Phys. Letters, 30B, No. 5, 332-333 (27 October, 1969). EXFOR-40503.

69VOR2 Vorobiev, A. A., Grachov, V. T., Komar, A. P., Kondurov, I. A., Nikitin, A. M., Seleverstov, D. M., "Light Nucleic Yield at $235 \mathrm{U}$ Fission Induced by Thermal Neutrons," At. Energ. (USSR) 27: 31-36 (July 1969). In Russian. NSA 23: 49410 (1969). 2H, 3H, 6He Isotopes per 100 Alpha Particles are 0.44 +/-0.04,6.3 +/- 0.2, and 1.4 +/-0.1, respectively. EXFOR-40243.

69VOR3 Vorob'jova, V. G., Gentosh, A. I., Kuz'minov, B. D., Sergachjov, A. I., Yadern. Fiz., 10(3), 491 (Sept 1969). EXFOR-40240.

69WAH1 Wahl, A. C., Norris, A. E., Rouse, R. A., and Williams, J. C., "Products from ThermalNeutron Induced Fission of 235U - A Correlation of Radiochemical Charge and Mass Distribution Data," Paper SM-122/116 presented at the IAEA Second Symposium on the Physics and Chemistry of Fission, Vienna (July 1969). COO-1162-35 STI/PUB/234 CONF-690708. p. 813.

69WAS1 Wasserburg, G. J., Huneke, J. G., Burnett, D. S., "Correlation Between Fission Tracks and Fission-Type Xenon From an Extinct Radioactivity," Phys. Rev. Lett., 22, 1198 (1969); J. Geophys. Res. 74, 4221 (1969). Meteoritic fission yields attributed to Pu-244 by 71ALE1. CSISRS-90404.

69WAT1 Watson, R. L., and Wilhelmy, J. B., "Calculated Fractional Independent Yields of Products Formed in the Spontaneous Fission of 252Cf," UCRL-18632, (February 1969). 40 pages, No experimental data, not coded. NSA 23: 23677 (1969).

69WEI1 Weiss, H. V., Ballou, N. E., Elzie, J. L., and Fresco, J. M., "Nuclear Charge Distribution in Symmetric Fission of $235 \mathrm{U}$ with Thermal Neutrons: Yields of $117 \mathrm{Ag}, 118 \mathrm{Ag}$ and 118Pd," Phys. Rev., 188, No. 4, 1893-1896 (20 Dec. 1969). CSISRS-90405.

69WEI2 Weiss, H. V., Ballou, N. E., Elzie, J. L., Fresco, J. M., "Radiochemical Studies in the Symmetric Region of 235U Fission," Paper SM-122/115 Second IAEA Symposium on the Physics and Chemistry of Fission, Vienna, (1969). Same data reported in 69WEI1.

69WEI3 Weinlaender, W., "Determination of the Neutron Fission Mass-Yield-Curves for 241 and 242m Americium," Paper SM-122/72 Second IAEA Symposium on the Physics and Chemistry of Fission, Vienna 1969 STI/PUB-234 Page 951. Abstract without data. 
69YEL1 Yellin, E., Ehrenberg, B., and Amiel, S., "Determination of Isomer Ratios," IA-1190, 99-100 (July 1969). Xe-135m -to- Xe-135 independent yield ratio for U235 thermal neutron induced fission. Independent yield ratio of $\mathrm{Xe} 135 \mathrm{~m} / \mathrm{Xe} 135=1.7 \pm 0.2$ so independent yield of $\mathrm{Xe135m}$ is $0.2427 \times 1.7 /(1.7+1.0)=0.1528$; $\mathrm{Xe} 135$ is $0.0899 \pm 0.0109$; And to confirm the arithmetic, $0.1528 / 0.0899=1.7$

70ALB1 Albinsson, H., and Lindow, L., "Prompt Gamma Radiation from Fragments in the Thermal Fission of U-235," AE-398 (1970).

70ALE1 Alexander, P., "Computer Analysis of Fission Data," Nucl. Instrum. Methods, 86, 99-107 (1970). NSA 24: 22149 (1970). Theoretical independent yields of Xe-133 and Xe-135. NSA 25: 05790 (1971).

70AMI1 Amiel, S., Feldstein, H., "A Semi-Empirical Treatment of Neutron Emission Probabilities from Delayed Neutron Precursors," Phys. Letters 318(2), 59-60 (Jan 19, 1970). This treatment is $\mathrm{Pn}=\mathrm{a}(\mathrm{QB}-\mathrm{BN})^{* *} \mathrm{~b}$.

70ARM1 Armani, R. J., Gold, R., Larsen, R. P., and Roberts, J. H., "Measured Fission Yields of 99Mo and $140 \mathrm{Ba}$ in Fast-Neutron-Induced Fission of 239Pu," ANS Transactions 13, No. 1, 90-91 (1970). See also ANL-7742, p. 5. CSISRS-90407.

70ART1 Artem'ev, Yu. M., Gromov, A. V., and Protopopov, A. N., "Mass Yields in the Fission of U235 by Thermal and Fast Neutrons at Fixed Kinetic Energies," Sov. J. Nucl. Phys., 11, No. 2, 162-5 (1970). NSA 24: 33149 (1970).

70BAC1 Bachmann, K., "Rare Earth Yields in the Interaction of 28-geV Protons with Uranium, Bismuth and Gold," J. Inorg. Nucl. Chem., 32, 1-15 (1970).

70BAL1 Balestrini, S. J., Forman, L., Wolfsberg, K., Jeter, T. R., “On-Line Technique for Measuring Independent Fission Yields," Proc. Int. Conf. Electromagn. Isotope Separators Tech. Appl., 7th (1973) BMBW FB70-28 pages 68-77 inclusive. CA 76: 09551g (1972). See 70FOR1 for identical data.

70BAR1 Barre, B., and Tourreil, R. de, "Concentration des Produits de Fission Apres une Fission Thermique de $235 \mathrm{U}$ et $239 \mathrm{Pu}$, et Apres une Fission Rapide (E ca $1 \mathrm{meV}$ ) de 235 U, 238U et 239Pu," CEA-N-1309 (Juin 1970). In French. Calculated fission product inventories, no experimental yields given. In author index, de Tourreil, R., reads (Tourreil, R. de ).

70BOW1 Bowles, B. J., and Willis, H. H., “The Absolute Yields of Selected Fission Isotopes from Fission of 235U in DFR," AERE R6393, (June 1, 1970).

70CAR1 Carraz, L. C., Compt. Rend. B 270, 358 (1970). Cited in 80MEI1.

70CHA1 Chaumont, J., “Fission Investigation Using On-Line Mass Spectrometry," NP-18829, 97 pages (1970). Thesis, Paris Univ., Orsay, France. Cross sections for production of $\mathrm{Cs}$ and $\mathrm{Rb}$ isotopes in fission of $\mathrm{U} 235$ by $156 \mathrm{meV}$ and $26 \mathrm{geV}$ protons. Not coded. See NSA 25: 36730 (1971). 
70CHO1 Choppin, G. R., and Tofe, A. J., "Charged Particle Fission of 232Th," J. Inorg. Nucl. Chem., 33, 1535-1541 (1971). Mass distribution in fission by $11.3 \mathrm{meV}$ protons and $11.5 \mathrm{meV}$ deuterons. Not coded.

70COW1 Cowan, G. A., Bayhurst, B. P., Prestwood, R. J., Gilmore, J. S., and Knobeloch, G. W., "Symmetry of Neutron-Induced $235 \mathrm{U}$ Fission at Individual Resonances. III," Phys. Rev. C, 2, 615-620 (August 1970). Radiochemical $115 \mathrm{Cd}$ to $99 \mathrm{Mo}$ at 38 resolved resonances show bimodal distribution; 24 group I avg 0.593 times thermal value and 14 group II avg 1.11 times thermal value. Not coded.

70COW2 Cowan, G. A., Bayhurst, B. P., Prestwood, R. J., Gilmore, J. S., Knobeloch, G. W., ANS Symposium on Engineering with Nuclear Explosives cited by 77CRO1. Published as 70COW1. CSISRS-90410.

70CRO1 Crouch, E. A. C., "A Library of Neutron Induced Fission Product Yields Maintained and Interrogated by Computer Methods," AERE-R-6642 (1970). Compiler. NSA 25: 23016 (1971).

70DEL1 Delucchi, A. A., and Greendale, A. E., "Fission Yields of Several Iodine Isotopes and Half-Life and Fission Yield of 135Te," Phys. Rev. C, 1, No. 4, 1491-1497 (April 1970). Isomeric independent yields are split per 76MAD2. CSISRS-90411.

70DEN1 Denschlag, H. O., and Qaim, S. M., "Unabhangige A usbeuten der Jodisotopen 133, 134, und 135 in der Spaltung von Thorium-232 mit Epi-Cd-Reaktor-Neutronen und mit 14-meV-Neutronen," Annual Report, 1969. Mainz Univ. West Germany. BMBW-FBK-7019, pp. 100-102 (Oct. 1970). Superseded by 71DEN2.

70DER1 Derengowski, M., and Melkonian, E., "Double-Energy Single-Time-of-Flight Measurements of Fission Fragments in Thermal-Neutron-Induced Fission of U235," Phys. Rev. 2, No. 4, C1554-C1569 (October 1970). Pre- and post-neutron mass yield graphically shown, no data given, not coded.

70EBE1 Ebersole, E. R., Laug, M. T., and Villarreal, R., "Burnup Measurements at EBR-II," USAEC Report, LA-4430-MS, pp. 140-144 (May 1970). CSISRS-90412. Superseded by 72EBE1.

70EIC1 Eichor, M., Troutner, D. E., Paper at 160th National Meeting of American Chemical Society Nuclear Section 74, Chicago, Illinois, Sept. 1970. CSISRS-90413.

70FAH1 Fahland, J., Lange, G., and Herrmann, G., "Independent Yields of 124Sb, 126Sb, and $160 \mathrm{~Tb}$ in Fission of $235 \mathrm{U}$ by Thermal Neutrons," (In German). J. Inorg. Nucl. Chem. 32, 3149-3157 (1970). NSA 25: 15038 (1971). EXFOR-22060 (Feb 7, 1989).

70FLY1 Flynn, K. F., and Glendenin, L. E., "Yields of Fission Products for Several Fissionable Nuclides at Various Incident Neutron Energies," ANL-7749 (Restatement of selected evaluators averages. No original data given. 1970). NSA 25: 23017 (1971).

70FOR1 Forman, L., Balestrini, S. J., Wolfsberg, K., and Jeter, T. R., "On-Line Investigation of Independent Cs Yields in 236U Fission: Identification of 145Cs," LA-DC-11500, 13 pages (October 31, 1970). CERN-70-30 Vol I pp. 589-599. NSA 25: 28307 (1971). International Conference on the Properties of Nuclei Far From Beta Stability, Leysin, Switzerland (31 August - 4 September 1970). CSISRS-90415. 
70FOR2 Ford, G. P., Wolfsberg, K., Unpublished preliminary results (Dec. 1970). Cited in 74 WOL1. Superseded by 84 FOR1. CSISRS-90416.

70GAZ1 Gazit, Y., Nardi, E., and Katcoff, S., "Emission of Particles of 3. LE. Z. LE. 8 in The Fission of Cf252," Phys. Rev. C, 1, 2101-2108 (June 1970). Yields per 1000 fission alpha

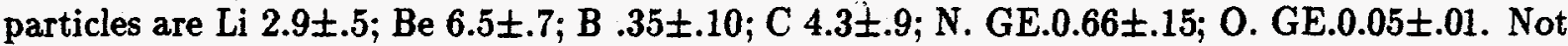
coded.

70GEV1 Gevaert, L. H., Jervis, R. E., and Sharma, H. D., "Cumulative Yields in the $14 \mathrm{meV}$ Neutron fission of $232 \mathrm{Th}$ and $238 \mathrm{U}$ in the Symmetric Region," Can. J. Chem, 48, 641-651, (1970). Dissertation Abstracts B 32, 3838 (Jan 1972). CSISRS-90417.

70GOE1 Goennenwein, F., und Pfeiffer, E., "Spaltung von Thorium und Uran mit $15 \mathrm{meV}$ Neutronen," Annual Report 1967/68 Physikalisches Institut der Universitat Tuebingen (West Germany) BMBW-FBK-70-14, pp. 35-48 (August 1970). U238(n, f), Th232(n, f). See 70PFE1.

70HAG1 Hagebo, E., "Some Yields and Isomeric Yield Ratios of Indium Isotopes Produced by High Energy Fission," J. Inorg. Nucl. Chem., 32, 2489-2500 (1970). Values reported in mbarns. NSA 25: 05784 (1971).

70HAR1 Harbour, R. M., Troutner, D. E., and Macmurdo, K. W., Paper at 161st ACS National Meeting, Los Angeles (1970). Cited in 72HAR2. CSISRS-90419.

70HAS1 Hasegawa, A., Tone, T., Katsuragi, S., “238Pu Fission Product Yields and Their Influence on Fast Reactor Characteristics," JAERI-MEMO-4165 (October 1970). In Japanese. Reviewer, Compiler, not coded. NSA 25: 20819 (1971).

70HEN1 Hensley, W. K., and Neuzil, E. F., "Helium Ion-induced Fission of 204Pb at 65, 41, 37, and 25 meV," J. Inorg. Nucl. Chem., 32, 1761-1766 (1970). NSA 24: 38256 (1970). CSISRS-90420.

70HOF1 Hofmann, P., “Fission Product Yield from the Fission of U-235, U-238, Pu-239 and Pu-241 with Neutrons of Different Energies," (In German) NP-18723 (November 12, 1970). Compiler. NSA 25: 28354 (1971). Formerly Kernforschungszentrum Karlsruhe-EXT6/70-2 (1970).

70HOR1 Horrocks, D. L., and White, E. B., "Tritium Yield in the Thermal Neutron Fission of 233U," Nuclear Physics, A151, 65-70 (1970).

70HUD1 Hudis, J., Kirsten, T., Stoenner, R. W., and Schaeffer, O. A., "Yields of Stable and Radioactive Rare-Gas Isotopes Formed by 3- and $29 \mathrm{geV}$ Proton Bombardment of $\mathrm{Cu}$, Hg, Au and U," Phys. Rev. C, 1, No. 6, 2019-29 (1970).

70IZA1 Izak, T., Thesis, The Hebrew University of Jerusalem (In Hebrew) 1970. Cited in 72IZA1.

70JAI1 Jain, H. C., Rengan, K., Ramaniah, M. V., B. A. R. C./I-62 (1973).

70KEM1 Kemmer, J., Kim, J. I., and Born H. J., "Study on the Reactor Neutron-Induced Fission of 231Pa," Radiochim. Acta, 13, No. 4, 181-187 (1970). NSA 25: 03656 (1971). 
70KHA1 Khan, A. H., Saha, G. B., and Yaffe, L., "Nuclear Charge Dispersion of Light-Mass Fission Products in the Fission of $235 \mathrm{U}$ and $238 \mathrm{U}$ by Medium Energy Protons," Can. J. Chem, 48, 1924-1933 (1970). NSA 24: 38254 (1970),

70KOB1 Kobayashi, K., Kimura, I., "Measurement of Average Cross Section for the 232Th (n, f) Reaction for the Fission-Type Reactor Spectrum," Annu. Rep. Res. Reactor Inst. Kyoto Univ. 3: 84-92 (1970). NSA 25: 28409 (1971). Relative fission yields for 5 nuclides $140 \mathrm{Ba}, 135 \mathrm{Xe}, 132 \mathrm{Cs}, 95 \mathrm{Zr}, 91 \mathrm{Sr}$. Mass 132 yield is here assumed to be for the chain yield because $\mathrm{Cs}$ is shielded and has an independent and cumulative yield of less than (9e-12)\%. EXFOR-20272.

70KON1 Konecny, E., Gunther, H., Rosler, H., Siegert, G., and Ewald, H., "Nuclear Charge Distribution of Heavy Fission Fragments from Thermal-Neutron-Induced Fission of 235U," Z. Physik, 231, 59-97 (1970).

70KRA1 Kratz, J. V., and Herrmann, G., "Half-Lives, Fission Yields and Neutron Emission Probabilities of $87 \mathrm{Se}$ and $88 \mathrm{Se}$, and Evidence for $87 \mathrm{As}$," J. Inorg. Nucl. Chem., 32, 3713-3723 (1970). NSA 25: 20327 (1971). EXFOR-22047 (Nov 18,1988).

70KRA2 Kratz, J. V., "Halbwertszeiten, Spaltausbeuten und Neutronemissionswahrscheinlichkeiten von Selen-87 und Selen-88," Annual Report, 1969. Mainz Univ. West Germany. BMBW-FBK-70-19, pp. 18-21 (Oct. 1970). See 70KRA1.

70KUZ1 Kuz'minov, B. D., Sergachev, A. I., Smirenkina, L. D., "Mass and Energy Distribution of Fragments at the Fission of Np237 by Neutrons," Yadern. Fiz., 11, No. 2, 297-303 (February 1970). EXFOR-40172.

70LIN1 Lin, C., and Wahl, A. C., "132Sn Half-Life and Fractional Cumulative Yield from Thermal Neutron Induced Fission of 235U," J. Inorg. Nucl. Chem., 32, 2501-2507 (1970). NSA 24: 49906 (1970). CSISRS-90427.

70LIS1 Lisman, F. L., Abernathey, R. M., Maeck, W. J., and Rein, J. E., "Fission Yields of Over 40 Stable and Long-Lived Fission Products for Thermal Neutron Fissioned 233U, 235U, 239Pu and 241Pu and Fast Reactor Fissioned 235U and 239Pu," Nucl. Sci. and Engr., 42, 191-214 (1970). This publication supersedes USAEC doc. IDO-14660, IDO-14676, IDO-14667, IN-1064, IN-1113, IN-1157, IN-1189, IN-1178, IN-1203, IN-1207, and IN-1215. NSA 25: 05765 (1971). U233 $\mathrm{Sm}$ adjusted by 73WAL1. U235F 85Rb yield corrected from 1.49 to 1.38 upon recalculation of $\mathrm{Kr}-85$ decay correction by authors, U235F $150 \mathrm{Nd}$ reduced $8.5 \%$ from 0.832 to 0.767 upon reanalysis by authors. PU239F estimates inferred by B. F. Rider to equal the group estimates for masses (119-124), (126-130), (155-160) by Lisman in heavy peak and to total $100 \%$ in light peak when combined with Lisman measurements. 70LIS1 errs in PU239T and U235T Xe-131, 132, 134, Ba-138, Nd-148 (75MAE1, 76MAE1). CSISRS-90428.

70LUM1 Lum-Hee, G., and Tomlinson, R. H., "Cumulative Fission Yields of Cadmium Isotopes," AECL-3776, 96-97 (September 30, 1970). Originally PR-CMA-14. Data from graph. NSA 25: 10583 (1971). See also INDC(CA)9, page 6 (1971). CSISRS-90429.

70LYL1 Lyle, S. J., and Wellum, R., "Some Cumulative Mass-Yields from the Fission of 238U by 3 meV Neutrons," Radiochim. Acta, 13, No. 3, 167-168 (1970). Sb-129 values are low by loss of $\mathrm{Sb}$ on evaporation with $\mathrm{HCl}$, corrected in 71BIR1. 
70MAN1 Manuel, O. K., Becker, V. J., Boulos, M. S., Srinivasan, B., "Radiochemical Determination of Heavy Elements and Contribution to Noble Gas Anomalies," Progress Report to the National Science Foundation, 15 June 1970, p. 12. Cited by 71ALE1, Cm244 relative yields read from graph. CSISRS-90431.

70MAR1 Marmol, P. del, Perricos, D. C., "Identification of 88Se and Search For Delayed Neutron Emission from 87Se and 88Se," J. Inorg. Nucl. Chem., 32, 705-712 (1970). NSA 24: 28758 (1970). In index, del Marmol, P., reads (Marmol, P. del ).

70MAS1 Mason, J. F., and Johns, M. W., “Decay of Kr-80 and Rb-80," Can. J. Phys., 48, 2056-2082 (1970). NSA 25: 20384 (1971).

70MCE1 Mc Elroy, W. N., Kellogg, L. S., Armani, R. J., Tochlin, E., and Zimmer, W. H., "Selection of Fission Yields," Trans. Amer. Nucl. Soc., 13, No. 2, 868-869, November 15-19, 1970. NSA 25: 05973 (1971). CSISRS-90433.

70NET1 Nethaway, D. R., and Mendoza, B., "Fission of U233 with 14.8-meV Neutrons," Phys. Rev. C, 2, No. 6, 2289-2296 (Dec. 1970). NSA 25: 07870 (1971). Data for U233 superseded by 72NET2.

70NIE1 Niece, L. H., Troutner, D. E., and Ferguson, R. L., "Independent Yields of 95Zr from Thermal-Neutron Fission of $235 U$ and 233U," Phys. Rev. C, 1, No. 1, 312-315 (January 1970). NSA 24: 24582 (1970). CSISRS-90434. A footnote to the introduction to the submitted manuscript (65NIE1) stated "The FRACTION of each isobar formed at fission (after emission but before beta decay) is called the independent yield . . ." This unusual redefinition of a good term was deleted by the journal editor without changing the text to the accepted nomenclature which is "Fractional Independent Yield." The author indeed did measure FI and labels them IN to the confusion of all readers. All A. C. Wahl and B. F.Rider publications correctly label these measurements as FI and have recommended CSISRS-90434 make a note of these as FI commenting that the author defines independent yield as FRACTIONAL for his purposes.

700HY1 Ohyoshi, E., Ohyoshi, A., and Shinagana, M., "Radiochemical Determination of Several Nuclides for 235U Fission," Radiochem. Radioanal. Ltrs., 3, 1-5 (1970). NSA 24: 16449 (1970).

700KA1 Okazaki, A., and Sokolowski, E. K., "Redetermination of the Thermal-Neutron Absorption Cross-Sections of Gross Fission Products of $233 \mathrm{U}, 23 \mathrm{U}$, and 239Pu," 2nd Int. Conf. on Nucl. Data for Reactors, Helsinki, 1970, Conf. 700605, Vol. 1, 703-719, STI/PUB-259 (Vol. 1). NSA 25: 15100 (1970).

70PAN1 Panontin, J. A., and Porile, N. T., "Charge Distribution and Recoil Properties in the Fission of $238 \mathrm{U}$ by $11.5 \mathrm{geV}$ Protons," J. Inorg. Nucl. Chem., 32, 1775-1790 (1970). Values reported are reaction cross-sections (mbarns). NSA 24: 47651 (1970). CSISRS-90436.

70PAN2 Panontin, J. A., and Sugarman, N., "Mass Yield and Charge Distribution in 450 meV Proton Fission of U-238," COO-1167-10 (1970). Not coded.

70PAN3 Panontin, J. A., and Sugarman, N., "High Energy Fission of Ta-181 and Bi-209 by 450 meV Protons," COO-1167-16 (1970). 
70PET1 Petrzhak, K. A., Teplykh, V. F., and Pan'yan, M. G., “Mass Spectrometric Determination of Xe Isotope Relative Yields from Natural Uranium Fission by $14.7 \mathrm{meV}$ Neutrons," Yadern. Fiz. 11, No. 6, 1178-1181 (June 1970). (U238 fission) See Sov. J. Nucl. Phys., 11, 654 (1970) for English translation.

70PFE1 Pfeiffer, E., "Thorium Fission by 4.8 and $14 \mathrm{meV}$ Neutrons," Z. Phys., 240: 403-419 (1970). NSA 25:17901 (1971). KE of fragments on surface barrier det. with distribution of mass given graphically. Not coded. NSA 25: 17901 (1971).

70QAI1 Qaim, S. M., and Denschlag, H. O., "Independent Yields of 133I, 134I, and 135I in the Thermal Neutron Induced Fission of 233U and 239Pu: Effect of the 82-Neutron Shell Closure on Charge Distribution," J. Inorg. Nucl. Chem., 32, 1767-1773 (1970). NSA 24: 47652 (1970). Supersedes 69DEN2. EXFOR-22067 (Feb 7, 1989). EXFOR-22067.

70RAB1 Rabotnov, N. S., Smirenkin, G. N., Soldatov, A. S., Usachev, L. N., Kapitsa, S. P., and Tsipeniuk, Iu. M., "Photofission of Thorium-232, Uranium-238, Plutonium-238, Plutonium240, and Plutonium-242 and Structure of the Fission Barrier," USAEC document LA-4385-TR Translated at Los Alamos by Helen J. Dahlby from the original Russian Institute of Physics and Energetics Report FEI-170 (1969). NSA 24: 38288 (1970).

70RAM1 Ramaniah, M. V., Jain, H. C., Mathew, K. A., and Avadhany, G. V. N., "99Mo Fission Yield in the Thermal Neutron Fission of 239Pu and 235U," Madras Symposium (1970). CONF-701148 (Vol 2) pp. 71-78. NSA 26: 32410 (1972). Superseded by 71JAI1, NSA 26: 49446 (1972).

70RID1 Rider, B. F., "Determination of Fission Yields for Cs-137 and Six Neodymium Nuclides from U-235 in the EBR-II Fast - Neutron Reactor Spectrum," Section 7.12 of Sodium Cooled Reactors: Fast Ceramic Reactor Development Program-35th Quarterly Report (May-July 1970), GEAP-10028-35, pp. 78-81 (1970). CSISRS-90439.

70ROW1 Rowe, M. W., Geochim. Cosmochim. Acta, 34, 1019 (1970). Meteoritic fission yields attributed by 71ALE1 to Pu-244. CSISRS-90440.

70RUN1 Runnalls, N. G., and Troutner, D. E., "Nuclear Charge Distribution in Fission: Independent Yields of 132I, 133I, and 134I from Thermal Neutron Fission of 233U," Phys. Rev. C, 1(1), 316-322 (Jan 1970). NSA 24:24581 (1970). CSISRS-90441.

70SAK1 Sakata, H., Nagayama, S., Otake, I., "Study for Decay Chain of Fission Products," JAERI-1194 (September 1970). Study by Reactor Constant Subcommittee, Japanese Nuclear Data Committee. Compilation of fission product yields, decay constants, sigmas, and resonance integrals. Not coded.

70SK01 Skovorodkin, N. V., Sorokina, A. V., Bugorkov, S. S., Krivokhatskii, A. S., and Petrzhak, K. A., "Radiochemical Determination of Yields of Rare Earth Elements During Fission of 239Pu and 241Pu by Slow Neutrons. II. Yields of Isotopes of Rare Earth Elements with Half-Lives of More than Ten Days," Radiokhimiya 12, 492-496 (1970). NSA 25:15689- 15690 (1971). In Russian; Sov. Radiochem., 12, 463-467 (1970) is the English translation. Updated by 71SOR1. 
70SKO2 Skovorodkin, N. V., Sorokina, A. V., Bugorkov, S. S., Krivokhatskii, A. S., and Petrzhak, K. A., "Radiochemical Determination of Yields of Rare Earth Elements During Fission of 239Pu and 241Pu by Slow Neutrons. I. Yields of Isotopes of Rare Earth Elements with Half-Lives of Less than Ten Days," Radiokhimiya 12, 487-492 (1970). NSA 25: 15690 (1971). In Russian; Sov. Radiochem. 12, 458-463 (1970), English translation.

70TAL1 Talat-Erben, M., and Dagsoz, B., "Discontinuity in Isotopic Mass Dispersion in ThermalNeutron-Induced Fission of 235U," Phys. Rev. C, 2, 2403-2405 (December 1970). Theoretical no experimental yields given.

70TRA1 Tracy, B. L., and Thode, H. G., "Independent Yields of 80Br, 82Br, $128 \mathrm{I}$ and $130 \mathrm{I}$ from the Thermal Neutron Fission of $235 \mathrm{U}, 239 \mathrm{Pu}, 233 \mathrm{U}$, and 238Np," Can. J. Phys., 48, No. 14, 1708-1715 (1970). Isomeric independent yields are here split per 76MAD2. CSISRS-90443.

70TRO1 Troutner, D. E., Eichor, M., and Pace, C. H., "Nuclear Charge Distribution in Fission: Independent Yields of Ag-112, I-134 and Cs-138 from Spontaneous Fission of Cf252," Phys. Rev. C, 1, No. 3, 1044-1052 (1970). Replaces ORNL-4306, Page 34 (1968). CSISRS-90444.

70TRO2 Troutner, D. E., Eichor, M., Harbour, R. M., Pace, C. H., and Runnalls, N. G., "Fractional Independent and Cumulative Yields of Some Products of Spontaneous Fission of 252-Californium," Paper 23, Nuclear Section, 159th National Meeting American Chemical Society, Houston, Texas, (Feb. 22-27, 1970). Supersedes ORNL-4306-34 page 5 (1968). CSISRS90445. A few of these cited values have been updated by Troutner by private communication to B. F. Rider (1970).

70VAR1 Varteressian, K. A., and Burris, L., "Fission-Product Spectra from Fast and Thermal Fission of U-235 and Pu-238," ANL-7678 (1970). Compiler/theoretical.

70WAL1 Walker, W. H., "The Evaluation of Fission Product Yields," AECL-3610 (1970). Nuclear Data for Reactors Vol I, Vienna, IAEA (1970). CONF-700605(Vol I). NSA 25: 15099 (1971). Superseded by 73WAL1.

70WAT1 Watson, R. L., Wilhelmy, J. B., Jared, R. C., Rugge, C., Bowman, H. R., Thompson, S. G., and Rasmussen, J. O., "A Study of the Low-energy Transitions Arising from the Prompt De-Excitation of Fission Fragments," Nuc. Phys., A141, 149-480 (1970). Graphical not coded.

70WEI1 Weinreich, R., Kiefer, W., Herrmann, G., German Chemical Society Meeting on General Radiochemistry, Nuclear-, Radio and Radiation Chemistry Group, Karlsruhe, May 4-6, 1970. Isomeric independent yields are here split per 77MAD2.

70WOL1 Wolfsberg, K., Ford, G. P., Unpublished Cf254S results 1970, Privately communicated to B. F. Rider. CF254S data later released as 74WOL3.

70ZAK1 Zakharova, V. P., Korostylev, V. A., and Ryazanov, D. K., “Investigation of Cf-252 Spontaneous Fission," IAE-2036 10 p. in Russian (1970). Kinetic energy distribution (not coded). 
71AKI1 Akimov, N. I., Vorob'eva, V. G., Kabenin, V. N., Kolosov, N. P., Kuz'minov, B. D., Sergachev, A. I., Smirenkina, L. D., Tarasko, M. Z., "Effect of Excitation Energy on Yields and Kinetic Energies of Fragments at the Fission of Pu-239 by Neutrons," Yadern. Fiz. 13(3), 484 (1971). EXFOR-40144.

71ALE1 Alexander, E. C., Jr., Lewis, R. S., and Reynolds; J., "Plutonium-244: Confirmation as an Extinct Radioactivity," Science, 172, 837-840 (21 May 1971). CSISRS-90447.

71ART1 Artyukh, A. G., Avdeichikov, V. V., Vilchinski, Ya., “Nuclei of Light Elements with Larger Neutron Excess," Isz. Akad. Nauk. SSSR Ser. Fiz. 35: No. 1, 2-17 (Jan. 1971). In Russian. NSA 25: 56788 (1971). Elements $\mathrm{O}, \mathrm{N}, \mathrm{C}, \mathrm{B}$ from irradiating Th-232 with N-15, Ne-22, 0-18. Not coded.

71BAB1 Baba, S., Umezawa, H., Baba, H., "Mass Distribution and the Total Cross Section in the Fission of $238 \mathrm{U}$ with Protons of Energies Ranging Between 13 and $55 \mathrm{meV}$," Nuclear Physics A175, 177-198 (1971). Proton-induced fission not coded.

71BER1 Berge, L. A., Private communication of preliminary results to 71TRO3. See 72BER1 for final results. CSISRS-90448.

71BIR1 Birgul, O., and Lyle, S. J., "The Radiochemical Determination of Cumulative Yields at Mass 129 in Nuclear Fission," Radiochim. Acta, 16, No. 2, 103-104 (1971). NSA 26: 19385 (1972). CA 76: 93278d (1972).

71BIR2 Birgul, 0., Lyle, S. J., and Wellum, R., "Some Cumulative Mass-Yields and Prompt Neutron Yields for Selected Masses from $14.8 \mathrm{meV}$ Neutron Fission of 238U," Radiochim. Acta, 16, No. 2, 104-106 (1971). NSA 26: 24324 (1972). EXFOR-22039.

71BLA1 Blachot, J., Cavallini, P., Chauvin, C., "Mass Yields in the $14 \mathrm{meV}$ Fission of 233U, 235U, 238U, 232Th," Page 13, U. of Kent, Canterbury, UK Conference, Chemical Nuclear Data: Measurements and Applications, (September 20-22 1971), British Nuclear Energy Society, 1-7 Great George Street, London SWI. Results updated by author on April 5, 1972 are still preliminary pending completion of experiments. NSA 26: 32413 (1972). See 74BLA1 for later values on $\mathrm{U} 235 \mathrm{~T}$ and $\mathrm{U} 238 \mathrm{HE}$.

71BLA2 Blachot, J., Carraz, L. C., Cavallini, P., Monnand, E., Schussler, F., "Direct Gamma Spectroscopy Analysis of Fission Products from U-235; Application to the Mass Distribution of Fission Products from Fission of Th-232 by $14 \mathrm{meV}$ Neutrons," J. of Radioanal. Chem. 7, 309 (1971). Abstracted in NEANDC(E)-1614 (1976). EXFOR-21561.

71BOC1 Bocquet, J. P., "Distributions de Produits de Fission Etudiees par Separation Isotopique en Ligne," Thesis, Sc. Phys., Grenoble, France 1971. Report No. FRNC-TH-236, (In French) 164 pp. Later published as 72BOC1. NSA 27: 23597 (1973).

71BRI1 Brissot, R., "Measurement of Fission Yields Using On-Line Isotope Separation," 64 pages. Thesis Grenoble Univ., Grenoble France, FRNC-TH-92, in French. Cumulative rare gas yields in $14 \mathrm{meV}$ fission of Th232, U235, U238 for Xe-137, 138, 139, 140 and $\mathrm{Kr}-87,88,89,90$, 91. NSA 25: 26551 (1971). Cited by 71BLA1. See also NSA 26: 03578 (1972). CA 76: 147868s (1972). 
71CHE1 Cheifetz, E., Wilhelmy, J. B., Jared, R. C., Thompson, S. G., "Determination of the Charge and Mass Distribution in the Fission of 252Cf," Phys. Rev. C 4, 1913-1926 (1971). CSISRS-90454. It is assumed by the author that all disintegrations go through the $2+$ to $0+$ transformation he measured. His arguments that no more than 2-7\% of the events bypass his measurements seems persuasive so that his gamma intensity measurements are no than $2-7 \%$ less than the independent yields which are within his experimental errors.

71CLI1 Cline, J. E., "Gamma Rays Emitted by the Fissionable Nuclides and Associated Isotopes," IN-1448 rev. (1971).

71COM1 Communeau, F., Commissariat a l'Energie Atomique, Paris, Unpublished French work cited by 71BLA1.

71CON1 Conde, H., Holmberg, M., "Prompt Nubar in Spontaneous and Neutron Induced Fission of 236U and Its Half-Life for Spontaneous Fission," J. Nucl. Energy 25(8) 331-338 (Aug 1971).

71CR01 Crouch, E. A. C., "The Assessment of Fission Product Yields," Paper 1, U. of Kent, Canterbury, UK conference, Chemical Nuclear Data: Measurements and Applications, (September 20-22 1971), British Nuclear Energy Society, 1-7 Great George Street, London SWI. NSA 26: 32412 (1972).

71CRO2 Crouch, E. A. C., "The Determination of the Capture to Fission Ratio and the Fission Product Yields of Fissile Materials Irradiated in the Dounreay Fast Reactor," Paper 18, U. of Kent, Canterbury, UK Conference, Chemical Nuclear Data: Measurements and Applications, (September 20-22, 1971), British Nuclear Energy Society, 1-7 Great George Street, London SWI, NSA 26: 32418 (1972).

71CRO3 Crouthamel, C. E., and Larsen, R. P., "Burnup Analysis and Fission Yields for Fast Reactors: Determination of Fast-Fission Yields and Nonfission Nuclear Transformations in a Fast Reactor," ANL-7887 pages 7.32-7.34 (November 1971). This data together with additional data appears in 71LAR3 and 72LAR1. CSISRS-90457. Superseded by 74LAR1.

71CRO4 Crouthamel, C. E., "Reactor and Analytical Chemistry," ANL-7775 pp. 87-102 (April 1971). Chemical Engineering Division Annual Report for 1970. NSA 26: 30212 (1972). Data summarized and updated in 72LAR1.

71CUN1 Cuninghame, J. G., "Fission Yields in Accelerator Neutron Spectra," Paper 6, U. of Kent, Canterbury, UK Conference, Chemical Nuclear Data: Measurements and Applications, (September 20-22 1971), British Nuclear Energy Society, 1-7 Great George Street, London SWI. NSA 26: 32415 (1972).

71DEN1 Denschlag, H. O., "Concerning Shell Effects on the Charge Distribution in Nuclear Fission," Habilitationsschrift Universitat Mainz 1971.

71DEN2 Denschlag, H. O., and Qaim, S. M., "Independent Yields of 133I, 134I, and 135I in the Fission of 232Th Induced by Reactor Neutrons and $14.8 \mathrm{meV}$ Neutrons: Charge Distribution in Low and Medium Energy Fission," J. Inorg. Nucl. Chem., 33, 3649-3661 (1971). NSA 26: 14159 (1972). CA 76: 40449d (1972). EXFOR-22056 (Feb 7, 1989). 
71DIE1 Dierckx, R., Maracci, G., Rustichelli, F., "Measurement of the 140La Fission Product Yield for Fissions in 238U in a Thermal Reactor Type Spectrum," J. Nucl. Energ., 25, 85-89 (1971). NSA 25: 39033 (1971). EXFOR-22058 (Aug 5, 1988).

71DYA1 D'yachenko, P. P., Kuz'minov, B. D., Kutsaeva, L. S., and Piksaikin, V. M., "Energy Dependence of Yields and Kinetic Energies of $235 \mathrm{U}$ Fission Fragments," Yadern. Fiz. 14, No. 6, 1129-1133 (December 1971). In Russian. NSA 26: 24334 (1972). 39 values graphically given for peak (137-141) and valley (118-121) yields at neutron energies 0.12- $6 \mathrm{meV}$. Consolidates 68DYA1 and 70DYA1. By integrating over a fission spectrum in 7 groups of $1 \mathrm{meV}$ each, effective valley yields for $\mathrm{U} 235 \mathrm{~F}=0.034 \pm 0.005$. Effective peak yields for $\mathrm{U} 235 \mathrm{~F}=6.28 \pm 0.05$. EXFOR-40234.

71EIC1 Eichor, M., and Troutner, D. E., "Nuclear Charge Distribution in Fission: Independent Yields of 139Ba and 140Ba," J. Inorg. Nucl. Chem., 33, 1543-1552 (1971). NSA 25: 48089 (1971). Dissertation Abstracts B 34, 1034 (Sept 1973). CSISRS-90463.

71ERD1 Erdal, B. R., Wahl, A. C., and Ferguson, R. L., "Modes of Formation of Tin Fission Products," J. Inorg. Nucl. Chem. 33, 2763-2781 (1971). NSA 26: 03619 (1972). Isomeric independent yields here split per 76MAD2. CSISRS-90464.

71FLU1 Fluss, M. J., and Dudey, N. D., "Tritium and Helium Yields in Fast Fissions of U235," Trans. Amer. Nucl. Soc., 14, No. 2, 809 (October 1971). ANS meeting, 17 October, 1971, Miami Beach, Fla., CONF 711009, NSA 26: 3628(1971). Same data also appears in 71LAR2.

71FRA1 Franz, E. M., and Friedlander, G., "Cross Sections for the Production of 84Rb, 86Rb, $132 \mathrm{Cs}$, and $136 \mathrm{Cs}$ from Thorium and Uranium by $28.5 \mathrm{geV}$ Protons," Phys. Rev. C, 4, 1671-1673 (1971). CSISRS-90465.

71FUD1 Fudge, A. J., "Requirements of Nuclear Data and Procedures for the Determination of Burnup by Chemical Methods," Paper 9, U. of Kent, Canterbury, UK Conference, Chemical Nuclear Data: Measurements and Applications, September 20-22 1971, British Nuclear Energy Society, 1-7 Great. George Street, London SWI. (Compilation of yields for common burnup monitors $\mathrm{Ce}-144, \mathrm{Cs}-137, \mathrm{Nd}-148$, and $\mathrm{Zr}-95$ ).

71GRI1 Grimm, W., "Untersuchungen zur Ladungsverteilung im Gebiet der Halogen-Neutronenstrahler bei der Neutron Induzierten Spaltung von Thorium-, Uran- und Plutonium-Isotopen," Dissertation zur erlangung des Doktorgrades. Johannes Gutenberg Universitat, Mainz, 1971: Page 195.

71GUN1 Gunten, H. R. von, "Radiochemical Methods in Fission Yield Determinations," Paper 2, U. of Kent, Canterbury, UK Conference, Chemical Nuclear Data: Measurements and Applications, (September 20-22 1971), British Nuclear Energy Society, 1-7 Great George Street, London SWI. NSA 26:30746 (1972). In author index, von Gunten, H. R., reads (Gunten, H. R. von ).

71HAR1 Harbour, R. M., and Troutner, D. E., "Fractional Cumulative Yield of 2.6 min 99m Nb from Low Energy Neutron Fission of 233U, 235U, 238U and Spontaneous Fission of 252Cf," J. Inorg. Nucl. Chem., 33, 1-8 (1971). NSA 25: 20406 (1971). Dissertation Abstracts B 30, 1578 (Oct 1969). CSISRS-90467. 
71HAR2 Harbour, R. M., Eichor, M., and Troutner, D. E., "Mass Yields from the Spontaneous Fission of Cf-252," Radiochim. Acta, 15, No. 3, 146-150 (1971). NSA 25: 34147 (1971). Normalized by 75FLY2. CSISRS-90468.

71HAW1 Hawkings, R. C., Edwards, W. J., and Olmstead, W. J., "Independent Yields of Mass-135 Xenons in the Thermal Neutron Fission of U-233, U-235, Pu-239, and Pu-241," Can. J. Phys., 49, 785-803 (1971). NSA 25: 34087 (1971). Supersedes EANDC-CAN32L (1967). Independent yields of Xe-135 divided between isomers per 76MAD2. CSISRS-90469. It is possible to estimate $\mathrm{I}-135$ cumulative yield by subtracting this $\mathrm{Xe}-135$ independent yield from the cumulative yield of $\mathrm{Xe}-135$ (see 73WAL3). This is a useful evaluators tool to improve the difficult to measure I-135 cumulative yield.

71HOL1 Holubarsch, W., Pfeiffer, E., and Goennenwein, F., "Fragment Kinetic Energies in meV Neutron-Induced Fission of 232Th," Nucl. Phys., A171, No. 3,631-640(1971). Peak/valley $=510 / 1,122 / 1,45 / 1$ for neutron energies $1.90,2.97$ and $4.81 \mathrm{meV}$. NSA 26: 14117 (1972).

71HYD1 Hyde, E. K., Butler, G. W., and Poskanzer, A. M., "Characteristics of Fragments Produced in the Interaction of 5.5 geV Protons with Silver," Phys. Rev. C, 4, 1759-1778 (1971). Review article with 78 refs. Extensive compilation of literature measurements.

71IYE1 Iyer, M. R., Ganguly, A. K., “Nuclear Charge Distribution in Fission Fragments," Phys: Rev. C, 3(2), 785-796 (1971). NSA 25: 28508 (1971).

71JAI1 Jain, H. C., and Ramaniah, M. V., "99Mo Fission Yield in the Thermal NeutronInduced Fission of 239Pu and 235U," Government of India AEC Report BARC-584 (1971). NSA 26: 49446 (1972). Earlier memo NSA 30: 05100 (1974). CA 77: 121261j (1972). Comparative method values reduced $9.1 \%$ per $73 \mathrm{LAM} 1$ to adjust authors $2200 \mathrm{~m} / \mathrm{s}$ fission cros sections to effective fission cross sections at an assumed 40 degrees $C$. neutron temperature and epithermal index $=.0022$. This topical report later published as journal article in 73JAIl. EXFOR-30504.

71JOH1 John, W., Hulet, E. K., Lougheed, R. W., Wesolowski, J. J., "Symmetric Fission Observed in Thermal-Neutron-Induced and Spontaneous Fission of 257Fm," Phys. Rev. Letters, 27, No. 1, 45-48 (5 July 1971). Graphical presentation. Not coded.

71KEM1 Kemmer, J., Kim, J. I., and Born, H. J., "Study on the Reactor Neutron Induced Fissics of U-232," Radiochimica Acta, 15, No. 3, 113-117 (1971). NSA 25: 34148 (1971).

71KOR1 Korostylev, V. K., Rjazanov, D. K., Safonov, V. A., "Fine Structure in Mass Fraction Distribution of Fission of U-235," Conf. 71Kiev, 2, 78 (May 1971). EXFOR-40293. Incomplete mass resolution (not coded).

71KRA1 Krappel, W., Seufert, H., and Stegemann, D., "A Method of Nondestructive Measurement of the Absolute Cumulative Barium-140 Yield of Uranium Irradiated in Thermal and Fast Neutron Spectra," Nuclear Technology, 12, 226-234 (Oct. 1971). NSA 25. 50733 (1971). Ba-140 U235T error assigned as in Table C1 73WAL3.

71KRA1 Kratz, J. V., Private communication to 71DEN1 (1971). 
71KUG1 Kugler, G., and Clarke, W. B., "Mass Spectrometric Search for Neon and Argon Isotopes in Ternary Fission of 235U," Phys. Rev. C, 3, 849-853 (February 1971). Upper limits in atoms per binary fission are: $20 \mathrm{Ne}$ 5.8-08; $21 \mathrm{Ne} 3.4-10 ; 22 \mathrm{Ne} 5.9-09 ; 36 \mathrm{Ar} 2.4-09 ; 37 \mathrm{Ar}$ $2.2-09 ; 38 \mathrm{Ar} 2.0-09 ; 39 \mathrm{Ar} 7.8-10 ; 40 \mathrm{Ar} 6.9-07 ; 42 \mathrm{Ar} 2.2-09$. Not coded. NSA 25: 28419 (1971).

71KU11 Kuiken, R., “Fission of Oriented Nuclei by Low Energy Neutrons," RCN-142 (1971). 167 pages. No yields given.

71KUR1 Kurchatov, B. V., Morozov, L. N., Novgorodtseva, V. I., et al., "Mass Distribution of the Fission Products from 249Cf Exposed to Slow Neutrons," Yadern. Fiz., No. 5, 943-949 (November 1971). NSA 26: 10973 (1972). EXFOR-40145.

71LAR1 Larsen, R. P., Heinrich, R. R., Dudey, N. D., Oldham, R. D., and Popek, R. J., "235U and 239Pu Fast Fission Yields as a Function of Neutron Energy," Trans. Amer. Nucl. Soc. 14, No. 1, p. 370 (June 1971). Same data appears in ANL-7750 pp. 82-85 (1971). NSA 25: 41241 (1971). See 74LAR1.

71LAR2 Larsen, R. P., Dudey, N. D., Fluss, M. J., Heinrich, R. R., Malewicki, R. L., Oldham, R. D., Popek, R. J., Williams, J., Crouthamel, C. E., and Vogel, R. C., "Chemical Engineering Division Cross Sections and Burnup Semiannual Report," January-June 1971 ANL7824 pp. 7-12 (August 1971). Tritium fission yields U235T and U235F (420,483, $539 \mathrm{keV})$ and U233F (483 keV). These early measurements are high because I-131 in the system introduced

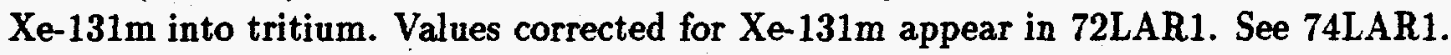

71LAR3 Larsen, R. P., Private communication to B. F. Rider December 6, 1971 to be published in ANL-7879 (May 1972). NSA 26: 49812 (1972). Supersedes ANL-7750. See 74LAR1.

71LAR4 Larsen, R. P., Private communication from Argonne National Laboratory to ASTM Committee E-10.05 on neutron dosimetry (1971). See 74LAR1. CSISRS-90475.

71LEM1 Lemmel, H. D., "The Present Status of Knowledge of the Thermal Cross-Sections of the Main Pu-Isotopes and Their Fission-Products," INDC(NDS)-40 (draft) (June 1971).

71LIS1 Lisman, F. L., Abernathey, R. M., Foster, R. E., Jr., Maeck, W. J., "Fission Yields and Branching Ratios of $85 \mathrm{Kr}$ in Thermal Neutron Fission of $233 \mathrm{U}, 235 \mathrm{U}, 239 \mathrm{Pu}$, and 241Pu," J. Inorg. Nucl. Chem., 33, 643-649 (1971). NSA 25: 28383 (1971). CSISRS-90476.

71MAE1 Maeck, W. J., "Fast Breeder Reactor Burn-up and Fission Yield Measurement Program," Paper 5, U. of Kent, Canterbury, UK Conference, Chemical Nuclear Data: Measurements and Applications, September 20-22 1971, British Nuclear Energy Society, 1-7 Great George Street, London SWI. NSA 26: 32793 (1972).

71MAR1 Marmol, P. del, Fettweis, P., Perricos, D. C., "On the Delayed Neutron Yields of the Longer-Lived Halogen Precursors in Thermal Neutron Fission of 235U," Radiochim. Acta, 16, No. 1, 4.7 (1971). In author index, del Marmol, P., reads (Marmol, P. del ).

71MAT1 Mathews, D. R., and Mc Gehee, B. G., "Review of Fission Product Yields and Cross Sections," GULF-GA-B-12071 (September 1971). 11 pp. NSA 27: 10973 (1973). Compilation. 
71MCL1 Mc Laughlin, T. P., "Measurements of Absolute Fission Product Yields from Thermal Fission of 235U," Univ, of Arizona Thesis (September 14, 1971). University Microfilm Catalog No. 71-14509. NSA 25: 53800 (1971). Ba-140 U235T error assigned as in Table C1 of 73WAL3. CSISRS-90478.

71MEY1 Meyer, E. F. Jr., Button, W. H., and Arnold, K.," "Mass Distribution in the Fission of 238U with 3He Ions," J. Inorg. Nucl. Chem., 33, 2745-2750 (1971). Cumulative yields of 13 fission products from $14-30 \mathrm{meV} 3 \mathrm{He}$ ion induced fission of $238 \mathrm{U}$. Not coded.

71NAK1 Nakahara, H., Fujiwara, I., Okamoto, H., Imanishi, N., Ishibashi, M., and Nishi, T., "Mass Yields of 241Am Thermal-Neutron Fission," J. Inorg. Nucl. Chem., 33, 3239-3250 (1971). EXFOR-22064 (Feb 7, 1989).

71NEI1 Neidhart, B., and Bachmann, K., "Untersuchung der bei der Wechselwirkung von Hochenergetischen Protonen mit Tantalkernen Entstehenden Lanthanidruckstrossprodukte-I: Bildungsquerschnitte der Lanthanide bei $580 \mathrm{meV}$ Protonenenergie," J. Inorg. Nucl. Chem., 33, 2751-2761 (1971). Independent or cumulative cross sections of 30 spallation products of Ta irradiated with $580 \mathrm{meV}$ protons. Not coded.

710KA1 Okazaki, A., Walker, W. H., and Bigham, C. B., "Erratum: The Ratio of the Direct to the Cumulative Yield of $135 X e$ in the Thermal Neutron Fission of 233U, 235U, 239Pu, and 241Pu," Can. J. Phys., 49, 498-499 (1971). Supersedes, corrects, and replaces 65BIG1 and 66OKA1. Independent yields of Xe-135 divided between isomers per 76MAD2. CSISRS-90480.

71PAC1 Pace, C. H., M. A. Thesis, University of Missouri (December 1971). Cited in 70TRO2. CSISRS90481.

71PET1 Petrzhak, K. A., Teplykh, V. F., Pan'yan, M. G., and Demin, V. A., "Relative Yields of Xenon Isotopes at Photofission of 238U," Yadern. Fiz., 14, No. 5, 950-952 (November, 1971). NSA 26: 10974 (1972).

71POD1 Poddar, R. K., Basu, B., "Annual Report for 1970 of Saha Inst. of Nucl. Phys, Calcutta, India," NP-19438 (July 1971) page 37. I-133, Mo-99 in U238 spontaneous fission. NSA 27: 01580 (1973). Not coded.

71QAI1 Qaim, S. M., and Denschlag, H. O., "Independent Yields of 133I, 134I, and 1351 in the Epicadmium Reactor Neutron and $14 \mathrm{meV}$ Neutron Induced Fission of 232Th: Charge Distribution in Medium Energy Fission Reactions," Paper 8, U. of Kent, Ca: terbury, UK Conference, Chemical Nuclear Data: Measurements and Applications, September 20-22 1971, British Nuclear Energy Society, 1-7 Great George Street, London SWI. NSA 26: 32417 (1972).

71RAM1 Ramaniah, M. V., Private communication to B. F. Rider, June 5, 1971. A substantial extensice of work reported in 69DAN1. See also NSA 26: 32410 (1972), 26:49446 (1972). Absolute Movalues reduced $9.1 \%$ per $73 \mathrm{LAM} 1$ to adjust authors $2200 \mathrm{~m} / \mathrm{s}$ fission cross sections to effectime fission cross sections at an assumed 40 degrees $C$. neutron temperature and epithermal index = .0022. Data now published in 71JAI1. Xe-135 yield corrected by 78 WAL1 for $15 \%$ direct yie of Xe-135 assuming 15 minute irradiation and the stated 2 days before counting using equation 2 of 710KA1. 
71REI1 Reisdorf, W., Unik, J. P., Griffin, H. C., Glendenin, L. E., "Fission Fragment K X-ray Emission and Nuclear Charge Distribution for Thermal Neutron Fission of 233U, 235U, 239Pu and Spontaneous Fission of 252Cf." Nucl. Phys. A177, 337 (Dec 1971). Elemental yields for 24 elements from arsenic to dysprosium. EXFOR-13456.

71RIC1 Richardson, P. J., "Methods for Determining Burnup in Enriched Uranium-235 Fuel Irradiated by Fast Reactor Neutrons," NASA TM X-2153 (Jan. 1971).

71ROB1 Robin, M., Bouchard, J., Darrouzet, M., "Determination of the Nd-148 Yield in the Fission of U235, U238, and Pu239 by Thermal and Fast Neutrons," Page 19, U. of Kent, Canterbury, UK Conference, Chemical Nuclear Data: Measurements and Applications, September 20-22 1971, British Nuclear Energy Society, 1-7 Great George Street, London SWI. NSA 26: 32414 (1972). EXFOR-22050.

71ROC1 Roche, M. F., Troutner, D. E., and Ferguson, R. L., "The Independent yield of $112 \mathrm{Ag}$ from Thermal Neutron Induced Fission of $233 \mathrm{U}$ and $235 \mathrm{U}$," Radiochim. Acta 16, No. 2, 66-71 (1971). NSA 26: 14158 (1972). CA 76: 93270v (1972). CSISRS-90485.

71SAB1 Sabu, D. D., "On Mass-Yield of Xenon and Krypton Isotopes in the Spontaneous Fission of Uranium," J. Inorg. Nucl. Chem., 33, 1509-1513 (May 1971). NSA 25: 46082 (1971). CSISRS-90486. It is not known why all $\mathrm{Kr}$ isotopes appear greater than several other published values while Xe isotopes agree with other published values.

71SIN1 Sinclair, V. M., and Davies, W., "Measurement of Fission Yields in the Dounreay Fast Reactor," Paper 7, U. of Kent, Canterbury, UK Conference, Chemical Nuclear Data: Measurements and Applications, September 20-22 1971, British Nuclear Energy Society, 1-7 Great George Street, London SWI. NSA 26: 32416 (1972). EXFOR-22066 (Aug 5, 1988).

71SOR1 Sorokina, A. V., Skovorodkin, N. V., Bugorkov, S. S., Krivokhatskii, A. S., Petrzhak, K. A., "Radiochemical Determination of Absolute Cumulative Yields of Fission Fragments in Slow-Neutron Fission of 241Pu and 239Pu," Atomnaya Energiya, 31, No. 2, 99-102 (August 1971). In Russian; English abstract ibid. Page 132; NSA 26: 1325 (1972).

71STA1 Staff, "Annual Report 1970," 131 pages (October 1971). Mainz University (West Germany) Institute fur Anorganische Chemie und Kernchemie BMBW-FBK-71-12. NSA 26: 8878 (1972). Charge distribution determined for 132 U235T and 133, 134, 135 for Th232 $3 \mathrm{meV}$ and $15 \mathrm{meV}$. Replaced by 71QAI1, 71DEN2.

71SUR1 Surin, V. M., Sergachev, A. I., Rezchikov, N. I., and Kuz'minov, B. D., "Yields and Kinetic Energies of Fragments at the Fission of $233 \mathrm{U}$ and $239 \mathrm{Pu}$ by 5.5 and $15 \mathrm{meV}$ Neutrons," Yadern. Fiz., 14, No. 5, 935-938 (November 1971). NSA 26: 10981 (1972). EXFOR-40112.

71SWI1 Swindle, D. L., Wright, R. J., and Kuroda, P. K., "Yields of Ruthenium Isotopes from the Spontaneous Fission of 238U," J. Inorg. Nucl. Chem., 33, 876-879 (1971). NSA 25: 28384 (1971). CSISRS-90488.

71SWI2 Swindle, D. L., Wright, R. J., Ward, T. E., and Kuroda, P. K., "Distribution of Low Mass Fission Yields in the $14.8 \mathrm{meV}$ Neutron Induced Fission of $238 \mathrm{U}$ and $232 \mathrm{Th}$," J. Inorg. Nucl. Chem., 33, 651-656 (1971). NSA 25: 28385 (1971). CSISRS-90489. 
71SWI3 Swindle, D. L., Moore, D. T., Beck, J. N., and Kuroda, P. K., "Mass Distribution of $3 \mathrm{meV}$ Neutron-Induced Fission of 232Th," J. Inorg. Nucl. Chem., 33, 3643-3647 (1971). NSA 26: 14162 (1972). CSISRS-90490. 153Pm is a typo in the article and should read 151Pm (28 hr).

71TAS1 Tasaka, K., Seki, Y., "Method for Calculating Energy Dependence of Mass Chain Yield of Fission," JAERI-MEMO-4383 (Mar. 1971). NSA 25: 28409 (1971). Calc'd yields of U235 at 1.95, 15meV agree with exp't. Not coded. NSA 25: 56816 (1971).

71TOM1 Tomlinson, L., and Hurdus, M. H., "Delayed Neutron Precursors - IV: 87Se, 88Se and 89Se; Half-Lives, Neutron Emission Probabilities and Fission Yields," J. Inorg. Nucl. Chem., 33, 3609-3620 (1971). NSA 26: 24326 (1972).

71TR01 Troutner, D. E., and Runnalls, N. G., "Nuclear Charge Distribution in Fission: Fractional Cumulative Yields of 131Sb and $131 \mathrm{~m}$-Te from Spontaneous Fission of 252Cf," J. Inorg. Nucl. Chem., 33, 2271-2279 (1971). CSISRS-90492.

71TRO2 Troutner, D. E., Harbour, R. M., "Nuclear Charge Distribution in Fission: Fractional Cumulative Yields of 132Te and 134Te from Thermal-Neutron-Induced Fission of 245Cm and 249Cf," Phys. Rev. C, 4(2), 505-507 (August 1971). NSA 25: 50755 (1971). CSISRS-90493.

71TRO3 Troutner, D. E., "Comparison of Nuclear Charge Distribution in Thermal-Neutron Induced Fission of 233U and 235U," J. Inorg. Nucl. Chem. 33, No. 12, 4327-4333 (Dec 1971). NSA 26: 14161 (1972). CA 76: 93597d (1972). CSISRS-90494. A review paper giving averages of other peoples work.

71TRO4 Troutner, D. E., Paper at 161st ACS Meeting (1971). Cited in 74WOL1.

71UME1 Umezawa, H., "Nuclear Charge Distribution in Proton-Induced Fission of 238U," J. Inorg. Nucl. Chem., 33, 2731-2743 (1971). Fractional chain yields of $86 \mathrm{Rb}, 132 \mathrm{Te}, 134 \mathrm{Cs}$, $136 \mathrm{Cs}, 140 \mathrm{Ba}, 148 \mathrm{Pm}, 160 \mathrm{~Tb}$ from $13-55 \mathrm{meV}$ proton induced fission. Not coded.

71VOG1 Vogel, R. C., Burris, L., Tevebaugh, A. D., Webster, D. S., and Proud, E. R., "Chemical Engineering Division Research Highlights, January-December 1971," ANL-7850 pp. 103-111 (1971) Duplicates data published as 72LAR1.

71WAL1 Walker, W. H., Private communication to H. D. Lemmel (1971) cited in 71LEM1.

71WAL2 Walker, W. H., "Yields from Thermal Neutron Fission of Pu239 and U233," Chemistry and Materials Division Progress Report, January 1 - March 31, 1971, AECL-3909, 92 pages (1971). NSA 25: 54587 (1971).

71WOL1 Wolfsberg, K., and Ford, G. P., "Thermal-Neutron Fission of Am-242m: Mass and Charge Distribution," Phys. Rev. C, 3, No. 3, 1333-1337 (March 1971). Also published as LA-DC-12142 (June 1, 1970). NSA 25: 31373 (1971). CSISRS-90495.

71WOL2 Wolfsberg, K., "A New Nuclide, Xe-145: Measurement of Its Fractional Cumulative Yield from Thermal Neutron Fission of U-235," J. Inorg. Nucl. Chem., 33, 587-589 (1971). NSA 25: 20470 (1971). CSISRS-90496. 
72ALE1 Alexander, P., "Independent Yields of 133, 135Xe in the Fission of 235U, 238U, and 239Pu by 14 meV Neutrons," Nucl. Phys., A198, No. 1, 228-236 (1972). NSA 27: 08665 (1973). Independent yields of Xe-133, 135 divided between isomers per 76MAD2. EXFOR22038 (Feb 7, 1989). CSISRS-90497.

72ALE2 Alexander, P., "Direct Population of the H 1/2 States in 133Xe and 135Xe Through Thermal Neutron Fission," pp. 1225-1230 of Radioactivity in Nuclear Spectroscopy Vol 2, Hamilton, J. H. (ed), New York, Gordon and Breach Science Publishers (1972). See CONF690818 (Vol 2). NSA 27: 26356 (1973).

72ALK1 Alkhazov, I. D., Kovalenko, S. S., Kostochkin, O. I., Malkin, L. Z., Petrzhak, K. A., Shpakov, V. I., Yadern. Fiz., 15(1), pg 22 (Jan 1972). EXFOR-40176.

72AND1 Anderson, D. G., "Fission Product Mass-Yield Measurements from Intermediate Energy Neutron Fission of Plutonium-239 and Plutonium-241," $\mathrm{Ph}$. D. Thesis, Univ. of Texas, Austin, Tex. (1972). 134 pages, NSA 28: 28893 (1973). University Microfilm Order No. 73-18394. Yields are not quantitative. Author feels unknown losses of volatile atoms from catcher foil accounts for 5-83\% low results on both $\mathrm{Pu}-239$ and $\mathrm{Pu}-241$ yields. Not coded. CSISRS-90498.

72BAL1 Ballou, N. E., K.'e, J. H., and Reeder, P. L., "Fission Yields of Alkali Metal and Rare Gas Isotopes in Thermal Neutron Fission of 235U," Paper 2, Charles D. Coryell Memorial Symposium, 163rd ACS National Meeting, Boston, Mass. (April 9-14, 1972). Isomeric independent yields are here split per 76MAD2. Superseded by 74BAL1. Reviewed by 75REE1. CSISRS-90499.

72BER1 Berge, L. A., Ph. D. Thesis, University of Missouri (1972). Dissertation Abstracts B 34, 1034 (Sept 1973). Order 73 - 21,396 from University Microfilms, a Xerox Company, Ann Arbor, Michigan. CSISRS-90500.

72BOC1 Bocquet, J. P., Brissot, R., Crancon, J., Moussa, A., “On-Line Measurements of Rare-Gas Fission Yields in $14 \mathrm{meV}$ Neutron Fission," Nucl. Phys. A189, 556-576 (1972). NSA 26: 47030 (1972). For full $164 \mathrm{pg}$. Thesis see 71BOC1. EXFOR-21734.

72CAM1 Cambiaghi, M., Cavallari, F., Fossati, F., "Experimental Results Concerning LongRange Light Particles and Fragments in the Ternary Fission of 233U, 235U, 239Pu, and 252Cf," Nuovo Cim., 11A, No. 3, 716-724 (Oct 1972). NSA 27: 01642 (1973). Not coded. Includes $6 \mathrm{He}$ in thermal $233 \mathrm{U}, 235 \mathrm{U}, 239 \mathrm{Pu}$; $3 \mathrm{H}$ in $235 \mathrm{U}$ thermal; $4 \mathrm{He}$ in $239 \mathrm{Pu}$ at $6 \mathrm{meV}$; $3 \mathrm{H}$, $4 \mathrm{He}, 6 \mathrm{He}$ in $\mathrm{Cf} 252$ spontaneous fission.

72CHA1 Chaumont, J., On-Line Mass Spectrometry Technique (see 70CHA1) cited by 72BAL1. Isomeric independent yields are here split per 76MAD2.

72CUN1 Cuninghame, J. G., Goodall, J. A. B., and Willis, H. H., “Anomalous Effect of Change of Neutron Spectrum on Mass Yields in Pu-239 Neutron Fission,” AERE-R-6555 (May 1972). NSA 26: 39887 (1972).

72CUN2 Cuninghame, J. G., Goodall, J. A. B., Willis, H. H., "Absolute Yields in the Fission of 235U, 238U, and 239Pu Irradiated in DFR," AERE-R-6862(rev) (May 1972). 17 pp. NSA 26: 50002 (1972). NSA 27: 13546 (1973). EXFOR-20768. 
72DEB1 Debertin, K., "Relative Yields of Some Gamma-Ray Emitting Nuclides from Thermal Neutron Fission of 235U," Radiochim. Acta 18, No. 4, 202-206 (1972). CA 80: 430635s (1974). NSA 29: 14293 (1974). EXFOR-22054 (Feb 7, 1989). EXFOR-22054.

72DUD1 Dudey, N. D., Malewicki, R. L., Rymas, S. J., "Tritium Yields from Fast-Neutron Fission of $235 \mathrm{U}$ (200-800 keV)," Transactions Amer. Nucl. Soc., 15, No. 1, 483 (June 18-22, 1972). Same data as 71LAR1. NSA 26: 44311 (1972).

72DUD2 Dudey, N. D., Fluss, M. J., Malewicki, R. L., "IV. Fast-Neutron Fission Yields of Tritium," ANL-7924, pp. 18-21 (Sept. 1972).

72DYA1 D'yachenko, P. P., Kabenin, V. N., Kolosov, N. P., Kuz'minov, B. D., Sergachev, A. I., “Yields and Kinetic Energy of the Fragments During Spontaneous and Induced Fission of Plutonium-242," FEI-366, 16 pages (in Russian) (1972). NSA 29: 01721 (1974). EXFOR40194 (Jan 15,1986).

72EBE1 Ebersole, E. R., Argonne National Laboratory, "EBR-II Burnup Measurements - Comparison of 139La and $148 \mathrm{Nd}$ as Monitors," Private communication to W. B. Loewenstein (Nov. 16, 1972). EBR-I, Mark III U235F MSRE; 139La:137Cs $=1.033 \pm 0.002 ; 148 \mathrm{Nd}: 137 \mathrm{Cs}$ $=0.2745 \pm 0.0013 ; 139 \mathrm{La}: 148 \mathrm{Nd}=3.763 \pm 0.018$ for 5 to 6 determinations. In EBR-II Fissium U235F, MSRE; 139La:148Nd = 3.797 \pm 0.011 for average of 13 determinations. Thermat Neutron-Induced fission yields for U235 (U235T) are $139 \mathrm{La}=(6.44 \pm 0.01) \% ; 148 \mathrm{Nd}=(1.69$ $\pm 0.01) \%$ from 1964 measurements on 11 rnup $235 \mathrm{U}$ where fissions were determined from isotopic change in uranium. CSISRS-9050: supersedes 70EBE1

72EHR1 Ehrenberg, B., Amiel, S., "Independent Yields of Krypton and Xenon Isotopes in Thermal-Neutron Fission of 235U. Observation of an Odd-Even Effect in the Element Yield Distribution," Phys. Rev. C, 6, No. 2, 618-628 (Aug 1972). CA 77: 95524a (1972).

72EIC1 Eichor, M., “Nuclear Charge Distribution in Fission: Fractional Independent Yields of $139,140 \mathrm{Ba}$ from Thermal-Neutron Induced Fission of $233 \mathrm{U}$ and Fractional Independent Yields of $138 \mathrm{Cs}$ from Spontaneous Fission of $249 \mathrm{Cf}, \mathrm{Ph}$. D. Thesis, Univ. of Missouri, Columbia, Mo. (1972) 105 pages University Microfilm Order No. 73-21776. NSA 28: 28818 (1973). published as 71EIC1. CSISRS-90508.

72FLU1 Fluss, M. J., Dudey, N. D., Malewicki, R. L., "The Yield of Tritium from the Fast Fission of U235," Argonne National Laboratory, 9700 S. Cass Avenue, Argonne, Illinois, Paper 73, General papers session Sept. 1, 1972, American Chemical Society Division of Nuclear Chemistry and Technology, 164th ACS National Meeting, New York, N. Y. August 28 - Sept. 1, 1972. Detailed values given in 72LAR1.

72FLU2 Fluss, M. J., Dudey, N. D., Malewicki, R. L., "Tritium and Alpha-Particle Yields in Fast and Thermal Neutron Fission of 235U," Phys. Rev., C, Vol 6, No. 6, 2252-2259 (Dec. 1972). NSA 27: 06541 (1973).

72FlY1 Flynn, K. F., Horwitz, E. P., Bloomquist, C. A. A., Barnes, R. F., Sjoblom, R. K., Fields, P. R., Glendenin, L. E., "Distribution of Mass in the Spontaneous Fission of 256Fm," Phys. Rev. C 5, 1725-1729 (1972). NSA 26: 34979 (1972). CSISRS-90509. It is assumed that Flynn's later publication with Unik (73UNI1) supersedes this 1972 dated study. 
72FLY2 Flynn, K. F., Srinivasan, B., Manuel, O. K., Glendenin, L. E., "Distribution of Mass and Charge in Spontaneous Fission of 244Cm," Phys. Rev. C, 6, 2211-2214 (1972). CSISRS90510.

72FOW1 Fowler, M. M., "Nuclear-Charge and Mass Distribution in Fission: Yields and Genetic Histories of $128 \mathrm{Sn}, 129 \mathrm{Sb}$, and $130 \mathrm{Sb}$ from Thermal-Neutron Fission of $235 \mathrm{U}$," $\mathrm{Ph}$. D. Thesis, 142 pages, Washington University, Saint Louis, Missouri (January 1972). NSA 26: 49451 (1972). DA 32B, 6899 (1972). University Microfilm Order No. 72- 17951, 149 pp. CSISRS-90511.

72GUN1 Gunther, H., Siegert, G., Ferguson, R. L., Ewald, H., Konecny, E., "Fission-Fragment Charge Distribution in Thermal-Neutron Induced Fission of 239Pu and 235U," Nucl. Phys., A196, No. 2, 401-411 (1972). NSA 27: 13566 (1973). Mass region 131-140 especially 132-Sn. (doubly magic). Data are inconsistent with all other values so none are used here. The reason for unreliable results is now known. Detector thickness variation was too large. The same detector was later masked down from 25 to 2 square $\mathrm{mm}$ using 100 -fold more flux with excellent results (see 74SIE2). Isomeric independent yields are here split per 76MAD2.

72HAR1 Harbour, R. M., Macmurdo, K. W., Troutner, D. E., and Vaughn, M. A., "Mass and Nuclear Charge Distribution from Spontaneous Fission of 254-Fm," Paper 29, Charles D. Coryell Memorial Symposium, 163rd ACS National Meeting, Boston, Mass. (April 9-14, 1972). Supersedes ORNL-4306-34 page 5 (1968).

72HAR2 Harbour, R. M., and Macmurdo, K. W., "Mass Yields from Thermal-Neutron Induced Fission of 245Cm," J. Inorg. Nucl. Chem. 34, 2109-2114 (1972). NSA 26: 47027 (1972). CSISRS-90513.

72HOF1 Hoffman, D. C., Sattizahn, J. E., Private communication to 74BAL1 (August 1972). CSISRS90514.

72HOL1 Holden, N. E., See 72WAL2.

72IZA1 Izak, T., Amiel, S., "Half Lives and Gamma Rays of Tin Isotopes of Masses 129, 130, 131 and 132," J. Inorg. Nucl. Chem. 34, 1469-1477 (1972). NSA 26: 34911 (1972).

72KAY1 Kay, M. A., Gordon, G. E., and Harvey, J. W., "The Mass Yield Curve for the Thermal Neutron Fission of Th229," Paper 9 Charles D. Coryell Memorial Symposium, 163rd ACS National Meeting, Boston, Mass. (April 9-14, 1972).

72KNO1 Knobeloch, G. W., The Los Alamos Scientific Laboratory, University of California, unpublished results (1972). Cited in 74WOL1. CSISRS-90515.

72KRY1 Kryger, B., “On a Discrepancy Between the Theoretical and Experimental Fast Neutron Fission Yields of Rare Gases. Demonstration in the Fuel of the Rapsodie Reactor," Panel on The Behavior and Chemical State of Fission Products in Irradiated Fuel, Vienna, 7-11 August 1972. CEA Fontenay-Aux-Roses, Nuclear Research Center, France. Paper 4B. Transl. from French by STS Inc. (Oct. 1972) see ANL-TRANS-918. Total Xe+Kr (83, $84,85,86 \mathrm{Kr}+131,132,134,136 \mathrm{Xe})$ cumulative fast fission yield $=(29 \pm 1) \%$ in Fortissimo 11-11-002 Assembly, Pin 22 with mean neutron energy of $700 \mathrm{keV}$. Because 70LIS1 finds this total $=25 \%$ for both $235 \mathrm{U}$ and $239 \mathrm{Pu}$ fast fission after 4 years storage acknowledging possible 
gas loss, 70LIS1 gas loss appears to be $16 \%(29 / 25=1.16)$ well above our $3 \%$ error. $\mathrm{Kr}$, Xe yields taken as $1.16 \times 70 \mathrm{LIS1}$ values which are still relative to $\mathrm{Nd}-148=1.75 \%$. NSA $27: 14251$ (1973). CA 79:12549e (1973). 70LIS1 Xe loss is real (75MAE1).

72KUG1 Kugler, G., and Clarke, W. B., "Mass-Spectrometric Measurements of 3H, 3He, and 4He Produced in Thermal-Neutron Ternary Fission of 235U: Evidence for Short Range 4He," Phys. Rev. C, 5, No. 2, 551-560 (February 1972). NSA 26: 21855 (1972).

72KUR1 Kurchatov, B. V., Morozov, L. N., Novgorodtseva, V. I., et. al., "Mass Distribution of the Fission Products from 249Cf Exposed to Slow Neutrons," Yad. Fiz. 14, 943 (1972). (In Russian). See Sov. J. Nucl. Phys. 14, 528 (1972) for English translation.

72LAR1 Larsen, R. P., Dudey, N. D., Crouthamel, C. E., Tevebaugh, A. D., and Vogel, R. C., "Chemical Engineering Division Burnup, Cross Sections, and Dosimetry Semiannual Report for July-December 1971," ANL-7879, (May 1972). Duplicates 71VOG1 ANL-7850, pp. 103-111 (1972). NSA 26: 38329 (1972). See 74LAR1. CSISRS-90518.

72LAR2 Larsen, R. P., Dudey, N. D., Heinrich, R. R., Armani, R. J., Gold, R., Oldham, R. D., Popek, R. J., "235U, 238U, and 239Pu Fast Fission Yields of Short-Lived Gamma-Active Nuclides," Trans. Amer. Nucl. Soc. 15, No. 1, 483-484 (June 18-22, 1972). Same data as 72LAR1. NSA 26: 44312 (1972). See 74LAR1.

72LAR3 Larsen, R. P., Private communication to B. F. Rider December 4, 1972. Superseded by 73LAR4. See 74LAR1.

72LAR4 Larsen, R. P., Dudey, N. D., Crouthamel, C. E., Tevebaugh, A. D., Vogel, R. C., “Chemical Engineering Division; Burnup, Cross Sections and Dosimetry Semiannual Report, July-December 1971," ANL-7879 (May 1972). NSA 26: 49812 (1972). See data under 71LAR3. See 74LAR1.

72LAR5 Larsen, R. P., Dudey, N. D., Crouthamel, C. E., Tevebaugh, A. D., Levenson, M., Vogel, R. C., "Chemical Engineering Division Burnup, Cross Sections, and Dosimetry Semiannual Report January-June 1972," ANL-7924 (September 1972). NSA 27: 11702 (1973). See 74LAR1.

72LIN1 Lin, C., and Wahl, A. C., “Cumulative Yields of 40.1-Min. 123Sn, 9.6-Min. 125Sn and 128Sn from Thermal-Neutron Induced Fission of $235 \mathrm{U}$," J. Inorg. Nucl. Chem., 34, 1479-1483 (1972). NSA 26: 34972 (1972). CA 77: 41557v (1972). CSISRS-90519.

72MAR1 Marmol, P. del, Fettweis, P., “Identification of New Germanium Isotopes in Fission: Decay Properties and Nuclear Charge Distribution in the $A=78$ to 84 Mass Region," Nucl. Phys. A194, 140-160 (1972). EXFOR-22055 (Dec 22, 1988).

72MAT1 Mathews, C. K., Tomlinson, R. H., "Cumulative Yields of the Heavy Fragments in the Fast Neutron Fission of 238U," Can. J. Phys., 50, No. 24, 3100-3110 (15 Dec 1972). NSA 27: 26363 (1973). Publication of 64MAT1. CSISRS-90521.

72MAT2 Matsumoto, W. Y., Mc Elroy, W. N., “Experimental Fission Yields for 137Cs and 148Nd Relative to $140 \mathrm{Ba}$ from Neutron Dosimetry Fission Foil Irradiations in EBR-II," HEDL-TME-72-90 pp. HEDL.9-HEDL.11 (Jun 1972). NSA 32: 21266 (1975). Superseded by 75ZIM1. 
72MEE1 Meek, M. E., Rider, B. F., “Compilation of Fission Product Yields, Vallecitos Nuclear Center - 1972," General Electric Company Report NEDO-12154 (January 1972). CA 77: 120920m (1972). NSA 26(14): 34967 (1972).

72NAE1 Naeumann, R., Folger, H., and Denschlag, H. O., "Determination of the Nuclear Charge Distribution in the Chain 132 from Thermal Neutron Fission of $235 \mathrm{U}$ and $233 \mathrm{U}$," J. Inorg. Nucl. Chem., 34, 1785-1797 (1972). NSA 26: 44324 (1972).

72NET1 Nethaway, D. R., and Mendoza, B., "Fission of 234U and 236U with 14.8-meV Neutrons," UCRL-73937 (January 1972). Published in Phys. Rev. C, 6, No. 5, 1821-1826 (Nov 1972). NSA 27: 08671 (1973). CSISRS-90523.

72NET2 Nethaway, D. R., Mendoza, B., "Comparison of Yields from Fission of 233U, 234U, 235U, 236U, and 238U with 14.8-meV Neutrons," UCRL-73936 (Apr 1972) Published in Phys. Rev. C. 6(5), 1827-1837 (Nov 1972). Isomeric independent yields are here split per 76MAD2. CSISRS-90524.

72PAN1 Panontin, J. A., and Sugarman, N., "Mass Yield Distribution and Charge Dispersion in 450 meV Proton Fission of 238U," J. Inorg. Nucl. Chem. 34, 1485-1502 (1972). NSA 26: 34973 (1972). Not coded.

72PET1 Petrzhak, K. A., Pan'yan, M. G., Teplykh, V. F., Kondrat'ko, M. Ya., "Relative Yields of Xenon Isotopes from the Neutron Fission of 239Pu and 233U," At. Energ. (USSR) 33, No. 2, 709-710 (Aug 1972). (In Russian). NSA 26: 57716 (1972).

72PET2 Petrzhak, K. A., Pan'yan, M. G., Teplykh, V. F., Aleksandov, B. M., "Relative Yields of Xenon Isotopes in 237Np and 238Np Fission by Neutrons," (Leningrad Technological Inst.) Yadern. Fiz. 15: No. 5, 860-862 (May 1972). In Russian. NSA 27:01648 (1973). Aleksandov, B. M., is transliterated Alexandrov, B. M., elsewhere. EXFOR-40019.

72PRI1 Prindle, A., Lawrence Livermore Laboratory of the Univ. of California, Private communication to 74WOL1. CSISRS-90527.

72RAM1 Ramaniah, M. V., Bhabha Atomic Research Centre, Trombay, Bombay, India. Private communication to W. H. Walker (May 11, 1972).

72RA01 Rao, S. A., "Charge Distribution in the Fission of Th232," Phys. Rev. C, 5, No. 1, 171-177 (January 1972). NSA 26: 14150 (1972). Dissertation Abstracts B 34, 1934 (Nov 1973). Isomeric independent yields are here split per 75MEE2. Nb96 independent yield seriously in error (see 75LAM1). CSISRS-90528.

72RAO2 Rao, S. A., Kuroda, P. K., "Distribution of Charge in the Fission of Th232," Paper 28, Charles D. Coryell Memorial Symposium, 163rd ACS National Meeting, Boston, Mass. (April 9-14, 1972). CSISRS-90529.

72RAO3 Rao, S. A., "Isomer Yield ratios of $115 \mathrm{~m}-\mathrm{Cd}$ and $115 \mathrm{~g}-\mathrm{Cd}$," J. Inorg. Nucl. Chem. 34, 2405-2411 (1972). NSA 26: 54628 (1972). CA 77: 82372z (1972). Dissertation Abstracts B 34, 1934 (Nov 1973). Supersedes ORO-3235-55 page 10 (1970).

72REE1 Reeder, P. L., Battelle-Northwest Pacific Northwest Laboratories, Private communication to 73AMI1, (1972). CSISRS-90531. 
72RID1 Rider, B. F., Relative radiochemical yields in SEFOR fast reactor, Core I. Core I contained BeO filled tightner rods to soften the neutron spectrum to that typical of a large liquid metal fast breeder reactor. Data summarized in Appendix H of "Experimental Program Results in SEFOR Core II,” by L. D. Noble, G. Kussmaul, and S. L. Derby, GEAP-13838 (June 1972). $\mathrm{Ge}(\mathrm{Li})$ measurements on unseparated samples. CSISRS-90532.

72RID2 Rider, B. F., Relative radiochemical yields in SEFOR fast reactor, Core II which differs from Core I by replacing $\mathrm{BeO}$ filled tightner rods with stainless steel, resulting in a harder neutron spectrum for Core II, typical of a small fast reactor. Data summarized in Appendix $\mathrm{H}$ of “Experimental Program Results in SEFOR Core II," by L. D. Noble, G. Kussmaul, and S. L. Derby, GEAP-13838 (June 1972). Ge(Li) measurements on unseparated samples. CSISRS-90532. Table H-14 lines 6 and 7 the exponents for Ce141 and Ru106 should read 12 and not 13 because all Atoms/Capsule are e+12 and all Fissions/Capsule are e+13. These are typographical errors.

72RID3 Rider, B. F., Unpublished work on SEFOR fast reactor Core 1. (1972). These data on long lived nuclides Cs-137 and Ce-144 were taken after 9 months cooling since publication of $72 \mathrm{RID} 1$. For data taken from 2 to 32 weeks cooling on shorter lived nuclides see 72RID1. Ge(Li) measurements on unseparated samples. CSISRS-90532.

72RUD1 Ruddy, F. H., and Alexander, J. M., "Long-Range Fragments from Fission of U236*," Phys. Rev. C, 5, No. 2, 549-551 (February, 1972). Not coded. NSA 26: 21854 (1972).

72SCH1 Scholtyssek, W., "Gamma Spectroscopy of Fission Products for Rate Determination: Relative Fission Product Yields of U235 in Fast and Thermal Neutron Spectra," KFK 1272/1 Vierteljahresbericht Projekt Schneller Brueter (1972).

72SCH2 Schroeder, I. G., "Emission of Long Range Alpha-Particles in the Subthermal-, Thermal-, and Resonance-Neutron Fission of 238Pu," Nucl. Phys. A195(1) 257-268 (1972). NSA 27: 6538 (1973). Binary-to-ternary fission ratio is $412 \pm 11$ for PU239T.

72SER1 Sergachev, A. I., D'jachenko, N. P., Kovalev, A. M., Kuz'minov, B. D., "Influence of Excitation Energy on the Distribu tion of Fission Fragments of U-233 in Masses and Kinetic Energy," Yadern. Fiz., 14(5), 935 (May 1971). EXFOR-40106.

72SID1 Sidebotham, E. W., "Fission Product Yield Data Extrapolated for Some Actinides," The Reactor Group TRG-Report-2143(R), Risley, Warrington, Lancashire, UK (1972). CA 77:12828d (1972). NSA 26:32407 (1972). Mean mass shift method used to estimate yield of masses 72-163 for fast fission of Th232, U233, U234, U235, U236, U237, U238, Np237, Np238, Pu238, Pu239, Pu240, Pu241, Pu242, Am241, Am243, Cm242 and thermal fission of U233, U235, U237, Np237, Pu239, Pu240, Pu241, Pu242, Am241.

72SWI1 Swindle, D. L., "Radiochemical Study of $238 \mathrm{U}$ and $232 \mathrm{Th}$ Fission Induced by Neutrons of 2.95 and $14.8 \mathrm{meV}$," Univ. of Arkansas Thesis $1972.80 \mathrm{p}$. Univ. Microfilms Order No. 72-10193. Data published already in 71SWI2 and 71SWI3. NSA 26: 34971 (1972). Dissertation Abstracts B 32, 5093 (Mar 1972).

72TRO1 Troutner, D. E., and Harbour, R. M., "Nuclear Charge Distribution in Fission: Fractional Independent Yield of 140La from Thermal-Neutron Induced Fission of 249Cf," J. Inorg. Nucl. Chem., 34, 801-809 (1972). NSA 26: 29721 (1972). CSISRS-90537. 
72VID1 Vidal, R., Progress Report EANDC(E)-150U (May 1972). Cited in 74IAE1. Superseded by coauthored 73ROB1.

72VOR1 Vorobiev, A. A., Seleverstov, D. M., Grachov, V. T., "Light Nuclei from 235U Neutron Fission," Phys. Lett. 40B, No. 1, 102-104 (12 Jun 1972). Yields H, He, Li, Be, B, C, and O isotopes in $235 \mathrm{U}$ fission. Not coded.

72WAH1 Wahl, A. C., Denig, R., “Cumulative Yield of 83-Sec 136I from Thermal-Neutron Induced Fission of 235U," J. Inorg. Nucl. Chem. 34, 2413-2418 (1972). NSA 26: 54626 (1972). CA 77: 83371y (1972). CSISRS-90538.

72WAL1 Walker, W. H., 1972 preprint subsequently printed as 73WAL1.

72WAL2 Walker, F. W., and Holden, N. E., General Electric Chart of the Nuclides, 11th edition, 1972. Available from the General Electric Company, Knolls Atomic Power Laboratory, Schenectady, New York. Reference data covered to April 1972.

72WEI1 Weinlaender, W., Born, H. J., "Bestimmung der Spaltausbeuteverteilung fur die Durch Reactorneutronen Induzierte Spaltung von 241Am," Radiochim. Acta, 17, (1) 18-27 (1972) in German. EXFOR-22073 (Aug 5, 1988).

72WOL1 Wolfsberg, K., Ford, G. P., The Los Alamos Scientific Laboratory, University of California, preliminary unpublished values (December 1972). Cited in 74WOL1. Later released as 74WOL2. Values are preliminary subject to further refinement. CSISRS-90540.

72ZAK1 Zakharova, V. P., Rjazanov, D. K., Basova, B. G., Rabinovich, A. D., Korostyljov, V. A., "Investigation of Uranium-235 Fission by the Thermal Neutrons," Yadern. Fiz. 16(4), 649 (1972). EXFOR-40200.

73AMI1 Amiel, S., Feldstein, H., “A Systematic Odd-Even Effect in the Independent Yield Distributions of Nuclides from Thermal Neutron Fissions of 235U," IAEA/SM-174/25, IAEA Third Symposium on the Physics and Chemistry of Fission, Rochester, N.Y. USA. Page 76 (August 13-17, 1973). NSA 31: 04988 (1975).

73AMI2 Amiel, S., "Status of Delayed Neutron Data," Review Paper, IAEA Panel on Fission Product Nuclear Data, Bologna, Italy, (November 26-30, 1973). Printed 74AMI3.

73BAL1 Balestrini, S. J., Forman, L., "Developments in Areas of On-Line Fission-Yield and Direct Mass Measurements at the Los Alamos Scientific Laboratory," LA-UR-73-838 (1973). NSA 28:14102 (1973). CA 80: 21537 (1974). These relative Cs independent yields are converted to fractional independent yields in 74WOL1 (which see). Made absolute 74WOL4. CSISRS-90541.

73BAL2 Balestrini, S. J., Personal communication to K. Wolfsberg September 1973 of relative independent yields converted to absolute values with Meek and Rider yields of 9-73 and unpublished Wolfsberg results of 12-72. Cited in 73WOL1. These relative $\mathrm{Rb}$ independent yields are converted to fractional independent yields in 74WOL1 (which see) and made absolute in 74 WOL 4 .

73BAL3 Ballou, N. E., Kaye, J. H., Reeder, P. L., Anderl, R. A., Stoffels, J. J., "Final Report on ARPA Fission Yield Project Work at Battelle-Northwest, April 1970 -April 1973," BNWL-B -281 (1973). See 74BAL1. CSISRS-90589. 
73BAT1 Battles, L. R., Chittenden, D. M., "Yields of Low Mass Products in the Fission of 232Th by 14.8 meV Neutrons," J. Inorg. Nucl. Chem. 35, No. 9, 3075-3078 (Sept 1973). NSA 28: 28888 (1973). CA 79: 131615h (1973). CSISRS-90542.

73BRA1 Brasca, M., Cesana, A., Sangiust, V., Terrani, M., “232Th Fission Yields Determined by Gamma Ray Spectroscopy on Unseparated Samples," Energ. Nucl. (Milan) 20(12), 691-698 (Dec 1973). NSA 29: 19792 (1974). CA 80: 90012j (1974).

73BR01 Browne, E., Personal communication on fission product genetics, Table of the Isotopes Project, Lawrence Berkeley Lab. (July 10, 1973).

73CHA1 Chauvin, C., "Mesure de Quelques Rendements dans la Fission du 232Th par Neutrons de $14 \mathrm{meV}$ et Par des Neutrons de Reacteur," Thesis, Grenoble (Sept. 27, 1973). Some yield measurements in the fission of $232 \mathrm{Th}$ by $14-\mathrm{meV}$ neutrons and reactor neutrons. (FRNC-TH-470). Nucl. Sci. Abstr. 30: 8491 (1974).

73CHI1 Chitambar, S. A., Jain, H. C., Kavimandan, V. D., Mathews, C. K., “Fission Yields in the Thermal Neutron Fission of Plutonium-241," BARC-690, pp. 134-137 (1973). CA 81: 144346n (1974). NSA 29: 14271 (1974). EXFOR-30514.

73CR01 Crouch, E. A. C., "Fission Product Chain Yields from Experiments in Thermal Reactors," Paper IAEA/SM-170/94 Symposium on Applications of Nuclear Data in Science and Technology, Paris, March 12-16, 1973. Originally AERE-R 7209 (Jan. 1973). NSA 29: 17307 (1974). NSA 30: 25661 (1974).

73CRO2 Crouch, E. A. C., "Fission Product Chain Yields from Experiments in Reactors and Accelerators Producing Fast Neutrons of Energies up to $14 \mathrm{meV}$," AERE-R 7394 (May 1973).

73CRO3 Crouch, E. A. C., Brownsword, M., Mc Kean, I. C., Unpublished work cited in 73CRO2.

73CUN1 Cuninghame, J. G., "Review of Fission Product Yield Data for Fast Neutron Fission," AERE - R 7548 (September 1973). Printed in 74CUN2. NSA 29: 3979 (1974), CA 80: 126843 (1974).

73DAR1 Daroczy, S., Raics, P., Nagy, S., "Compiled Fission Product Yields of U238 for $14 \mathrm{meV}$ Neutrons," Unpublished work summarized in 73CUN1. Full text in 74DAR2. Superseded by 75 DAR1.

73DAR2 Daroczy, S., German, E., Raics, P., Nagy, S., Csikai, J., "Application of Ge(Li) Detector to the Measurement of U-238 Fission Yields for $14.4 \mathrm{meV}$ Neutrons," Proceedings of the 2nd All-Union Conference on Neutron Physics, Kiev, 28 May - 1 June 1973, Vol. III, Pages 323-328 (1973). Superseded by 75DAR1.

73DAR3 Daroczy, S., Nagy, S., Kover, L., Raics, P., Csikai, J., To be published (1973). Private communication to B. F. Rider (November 30, 1973). Cited in 73DAR1 as ref. Da73A. Superseded by 75DAR1.

73DAR4 Daroczy, S., German, E., Raics, P., Nagy, S., Csikai, J., Institute of Exp. Phys. Kossuth Univ., Debrecen, Hungary, "Some U-238 Fission Yields for $14.4 \mathrm{meV}$ Neutrons," Privately communicated to B. F. Rider (November 30, 1973). Superseded by 75DAR1. USA National Nuclear Data Center Accession No. 30267 (March 8, 1985). 
73DEE1 Deen, J. R., Draper, E. L., Jr., "Measurement of Fission Product Yields from 232Th in a 252Cf Fission Neutron Spectrum," Trans. Amer. Nucl. Soc. 17, 531-532 (November 1973). NSA 29: 06593 (1974). CA 80: 21825q (1974). Thesis Univ. Texas, Austin, Tex. Diss. Abstr. Int. B 34(5), 2045 (1973). Univ. Microfilms, Ann Arbor, Mich. Order No. 73-25996. Thesis NSA 29: 11556 (1974). Later published as 75DEE1. CSISRS-90551.

73DEN1 Denschlag, H. O., Private communication to 86WAH1.

73DEV1 Devillers, C., Blachot, J., Lott, M., Nimal, B., Van Dat, N., Noel, J. P., Tourreil, R. de, “Bibliotheque de Donnees Relatives aux Produits de Fission," IAEA-SM-170/63 IAEA Symposium on the Applications of Nuclear Data in Science and Technology, Paris, March 12-16, 1973. Describes file format, no yields given.

73DUB1 Dubrovina, S. M., Novgorodtseva, V. I., Morozov, L. N., Pchelin, V. A., Chistyakov, L. V., Shigin, V. A., Shubko, V. M., "Changes of Mass Distribution of Fragments from Th232 Fission with Neutron Energy," Yadern. Fiz. 17, 470-474 (1973). CA 78: 142555u (1973). Measured As77, Cd115, Sr89. NSA 28: 07045 (1973). EXFOR-40193.

73DUD1 Dudey, N. D., Argonne National Laboratory, Private communication to B. F. Rider, (December 12, 1973). CMRMF reactor measurements at $6 \mathrm{kw}$. (neutron spectrum is unfortunately somewhat harder than the future LMFBR which it was desired to simulate but this reactor has the advantage of sufficient fluence to give good statistical data with desireably thin foils). Judged $5 \%$ accurate. CSISRS-90553.

73DUD2 Dudey, N. D., Argonne National Laboratory, Private communication to B. F. Rider, (December 12, 1973). Engineering mockup critical assembly of fast test reactor at Hanford, Washington (representative spectrum of future LMFBR but very low power level which makes low level counting statistically limiting).

73EDE1 Eder, O. J., Lammer, M., "Influence of Uncertainties in Fission Product Nuclear Data on the Interpretation of Gamma Spectrometric Measurements on Burnt Fuel Elements," IAEA/SM-170/12 IAEA Symposium on Applications of Nuclear Data in Science and Technology, Paris, March 12-16, 1973. Details need for fission yield compilation and impact of errors in yields.

73FLY1 Flynn, K. F., Gindler, J. E., Sjoblom, R. K., Glendenin, L. E., "Spontaneous Fission of 253Es and Neutron-Induced Fission of 255Fm and 251Cf," ANL-7996, Pages 8-9 (1973).

73FOR1 Ford, G. P., Wolfsberg, K., Los Alamos Scientific Laboratory, University of California, unpublished preliminary results (April, June, September 1973). Cited in 74WOL1. CSISRS-90555.

73FOR2 Forman, L., Balestrini, S. J., Wolfsberg, K., Jeter, T. R., Proceedings of the International Conference on the Properties of Nuclei Far from Beta Stability (1973). Cited in 74WOL1. CSISRS-90556.

73FOW1 Fowler, M. M., Wahl, A. C., "Yields and Genetic Histories of 128Sb, 129Sb, and 130Sb from Thermal-Neutron Induced Fission of 235U," Submitted to J. Inorg. Nucl Chem. Published as 74FOW1. 
73FOW2 Fowler, M. M., Goth, G. W., Lin, C.-C., Wahl, A. C., "Half Lives of Tin and Antimony Fission Products with $A=128-133, "$ Submitted to J. Inorg. Nucl. Chem. 6-14-73. Published as 74FOW2.

73FUD1 Fudge, A. J., UKAEA, Harwell, Private communication to B. F. Rider (August 2, 1973). From weighted average of relative measurements on $12 \mathrm{Th}-232 \mathrm{flux}$ monitors.

73GAE1 Gaeggeler, H., "Unabhangige Ausbeuten von Pm-150 in der Thermischen Neutronenspaltung von U-233, U-235, Pu-239 und Cf-249, Sowie in der Gammaindurierten Spaltung von U-238," Ph. D. Thesis, Universitat Bern, Switzerland (1973). Superseded by 76GAE1. Initial progress report was EANDC(OR)-133L (June 1973).

73GRI1 Grimm, W., "Charge Distribution of Halogen Neutron Emitters from Neutron Induced Fission of Thorium, Uranium, and Plutonium Isotopes," Mainz Univ. Thesis (1973). 270 p. (In German). NSA 28: 19990 (1973). Cited in 74WOL1.

73GUN1 Gunten, H. R. von, Eidg. Institut fur Reactorforschung, Wurenlingen, Switzerland. Personal communication to K. Wolfsberg, University of California, Los Alamos Scientific Laboratory (August 1973). In author index, von Gunten, H. R., reads (Gunten, H. R. von). Cited in 74WOL1. Superseded by 76GAE1.

73HAR1 Harbour, R. M., Macmurdo, K. W., Troutner, D. E., Hoehn, M. V., “Mass and Nuclear Charge Distributions from Spontaneous Fission of 254Fm," Phys. Rev. C, 8, 14881493 (October 1973). NSA 29: 14283 (1974). CSISRS-90558.

73HAR2 Harbour, R. M., Troutner, D. E., Macmurdo, K. W., “Nuclear Charge Distribution in Fission; Fractional Independent Yields," DP-MS-73-29 (September 27, 1973). Paper at 166th ACS Meeting, Chicago (1973). Independent yields of Xe-135 divided between isomers per 74WOL2. Later published as 74HAR1. CSISRS-90559.

73HER1 Herrmann, G., Private communication to A. C. Wahl (November 1973). Cited in 74WOL1.

73IAE1 IAEA, CINDA73, An index to the literature on microscopic neutron data, a thorough bibliography of literature on yields through 1972 listed under the fissionable nuclide such as $\mathrm{U}-235$, Pu-239 etc.

73JAI1 Jain, H. C., Ramaniah, M. V., "Fission Yield of Molybdenum-98 in the Thermal Neutron Fission of Plutonium-239," Radiochimica Acta, 19(2), 90-93 (1973). These values must be reduced $9.1 \%$ per 73LAM1 by using effective cross sections at 40 degrees $C$. and epithermal index of 0.0022 instead of $2200 \mathrm{~m} / \mathrm{s}$ fission cross sections author used in the comparative method. These neutron temperature corrected values have been listed in this compilation under 71JAI1. NSA 31: 04973 (1975). CA 81: 56787 (1974). Based on earlier report NSA 30: 05101 (1974). EXFOR-30504.

73KIK1 Kikuchi, M., Church, L. B., "A New Method for the Determination of Fractional Fission Yields: The Reaction of Gaseous Methyl Iodide with Fission-Produced Iodine," Radiochimica Acta 19, No. 2, 54-58 (1973). Fractional indirect yield of I-135 [i. e, fractional cumulative yield of Te-135] in U235T is $(0.27 \pm 0.04 / 0.54 \pm 0.04$ ) that of $\mathrm{I}-131$ (taken as 0.997$)=0.499 \pm 0.082$ and fractional independent yield ratio of $\mathrm{I}-135$ to $\mathrm{I}-133$ is ratio of slopes $(-1.36 \pm 0.04 /-0.056 \pm 0.04)$. Assuming sum of both FI's $=0.504$ [i. e. from Table I line 2 Col. 1 
$\& 2$ [one finds that fractional direct fission yields for $135 \mathrm{I}$ and $133 \mathrm{I}$ of $0.481+0.023=0.504$ ] then $(.056 /(1.36+.056))^{*} .504=0.020 \pm 0.014 \mathrm{FI}$ for $\mathrm{I}-133 \mathrm{U} 235 \mathrm{~T}$ and $(1.36 /(1.36+.056)) * 0.504=$ $0.484 \pm 0.024$ FI for I-135 U235T. CA 81:56776 (1974). NSA 30: 15012 (1974). For thesis see NSA 30: 15009 (1974). CSISRS-90561.

73KIR1 Kirouac, G. J., Eiland, H. M., Slavik, C. J., "Fast Neutron Flux Measurement," KAPLP-4005 (1973). 13 pages (preliminary yields of several 237Np fission products). NSA 28: 21196 (1973). EXFOR-10985. CSISRS-90562.

73KOC1 Koch, L., Karlsruhe, Relative Nd yields for neutron spectrum approximating DFR, Rapsodie, and EBR-2. Privately communicated to W. J. Maeck (Nov. 1973).

73KRA1 Kratz, J. V., Franz, H., Herrmann, G., "Delayed-Neutrons from Arsenic Isotopes 84As, 85As, and 86As," J. Inorg. Nucl. Chem. 35, 1407-1417 (1973). NSA 28: 01335 (1973). EXFOR-20521.

73KRA2 Kratz, J. V., Herrmann, G., "Yields of Short-Lived Fission Products in the 50-Neutron Shell Region in Thermal-Neutron Induced Fission of 235U," IAEA/SM-174/14, Page 95 in IAEA Third Symposium on the Physics and Chemistry of Fission, Rochester, N. Y. USA (August 13-17, 1973). For thesis see NSA 29: 19786 (1974). NSA 31: 04989 (1975).

73KRA3 Kratz, K. -L., "Determination of the Fission Yields of Short-Lived Halogen Isotopes;" BMFT-FBK-73-22 (Aug 1973). Jahresbericht, Universitat Mainz. Cited in 73WAL3. NSA 29: 11533 (1974).

73KRA4 Kratz, K. -L., Herrmann, G., "Systematics of Neutron Emission Probabilities from Delayed Neutron Precursors," Z. Physik 263, 435-442 (1973).

73LAM1 Lammer, M., and Eder, O. J., "Discussion of Fission Product Yield Evaluation Methods and a New Evaluation," Paper IAEA/SM-170/13, Symposium on Applications of Nuclear Data in Science and Technology, Paris, March 12-16 1973. NSA 28: 23025 (1973). CA 79: 48376k (1973). NSA 29: 17309 (1974).

73LAR1 Larsen, R. P., Argonne National Laboratory, Private communication to B. F. Rider (Jan. 12, 1973). See 74LAR1.

73LAR2 Larsen, R. P., Dudey, N. D., Heinrich, R. R., Oldham, R. D., Armani, R. J., Popek, R. J., Gold, R., "Fast and Thermal Fission Yields of Short-Lived Gamma-Active Nuclides and Their Neutron Energy Dependence," Submitted to Nucl. Sci. and Engr. (1973). See 74LAR1 for values. Supersedes ANL-7979 pp. 7-11, 72LAR1 and 72LAR3.

73LAR3 Larsen, R. P., "LMFBR Reaction Rate and Dosimetry 7th Quarterly Progress Report," December 1972, January, February 1973. (March 1973). See ANL section. HEDLTME-73-31 (1973). See 74LAR1.

73LAR4 Larsen, R. P., Dudey, N. D., Crouthamel, C. E., Levenson, M., Vogel, R. C., "Chemical Engineering Division Burnup, Cross Sections and Dosimetry Semiannual Report, June 1972 - December 1972," ANL-7979 (March 1973). See 74LAR1. CSISRS-90567.

73LAS1 Laskiewicz, R. A., Golden, G. H., "Comparison of Measured with Predicted Burnups in EBR-II," Trans. Amer. Nucl. Soc. 17, 106-107 (June 1973). 
73LIN1 Lin, C., Wahl, A. C., “Chemical Behavior of Short-Lived Tin Fission Products,” J. Inorg. Nucl. Chem. 35, 1-9 (1973).

73MAE1 Maeck, W. J., "Burnup Determination for Fast Reactor Fuels: 235U Fast Fission Yields," USAEC Report ICP-1050-III (August 27, 1973). Superseded by 74MAE1.

73MAE2 Maeck, W. J., Preliminary results privately communicated to B. F. Rider (November 30, 1973). Superseded by 74MAE1.

73MAN1 Mandler, J. W., Reed, J. H., Moler, R. B., "Relative Yields from Fission of U235 and U238 with 1-, 2-, 4-, 6-, 8-, and 14-meV Neutrons," Paper presented at 1973 Summer Meeting of the American Physical Society, East Lansing, Michigan, (June 18-20, 1973). As cited in 73CUN1. For abstract see Bull. Am. Phys. Soc. 18, 768 (May 1973).

73MAN2 Manohar, S. B., Venkatesan, P. P., Deshmukh, S. M., Prakash, S., Ramaniah, M. V., "Mass Distribution and Recoil Range Measurements in the Reactor Neutron Induced Fission of 232U," BARC-690 pp. 132-134, (1973).

73MAR1 Marmol, P. del, "Identification of New Germanium Isotopes in Fission: Decay Properties and Nuclear Charge Distribution in $A=78$ to 84 Mass Region," Nucl. Phys. A194: No. 1, 140-160 (1972). NSA 27:18710 (1973). In index, del Marmol, P., reads (Marmal, P. del).

73MCE1 Mc Elroy, W. N., Hanford Engineering Development Laboratory, USAEC, Private communica tion to B. F. Rider, Oct 2, 1973. CSISRS-90570.

73MCE2 Mc Elroy, W. N., (Ed.) “LMFBR Reaction Rate and Dosimetry 7th Quarterly Progres Report," HEDL-TME 73-31, Hanford Engineering Development Laboratory (Mar. 1973). Gives Np237F yields in EBR-II later cited in Table XV, Col 5 of 75MCE1. In 75MCE1, however, the results are normalized to the $\mathrm{Ba}-140$ CFRMF value of $\mathrm{Col} 4$ (a slight $2.4 \%$ adjustmen well within the experimental error.)

73MUS1 Musgrove, A. R. del, Cook, J. L., Trimble, G. D., "Prediction of Unmeasured Fission Product Yields," Paper at IAEA Panel on Fission Product Nuclear Data, Bologna, Itał (November 26-30, 1973). Printed 74MUS1. In author index, del Musgrove, A. R., reads (Mus grove, A. R. del ).

73NET1 Nethaway, D. R., Lawrence Livermore Laboratory, University of California, Unpublished wort 1973. Cited in 74NET1. Isomeric independent yields are here split per 77MAD2. CSISRS90571.

73NET2 Nethaway, D. R., Barton, G. W., "A Compilation of Fission Product Yields in Use at the Lawrence Livermore Laboratory," UCRL-51458 (October 3, 1973). CA 81: 84 (1974). NSA 29: 11541 (1974).

73PAR1 Parsons, R. W., Sharma, H. D., "Method for the Determination of Independent Yield. in Fission with a Ge(Li) Detector," Radiochem. Radioanal. Lett. 15(4-5), 335-345 (N 20, 1973). Univ. of Waterloo, Ont. Replaces 73SHA1 in part. NSA 29: 09832 (1974). CSISRS 90576. Also CSISRS-90572. 
73PLE1 Pleasonton, F., Ferguson, R. L., Plasil, F., Bemis, C. E., "Fragment-Mass and KineticEnergy Distributions from the Spontaneous Fission of $246 \mathrm{Cm}$," Phys. Rev. C, 8 , 1018-1022 (September 1973). Graphic presentation of pre-neutron emission mass distribution. See original paper for data.

73PUR1 Purkayastha, B. C., Bhattacharyya, D. K., "The Ràdiochemical Yield in the Spontaneous Fission of 238U," J. Inorg. Nucl. Chem. 35, 1793-1796 (1973). NSA 28: 09695 (1973).

73RAO1 Rao, S. A., Kuroda, P. K., "Charge Distribution in Neutron-Induced Fission of Thorium," J. Inorg. Nucl. Chem. 35, 1443-1449 (1973). NSA 28: 01384 (1973). CA 78: 153829n (1973). Isomeric independent yields are here split per 76MAD2. CSISRS-90574.

73ROB1 Robin, M., Hageman, R., Bouchard, J., Frejaville, G., Vidal, R., "Importance des Constantes Nucleaires des Produits de Fission dans la Determination du Taux de Combustion," French paper contributed to review 73MAE1. Full text in 74ROB1.

73RUD1 Rudstam, G., Grapengiesser, B., Lund, E., "Evaluation of Half-Life Data for Short-Lived Fission Products," Appendix B, Review Paper No. 12, LAEA Panel on Fission Product Nuclear Data, Bologna, Italy (November 26-30, 1973). Full text in 74RUD1.

73SAN1 Santos, et. al. Progress Report of $14.7 \mathrm{meV}$ TH232H Ag-115 Yield, Short Note, Table in INDC(SEC)-35 (Sept 1973). Institute de Engenharia Nuclear, Rio de Janeiro, Brazil. Cited in 74IAE1. Not coded.

73SHA1 Sharma, H. D., Personal communication to D. Troutner (September 20, 1973). Cited in 74WOL1.

73SK01 Skovorodkin, N. V., Lozhkomoev, K. A., Petrzhak, K. A., Sorokina, A. V., Alexandrov, B. M., Krivokhatskii, A. S., "Fragment Yields from Spontaneous Fission of 252Cf," Atomnaya Energiya, 34, 365-371 (1973). NSA 28: 19988 (1973). Alexandrov, B. M., is transliterated Aleksandov, B. M., elsewhere. EXFOR-40205.

73SKO2 Skovorodkin, N. V., "Fragment Yield Curves from Slow Neutron Fission of 241Am and 241Pu," Atomnaya Energiya, 35, 409-416 (1973). NSA 29: 23021 (1974). EXFOR-40389.

73TON1 Tong, S. L., Fritze, K., Prestwich, W. V., "Mass-Yield Variations in the Thermal and Epithermal Fissions of 239Pu," J. Inorg. Nucl. Chem. 35, No. 9, 3079-3086 (Sept 1973). NSA 28: 28889 (1973). CSISRS-90579.

73TRA1 Tracy, B. L., Pleva, J. F., Thode, H. G., "Cumulative Krypton and Xenon Yields from the Neutron-Induced Fission of $237 \mathrm{~Np}, 238 \mathrm{~Np}, 241 \mathrm{Am}$, and $242 \mathrm{Am}$," J. Inorg. Nucl. Chem., 35, 2639-2651 (1973). CA 79:120609h (1973). NSA 28:23032 (1973). CSISRS-90580.

73TRO1 Troutner, D. E., Harbour, R. M., "Fractional Independent Yields of $111 \mathrm{~m}-\mathrm{Pd}$ and 112 Ag from Thermal-Neutron-Induced Fission of 249Cf," J. Inorg. Nucl. Chem., 35, 11-17 (1973). NSA 27: 21154 (1973). CSISRS-90581.

73TR02 Tromp, R. L., Emel, W. A., Kussy, M. E., Erikson, A. L., Delmore, J. E., Maeck, W. J., "Absolute Fast Reactor Fission Yields for 235U," A paper presented at the Seventeenth Conference on Analytical Chemistry in Nuclear Technology, October 23-25, 1973 at Gatlinburg, Tennessee. Exact duplicate of data in 73MAE1. See 73MAE1 for values. 
73TRO3 Troutner, D. E., Personal communication to K. Wolfsberg, (October 12, 1973). Cited in 74WOL1. CSISRS90582.

73UME1 Umezawa, H., "Independent Isomeric Yield Ratio of 148Pm in the Thermal-Neutron Induced Fission of 233U," J. Inorg. Nucl. Chem. 35, 353 (1973). NSA 27: 23609 (1973). EXFOR-22069 (Dec 22, 1988).

73UNI1 Unik, J. P., Gindler, J. E., Glendenin, L. E., Flynn, K. F., Gorski, A., Sjoblom, R. K., "Fragment Mass and Kinetic Energy Distributions for Fissioning Systems Ranging from Mass 230 to 256," IAEA-SM-174/209. Third IAEA Symposium on the Physics and Chemistry of Fission, Rochester, N. Y. (August 13-17, 1973). Appears as "Physics and Chemistry of Fission 1973," Vol. II, 19-45 (1974). Supersedes USNDC-9, 36 (February 1973). CSISRS90584. It is assumed that this supersedes 72FLY1 because of its later publication date. Es254T, Cf251T and Fm254S data superseded by 76FLY1, 75FLY3 and 77GIN1, respectively.

73VAN1 Van Assche, P. H. M., Vandenput, G., Jacobs, L., van den Cruyce, J. M., Silvernans, R., "The Mass Distribution of Neutron Induced Fission for $\mathrm{Pu}-239$ at the $0.287 \mathrm{eV}$ Resonance," RCN-203, 95 (Dec 1973). EXFOR-21560.

73VEN1 Venezia, A., Ph. D. Thesis, Hebrew University, Jerusalem. (1973). Cited in 73AMI1. See earlier progress in Israel Annual Report IA-1262 page 80 (December 1972).

73VID1 Vidal, R., Neodymium yields in Pu-241 fast neutron fission EANDC(E)157U2 cited in 74IAE1. Superseded by coauthored 73ROB1.

73VOR1 Vorob'yeva, V. G., D'jachenko, N. D., Kuz'minov, B. D., Sergachev, A. I., Surin, V. M., "Mass Yields and Kinetic Energy of Fragments for Fission of Plutonium Isotopes," Conf. 73Kiev 3, 270 (May 1973). EXFOR-40284. Monoenergetic Neutrons.

73WAG1 Wagemans, C., Deruytter, A. J., "Ratio of the Ternary to Binary Fission Induced by Thermal and Resonance Neutrons in 239Pu," Nucl. Phys. A212: No. 3, 556-572 (1973). NSA 28: 28898 (1973).

73WAH1 Wahl, A. C., "Radiochemical Studies of Nuclear Fission and Search for Element 118 (Eka-Radon)," CO0-1162-46 Progress Report April 1, 1972-March 31, 1973. NSA 27: 18764 (1973).

73WAH2 Wahl, A. C., Private communication to B. F. Rider (November, 1973).

73WAL1 Walker, W. H., "Fission Product Data for Thermal Reactors; Part II-Yields," Chalk River Nuclear Laboratories, Chalk River, Ontario, AECL-3037 Part II (April 1972). Supersedes 70WAL1. NSA 28: 19956 (1973). CA 80: 32537y (1974).

73WAL2 Walker, W. H., "Yields in Thermal Neutron Fission-Some Results and Recommendations Based on a Recent Evaluation," AECL-4453 (March 1973). Selected high cross section nuclides cited from 73WAL1. NSA 28: 04200 (1973).

73WAL3 Walker, W. H., "Status of Fission Product Yield Data for Thermal Reactors," AECL4704, IAEA Panel on Fission Product Nuclear Data, Bologna, Italy, (November 26-30, 1973). See 74WAL1. NSA 30: 08488 (1974). It is suggested on pg. 40, para. 4, sentence 2 that cumulative yield of $\mathrm{I} 135$ can be calculated from cumulative yield of Xe135 minus the independent 
yield of Xe135 < for U235T $1.00-0.035=0.965$ as seen in Table 4 Cols. $1 \& 2>$. This is useful because it is not possible to determine $\mathrm{I135}$ radiochemically to so great an accuracy as it is possible by this small correction to an accurately done mass spectrometric measurement of the cumulative yield of Xe135.

73WOL1 Wolfsberg, K., Ford, G. P., Erdal, B. R., Private communication to B. F. Rider, October 1973. CSISRS-90587.

73ZAK1 Zakharova, V. P., Rjazanov, D. K., Basova, B. G., Rabinovich, A. D., Korostylev, V. A., Yadern. Fiz. 18(6), 1145 (Jun 1973). EXFOR-40232.

74AMI1 Amiel, S., Feldstein, H., "Odd-Even Systematics in Neutron Fission Yields of Uranium Isotopes 233 and 235," Submitted to Phys. Rev. C (July 1974). See 75AMI1.

74AMI2 Amiel, S., Izak-Biran, T., To be published (1974). Cited in 74AMI1.

74AMI3 Amiel, S., "Status of Delayed Neutron Data," Print of 73AMI2. In "Proceedings of a Panel on Fission Product Nuclear Data, held in Bologna, 26-30 November 1973," Technical Report IAEA-169, Vol. II, 33-52 (1974). Available by $\$ 0.65$ prepayment from INIS Microfiche Clearinghouse, IAEA, Kaertner Ring 11, P. O. Box 590, A-1011, Vienna, Austria.

74BAL1 Ballou, N. E., Kaye, J. H., Reeder, P. L., Anderl, R. A., Stoffels, J. J., "Independent and Cumulative Fission Yields for Several Fission Systems," Paper 16, Nuclear Chemistry and Technology Div., 167th American Chemical Society National Meeting, Los Angeles, April 1-4, 1974. From "Final Report on ARPA Fission Yield Project work at BattelleNorthwest, April 1970 - April 1873," BNWL-B - 281 (1973). NSA 32: 23474 (1975). INIS Atomindex 7: 223087 (1976). CSISRS-90589. EXFOR-13274.

74BAL2 Balestrini, S. J., Forman, L., "Independent Fission Yields of Rb and Cs from $238 \mathrm{U}$ by Fission-Spectrum Neutrons," Phys. Rev. C, 10, No. 5, 1872-1879 (November 1974). NSA 31: 12958 (1975). CSISRS-90590.

74BLA1 Blachot, J., Carraz, L. C., Cavallini, P., Chauvin, C., Ferrieu, A., Moussa, A., “Distribution en Masse dans la Fission de $238 \mathrm{U}$ par des Neutrons de $14 \mathrm{meV}$, J. Inorg. Nucl. Chem. 36, 495-501 (1974). Supersedes 71BLA1. NSA 30: 20191 (1974). EXFOR-21590, -21736.

74CRO1 Crouch, E. A. C., "Assessment of Known Independent Yields and the Calculation of Those Unknown in the Fission of 232Th, 233U, 235U, 238U, 239Pu, 240Pu and 241Pu," AERE - R 7680 (May 1974). NSA 30: 31008 (1974). NSA 31: 31536 (1975).

74CUN1 Cuninghame, J. G., Goodall, J. A. B., and Willis, H. H., "Absolute Yields in the Fission of $235 \mathrm{U}$ by Mono-Energetic Neutrons of Energy 130-1700 keV," J. Inorg. Nucl. Chem. 36, 1453-1457 (1974). CA 81: 144373u (1974). EXFOR-20769.

74CUN2 Cuninghame, J. G., "Review of Fission Product Yield Data for Fast Neutron Fission," Print of 73CUN1. In "Proceedings of a Panel on Fission Product Nuclear Data, held in Bologna, 26-30 November 1973," Technical Report IAEA-169, Vol. I, 353-434 (1974). Available by $\$ 0.65$ prepayment from INIS Microfiche Clearinghouse, IAEA, Kaertner Ring 11, P. O. Box 590, A-1011, Vienna, Austria. NSA 33: 27722 (1976). 
74DAN1 Daniels, W. R., Ford, G. P., Hoffman, D. C., Wolfsberg, K., "Branching Ratio for the Decay of $135 I$ to the Isomers of $135 X e$," J. Inorg. Nucl. Chem., 36, 201-203 (1974).

74DAR1 Daroczy, S., German, E., Raics, P., Nagy, S., Csikai, J., "Some U-238 Fission Yields for $14.4 \mathrm{meV}$. Neutrons," EXFOR30267. Data on cumulative yields for 20 nuclides, revised data cited in CINDA74 supplement (December 1974). Numerical data on file and available from all 4 neutron data centers (USA, USSR, NEA, IAEA) on request. Supersedes 73DAR2 and INDC(HUN)-11, 26, 7309. From Csikai at Smolenice, Czech. CONF 741022c (1 Sept 1974). NSA 33: 10928 (1976). Superseded by 75DAR1.

74DAR2 Daroczy, S., Raics, P., Nagy, S., "Compiled Fission Product Yields of U238 for $14 \mathrm{meV}$ Neutrons," Print of 73DAR1. In "Proceedings of a Panel on Fission Product Nuclear Data, held in Bologna, 26-30 November 1973," Technical Report IAEA-169, Vol. III, 281-322 (1974). Available by $\$ 0.65$ prepayment from INIS microfiche clearinghouse, IAEA, Kaertner Ring 11, P. O. Box 590, A-1011, Vienna, Austria. NSA 33: 27729 (1976). Superseded by 75DAR1.

74DJA1 D'Jachenko, N. P., Kabenin, V. N., Kolosov, N. P., Kuz'minov, B. D., Sergachjov, A. I., “Yields and Kinetic Energies of Fission Fragments by Spontaneous and Induced Fission of Pu-242," (YFI-17,3,7408). EXFOR-40195. Monoenergetic neutrons. EXFOR-40194.

74EGG1 Egger, C., Gunten, H. R. von, Schmid, A., Pruys, H. S., "Absolute Yields of 89 Mo in the Thermal Neutron-Induced Fission of $233 \mathrm{U}$ and 239Pu," Radiochim. Acta 21(3/4), 200-202 (1974). INIS Atomindex 7: 239341 (1976). NSA 33: 31875 (1976). EXFOR-22059 (Dec $11,1987)$.

74EIC1 Eichor, M., "The Independent Yield of 138Cs from Thermal-Neutron-Induced Fission of 249Cf," J. Inorg. Nucl. Chem., 36, 3880-3882 (1974). CSISRS-90596.

74FOR1 Ford, G. P., Wolfsberg, K., and Erdal, B. R., "Independent Yields of $133 \mathrm{~m} \mathrm{Xe,} \mathrm{133g} \mathrm{Xe,}$ and $135 \mathrm{~m}$ Xe and $135 \mathrm{~g}$ Xe from Fission," Paper 19, Nuclear Chemistry and Technology Div., 167th American Chemical Society National Meeting, Los Angeles, April 1-4, 1974.

74FOW1 Fowler, M. M., Wahl, A. C., "Yields and Genetic Histories of 128Sb, 129Sb and 130Sb from Thermal-Neutron Induced Fission of 235U," J. Inorg. Nucl. Chem. 36, 1201-1212 (1974). CA 81: 84634 (1974). NSA 30: 17394 (1974). CSISRS-90597.

74FoW2 Fowler, M. M., Goth, G. W., Lin, C., Wahl, A. C., "Half Lives of Tin and Antimony Fission Products with $A=128-133, "$ J. Inorg. Nucl. Chem. 36, 1191-1198 (1974). NSA 30: 17380 (1974). CSISRS-90598.

74GRA1 Grapengiesser, B., Lund, E., Rudstam, G., "Survey of Short - Lived Fission Products Obtained Using the Isotope - Separator - On - Line Facility at Studsvik," J. Inorg. Nucl. Chem., 36, 2409-2431 (1974).

74HAR1 Harbour, R. M., Troutner, D. E., Macmurdo, K. W., "Nuclear Charge Distribution in Fission: Fractional Independent Yield of 135Xe from Thermal- Neutron- Induced Fission of $245 \mathrm{Cm}$ and 249Cf," Phys. Rev. C 10, 769-773 (August 1974). With 135Xe metastable/ground states split per 76MAD2. Supersedes 73HAR2. CA 81: 129994d (1974). NSA 30: 28529 (1974). CSISRS-90599. 
74HAW1 Hawa, A. H., Baeckmann, A. von, "Simultaneous Determination of 235U and 239Pu Traces by Gamma-Spectrometric Analysis of Noble Fission Gases Following Neutron Activation," KFK-1888 (January 1974). Thesis 59 pages (In German). NSA 29: 28969 (1974). EXFOR-21558.

74LAE1 IAEA, CINDA74, An index to the literature on microscopic neutron data.

74JAM1 James, W. D., Adams, D. E., Beck, J. N., Kuroda, P. K., "Investigation of the Reported Mass Distribution Curves in the Fission of $233 \mathrm{U}$ with $14.8 \mathrm{meV}$ Neutrons," ORO4282-14 (1974). Preprint of 75JAM1.

74JEN1 Jensen, C. M., Rogers, V. C., Jensen, G. L., "Relative Thermal and 450-keV Fission Product Yields in 239-Pu," Trans. Am. Nucl. Soc. 19, 397-398 (1974). NSA 31: 07403 (1975). CSISRS-90601.

74KIR1 Kirouac, G. J., Eiland, H. M., Slavik, C. J., "Fast Neutron Flux Measurement," Report No. KAPL-P-4005 (January 1974). USA Knolls Atomic Power Laboratory. Contains CF252S yields. Lexan and mica detectors used to measure fissions; $\mathrm{Ge}(\mathrm{Li})$ for gamma detection of fission products. USA National Nuclear Data Center Accession No. 10985 (March 5, 1982). Sr-91, Sr-92, Zr-97, I-132 appear to be over estimated possibly by under correction for Compton scattering background from higher energy unseparated fission products. Ba-140 appears to be a good measurement possibly because the $1.596 \mathrm{meV}$ gamma ray from its La-140 daughter is virtually the highest energy gamma ray present, hence immune to such interference. EXFOR10985.

74LAM1 Lammer, M., "Yields in Fast Neutron Fission of U-238 and Th-232," in "Proceedings of a Panel on Fission Product Nuclear Data, held in Bologna, 26-30 November 1973," Technical Report IAEA-169, Vol. III, 245-279 (1974). Available by $\$ 0.65$ prepayment from INIS Microfiche Clearinghouse, IAEA, Kaertner Ring 11, P. O. Box 590, A-1011, Vienna, Austria. NSA 33: 27728 (1976).

74LAM2 Lammer, M., "Evaluation of Fractional Yields," in "Proceedings of a Panel on Fission Product Nuclear Data, held in Bologna, 26-30 November 1973," Technical Report IAEA-169, Vol. II, 282-296 (1974). Available by $\$ 0.65$ prepayment from INIS Microfiche Clearinghouse, IAEA, Kaertner Ring 11, P. O. Box 590, A-1011, Vienna, Austria.

74LAR1 Larsen, R. P., Dudey, N. D., Heinrich, R. R., Oldham, R. D., Armani, R. J., Popek, R. J., Gold, R., "The Yield of Short-Lived Gamma-Ray Emitting Nuclides from Fast- and Thermal-Neutron Fission," Nucl. Sci. and Engr. 54, 263-272 (1974) CA 81: 57255p (1974). NSA 30: 20194 (1974). Isomeric independent yields are here split per 76MAD2. EXFOR-12771. CSISRS- 90603. EXFOR-13271.

74LAR2 Larsen, R. P., Argonne National Laboratory, Private communication to W. J. Maeck of EBR-2 Row 2 and 4 data at $11.5 \%$ Burnup of highly enriched U-235 by fast fission (harder spectrum than commercial LMFBR) (October 1974). Cited in 74MAE1. CSISRS-90604.

74MAE1 Maeck, W. J., "Fast Reactor Fission Yields for 233U, 235U, 238U, 239Pu and Recommendations for the Determination of Burnup on FBR Mixed Oxide Fuels: An Interim Project Report," ICP-1050-1 (1974). Preprint. PU239F values are preliminary. Presented also as a paper at conference: 75 WASH,1,378,7503. CSISRS-90605. 
74MAE2 Maeck, W. J., Emel, W. A., Tromp, R. L., "Revised Fission Yield Data for Light and Heavy Mass Peaks for Fast Reactor EBR-I Fissioned Uranium-235," ICP-1058 (October 1974). NSA 31: 10284 (1975). Superseded by 74MAE1.

74MCE1 Mc Elroy, W. N., Hanford Engineering and Development Laboratory, Private communication to B. F. Rider, March 13, 1974. CSISRS-90606.

74MEE0 Meek, M. E., Rider, B. F., Th-232 estimates based on all measured values plus the assumption that the light and heavy mass peaks should each sum to $100 \%$ and nubar (from ENDF/B-IV) measures 2.49 neutrons per fission experimentally.

74MEE1 Meek, M. E., Rider, B. F., "Compilation of Fission Product Yields, Vallecitos Nuclear Center 1974," General Electric Company Report NEDO-12154-1 (January 26, 1974). NSA 30: 17391 (1974). INIS Atomindex 28(20): 334131 (1977).

74MEE2 Meek, M. E., Rider, B. F., Estimated cumulative yield from Wahl model (normally found from calculation on a previous iteration) to prevent distortion of independent yields where a measured independent yield is larger than the measured cumulative yield of that nuclide which would otherwise infer zero independent yields from all precursors.

74MEE3 Meek, M. E., Rider, B. F., Estimated independent yield from Wahl model where conflicts in data infer large independent yields in obvious disagreement with charge distribution. (e. g., where a chain yield is measured to be $6.5 \%$ yield and its precursor is measured to have a cumulative yield of $6.1 \%$, an independent yield of 6.5 minus $6.1=0.4 \%$ is inferred but the Wahl model may predict $1.0 \mathrm{e}-9 \%$ for this stable $\mathrm{Z}$. In this case we believe the model prediction that the two final chain members cumulative yields should differ by only $1.0 \mathrm{e}-9 \%$ ).

74MEE4 Meek, M. E., Rider, B. F., Fast yields estimated from thermal yield for U235F and PU239F only. Note the wing and valley yields increase with compensating slight decrease in peak region. Smooth curves through measured fast/thermal yield ratios are within $10 \%$ (1 sigma) of all data. In the range $500 \pm 400 \mathrm{keV}$ median fission energy, fast yields are almost independent of neutron energy in peak regions.

74MEE5 Meek, M. E., Rider, B. F., Estimated fractional fission yield from charge distribution model (normally computed in previous iteration).

74MEE6 Meek, M. E., Rider, B. F., More valley yields are unmeasured than in any other region. We have estimated valley yields assuming no fine structure in the valley. This should result in a smooth shaped valley. To keep from influencing the absolute peak-to-valley ratio, these smooth estimates are usually made relative to the most accurately measured valley nuclide.

74MEE7 Meek, M. E., Rider, B. F., It is assumed that where the independent yield of the final chain members is negligible, the branching fraction for radioactive decay is independent of the fissioning nuclide. In the case of $\mathrm{Kr}-85$, the independent yield of $\mathrm{Kr} 85$ is not negligible. In U235T fission, the total cumulative yield for $\mathrm{Kr} 85 \mathrm{~g}$ is $0.28519 \%$, of which the cumulative yield for $\mathrm{Kr} 85 \mathrm{~g}$ from its independent yield is $\mathbf{0 . 0 2 7 4 7 \%}$, leaving the cumulative yield of $\mathrm{Kr} 85 \mathrm{~g}$ from radioactive decay from $\mathrm{Kr} 85 \mathrm{~m}=0.25772 \%$. The total cumulative yield of $\mathrm{Kr} 85 \mathrm{~m}=1.28996 \%$. Hence, the true radioactive branching fraction of $\mathrm{Kr} 85 \mathrm{~m}$ to $\mathrm{Kr} 85 \mathrm{~g}$ is $(0.25772 / 1.28996)=0.19979$. By adding back the independent yield of $\mathrm{Kr} 85 \mathrm{~g}$ to the $\mathrm{Kr} 85 \mathrm{~g}$ from radioactive decay of $\mathrm{Kr} 85 \mathrm{~m}$, as it appears in actual fission product samples, one obtains an observable ratio of cumulative yield 
of $\mathrm{Kr} 85 \mathrm{~g}$-to- cumulative yield of Rb85 of $0.2164,0.2237$, and 0.2312 for $\mathrm{U} 235 \mathrm{~T}$, PU239T, and $\mathrm{U} 233 \mathrm{~T}$, respectively. This computed U235T ratio of 0.2164 is in reasonable agreement with the value of $0.2160 \pm 0.0019$ measured by ( $80 \mathrm{JAF} 1$ ). The $\mathrm{Kr} 85$ independent yields used in these computations are an average of the mass 85 independent yields computed from the $\mathrm{Zp}$ model and the A'p model for charge distribution in fission by (86WAH1) split between Kr85m and $\mathrm{Kr} 85$ isomers according to the angular momentum model described by (76MAD2).

74MEE8 Meek, M. E., Rider, B. F., Estimated yields for U235HE based on the assumption that the light and heavy mass peaks should each sum to $100 \%$ and that nubar (from ENDF/B-IV) measured experimentally to be 4.38 neutrons per fission.

74MEE9 Meek, M. E., Rider, B. F., Estimated yields for U238HE based on the assumption that the light and heavy mass peaks should each sum to $100 \%$ and that nubar (from ENDF/B-IV) measured experimentally to be 4.41 neutrons per fission.

74MUS1 Musgrove, A. R. del, Cook, J. L., Trimble, G. D., "Prediction of Unmeasured Fission Product Yields," Print of 73MUS1. in "Proceedings of a Panel on Fission Product Nuclear Data, held in Bologna, 26-30 November 1973," Technical Report IAEA-169, Vol. II, 163-200 (1974). Available by $\$ 0.65$ prepayment from INIS microfiche clearinghouse, IAEA, Kaertner Ring 11, P. O. Box 590, A-1011, Vienna, Austria. NSA 33: 27724 (1976).

74NET1 Nethaway, D. R., "Variation of Zp in Fission with Changes in Excitation Energy and Compound Nucleous," UCRL-51538 (Feb. 6, 1974). NSA 30: 01682 (1974).

74NET2 Nethaway, D, R., "Tables of Values of Zp, The Most Probable Charge in Fission," UCRL-51640 (July 2, 1974).

74PAF1 Paffrath, G., "Determination of the Charge Distribution in the 132 Chain by the Fission of Plutonium-239 with Thermal Neutrons," NP-20343 1973 Annual Report University of Mainz, Pages 85-90 (15 Jun 1974). Sb-132g and $\mathrm{m}$ values are incorrect due to an arithmetic error. Those values are superseded and corrected by 76PAF1 and 77DEN1. NSA 32: 15933 (1975).

74PAR1 Parsons, R. W., Sharma, H. D., "Nuclear Charge Distribution in Mass Chains 131-134 in Thermal Neutron Fission of 235U," J. Inorg. Nucl. Chem. 36, 2392-2396 (1974). NSA 31: 4972 (1975). Replaces 73SHA1 in part. CSISRS-90608.

74RA01 Rao, V. K., Bhargava, V. K., Marathe, S. G., Sahakundu, S. M., Iyer, R. H., "Search for Low Yield Products in the Neutron-Induced Highly Asymmetric Fission of Uranium," Phys. Rev. C, 9, No. 4, 1506-1514 (April 1974). Data on $66 \mathrm{Ni}, 67 \mathrm{Cu}, 72 \mathrm{Zn}, 161 \mathrm{~Tb}, 172 \mathrm{Er}$, $175 \mathrm{Yb}, 177 \mathrm{Lu}$ in U235T and U238F. Supersedes INDC(SEC)-28 (September 1972). NSA 30: 14327 (1974). EXFOR-30496.

74ROB1 Robin, M., Hageman, R., Bouchard, J., Frejaville, G., Vidal, R., “Importance des Constantes Nucleaires des Produits de Fission dans la Determination du Taux de Combustion," Print of 73ROB1. In "Proceedings of a Panel on Fission Product Nuclear Data, held in Bologna, 26-30 November 1973," Technical Report IAEA-169, Vol. III, 5969 (1974). Available by $\$ 0.65$ prepayment from INIS Microfiche Clearinghouse, IAEA, Kaertner Ring 11, P. O. Box 590, A-1011, Vienna, Austria. EXFOR-22050 (Nov 18, 1988). 
74RUD1 Rudstam, G., Grapengiesser, B., Lund, E., "Evaluation of Half-Life Data for Short-Lived Fission Products," Print of 73RUD1. In "Proceedings of a Panel on Fission Product Nuclear Data, held in Bologna, 26-30 November 1973," Technical Report IAEA-169, Vol. II, 1-32 (1974). Available by $\$ 0.65$ prepayment from INIS Microfiche Clearinghouse, IAEA, Kaertner Ring 11, P. O. Box 590, A-1011, Vienna, Austria.

74SIE1 Siegert, G., Wollnik, H., Greif, J., Fiedler, G., Asghar, M., Bailleul, G., Bocquet, J. P., Gautheron, J. P., Schrader, H., Ewald, H., Armbruster, P., "Direct Determination of the Nuclear Charge Distribution of Mass Separated Fission Products from $235 U(n$ th, $f)$," Phys. Lett. 53B, No. 1, 45-47 (11 Nov 1974). NSA 31: 15499 (1975).: Isomeric independent yields are here split per 75MEE2 and 77MAD2. EXFOR-21594.

74SIE2 Siegert, G., Wollnik, H., Greif, J., Fiedler, G., Asghar, M., Bailleul, G., Bocquet, J. P., Gautheron, J. P., Schrader, H., Ewald, H., Armbruster, P., "Nuclear Charge Distribution in the Isobars 89 to 99 Resulting From Thermal Neutron Fission of Uranium-235," Institute Laue-Langevin, Grenoble. A detector of 8 micrometers thickness was chosen [but] the thickness variation was still too large. Thus we limited the detector area in steps down to 2 square $\mathrm{mm}$ which yielded a reasonable resolution for adjacent nuclear charges. The small area limited the count rate to a few counts per second. This limitation in detector area explains, why an earlier experiment with the same detector did not yield significant results at the mass spectrometer for fission products in Munich (72GUN1), where the density of fission products was two orders of magnitude smaller than at the Lohengrin. Isomeric independent yields are here split per 76MAD2.

74SIL1 Silva, C. Feu Alvim da, Crancon, J., "Measurement of Some Cumulative Fraction in the 238U Fission Induced by 14-meV Neutrons," CEA-N-1784, Page 2, NSA 32: 21261 (1975). U238HE yields by Wolfsberg's method. Superseded by 77FEU1. Cross-indexed under (Feu Alvim, C. A.). EXFOR-21591.

74VOR1 Vorob'eva, V. G., D'yachenko, P. P., Kolosov, N. P., Kuz'minov, B. D., Sergachev, A. I., Surin, V. M., "Yields and Kinetic Energies of Fragments from Fission of the 241-Pu Nuclei Induced by Fast Neutrons," Yadern. Fiz. 29: No. 6, 1216-1221 (June 1974). In Russian. NSA 30: 22858 (1974). EXFOR-40282.

74VOR2 Vorob'eva, A. A., Grachev, V. T., Kondurov, I. A., Miroshnichenko, Yu. A., Nikitin, A. M., Seliverstov, D. M., and Smirnov, N. N., "Ternary Fission of 239Pu and $242 \mathrm{~m}-\mathrm{Am}$ by Neutrons," Yadern. Fiz. 20, 461-466 (Sept 1974). (In Russian). Ternary Fission products in Pu239T (2H, 3H, 6He, 8He, through 200 with upper limits through Fe-60). English translation is Soviet Journal of Nuclear Physics 20(3), 248-250 (Mar 1975).

74WAL1 Walker, W. H., "Status of Fission Product Yield Data for Thermal Reactors," Print of 73WAL3. In "Proceedings of a Panel on Fission Product Nuclear Data, held in Bologna, 26-30 November 1973," Technical Report IAEA-169, Vol. I, 285-352 (1974). Available by $\$ 0.65$ prepayment from INIS Microfiche Clearinghouse, IAEA, Kaertner Ring 11, P. O. Box 590, A-1011, Vienna, Austria.

74WOL1 Wolfsberg, K., "Estimated Values of Fractional Yields from Low Energy Fission and a Compilation of Measured Fractional Yields," USAEC Report LA-5553-MS (1974). Available as printed copy $\$ 7.60$, Microfiche $\$ 1.45$ from NTIS, U. S. Dept. of Commerce, 5285 Port 
Royal Road, Springfield, Virginia, 22151 USA. NSA 30: 17390 (1974). Isomeric independent yields are here split per 76MAD2.

74WOL2 Wolfsberg, K., Private communication to B. F. Rider (April 10, 1974) of current measurements. These include $133 \mathrm{Xe}$ and $135 \mathrm{Xe}$ from $238 \mathrm{U}, 239 \mathrm{Pu} 233 \mathrm{U}, 235 \mathrm{U}, 242 \mathrm{~m}-\mathrm{Am}$ at various neutron energies (thermal, fast, and $14 \mathrm{MeV}$ ) and $254 \mathrm{Cf}$ in spontaneous fission. These data were shown as slides in the presentation: "Fractional Cumulative Yields of Some Krypton and Xenon Nuclides from Fission-Spectrum Neutron Induced Fission of $235 U$ and 238U," Paper 18, Nuclear Chenistry and Technology Div., 167th American Chemical Society National Meeting, Los Angeles, April 1-4, 1974. Xe-133 and Xe-135 metastable to ground state independent yield ratios are $2.93 \pm 0.28$ and $1.41 \pm 0.13$, respectively, for all fissioning nuclides. The complete paper No. 18 is published as 75WOL1. CSISRS-90612.

74WOL3 Wolfsberg, K., and Ford, G. P., "Mass Yields from Spontaneous Fission of 254Cf," Paper 21, Nuclear Chemistry and Technology Div., 167th American Chemical Society National Meeting, Los Angeles, April 1-4, 1974. CSISRS-90613. EXFOR-13467. 143Xe yield of Cf254S does not appear in the paper but was taken from private communication 74WOL2 above.

74WOL4 Wolfsberg, K., Relative independent yields of 73BAL1 and 73BAL2 made absolute by normalizing to one or more measured or estimated independent yields on page $16174 \mathrm{WOL} 1$ and used here because the present computer code for NEDO-12154 does not yet handle relative independent yields. CSISRS-90614.

74YUR1 Yurova, L. N., Bushuev, A. V., Kozhin, A. F., "Determination of Certain Fragment Yields During Fission of Uranium-238 by Reactor Spectrum Neutrons," At. Energ. 36(1), 66-69 (1974). (Russian). CA 80: 102806m (1974). First appeared in Proceedings of the 2nd All-Union Conference on Neutron Physics, Kiev, 28May - 1June 1973, Vol. III, Pages 315-322 (1973). (In Russian). Sov. J. At. Energ. 36, 75 (July 1974). English translation of Russian. NSA 33: 10927 (1976). EXFOR-40206.

75ADA1 Adams, D. E., James, W. D., Beck, J. N., Kuroda, P. K., "Distribution of Fission Yields in the $14.8 \mathrm{meV}$ Neutron-Induced Fission of 238U," J. Inorg. Nucl. Chem. 37, 419-424 (1975). NSA 32: 07829 (1975). Based on Thesis, University Microfilm Order No. 75-27644, NSA 33: 21723 (1976). INIS Atomindex 7: 250642 (1976). CSISRS-90616.

75AJI1 Ajitanand, N. N., Choudhury, R. K., Kapoor, S. S., "Determination of Fragment Isotopic Yields in the Fission of 252Cf Accompanied by Light Charged Particles," Nucl. Phys. A246, 505-514 (1975). EXFOR-30418.

75AMI1 Amiel, S., Feldstein, H., "Odd-Even Systematics in Neutron Fission Yields of $233 \mathrm{U}$ and 235U," Phys. Rev. C, 11, No. 3, 845-858 (March 1975). NSA 32: 02075 (1975).

75BAL1 Balestrini, S. J., Forman, L., "Independent Fission Yields of Rb and Cs from ThermalNeutron-Induced Fission of 239Pu," Phys. Rev. C, 12(2), 413-416 (August 1975). NSA 32: 27109 (1975). INIS Atomindex 7: 227222 (1976). CSISRS-90617.

75BLA1 Blachot, J., Cavallini, P., Ferrieu, A., Louis, R., "The Cumulative Decay Yield of 252Cf," J. Radioanal. Chem. 26(1), 107-125 (1975). INIS Atomindex 7: 247695 (1976). EXFOR-21592. 
75BRI1 Brissot, R., Crancon, J., Ristori, Ch., Bocquet, J. P., Moussa, A., "Distributions Isotopiques des Gaz rares dans la Fission par Neutrons Thermiques de $235 \mathrm{U}$ et $233 \mathrm{U}$," Nucl. Phys. A 255(2), 461-471 (22 Dec 1975). INIS Atomindex 7: 239325 (1976). EXFOR-21550.

75CAV1 Cavallini, P., Thesis: University of Grenoble 205142.1975.54 Cited by 77CRO1.

75CLE1 Clerc, H. -G., Schmidt, K. -H., Wohlfarth, H., Lang, W., Schrader, H., Pferdekaemper, K. E., Jungmann, R., Asghar, M., Bocquet, J. P., Siegert, G., "Nuclear Charge Distribution of Mass-Separated Isobars from Thermal-Neutron Induced Fission of $235 \mathrm{U}$," Nucl. Phys. A, 247 (1) 74-90 (28 July 1975). NSA 32: 29882 (1975). Superseded by 75CLE2. EXFOR-21597.

75CLE2 Clerc, H. -G., Lang, W., Wohlfarth, H., Schmidt, K. -H., Pferdekaemper, K. E., Jungmann, R., "The Influence of Pairing and Nuclear Structure on the Thermal Neutron-Induced Fission of 235U," Institute fur Kernphysik, Darmstadt, IKDA- 75/10, 39 pages (June 27, 1975). Isomeric independent yields are here split per 76MAD2. Later published Z. Phys. A 274, 203 (1975). EXFOR-21557.

75CRO1 Crouch, E. A. C., "Chain and Independent Fission Product Yields Adjusted to Conform with Physical Conservation Laws," AERE-R7785 (February 1975). NSA 32: 21257 (1975).

75DAR1 Daroczy, S., Raics, P., Nagy, S., Csikai, J., Kover, L., Hamvas, I., "Experimental Results on Mass Distribution of 238U Fission Induced by $14 \mathrm{meV}$ Neutrons," Paper at Symposium on Fast Neutron Interactions and on the Problems of High Current Neutron Generators, 27-30 August 1975, Debrecen, Hungary. Published in journal "Atomki Kozlemenyek" 18(2), Page 315 or 317 (1976) (Proceedings) and (detailed article) according to INDC(NDS)75/G+P page 10-11 (May 1976) to be published perhaps in J. Inorg. and Nucl. Chem. Supersedes 73DAR1, -2, -3, -4, 74DAR1 and -2. Isomeric independent yields of Xe-135 split per 76MAD2. EXFOR-30639.

75DEE1 Deen, J. R., "Measurement of Fission Product Yields and the Energy Integral Fission Cross Section of Thorium-232 in a Californium-252 Fission-Neutron Spectrum," Nucl. Technology, 25, 416-422 (1975). NSA 31: 24343 (1975). See 73DEE1. NSA 31: 24343 (1975). CSISRS-90624.

75DON1 Donichkin, A. G., Smirnov, A. N., Solov'jov, S. M., Ejsmont, V. P., "Yield Determination of Some Fission-Fragments of Cf252s," Conf. 75Kiev, 5, 82 (Jun 1975). EXFOR-40459.

75DUD1 Dudey, N. D., Popek, R. J., Greenwood, R. C., Helmer, R. G., Rogers, J. W., Kellogg, L. S., Zimmer, W. H., "Fission-Product-Rate Measurements and Yields," Nucl. Technology, 25, 294-304 (1975). Supersedes 73DUD1. Measured in EMC. CSISRS-90625.

75DUD2 Dudey, N. D., Popek, R. J., Greenwood, R. C., Helmer, R. G., Rogers, J. W., Kellogg, L. S., Zimmer, W. H., “Fission-Product-Rate Measurements and Yields," Nucl. Technology, 25, 294-304 (1975). Supersedes 73DUD2. Measured in CFRMF. CSISRS-90625.

75EME1 Emel, W. A., Erikson, A. L., Kussy, M. E., Tromp, R. L., Maeck, W. J., "Burnup Methods for Fast Breeder Reactor Fuels," IDO-1063, 156-170 (January 1975). Progress report of preliminary but superseded data before error analysis. 
75ENG1 England, T. R., "Delayed Neutron Emission," Calculated values prepared for 4th Meeting of the "Task Force on Nuclear Data," ENDF/B-IV Fission Product Decay Files, Hanford Engineering Development Laboratory April 8-9, 1974. Private communication to B. F. Rider Letter File No. T-2-L-1278.

75ENG2 England, T. R., Schenter, R. E., “ENDF/B-IV Fission-Product Files: Summary of Major Nuclide Data," (ENDF-223) LA-6116-MS (October 1975).

75FET1 Fettweis, P., Marmol, P. del, "Study of Short Lived Ru Isotopes Produced in Thermal Neutron Fission of U-235," Z. Phys. A, 275, 359-367 (Dec 1975). USA National Nuclear Data Center CSISRS Accession Number 21530. Publication of INIS Atomindex 7(4): 224691 (15 Feb 1976). Abstracted in INIS Atomindex 7(18): 263631 (15 Sept 1976) EXFOR-21530.

75FEU1 Feu Alvim, C. A., "Mesure des Distributions en Masse et en Charge Dans la Fission a Moyenne Energie Par une Methode d'Emanation," These presentee a l'Universite Scientifique et Medicale de Grenoble pour obtenir le grade de Docteur es-Sciences Physiques. Soutenue le 12 Juin 1975 Devant la Commission de'Examen. No. d'Ordre: A. O. 11.376. (In French). EXFOR-21567.

75FLY1 Flynn, K. F., Glendenin, L. E., "Independent Yields of 84Rb, 86Rb, and 136Cs for Thermal-Neutron-Induced Fission of $233 \mathrm{U}, 235 \mathrm{U}$, and $239 \mathrm{Pu}$," J. Inorg. Nucl. Chem., 37, 869-872 (1975). Isomeric independent yields are here split per 76MAD2. EXFOR-10517. CSISRS-90628.

75FLY2 Flynn, K. F., Gindler, J. E., Glendenin, L. E., “The Mass Distribution for Spontaneous Fission of 252Cf," J. Inorg. Nucl. Chem., 37, 881-885 (1975). NSA 32: 07809 (1975). Article typo $\mathrm{Kr}-85$ should read $\mathrm{Kr} 85 \mathrm{~m}$.

75FLY3 Flynn, K. F., Gindler, J. E., Sjoblom, R. K., Glendenin, L. E., "Mass Distributions for Thermal-Neutron-Induced Fission of 255Fm and 251Cf," Phys. Rev. C. 11, (5), 16761680 (May 1975). EXFOR-10516. Supersedes 73UNI1. CSISRS-90630.

75FLY4 Flynn, K. F., Nagy, S., Glendenin, L. E., Gindler, J. E., “Monoenergetic Neutron Fission of 238U," Trans. Am. Nucl. Soc. 22: 677-679 (November 1975). NSA 33: 21710 (1976). INIS Atomindex 9(2): 356774 (1978). CSISRS-90631. Monoenergetic $2 \mathrm{meV}$ neutron data is pooled with U238F for ENDF evaluations. Superseded by 78NAG1.

75 FLY5 Flynn, K. F., Gindler, J. E., Glendenin, L. E., "Distribution of Mass in Thermal Neutron Induced Fission of 257Fm," Phys. Rev. C 12 (5) 1478-1482 (Nov 1975). INIS Atomindex 7: 239353 (1976).

75GOE1 Goektuerk, H., Birgul, O., Erten, H. N., Aras, N. K., "Yields of Some Ruthenium and Rhodium Isotopes in Spontaneous Fission of 252Cf," J. Inorg. Nucl. Chem. 37, 22472248 (1975).

75HAR1 Harvey, J. T., Adams, D. E., James, W. D., Beck, J. N., Meason, J. L., Kuroda, P. K., "Distribution of Fission Yields in the $3.0 \mathrm{meV}$ Neutron-Induced Fission of $238 \mathrm{U}$," J. Inorg. Nucl. Chem. 37, 2243-2246 (1976). CSISRS-90634. EXFOR-13469.

75IZA1 Izak-Biran, T., Amiel, S., "Reevaluation of the Emission Probabilities of Delayed Neutrons from Fission Products," Nucl. Sci. and Engr. 57 117-121 (1975). 
75JAM1 James, W. D., Adams, D. E., Beck, J. N., Kuroda, P. K., "Investigation of the Reported Mass Distribution Curves in the Fission of 233U with $14.8 \mathrm{meV}$ Neutrons," J. Inorg. Nucl. Chem., 37, 1341-1342 (1975). EXFOR-10433. CSISRS-90635.

75LAE1 Laeter, J. R. de, Thode, H. G., "Relative Yields of Stable Cadmium Isotopes in Neutron Induced Fission," Can. J. Phys. 53, (8), 775-785 (1975). Cross-indexed (de Laeter, J. R.).

75LAM1 Lammer, G., "Progress in Fission Product Nuclear Data No. 1," INDC(NDS)-70/G+P page 35 (November 1975). International Atomic Energy Agency, Vienna.

75MAE1 Maeck, W. J., Private communication to B. F. Rider: For PU239T, the Xe-131, 132, 134 in 70LIS1 are low $8.7 \%$ by failure to recover completely the Xe from column trap into chromatograph for measurement; $\mathrm{Ba}-138$ is low by 0.6 absolute $\%(5.4+0.6=6.0)$ by overcorrection for natural barium-138; Nd-148 is high by $2-3 \%$ by neutron absorption on Nd-147. So heavy peak would total 101.93. All masses renormalized then to 100.00 INIS Atomindex 7(20): 267581 (1976). See 76MAE1 for publication. CSISRS-90637. See 76MAE1 for published copy of this private communication. Yields are superseded by 79MAE2.

75MCE1 Mc Elroy, W. N., Kellogg, L. S., "Evaluated Fission Yields," Pages 202-207 in "Fuels and Materials Fast-Reactor Dosimetry Data Development and Testing," Nucl. Technology, 25, 180-223 (1975). NSA 31: 24992 (1975).

75MEE1 Meek, M. E., Rider, B. F., Estimated by interpolation or extrapolation.

75MEE2 Meek, M. E., Rider, B. F., Independent yields of isomers depend on the total nuclear angular momentum of each state where probability $p(j)=(2 j+1) \exp \left(-(j+1 / 2)^{* *} 2 /<j^{* *} 2>\right)$ from 69RAS1 as recommended by 76MAD2.

75MEE3 Meek, M. E., Rider, B. F., Estimated yields for TH232H based on all measured values plus the knowledge that average nu (77CRO1) measured experimentally to be $4.08+/-0.20$ neutrons per fission.

75MEE4 Meek, M. E., Rider, B. F., Estimated yields for NP237F based on all measured values plus the knowledge that average nu (64HYD1 pg. 215) measured experimentally to be $2.81+/-0.09$ neutrons per fission.

75MEE5 Meek, M. E., Rider, B. F., Estimated yields for PU239H based on all measured values plus the knowledge that average nu (64HYD1 pg. 215) measured experimentally to be $4.75+/-0.40$ neutrons per fission.

75RAJ1 Rajagopalan, M., Pruys, H. S., Gruetter, A., Gunten, H. R. von, Hermes, E. A., Richmond, R, Roessler, E., Schmid, A., Wydler, P., "Mass Yields in the Fission of $235 \mathrm{U}$ and $239 \mathrm{Pu}$ in Neutron-Spectrum of a Gas Cooled Fast Reactor," Nucl. Sci. and Engr., 58(4) 414-419 (1975). NSA 33: 8732 (1976). INIS Atomindex 7: 237767 (1976) EXFOR-21595.

75REe1 Reeder, P. L., Wright, J. F., Anderl, R. A., "Fission Product Nuclear Data Obtained by Use of an On-Line Mass Spectrometer," Proceedings of a Conference on Nuclear Cros Sections and Technology Vol. 1, Washington, D. C. March 3-7, 1975, Pages 401-404, U. S. Dept. of Commerce, National Bureau of Standards (issued October 1975). Relative independent yiels of U235T normalized for this work to $\mathrm{Rb}-93=3.096 \%$; Cs-141 $=3.134 \%$; Br-88 $=1.575 \%$. NSA 32: 10548 (1975). See BNWL-SA-5243. CSISRS-90639. 
75RUD1 Rudolph, W., Kratz, K. -L., Ohm, H., "Neutronenemissionswahrscheinlichkeit und Kumulierte Spaltausbeute von Antimon-134, -135 und -136," Jahresbericht 1974, Institut fur Kernchemie der Universitat Mainz, Seite 73-76 (2 Juni 1975). NP-20507 (in German) NSA 32: 23491 (1975).

75SAD1 Sadler, G., Khan, T. A., Sistemich, K., Grueter, J. W., Larwin, H., Lauppe, W. D., Selic, H. A., Shaanan, M., "Studies of the Beta-Decay of $96 \mathrm{Y}$ and the Level Scheme of $96 \mathrm{Zr}$," Nucl. Phys. A, 252(2), 365-380 (10 Nov 1975). INIS Atomindex 7(7): 232578 (1976).

75SIE1 Siegert, G., Greif, J., Wollnik, H., Fiedler, G., Decker, R., Asghar, M., Bailleul, G., Bocquet, J. P., Gautheron, J. P., Schrader, H., Armbruster, P., Ewald, H., "Nuclear Charge Distribution in Isobars 92-100 Resulting from Thermal Neutron Fission of Uranium-235," Phys. Rev. Lett. 34(16), 1034-1036 (21 Apr 1975). NSA 32: 10552 (1975). Fractional independent yields seem valid above $2.0 \%$. Smaller values best considered upper limits where values confound with system background. Isomeric independent yields are here split per 76MAD2. EXFOR-21562.

75SPI1 Spinrad, B. I., "Evaluation of Fission Product After-Heat," Annual Report May 1, 1974 - June 30, 1975. Department of Nuclear Engineering, Oregon State University (1975).

75VOR1 Vorobiev, A. A., Grachov, V. T., Kondurov, I. A., Miroshnichenko, Yu. A., Nikitin, A. M., Seleverstov, D. M., Smirnov, N. N., "Neutron-Induced Ternary Fission of 238Pu and 242m-Am," Yad. Fiz. 20, 461 (1974). NSA 31: 4976 (1975). EXFOR-40503.

75WAG1 Wagemans, C., Deruytter, A. J., "Ratio of the Ternary-to-Binary Fission Cross Sections Induced by Thermal and Resonance Neutrons in 241Pu," Z. Phys. A275, 149 (1975). NSA 31: 24338 (1975). EXFOR-21529.

75WEI1 Weis, M., "Ladungsverteilung in der Kette 99 bei der Spaltung von Uran-235 mit Thermischen Neutronen," Jahresbericht 1974, Institut fur Kernchemie der Universitat Mainz, Seite 108-111 (2 Juni 1975). NP-20507 (in German) NSA 32: 23492 (1975).

75WES1 Westgaard, L., Aleklett, K., Nyman, G., Roeckl, E., “Beta-Decay Energies and Masses of Short-Lived Isotopes of Rubidium, Caesium, Francium, and Radium," Z. Physik, A275, 127 (1975).

75WOL1 Wolfsberg, K., "Nuclear Charge Distribution in Fission-Spectrum-Neutron Fission: Fractional Cumulative Yields of Rare Gas Nuclides from Fission of 235U and 238U," J. Inorg. Nucl. Chem., 37, 1125-1128 (1975). Also see 74WOL2. NSA 32: 07812 (1975). CSISRS-90643.

75ZIM1 Zimmer, W. H., Heinrich, R. R., Kellogg, L. S., Matsumoto, W. Y., “High Flux Level Reaction Rate Measurements," Nucl. Technology, 25, 289-293 (1975). NSA 31: 24342 (1975). CSISRS-90644. Errors given by author do not include uncertainty in the standard. ENDF evaluation takes into consideration the uncertainty in the standard.

76ALE1 Aleklett, K., Lund, E., Rudstam, G., “Total Binding Energy of the Doubly Closed Shell Nuclide 132-Sn," The Swedish Research Council Laboratory Research Report LF-73 (1976).

76ALE2 Alexandrov, B. M., et. al., Nejtronnaya Fiz., Moscow, Part 6 pg. 97 (1976). Ternary yields in Pu239t (4He etc.). 
76ARM1 Armbruster, P., "Separation of Fast Nuclear Reaction Products," From Proceedings of 3rd International Conference on Nuclei Far from Stability, 19-26 May 1976, Corsica (France), CERN 76-13 (15 July 1976), pp. 3-14. See pg. 10 for Y-97 Y-97m decay data.

76BRI1 Brissot, R., Crancon, J., Ristori, Ch., Bocquet, J. P., Moussa, A., "Distributions Isotopiques des Gaz Rares et de Leurs Precurseurs dans la Fission Thermique de 239, 241 Pu. Et ude de l'Effet 'Pair-Impair'," Submitted to Nuclear Physics (1976). Appeared as 77BRI1.

76BRI2 Brissot, R., Crancon, J., Ristori, Ch., Moussa, A., "Distributions Isotopiques des Gaz Rares dans la Fission par Neutrons Thermiques de 239Pu et 241Pu: Evaluation de l'Effet de Parite en Protons," J. Phys. (Paris) Lett., 37(10), L.241- L.242 (Oct 1976). (In French). INIS Atomindex 8(7): 298054 (1 April 1977). Isotopic distribution of rare gases in $239 \mathrm{Pu}$ and $241 \mathrm{Pu}$ thermal fission. See 77BRI1.

76BUZ1 Buzzelli, G., Langer, S., Jones, C., Gainey, B., “Tritium: Fast Fission Yields of 238U and 232Th," Trans. Am. Nucl. Soc. 24, 458-459 (Nov 1976). INIS Atomindex 8(10): 305341 (1977).

76CLE1 Clerc, H. -G., Lang, W., Wohlfarth, H., Schmidt, K. -H., Schrader, H., "Nuclear Charge and Mass Yields for 235U ( $\mathrm{th}, \mathrm{f}$ )," IKDA 76/5 (May 1976). Values are for higher than average energies so are not coded here.

76CLE2 Clerc, H. -G., Lang, W., Wohlfarth, H., Schmidt, K. -H., Schrader, H., uNuclear Charge and Mass Yields for $235 U(n t h, f)$," Independent yields and nuclear charge distribution parameters $z$ (bar) and sigma at a kinetic energy of $108 \mathrm{meV}$ (not coded because only the most probable kinetic energy is desired for ENDF/B-V). From Proceedings of 3rd International Conference on Nuclei Far from Stability, 19-26 May 1976, Corsica (France), CERN 76-13 (15 July 1976), pp. 509-516.

76CRA1 Crancon, J., Private communication to B. F. Rider October 1976. Submitted to J. Inorg. Nucl. Chem. Supersedes 74SIL1.

76CR01 Crouch, E. A. C., "Chain and Independent Fission Product Yields Adjusted to Conform with Physical Conservation Laws. Part 2," AERE- R8152 (January 1976). INIS Atomindex 7: 252345 (1976).

76DAV1 David, P., Debrus, J., Luebke, F., Mommsen, H., Schoenmackers, R., "Total Kinetic Energies and Mass Yield Distributions of 252 Fission Fragments," Phys. Letters, 60B(5) 445 (16 Feb 1976). EXFOR-21914.

76DEB1 Debertin, K., "Fission Product Yields in 235U Fission by Fast and Thermal Nettrons," To be published in Physikalisch Technische Bundesanstalt Jahresbericht 1976.

76DEN1 Denschlag, H. O., Fischbach, G., Meixler, H., Paffrath, G., Rudolph, W., Weis, M., "Charge Distribution in Thermal Neutron Induced Fission Reactions," Institute fur Kernchemie Johannes Gutenberg-Universitat Mainz. From Progress Report on Nuclear Data Research in the Federal Republic of Germany, NEANDC (E)-172U Vol. 5 INDC(Ger)-18L+special (July 1976). pp. 47-49. 
76DII1 Diiorio, G., Wehring, B. W., "Direct Physical Measurement of Mass Yields for 235U (n th, f)," Paper at Amer. Nucl. Soc. Winter Meeting, Washington, D. C. (November 15-19, 1976). Adjusted to post-delayed neutron emission yields. Ph. D. Thesis, Univ. of Ilinois, Urbana. University Microfilms Order No. 77-8975. INIS Atomindex 9(7): 364762 (1978). CSISRS- 90647.

76DI12 Diiorio, G., "Direct Physical Measurement of Mass Yields in Thermal Fission of Uranium-235," Ph. D. Thesis, University of Mlinois at Urbana-Champaign (1976).

76FIS1 Fischbach, G., Denschlag, H. O., Jahresbericht 1975, Institute fur Kernchemie, Universitat Mainz, p. 108 ff. (1976).

76FLY1 Flynn, K. F., Gindler, J. E., Glendenin, L. E., Sjoblom, R. K., "Mass Distributions for The Spontaneous Fission of 253Es and the Thermal-Neutron-Induced Fission of 254Es," J. Inorg. Nucl. Chem., 38, 661-664 (1976). Supersedes 73UNI1. CSISRS-90649.

76FOR1 Ford, G. P., Norris, A. E., "A Compilation of Yields from Neutron-Induced Fission of 232Th, 235U, 236U, 237Np, 238U, and 239Pu Measured Radiochemically at Los Alamos," LA-6129-MS (Feb 1976). 43 pages. INIS Atomindex 7(20): 267508 (1976). CSISRS-90252.

76GAE1 Gaeggeler, H., Gunten, H. R. von, Pruys, H. S., "Independent Yields of 150-Pm in the Thermal Neutron-Induced Fission of 233-U, 235-U, 239-Pu and 249-Cf and CrossSection for the 149-Pm(n, gamma)150-Pm Reaction,". J. Inorg. Nucl. Chem. 38, 205-210 (1976). INIS Atomindex 7: 239356 (1976). EXFOR-21552.

76GIL1 Gilliam, D. M., “Proposed ILRR Consensus Fission Yields,” (July 20, 1976). Weighted average values of ANC, ANL, HEDL, ARHCO Labs on Big-10 and CFRMF Fast Irradiations and NBS thermal column irradiations. Private communication from D. M. Gilliam, National Bureau of Standards, to B. F. Rider. INIS Atomindex 9(2): 356770 (1978). BNL - NCS - 22500 (March 1977). CSISRS-90653. Superseded by 77GIL1.

76GIL2 Gilliam, D. M., See 76GIL1. Individual laboratory averages for Big-10 Fast Irradiations. CSISRS-90654. Superseded by 77 GIL1.

76GIL3 Gilliam, D. M., Individual laboratory averages for CFRMF fast irradiation. See 76GIL1. CSISRS-90655. Superseded by 77GIL1.

76GIL4 Gilliam, D. M., See 76GIL1. Individual laboratory averages for NBS thermal column irradiations. CSISRS-90656. Superseded by 77GIL1.

76IMA1 Imanishi, N., Fujiwara, I., Nishi, T., "Independent Isomer Yields of Sb and Te Isotopes in Thermal-Neutron Fission of 233U, 235U, and 239Pu," Nuclear Physics A263, 141-149 (1976). INIS Atomindex 7(18): 262370 (1976). EXFOR-20589.

$76 \mathrm{KLO} 1 \mathrm{Klonk}$, H., “Untersuchen von Eigenschaften Kurzlebige Spaltprodukte aus der Spontanspaltung des Cf-252," (In German). Investigation of short-living fission products from spontaneous fission of Cf-252. INIS Atomindex 8(11): 309371 (1977). 129 pages. Thesis, Darmstadt Tech. Hochschule (January 12, 1976). Thesis: HS-517428-A. See page 64. EXFOR-21559.

76KOC1 Koch, L., Private communication to W. J. Maeck (1976). 
76KOC2 Koch, L., "Cumulative Fission Yields for Fast Fission of 240Pu and 242Pu," Institute fur Transurane, Karlsruhe, Progress Report 21 1976, Page 98.

76LAV1 Lavi, N., Nir-El, Y., "Isomer Yield Ratios of $132 \mathrm{~m}$ I and 134m I," J. Inorg. Nucl. Chem. 38, 2133-2134 (1976). EXFOR-30365.

76LIP1 Lipinski, R. J., Wehring, B. W., "Element Yields in Californium-252 Spontaneous Fission Determined from Measured X-Ray Multiplicities," Paper Amer. Nucl. Soc. Winter Meeting (November 15-19, 1976). Cf-252S odd-even proton effect is $5 \%$ deviation in independent yields from "Normal" as calculated from Gaussian Wahl model.

76LIS1 Lisin, S. K., Morozov, L. N., Pchelin, V. A., Chistyakov, L. V., Shigin, V. A., Shubko, V. M., "Barriers for Fragments in Fission of 232Th by Neutrons," Sov. J. Nucl. Phys. 24(6) 570-575 (1976). EXFOR-40428.

76MAD1 Madland, D. G., England, T. R., "The Influence of Pairing on the Distribution of Independent Yield Strengths in Neutron Induced Fission," ENDF-240 (1976). Also known as LA-6430-MS and its Supplement 1. Finally as a paper (LA-UR-76-1488) at Amer. Nucl. Soc. Winter Meeting (Nov. 15-19 1976). INIS Atomindex 8(5): 292101 (1977).

76MAD2 Madland, D. G., England, T. R., "Distribution of Independent Fission-Product Yields to Isomeric States," ENDF-241 (1976). Also known as LA-6595-MS. Published finally as a paper (LA-UR-76-1500) at Amer. Nucl. Soc. Winter Meeting (Nov 15-19, 1976). [See also 77MAD2].

76MAE1 Maeck, W. J., Duce, F. A., Dickerson, L. L., Keller, J. H., Tromp, R. L., “Discrepancies and Comments Regarding 235U and 239Pu Thermal Fission Yields and the Use of 148Nd as a Burnup Monitor," ICP-1092. (Xe-136 here obtained from Xe-136 + Cs-135 taking $0.53827 \pm 0.00754$ and $0.35994 \pm 0.00504$ as $\mathrm{Cs}-135$ to $\mathrm{Nd}-143+144+145+146$ ratio from other literature sources for PU239T and U235T, respectively). CSISRS-90662.

76MAE2 Maeck, W. J., Preliminary data by isotope dilution gas mass spectrometry relative to data in 76MAE1. CSISRS-90663.

76MAE3 Maeck, W. J., Private communication to CSEWG Fission Yield Subcommittee Meeting of preliminary data specifically for inclusion in ENDF/B-V (December 8, 1976). CSISRS-90664. Original 76MAE3 coding is retained in this work. Later published as 79MAE1 which is considered here as a duplicate and not coded. Data is in EXFOR-10845.

76MEI1 Meixler, H., "Test of an Apparatus for the Determination of the Cumulative Inert Gas Yield for 249-Cf(n(th), f)," Doctoral Thesis, Univ. of Mainz. To be published. Cited in 76DEN1. See Phys. Rev. (126), 1112 (1962) for exper. method. See Univ. Mainz Ann. Rept. 1976 page 113 (June 1976). EXFOR-20711.

76MON1 Monnand, E., Blachot, J., Schussler, F., Bocquet, J. P., Pfeiffer, B., Sadler, G., Selic, H. A., Khan, T. A., Lauppe, W. D., Lawin, H., Sistemich, K., "Recent Studies of the OddMass Sr, Zr, Y and Nb Fission Products $(A=95,07,99)$," from Proceedings of 3rd International Conference on Nuclei Far from Stability, 19-26 May 1976, Corsica (France), CERN 76-13 (15 July 1976), pp. 477-487. 
76NAD1 Nadkarni, D. M., Choudhury, R. K., Rama, P. N., Rao, S. S., Kapoor, S. S., "Energy Spectra and Yields of Alpha Particles in Ternary Fission of U-235 by Neutrons with Energies Ranging to 1.75 MeV," BARC-878 pg 47 (1976). EXFOR-30509.

76PAF1 Paffrath, G., Denschlag, H. O., Jahresbericht 1975, Institute fur Kernchemie, Universitat Mainz, p. 90-94 (1976) and see G. Paffrath, Dissertation, Univ. of Mainz (1976).

76PET1 Petrzhak, K. A., Platygina, E. V., Teplykh, V. F. "Relative Yields of Xenon Isotopes in Photofission of 237Np and 235U," Atomnaya Energia, 41(1), 44-45 (July 1976).

76RAJ1 Rajagopalan, M., Pruys, H. S., Gruetter, A., Hermes, E. A., Gunten, H. R. von, "Mass Yields in the $14 \mathrm{meV}$ Neutron-Induced Fission of 238U," J. Inorg. Nucl. Chem., 38, 351-352 (1976). INIS Atomindex 7: 235899 (1976). EXFOR-21554.

76RIS1 Ristori, Ch., Asghar, M., Crancon, J., Gautheron, J. P., "Nuclear Charge Distributions from 4pi-Beta Measurements on Mass Separated Fragments from 235U(n th, $f$ )," Z. Physik A277, 71-75 (1976). EXFOR-21556.

76ROS1 Rose, P. F., Burrows, T. W., "ENDF/B Fission Product Decay Data," USERDA Report BNL-NCS-50545 (ENDF-243) Vols. I and II (August 1976). Short half-lives consist of estimates for ENDF/B-IV by R. Schenter (9/73) from theory where values are required for decay heat summation codes.

76RUD1 Rudolph, W., Dissertation Mainz (1976). Values partly recalculated by 77DEN1.

76RUD2 Rudstam, G., "Characterization of Delayed-Neutron Spectra," Swedish Research Council Report LF-70 (1976) (revised). INDC(SWD) 9/G+P (1976).

76SHA1 Sharma, D. N., Iyer, M. R., Ganguly, A. K., "Charge Distribution, Neutron Evaporation, and Energy Distribution in Higher Energy Binary Fission," Phys. Rev. C, 14 (1) 181 194 (July 1976).

76SIE1 Siegert, G., Wollnik, H., Greif, J., Decker, R., Fiedler, G., Pfeiffer, B., "Nuclear Charge Distribution of Fission Products from 235U (n th, $f$ ) of the Masses 79 to 100," Phys. Rev. C 14(5), 1864-1873 (Nov 1976). INIS Atomindex 8(6): 295068 (1977). EXFOR21605.

76THI1 Thierens, H., Frenne, D. de, Jacobs, E., Clercq, A. de, D'hondt, P., Deruytter, A. J., "Study of the Catcherfoil Technique with the Aid of Cf-252 Spontaneous Fission and U235 Thermal Neutron Fission," Nucl. Instrum. Methods, 134, 299-308 (April 1976). USA National Nuclear Data Center CSISRS Accession Number 21531. INIS Atomindex 7(18): 263631 (15 Sept 1976). EXFOR-21531.

76UME1 Umezawa, H., Private communication to B. F. Rider October 25, 1976.

76VIN1 Vine, E. N., "Fractional Independent Yields of 104Tc and $105 \mathrm{Tc}$ from Thermal Fission of 235U," Diss. Abst. 37(12) 61213, Washington Univ. (December 1, 1976). INIS Atomindex 10(10): 451269 (1979). Univ. Microfilms Order Number 77-12486. Published as 81VIN1. CSISRS-90673. 
76WAL1 Walker, W. H., "The Use of Averages and Other Summation Quantities in the Testing of Evaluated Fission Product Yield and Decay Data. Applications to ENDF/B-IV," AECL-5259 (January 1976).

76WEI1 Weis, M., Denschlag, H. O., Jahresbericht 1975, Institute fur Kernchemie, Universitat Mainz, pp. 84-90 (1976).

76WEI2 Weis, M., Denschlag, H. O., Values from 76WEI1 plus subsequent work, to be published.

76WOH1 Wohlfarth, H., Lang, W., Clerc, H. -G., Schrader, H., Schmidt, K. -H., Dann, H., "Mass Distributions of $235 \mathrm{U}$ ( $\mathrm{n}$ th, $\mathrm{f}$ ) Fission Products as a Function of their Kinetic Energy," IKDA 76/9 (June 1976). Not coded because the average Energy already published as 75CLE2 which see.

76WOH2 Wohlfarth, H., "Massausbeuten bei der Reaction 235 (n th, f) als Funktion der Kinetischen Energie und der Ionladung der Spaltprodukte," Dissertation Doktors der Naturwissenschaften, Darmstadt (Dezember 1976). (In German). Mass distributions summed over the energy of the fission products, before delayed neutron emission corrected here by a normalization value to become after delayed neutron yields. Normalization value is the ratio of the yield as measured to the delayed neutron corrected yield values in 76DII1. EXFOR-21054.

76WOL1 Wollnik, H., Siegert, G., Greif, J., Fiedler, G., "The Nuclear Charge Distribution of Fission Products of Thermal Neutron Induced Fission of $235 U$," From Proceedings of 3rd International Conference on Nuclei Far From Stability, 19-26 May 1976, Corsica (France), CERN 76-13 (15 July 1976), pp. 517-527.

77ALE1 Aleklett, K., "Total Beta Decay Energies and Atomic Masses in Regions Far From Stability," On-Line isotope separator investigations in mass regions $A=75-87,120-135,222$ 229. Doctoral Thesis, University of Gothenburg, Sweden (1977).

77ALE2 Aleklett, K., Lund, E., Nyman, G., Rudstam, G., “Total Beta-Decay Energies and Masses of Short-Lived Isotopes of Zinc, Gallium, Germanium, and Arsenic," The Swedish Research Council Laboratory Research Report LF-74 (1977).

77ALE3 Aleklett, K., Lund, E., Rudstam, G., “Total Beta-Decay Energies and Masses of Strongly Neutron-Rich Indium Isotopes Ranging from $A=120$ to 129," The Swedish Research Council Laboratory Research Report LF-76 (1977).

77AMI1 Amiel, S., Feldstein, H., Izak-Biran, T., "Distributions of Fission Products from Various Low-Energy Fission Reactions and the Systematics of the Odd-Even Fluctuations," Phys. Rev. C 15(6) 2119-2126 (June 1977).

77AND1 Andreev, V. N., Nedopekin, V. G., Rogov, V. I., "Thermal Neutron Fission of U-233 and U-235 Accompanied by Alpha Particles Produced at Small Angles with Respect to the Fission Axis," Yadern. Fiz., 29(2), 293 (Feb 1979). EXFOR-40527.

77ASG1 Asghar, M., Hondt, P. D', Guet, C., Perrin, P., Wagemans, C., "Fission Fragment Energy Correlation Measurements for the Thermal Neutron Sub-Barrier Fission of 237Np and Above-The-Barrier Fission of 235U," Nucl. Phys. A292 (1977) 225-236. EXFOR21543. 
77AUM1 Aumann, D. C., Gueckel, W., Zeising, H., "Independent Yields of 148m-Pm, 148g-Pm, and $146 \mathrm{Pm}$ for Thermal-Neutron-Induced Fission of $233 \mathrm{U}$ and $235 \mathrm{U}$," J. Inorg. Nucl. Chem. 39, 1217-1223 (1977) EXFOR-21532.

77BEM1 Bemis, C. E. Jr., Ferguson, R. L., Plasil, F., Silva, R. J., O’Kelly, G. D., Kiefer, M. L., Hahn, R. L., Hensley, D. C., Hulet, E. K., Lougheed, R. W., "Mass Asymmetry and Total-KineticEnergy Release in the Spontaneous Fission of 262[105]," Phys. Rev. Ltrs., 39(20), 1246 (Nov 14 1977). EXFOR-10832.

77BJE1 Bjerke, M. A., Holm, J. S., Shay, M. R., "A Review of Short-Term Fission-ProductDecay Power," Nucl. Safety 18(5), 596-616 (Sept-Oct 1977).

77BLA1 Blachot, J., Ferrieu, A., Private communication to B. F. Rider October 4, 1976. Thesis by A. Ferrieu at the Universite Scientifique et Medicale de Grenoble. To be published in 1977. Uranium targets were irradiated in system "Caramel" placed near the "Melusine" reactor heart at the Centre d'Etudes Nucleaires de Grenoble. Caramel removes thermal and epithermal neutrons and gives a fission spectrum of $7 \mathrm{e}+11 \mathrm{n} / \mathrm{cm}^{* *} 2 / \mathrm{sec}$. Procedure same as 74BLA1 and 75BLA1. EXFOR-21736.

77BLA2 Blachot, J., Ferrieu, A., Lhospice, G., "Cumulative Fission Yields for Fast Neutron Fission of 237Np," Proceedings of the Second Advisory Group Meeting on Fission Product Nuclear Data, Petten, Netherlands, (September 5-9 1977). Contribution to 77CUN2, as corrected by private communication from J. Blachot to B. F. Rider (2 Nov 1977).

77BRI1 Brissot, R., Crancon, J., Ristori, Ch., Bocquet, J. P., Moussa, A., "Distributions Isotopiques des Gaz Rares et de Leurs Precurseurs dans la Fission Thermique de 239, $241 \mathrm{Pu}$. Etude de l'Effet 'Pair-Impair'," (In French). Nucl. Phys. A. 282(1), 109-124 (16 May 1977). INIS Atomindex 8(18): 329671 (1977). EXFOR-21549.

77BUZ1 Buzzelli, G., Langer, S., Trans. Am. Nucl. Soc., Vol 27, 283-284 (1969). U235F tritium yield.

77CRO1 Crouch, E. A. C., "Fission-Product Yields from Neutron-Induced Fission," Atomic Data and Nuclear Data Tables, 19(5), 417-532 (May 1977). Supersedes 73CRO1, 73CRO2, 74CRO1, 76CR01. Data : hrough 1975 (with some 1976 results) from 386 selected references. All non-U235T published errors are increased to at least 5\%. That is, measurements accurate to $0.5 \%$ are treated as though they were uncertain to $5.0 \%$ even in the case of relative mass spectrometric results of a single element. This is a very pessimistic view of the accuracy with which fission yields of many fission products are known.

77CUN1 Cuninghame, J. G., Willis, H. H., "Absolute Yields in the Fission of 239Pu by MonoEnergetic Neutrons of Energy 130-1700 keV," J. Inorg. Nucl. Chem. 39, 383-386 (1977). EXFOR-20771.

77CUN2 Cuninghame, J. G., "The Status of Fission Product Yield Data (FPND) in 1977," AERE-R-8753 (May 1977), INIS Atomindex 28(20): 334174 (1977).

77DEB1 Debertin, K., "Fission Yields in U235: Fission by Fast and Thermal Neutrons," Second Advisory Group Meeting on Fission Product Nuclear Data, Petten, Netherlands, 5-9 September 1977. INDC(NDS)-87,211, 7805. Data appears in Figure 40, Page 15, 77CUN1. EXFOR-21624. 
77DEN1 Denschlag, H. O., Fischbach, G., Meixler, H., Paffrath, G., Rudolph, W., Weis, M., Wolfsberg, K., "Charge Distribution in Thermal Neutron Induced Fission Reactions," Institute fur Kernchemie, Johannes Gutenberg-Universitat Mainz. contribution to "Progress Report on Nuclear Data Research in Federal Republic of Germany," NEANDC(E)-182U, Vol V, p. 65, (1976). Later published in J. Inorg. Nucl. Chem. (39), 753 (1977). Supersedes values in 76DEN1. EXFOR-20878.

77DEN2 Denschlag, H. O., "Prediction of Unmeasured Fission Product Yields by Nuclear Theory or Systematics," Review Paper No. 11 at the Second Advisory Group Meeting on Fission Product Nuclear Data held at Petten, Netherlands (Sept 5-9, 1977).

77DII1 Diiorio, G., Wehring, B. W., "Hiawatha, a Fission-Fragment Recoil Mass Spectrometer," Nucl. Instr. and Methods, 147, 487-499 (1977). Tabulates U235T yields which were measured to be different from 74MEE1 and other evaluations.

77DYA1 D'yachenko, N. P., Kuz'minov, B. D., Mitrofanov, V. F., Sergachjov, A. I., "Fine Structure of Fission Fragments Distribution on Masses by Th-232 Fission," Yadern. Fiz. 26(4), 691 (Oct 1977). EXFOR-40570.

77ENG1 England, T. R., “Delayed Neutron Precursors and Pn Values for ENDF/B-V," Private communication (Letter File No. T-2-L-2299) to R. E. Schenter (April 19, 1977).

77ENG2 England, T. R., "Delayed Neutron Calculations," Table of 74 delayed neutron precursors and $\mathrm{Pn}$ values for ENDF/B-V included. Delayed neutron yields per 100 fissions calculated values using preliminary ENDF/B-V(C) yields and 74 Pn values vs. ENDF/B-IV evaluation (best values) for U235 thermal, fast and high energy, respectively are: 1.61 vs. $1.67 \pm .07 ; 1.68$ vs. $1.67 \pm .07 ; 0.909$ vs. 0.90 . For Pu239: 0.693 vs. $0.645 \pm .04 ; 0.642$ vs. $0.645 \pm .04 ; 0.399$ vs. 0.43 . In worst cases $\mathrm{U} 238 \mathrm{~F}$ and $\mathrm{TH} 232 \mathrm{~F}$ were low $36 \%$ and $24 \%$. Other agreements are intermediate 3-24\%. Better agreement is obtained than with ENDF/B-IV yields inferring ENDF/B-V yields are improved over ENDF/B-IV. Private communication to R. E. Schenter (Letter File No. T-2-L-2311) (April 29, 1977).

77ENG3 England, T. R., “ENDF/B-V Delayed Neutron Precursor Branchings (Pn)," Table of 102 delayed neutron precursors, their $\mathrm{Pn}$ and half-lives. The result of a revision by the 1977 IAEA Advisory Panel on Fission Product Nuclear Data at Petten Netherlands, Delayed Neutron Subcommittee (G. Rudstam, Chm., K. L. Kratz, P. Delmarmol, T. England, secretary) of 76RUD2 and 77RUD2. Updates 77ENG1 and 77ENG2. Private communication to CSEWG decay and actinide subgroup (Los Alamos Letter File T-2-L-2520) (October 11, 1977). Half life values listed with 0.0 error are from $76 R O S 1$ and consist of estimates for ENDF/B-IV by R. Schenter $(9 / 73)$ from theory where values are required for decay heat summation codes. Published as 78ENG1.

77ENG4 England, T. R., "Delayed Neutron Yields," From a table of 102 delayed neutron precursor yields resulting equilibrium values of the delayed neutron emission have been calculated. Private communication to $R$. E. Schenter (letter T-2-L-2608, December 12, 1977). Published as $78 \mathrm{ENG1.}$

77FER1 Ferrieu, A., Blachot, J., Lhospice, G., Thesis, Univ. of Grenoble (1977). 
77FEU1 Feu Alvim, C. A., Bocquet, J. P., Brissot, R., Crancon, J., Moussa, A., "Rare-Gas Yields in 238U and 232Th Fission by $14 \mathrm{meV}$ Neutrons Measured by an Emanating Method," (In French) J. Phys. (Paris) 38, 273-281 (Mar 1977). Publication of 76CRA1. Cross indexed under Silva, C. Feu Alvim da . INIS Atomindex 8(18): 298054 (1977). EXFOR-21591.

77FIS1 Fischbach, G., Denschlag, H. O., Jahresbericht 1976, Institute fur Kernchemie, Universitat Mainz (1977).

77FLY1 Flynn, K. F., Gindler, J. E., Glendenin, L. E., "Mass Distributions for the Spontaneous Fission of 248Cm and 250Cf," J. Inorg. Nucl. Chem. 39, 759-762 (1977). EXFOR-10979. CSISRS-90687.

77GAB1 Gabeskiriya, V. Ya., Gryzina, V. V., Novikov, Yu. B., Prokopenko, V. S., Prokopev, V. M., Tikhomirov, V., Chetverikov, V. A., "Fission Product Yields during Irradiation of Uranium-235 and Plutonium-239 in Reactor BOR-60," Atomnaya Energiya 43(1), 59-60 (1977). INIS Atomindex 9(16): 392404 (1978).

77GAE1 Gaeggler, H., Gunten, H. R. von, "Cumulative Yields of Rare Earth Elements in the Thermal-Neutron-Induced Fission of 249-Cf," J. Inorg. Nucl. Chem. 39, 1105-1106 (1977). EXFOR 21553.

77GIL1 Gilliam, D. M., Helmer, R. G., Greenwood, R. C., Rogers, J. W., Heinrich, R. R., Popek, R. J., Kellog, L. S., Lippincott, E. P., Hansen, G. E., Zimmer, W. H., "Reference and Standard Benchmark Field Consensus Fission Yields for U. S. Reactor Dosimetry Programs," Proceedings of the Second ASTM-EURATOM Symposium on Reactor Dosimetry, October 3-7, 1977 Palo Alto, Calif., NUREG/ CP-0004 Vol. 3, 1289-1306 (1977). CSISRS-90690. Supersedes 76GIL1, 76GIL2, 76GIL3, and 76GIL4. EXFOR-13335,-13478,-13479.

77GIN1 Gindler, J. J., Flynn, K. F., Glendenin, L. E., Sjoblom, R. K., "Distribution of Mass, Kinetic Energy, and Neutron Yield in the Spontaneous Fission of 254Fm," Phys. Rev. C, 16, 1483, (1977). EXFOR-10642. Supersedes 73UNI1.

77GUD1 Gudkov, A. N., Zhivun, V. M., Zhukov, I. V., Zvonarev, A. V., Kovalenko, V. V., Koldobskii, A. B., Koleganov, Yu. F., Kolobashkin, V. M., Kulakovskij, M. Ya., Piven, N. S., "Determination of 232Th, 233U, 235U, 238U, 239Pu Fast Neutron Fission Product Yields," INIS MF-4786 (1977). Fourth All-Union Conference on Neutron Physics, Kiev, Ukrainian SSR 18-22 Apr 1977. INIS Atomindex 10(7): 440161 (1979). EXFOR-40554.

77HAR1 Harris, D. C., Beck, J. N., Raines, W. L., Harvey, J. T., Inn, K. G. W., Meason, J. L., Wright, H. L., "Distribution of Fission Yields for Fission Spectrum Neutron-Induced Fission of Uranium-238," Nucl. Sci. and Engr. 63, 504-507 (1977). INIS Atomindex 9(11): 372763 (1978). EXFOR-10683. CSISRS-90692.

77HOW1 Howerton, R. J., “Nubar Revisited," Nucl. Sci. and Engr. 62, 438 -454 (1977). Latest method of predicting nubar tested against available experimental data.

77IZA1 Izak-Biran, T., Amiel, S., "Independent Yield of Fast Neutron Fission of 232Th," Phys. Rev. C, 16, 266-272 (1977). INIS Atomindex 9(2): 351374 (1978). EXFOR-30425. 
77KEL1 Kellogg, L. S., Davis, A. I., Lippincott, E. P., Ruggles, J. M., “EBR-II Validated, Key Fission Product Yields for Fast Reactor Application," Proceedings of the Second ASTMEuratom Symposium on Reactor Dosimetry October 3-7 1977 Palo Alto, Calif. NUREG/ CP0004 Vol. 3, 1307-1321 (1977). CSISRS-90694. EXFOR-13342.

77KOC1 Koch, L., "Status Report of Fast Reactor Fission Yields in the TACO Experiment," Paper presented to IAEA Second Advisory Group Meeting on Fission Product Nuclear Data, Petten, Netherlands (September 5-9, 1977). Irradiations in Rapsodie Fast Reactor. EXFOR21155.

77KRA1 Kratz, K. -L., "Independent Fission Yields and Neutron Emission Probabilities of Short-Lived Halogen Isotopes," Univ. Mainz, Jahresbericht-1976, Page 34 (June 1977). EXFOR-21058. Superseded by 78KRA1.

77KRE1 Kreiner, H. J., "Nuclear Charge Distribution of Light Fission Products Measured by Direct Gamma Spectrometry," Radiochim. Acta. 24(1), 8-10 (1977).

77KRI1 Krishnarajulu, B., Mehta, G. K., et. al., Pramana 8(4), 315-321 (1977). U235F ternary fission (4He etc.). EXFOR-30497.

77LUN1 Lund, E., Aleklett, K., Rudstam, G., "Total Beta-Decay Energies and Masses of Tin, Antimony, and Tellurium Isotopes in the Vicinity of 132-Sn," The Swedish Research Council Laboratory Research Report LF-75 (1977).

77MAC1 Macedo Grassi, G. M. de, Bellido, A. V., “Non Lanthanides Fission Product Yields, in the 238U Fission with 14 meV Neutrons," Cienc. Cult. (Sao Paulo) Supl. (Jul 1977) V. 29(7) p. 275 INIS Atomindex 9(16): 394076 (1978).

77MAD1 Madland, D. G., Stewart, L., "Light Ternary Fission Products: Probabilities and Charge Distributions," LA-6783-MS (ENDF-247) ENDF/B-V preliminary evaluation (1977). Trans. Amer. Nucl. Soc., 27, 874-875 (Nov 1977).

77MAD2 Madland, D. G., England, T. R., "The Influence of Isomeric States on Independent Fission Product Yields," Nucl. Sci. and Engr. 64, 859-865 (1977). Publication of 76MAD2.

77MAE1 Maeck, W. J., "Fast Reactor Fission Yields for 239Pu and 241Pu," USERDA Report ICP-1050-II (August 1977). INIS Atomindex 9(7): 365982 (1978). These Pu241 fast yields are subject to less uncertainties than Pu241 thermal yields (e.g., less Xe-135 neutron capture). Although valley and wing yields slightly increase with increasing energy of the fission inducing neutron, there is good reason for believing that PU241F peak yields differ from PU241T yields by less than their quoted uncertainties so they were used (77CUN2,79WAL1) as estimates of thermal yields, mainly to help resolve discrepancies.

77MAE2 Maeck, W. J., Emel, W. A., Erikson, A. L., Delmore, J. E., Meteer, J. W., “Fast Reactor Fission Yields for 237Np," ICP-1050 III (September 1977). INIS Atomindex 9(11): 374256 (1978). EXFOR-10723.

77MAK1 Maksyutenko, B. P., Shimanskii, A. A., Balakshev, Yu F., "Symmetric and Asymmetric Modes of Spontaneous Fission of 252Cf," Sov. J. Nucl. Phys. 25(5) 503-506 (May 1977). For Russian Yad. Fiz. 25(5) 945-970 (May 1977). Yields of four iodine isotopes, 135Sb, and 143Cs by Counting Delayed Neutrons. INIS Atomindex 9(15): 385332 (1978). 
77MAT1 Mathews, C. K., "Fission Yields in the Symmetric Region: Yields of Stable Isotopes of Tin and Cadmium in the Thermal Neutron Fission of $235 U$ and 239Pu," Phys. Rev. C, 15(1), 344-351 (Jan 1977). EXFOR-30508. CSISRS-90700.

77MEI1 Meixler, H., Wolfsberg, K., Denschlag, H. O., Jahresbericht 1976, Institute fur Kernchemie, Universitat Mainz (1977). Cited in 77DEN1.

77MEI2 Meixler, H., Wolfsberg, K., Denschlag, H. O., Jahresbericht 1977, Institute fur Kernchemie, Universitat Mainz (1977). cited in 77DEN1.

77MYE1 Myers, W. A., Kantelo, M. V., Osborne, R. L., Prindle, A., Nethaway, D. R., "Fission of 240Pu with Neutrons Having a Fission-Spectrum Energy Distribution," UCRL80020 (September 8, 1977). Published later in Phys. Rev. C 18, 1700 (1978). (see 78MYE1). $\mathrm{Sm}-153$ decay scheme is in error in 77MYE1. In 79PRI1 this has been corrected to read $5.48 \mathrm{e}-3$ and not 5.22e-3 atoms/fission for Sm-153 yield. CSISRS-90703.

77NET1 Nethaway, D. R., Prindle, A., Myers, W. A., Fuqua, W. C., Kantelo, M. V., "Fission of 240Pu with 14.8 meV Neutrons," UCRL-79335 (Mar. 2, 1977). Published in Phys. Rev, C, 16(5), 1907-1918 (Nov 1977). Sm-153 decay scheme is in error. Sm-153 yield is corrected in 79PRI1 to read $5.8 \mathrm{e}-3$ and not $6.55 \mathrm{e}-3$ atoms/fission. Also $88 \mathrm{Kr}$ is changed to read 0.0139 atoms/fission and not 0.0128 to reflect changed photon abundances. (See 79PRI1, pg. 1825, Col 2, lines 16-20, for these corrections to Sm-153 and Kr88 in 77NET1). These are important and hard to find corrections not in EXFOR. EXFOR-10712. CSISRS-90704.

77NIS1 Nishi, T., Fujiwara, I., Imanishi, N., "Independent Fission Yields of Several Isotopes in the Thermal Neutron Induced Fission of U-233, U-235, and Pu-239," Table 1 NEANDC(J)-51, 38-39 (Sept 1977). Cited in USA NNDC Accession 20848. Same as INDC (Jap)-37/U superseded. EXFOR-20848.

77PET1 Petrzhak, K. A., Platygina, E. V., Teplykh, V. F., "The Fine Structure of Heavy Nuclei Fission Fragment Yields," Atomnaya Energiya 42(4) 337-338 (April 1977). INIS Atomindex 9(13): 381705 (1978).

77RUD1 Rudolph, W., Kratz, K. -L., Herrmann, G., "Half Lives, Fission Yields and Neutron Emission Probabilities of Neutron Rich Antimony Isotopes," J. Inorg. Nucl. Chem. 39, 753-758 (1977). And W. Rudolph, Dissertation, Univ. of Mainz (1976). EXFOR-20879.

77RUD2 Rustam, G., "Status of Delayed Neutron Data," Review paper at LAEA Second Advisory Group Meeting on Fission Product Nuclear Data, Petten Netherlands, Sept 5-9, 1977.

77SCH1 Schenter, R. E., Schmittroth, F., England, T. R., "Integral Determination of Fission Product Inventory and Decay Power," Review paper at IAEA Second Advisory Group Meeting on Fission Product Nuclear Data, Petten Netherlands, Sept. 5-9, 1977.

77SHM1 Shmid, M., Amiel, S., "Yields of Rb, Cs, Sr, and Ba Isotopes in Thermal Fission of 235U," IA-1338 pages 97-99 (June 1977). Israel Atomic Energy Comm. Tel Aviv. Research Laboratories Annual Report 1976. INIS Atomindex 9(7): 364719 (1978).

77SPI1 Spinrad, B. I., "The Sensitivity of Decay Power to Uncertainties in Fission Product Yields," Nucl. Sci. and Engr. 62, 35-44 (1977). ERDA Energy Research Abstracts 2(9): 22380 (1977). 
77STR1 Strittmatter, R. B., Wehring, B. W., “Direct Physical Measurement of Nuclide Yields for 235 U (n th, f)," Paper at 1977 Winter Meeting of the American Nuclear Society, San Francisco, CA. (Nov 23-Dec 2, 1977). Trans. Am. Nucl. Soc. 27, 862-863 (Nov 1977). INIS Atomindex 9(13): 381727 (1978). EXFOR-10722. CSISRS-90708.

77TOB1 Tobias, A., "Decay Heat Testing of the UK-ENDF/B-IV Format Fission Product Decay Data File," U. K. Central Electricity Generating Board, Research Division, Berkeley Nuclear Laboratories, RD/B/N4179 (Dec 1977).

77WAG1 Wagemans, C., Asghar, M., D'Hondt, P., DeRuytter, A. J., Emsallem, A., "Study of Some Characteristics of the Thermal Neutron-Induced Sub-Barrier Fission of $231 \mathrm{~Pa}$, 232Th, and 237Np," Nucl. Phys. A285 32-44 (1977). EXFOR-20824.

77WAH1 Wahl, A. C., “Nuclear-Charge Distribution in Fission - Investigation of Systematics and Methods for Estimation of Independent Yields," Contribution to Review Paper No. 11 for the IAEA Petten Panel on Fission Product Nuclear Data September 5 -9, 1977 (submitted July 15, 1977).

77WAL1 Walker, F. W., Kirouac, G. J., Rourke, F. M., "General Electric Company Chart of the Nuclides," 12th edition, Revised to April 1977. (October 1977).

77WEI1 Weis, M., Denschlag, H. O., Jahresbericht 1976, Institute fur Kernchemie, Univeritat Mainz (1977).

77WUN1 Wunsch, K. D., Wollnik, H., Contribution to the Isotope Separator On-Line Workshop, Brookhaven (Oct 31, 1977). Cited by 79PEU1.

77YAR1 Yarnell, J. L., Bendt, P. J., "Decay Heat from Products of 235U Thermal Fission by Fast-Response Boil-Off Calorimetry," Report LA-NUREG-6713 (Feb 1977).

78ASG1 Asghar, M., Caitucoli, F., Perrin, P., Guet, C., Hondt, P. D', “Fission Fragment Energy Correlation Measurements for the Thermal Neutron Sub-Barrier Fission of 231Pa," Nucl. Phys. A311 (1978) 413-420. EXFOR-21313.

78ASG2 Asghar, M., Caitucoli, F., Perrin, P., Wagemans, C., "Fission Fragment Energy Correlation Measurements for the Thermal Neutron Fission of $239 \mathrm{Pu}$ and $235 \mathrm{U}$," Nucl. Phys. A311 (1978) 205-218. EXFOR-21544.

78AUM1 Aumann, D. C., Weismann, D., "Independent Isomeric Yield Ratios of 90Rb and 138Cs in the Spontaneous Fission of 252Cf," J. Inorg. Nucl. Chem., 40, 1611-1618 (1978). EXFOR-21579.

78BYA1 Byalko, A. A., Gudkov, A. N., Zhivun, V. M., et. al. "U-235 and Pu239 Fission Product Yields by Neutrons of the BR-1 Fast Reactor Spectrum," INIS-SU-38 (1978). MF available from INIS. (in Russian) INIS Atomindex Vol 13(4): 652658 (15 Feb 1982).

78CHA1 Chapman, T. C., Anzelon, G. A., Spitale, G. C., Nethaway, D. R., “Fission Product Yields from 6-9 meV Neutron-Induced Fission of 235U and 238U," Phys. Rev. C 17(3), 1089-1097 (March 1978). INIS Atomindex 11(24): 568355 (15 Dec 1980). Univ. Microfilm Order No. 79-00106 for 88 page thesis. EXFOR-10828 (Sept 14,1988). CSISRS-90713. 
78CHO1 Choudhury, R. K., Nadkarni, D. M., "Measurement of Relative Yields and Energy Spectra of Tritons and Alpha Particles Emitted in Thermal Neutron Fission of U-235," BARC-978, 56 (1978). EXFOR-30526. Tritium Yield included.

78CUN1 Cuninghame, J., Proceedings of 11th Advisory Group Meeting on Fission Product Nuclear Data, Petten, 1977 Vol. 1, p. 351, Vienna (1978). Quoted in 83GUD1.

78DEB1 Debertin, K., "Fission Product Yields in 238U Fission by 252Cf-Neutrons," Paper at International Conference on Neutron Physics and Nuclear Data for Reactors and Other Applied Purposes. Harwell (September 25-29 1978). EXFOR-21306.

78DEN1 Denschlag, H. O., "Fission Yield Data," International Conference on Neutron Physics and Nuclear Data for Reactors and Other Applied Purposes, Harwell, (25-29 September 1978).

78DEN2 Denschlag, H. O., Fischbach, G., Weis, M., "Charge Distribution in Thermal Neutron Induced Fission Reactions," NEANDC(E)-192/U, 5, 70 (April 1978). EXFOR-21008.

78ENG1 England, T. R., "Delayed Neutron Calculations," LA-UR-78-688. Data dated February 10, 1978. 102 delayed neutrons from 77ENG3, 77ENG4. Report dated (March 9, 1978).

78ERT1 Erten, H. N., Birgul, O., Aras, N. K., "Yields and Isomer Ratio in A=134 Chain for the Spontaneous Fission on 252Cf," J. Inorg. Nucl. Chem. 40, 183-185 (1978). INIS Atomindex 9(11): 375172 (1978).

78FAS1 Fassbender, Th., Kaffrell, N., Trautmann, N., “Bestimmung des Unabhangig Gebildeten Anteils an der Fractionalen Kumulierten Ausbeute fur die Isotope $104 \mathrm{Tc}$ bis $108 \mathrm{Tc}$ bei der Spaltung von $235 \mathrm{U}$ und 238Pu durch Thermische Neutronen," Univ. Mainz, F. R. Germany, Inst. Fur Kernchemie, Jahresbericht 1978. Kernspaltung Teil C, INIS-mf-5838 pp. 97-102, Dec. 1979 available from IAEA, P. O. Box 100, Vienna, Austria.

78FIS1 Fischbach, G., Denschlag, H. O., Jahresbericht 1977, Institut fur Kernchemie, Univ. Mainz (1978) cited in NEANDC(E)-192U Vol. V, Page 70. INDC(Ger)-L+special (1978).

78GAE1 Gaeggeler, H., Gunten, H. R. von, "Charge Distribution in the Thermal-NeutronInduced Fission of 249Cf: Independent and Fractional Cumulative Yields of Isotopes of Nb, I, and Cs," Phys. Rev. C, 17(1), 172-176 (1978). INIS Atomindex 9:397336 (1978). EXFOR-21604.

78GIN1 Gindler, J. E., Glendenin, L. E., “Calculated Fission Product Yields for Fast-Neutron Fission of 238U," Trans. Am. Nucl. Soc. 28, 747-748 (June 1978).

78HIL1 Hill, J. C., Wohn, F. K., Vary, J. P., Williams, S. A., "Tristan - The Nuances of Nuclear Fission," Changing Scene Highlights III, Ames Laboratory, Iowa State U., Ames, Iowa (1978).

78IZA1 Izak-Biran, T., Amiel, S., "Cumulative and Independent Yields of Krypton and Xenon Isotopes from Reactor Neutron Induced Fission of 232Th," J. Inorg. Nucl. Chem. 40(5), 757-763 (1978). INIS Atomindex 9(23): 413578 (1978). EXFOR-30425.

78IZA2 Izak-Biran, T., Amiel, S., "Yields of Tin and Antimony Isotopes in the Vicinity of the Closed Shell $\mathbf{N}=82$ from Reactor Neutron Induced Fission of 232Th," J. Inorg. Nucl. Chem. 40, 937-943 (1978). EXFOR-30425. 
78KAI1 Kaiser, T., Gunten, H. R. von, "Independent Yields of $96 \mathrm{Nb}$ and $51 \mathrm{~min} 98 \mathrm{Nb}$ in the Thermal Neutron-Induced Fission of $233 \mathrm{U}, 235 \mathrm{U}$ and 239Pu," J. Inorg. Nucl. Chem. 40, 377-379 (1978). INIS Atomindex 9(15): 385322 (1978). EXFOR-21218.

78KAI2 Kaiser, T., Gunten, H. R. Von, "Cumulative Mass Yields in the Neutron-Induced Fission of 239Pu at the Resonance Energy of $0.3 \mathrm{eV}, "$ Phys. Rev. C, 17(4) 1510-1512 (April 1978). EXFOR-21745.

78KRA1 Kratz, K. -L., "Independent Fission Yields and Neutron Emission Probabilities of Short-Lived Halogen Isotopes," Radiochim. Acta 25, 1-7 (1978). EXFOR-21058.

78MAE1 Maeck, W. J., Emel, W. A., Duce, F. A., Tromp, R. L., Meteer, J. W., ICP-1142, “A bsolute Thermal Fission Yields for 235U," (September 1978). Zr yields on capsule 7 increased 6.4\%. This adjustment suggested by author in section 7. EXFOR-10865. CSISRS-90725.

78MAN1 Manohar, S. B., Datta, T., Rattan, S. S., Prakash, S., Ramaniah, M. V., "Charge Distribution in Nuclear Fission: Determination of Fractional Yields of 134Te and 1351 in the Spontaneous Fission of 252Cf," Phys. Rev. C, 17(1) 188-194 (January 1978). INIS Atomindex 9(18): 397369 (1978). EXFOR-30516.

78MAR1 Marathe, S. G., Rao, V. K., Bhargava, V. K., Sahakundu, S. M., Iyer, R. H., "Absolute Yields of $99 \mathrm{Mo}$ and $111 \mathrm{Ag}$ in the Reactor Neutron Induced Fission of $238 \mathrm{U}$," J. Inorg. Nucl. Chem. 40, 1981-1982 (1978). EXFOR-30439.

78MON1 Monzyk, M. A., Troutner, D. E., “Independent Yield of 139Ba and 142La from Fission of 248Cf," Paper at 176 ACS National Meeting, Miami Beach, Florida (Sept 11-15 1978). Published in Phys. Rev. C, 20, 212 (July 1979). CSISRS-90729.

78MYE1 Myers, W. A., Kantelo, M. V., Osborne, R. L., Prindle, A., Nethaway, D. R., "Fission of 240Pu with Neutrons Having a Fission-Spectrum Energy Distribution," UCRL-80020 (September 8, 1977). Published here in Phys. Rev. C, 18, 1700 (1978). (see 77MYE1). Sm-153 decay scheme is in error in 78MYE1. In 79PRI1, this has been corrected to read $5.48 \mathrm{e}-3$ and not 5.22e-3 atoms/fission for Sm-153 yield. EXFOR-10821.

78NAG1 Nagy, S., Flynn, K. F., Gindler, J. E., Meadows, J. W., Glendenin, L. E., “Mass Distribution in Monoenergetic-Neutron Induced Fission of 238U," Phys. Rev. C., 17(1), 163171(Jan 1978). Monoenergetic Neutron Fission. Fission spectrum yields are computed from these measurements in 78GIN1. EXFOR-10798. CSISRS-90730.

78RAM1 Ramaswami, A., Natarajan, V., Kumar, R. S., Iyer, R. H., Paper presented in the Symposium on Nuclear Physics and Solid State Physics held at I. I. T. Bombay, India (1979). Published as 80RAM2.

78SCH1 Schoenig, F. C., Moore, W. E., Submitted to Nucl. Sci. and Engr. Cited by 78WAL2.

78SHI1 Shima, M., Thode, H. G., Tomlinson, R. H., "Cumulative Yields of Stable and Long-Lived Isotopes of Ruthenium and Palladium in Neutron-Induced Fission," Can. J. Phys. 56, 1340-1352 (1978). INIS Atomindex 10(10): 431379 (1979). EXFOR-10864. CSISRS-90732. 
78SIN1 Singh, S., Datta, T., Trehan, R., Venkatesan, P. P., Dange, S. P., Manohar, S. B., Nair, A. G. C., Prakash, S., Ramaniah, M. V., "Nuclear Charge Distribution in Fission: Determination of Fractional Cumulative Yields of $99 \mathrm{Mo}, 112 \mathrm{Pd}$, 132Te in the Spontaneous Fission of 252Cf and of $99 \mathrm{Mo}$ and $135 \mathrm{I}$ in Thermal Neutron Induced Fission of $245 \mathrm{Cm}$," Proceedings of the Nuclear Physics and Solid State Physics Symposium, Pune, Dec 26-30, 1977, B. K. Jain, convener, Dept. of Atomic Energy, Govt. of India (INSDOC, New Delhi, 1978). Nuclear Physics, 20B, 249 (1978).

78SPI1 Spinrad, B. I., Wu, C. H., "A General Correlation for Independent Fission Product Yield Uncertainties," Nucl. Sci. and Engr. 66(3), 421-424 (1978). INIS Atomindex 9(22): 410465 (1978).

78STR1 Strittmatter, R. B., Wehring, B. W., "Direct Measurement of Nuclide Yields in ThermalNeutron Fission using Hiawatha," Summary of a paper to be submitted for presentation at the International Conference on Nuclear Physics and Nuclear Data for Reactors and Other Applied Purposes (1978). EXFOR-10722. CSISRS-90733.

78STR2 Strittmatter, R. B., “Nuclide Yields for Thermal Fission of Uranium 235," Ph. D. Thesis, Univ. of Illinois at Urbana Champaign, 1978.

78VAl1 Valenta, V., Hep, J., "Fission Product Yields," ZJE - 211 (1978). Skoda works, Nuclear Power Construction Division, Information Center, Plzen, Czechoslovakia. This is a yield evaluation for U235T, PU239T, PU241T, U235F, U238F, PU239F, PU241F for the 5th Version of the library "Yields" supplementing the "BIBFP" program. Fractional independent yields (without odd-even effect), cumulative and isobaric yields. Partly illegible. INIS Atomindex 10(21): 483809 (1979).

78WAH1 Wahl, A. C., Private communication to B. F. Rider, March 22, 1978. Regarding error assignments which should be put on modelled independent yields. At low FI $(<1 . e-4)$, it is difficult to predict to better than a factor of 2 and at very low yields $($ FI $<1 . e-9)$ to a factor of 100 . Low FI Lohengrin data $<0.02$ should have large uncertainties or be upper limits. 76SIE1 and 76 WOL1 are the same experiment (only one entry should be averaged in).

78WAH2 Wahl, A. C., Wolfsberg, K., Denschlag, H. O., “1877 Compilation of Nuclear-Charge Distribution Data for Thermal Neutron Induced Fission of U235, U233, and Pu238 and for Spontaneous Fission of Cf252," (June 1, 1978).

78WAL1 Walker, W. H., Private communication to B. F. Rider, April 18, 1978, regarding Xe-135 yields for PU239T in 71RAM1 and also for U233T in 66GOR1 and the need for correcting for direct yield of Xe-135 as well as that from decay of I-135 according to equation 2 of 710KA1. Other corrections to NEDO-12154-2D were included. NEDO-12154-2E embodies these corrections. The evaluated data in this communication are published in 78WAL2.

78WAL2 Walker, W. H., "Cross Sections and Yields Important for Fission Product Absorption in Thermal Reactors," A review paper before CSEWG (May 1978).

78WEH1 Wehring, B. W., Private communication to B. F. Rider (June 13, 1978).

78WEI1 Weis, M., Denschlag, H. O., Jahresbericht 1977, Institute fur Kernchemie, Univ. Mainz (1978). Cited in NEANDC(E)-192/U Vol. V page 70 (1978). INDC(Ger)-20/L + special. 
78ZAM1 Zamjatnin, Ju. S., Basova, B. G., Rjazanov, D. K., Rabinovich, A. D., Korostyljov, V. A., "Structure of Mass Distribution of Fragments of U-235, Pu-239 Fission by Thermal Neutrons and From Spontaneous Fission of Cf-252 and Pu-240," Yadern. Fiz. 27(1), 60 (Jan 1978). EXFOR-40479. Monoenergetic neutrons.

78ZWI1 Zwicky, H. U., Gunten, H. R. von, "Independent Yields of 148m Pm and 148g Pm in the Thermal Neutron-Induced Fission of $233 \mathrm{U}$ and 239Pu," NEANDC(OR)-151"L" Part B; INDC(SWT)-12/G June 1978. EXFOR-21002.

79BAL1 Balestrini, S. J., Decker, R., Wollnik, H., Wunsch, K. D., Jung, G., Koglin, E., Siegert, G., "Independent Yields of $\mathrm{Rb}$ and $\mathrm{Cs}$ Isotopes from Thermal Neutron Induced Fission of 235U," LA-UR-79-739, Phys. Rev. C, 222-244 (1979). EXFOR-21641.

79BLA1 Blachot, J., Private communication to $86 \mathrm{WAH} 1$. Here independent yields are split between isomers per 76DEN2 (or 77DEN1 if measured).

79BRA1 Braun, H., Izak-Biran, T., Alfassi, Z. B., Lauppe, W. D., Denschlag, H. O., "Some Decay Properties and Yields in Fission Product Chains 131, 133, and 135," NEANDC(E)202U, 5, 74 (June 1979). Univ. Mainz. EXFOR-21662.

79CLE1 Clerc, H. -G., Lang, W., Wohlfarth, H., Schrader, H., Schmidt, K. -H., "Detailed Study of the Nuclide Yields in $235 U$ (n sub th, $f$ ) and Their Relation to the Properties of the Scission Configuration and the Dynamics of the Fission Process," IKDA 8/79 IAEA SM - 241 -F-2. Contribution to the "International Symposium on Physics and Chemistry of Fission," Juelich, Federal Republic of Germany, May 14-18, 1979. INIS Atomindex 10(21): 483922 (1979).

79DEN1 Denschlag, H. O., Braun, H., Faubel, W., Fischbach, G., Meixler, G., Paffrath, G., Poersd, W., Weis, M., Schrader, H., Siegert, G., Blachot, J., Alfassi, Z. B., Erten, H. N., Izak-Biran, T., Tamai, T., Wahl, A. C., Wolfsberg, K., Conf. 79Juelich, 2, 153 (May 18, 1979). EXFOR-21701. Also same title offered at later Conference 80DEN1. Partial yields.

79DIC1 Dickens, J. K., "Fission Yields for Thermal-Neutron Fission of Plutonium-241," Nud. Sci. and Engr., 70, 177-183 (1979). EXFOR-10894. CSISRS-90740.

79ENG1 England, T. R., Schenter, R. E., Rider, B. F., Liaw, J. R., "Status of ENDF/B-V Fission Yields," LA-UR 79-2703 Paper at Third ASTM-Euratom Symposium on Reactor DosimetTy, Ispra (Varese), Italy (Oct 1-5, 1979).

79ENG2 England, T. R., Schenter, R. E., Schmittroth, F., "Delayed Neutron Calculations Using ENDF/B-V Data," LA-UR 79-2890 Paper at International Conference on Nuclear Cros Sections for Technology, Knoxville, Tenn. (Oct 22-26, 1979) 105 DN precursors with 18 new Ph measurements for the first time since 77ENG3 and 18 other Pn values remeasured with smalkr or more realistic errors reported by $R$. J. Tuttle at LAEA Consultants Meeting on Delaye Neutron Properties, Vienna, Austria (March 26-30, 1979).

79ERT1 Erten, H. N., Aras, N. K., "Charge Distribution in the Spontaneous Fission of 252Cf" J. Inorg. Nucl. Chem. 41, 149-154 (1979). 
79FEL1 Feldstein, H., "Heavy Isotopes of Krypton and Xenon in Thermal Induced Fission of 235U," IA-1333 Israel Atomic Energy Commission, Soreq Nuclear Research Center, Yavne, Israel (July 1979). Thesis 64 pages; 89 refs; 18 figs.; 9 tables. INIS Atomindex 11(22): 561463 (15 Nov 1980). Kr94 and Xe134 yields given which indicate a $(22 \pm 7) \%$ odd-even neutron pairing effect in agreement with previous work (73AMI1).

79HUL1 Hulet, E. K., Wild, J. F., Lougheed, R. W., Baisden, P. A., Dougan, R. J., Mustafa, M. G., "The Kinetic Energy Deficit in the Symmetric Fission of 259Md," Conf 79Juelich, 2, 299, (May79). EXFOR-10893. See 80HUL1.

79KRI1 Krishnarajulu, B., Sen, S., Mehta, G. K., "Light-Charged-Particle Emission in keV Neutron-Induced Fission of 239Pu," J. Phys. G: Nucl. Phys. 5(2) (1979) Pg 319. EXFOR30498.

79MAE1 Maeck, W. J., Eggleston, R. L., Erikson, A. L., Tromp, R. L., "Fast Reactor Fission Yields for 240Pu and 242Pu," ICP-1050-IV (February 1979). EXFOR-10845. This is open literature publication of 76MAE3. Because 76MAE3 is coded in this work and 79MAE1 is an exact duplicate, 79MAE1 data are not duplicated here. For data see 76MAE3.

79MAE2 Maeck, W. J., Duce, F. A., Eggleston, R. L., Tromp, R. L., Meteer, J. W., Emel, W. A., "Absolute Thermal Fission Yields for 239Pu," ENICO-1001 (Sept 1979). EXFOR-10898. CSISRS-90741.

79MAN1 Manohar, S. B., Venkatesan, P. P., Deshmukh, S. M., Prakash, S., Ramaniah, M. V., "Mass Distribution in the Reactor Neutron Induced Fission of U-232," Phys. Rev. C, 19, 1827-1831 (May 1979). Final results superseding 73MAN2 as well as BARC-690 pg 132 (1973), BARC-821 pg 183 (1975), and BARC-872 pg 101 (1976). EXFOR-30511 -30514 -30516.

79MAR1 Martin, G. C., Private communication to B. F. Rider. CSISRS-90743.

79MEI1 Meixler, H., Denschlag, H. O., Wolfsberg, K., "Fractional Cumulative Yields of Kr and Xe Nuclides from Thermal-Neutron Fission of Cf-249 and Spontaneous Fission of Cf-250," Paper, ACS/CSJ Chemical Congress, Honolulu, Hawaii (April 2-6, 1979).

79MON1 Monzyk, M. A., Troutner, D. E., "Fractional Independent Yields of 139Ba and 142La from Thermal-Neutron-Induced Fission of 249Cf," Phys. Rev. C, 20(1), 212-215 (July 1979). Publication of $78 \mathrm{MON} 1$ paper. Private communication to 80MEI1. EXFOR-10897. CSISRS-90744.

79PEU1 Peuser, P., Otto, H., Weis, M., Nyman, G., Roeckl, E., Bonn, J., Reisky, L. von, Spath, C., "Half-Lives, Neutron Emission Probabilities and Fission Yields of Neutron-Rich Rubidium Isotopes in the Mass Region $A=96$ to $A=100$," Z. Phys. A 289(2), 219-224 (Jan 1979). EXFOR-21581.

79PRI1 Prindle, A., Sisson, D. H., Nethaway, D. R., Kantelo, M. V., Sigg, R. A., "Fission of 241Am with 14.8 meV Neutrons," UCRL-82601 (May 25, 1979). Page 20 corrects former publication 77NET1 PU240H Pd-111m Yield to $4.80 \mathrm{e}-3$ Atoms per Fission. The 9.9e-3 cited was Pd$111 \mathrm{~m} /$ Mo99 ratio not yield in atoms per fission as quoted. Published in Phys. Rev. C, Vol. 20, 1824 (1979). EXFOR-10913. CSISRS-90746. 
79RAM1 Ramaswami, A., Natarajan, V., Srivastava, B. K., Sampathkumar, R., Chaudhuri, N. K., Iyer, R. H., "Absolute Fission Yield Measurements by Track-Etch-Cum Gamma Spectrometry," J. Inorg. Nucl. Chem. 41, 1531-1534, (1979). EXFOR-30495.

79RAM2 Ramaswami, A., Dange, S. P., Satyaprakash, K., Ramaniah, M. V., “Mass Yield from Thermal Neutron Induced Fission of 245Cm," J. Inorg. Nucl. Chem. 41, 1649-1653 (1979). EXFOR-30437.

79RAM3 Ramaswami, A., Natarajan, V., Sampathkumar, R., Iyer, R. H., “Absolute Yields of ShortLived Fission Products in the Neutron Fission of Actinide Isotopes," Proceedings of the Nuclear Physics and Solid State Physics Symposium, Bombay (December 28-31, 1978), Vol 21B, pp. 233-236, Bombay Dept of Atomic Energy 1979. INIS Atomindex 12(3): 577093 (1 Feb 1981). Superseded by 80RAM1. EXFOR-30575.

79RA01 Rao, V. K., Bhargava, V. K., Marathe, S. G., Sahakundu, S. M., Iyer, R. H., "Shoulders in the Very Asymmetric Mass Distribution for Reactor-Neutron-Induced Fission of 238-U," Phys. Rev. C, 19, (4), 1372-1379 (April 1979). EXFOR-30496.

79RID1 Rider, B. F., England, T. R., Madland, D. G., Liaw, J. R., Schenter, R. E., "Evaluation of Fission Product Yields for the U. S. National Data Files," Paper at 177 National ACS Meeting, Honolulu (April 2-6, 1979).

79RUD1 Rudstam, G., "Review of Delayed Neutron Branching Ratios," Proc. Consultants" Mtg. on Delayed Neutron Properties, Vienna, Austria, March 1979, INDCNDS-107/G-Special, International Atomic Energy Agency, Vienna (1979).

79SHA1 Sharma, S. C. L., et. al, Proc. Nucl. Phys. Symposium at Madras Dec. 26-30 (1979), Vol $22 B$. INIS Atomindex 14:774731. Ternary Fission U235F (1H, 4He etc.).

79SHM1 Shmid, M., "Measurement of Fission Yields Far from the Center of Isotopic Distributions in the Thermal Neutron Fission of 235U," IA-1345 Israel Atomic Energy Commission, Yavne. Soreq Nuclear Center (Aug 1979). Available from USA Dept of Commerce, National Technical Information Service, 5285 Port Royal Road, Springfield, Virginia, 22161, U. S. A.

79STR1 Strittmatter, R. B., Wehring, B. W., "Fragment Atomic-Number Identification Using a Gas Ionization Chamber in Fission Yield Measurements," Nucl. Instr. and Meth. 166, 473-481, (1979).

79TEP1 Teplykh, V. F., Platygina, E. V., Petrzhak, K. A., Markov, B. M., Kozinets, V. I., "Fine Structure in Mass Yields of Neutron-Induced Fission Products of 237Np," Yadernaya Fizika 29, 293-295 (1979). EXFOR-40945.

79TOP1 Toppare, L., Erten, H. N., Aras, N. K., "Yields of Products in the Spontaneous Fission of 252Cf by Direct Gamma Ray Spectrometry," Paper at 178 ACS National Meeting (Sept 10-14, 1979). Published later as 80TOP1. EXFOR-21700.

79TRO1 Trochon, J., Abou Yehia H., Brisard, F., Pranal, Y., "Fission Properties of 233Th," Nud. Phys. A318 (1979) 63-76. EXFOR-21310. 
79UME1 Umezawa, U., Suzuki, T., Ichikawa, S., Yamashita, T., "Independent Yield of Cs-134 in the Thermal Neutron Fission of U-235," NEANDC(J)-51, 33 (Sept 1977). As cited in the USA National Nuclear Data Center (CSISRS) Accession No. 20847 (March 19,1979). EXFOR20847.

79WAG1 Wagemans, C. M. C., Wegener-Penning, G., Weigmann, H., Barthelemy, R., Conf. 79Juelich, 2, 143 (May 1979). EXFOR-21744.

79WAL1 Walker, W. H., A. E. C. L. Private communication to B. F. Rider (Jan. 11, 1979). PU241T evaluation Mark III.

79WEI1 Weis, M., "Ladungs-, Isotopen-, und Spinverteilung der Reaction $235 \mathrm{U}(\mathrm{n}$ th, f) im Gebiet um $A=$ ca 100," Doctoral Thesis, Mainz (1979). EXFOR-21687.

79YUR1 Yurova, L. N., Bushuev, A. V., Ozerkov, V. N., Chachin, V. V., Zvonaryov, A. V., Liforov, Yu. G., Koleganov, Yu. F., Miller, V. V., Gorbatyuk, O. V., "The Yield of Several Fragments During Fission of $235 \mathrm{U}, 238 \mathrm{U}$, and 239Pu due to Neutrons of the Reactor BP-1 Spectrum," Atomnaya Energiya, 47(1), 26-28 (1979). EXFOR-40489.

79ZAK1 Zakharova, V. P., Rjazanov, D. K., Basova, B. G., Rabinovich, A. D., Korostyljov, V. A., Yadern. Phys. 30(7), 36 (Jul 1979). EXFOR-40420.

79ZIJ1 Zijp, W. L., Baard, J. H., “Nuclear Data Guide for Reactor Neutron Metrology," Part 2: Fission Reactions (1979 Edition), ECN-71 (August 1979).

80ASG1 Asghar, M., Perrin, P., Barreau, G., "Fission Fragment Energy Correlation Measurements for the Subbarrier Thermal Fission of 241Am and 243Am," Nucl. Phys. A 334(2) 327-335 (Feb 1980). INIS Atomindex 11(11): 526992 (1 June 1980). EXFOR-21545.

80ASG2 Asghar, M., Caitucoli, F., Perrin, P., Doan, T. P., Barreau, G., Leroux, B., "Structures in Far-Out Asymmetric Mass Distributions in Low Energy Fission," Nucl. Phys. A 341(3), 388-396 (June 1980). INIS Atomindex 11(20): 554306 (15 Oct 1980).

80AUM1 Aumann, D. C., Zeising, H., "Search for Be-7 and Si-32 as Products of Ternary Fission of U-235 by Thermal Neutrons," Inorg. and Nucl. Chem. Ltrs., 16, 171 (1980). EXFOR21884. Ternary fission of U235T (7Be, 10Be, 32Si etc.).

80CHI1 Chinese Cumulative Yield Group, "The Absolute Determination of Cumulative Yield of Several Nuclides from Thermal Neutron and Fission Spectrum Neutron Induced Fission of 235U," J. Nucl. Radiochem. 2(1), 1-8, and page 42 (Feb 1980). In Chinese. Institute of Atomic Energy, P. O. Box 275, Beijing.

80CHI2 Chinese Cumulative Yield Group, "The Absolute Determination of Cumulative Yields of Several Nuclides from 14.9 MeV Neutron-Induced Fission of 238U," J. Nucl. Radiochem. 2(4), 193-198 (Nov 1980). In Chinese. Institute of Atomic Energy, P. O. Box 275, Beijing.

80DAT1 Datta, T., Dange, S. P., Manohar, S. B., Nair, A. G. C., Prakash, S., Ramaniah, M. V., “Charge

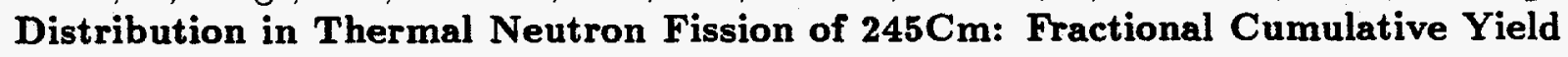
of 135I and 140Ba," Phys. Rev. C, 21(4), 1411-1415 (April 1980). INIS Atomindex 11(19): 551305 (1 Oct 1980). 
80DEN1 Denschlag, H. O., Braun, H., Faubel, W., Fischbach, G., Meixler, H., Paffrath, G., Porsch, W., Weis, M., Schrader, H., Siegert, G., Blachot, J., Alfassi, Z. B., Erten, H. N., Izak-Biran, T., Tamai, T., Wahl, A. C., Wolfsberg, K., "Distribution of Nuclear Charge and Angular Momentum in Chains 132-137, 99, and 102 of $235 \mathrm{U}(\mathrm{n}$ th, $f$ ) at Various Kinetic Energies and Ionic Charge States of the Fragments," IAEA-SM-241/F9, Physics and Chemistry of Fission (1979), Vol II, Vienna, 1980. Partial yield. See also 79DEN1 EXFOR21701.

80DIC1 Dickens, J. K., Mc Connell, J. W., "Fission Product Yields for Thermal Fission of Plutonium-239," Nucl. Sci. and Engr. 73, 42-55 (1980). EXFOR-10890. CSISRS-90758.

80DIC2 Dickens, J. K., Mc Connell, J. W., "Yields of Fission Products Produced by ThermalNeutron Fission of $245 \mathrm{Cm}$," To be published in 1980 . Actually appeared as $81 \mathrm{DIC} 2$. CSISRS-90759.

80DIC3 Dickens, J. K., McConnell, J. W., Northcutt, K. J., "Yields of Short-Lived Fission Products Produced by Thermal-Neutron Fission of Plutonium-239," Submitted to Nucl. Sci. and Engr. Published as 81DIC1.

80ENG1 England, T. R., Wilson, W. B., Whittemore, N. L., "Delayed Neutron Data and Calculations," From LA-8298-PR pages 16-21 (March 1980). 105 DN Emitters on page 18; ENDF/B$\mathrm{V}(\mathrm{E})$ calculated DN yields on page 19.

80ENG2 England, T. R., "FP Decay Data Summary Based on Official BNL File," Private communication to B. F. Rider of decay data processed from the Official BNL ENDF/B-V (Evaluated Nuclear Data File, Format B, Version V) (October 15, 1980).

80FON1 Fontenla, C. A., Fontenla, D. P., "Evidence for Nuclear Superfluidity in 236 U Isomericand Prompt-Fission Modes," Phys. Rev. Ltrs. 44(18), 1200 (May 5, 1980). EXFOR-10937. No data.

80GLE1 Glendenin, L. E., Gindler, J. E., Ahmad, I., Henderson, D. J., Meadows, J. W., "Mass Distributions in Monoenergetic Neutron Induced Fission of 232-Th," Phys. Rev. C, 22(1) 152-159 (July 1980). INIS Atomindex 11(21): 557569 (1 Nov 1980). EXFOR-10920. CSISRS-90760.

80GUD1 Gudkov, A. N., Zhivun, V. M., Zvonaryov, A. V., Kovalenko, V. V., Koldobskii, A. B., Koleganov, Yu. F., Kolobashkin, V. M., Liforov, V. G., Piven, N. S., Tolstikov, V. A., Tipunkov, A. O., "Measurement of the Yields of 236 U Fission by Fast Reactor Neutrons," INIS Atomindex 12(5): 585934 (Mar 1, 1981). Atomnaya Energiya 48(6), 395-396 (Jun 1980).

80HAM1 Hamelin, C., Crancon, J., Blachot, J., Lhospice, G., Bocquet, J. P., Moussa, A., "Determination Experimentale du Rapport Isomerique du 1361 dans la Fission Thermique et par Neutrons de $3 \mathrm{meV}$ de 235U," J. Phys. (Paris) Lett. 41, 1223-1225 (1 May 1980). INIS Atomindex 11(20): 554296 (15 Oct 1980). YI 136I (45sec)/ YI 136I $(45 \mathrm{sec}+83 \mathrm{sec})=$ $0.55 \pm 0.05$ for $\mathrm{U} 235 \mathrm{~T}$ assumed here to be $1.681 / 3.057=0.55$. For $3 \mathrm{meV}$ this ratio is $0.66 \pm 0.07$. EXFOR-21903.

80HOF1 Hoffman, D. C., Lee, D., Ghiorso, A., Nurmia, M., Aleklett, K., “Fragment Mass and Kinetic-Energy Distributions from Spontaneous Fission of the Neutron-Deficient 
Isotopes 1.2-s 246Fm and 38-s 248Fm," Phys. Rev. C, 22(4), 1581 (October 1980). EXFOR-10925.

80HOF2 Hoffman, D. C., Wilhelmy, J. B., Weber, J., Daniels, W. R., Hulet, E. K., Lougheed, R. W., Landrum, J. H., Wild, J. K., Dupzyk, R. J., "12.3-min 256Cf and 43-min 258Md and Systematics of the Spontaneous Fission Properties of Heavy Nuclides," Phys. Rev. C, 21(3), 972 (March 1980). EXFOR-10923.

80HON1 Hondt, P. D', Wagemans, C., DeClercq, Barreau, G., DeRuytter, A., “Energy Distributions and Absolute Yields of the Charged Light Particles Emitted During the Thermal Neutron Induced Ternary Fission of 235U," Nucl. Phys. A346, 461-472 (1980). See also 81HON1 EXFOR-21705.

80HUL1 Hulet, E. K., Lougheed, R. W., Landrum, J. H., Wild, J. F., Hoffman, D. C., Weber, J., Wilhelmy, J. B., "Spontaneous Fission of 259Fm," Phys. Rev. C, 21(3), 966-971 (Mar 1980). INIS Atomindex 11(15): 540339 (1 Aug 1980). EXFOR-10924.

80JAF1 Jaffey, A. H., Steinberg, E. P., Gindler, J. E., Gray Jr., J., Horwitz, E. P., Hughes, J. P., Schmitz, F. J., "Branching Fraction in the Radioactive Decay of $85 \mathrm{~m}-\mathbf{K r}$," ANL-79107 (February 1980). Absolute gas count of $\mathrm{Kr}-85$ and isotopic dilution mass spectrometry of $\mathrm{Rb}-85$. Value is: $\mathrm{Kr} 85 /(\mathrm{Kr} 85+\mathrm{Rb} 85)=0.2160 \pm 0.0019$. INIS Atomindex 11(18): 547986 (15 Sept 1980).

80LAM1 Lam, S. T., Yu, L. L., Fielding, H. W., Dawson, W. K., Neilson, G. C., Sample, J. T., "Fast Neutron Induced Fission of 238U," Phys. Rev. C, 22(6), 2485 (December 1980). EXFOR10964. Monoenergetic Neutrons.

80LAN1 Lang, W., Clerc, H. -G., Wohlfarth, H., "Nuclear Charge and Mass Yields for 235U(n (sub) th, f) as a Function of the Kinetic Energy of the Fission Products," Nucl. Phys. A345 (1980) 34-71, Supersedes works 75CLE2, 76SIE1, 76WOL1, and 79CLE1. Isomeric independent yields here split per 76MAD2. EXFOR-21689.

80LEE1 Lee, S., Wehring, B. W., “Model for Estimating Independent Yields of Fission Products," Trans. Am. Nucl. Soc. (1980) Vol 35 pp. 552-553. INIS Atomindex 14(7): 740536 (1 Apr 1983). Graphic data only.

80LI 1 Li Wenxin, Sun Tongyu, Sun Xiuhua, Zhang Tianmei, Zheng Manjiao, Dong Tianrong, Fu Ming, "Charge Distribution in the Fission of $238 \mathrm{U}$ by 14.7 MeV Neutrons," J. Nucl. Radiochem., 2 (1), 9-16 (Feb 1980). In Chinese. EXFOR 30912 (Dec 23, 1988).

80MAE1 Maeck, W. J., Erikson, A. L., Tromp, R. L., "Fast Reactor Fission Yields for 233U and 235U Irradiated in Row 4 of EBR-II," ENICO-1028 (February 1980).

80MAE2 Maeck, W. J., "Natural Fission Reactor Studies: 238U Spontaneous Fission Yields," (Updated) Reported in INDC(NDS)-113/G+P page 68, Progress in Fission Product Nuclear Data, No. 6 (June 1980).

80MAE3 Maeck, W. J., "The Correlation of 235U Thermal and Fast Reactor Fission Yields with Neutron Energy," ENICO-1065 (December 1980). Graphic representation of fast reactor yields relative to thermal yields as a function of spectral index ( $\mathrm{Nd150} / \mathrm{Nd} 143$ ratio in the 
sample). This index for ENDF U235F $=0.1204$ average. For U235T $=0.1091$. Yields for any spectral index can be obtained by multiplying the thermal yield by the fast/thermal yield ratio obtained from graphs. This is a powerful technique applicable to fuel irradiated in any neutron spectrum (from thermal to fast) requiring only that $\mathrm{Nd} 143$ and $\mathrm{Nd} 150$ be analyzed in addition to $\mathrm{Nd} 148$ or other fission product used for analysis.

80MAE4 Maeck, W. J., Erikson, A. L., Tromp, R. L., "Fast Reactor Fission Yields for Pu-241 and Relative Fission Product Isotopic Data for Pu-239 Irradiated in Row 4 of EBRII," ENICO-1046 (July 1980). EXFOR-10917. INIS Atomindex 11(24): 571450 (15 Dec 1980). EXFOR-10917.

80MEI1 Meixler, H., "Fraktional Kumulierte Edelgasausbeuten bei den Reaktionen 249Cf (n (sub) th, f) und 250Cf ( $(\mathrm{sub}) \mathrm{sp}, \mathrm{f}$ ), Ladungsverteilung in der Kette 137 bei $235 \mathrm{U}$ (n th, f)," Doctoral Thesis, Univ. Mainz. (Juni 1980). EXFOR-21686. Published later as 83MEI1.

80NAI1 Nair, A. G. C., Datta, T., Dange, S. P., Burte, P. P., Manohar, S. B., Das, S. K., Prakash, S., "Charge Distribution in Thermal Neutron Induced Fission of 241Pu: Determination of Fractional Cumulative Yields of $135 \mathrm{I}$ and 140Ba," Proceedings of the Nuclear Chemistry and Radiochemistry Symposium (held at) Waltair, Feb 25-28, 1980, Bombay, India. pp. 150-154. INIS Atomindex 15(5): 016664. (1 Mar 1984). (Data).

80NAQ1 Naqvi, A. A., "Fragment Properties in Fission of Np-237 with Fast Neutrons-An Experimental Investigation of Fission Dynamics," KFK-2919 (March 1980). Kernforschungszentrum, Karlsruhe. Pulse proton beam on Li-7 for $800 \mathrm{keV}$ data. EXFOR-21661.

80NET1 Nethaway, D. R., Richardson, A. E., "Independent Yields of $148 \mathrm{~m}-P \mathrm{~m}, 148 \mathrm{~g}-\mathrm{Pm}$, and $150-P m$ from Fission of $235 \mathrm{U}$ and $238 \mathrm{U}$ with 14.8-meV Neutrons," UCRL-84054 (March 27, 1980). J. Inorg. Nucl. Chem. 43, 889-893 (1981).

80NIS1 Nishi, T., Fujiwara, I., Imanishi, N., Moriyama, H., "Independent Isomer Yields of I132, I-134, Xe-135, and Cs-138 Produced in Thermal Fission of U-233, U-235, and Pu-239," Kyoto Univ. Institute of Atomic Energy. Nishi, T. Private communication August 22,1980 with numerical data and text of an unpublished article supersedes 77NIS1. CSISRS Accession No. 20848. EXFOR-20848.

80PEU2 Peuser, P., Otto, H., Nyman, G., Roeckl, E., "Decay Properties of Neutron-Rich Isotopes of $\operatorname{Rb}(Z=37)$ to $\operatorname{Tc}(Z=43)$ and the Mass Energy Surface Around $A=100$," Nuclear Spectroscopy of Fission Products, Chapter 5, pages 288-296 (1980). ISBN 085498 142X Bristol. The Institute of Physics Conf. Series 51. INIS Atomindex 11(23): 564215 (1 Dec 1980).

80RAM1 Ramaswami, A., Natarajan, V., Iyer, R. H., "Absolute Yields of Short-Lived Fission Products in the Thermal Neutron Induced Fission of $235 \mathrm{U}$ and $239 \mathrm{Pu}$," J. Inorg. Nucl. Chem., 42 1213-1216 (1980). INIS Atomindex 12(3): 577100 (1 Feb 1981). EXFOR-30575 (Dec 14, 1987).

80RAM2 Ramaswami, A., Raghuraman, K., Srivastava, B. K., Natarajan, V., Iyer, R. H., Jadhav, A. V., Sivaramakrishnan, C. K., "Determination of Absolute Fission Yields in Spontaneous Fission Systems: Yields of $99 \mathrm{Mo}$ and $140 \mathrm{Ba}$ in Spontaneous Fission of $244 \mathrm{Cm}$," 
Proceedings of the Nuclear Chemistry and Radiochemistry Symposium (held at) Waltair. Feb 25-28, 1980. INIS Atomindex 15(5): 016663 (1 Mar 1984). Published later as 82RAG1.

80RAT1 Rattan, S. S., Reddy, A. V. R., Singh, R. J., Prakash, S., Ramaniah, M. V., "Studies on Charge Distribution in the Thermal-Neutron-Induced Fission of 229Th," pp. 155158 Proceedings of the Nuclear Chemistry and Radiochemistry Symposium (held at) Waltair Feb 25-28 (1980) Bombay, India. INIS Atomindex 15(5): 016665 (1 Mar 1984). (Data).

80RID1 Rider, B. F., "Compilation of Fission Product Yields, Vallecitos Nuclear Center, 1880," Available either as General Electric Company Report NEDO-12154-3(C) (October 1981), or as Brookhaven National Laboratory, Evaluated Nuclear Data File Report ENDF-292.

80RID2 Rider, B. F., England, T. R., Madland, D. G., Liaw, J. R., Schenter, R. E., "Evaluation of Fission Product Yields for the U. S. National Nuclear Data Files," Paper for presentation at the Workshop on Evaluation Methods and Procedures for Applied Data, Sept 22-26, 1980, Upton, N. Y. To be published in Proceedings of Conference as a U. S. A. DOE-BNL Report see (81RID1).

80SIG1 Sigg, R. A., Kantelo, M. V., Sisson, D. H., Prindle, A. L., Nethaway, D. R., "Fast Fission of 241 Am," UCRL-85195 (Dec 1, 1980). Later published as 83SIG1 (which see).

80SRI1 Srinivasan, B., Flynn, K. F., "Kr and Xe Isotopes from Spontaneous Fission of (Cm248,a dn Cf250): Comparison with Noble Gases in Carbonaceous Chondrites," Earth Planet. Sci. Lett., 47, 235 (1981). EXFOR-12711. Cf250S yields for masses $83 \mathrm{Kr}, 84 \mathrm{Kr}$, $85 \mathrm{Kr}, 86 \mathrm{Kr}, 101 \mathrm{Tc}, 104 \mathrm{Tc}, 105 \mathrm{Ru}, 107 \mathrm{Rh}, 131 \mathrm{Xe}, 132 \mathrm{Xe}, 133 \mathrm{Xe}, 134 \mathrm{Xe}, 136 \mathrm{Xe}, 139 \mathrm{Ba}$ and 142La. Cm248S yields for $83 \mathrm{Kr}, 84 \mathrm{Kr}, 85 \mathrm{Kr}, 86 \mathrm{Kr}, 131 \mathrm{Xe}, 132 \mathrm{Xe}, 134 \mathrm{Xe}$, and $136 \mathrm{Xe}$.

80THI1 Thierens, H., Jacobs, E., D'hondt, P., De Frenne, D., De Clercq, A., De Gelder, P., Deruytter, A. J., Blachot, J., Perrin, P., "The Thermal Neutron Sub-Barrier Fission of Np237," Nucl. Phys. A 342(2), 229-238 (June 1980). INIS Atomindex 11(22): 561541 (15 Nov 1980). EXFOR-21651.

80TOB1 Tobias, A., Davies, B. S. J., "UKFPDD-2: A Revised Fission Product Decay Data File in ENDF/B-IV Format," Central Electricity Generating Board Research Division Report RD/B/N4942 (Nov 1980). Berkeley Nuclear Laboratories, U. K.

80TOP1 Toppare, L., Erten, H. N., Aras, N. K., "Yields of Products in the Spontaneous Fission of 252Cf by Direct Gamma Ray Spectrometry," Tech. J. Ankara Nucl. Res. Cent. 7(1) 8-14 (April 1980). INIS Atomindex 11(22): 561441 (15 Nov 1980).

80WAH1 Wahl, A. C., "Systematics of Nuclear Charge Distribution in Fission; The Zp Model," J. Radioanal. Chem., 55(1), 111-123 (1983).

80WAN1 Wang Yusheng, Tang Funan, Zhou Ling, Tang Peijia, Kang Jingxiu, "The Measurement of Cumulative Fission Yields of $235 U$ Induced by Thermo- and Fission Spectrum Neutrons by Using Ge(Li) Detector," J. Nucl. Radiochem., 2(3), 129-138 (Aug 1980). In Chinese. Institute of Atomic Energy, P. O. Box 275, Beijing.

80WEH1 Wehring, B. W., Lee, S., Swift, G., "Light-Fragment Independent Yields for Thermal Neutron Fission of U-233," Univ. of Illinois, Urbana, Ill. Document UILU-ENG-80 -5312 (May 1, 1980). See Trans. Am. Nucl. Soc. (35), 551 (November 1980). EXFOR-10918. 
80ZHE1 Zheng Yung-Foh, et. al., "Relative Fission Product Yields in $14 \mathrm{MeV}$ Neutron-Induced Fission of 238U by Non-Destructive Ge(Li) Gamma Ray Spectrometry," Page 151 (1980). Nuclear Energy Publishers, Beijing (In Chinese). Cited in 85LIU1.

80ZWI1 Zwicky, H. U., Gunten, H. R. von, "Independent Yields of 148m Pm and $148 \mathrm{~g}$ Pm in the Thermal-Neutron-Induced Fission of $233 \mathrm{U}$ and 239Pu," Radiochim. Acta 27, 121-124 (1980). INIS Atomindex 11(22): 561574 (15 Nov 1980). EXFOR-21706.

81ASG1 Asghar, M., Caitucoli, F., Leroux, B., Perrin, P., Barreau, G., "Fission-Fragment Energy Correlation Measurements for $(n$ th, $f$ ) of $232 U$ and 233U," Nucl: Phys. A368, 328-336 (1981). EXFOR-21771.

81ASG2 Asghar, M., Caitucoli, F., Leroux, B., Maurel, M., Perrin, P., Barreau, "Fission Fragment Energy Correlation Measurements for 238Pu(n th, f)," Nucl. Phys. A368, 319-327 (1981). EXFOR-21774.

81AUM1 Aumann, D. C., Weismann, D., and Zeising, H., "Independent Yields of 102m-Rh, 102g$\mathrm{Rh}$ and 101g-Rh for Thermal-Neutron-Induced Fission of $235 \mathrm{U}$, J. Inorg. Nucl. Chem. Vol 43(10) pp. 2223-2226 (1981). INIS Atomindex 13(4): 652703 (15 Feb 1982). EXFOR-21754.

81BAY1 Bayer, R., Dlouhy, Z., Svanda, J., Wilhelm, I., “A Multiparameter System for Heavy Nuclei Fission Study," Czech. J. Physics B 31, 1273-1285 (1981). Ref. 2021 in 89MIL2. Results of light particle emission from spontaneous fission of $252 \mathrm{Cf}$ and from neutron induced fission of $235 \mathrm{U}$ are presented.

81CAI1 Caitucoli, F., Wagemans, C., Perrin, P., Allaert, E., D'Hondt, P., “Mass and Energy Characteristics of the 241Pu(n th, f) Fragments," Nucl. Phys. A369 15-24 (1981). EXFOR21758.

81CUM1 Cumpstey, D. E., Vass, D. G., “The 6He Emission Accompanying the Spontaneous Fission of 252Cf," Nucl. Phys. A359 (1981) 377-385. EXFOR-21699.

81DIC1 Dickens, J. K., Mc Connell, J. W., Northcutt, K. J., "Yields of Short-Lived Products Produced by Thermal-Neutron Fission of Plutonium-239," Nucl. Sci. and Engr. 77, 146-152 (1981). EXFOR-10961.

81DIC2 Dickens, J. K., Mc Connell, J. W., "Yields of Fission Products Produced by ThermalNeutron-Fission of 245Cm," Phys. Rev. C, 23(1): 331-350 (Jan 1981). Publication of 80DIC2 which see. EXFOR-10962.

81DIC3 Dickens, J. K., Mc Connell, J. W., "Yields of Fission Products Produced by Thermal Neutron Fission of Cf-249," Phys. Rev. C, V24(7), pp. 192-205 (1981). EXFOR-12705.

81ERT1 Erten, H. N., Gruetter, A., Roessler, E., Gunten, H. R. von, “Mass Distribution in the Reactor Neutron Induced Fission of Thorium-232," Nucl. Sci. and Engr. 79, 167-174 (1981). EXFOR-21712, -21723.

81GIN1 Gindler, J. E., Glendenin, L. E., Henderson, D. J., "Fission Yields for Spontaneous Fission of $246 \mathrm{Cm}$ and Thermal-Neutron-Induced Fission of $229 \mathrm{Th}$ and $236 \mathrm{~Np}$," J. Inorg. Nucl. Chem. 43, 1433-1437 (1981). EXFOR-10966. 
81GIN2 Gindler, J. E., Glendenin, L. E., Krapp, E. L., Fernandez, S. J., Flynn, K. F., Henderson, D. J., "Mass Distribution in Thermal-Neutron-Induced Fission of Long Lived 236Np," J. Inorg. Nucl. Chem. 43, pp. 445-448 (1981). EXFOR-10967.

81GIN3 Gindler, J. E., Henderson, D. J., Glendenin, L. E., "Spontaneous Fission Yields for 246Cm," J. Inorg. Nucl. Chem. 43, 895-896 (1981). EXFOR-10986.

81GIN4 Gindler, J. E., Glendenin, L. E., Henderson, D. J., "Mass Distributions for the Fission of 250Cf," J. Inorg. Nucl. Chem. 43, 1743-1749 (1981). EXFOR-12710.

81GLE1 Glendenin, L. E., Gindler, J. E., Henderson, D. J., Meadows, J. W., "Mass Distributions for Monoenergetic-Neutron-Induced Fission of 235U," Phys. Rev. C, 24(6), 2600-2605 (Dec 1981). EXFOR-12729. Monoenergetic neutrons.

81GUD1 Gudkov, A. N., Kazantsev, V. U., Kovalenko, V. V., Koldobskii, A. B., Kolobashkin, V. M., Slyusarenko, A. I., "Measurement of Short Lived Fission Products Yield of 233U Thermal Fission by Gamma Spectrometric Method in a Cyclic Regime," (in Russian). INIS-SU-122 (1981) pp. 49-52. MF available from INIS. INIS Atomindex 13(4): 704721 (1982).

81HON1 Hondt, P. D', Wagemans, C., Clercq, A. De, Barreau, DeRuytter, A., “Energy Distributions and Absolute Yields of the Charged Light Particles Emitted During the Thermal Neutron Induced Ternary Fission of 235U," Talk based on Nucl. Phys. A346 (1980) 461-472. EXFOR-21705. See 80HON1.

81HSU1 Hsu, S. S., Lin, J. T., Yang, M., Yu, Y. M., "Yields and Isomeric Ratios of Xenon and Krypton Isotopes from Thermal Fission of U-235," Phys. Rev. C, 24(2), 523-528 (August 1981). USA National Nuclear Data Center Accession No. 30666 (March 26, 1985). EXFOR-30666.

81JOS1 Jost, D. T., and Gunten, H. R. von, "Independent Yields of $92 \mathrm{~m}-\mathrm{Nb}$ in the Thermal NeutronInduced Fission of 233U, 235U and 239Pu," J. Inorg. Nucl. Chem. Vol 43(11) pp. 2629-2631. INIS Atomindex 13(7): 660958 (1 Apr 1982). EXFOR-21711.

81KOC1 Koch, L., "Systematics of Fast Cumulative Fission Yields," Radiochim. Acta. (1981) Vol 29(2/3), 61-63. INIS Atomindex 13(14): 682811 (15 July 1982). For details see Koch, L. et. al. EUR-6738 (1980) cited as 81KOC2. EXFOR-21155.

81KOC2 Koch, L., Kammerichs, K., Cottone, G., Steinert, D., De Meester, R., Heitz, J., Molinet, R., Rijkebor, C., "Cumulative Fast Reactor Fission Yields of $233 \mathrm{U}, 235 \mathrm{U}, 236 \mathrm{U}, 238 \mathrm{U}$, 237Np, 239Pu, 240Pu, 241Pu, 242Pu, 241Pu," European Appl. Res. Report.-Nucl. Sci. Technol. Vol 3, Nos. 1 and 2 (1981), pp. 1-22. EUR-6738 EN.

81LAU1 Laurec, J., Adam, A., de Bruyne, Th., "Determination par une Methode Radiochimique des Rendements de Fissions Induites par un Spectre de Neutrons de Fission et des Neutrons de 14.7 meV Dans 233U, 235U, 238U, 239Pu," To be published 1981. Republique Francaise Commissariat a l'Energie Atomique, 29-33, Rue de la Federation, Paris (XVE); CEA, B. P. 51175752 Paris Cedex 15, RCP No. 231 (30 Mars 1981). EXFOR-21707, -21708 . 
81MAE1 Maeck, W. J., Tromp, R. L., "Revised EBR-II Fast Reactor Fission Yields for 233U, 235U, and 238U," ENICO-1091 (August 1981). Major revision of masses 139, 140, 141, and 142 from 74MAE1 by improved methods and background corrections.

81MAE2 Maeck, W. J., Private communication to B. F. Rider (October 20, 1981). Neutron energy effect on the fission yields of Pu239. To be published as ENICO-1099.

81MAR1 Mariolopoulos, G., Hamelin, Ch., Blachot, J., Bocquet, J. P., Brissot, R., Crancon, J., Nifenecker, H., Ristori, Ch., "Charge Distributions in Low-Energy Nuclear Fission and Their Relevance to Fission Dynamics," Nucl. Phys. A. Vol 361(1) pp. 213-241 (18 May 1981). INIS Atomindex 12(18): 624112 (1 Sept 1981). Isomeric independent yields here split per 76MAD2. EXFOR-21743.

81MUE1 Mueller, R., Naqvi, A. A., Kaeppeler, F., Bao, Z. Y., "Numerical Results of a (2E, 2v)Measurement for Fast Neutron Induced Fission of $235 \mathrm{U}$ and 237Np," KfK-3220 (Dezember 1981). EXFOR-21834. Energy dependent yields.

81NET1 Nethaway, D. R., Richardson, A. E., "Independent Yields of $148 \mathrm{~m}-\mathrm{Pm}, 148 \mathrm{~g}-\mathrm{Pm}$, and 150-Pm from Fission of $235 \mathrm{U}$ and $238 \mathrm{U}$ with $14.8 \mathrm{meV}$ Neutrons," J. Inorg. Nucl. Chem. 43, 889-893 (1981). Publication of 80NET1 UCRL Report (which see). EXFOR-10994.

81NIC1 Nicoli, I. G., "Determination of the Fission Products Yields, Lanthanum and Yttrium, in the Fission of $238 \mathrm{U}$ with Neutrons of Fission Spectra," INIS-BR-27 June 1981. MF available INIS. (Data).

81PAL1 Pal, D. K., Troutner, D. E., "Fractional Independent Yields of La-141 and La-142 from Thermal-Neutron-Induced Fission of U-233," J. Inorg. Nucl. Chem. 43, 885-888 (1981). Cited in INDC(NDS)-113/G+P, pg. 78 (June 1980). Data privately communicated to B. F. Rider from D. E. Troutner (Jan. 19, 1981). EXFOR-10995.

81PAL2 Pal, D. K., "Nuclear Charge Distribution in Fission: Independent Yields of 141La, 142La, 92Y, and $93 \mathrm{Y}$ from Thermal-Neutron Fission of 249Cf," Missouri Univ., Columbia, Missouri (USA) Thesis Ph. D. 1981104 pp. Univ. Microfilms Order No. 82-23, 467. INIS Atomindex 14(23): 801615 (1 Dec 1983). (data).

81RAM1 Ramaswami, A., Natarajan, V., Srivastava, B. K., Iyer, R. H., "Absolute Yields of the Fission Products in the Neutron Induced Fission of $232 \mathrm{Th}$ and $233 \mathrm{U}$," J. Inorg. Nucl. Chem. Vol 43(12) pp. 3067-3069 (1981). INIS Atomindex 13(18): 694748 (15 Sept 1982).

81RID1 Rider, B. F., England, T. R., Madland, D. G., Liaw, J. R., Schenter, R. E., "Evaluation of Fission Product Yields for the U. S. National Nuclear Data Files," Proceedings of the Conference on Nuclear Data Evaluation Methods and Procedures, Brookhaven National Laboratory, Upton, N. Y. September 22-25, 1980. Information Analysis Center Report BNLNCS-51363 Vol II of II, Pages 797-834 (March 1981). This report also is designated as DOENDC-23 and NEANDC(US)-209 as well as INDC(USA)-85. Obtainable from National Technical Information Service, U. S. Dept. of Commerce, 5285 Port Royal Road, Springfield, Va 22161, U. S. A. Fiche copy price code A01.

81SEH1 Sehr, R., Denschlag, H. O., "Measurement of the Fractional Cumulative Yield of 77 Ga in the Thermal-Neutron-Induced Fission of 235U," 1980 Annual Report, University of Mainz (1981). From Diplomarbeit (1980). 
81SHM1 Shmid, M., Nir-el, Y., Engler, G., Amiel, S., "Fission Yield Measurements of Rb, Sr, Cs, and $\mathrm{Ba}$ Isotopes Far From the Center of the Isotopic Yield Distributions in $235 \mathrm{U}$ (n sub th, f)," J. Inorg. Nucl. Chem., 43, 867-875 (1981).

81TAN1 Tanner, J. E., PNL-3563, UC-11 (Mar 1981) pp. 3-4. Tritium yields Pu239T, U233HE, U235F, Pu241T.

81VIN1 Vine, E. N., Wahl, A. C., "Fractional Independent Yields of 104Tc and 105Tc from Thermal-Neutron-Induced Fission of $235 \mathrm{U}$ and 239Pu," J. Inorg. Nucl. Chem. 43, 877883 (1981). Partly from 76VIN1 Thesis Washington University plus much new data including that from Pu239 from later LASL work. EXFOR-10996.

81WAG1 Wagemans, C., Allaert, E., Caitucoli, F., Hondt, P. D', Barreau, G., Perrin, P., "Experimental Study of Some Important Characteristics of the Thermal Neutron Induced Fission of $237 \mathrm{~Np}$," EXFOR-21752.

81WAL1 Waldo, R. W., Karam, R. A., Meyer, R. A., "Delayed Neutron Yields: Time Dependent Measurements and a Predictive Model," Phys. Rev. C, 23(3), 1113-1127 (Mar 1981). EXFOR-12926.

81WEB1 Weber, J., Britt, H. C., Wilhelmy, J. B., "Comparison of 252Cf Spontaneous Fission with 250Cf(t, pf)," Phys. Rev C, 23(5), 2100 (May 1981). EXFOR-12709.

81 WEI1 Weis, M., Denschlag, H. O., "Fractional Independent Yields of 99Y, 99Zr, 99m-Nb, $99 \mathrm{~g}-\mathrm{Nb}$ in the Thermal Neutron Induced Fission of $235 \mathrm{U}$," J. Inorg. Nucl. Chem. 43, 437-444 (1981).

82ALL1 Allaert, E., Wagemans, C., Wegener-Penning, G., DeRuytter, A. J., Barthelemy, R., “Energy and Mass Distributions for 241Pu(n th, f), 242Pu(s.f.) and 244Pu(s.f.) Fragments," EXFOR-21788.

82ASG1 Asghar, M., Caitucoli, F., Leroux, B., Maurel, M., Perrin, P., Barreau, G., "Fission Fragment Energy Correlation Measurements for 228Th(n th, f)," Nucl. Phys. A373 (1982) 225236. EXFOR-21776.

82AUM1 Aumann, D. C., Friedmann, L., “Cumulative Yield of 1.6x10(superscript 7)-year 129I from Thermal Neutron Induced Fission of 235U," Radiochim. Acta (1982) V30(1) pp. 19-20. INIS Atomindex 13(23): 710110 (1 Dec 1982). EXFOR-21917.

82BAIl Baisden, P. A., Wild, J. F., Dougan, R. J., Lougheed, R. W., Hulet, E. K., Progress Rpt. UCAR-10062-81-1,153 (1982). EXFOR-12814. Concerning ternary fission; contains $4 \mathrm{He}$ yield for $\mathrm{Fm}-257$ spontaneous fission.

82BRE1 Breederland, D. G., "Fission Product Yields for Thermal Neutron Fission of Curium243,” ORNL/TM-8168 (Jan 1982). INIS Atomindex 13(17): 691219 (1 Sept 1982).

82CAI1 Caitucoli, F., Asghar, M., Leroux, B., Barreau, G., Hamadache, K., Sicre, A., "FissionFragment Energy Correlation Measurement for the Spontaneous Fission of 244Cm," Nucl. Phys. A394 (1983) 360-368. EXFOR-21844 and -21853. 
82CHI1 Chinese Cumulative Yield Group, "The Absolute Determination of Cumulative Yield of Several Nuclides from Induced Fission of $235 \mathrm{U}$ by Neutrons of Spontaneous Fission of 252Cf," J. Nucl. Radiochem. 4(1), 44-47 (Feb 1982). In Chinese. Institute of Atomic Energy, P. O. Box 275, Beijing.

82DAT1 Datta, T., Dange, S. P., Nair, A. G. C., Prakash, S., Ramaniah, M. V., "Fission Fragment Angular Momentum: Ratios of Independent Yields of Isomers of $95 \mathrm{Nb}$ and $132 \mathrm{I}$ in Thermal-Neutron-Induced Fission of 233U," Phys. Rev. C, 25(1), 358 (1982).

82DIC1 Dickens, J. K., Mc Connell, J. W., Northcutt, K. J., "Yields of Short-Lived Fission Products of Thermal-Neutron Fission of Thorium-229," Nucl. Sci. and Engr. Vol 80, 455-461 (March 1982). Oak Ridge National Laboratory. USA National Nuclear Data Center Accession No. 12734 (April 26, 1983). EXFOR-12734.

82ERT1 Erten, H. N., Gruetter, A., Roessler, E., Gunten, H. R. von, "Charge Distribution in the Reactor Neutron Induced Fission of 232Th," Phys. Rev. C, V25(5) pp. 2519-2523. INIS Atomindex 14(4): 727083 (15 Feb 1983). EXFOR-21805.

82GAR1 Garlea, T., Miron, C., Musat, T., Roth, C., "Determination of Some Fission Yields in the Spectrum for the Reactions $235 U(n, f), 239 P u(n, f), 238 U(n, f), " \operatorname{INDC}(R O M)-014$, 2, (Dec 1982). EXFOR-30752 (Dec 14,1987).

82GOL1 Golovanov, A. G., Gudkov, A. N., Kazantsev, V. V., Kovalenko, V. V., Koldobskii, A. B., Kolobashkin, V. M., Lifanov, S. I., and Slyusarenko, A. I., "Determination of the Cumulative Yields of Short-Lived Products of Thermal Neutron 239Pu Fission by Means of the Gamma Spectrometric Method Under Cyclic Conditions," At. Ehnerg. (Aug 82) Vol 53(2) p. 117-118. INIS Atomindex 14(17): 785835 (1 Oct 1983).

82LAE1 Laeter, J. R. de, Rosman, K. J. R., and Boldeman, J. W., "Relative Yields of Stable Tellurium Isotopes in Neutron Fission," Aust. J. Phys. (1982) Vol 35, pp. 385-391. INIS Atomindex 14(2): 718883 (1983). EXFOR-30650.

82LI 1 Li Wenxin, Sun Tongyu, Sun Xiahua, Zhang Tianmei, Zheng Manjiao, Dong Tianrong, Fu Ming, "Charge Distribution in the Fission of Th-232 by $14 \mathrm{MeV}$ Neutrons," Phys. Energ. Fort. et Phys. Nucl. 6, 365 (May 1982). In Chinese. EXFOR 30695 dated 1985-12-10.

82NAI1 Nair, A. G. C., Srivastava, A., Srivastava, B. K., Prakash, S., Ramaniah, M. V., "Cumulative Yields of Short-Lived Ruthenium Isotopes in the Spontaneous Fission of 252Cf," (Paper No. NC-2) of Radiochemistry and Radiation Chemistry Symposium held at Pune (India) Dec 7-11, 1982. (Preprint Volume) 3 pages. 105, 107-109 Ru values. See 84NAI1. INIS Atomindex 17(18): 061256 (15 Sept 1986). Later published as 84NAI1 (which see).

82NAK1 Nakagomi, Y., Kobayashi, S., Yamamoto, S., Kanno, I., “Measurement of Mass Distribution of U-235 Fission Products in the Intermediate Neutron Region," UTNL-R-0122 Feb 1982126 p. MF available INIS. This region not treated here. INIS Atomindex 15(18): 055108 (15 Sept 1984).

82POE1 Poersch, W., Braun, H., Denschlag, H. O., Ditz, W., Faubel, W., Faust, H., Sohnius, B., "Charge Distribution and Isomeric Ratios in the Chains $A=130,131$ in U235 
(n(th),f)," Ann. Rept. 1981 (Mainz) 25 Nov 1982165 p. MF available INIS. INIS-MF8160. INIS Atomindex 14(11): 757529 through 757531 (1 Jun 1983). Yields not yet summed over energy. Entry 757530 covers $A=140$. Entry 757532 covers $A=142$.

82RAG1 Raghuraman, K., Ramaswami, A., Sivaramakrishnan, C. K., Iyer, R. H., “Absolute Yields of 99Mo and 140Ba in Spontaneous Fission of $244 \mathrm{Cm}$," Radiochim. Acta (1982) V31(1/2) pp. 65-67. INIS Atomindex 14(5): 736023 (15 Mar 1983). This is the Journal publication of Waltair paper cited in INIS Atomindex 15(5): 016663 (1 Mar 1984).

82RAM1 Ramaswami, A., Srivastava, B. K., Manohar, S. B., Prakash, S., Ramaniah, M. V., "Charge Distribution in the Spontaneous Fission of 252Cf: Determination of Fractional Cumulative Yields of $138 \mathrm{Xe}$ and 139Cs," Radiochim. Acta (1982) V30(1) pp. 15-17 INIS Atomindex 13(23): 710109 (1 Dec 1982). EXFOR-30629.

82RAM2 Ramaswami, A., Raghuraman, K., Srivastava, B. K., Iyer, R. H., "Absolute Yields of Fission Products in the Thermal-Neutron Induced Fission of $245 \mathrm{Cm}$," Radiochim. Acta (1982) V. 30(1) pp. 11-14. INIS Atomindex 13(23): 710108 (1 Dec 1982). EXFOR-30631.

82RAM3 Ramaswami, A., Srivastavia, B. K., Manohar, S. B., Prakash, S., Ramaniah, M. V., "Charge Distribution in the Spontaneous Fission of 252Cf: Determination of Fractional Cumulative Yields of $138 \mathrm{Xe}$ and 139Cs," Radiochim. Acta (1982) V. 30(1), 15-17. INIS Atomindex 13(23): 710109 (1 Dec 1982).

82RAT1 Rattan, S. S., Reddy, A. V. R., Singh, R. J., Prakash, S., Ramaniah, M. V., "Charge Distribution in the Thermal Neutron Induced Fission of 229Th," Paper No. NC-4 of Radiochemistry and Radiation Chemistry Symposium held at Pune (India) Dec. 7-11, 1982 (Preprint Volume) 4 pages. Rb88 FC $=0.77 \pm 0.08$ and $\mathrm{I}-134 \mathrm{FC}=0.77 \pm 0.15$ for $\mathrm{TH} 229 \mathrm{~T}$. INIS Atomindex 17(18): 061255 (15 Sept 1986).

82REN1 Rengan, K., Lin, J., Meyer, R. A., "Fission Yields by Continuous Chemistry," University of California Lawrence Radiation Laboratory UCAR-10062-81-1,111,82. EXFOR-12740.

82SIN1 Singh, R. J., Rattan, S. S., Reddy, A. V. R., Venkatasubramani, C. R., Ramaswami, S., Prakash, S., Ramaniah, M. V., "Mass Distribution in the Thermal Neutron Induced Fission of 229Th," Radiochim. Acta, (1982) 31(1/2) pp. 69-73. INIS Atomindex 14(5): 736024 (15 Mar 1983).

82SRI1 Srivastava, A., Nair, A. G. C., Srivastava, B. K., Manohar, S. B., Prakash, S., Ramaniah, M. V., "Nuclear Charge Distribution in the Spontaneous Fission of 252Cf," (Paper No. NC-3) of Radiochemistry and Radiation Chemistry Symposium held at Pune (India) December 7-11, 1982 (Preprint Volume) 4 pages. INIS Atomindex 17(18): 061258 (15 Sept 1986).

82VIN1 Vine, E. N., Wahl, A. C., "The Chemical Behavior of Tracer-Level Technetium Isotopes," International J. Appl. Radiat. Isot. (UK) Vol 33(10), 861-866 (October 1982). INIS Atomindex 14(10): 753982 (15 May 1983).

82WAN1 Wang Lianbi, and the Fission Yield Group, "Absolute Cumulative Yields of Several Nuclides from U-235 Fission Induced by Cf-252 Spontaneous Fission Neutrons," J. Nucl. Radiochem., 4(1), 44 (Feb 1982). Inst. of Atomic Energy, P. O. Box 275, Beijing. EXFOR 30744 Dated 1987-12-14. 
82WEN1 Wenxin, L., Tongyu, S., Xiuhua, S., Tianmei, Z., "Charge Distribution in the Fission of Th232 by $14 \mathrm{meV}$ Neutrons," Abstr. of the International Conference on Nuclear Physics, Berkeley, California, USA (August 24-30 1980). Lawrence Berkeley Laboratory LBL$1118,928,8008$ (August 1980). Preliminary results superseded by journal publication (in Chinese) High Energy Physics, 6, page 365 (May 1982). EXFOR 30695 (December 10, 1985). See also 87LI 1, High Energy Physics and Nuclear Physics 11(3), 376-383 (May 1987). EXFOR30695.

83DIC1 Dickens, J. K., and Mc Connell, J. W., "Yields of Fission Products Produced by ThermalNeutron Fission of 229Th," Phys. Rev. C., Vol 27(1), 253-264 (January 1983). EXFOR12807.

83ENG1 Engler, G., Rapaport, M. S., "Independent Fission Yields of Short-Lived Br and I Isotopes in Thermal Neutron Fission of 235U," Z. Phys. A, Vol 314, 59-61 (1983). INIS Atomindex 15(1): 001071 (1 Jan 1984).

83ENG2 England, T. R., Rider, B. F., "Status of Fission Yield Evaluations," Paper from NEANDC Specialists' Meeting on Yields and Decay Data of Fission Product Nuclides, held at Brookhaven National Laboratory, October 24-27, 1983. (Published BNL-51778).

83GIN1 Gindler, J. E., Glendenin, L. E., Henderson, D. J., Meadows, J. W., "Mass Distributions in Monoenergetic-Neutron-Induced Fission of 239Pu," Phys. Rev. C, 2058-2062 (May 1983). EXFOR-12817. Monoenergetic neutrons.

83GUD1 Gudkov, A. N., Zhivun, V. M., Zvonarjov, A. V., Zolotov, A. F., Koldobskii, A. B., Koleganov, Yu. F., Kolobashkin, V. M., Krivasheev, S. V., and Piven', N. S., "Determination of the Yield Figures of the Products Resulting from the 242Pu and 241Am Fission by Fast Neutrons with the Aid of Semiconductor Spectrometry," At. Ehnerg. (Jun 83) V54(6) pp. 404-406. INIS Atomindex 15(17): 051773 (1 Sept 1984). EXFOR-40678.

83GUD2 Gudkov, A. N., Zhivun, V. M., Zvonarjov, A. V., Zolotov, A. F., Koldobskyj, A. B., Koleganov, Ju. F., Kolobashkin, V. M., Krivasheev, S. V., and Piven', N. S., Presumed title is “Determination of the Yield Figures of the Products Resulting from the 237Np Fission by Fast Neutrons with the aid of Semiconductor Spectrometry," Jour. YK, 1/50,48, (Mar 1983). YK is an abbreviation for Yadernaya Konstanty (Nuclear Constants). Full title of journal is "Voprocy Atomnoj Nauki I Tekhniki, Serija Jadernye Konstanty." EXFOR-40677. Twenty eight fission product yields for $\mathrm{Np237}$ ( $\mathrm{n}$ fast, $\mathrm{f}$ ).

83HON1 Hondt, P. D', Wagemans, C., et. al., Nuclear Data for Sci. and Techn. Proc. (1983) pp. 147-9, EUR-8355, INIS Atomindex 15: 041688 (1984). Ternary Fission.

83LAM1 Lam, S. T., Yu, L. L., Fielding, H. W., Dawson, W. K., Neilson, G. C., "Neutron-Induced Fission of 232Th near Threshold," Phys. Rev. C, 28(3), 1212 (Sept 1983). EXFOR-12843. Monoenergetic neutrons.

83LI 1 Li Ze, Liu Conggui, Lu Huijun, Liu Yonghui, Wang Lianbi, "The Measurement of Absolute Fission Product Yield for Spontaneous Fission of Cf-252," Chinese J. of Nucl. Phys. (Peking), 5(3), 226 (Aug 1983). In Chinese. Inst. of Atomic Energy, P. O. Box 275, BeijingEXFOR 30691 Dated 1985-03-07. EXFOR-30691. 
83LI 2 Li Wenxin, Sun Tongyu, Zhen Manjiao, Dong Tianrong, Sun Xiuhua, "Determination of the Yields for the Rare-Earth Nuclides from $14 \mathrm{MeV}$ Neutron Fission of $238 \mathrm{U}$ Using Ge(Li) Detector," J. Nucl. Radiochem., 5(2), 176-180 (May 1983). In Chinese. Inst. of Modern Physics Academia Sinica, Lanchou.

83MEI1 Meixler, H. H., Wolfsberg, K., Denschlag, H. O., "Nuclear Charge Distribution in Fission: Fractional Cumulative Yields of Isotopes of Krypton and Xenon in Cf249(n th, $f$ ) and Cf250(sp, f)," Can. J. Chem., 61(4), 665-670 (Apr 1983). INIS Atomindex 15:043253 (1984). Publication of 80MEI1.

83QUA1 Quade, U., "Measurements of the Yields of the Light Fission Products from the Reaction $233 U$ (n (th), f) by an Ionization Chamber," Muenchen Univ. (Ger F. R. ) Dissertation. Masses 79-106 measured. (in German) INIS-MF-9826 Dec 15, 1983. 276 p. INIS Atomindex 17: 001576 (1986). Isomeric independent yields here split per 76MAD2.

83RAT1 Rattan, S. S., Reddy, A. V. R., Singh, R. J., Prakash, S., Ramaniah, M. V., "Charge Distribution in the Thermal-Neutron-Induced Fission of 229Th," Radiochim. Acta 33, 189-193 (1983).

83ROS1 Rosman, K. J. R., De Laeter, J. R., Boldeman, J. W., Thode, H. G., "Cumulative Yields of Stable and Long-Lived Isotopes of Tin in Neutron-Induced Fission," Can. J. Phys. Vol 61, 1490-1497 (1983). Supersedes 69LAE1. As evaluated by 86WAH1.

83SCH1 Schmidt, R., Henschel, H., "Comparison of the Spontaneous Fission of $244 \mathrm{Cm}$ and 252Cf (I): Fragment Masses and Kinetic Energies," Nucl. Phys. A395 (1983) 15-28. EXFOR-21851.

83SCH2 Schmidt, R., Henschel, H., "Comparison of the Spontaneous Fission of $244 \mathrm{Cm}$ and 252Cf (II): Prompt Neutron Emission," Nucl. Phys. A395 (1983) 29-43. EXFOR-21859.

83SHM1 Shmid, M., Engler, G., "Fission Yields of In Isotopes in the Thermal Neutron Fission of 235U," Z. Phys. A, Vol 311, 113-116 (1983). INIS Atomindex 16(4): 012681 (15Feb 1985). The average evaluated FI values for $127 \mathrm{In}$ and $128 \mathrm{In}$ are lower than values derived from isotopeseparator-on-line (IS) data alone; About $70 \%$ for values from 83 SHM1. Because ratios of (IS) yields should be more reliable than absolute values, yields for $129 \mathrm{In} 130 \mathrm{In} 131 \mathrm{In}$ and $132 \mathrm{In}$ from $83 \mathrm{SHM} 1$ are multiplied by $0.7 \pm 0.2$ per $87 \mathrm{WAH} 1$; The uncertainties are estimated by $87 \mathrm{WAH} 1$.

83SIG1 Sigg, R. A., Kantello, M. V., Sisson, D. H., Prindle, A. L., Nethaway, D. R., "Fast Neutron Fission of 241Am," Phys. Rev. C, 27(1), 245-252 (Jan 1983). This is a publication of an earlier report called 80SIG1.

83THI1 Thierens, H., Clercq, A. De, Jacobs, E., Piessens, M., Hondt, P. D', Frenne, D., "Kinetic Energy and Fragment Mass Distributions for the Spontaneous and Photon-Induced Fission of 244Pu," Phys. Rev. C, 27(3), 1117 (Mar 1983). EXFOR-21860.

83VEN1 Venema, W. Z., Klinken, J. van, Pfeiffer, B. "EO Transitions After Fission Processes," Kernfysisch Versneller Instituut der Ruksuniversitett, Groningen, The Netherlands. KVI Annual Report 1983. 
84CHE1 Chen Qingjiang, Su Shuxin, et. al., "The Absolute Determination of the Spontaneous Fission Yield of 252Cf by Radiochemical Method," J. Nucl. Radiochem. 6(1), 24 (August 1984). In Chinese. Inst. of Atomic Energy, P. O. Box 275, Beijing. EXFOR-30793.

84CHI1 Chinese Cumulative Yield Group, "The Absolute Determination of Cumulative Yields of Several Nuclides from 14.9 meV Neutron-Induced Fission of $235 U$," J. Nucl. and Radiochem. (November 1984) V. 6(4) pp. 229-231. Institute of Atomic Energy, P. O. Box 275, Beijing (China). (In Chinese). 95Zr, 97Zr, 106Ru, 143Ce, 144Ce, 140Ba, 147Nd in U235HE $14.9 \mathrm{meV}$ fission by RC method. INIS Atomindex Vol 16(12): 040866 (15 Jun 1985).

84CHI2 Chitambar, S. A., Acharya, S. N., Jain, H. C., Mathews, C. K., "Fission Yields of LongLived and Stable Fission Products in Thermal Neutron Fission of 241Pu," Proceedings of the Nuclear Chemistry and Radiochemistry Symposium held at Waltair, Feb 25-28, 1980. INIS Atomindex Vol 15(5): 016662 (1 Mar 1984). Superseded by 86CHI1.

84CH13 Chinese Cumulative Yield Group, "The Absolute Determination of Cumulative Yield of Several Nuclides from Induced Fission of $238 \mathrm{U}$ by $5 \mathrm{MeV}$ Neutrons," J. Nucl. Radiochem., 6(3), 183-185 (Aug 1984).

84CHU1 Chung, C., Hasan, A. A., Sahin. S., "Cumulative Yields of Short-Lived Fission Products in Thermal-Neutron Fission of 235U," Radiochim. Acta. 37(3), 131-135 (1984). INIS Atomindex Vol 16(21): 073732 (1 Nov 1985).

84DEN1 Denschlag, H. O., Alfassi, Z., Braun, H., Ditz, W., Faubel, W., Faust, H., Hoener, St. Poersch, W., Sehr, R., Schrader, H. Sohnius, B., "Nuclear Charge Distribution of Heavy Mass Fission Products in U-235(n, f)," NEANDC (E)-252U, (5), 38 (June 1984). Also Nuclear Data Research in Federal Republic of Germany Progress Report for the Year (1984) pages 38-42. EXFOR- 21939. Independent yields measured for each mass are summed and normalized to $100 \%$. We cite independent yields measured plus Wahl model estimates of the 9 unmeasured charges normalized to unity as cited by 86 WAH1.

84DJE1 Djebara, M., Asghar, M., Bocquet, J. P., Maurel, M., Nifenecker, H., Ristori, C., Brissot, $\mathbb{R}$, "Measurement of Charge Distribution for $229 \mathrm{Th}(\mathrm{n}(\mathrm{th}), \mathrm{f})$ and $232 \mathrm{U}(\mathrm{n}(\mathrm{th}), \mathrm{f})$," Nucl. Phys. A (20 Aug 1984) Vol. 425(1) pp. 120-140. EXFOR-21919.

84DOB1 Dobreva, E., Nenoff, N., "Yields of Fission Products with Masses $A=131$ to $A=135$ for the Fast Neutron Induced Fission of U-238," J. Radioanal. and Nucl. Chem., Articles, V81(1) pp. 29-36 (1984). INIS Atomindex Vol 15(15): 045842 (1 Aug 1984). Isomeric independent yields here split per 76MAD2.

84FOR1 Ford, G. P., Wolfsberg, K., Erdal, B. R., "Independent Yields of the Isomers of 133Xe and $135 \mathrm{Xe}$ for Neutron-Induced Fission of $233 \mathrm{U}, 235 \mathrm{U}, 238 \mathrm{U}, 239 \mathrm{Pu}$, and $242 \mathrm{mAm}$," Phys. Rev. C, Vol 30(1) pp. 195-213 (Jul 1984). INIS Atomindex Vol 16: 049175 (15 Jul 1985). EXFOR-12895.

84GUD1 Gudkovt, A. N., et. al. "Fission Product Yields from the $1050 \mathrm{keV}$ Neutron Induced Fission of the $238 \mathrm{U}$ and $960-3300 \mathrm{keV}$ Neutron Induced Fission of 236U," Proceedings of the 6th All-Union Conference Vol 2. (held at) Kiev 2-6 Oct 1983. MF available INIS. INIS-SU-305 pp. 259-263 (1984). 7 ref. 1 table. INIS Atomindex Vol 16(16): 056796 (15 Aug 1985). EXFOR- 40878. 
84HIC1 Hicks, H. G., "Fission Yield of 161-Tb from Fission Spectrum Neutrons on Plutonium 239," University of California Lawrence Radiation Laboratory Publ. UCAR-10062,66,84 (1984). USA National Nuclear Data Center Accession No. 12925 (August 12, 1985). Yield entry approved (proofread) by H. G. Hicks (September 16, 1985). EXFOR-12925.

84JAI1 Jain, H. C., Ramaniah, M. V., "Fission Yields in the Thermal Neutron Fission of Plutonium-239," Radiochim. Acta (37), 63-67 (1984). Fission yields of 27 masses. This paper takes account of neutron temperature on $\mathrm{Pu}$ fission cross section and compares to two recent compilations. INIS Atomindex Vol 16(20): 070131 (15 Oct 1985).

84LIN1 Lindner, M., Seegmiller, D., "Fission Product Mass Yield, Thermal Neutron Fission Cross section and Nu-Bar of the Long Lived Np-236 Isomer," Progress Rpt. UCAR10062,164,(1984). EXFOR-12919. No Data.

84MAN1 Mann, F. M., Schreiber, M., Schenter, R. E., and England, T. R., "Evaluation of Delayed Neutron Emission Probabilities," Nuclear Science and Engineering, Vol 87, 418 (1984).

84NAI1 Nair, A. G. C., Srivastava, A., Srivastava, B. K., Prakash, S., Ramaniah, M. V., "Cumulative Yields of the Short-Lived Ruthenium Isotopes in the Spontaneous Fission of 252Cf," J. Radioanal. and Nucl. Chem., Articles, V82(2), 263-267 (1984). INIS Atomindex 15(15): 063466 (1 Aug 1984).

84SCH1 Schmitt, C., Guessous, A., Bocquet, J. P., Clerc, H. G., Brissot, R., Engelhart, D., Faust, H. R., Goennenwein, F., Mutterer, M., Nifenecker, H., Pannicke, J., Ristori, Ch., Theobald, J. P., "Fission Yields at Different Fission-Product Kinetic Energies for Thermal-NeutronInduced Fission of 239-Pu," Nucl. Phys. A, (430), 21-60 (1984). USA National Nuclear Data Center Accession No. 21928 (November 8, 1985). From Institute Laue Langevin, Grenoble experimental site high flux reactor and Lohengrin mass spectrometer in cooperation with Techn. Hochschule Darmstadt and Centre d'Etudes Nucleaires Grenoble and Kernforschungszentrum Karlsruhe. Only fractional independent fission yields summed over the kinetic energy are cited for this compilation of fission product yields. For data at individual energies see the (Nucl Phys. A) article directly. Here cited as evaluated by $86 \mathrm{WAH} 1$. Independent yields are here split between isomers per 76MAD2. Evaluator uncertainties are estimated to be \pm 0.02 on fractional independent yields or $\pm 5 \%$ of the value, whichever is larger. EXFOR-21928.

84SEM1 Semkow, T. M., Wahl, A. C., Robinson, L., "Yields of In and Sn Products from Thermaland 14 meV-Neutron-Induced fission of $235 U$," Phys. Rev. C, 30(6), 1966-1975. INIS Atomindex Vol 16: 083749 (1985). Thesis. EXFOR-12813.

84SRI1 Srivastava, A., Nair, A. G. C., Srivastava, B. K., Manohar, S. B., Prakash, S., Ramaniah, M. V., "Nuclear Charge Distribution in the Spontaneous Fission of 252Cf: Isotopic Yield Distribution for Technetium Isotopes," Radiochim. Acta Volume 35, 15-21 (1983). CF252S yields for 101, 103, 104, 105-Tc. Publication of 82SRI1.

84TEP1 Teplykh, V. F., Platygina, E. V., Petrzhak, K. A., Smirnov, A. V., Nebogatikov, V. V., Solonkin, A. A., "Measurement of the 135Xe Yield in the Fission of Uranium Isotopes," Proceedings of the 6th All-Union Conference V. 2 (held at) Kiev 2-6 Oct, 1983. MF available INIS. INIS-SU-305 p. 251-253 (1984) 5 refs. 1 tab. INIS Atomindex Vol 16(16): 056796 (15 Aug 1985). EXFOR-40877. 
84THI1 Thierens, H., Jacobs, E., Hondt, P. D', Clercq, A. De, Piessens, Frenne, D., "Fragment Mass and Kinetic Energy Distributions for 242Pu(sf), 241Pu(n th, f) and 242Pu(gamma, f)," Phys. Rev. C, 29(2), 498 (1984). EXFOR-21915.

84WIN1 Winkelmann, I., Aumann, D. C., "Fission of Pu242 with 15.1 meV Neutrons," Phys. Rev. C, Vol 30(3), 934-940 (September 1984). USA National Nuclear Data Center Accession No. 21983 (March 25, 1986). EXFOR-21983.

85AKE1 Aker, E., Engelhardt, D., Brissot, R., Geltenbort, P., Goennewein, F., Oed, A., Gindler, T., Wilkens, B., "Mass and Energy Distribution of Fission Fragments in 249-Cf(n-th, $f$ )," International Conference on Nuclear Data for Basic and Applied Science, Santa Fe, New Mexico (May 13-17, 1985). Paper (BC05) (1985). USA National Nuclear Data Center Accession No. 21967 (February 6, 1986). CONF-850507-61 INIS Atomindex Vol 17:080174 (1986). EXFOR21967.

85BLA1 Blachot, J., and Brissot, R., "Systematics of Neutron-Induced Fission Yields," BNL51778 (1985) pages 65-89.

85BRA1 Braun, H., Denschlag, H. O., "Cumulative Yields of Te-131m and Te-133m in Fission of 235U," Radiochim. Acta, (1985) V38(4) p. 169-171. INIS Atomindex 17(15): 049590 (1 Aug 1986). Te131m $=(0.50 \pm 0.05) \%$ and $\mathrm{Te} 133 \mathrm{~m}=(3.61 \pm 0.15) \%$. EXFOR-22040 (Feb 7 , 1989).

85BUG1 Bugrov, V. P., Bjalko, A. A., Volkov, N. G., Kolobashkin, V. M., Sljusarenko, A. I., (J, YK, (1), 66,8503) EXFOR-40895. YK is an abbreviation for Yadernaya Konstanty (Nuclear Constants). Russian journal title in full is "Voprocy Atomnoj Nuaki I Tekhniki, Serija Jadernye Konstanty."

85CHA1 Chapman, T. C., Tromp, R. L., Emel, W. A., "Shielded Mass 136 Yield from Fast Fission of U-233, U-235, Np-237, and Pu-239 through 242," BNL-51788 (1985) pp. 133-136. INIS Atomindex Vol 16: 049153 (15 July 1985). EXFOR-10974.

85CHI1 Chinese Cumulative Yield Group, "Distribution of Fission Yields in the $300 \mathrm{meV}$ Neutron Induced Fission of 238U," J. Nucl. and Radiochem. (Feb 1985) V. 7(1) pp. 1-6 Institute of Atomic Energy, P. O. Box 275, Beijing, China. (In Chinese). 38 mass chains by RC and direct $\mathrm{Ge}(\mathrm{Li}) .125$ and 128 reported for first time. INIS Atomindex Vol 17: 011833 (1986). Each peak $=100.1 \%$. Peak to valley $=179$. The measured yields $=114.4 \%$ out of expected $200 \%$. EXFOR 30743 Dated 1987-12-14.

85CHU1 Chung, C., Pan, L., "Cumulative Yields of 33 Fission Products in Fast Neutron Induced Fission of 232Th," Radiochim. Acta (1985) V38(4) pp. 173-179. 22 measured for the first time. INIS Atomindex 17(15): 049591 (1 Aug 1986).

85DEN1 Denschlag, H. O., “Independent Fission Yield Measurements,” BNL-51778 (1985) pages $7-31$.

85DOA1 Doan, T. P., et. al., Rapport D'activites pour L'Annnee 1984, NEANDC(E) 262[L] INDC(FR) 66/L. Ternary fission ( $9 \mathrm{Li}, 10 \mathrm{Be}, 6 \mathrm{He}, 12 \mathrm{Be}$ etc.).

85FUD1 Fudge, A. J., “Fission Yield Data for Dosimetry," BNL-51778 (1985) pp. 121-132. Nucl. Sci. \& Engr. 94, 337-352 (1986). 
85GEL1 Geltenbort, P., Goennewein, F., Oed, A., (Conf. 85Santa Fe, 1985). EXFOR-21981.

85GUD1 Gudkov, A. N., Zhivun, V. M., Kovalenko, V. V., Koldobskii, Kolobashkin, V. M., et. al., "Measurement of Cumulative and Independent Yields of Fission Products from Thermal-Neutron Fission of 242m-Am," Yad. Fiz. 41, 573-578 (Mar 1985). For English translation see Sov. J. Phys. (Mar 1985) Vol 41(3), pp. 365-368. INIS Atomindex 17: 015405 (1986). EXFOR-40869.

85HAD1 Haddad, M., Asghar, M., Crancon, J., Hamelin, C., Lhospice, G., Blachot, J., "Post-Neutron Mass Distribution for 232U (n th, f)," CEA-CONF-8034 (May 1985). CEA Centre d'Etudes Nucleaires de Grenoble, 38 (France). MF avail from INIS. International Conference on Nuclear Data for Basic and Applied Science, Santa Fe, New Mexico (USA) (May 13-17, 1985). USA National Nuclear Data Center Accession No. 21972 (February 6, 1986). INIS Atomindex 18(5): 017392 (1 March 1987). and 18(9): 034835 (1 May 1987). Now published as 87HAD1 in Radiochim. Acta, 42(4), 165-168 (1987). INIS Atomindex 19(14): 057655 (15 July 1988). EXFOR-21972.

85HAS1 Hasan, A. A., Chung, C., Sahin, S., "Yields of Short-Lived Fission Products in $235 U$ (n th, f)," Trans. Amer. Nucl. Soc. 49, 209-211 (1985). INIS Atomindex 17(9): 027080 (1 May 1986). Conf. on "Nuclear Data for Basic and Applied Science," Vol. 1 (1985). Yields of Ce-148, $\mathrm{Kr}-89, \mathrm{I}-136 \mathrm{~m}$, La-144, Kr-90, Xe-139, Xe-137, and Cs-140. INIS Atomindex 18(9): 034825 (1 May 1987). INIS Atomindex 18(5): 017400 (1 March 1987) and 18(9): 034825 (1 May 1987).

85LI 1 Li Ze, Zhang Chunhua, Liu Conggui, Wang Xiuzhi, Qi Linkun, Ju Changxin, Liu Daming, Tang Peija, Meng Jiangchen, "Mass Distribution in $8.3 \mathrm{MeV}$ Neutron-Induced Fission of U-238," Chinese J. of Nucl. Phys. (Peking), 7(2), 97 (May 1985). In Chinese. Inst. of Atomic Energy, P. O. Box 275, Beijing. EXFOR 30751 Dated 1988-12-05.

85LIU1 Liu Conggui, Lu Huijun, Li Ze, Liu Yonghui, “The Mass Distribution in $14.9 \mathrm{MeV}$ Neutron-Induced Fission of 238U," Chinese Journal of Nuclear Physics, 7 (3), 235-241 (Aug 1985). Institute of Atomic Energy, P.O.Box 275, Beijing. (In Chinese). EXFOR 30788 (Dec 22, 1988).

85LUN1 Lund, E., Rudstam, G., Aagaard, P., and Zwicky, H. -U., "Gamma Branching Ratios for Fission Products: Application to the Study of the Yield Distribution from $235 U$ (Thermal Neutrons, Fission)," BNL-51778 (1985), Pages 137-151. This paper is a presentation of data which appeared as NFL-42 (1985). See 85RUD1.

85MAE1 Maeck, W. J., "Determination and Correlation of Fast Reactor Fission Yields with Neutron Energy," BNL-51778 (1985) pages 91-120. A further development of work started in 80MAE3 and 81MAE2.

85NAI1 Nair, A. G. C., Srivastava, A., Goswami, A., Srivastava, B. K., “Cumulative Yields of ShortLived Ruthenium Isotopes in the Thermal-Neutron-Induced Fission of $233 \mathrm{U}, \mathbf{2 3 5 U}$, and 239Pu," J. Radioanal. Nucl. Chem., Vol 91(1) pp. 73-79 (Aug 1985). INIS Atomindex Vol 17: 008502 (1986).

85RAM1 Ramaniah, M. V., "Radiochemical Studies on Fission of Actinides," Pramana, Vol. 24, Nos. 1 \& 2 (January \& February 1985) pp. 137-153. (India). 
85REE1 Reeder, P. L., Warner, R. A., Ford, G. P., Willmes, H., "Independent Isomer Yield Ratio of 90Rb from Thermal Neutron Fission of 235U," Phys. Rev. C, V32(4), 1327-1334 (Oct 1985). Rb90m/Rb90 = 8.7 \pm 1.0. INIS Atomindex 17(5): 015403 (1 Mar 1985). (i. e., $0.7528 / 0.0864=8.7$ using 81 RID1 independent yield value of $0.8392 \%$ and an isomer ratio of 8.7). EXFOR-12945.

85RIM1 Rimpault, G., and Martin-Deidier, L., "Uncertainties on the Pseudo Fission Product for Fast Power Reactors Due to the Yields Library," BNL-51778 (1985) pages 153-156.

85ROB1 Robinson, L., Wahl, A. C., Semkow, T. M., Norris, A. E., "Nuclear Charge Distribution for $A=121$ from Thermal Neutron Induced Fission of 235U," Phys. Rev. C, Vol 31(4) pp. 1334-1339. INIS Atomindex Vol 16: 073730 (1 Nov 1985). See INIS Atomindex Vol 17: 011876 (1986) for abstract. Thesis available from Univ. Microfilms Order No. (85-03, 133). EXFOR-12931.

85RUD1 Rudstam, G., Aagaard, P., Zwicky, H. -U., "Yields of Products from Thermal Neutron Induced Fission of 235 U," Research Report NFL-42 (1985) 79 pp. The Studsvik Science Research Laboratory, S-611 82 Nykoping, Sweden. The average evaluated FI values for 127In and 128In are lower than values derived from isotope-separator-on-line data alone about $60 \%$ from 85RUD1. Because ratios of yields should be more reliable than absolute values, yields for $129 \mathrm{In}$ and $130 \mathrm{In}$ from $85 \mathrm{RUD} 1$ are multiplied by $0.60 \pm 0.20$ as recommended by 87 WAH1. EXFOR-22003. Superseded by 89RUD2.

85STR1 Straede, C. A., Aarhus Univ. (Denmark) Thesis (Dr. Phys.) "Neutron Induced Fission of 235sub(U)," INIS MF 10460 May 1985146 pages 82 refs. Available from INIS. INIS Atomindex 17(16): 053771 (15 August 1986). Contains mass, energy, angular distributions, and the mass versus energy and mass versus angular distribution correlations as a function of excitation energy from thermal to $6.0 \mathrm{meV}$ using a double Frisch-gridded ionization chamber.

85TOM1 Tomar, B. S., Naik, H., Ramaswami, A., Prakash, S., "Yields of Rare Earth Fission Products in the Spontaneous Fission of Californium-252," J. Radioanal. Nucl. Chem. V91(2) pp. 291-296 (Sept 1985). INIS Atomindex Vol 17: 008503 (1986).

85TOM2 Tomar, B. S., Goswami, A., Das, S. K., Datta, T., Prakash, S., Ramaniah, M. V., "Fission Fragment Angular Momentum: Independent Isomeric Yield Ratio of 138Cs in Thermal Neutron Induced Fission of 233U, 239Pu, and 241Pu," Radiochim. Acta (1985), V. 39(1), pp. 1-3. INIS Atomindex 17: 064388 (1986). IN(Cs138m)/ [IN(Cs138m) + $\mathrm{IN}(\mathrm{Cs138 \textrm {g } )})=0.72 \pm 0.04,0.67 \pm 0.06$, and $0.65 \pm 0.11$ for U233T, PU239T and PU241T fission, respectively. Independent yields are here derived from 81RID1 values for the sum of independent yields for Cs138 isomers multiplied by these ratios. INIS Atomindex 17 (19): 064388 (1 October 1986).

85WAH1 Wahl, A. C., "Nuclear Charge Distribution Near Symmetry for Thermal Neutron Induced Fission of 235U," Phys. Rev. C, Vol 32(1) pp. 184-194. INIS Atomindex Vol 17: 001408 (1986).

85WAN1 Wang Dao, Tang Peijia, Ju Changxin, Liu Daming, Wang Qing, “Determination of 99Mo Cumulative Yield of $235 \mathrm{U}$ Fission by the Spontaneous Fission Neutrons of $252 \mathrm{Cf}$ Source," Journal of Nucl. and Radiochem. 7(4), 234-238 (Nov 1985). Work done at the 
Institute of Atomic Energy, P. O. Box 275, Beijing, China. Uses R-value method. EXFOR 30786 (Dec 23, 1988).

86BAN1 Banai, J. "Proposed New Data Base for Neutron Induced Fission Product Yields," Dept. of Physics, Univ. of Birmingham, England. Paper given at Specialists Meeting on Delayed Neutrons held at Univ. of Birmingham, England, September 15-19, 1986.

86CHE1 Chen Qingjiang, Su Shuxin, Yang Jingxia, Chen Yundong, Li Xueliang, Zhang Hongdi, Lin Fa, Sun Shuying, Zhang Shulan, Guo Jingru, "The Absolute Determination of the Spontaneous Fission Yield of $\mathbf{2 5 2 C f}$ by the Radiochemical Method," Atomic Energy Science and Technology, 20 (2), 161-166 (Mar 1986). Institute of Atomic Energy, P.O.Box 275, Beijing. EXFOR 30793 (1988).

86CHI1 Chitambar, S. A., Jain, H. C., Ramaniah, M. V., "Fission Yields in the Thermal Neutron Fission of 233U, 235U, 239Pu, and 241Pu," Private communication to B. F. Rider, July 23, 1986. This is a summary of the work submitted as a $\mathrm{Ph}$. D. Thesis, Univ. of Bombay, India (1984) by S. A. Chitambar. This supersedes the Waltair paper preliminary results $84 \mathrm{CHI} 2$. Now published in Radiochim. Acta 42(4), 169-178 (1987). INIS Atomindex 19(14): 057656 (15 July 1988) as $87 \mathrm{CHI} 1$.

86DH01 D'Hondt, P., Fabry, A., “Application of Some Fission Properties to Neutron Dosimetry," Semi-annual Report BLG-586, 43 (May 1986). EXFOR-22057 (Feb 7, 1989). U235F fast reactor spectrum in French MASURCA Fast Critical Facility in Caderache. $\mathrm{Ge}(\mathrm{Li})$ detector used for fission product activity measurements.

86DIC1 Dickens, J. K., and Mc Connell, "Yields of Fission Products Produced by ThermalNeutron Fission of Cm-243," Phys. Rev. C, 34(2), 722-725 (August 1986). Measured 69 fission products for 41 mass chains. INIS Atomindex 17(24): 086214 (15 December 1986). EXFOR-13102 (Aug 13, 1987).

86DIC2 Dickens, J. K., and Raman, S., "Fission-Product Yield Data from the US/UK Joint Experiment in the Dounreay Prototype Fast Reactor," ORNL-6266, Oak Ridge National Laboratory (1986). Unclassified report. Only data extracted by 87DIC1 are good enough for fission yield work. In $87 \mathrm{DIC} 1$ these data are used to 'predict' unmeasured mass yields of $\mathrm{Cm}$ isotopes.

86ENG1 England, T. R., Brady, M. C., Arthur, E. D., Labauve, R. J., Mann, F. M., "Status of Evaluated [Delayed Neutron] Precursor and Aggregate [Delayed Neutron] Spectra," LA-UR-86-2693, Presentation at the Specialists' Meeting on Delayed Neutrons held at University of Birmingham, Birmingham, England, September 15-19, 1986. Pn values for 272 DN precursor nuclides. See also 79RUD1, 84MAN1, 86MAN1, 86LUN1 for other delayed neutron evaluations.

86ENG2 England, T. R., Private communication to B. F. Rider Nov. 17, 1986 updating 86ENG1 to Oct 10, 1986. The systematic Pn values are from the Kratz-Hermann equation using Fred Mann's fit for constants $\mathrm{A}=54.0$ and $\mathrm{B}=3.44$ from the Birmingham Meeting of Sept. 1986 mentioned in $86 \mathrm{ENG} 1$.

86GOS1 Goswami, A., Srivastava, B. K., Srivastava, A., Manohar, S. B., Prakash, S., Ramaniah, M. V., "Correlation Between Fractional Independent Yields and Neutron-to-Proton Ratio 
of Fission Products in Low Energy Fission," Pramana - J. Phys. Vol. 26, No. 3 (Mar 1986) pp. 179-189 (India). Extension of 69MUK1 model.

86HUL1 Hulet, E. K., Wild, J. F., Dougan, R. J., et. al., "Bimodal Symmetric Fission Observed in the Heaviest Elements," Phys. Rev. Lett. V56(4), 313-316 (27 Jan 1986). 258Fm, 259Md, $260 \mathrm{Md}, 258 \mathrm{No}, 260[104]$ fission yields graphically given. INIS Atomindex 17(15): 049589 (1 Aug 1986). Symmetric yield (no light or heavy peak and no valley; Just one symmetric hump). Data is given in Atomindex 18(5): 017384 (1 March 1987).

86JIA1 Jianquo, S., Jingru, G., Fangding, W., "Independent Yield of $88 Y$ from Thermal Neutron induced fission of 235U," Jour. Radioanal. Nucl. Chem., Letters Vol 108 (6), 347-356 (1986). Superseded by 87JIA1.

86LEI1 Leitz, C., Denschlag, H. O., Ditz, W., Guettler, U., Sohnius, B., Stumpf, P., and Faust, H., "Progress Report on Nuclear Data Research in the Federal Republic of Germany," S. M. Qaim, editor (1986), pp. 33-36. U233T independent yields. See 87WAH1 Table XA. "Comparison of New Experimental Data and Model Estimates for U233T," EXFOR-22016, EXFOR22017.

86LUN1 Lund, E., Rudstam, G., Aleklett, K., Ekstrom, B., Fogelberg, B. Jacobsson, L., "A Status Report on Delayed Neutron Branching Ratios of Fission Products and Delayed Neutron Programme at OSIRIS using the New Ion-source ANUBIS," Proceedings of the Specialists' Meeting on Delayed Neutron Properties, September 15-19, 1986, University of Birmingham, England. Editor D. R. Weaver, Dept. of Physics Radiation Centre, U. of Birmingham. ISBN-07044-0926-7 Pages 59-74.

86MAN1 Mann, F. M., “1886 Evaluation of Delayed-neutron Emission Probabilities," Proceedings of the Specialists' Meeting on Delayed Neutron Properties, September 15-19, 1986, Univ. of Birmingham, England (1986). Pages 21-36. Update of 84MAN1.

86NAI1 Naik, H., Dange, S. P., Datta, T., Prakash, S., Ramaniah, M. V., "Determination of Fractional Cumulative Yields of Sr-91, Sr-92 and Te-134 in the Thermal Neutron Induced Fission of U-233 and Pu-241," Radiochim. Acta 40(4), 175 (1986). EXFOR- 30766 (Feb 8, 1989).

86RED1 Reddy, A. V. R., Deshmukh, S. M., Burte, P. P., Manohar, S. B., Prakash, S., Ramaniah, M. V., "Isotopic Yield Distribution of Iodine in the Reactor-Neutron-Induced Fission of 237Np," Pages 28-29 of Dept. of Atomic Energy, Bombay, India, Symposium on Nuclear Physics; Contributed Papers Volume 26B, Bhabha Atomic Research Centre 1986, 388 pages, 3 tables. [Jaipur, (India) 16-20 December 1985]. 131-134 I independent yields and cumulative yields of 131-134Te given. INIS Atomindex 18(4): 012261 (15 February 1987).

86RED2 Reddy, A. V. R., Deshmukh, S. M., Burte, P. P., Manohar, S. B., Prakash, S., Ramaniah, M. V., "Isotopic Yield Distribution of Iodine in the Thermal Neutron Induced Fission of $245 \mathrm{Cm}$," Conf. on Radiochemistry and Radiation Chemistry at Tirupati (during) 13-17 Dec 1986. pp. 98-100. Independent yields of 131-134I and cumulative yields of 131-134Te in thermal neutron induced fission of $245 \mathrm{Cm}$. INIS Atomindex 20(3): 007412.

86RIC1 Richardson, A. E., Wright, H. L., Meason, J. L., Smith, J. R., "Mass Distribution in the Fission of Th-232 by Degraded Fission-Spectrum Neutrons,” Nucl. Sci. and Engr. 94, 
413-425 (1986). 25 mass chains including mass 138 for the first time for fast fission. Cumulative yields of Se-83g, Sb-130g were observed, the latter also for the first time for fast fission. Yields were slightly higher in inner portions of both heavy and light mass wings than from reactorneutron - induced fission of Th-232. This is expected since average energy of neutron spectrum is slightly above that of reactor neutron spectrum. INIS Atomindex 18(13): 054792 (1 July 1987). EXFOR-13103 (Mar 28, 1988).

86RID1 Rider, B. F., Estimated cumulative yield for CM246S and CM248S as suggested by 81 GIN3. This is in agreement with earlier observations that the average mass of the heavy mass group is nearly constant for low energy fissioning systems in the region from $\mathrm{U}$ through $\mathrm{Cm}$; Whereas, the average mass of the light mass group changes with the fissioning system (73UNI1).

86RUD1 Rudstam, G. "Isomeric Yields in the Fission of 235 U Induced by Thermal Neutrons," The Studsvik Neutron Research Laboratory Research Report NFL-50 (1986), a paper given at the Specialists' Meeting on Delayed Neutrons held at the Univ. of Birmingham, Birmingham, England September 17-19, 1986. Superseded by 89RUD2.

86SRI1 Srivastava, A., Goswami, A., Srivastava, B. K., Nair, A. G. C., Manohar, S. B., Prakash, S., Ramaniah, M. V., "Fragment Shell Effect in Low Energy Fission: Independent Yield of Technetium in the Thermal-Neutron-Induced Fission of 238Pu," Phys. Rev. C, Vol 33(3), pp. 969-973 (March 1986). INIS Atomindex 17(15): 049588 (1 August 1986). Independent yield of $101,103,104,105 \mathrm{Tc}$ by fast radiochemistry and gamma spectrometry.

86WAG1 Wagemans, C., D'hondt, P., Schillebeeckx, P., Brissot, R., "Triton and Alpha Emission in the Thermal-Neutron-Induced Ternary Fission of $233 \mathrm{U}, 235 \mathrm{U}, 239 \mathrm{Pu}$, and $241 \mathrm{Pu}$," Phys. Rev C, 33(3), 943 (March 1986).

86WAG2 Wagemans, C., Schillebeeckx, P., DeRuytter, A., Barthelemy, R., Rpt. BLG-592 (Nov 1986). (In French). EXFOR-22070. INIS-Atomindex 20(11): 036370 (1 June 1989). [DATA]. Available from INIS on Microfiche.

86WAH1 Wahl, A. C., Private communication to T. R. England, August 23, 1986, of Zp, sigma, mass yields, NU, EOZ, EON, and average $Z$ and RMS charge dispersion from U235T, U233T, PU239T, and CF252S fission. Here valued for its critical evaluation of $\mathrm{Zp}$ and RMS charge dispersion (sigma) for these fissioning systems.

87CHI1 Chitambar, S.A., Jain, H. C., Ramaniah, M. V., "Fission Yields in the Thermal Neutron Fission of 233U, 235U, and 241Pu," Radiochim. Acta, 42(4), 169-178 (1987). Yields of forty mass numbers by mass spectrometric, gamma-spectrometric, and radio chemical techniques with errors $1-3 \%$. However, radiochemical techniques gave about $4 \%$ error in asymmetric regions and $12 \%$ in symmetric regions. Publication of $86 \mathrm{CHI} 1$ (which see). INIS Atomindex 19(14): 057656 (15 July 1988).

87CHU1 Chien Chung, Ming-Yung Woo, "Fission Product Yields in the Fast-Neutron Fission of 238U," Jour. of Radioanal. and Nucl. Chem., Articles, Vol 109(1), 117-131 (1987). A radiochemical determination of 38 fission product yields from fast fission of U238.

87DIC1 Dickens, J. K., "Fission Product Yields for Fast-Neutron Fission of 243,244,246,248 Cm," Nucl. Sci. and Engr. 96, 8-16, (1986). Measurements of Zr-95, Sb-125, Cs-137, Ce-141, Ce-144, and Eu-155 from 86DIC2 were used to calculate the Five Gaussian Model to compute all 
unmeasured chain yields for these fissionable nuclides. Results are at least sufficiently accurate for first order estimates of the inventory of fission products in a fuel rod at any given time during its use. The author concludes: "Clearly, however, as engineering needs become more demanding, so will the need to obtain additional experimental data."

87GOS1 Goswami, A., Nair, A. G. C., Srivastava, B. K., Manohar, S. B., Prakash, S., Ramaniah, M. V., "Charge Distribution in the Thermal Neutron Fission of 241Pu: Fractional Cumulative Yield of 105Mo and 105Tc," Paper in Radiochemistry and Radiation Chemistry Symposium held at Kanpur during 9-13 Dec 1985. pp. 217-219. INIS Atomindex 18:071985 (1988). Additional data not in 87NAI1. See also BARC-1381 pp. 33-36 (1987).

87HAD1 Haddad, M., Crancon, J., Lhospice, G., Asghar, M., Blachot, J., “Post Neutron Mass Distribution of 229Th(n/sub T/F)," Radiochim. Acta, 42(4), 165-168 (1987). INIS Atomindex 19(14): 057655 (15 July 1988). Yields of 51 fission products of TH229T in 34 mass chains by gamma-spectroscopy including Sb-131, Sb-132, Cs-138, Cs-140, 1-134, Se-82, Se-84, Xe-135, $\mathrm{Xe}-138$ and Xe-139. [DATA]. Supersedes 85HAD1.

87JIA1 Jianguo, S., Jingru, G., Fangding, W., "Independent Yield of $88 Y$ from Thermal Neutron Induced Fission of $235 \mathrm{U}$," J. Radioanal. Nucl. Chem. Ltrs. 108(6), pp. 347-356 (27 Mar 1987). Using a liquid counting source on a 130 cubic centimeter $\mathrm{Ge}(\mathrm{Li})$ for $88 \mathrm{Y}$ measurement and a plastic beta scintillation detector for $91 \mathrm{Y}$, the activity ratio of $88 \mathrm{Y} / 91 \mathrm{Y}<=1.53$ e-8\% INIS Atomindex 18:084561 (1 Nov 1987). EXFOR 30790 (Nov 30, 1988).

87LEE1 Lee., C. H., Hwang, L. T.,Lin, Y. S., Yu, Y. W., "Isomeric Yield Ratio of 146La and 84Br in the Thermal Neutron Induced Fission of $235 \mathrm{U}, " \mathrm{~J}$. Radioanal. and Nucl. Chem., Letters $119(2), 101-107 \cdot(1987)$. Independent yield of $146 \mathrm{La}(\mathrm{g})=0.104 \pm 0.018$; Cumulative yield of $146 \mathrm{La}(\mathrm{m})=2.64 \pm 0.26 ;$ Independent yield of $84 \mathrm{Br}(\mathrm{m})=0.0195 \pm 0.0066$

87LI 1 Li Wen-xin, Sun Tong-yu, Sun Xiu-hua, Fu Ming, Dong Tian-rong, Zheng Man-jiao, "Charge Distribution in the 14.7 Mev Neutron-Induced Fission of 232Th: Independent Yields of Isotopes of Rh, Ag, In, and Sb," High Energy Physics and Nuclear Physics, 11(3), 376-383 (May 1987). Institute of Modern Physics, Academia Sinica, Lanzhou, China. EXFOR 30785 1988-12-06.

87NAI1 Nair, A. G. C., Das, S. K., Tomar, B. S., Goswami, A., Srivastava, B. K., Prakash, S., "Cumulative Yield of Short-lived Ruthenium Isotopes in Thermal Neutron-Induced Fission of Pu-241," Radiochim. Acta, Vol 42(1), pp. 7-8 (1987). Ru-107, 108, 109 yields. INIS Atomindex 19(8): 030835 (15 April 1988).

87NAI2 Naik, H., Dange, S. P., Datta, T., Prakash, S., Ramaniah, M. V., "Charge Distribution in 241Pu (n th, $f$ ): Determination of Fractional Cumulative Yields of 91, 92Sr," BARC-1381 pp. 37-39 (1987).

87NAI3 Nair, A. G. C., Srivastava, A., Goswami, A., Srivastava, B. K., "Cumulative Yields of Shortlived Ruthenium Isotopes in the Thermal Neutron induced Fission of $233 \mathrm{U}, \mathbf{2 3 5 U}$, and 239Pu," BARC-1381 pp. 57-58 (1987). Progress Report of data appearing Published in 85 NAI1. 
87RAM1 Ram, S., Singh, N. L., Bose, S. K., Rao, J. R., "Absolute Yields of Some Fission Products in the $14 \mathrm{MeV}$ Neutron Induced Fission of U-238," Nucl. Instr. Meth. Phys. Res. B24/25, 501 (May 1987). EXFOR-30768 (Dec 14, 1987).

87RAM2 Ramaswami, A., Rattan, S. S., Chakravarty, N., Singh, R. J., Prakash, S., Ramaniah, M. V., "Charge Distribution Study in the Neutron Induced Fission of Np-237: Fractional Cumulative Yield of Te-134, I-135 and Xe-138," Radiochim. Acta, 41(1), 9 (1987). EXFOR-30765 (Dec 14, 1987). Publication of BARC-1381 pp. 40-43 (1987) data.

87RAM3 Ramaswami, A., Rattan, S. S., Chakravarty, N., Singh, R. J., Manohar, S. B., Prakash, S., "Charge Distribution Study in the Neutron Induced Fission of 237Np: Fractional Cumulative Yield of 134Te, 135I, and 138Xe," BARC-1381 pp. 40-43 (1987).

87RED1 Reddy, A. V. R., Deshmukh, S. M., Manohar, S. B., Prakash, S., Ramaniah, M. V., "Isotopic Yield Distribution of Iodine in the Thermal Neutron Induced Fission of 241Pu," BARC-1381 pp. 25-32 (1987).

87RED2 Reddy, A. V. R., Deshmukh, S. M., Burte, P. P., Manohar, S. B., Prakash, S., Ramaniah, M. V., "Isotopic Yield Distribution of Iodine in the Reactor Neutron induced fission of $237 \mathrm{~Np}$," BARC-1381 pp. 44-46 (1987).

87RED3 Reddy, A. V. R., Deshmukh, S. M., Burte, P. P., Manohar, S. B., Prakash, S., Ramaniah, M. V., "Isotopic Yield Distribution of Iodine in the Reactor Neutron Induced Fission of 241Am," BARC-1381 pp. 47-50 (1987).

87RED4 Reddy, A. V. R., Deshmukh, S. M., Burte, P. P., Manohar, S. B., Prakash, S., Ramaniah, M. V., "Isotopic Yield Distribution of Iodine in the Thermal Neutron Induced Fission of $245 \mathrm{Cm}, "$ Radiochemistry and Radiation Chemistry Symposium (held at) Tirupati during (13-17 Dec 1986). p. 98-100. [IN of 131-134I; 131-134Te rel. to $135 \mathrm{I}$ assumed to be $11.4 \pm 2.5 \%$ ]. No data given in abstract.

87RID1 Rider, B. F., England, T. R., "Evaluation of Fission Product Yields for the U.S.A. National Nuclear Data Files," LA-UR-87 -2462. Paper presented at The Specialists Meeting on Data for Decay Heat Predictions, Studsvik, Sweden, September 7-10, 1987. Current evaluated chain yields given for twenty fissioning systems: U235T, U235F, U235HE, U238F, U238HE, PU239T, PU239F, PU241T, U233T, TH232F, U233F, U233HE, U236F, PU239H, PU240F, PU241F, PU242F, TH232H, NP237F and CF252S. This is an update of 81RID1 but with over 3200 new fission yield measurements published since 1981 included in the data base of 28400 published fission yield measurements from over 1371 literature publications. Over 110,000 individual independent and cumulative fission yields have been evaluated for ENDF/B-VI, which is scheduled to be released the last half of 1988. This paper (87RID1) appears in NEACRP-302'L' also known as NEANDC-245'U' pp. 21-36 (1987). Obtainable from NEA Data Bank, F91191 Gif-Sur-Yvette Cedex, France. INIS Atomindex 19(15): 061890 (1 Aug 1988).

87RID2 Rider, B. F., Estimated cumulative yield for CM243T and CM245T as suggested by 81 GIN3. This is in agreement with earlier observations that the average mass of the heavy mass group is nearly constant for low energy fissioning systems in the region from $\mathrm{U}$ through $\mathrm{Cm}$; Whereas, the average mass of the light mass group changes with the fissioning system (73UNI1). 
87SRI1 Srivastava, A., Nair, A. G. C., Goswami, A., Srivastava, B. K., Manohar, S. B., Prakash, S., Ramaniah, M. V., "Fragment Shell Effect in Low Energy Fission: Measurement of Isotopic Yield of Technetium Isotopes in the Thermal Neutron Induced Fission of 238Pu," BARC-1381 pp. 51-56 (1987).

87SRI2 Srivastava, A., Srivastava, B. K., Nair, A. G. C., Manohar, S. B., Prakash, S., Ramaniah, M. V., "Fractional Cumulative Yields of $140 \mathrm{Ba}$ and 142Ba, 146Ce in Spontaneous Fission of 252Cf," BARC-1381 pp. 62-62 (1987).

87TOM1 Tomar, B. S., Goswami, A., Das, S. K., Datta, T., Prakash, S., Ramaniah, M. V., BARC-1381, pp. 7-15 (1987). INIS Atomindex 19(20): 087215 (1988).

87TOM2 Tomar, B. S., Naik, H., Ramaswami, A., Prakash, S., Ramaniah, M. V., "Cumulative Yields of Rare-Earth Fission Products in the Spontaneous Fission of 252Cf," BARC-1381 pp. 59-61 (1987).

87WAH1 Wahl, A. C., "Nuclear-Charge Distribution and Delayed-Neutron Yields for ThermalNeutron-Induced Fission of $235 \mathrm{U}, 233 \mathrm{U}$, and 238Pu and for Spontaneous Fission of 252Cf," Department of Chemistry, Washington University, St. Louis, Missouri, 63130, USA. To be published. The data in this article are both more extensive and critically evaluated for the restricted set of four fission reactions than in earlier compilations. The systematics used for estimation of unmeasured independent yields for these and other compilations were not as fully developed as those presented here; and systematics near symmetry which have been determined for U235T only recently, differ significantly from those estimated earlier. The complete independent yield sets derived from earlier compilations and systematics do not achieve detailed charge balance for complementary elements. All data for both light and heavy products are treated together globally by least squares calculations for both $\mathrm{Zp}$ and A'p models. The A'p model offers a mathematical alternative, the sums of Gaussian functions representing charge distributions, to graphical procedures for interpolating and extrapolating $Y(A)$ values. This alternative procedure was used to derive the $\mathrm{Y}(\mathrm{A})$ set for CF252S. See 88WAH1 for publication.

87WAH2 Wahl, A. C., "Nuclear-Charge Distribution and Delayed-Neutron Yields for ThermalNeutron-Induced Fission of 235U, 233U, 239Pu, and 241Pu and for Fast-NeutronInduced Fission of 238U," Paper delivered at The Specialists Meeting on Data for Decay Heat Predictions, Studsvik, Sweden September 7-10, 1987. NEACRP-302'L' also called NEANDC-245'U' pp. 9-19 (1987). obtainable from NEA Data Bank, F91191 Gif-Sur-Yvette Cedex, France.

87WAN1 Wang Dao, Zhang Dongming, "Chinese Evaluated Fission Product Data Library 1987 (Preliminary)," IAEA-NDS-91(rev0) Oct 198732 p. INIS Atomindex 22(9):030810 (1 May 1991). Pu239, Pu241 Fission Yields.

88AFA1 Afarideh, H., Thesis, University of Birmingham (England) 1988. K. Randle Supervisor. "Investigation of Binary and Ternary Fission in $238 \mathrm{U}$ Induced by Monoenergetic Neutrons," pp. 987-990 in: Igarashi, S. (ed.) Japan Atomic Energy Research Inst., Tokyo (Japan) [30 May - 3 June 1988].Absolute yields heave been determined for 34 binary fission products representing 29 mass chains created during fission of $238 \mathrm{U}$ with monoenergetic neutrons. Neutron energies used for fission ranged from $1.75 \mathrm{MeV}$ to $5.8 \mathrm{MeV}$. Radiochemical measurements with $\mathrm{Ge}$ detector by direct counting of irrad. $238 \mathrm{U}$ foil. [Data]. These values are also found 
listed in 89MIL2 at 1.72, 2.16, 4.78, and $5.98 \mathrm{MeV}$. We pool 1.72 and $2.16 \mathrm{MeV}$ values with U238F. INIS Atomindex 20(14):048017 (15 July 1989). Published Ann. Nucl. Energy, (1989) v16(7) p. 313-325. INIS Atomindex 21(4): 013150 (15 Feb 1990).

88BHA1 Bhargava, V. K., Oak, M. S., Prakash, S., "Absolute Yields of Fission Products 99Mo and 132Te in the Spontaneous Fission of 252Cf," (Preprint No. NR-7) Radiochemistry and Radiation Chemistry Symposium held at Bombay on Feb 22-26, 1988. INIS Atomindex 20(11): 036376 (1 June 1989). [Data] RCCU 99Mo and 132Te in Cf252S are 2.70土0.15 and $1.88 \pm 0.17$, respectively. Later published in Radiochim. Acta 46(4), 177-179 (1989). INIS Atomindex 20(16):055032 (15 August 1989).

88HAD1 Haddad, M., Asghar, M., Crancon, J., Lhospice, G., "Post Neutron Mass Distribution for Pu238 (N (th), F)," Nucl. Phys. A 481(2), 333-339 (2 May 1988). INIS Atomindex 19(16): 065725 (15 Aug 1988). Fifty one fission yields in 36 chains by gamma spectrometry. [DATA].

88HOR1 Horner, St., Denschlag, H. O., Gabelmann, H., Kratz, K. L., Pfeiffer, B., Stohlker, U., Private Communication to M. F. James and R. W. Mills from Institut fur Kernchemie (1988).

88LAM1 Lammer, M., "Fission Yield Evaluation," INDC(NDS)-208/G+P (September 1988). Summary Report of a Specialists' Meeting organized by the International Atomic Energy Agency, held at Studsvik, Sweden, on September 11, 14 and 15 1987. Prepared by Meinhart Lammer, IAEA Nuclear Data Section, Wagramerstrasse 5, A-1400 Vienna. [No Data].

88LEE1 Lee, C., Chang, C., Chen, C., Yu, Y., "Computerized On-line Measurement of the Yield of Short-lived Bromine and Iodine Isotopes from Thermal Neutron Fission of 235U," J. Radioanalytical Chem Articles, 123(2) 607-611 (Aug 1988). INIS Atomindex 20(9):028534 (1 May 1989). Independent yields of $86 \mathrm{Br}, 134 \mathrm{~m}-\mathrm{I}, 134 \mathrm{~g}-\mathrm{I}, 236 \mathrm{~m}-\mathrm{I} 136 \mathrm{~g}-\mathrm{I}$, the cumulative yields of $87 \mathrm{Br}, 88 \mathrm{Br}, 137 \mathrm{I}, 138 \mathrm{I}$, and isomeric yield ratio of $134 \mathrm{I} / 136 \mathrm{I}$ determined.

88QUA1 Quade, U., Rudolph, K., Skorka, S., Armbruster, P., Clerc, H. G., Lange, W., Metterer, M., Schmitt, C., Theobald, J. P., Goennenwein, F., Pannicke, J., Schrader, H., Siegert, G., Englehardt, D., "Nuclide Yields of Light Fission Products from Thermal-Neutron Induced Fission of 233U at Different Kinetic Energies," Nucl. Phys. A487(1), 1-36 (3 Oct 1988). INIS Atomindex 20(2): 005144 (15 Jan 1989). Journal publication of 83QUA1 Thesis. OddEven proton effect is $22.1 \pm 2.1 \%$. Odd-Even neutron effect is $5.4 \pm 1.7 \%$.

88RAM1 Ramaswami, A., Manohar, S. B., Gubbi, G. K., Rattan, S. S., Singh, R. J., Chakravarty, N., Prakash, S., "Nuclear Charge Distribution in the Spontaneous Fission of 252Cf," (Preprint No. NR-6) Radiochemistry and Radiation Chemistry Symposium held at Bombay on (22-26 Feb 1988). INIS Atomindex 20(11): 036379 (1 June 1989). [Data]. RCFC 140,141,142-Ba in Cf252S.

88S0L1 Solonkin, A. A., Teplykh, V. F., Platygina, E. V., Petrzhak, K. A., Mosesov, A. V., "Fission Product Yields in 238Np Thermal Fission," At. Ehnerg. 64(6), 435-439 (Jun 1988). Relative cumulative fission yields of 23 products $A=131-155$ in thermal fission of short-live odd-odd $238 \mathrm{~Np}$ nuclide are determined using mass spectrometric method. Heavy peak plot. Absolute yields of heavy products are evaluated. INIS Atomindex 20(9):028532 (1 May 1989).

88WAH1 Wahl, A. C., "Nuclear-Charge Distribution and Delayed-Neutron Yields for ThermalNeutron-Induced Fission of 235U, 233U, and 239Pu and for Spontaneous Fission 
of 252Cf," Atomic Data and Nuclear Data Tables Vol. 39 No. 1, 1-156 (May 1988). The publication of 87WAH1. INIS Atomindex 19(19): 081572 (1 Oct 1988). Yields of individual isomers are not included. Because decay heat calculations require yields of both short and long lived isomers, yields for individual isomers are computed for ENDF/B-VI from Wahl's sum of isomers according to the model of 77MAD2.

89BHA1 Bhargava, V. K., Oak, M. S., Ramaswami, A., Prakash, S., ."Absolute Yields of 99Mo and 132Te in Spontaneous Fission of 252Cf," Radiochim. Acta, 46(4), 177-179 (1989). INIS Atomindex 20:055032 (15 Aug 1989). [CU of Mo99 = 2.70\%; CU of Te132 = 1.88\% by track etch cum gamma ray spectrometry].

89BRA1 Brady, M. C., England, T. R., "Delayed Neutron Data and Group Parameters for 43 Fissioning Systems," Nucl. Sci. and Eng. 103, 129-149 (1989).

89DJE1 Djebara, M., Asghar, M., Bocquet, J. P., Brissot, R., Crancon, J., Ristori, C., Aker, E., Englehardt, D., Gindler, J., Wilkins, B. D., Quade, U., Rudolf, K., "Mass and Nuclear-Charge Yields for Cf249 (N Sub(th), F) at Different Fission Product Kinetic Energies," Nucl. Phỳs. A496(2), 346-366 (29 May 1989). [Data]. INIS Atomindex 20(20):067686 (15 Oct 1989).

89ENG1 England, T. R., Rider, B. F., “Evaluation and Compilation of Fission Product Yields," ENDF-349 (1989). Documentation of fission product yields for the preliminary Evaluated Nuclear Data File (ENDF/B-VI). (Early draft of this report for IAEA CRP Committee-first release of ENDF/B-VI.)

89ENG2 England, T. R., Rider, B. F., Katakura, J. -I., "Summary Information for Yields and Fission Product and Actinide Subcommittees," Cross Section Evaluation Working Group (CSEWG) Meeting, April 3-6, 1989.

89HAD1 Haddad, M., Crancon, J., Lhospice, G., Asghar, M., "Post-Neutron Mass Distribution for $232 U$ (n th, f)," Radiochimica Acta 46, 23-24 (1989). 36 fission products in 27 chains produced by thermal neutron fission of $232 \mathrm{U}$ by gamma ray spectroscopy. INIS Atomindex 20(7): 021395 (1 Apr 1989).

89MAN1 Manohar, S. B., Ramaswami, A., Srivastava, B. K., Reddy, A. V. R., Nair, A. G. C., Gubbi, G. K., Srivastava, A., Prakash, S., "Nuclear Charge Distribution in the Spontaneous Fission of 252Cf," Nucl. Phys. A502 (9 Oct 1989) 307c-314c. INIS Atomindex 21: 009400 (1 Feb 1990). [FC 108,109Ru; 137,138,139Xe; 139,140,141,142Ba; 146Ce].

89MIL1 Mills, R. W., James, M. F., Weaver, D. R., "The Production of a New Evaluation of Fission Products," Paper presented at the Conference on 50 Years with Nuclear Fission, Washington, D. C. April 28, 1989.

89MIL2 Mills, R. W., James, M. F., Weaver, D. R., "New Fission Product Yield Evaluation: Chain Yields," CNDC(89)P34, Address James at: UKAEA, AEE Winfrith, Dorchester, Dorset DT2 8DH, England; Mills or Weaver at: Department of Physics, Birmingham University, PO Box 363, Birmingham, B15 2TT, England.

89NAI1 Nair, A. G. C., Reddy, A. V. R., Ramaswami, A., Prakash, Satya, "Cumulative Yields of Short-lived Xenon Isotopes in the Spontaneous Fission of 252Cf," (Preprint No. 
NR-07) Bhabha Atomic Research Center, Bombay. Jan 1989, 2p. from Symposium on Radiochemistry and Radiation Chemistry, Kalpakkam (India) (4-7 Jan 1989). INIS Atomindex 21(8):030295 (15 Apr 1990). See 90NAI1.

89RID1 Rider, B. F., Value estimated by interpolation or extrapolation by BFR, for instance, where no data is found in the data base.

89RUD1 Rudstam, G., Ekstrom, B., Lund, E., "Independent Yield Pattern in Thermal NeutronInduced Fission of 235U," Uppsala Univ. The Studsvik Neutron Research Laboratory S-611 82 Nykoping Sweden. Contribution to "Fifty Years with Nuclear Fission," April 25-28, 1989 held at Gaithersburg, MD, USA. Graphic not digital data given (not coded). See 89RUD2.

89RUD2 Rudstam, G., Aagaard, B., Ekstrom, B., Lund, E., Gokturk, H., and Zwicky, H. U., "Yields of Products from Thermal-Neutron Induced Fission of $235 U$," Internal Report (1989). Univ. of Uppsala, The Studsvik Neutron Research Laboratory, S-611 82 Nykoping, Sweden. Submitted to Radiochimica Acta for publication in 1990. Measured 280 independent and cumulative yields, for 191 nuclides, 68 of which have never been measured before. Supersedes 85RUD1, 86RUD1.

89SOL1 Solonkin, A. A., Teplykh, V. F., Platygina, E. V., Petrzhak, K. A., Mosesov, A. V., “Thermal Fission of Short Lived Odd-Odd 238Np Nuclide," At. Ehnerg. 64(6) 435-439 (Jun 1988). Using mass spectrometer method. Heavy peak plot; absolute yields of heavy product peak are evaluated. INIS Atomindex 20(9):028532 (1 May 1989).

89SRI1 Srivastava, A., Denschlag, H. O., "Nuclear Charge Distribution in the Reactor Neutron Induced Fission of 232Th: Fractional Cumulative Yields of the Isotopes of Krypton and Xenon," Radiochim. Acta, 46(1), 17-21, (1989). (91,92,93Kr; 139,140,141, 142,143Xe). INIS Atomindex 20(7): 021394 (1 Apr 1989).

89SRI2 Srivastava, A., Denschlag, H. O., Ditz, W., Guettler, U., Stumpf, P., "Isomeric Yield Ratio of $97 \mathrm{Y}$ in 239Pu (n-th,f) at Different Kinetic Energies of the Fission Fragments," Preprint No. NR-02. Symposium on Radiochemistry and Radiation Chemistry. Kalpakkam (India) (4-7 Jan 1989) 2 p. INIS Atomindex 21(8):030296 (15 Apr 1990).

89TSU1 Tsukada, K., Sueki, K., Ohtsuki, T., Kobayashi, T., Nishinaka, I., Nakahara, H., Shinohara, N., Ichikawa, S., Iimura, H., "Measurement of the Fission Yields of the Heavy Rare Earth Elements Using an Automatic Separation System," The 1989 International Chemical Congress of Pacific Basin Societies Abstracts Pt I \& II. Washington DC(USA) ACS 1989. 1700 p. (17-22 Dec 1989). Cf252S Yields. INIS Atomindex 22(13):045749 (1 Jul 1991).

89WAH1 Wahl, A. C., "Nuclear-Charge and Mass Distribution from Fission," Washington University, St. Louis, MO 63130 U.S.A. Telephone (314)-889-6578. A Paper presented at the Conference on 50 Years with Nuclear Fission, Washington, D. C. April 28, 1989. Additional Zp and Sigma values for the first 50 fissioning systems in this work were privately communicated to T. R. England (Sept 1989).

89WAL1 Walker, F. W., Parrington, J. R., Feiner, F., General Electric Chart of the Nuclides, 14th Edition (November 1989). 
90BOC1 Bocquet, J. P., Faust, H. R., Brissot, R., Fowler, M., Wilhelmy, J., Asghar, M., Djebara, M., "Characteristics of Mass and Nuclear Charge distribution of 228Th (n sub th, f): Implications for Fission Dynamics," Z. Phys. A.: Atomic Nuclei, 335(1), 41-48 (Jan 1990). INIS Atomindex 21: 013155 (15 Feb 1990).

90BUR1 Burrows, T., National Nuclear Data Center, Brookhaven National Laboratory. Private communication to T. R. England of MIRD2 (Medical Isotopes Radioactive Decay File-2) (Jan 23, 1990). The isotopes covered are largely longer half lived nuclides decaying by electron conversion, internal transition, positron emission or beta decay near the stable valley or on the neutron rich side of the stable valley.

90CHI1 Chien Chung, "Measurement and Evaluation of some Low-Energy Fission Yields," J. Radioanal. Nucl. Chem. Articles 142(1) 254 -264 (Sep 1990). INIS Atomindex 22(14): 049605 (15 Jul 1991). Pu239 Fission Yields.

90CHU1 Chung Chien, Huang Kweilon, "Cumulative Yields in the Thermal-Neutron Fission of 232U," Radiochim. Acta, 49(3), pp 113-117 (1990). INIS Atomindex 21: 056921 (1 Aug 19S0). Tsing Hua Univ., Hsinchu (Taiwan). Twelve CU in U232T measured for first time by direct gamma ray spectrometry.

90ENG1 England, T. R., Wilson, W. B., Rider, B. F., Reich, C. W., Mann, F. M., Schenter, R. E, Brady, M. C., Katakura, J., "Activation, Actinide, and Fission-Product Yields and Decay Data," Nuclear Theory and Applications Progress Report for the period January $\mathbf{1}$, 1989- April 1, 1990. pp. 96-113. Issued (December 1990).

90ENG2 England, T. R., Rider, B. F., Brady, M. C., "Fission Product Chain Yields and Delayed Neutrons: ANS Standards 5.2 and 5.8," Trans. Am. Nucl. Soc. 62, 529 (Nov 1990).

90HEN1 Hentzchel, R., Denschlag, H. O., "Isomeric Yield Ratios of 134I and 1361 and Indpendent Fractional Yields of Some Halogen Isotopes in the Fission of 232Th witl Reactor Neutrons," Radiochim. Acta, 50(1/2), p 1-4 (1990). Mainz Univ. (Germany). INis Atomindex 21: 081599 (1 Nov 1990). FI of $85 \mathrm{Br}=4.7 \%$; FI of $86 \mathrm{Br}=18.4 \%$; FI of $134 \mathrm{I}=$ $1.6 \%$; FI of $135 \mathrm{I}=16.2 \%$; FI of $136 \mathrm{I}=33.4 \%$.

90IYE1 Iyer, R. H., “Highly Asymmetric Binary Fission,”. J. Radioanal. Nucl. Chem. Artides 142(1) 265-277 (Sep 1990). U238 Fission Yields.

90LEM1 Lemmel, H. D., "JEF-1 Fission Product Yield Data," IAEA-NDS-123(rev0) Sept 1999 MF available from INIS. INIS Atomindex 22(9): 030807 (1 May 1991) 7 p. Final Data from ENDF/B-5 June 1985. Nuclear Data Collection.

90NAI1 Nair, A. G. C., Reddy, A. V. R., Ramaswami, A., Prakash, S., "Cumulative Yields of Shortlived Xenon Isotopes in the Spontaneous Fission of $252 \mathrm{Cf}$," J. Radioanal. Nucl. Chem. Articles 140(1) 215-218 (May 1990). INIS Atomindex 21(24):092441 (15 Dec 1990). Cf2525

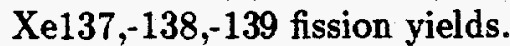

90OAK1 Oak, M. S., Ramaswami, A., Prakash, S. "Cumulative Yields of Short-lived Rare Earth Fission Products in the Spontaneous Fission of 252Cf," Paper No. NC-05 (Feb 1990). Symposium on Radiochemistry and Radiation Chemistry. Nagpur (India) (5-8 Feb 1990). INFS Atomindex 22(15): 054211 (1 Aug 1990). See 91RAM1 for Publication. 
90RAM1 Ramaswami, A., Rattan, S. S., Singh, R. J., Prakash, S., “Charge Distribution Study in the Thermal Induced Fission of 241-Pu: Fractional Yields of 134Te, 135I, 138Xe and 140Ba," (Paper No. NC-06) Symposium on Radiochemistry and Radiation Chemistry, Napur (India) (5-8 Feb 1990). INIS Atomindex 22(15): 054210 (1 Aug 1991).

90RUD1 Rudstam, G., Aagaard, P., Ekstrom, B., Lund, E., Gokturk, H., Zwicky, H. U., "Yields of Products from Thermal-Neutron-Induced Fission of $235 U$," Radiochimica Acta 49(4), p 155-191 (1990). The Studsvik Neutron Research Laboratory, S-61182 Nykoping, Sweden. INIS Atomindex 21: 081598 (1 Nov 1990).

90RUD2 Rudstam, G. Private communication to T. R. England of additional cumulative yields in U235 thermal fission made since 90RUD1 in cases where branching ratios have become available. These are Ge84 $=(0.034 \pm 0.009) \%$, Sr101 $=(0.0044 \pm 0.0016) \%, \operatorname{Ag} 123=(0.00055 \pm 0.00041) \%$, $\mathrm{Cd} 123 \mathrm{~m}=(0.0078 \pm 0.0033) \%, \mathrm{Cd} 123=(0.0037 \pm 0.0033) \%, \mathrm{Cd} 125 \mathrm{~m}=(0.0102 \pm 0.0033) \%, \mathrm{Cd} 125$ $=(0.0053 \pm 0.0024) \%, \operatorname{Sn} 134=(0.018 \pm 0.011)$. The presence of isomeric states in $\mathrm{Cd} 123$ and Cd125 was not known earlier. Great change is made in 2 yields because of newly available branching ratios: $\mathrm{As} 85=(0.220) \%, \mathrm{Ag} 113 \mathrm{~m}=(0.0050 \pm 0.0016) \%$. The independent yield is nearly the same since parent contribution is small.

90RUD3 Rudstam, G., England, T. R., “Test of Pre-ENDF/B-VI Decay Data and Fission Yields," LA-11909-MS (Oct 1990).

90SUN1 Sun Tongyu, Li Wenxin, Fu Min, Thao Lili, "Mass Distribution in the 14.7 MeV Neutron Induced Fission of 237Np," CNIC-1-004 (Jun 1990). 138 p. Available from INIS. Summary form only. INIS Atomindex 21(22): 085840 (15 Nov 1990).

91BRA1 Brady, M. C., Wright, R. Q., England, T. R., “Actinide Nuclear Data for Reactor Physics Calculations," ORNL/CSD/TM-266 (July 1991).

91HEN1 Hentzschel, R., Denschlag, H. O., Faust, H. R., Gindler, J. E., Wilkins, B., "Mass Yields with $A=69$ to 87 in the Fission of 249-Cf by Thermal Neutrons," Progress Report on Nuclear Data Research in the Federal Republic of Germany, NEANDC(E)-322-U-Vol. V (1991).

91HEN2 Hentzschel, R., Faust, H. R., Denschlag, H. O., Wilkins, B. D., Gindler, J., "Mass Yields in the Very Asymmetric Fission of 249-Cf(nth,f)," Proceedings of the International Conference on Nuclear Data for Science and Technology, Juelich, (May 1991). In Print.

91JAM1 James, M. F., Mills, R. W., Weaver, D. R., "A New Evaluation of Fission Product Yields and Production of a New Library (UKFY2) of Independent and Cumulative Yields. Part I. Methods and Outline of the Evaluation," AEA-TRS-1015 (1991).

91JAM2 James, M. F., Mills, R. W., Weaver, D. R., “A New Evaluation of Fission Product Yields and Production of a New Library (UKFY2) of Independent and Cumulative Yields. Part II. Tables of Measured and Recommended Fission Yields," AEA-TRS1018 (1991).

91JAM3 James, M. F., Mills, R. W., Weaver, D. R., "A New Evaluation of Fission Product Yields and Production of a New Library (UKFY2) of Independent and Cumulative Yields. Part III. Tables of Fission Yields with Discrepant or Sparse Data," AEA-TRS-1018 (1991). 
91KAT1 Katakura, J., and England, T. R., "A ugmentation of ENDF / B Fission Product GammaRay Spectra by Calculated Spectra," Los Alamos National Laboratory report LA-12125MS [ENDF-352], (November, 1991)

91LAM1 Lammer, M., “UKFY2, The Fission Product Yield Library, Version 2, 1991, Summary Documentation," IAEA-NDS-124 Rev. 0 (April 1991). 12 p. MF available INIS. In ENDF/B-VI Format, adopted for JEF-2 file. Available on magnetic tape costfree from International Atomic Energy Agency, Nuclear Section, P. O. Box 100, A-1400 Vienna, Austria. Includes 21 fissioning species INIS Atomindex 22(16):058683 (15 Aug 1991).

91LAM2 Lammer, M., Schwerer, O., "Handbook of Nuclear Data for Safeguards," INDC(NDS)248 June 1991. Nuclear Data Section, International Atomic Energy Agency, Vienna, Austria.

91MIL1 Mills, R. W., James, M. F., Weaver, D. R., "Study of the Delayed Neutron Yield and its Time Dependence by the Summation Method and the Sensitivity of the Yield to Parameters of the Independent Yield Model and the Decay Data," Proc. Conference on Nuclear Data for Bașic and Applied Science, May 1991, Juelich, Germany. (to be published).

91RAM1 Ramaswami, A., Oak, M. S., Prakash, S., "Cumulative Yields of Short-lived Rare Earth Products in the Spontaneous Fission of 252Cf," Radiochem. Acta 54(4) 163-164 (1991). 10 Fission Product with half lives from $40 \mathrm{sec}$. to a few minutes relative to $\mathrm{Nd}-149$ yield, most for the first time. INIS Atomindex 22(24):087862 (15 Dec 1991).

91ROS1 Rose, P. F., Dunford, C. L., "Data Formats and Procedures for the Evaluated Nuclear Data File ENDF/B-VI," ENDF-102 BNL-NCS- 44945 Revised (October 1991).

91RUD1 Rudstam, G., "Isomeric Yields in Fission," Research Report Draft (Sept 1991). University of Uppsala. The Studsvik Neutron Research Laboratory, S-611 82 Nykoping, Sweden.

91RUD2 Rudstam, G., "Pn-Values and Half Lives of Delayed-Neutron Precursors in the Fission Product Region," NFL-70/Rev. (1991).

92HEN1 Hentzschel, R., Doctoral Thesis, Univ. Mainz (1992).

92JAM1 James, M. F., Mills, R. W., Weaver, D. R., "The Use of the Normalized Residual in Averaging Experimental Data and in treating Outliers," AEA-RS-1082 (Jan 1992). AEA Reactor Services, Winfrith Technology Centre, Dorchester, Dorset DT2 8HD, U.K.

92JAM2 James, M. F., Mills, R. W., Weaver, D. R., "A New Evaluation of Fission Product Yields and the Production of a New Library (UKFY2) of Independent and Cumulative Yields," Progress in Nuclear Energy, Vol 26, No. 1, pp 1-29 (1991). Pergamon Press.

92ENG1 England, T. R., Rider, B. F., "ENDF/B Yields Evaluation for 1992: Methods and Content," and also "Yield Validation: Integral Comparisons," Specialists Meeting on Fission Product Nuclear Data, Tokai Research Establishment, Japan Atomic Energy Institute, Tokai-Mura, Japan. (May 25-27, 1992). NEA1/NSC1/DOC(92)9

92ENG2 England, T. R., Brady, M. C., Rider, B. F., "Recent Advances in the U. S. Fission Yield and Delayed Neutron Evaluations," Submitted to American Nuclear Society Transactions 1992. 
92ENG3 England, T. R., Katakura, J., Mann, F. M., Reich, C. W., and Wilson, W. B., "Decay Data Evalution for ENDF/B-VI," International Symposium on Nuclear Data Evaluation Methodology, Brookhaven National Laboratory, Upton, NY, USA, Ed. Charles L. Dunford, World Scientific, 12-16 October 1992 [LA-UR-92-3785]

93ENG1 England, T. R., and Rider, B. F., "Evaluation and Compilation of Fission Product Yields, 1993," ENDF-349, LA-UR-94-3106. Final document September, 1994 [This document will serve as the primary documentation for ENDF/B-VI yields.] 